\title{
The scattering of spinning hadrons from lattice QCD
}

\author{
Antoni James Woss \\ St. John's College

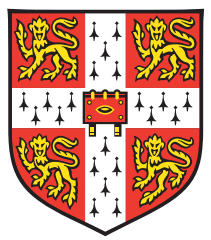 \\ University of Cambridge \\ Department of Applied Mathematics and Theoretical Physics \\ Faculty of Mathematics
}

Supervisor: Dr. Christopher Thomas

This thesis is submitted for the degree of

Doctor of Philosophy

July 2019 



\section{Declaration}

This thesis is the result of my own work and includes nothing which is the outcome of work done in collaboration except as declared in the Preface and specified in the text. It is not substantially the same as any that I have submitted, or, is being concurrently submitted for a degree or diploma or other qualification at the University of Cambridge or any other University or similar institution except as declared in the Preface and specified in the text. I further state that no substantial part of my thesis has already been submitted, or, is being concurrently submitted for any such degree, diploma or other qualification at the University of Cambridge or any other University or similar institution except as declared in the Preface and specified in the text. It does not exceed the prescribed word limit for the relevant Degree Committee.

Chapters 3 and 5 are based on the following works respectively:

- Woss, Antoni J. and Thomas, Christopher E. and Dudek, Jozef J. and Edwards, Robert G. and Wilson, David J., "Dynamically-coupled partial-waves in $\rho \pi$ isospin-2 scattering from lattice QCD", JHEP 07 (2018) 043, [arXiv:1802.05580]

- Woss, Antoni J. and Thomas, Christopher E. and Dudek, Jozef J. and Edwards, Robert G. and Wilson, David J., "The $b_{1}$ resonance in coupled $\pi \omega, \pi \phi$ scattering from lattice QCD", Phys.Rev. D100 (2019) no.5, 054506, [arXiv:1904.04136] 



\section{Acknowledgements}

First and foremost, I would like to thank the support of my supervisor, Christopher Thomas. Through your dedication, patience and teaching, I have thoroughly enjoyed an interesting and productive $\mathrm{PhD}$ programme. I would like to extend a huge thanks to David Wilson, Jo Dudek and Robert Edwards for insightful and extensive discussions. In particular, I would like to thank Jo Dudek for helping polish a number of the figures that appear in the works listed in the Preface and subsequently here in Chapters 3 and 5 . In addition, I would like to thank Raúl Briceño, Max Hansen, Mike Peardon, Ron Horgan, Luka Leskovec, Kostas Orginos, Sinéad Ryan, Graham Moir and Bipasha Chakraborty who I have had the pleasure of working alongside. Also, to my fellow lattice cohort and office mates, past and present, Gavin Cheung, Judd Harrison, Alex Abbott, Laurence Cooper, James Delaney, Ciaran Hughes, Roland Bittleston and Jack Williams, it has been a pleasure. To my family and fiancée Jessica, for your love, support and encouragement, I owe enormous gratitude.

I would like to thank the U.K. Science and Technology Facilities Council (STFC) and St. John's College, University of Cambridge, for financial support. This work has been partially supported by STFC consolidated grant ST/P000681/1.

Part of this research was performed using resources provided by the Cambridge Service for Data Driven Discovery (CSD3) operated by the University of Cambridge Research Computing Service (www.csd3.cam.ac.uk), provided by Dell EMC and Intel using Tier-2 funding from the Engineering and Physical Sciences Research Council (capital grant EP/P020259/1), and DiRAC funding from the Science and Technology Facilities Council (www.dirac.ac.uk). Part of this research was performed using the Cambridge Service for Data Driven Discovery (CSD3), part of which is operated by the University of Cambridge 
Research Computing on behalf of the STFC DiRAC HPC Facility (www.dirac.ac.uk). The DiRAC component of CSD3 was funded by BEIS capital funding via STFC capital grants $\mathrm{ST} / \mathrm{P} 002307 / 1$ and ST/R002452/1 and STFC operations grant ST/R00689X/1. DiRAC is part of the National e-Infrastructure. Part of this research was performed using the Darwin Supercomputer of the University of Cambridge High Performance Computing Service (www.hpc.cam.ac.uk), provided by Dell Inc. using Strategic Research Infrastructure Funding from the Higher Education Funding Council for England and funding from the Science and Technology Facilities Council. This research was also performed on clusters at Jefferson Lab under the USQCD Collaboration and the LQCD ARRA Project. I acknowledge support from the U.S. Department of Energy, Office of Science, Office of Advanced Scientific Computing Research and Office of Nuclear Physics, Scientific Discovery through Advanced Computing (SciDAC) program. Also acknowledged is support from the U.S. Department of Energy Exascale Computing Project. This research was supported in part under an ALCC award, and used resources of the Oak Ridge Leadership Computing Facility at the Oak Ridge National Laboratory, which is supported by the Office of Science of the U.S. Department of Energy under Contract No. DE-AC05-00OR22725. The software codes Chroma [1] and QUDA 2, 3] were used to compute the propagators required for this project. This research used resources of the National Energy Research Scientific Computing Center (NERSC), a DOE Office of Science User Facility supported by the Office of Science of the U.S. Department of Energy under Contract No. DEAC02-05CH11231. I acknowledge the Texas Advanced Computing Center (TACC) at The University of Texas at Austin for providing HPC resources that have contributed to the research results reported within this thesis. Gauge configurations were generated using resources awarded from the U.S. Department of Energy INCITE program at the Oak Ridge Leadership Computing Facility, the NERSC, the NSF Teragrid at the TACC and the Pittsburgh Supercomputer Center, as well as at Jefferson Lab. 


\section{Abstract}

Hadron spectroscopy is predominantly the study of resonances that decay via the strong interaction into a multitude of stable hadrons, such as the pion. The vast majority of resonances decay via an intermediate hadron with non-zero intrinsic spin. In this thesis, I will present the results of scattering calculations featuring mesons with non-zero intrinsic spin. Before doing so, I will first give a brief introduction to QCD and review the framework necessary to perform lattice QCD calculations in Chapters 1 and 2.

In Chapter 3, I present the first lattice calculation of $\rho \pi$ scattering in isospin-2. Here, $\rho \pi$ features in dynamically-coupled ${ }^{3} S_{1}$ and ${ }^{3} D_{1}$ partial-waves with $J^{P}=1^{+}$. No resonance enhancement is anticipated in the flavour exotic isospin-2 channel and as such it provides an ideal testing ground for this first calculation. I work at heavier than physical quark masses at the $\mathrm{SU}(3)_{\mathrm{F}}$ point where the up, down and strange quarks are mass degenerate. Finite-volume spectra are calculated and, utilising the relationship between the discrete energy spectrum and the infinite-volume scattering amplitudes, partial-wave amplitudes with $J \leq 3$ and the degree of dynamical mixing between the coupled ${ }^{3} S_{1}$ and ${ }^{3} D_{1}$ channels are determined.

In Chapter 4, I investigate $\rho \pi$ in isospin-1 where the $a_{1}$ axial-vector resonance is expected to feature. Here, I present a discussion on $G$-parity and Bose-symmetry at the $\mathrm{SU}(3)_{\mathrm{F}}$ point. Working at heavier than physical quark masses, the resulting finite volume spectrum suggests that the $a_{1}$ is a bound-state and that the ${ }^{3} S_{1^{-}}$and ${ }^{3} D_{1}$-wave, $\rho \pi$ scattering amplitudes are similar to those in isospin-2.

I present the first calculation of coupled $\pi \omega$ and $\pi \phi$ scattering in Chapter 5 where resonant enhancement is seen experimentally in the $J^{P}=1^{+}$channel. Working at a somewhat lighter pion mass than in previous chapters, the finite-volume spectra are determined and the scattering amplitudes are calculated. Analytically continuing the amplitudes into the complex energy plane, a resonance pole is found, interpreted as the analogue of the $b_{1}$ axial-vector, which couples dominantly to ${ }^{3} S_{1}$-wave $\pi \omega$, with a 
much-suppressed coupling to ${ }^{3} D_{1}$-wave $\pi \omega$, and a negligible coupling to $\pi \phi$.

In Chapter 6, the exotic $J^{P C}=1^{-+}$channel is studied. These quantum numbers are not allowed in the quark model but can be obtained, for example, through a gluonic excitation coupled to a quark-antiquark pair. In this exploratory calculation, performed at the $\mathrm{SU}(3)_{\mathrm{F}}$ point, the finite-volume spectra and coupled-channel scattering amplitudes are presented. A single resonance pole is found, interpreted as the exotic $\pi_{1}$, and couplings to meson-meson channels, including for example $\pi \eta\left\{{ }^{1} P_{1}\right\}, \pi \eta^{\prime}\left\{{ }^{1} P_{1}\right\}$ and $\rho \pi\left\{{ }^{3} P_{1}\right\}$, are calculated for the first time in lattice QCD.

In order to minimally present the contents of a unitary $n$-channel scattering matrix, I introduce, in Chapter 7, an $n$-channel generalisation of the traditional two-channel Stapp parameterisation. 


\section{Contents}

Declaration iii

\begin{tabular}{|lll}
\hline Acknowledgements & v
\end{tabular}

Abstract vii

1 Introduction 1

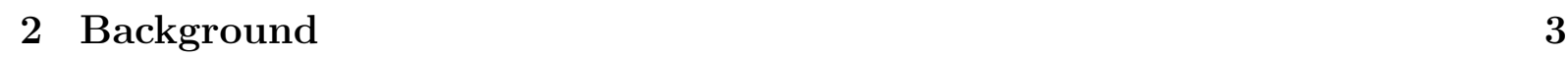

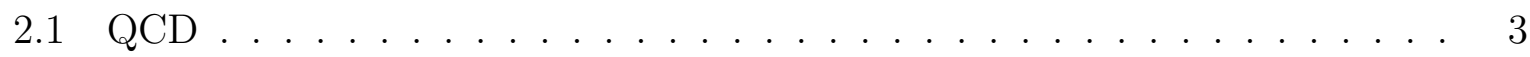

2.2 Lattice QCD $\ldots \ldots \ldots \ldots \ldots \ldots \ldots$

2.2 .1 Discretising the Fermion Action . . . . . . . . . . . . . . . . 6

2.2 .2 Discretising the Gauge Action . . . . . . . . . . . . . . . . . 8

$2.2 .3 \quad$ Symanzik-Improved Anisotropic Wilson Fermion Action . . . . . . 9

$2.2 .4 \quad$ Symanzik-Improved Anisotropic Gauge Action . . . . . . . . . . . . 11

2.3 Numerical Simulation . . . . . . . . . . . . . . . . . . . . . . . . . . . . . 13

2.4 Distillation Smearing . . . . . . . . . . . . . . . . . . . . . . . 14

2.5 Symmetries of the Lattice . . . . . . . . . . . . . . . . . . . . . . . . 18

$2.6 \quad$ Extracting Energies and Spin Identification . . . . . . . . . . . . . . . . . 20

2.7 Scattering Formalism . . . . . . . . . . . . . . . . . . . . . . . . . . . . 21

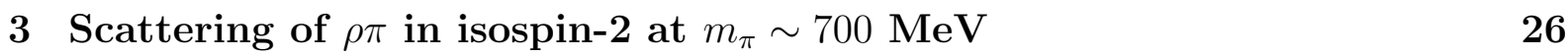

3.1 Vector-Pseudoscalar Scattering . . . . . . . . . . . . . . . . . 28

3.1 .1 Infinite Volume . . . . . . . . . . . . . . . . . . . . . . . . . . . . . 28

3.1 .2 Finite Volume . . . . . . . . . . . . . . . . . . . . . . . . . . . 30

3.2 Operator Construction . . . . . . . . . . . . . . . . . . 32

$3.2 .1 \quad$ Single-Meson Operators in SU(3) Flavour Representations . . . . . 33 
3.2 .2 Two-Meson Operators in SU(3) Flavour Representations . . . . . . 34

3.3 Computational Details . . . . . . . . . . . . . . . . . . . . . . 38

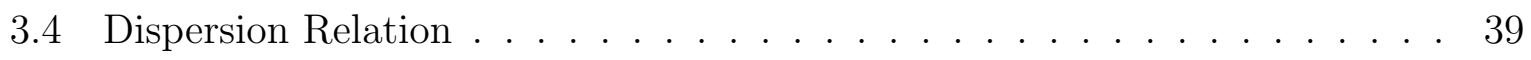

3.5 Finite-Volume Spectra ． . . . . . . . . . . . . . . . . . . . . . . . . . . . . 42

3.6 Scattering Amplitudes $\ldots \ldots \ldots$. . . . . . . . . . . . . . . . . . . . . . . . . . 48

$3.6 .1 \quad$ Uncoupled $P$-wave Scattering . . . . . . . . . . . . . . . . . . . . 50

3.6 .2 Coupled $S, P, D$-wave Scattering . . . . . . . . . . . . . . . . 51

3.6 .3 Constraints on the ${ }^{3} S_{1}-{ }^{3} D_{1}$ Mixing-Angle . . . . . . . . . . . . . 54

3.7 Summary $\ldots \ldots \ldots \ldots$

3.A Subduction Tables . . . . . . . . . . . . . . . . . . . . . . . . . . . 62

3.B Operator Bases . . . . . . . . . . . . . . . . . . . 64

3.C Additional Spectra . . . . . . . . . . . . . . . . . . . . 67

3.D Chew-Mandelstam Phase-Space . . . . . . . . . . . . . . . . . . . . 69

3.E $\quad$ Scattering Parameterisations . . . . . . . . . . . . . . . . . . . . . . . 71

$4 \quad$ Investigation of $\rho \pi$ in isospin-1 at $m_{\pi} \sim 700 \mathrm{MeV} \quad 73$

$4.1 \quad G$-Parity in $\mathrm{SU}(3)_{\mathrm{F}} \ldots \ldots \ldots \ldots \ldots . \ldots \ldots . \ldots \ldots$

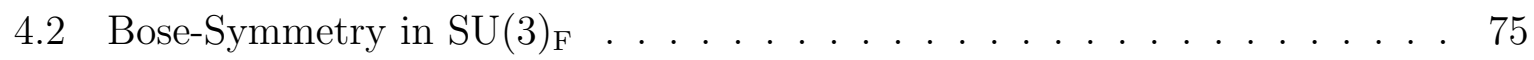

4.3 Operator Constructions in $[000] T_{1}^{+} \ldots \ldots \ldots \ldots$. . . . . . . . . 76

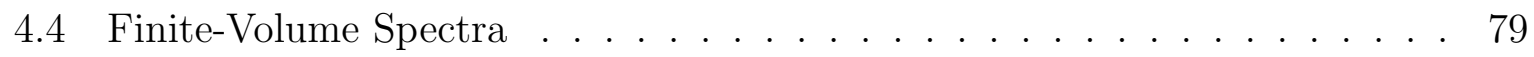

4.5 Summary $\ldots \ldots \ldots \ldots$

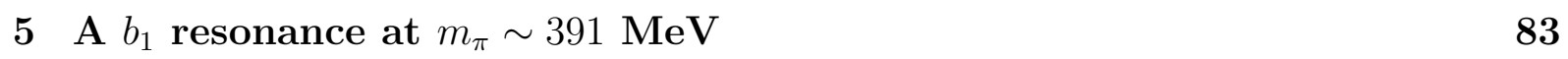

5.1 Operator Constructions . . . . . . . . . . . . . . . . . . 85

$5.1 .1 \quad$ Single-Meson Operators . . . . . . . . . . . . . . . . . 85

5.1 .2 Two-Meson Operators $\ldots \ldots$. . . . . . . . . . . . 86

5.1 .3 Three-Meson Operators . . . . . . . . . . . . . . 86

5.2 Lattice Details . . . . . . . . . . . . . . . . . . . . . . . . . . . . . . . 91

5.3 Operator Bases $\ldots \ldots \ldots \ldots$

5.4 Finite-Volume Spectra . . . . . . . . . . . . . . . . . . . . . . . . . 96

5.5 Scattering Analysis . . . . . . . . . . . . . . . . . . . . . . . 102

$5.5 .1 \quad$ Elastic $\pi \omega\left\{{ }^{3} S_{1}\right\}$ Scattering . . . . . . . . . . . . . . . . . . 104

5.5.2 Dynamically-Coupled $\pi \omega\left\{{ }^{3} S_{1}\right\}, \pi \omega\left\{{ }^{3} D_{1}\right\}$ Scattering . . . . . . . . . 105 
5.5.3 Coupled $\pi \omega\left\{{ }^{3} S_{1}\right\}, \pi \omega\left\{{ }^{3} D_{1}\right\}$ and $\pi \phi\left\{{ }^{3} S_{1}\right\}$ Scattering . . . . . . . . . 107

5.6 Pole Analysis for Coupled-Channel Amplitudes . . . . . . . . . . . . . . . 115

5.7 Systematic Tests . . . . . . . . . . . . . . . . . . . . . . 119

5.7 .1 Additional Partial-Waves . . . . . . . . . . . . . . . . . . . . . . . . 119

5.7.2 Spectrum dependence on $\pi \omega\left\{{ }^{3} P_{0}\right\}, \pi \omega\left\{{ }^{3} P_{2}\right\}$ and $\pi \omega\left\{{ }^{3} D_{1}\right\}$. . . . 120

5.7 .3 Three-body channels . . . . . . . . . . . . . . . 121

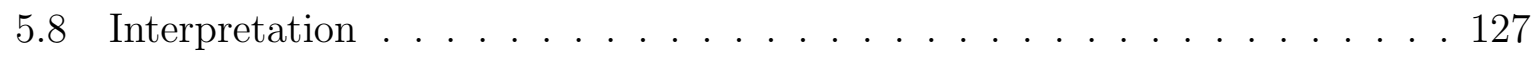

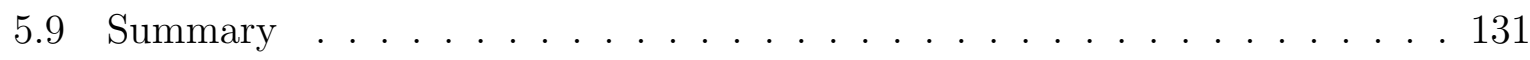

$5 . \mathrm{A}$ Operator Tables . . . . . . . . . . . . . . . . . . . . 133

5.B Scattering Parameterisations . . . . . . . . . . . . . . . . . . . . 138

$5 . \mathrm{C}$ Error Ellipses . . . . . . . . . . . . . . . . . . . . . . 142

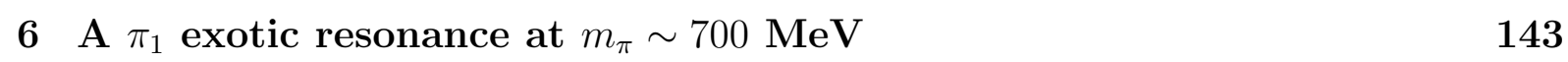

6.1 Computational Details . . . . . . . . . . . . . . . . . . . . . 145

6.2 Operator Bases ． . . . . . . . . . . . . . . . . . . . . . . . . . . . . . . . . 148

6.2 .1 Single-Meson Operators . . . . . . . . . . . . . . . . . . . 149

6.2 .2 Two-Meson Operators . . . . . . . . . . . . . . . . . . . 149

6.2 .3 Three-Meson Operators . . . . . . . . . . . . . . . . . 149

6.3 Finite-Volume Spectra ． . . . . . . . . . . . . . . . . . . . . . . . . . . 151

6.4 Scattering Analysis . . . . . . . . . . . . . . . . . . . . . . . . . 158

6.5 Pole Analysis . . . . . . . . . . . . . . . . . . . . . . . . . . . . . 169

6.6 Interpretation $\ldots \ldots \ldots \ldots \ldots \ldots \ldots$

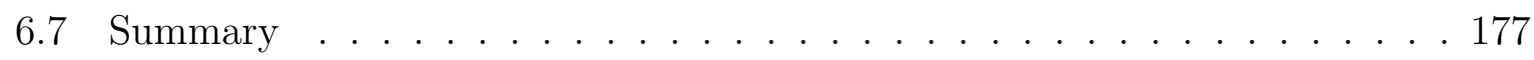

$6 . \mathrm{A} \quad \mathrm{SU}(3)_{\mathrm{F}}$ Octet Multiplets . . . . . . . . . . . . . . . . . 178

6.B Indistinguishable $(\omega)_{8}(\omega)_{1}$ Amplitudes $\ldots \ldots \ldots$. . . . . . . . 180

6.C Scattering Parameterisations . . . . . . . . . . . . . . . . . 181

\begin{tabular}{lll}
\hline 7 & Generalised Stapp-parameterisation & 185
\end{tabular}

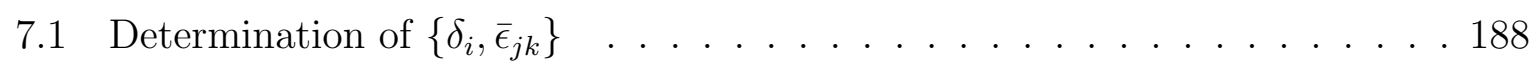

7.2 Properties of $S\left(\delta_{i}, \bar{\epsilon}_{j k}\right) \ldots \ldots \ldots \ldots \ldots$

7.3 Examples . . . . . . . . . . . . . . . . . . . . . . 189

$7.3 .1 f_{0} \ldots \ldots \ldots \ldots \ldots \ldots$

7.4 Summary $\ldots \ldots \ldots \ldots \ldots$ 
$\begin{array}{lll}8 & \text { Conclusion } & 194\end{array}$

\begin{tabular}{ll}
\hline Bibliography & 205
\end{tabular} 


\section{List of Figures}

2.1 Quark propagation relevant for the two-point correlation function constructed using a fermion bilinear operator, $\bar{\psi} \Gamma \psi$, at source and sink. Shown separately are the 'connected' and 'disconnected' diagrams. . . . . . . . . . . 17

3.1 (Left): quark content of flavour irrep 3. (Right): anti-quark content of $\overline{3}$. 34

3.2 Meson content of the $J^{P}=0^{-}$pseudoscalar octet (left) and the $J^{P}=1^{-}$ vector octet (right). Orange corresponds to $I=1$, green to $I=1 / 2$ and blue to $I=0 . \ldots \ldots \ldots \ldots \ldots$. . . . . . . . . . . . . . . . . . . . .

3.3 Upper panel: Momentum dependence of the $\pi$ and $\rho$ energies where both $|\lambda|=0,1$ helicity components of the $\rho$ are considered. The statistical errors on the energies are smaller than the points. Lines and numerical values show results of fits to determine $\xi$ using Eq. 3.10 , Lower panel: points show the effective momentum-dependent $\xi$ obtained via $\left[\left(\frac{2 \pi}{L / a_{s}}\right)^{2}|\vec{n}|^{2} /\left(\left(a_{t} E_{\vec{n}}\right)^{2}-\left(a_{t} m\right)^{2}\right)\right]^{1 / 2}$, with the two volumes $\left(L / a_{s}=20,24\right)$ and the different mesons offset slightly for clarity. The orange line and

band indicate, respectively, the value and uncertainty on $\xi$ we use when
investigating $\rho \pi$ scattering as described in the text. . . . . . . . . . . . . 41

3.4 Principal correlators, $\lambda_{\mathfrak{n}}\left(t, t_{0}=9\right)$, plotted as $e^{E_{\mathfrak{n}}\left(t-t_{0}\right)} \lambda_{\mathfrak{n}}\left(t, t_{0}\right)$, from a variational analysis of the $[000] T_{1}^{+}$irrep on the lattice with $L / a_{s}=24$. Curves show the results of fits described in Section 2.6 . The horizontal axes are in units of $t / a_{t} . \ldots \ldots \ldots \ldots$. . . . . . . . . . . . . . . . . 44 
3.5 Left: finite-volume energy levels in the [000] $T_{1}^{+}$irrep on the lattice with \begin{tabular}{|l}
$L / a_{s}=24$. Dashed lines indicate the location of non-interacting energies. \\
\hline
\end{tabular}

Right: histograms showing the corresponding operator-state overlaps, $Z_{i}^{\mathfrak{n}}=\left\langle\mathfrak{n}\left|\mathcal{O}_{i}^{\dagger}(0)\right| 0\right\rangle$, for the operators ordered as in Table $\mid 3.3$. The colours reflect the non-interacting energies associated with each operator. The overlaps are normalised such that the largest value for a given operator across all energy levels is equal to one. . . . . . . . . . . . . 45

$3.6 \quad$ Energy spectra in irreps at rest. Black and grey points, slightly displaced in $L / a_{s}$ for clarity, show the extracted energy levels below and above $a_{t} E_{\mathrm{cm}}=0.41$ respectively. Errorbars reflect the statistical uncertainties.

\begin{tabular}{|c|c|}
\hline Points in grey are not used in the subsequent analysis in Section 3.6 & .
\end{tabular} Dashed lines show the $\rho \pi$ and $\pi \pi \pi$ thresholds. Solid red curves indicate

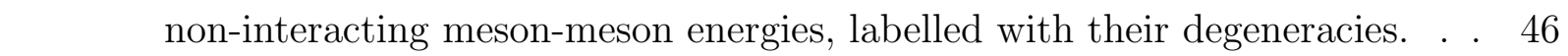

\begin{tabular}{|ll|l|l|l}
\hline 3.7 & As Figure & 3.6 & but for $A_{2}$ irreps with $\vec{P} \neq \overrightarrow{0}$. Dashed red curves indicate
\end{tabular}

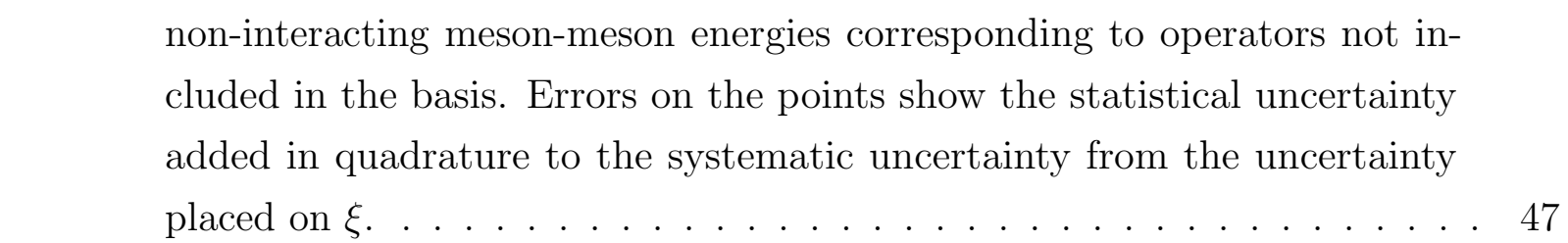

3.8 Phase-shifts for the ${ }^{3} P_{0},{ }^{3} P_{1}$ and ${ }^{3} P_{2}$ partial-waves. The points are as described in the text. Inner bands reflect the statistical uncertainties on the \begin{tabular}{|c|c|}
\hline phase-shifts from the fits in Eq. 3.17 and outer bands reflect the combined \\
\hline
\end{tabular} statistical and systematic uncertainties. . . . . . . . . . . . . 55

3.9 Volume-dependent spectra for irreps with $\vec{P}=\overrightarrow{0}$. Black and grey points, \begin{tabular}{|c|c|c|c|}
\hline slightly displaced in $L / a_{s}$ for clarity, are, as in Figure & 3.6 & , energy levels
\end{tabular} extracted from analyses of correlation functions. Orange points and bands \begin{tabular}{|c|c|}
\hline show solutions to Eq. 2.53 for the reference $\boldsymbol{K}$-matrix parameterisation \\
\hline
\end{tabular}

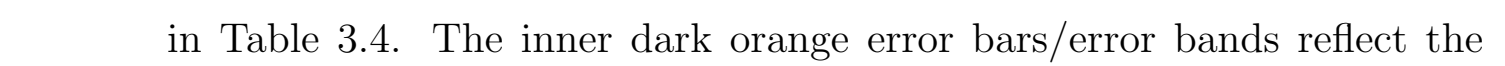
\begin{tabular}{|c|}
\hline statistical uncertainties and the outer lighter orange error bars/error bands \\
\hline
\end{tabular} also include systematic uncertainties. . . . . . . . . . . . . . . 56

3.10 As Figure 3.9 but for $A_{2}$ irreps with $\vec{P} \neq \overrightarrow{0} . \ldots \ldots \ldots$. . . . . . . . 57 
3.11 Phase-shifts for partial-waves, ${ }^{3} \ell_{J}$, and ${ }^{3} S_{1}-{ }^{3} D_{1}$ mixing angle, $\bar{\epsilon}$, as described \begin{tabular}{|c|c|}
\hline in the text. Each curve corresponds to a parameterisation in Table $\mid 3.12$ & \\
\hline
\end{tabular} with $\chi^{2} / N_{\text {dof }} \leq 1.5$. The darker inner band (typically thinner than the

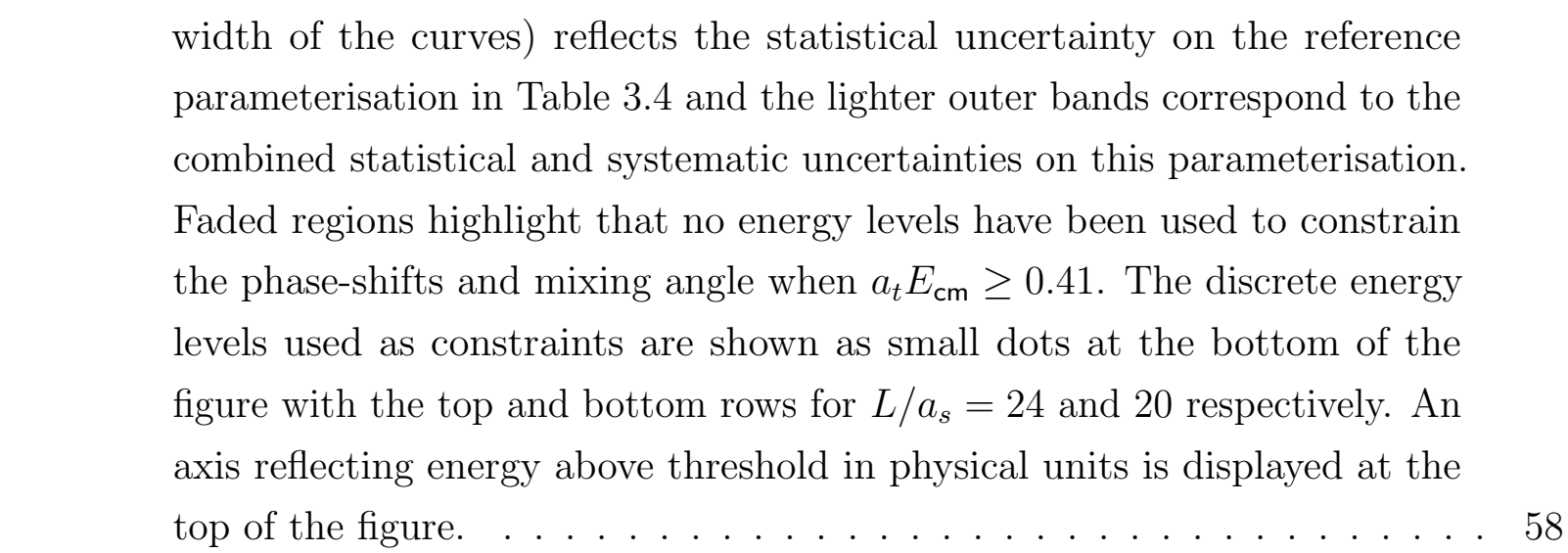

3.12 Upper: Finite-volume spectra in the [000] $T_{1}^{+}$and $\vec{P} \neq \overrightarrow{0} A_{2}$ irreps on the $L / a_{s}=24$ lattice as a function of $c_{0}\left(\left.{ }^{3} S_{1}\right|^{3} D_{1}\right)$ as described in the text. Black and grey points are, as in Figure $\mid 3.6$, energy levels extracted from correlation functions, plotted at $c_{0}\left(\left.{ }^{3} S_{1}\right|^{3} D_{1}\right)=-5.28$ the value in the reference amplitude parameterisation in Table 3.4. Grey bands are to guide the eye and show the combined statistical and systematic uncertainties on the black points. Orange curves show the finite-volume spectra from the reference amplitude when $c_{0}\left({ }^{3} S_{1} \mid{ }^{3} D_{1}\right)$ is varied with the other parameters fixed. Lower: $\delta_{{ }^{3} S_{1}}\left(E_{\mathrm{cm}}\right), \delta_{{ }^{3} D_{1}}\left(E_{\mathrm{cm}}\right)$ and $\bar{\epsilon}\left(E_{\mathrm{cm}}\right)$ for the reference amplitude with a selection of values for $c_{0}\left({ }^{3} S_{1} \mid{ }^{3} D_{1}\right)$. The shaded bands shows the combined statistical and systematic uncertainties of the reference amplitudes, i.e. when $c_{0}\left(\left.{ }^{3} S_{1}\right|^{3} D_{1}\right)=-5.28$. . . . . . . . . . . . . . . . . . 59

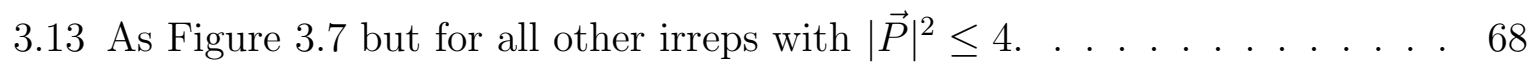

3.14 A sketch of the contour $\mathcal{C}_{a}$ in the complex $s$-plane. The conventional choice of branch cut from $s_{\text {thr. }}^{(a)}=\left(m_{1}^{(a)}+m_{2}^{(a)}\right)^{2}$ towards infinity along the positive real axis is shown. . . . . . . . . . . . . . . . . . . 70 
4.1 Finite-volume spectrum for the $\left(\boldsymbol{f}_{\mathbf{1}}\right)_{\mathbf{8}}$ flavour multiplet in the $[000] T_{1}^{+}$ irrep on each lattice volume. Energy levels are coloured according to the largest operator-state overlap factors. Black points correspond to energy levels with dominant operator-state overlap factors onto $q \bar{q}$-like operators, red are $(\omega)_{8}(\eta)_{8}$-like, blue are $(\boldsymbol{\eta})_{8}\left(f_{0}\right)_{1}$-like and green are $(\omega)_{8}(\omega)_{1}$-like. Errorbars reflect statistical uncertainties only. Points are slightly displaced horizontally for clarity. Solid curves reflect non-interacting energies corresponding to meson-meson operators included in the basis, as shown in Table 4.3 , and dashed lines are thresholds. . . . . . . . . . . . . . 81

5.1 Quark propagation lines (black are light quarks, green are strange quarks) from operator constructions featuring in an optimised $a_{0}$-like operator. . . 91

5.2 Wick contraction topologies for $b_{1}-a_{0} \pi$. Left meson resembles the $b_{1}$, upper right meson the $\pi$ and the remaining one or two mesons the $a_{0}$ (only a subset of the topologies in Figure $\mid 5.1$ is relevant here). . . . . . . . . . . . 91

5.3 Momentum dependence of $\omega$ and $\phi$ energies and fits to Eq. 3.10. Blue and red lines correspond to the $\omega$ meson with $|\lambda|=0$ and 1 respectively. Similarly, green and orange lines correspond to the $\phi$ meson with $|\lambda|=0$ and 1. Points are shown with statistical uncertainties and grey points show the $\left(L / a_{s}\right)=16$ in-flight energies which are not included in the fit. . . . . . 93 
5.4 Left: Finite-volume spectrum in the $[000] T_{1}^{+}$irrep on three lattice volumes. Black points give the energy levels, including statistical uncertainties, from a variational analysis using the operator bases in Table 5.3 . Solid curves are two-meson non-interacting energies, $a_{t} E_{\text {n.i. }}^{(2)}$, short dashed horizontal lines are $a_{t} E_{\text {n.i. }}^{(2+1)}$, and long dashed horizontal lines show the two-, three-, and \begin{tabular}{|l}
\hline four-meson thresholds. Multiplicities (if greater than one) are shown as \\
\hline$\{n\}$. For each energy level on the largest volume, we show the principal
\end{tabular} correlators, plotted as $\lambda_{\mathfrak{n}}\left(t, t_{0}\right) e^{E_{\mathfrak{n}}\left(t-t_{0}\right)}$ for $t_{0}=10 a_{t}$ so that a horizontal line is observed when a single exponential dominates. Points show $\lambda_{\mathfrak{n}}(t, 10)$ and error bars correspond to the one-sigma statistical uncertainty. Curves show fits to the form described in the text; the curves show the fit range and grey points are not included in the fit. The histograms show the operatorstate overlap factors, $Z_{i}^{\mathfrak{n}}=\left\langle\mathfrak{n}\left|\mathcal{O}_{i}^{\dagger}(0)\right| 0\right\rangle$, for each energy level on the largest volume for the $\mathbb{M M}=\pi \omega$ (dark blue), $\pi \phi$ (green) and $\mathbb{R M}=\rho \eta$ (bluegreen), $K^{*} \bar{K}$ (purple) operators along with a sample set of single-meson operators subduced from $J^{P}=1^{+}$(red) and $J^{P}=3^{+}$(orange). The overlaps are normalised such that the largest value for any given operator across all energy levels is equal to one. Right: The spectrum extracted when $\rho \eta$ and $K^{*} \bar{K}$ operators are excluded from the basis (black) compared with the complete spectrum (grey). . . . . . . . . . . . . . . . . . 99

$5.5 \quad$ Finite-volume energy levels in the cm-frame for [000] $T_{1}^{+}$and $\vec{P} A_{2}$ below the lowest $E_{\text {n.i. }}^{(2+1)}$ or $E_{\text {n.i. }}^{(3)}$. Black points are used in the scattering analysis in Section 5.5 while grey points are excluded from the main analysis as discussed in the text. Solid curves are two-meson non-interacting energies, $a_{t} E_{\text {n.i. }}^{(2)}$, short solid grey horizontal lines show the lowest $E_{\text {n.i. }}^{(2+1)}$ or $E_{\text {n.i. }}^{(3)}$, and long dashed horizontal lines show the two-, three-, and four-meson thresholds. Multiplicities (if greater than one) are shown as $\{n\}$. The horizontal axes are in units of $L / a_{s} . \ldots \ldots$. . . . . . . . . . 100

\begin{tabular}{|ll|l|l|l}
5.6 & As Figure & 5.5 & but for irreps $[000] T_{2}^{+},[000] E^{-},[001] B_{1}$ and $[001] B_{2}$ on
\end{tabular} the largest lattice volume. Dashed curves show non-interacting two-meson energies where the corresponding operator was not included in the basis. . 101 
$5.7 \pi \omega\left\{{ }^{3} S_{1}\right\}$ elastic phase-shift assuming no ${ }^{3} D_{1}$ amplitude. The blue line shows

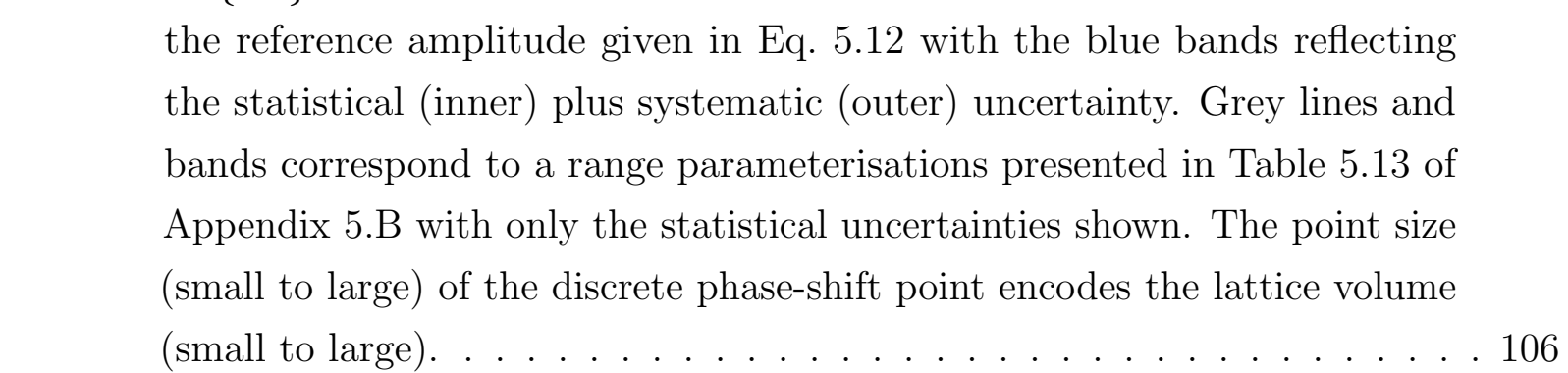

5.8 Upper: $\pi \omega\left\{{ }^{3} S_{1}\right\}$ (blue) and $\pi \omega\left\{{ }^{3} D_{1}\right\}$ (purple) phase-shifts for the reference

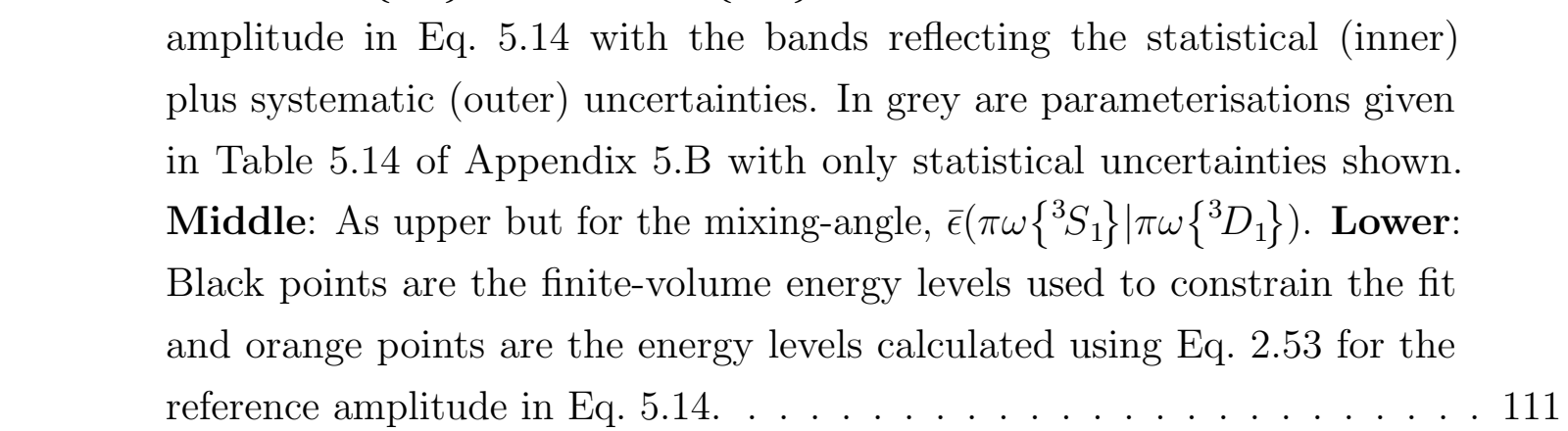

5.9 Upper: As in Figure 5.8 but for the $\pi \omega\left\{{ }^{3} S_{1}\right\}$ (blue), $\pi \omega\left\{{ }^{3} D_{1}\right\}$ (purple) \begin{tabular}{|c|}
\hline and $\pi \phi\left\{{ }^{3} S_{1}\right\}$ (green) phase-shifts for the reference amplitude in Eq. $|5.16|$ \\
\hline \hline and for other parameterisations presented in Table $|5.15|$ of Appendix $|5 . \mathrm{B}|$ \\
\hline \hline (grey). Middle: As upper but for the mixing-angle $\bar{\epsilon}\left(\pi \omega\left\{{ }^{3} S_{1}\right\} \mid \pi \omega\left\{{ }^{3} D_{1}\right\}\right)$. \\
\hline \hline The other mixing-angles, $\bar{\epsilon}\left(\pi \omega\left\{{ }^{3} S_{1}\right\} \mid \pi \phi\left\{{ }^{3} S_{1}\right\}\right)$ and $\bar{\epsilon}\left(\pi \omega\left\{{ }^{3} D_{1}\right\} \mid \pi \phi\left\{{ }^{3} S_{1}\right\}\right)$, are \\
\hline \hline extremely small and consistent with zero for all parameterisations and are \\
\hline not plotted. Lower: The energy levels used to constrain the scattering \\
\hline amplitude (black) and their corresponding description by the reference \\
\hline \hline amplitude in Eq. 5.16 (orange). . . . . . . . . . . . . . . . . . . 112 \\
\hline \hline
\end{tabular}

5.10 As Figure $|5.9|$ but for $\rho_{a} \rho_{b}\left|t_{\ell J a, \ell^{\prime} J b}\right|^{2}$. Coloured curves illustrate the ref\begin{tabular}{|l|}
\hline erence amplitude in Eq. $|5.16|$ with bands reflecting the statistical (inner) \\
\hline plus systematic (outer) uncertainty. Other parameterisations presented \\
\hline in Table 5.15 of Appendix $|5 . \mathrm{B}|$ are in grey with bands reflecting only the \\
\hline statistical uncertainties. $\rho_{a} \rho_{b}\left|t_{\ell J a, \ell^{\prime} \mathrm{Jb}}\right|^{2}$ not plotted are significantly smaller \\
\hline
\end{tabular} than those shown and are consistent with zero. . . . . . . . . . . . . . . . . 113 
5.11 As Figure 5.5 but including, as orange bands, the energy levels calculated \begin{tabular}{|c|c|c|c|c|}
\hline from the reference amplitude in Eq. & 5.16 & using Eq. & 2.53 & as a function $L / a_{s}$.
\end{tabular} The thickness of the bands reflect the combined statistical and systematic uncertainties. The vertical red band on the right of the figure indicates the position of the resonant pole of $m_{R}$ and width $\Gamma_{R}$ as determined in Section 15.6 . The red horizontal line at the resonant mass is shown in each irrep to guide the eye. . . . . . . . . . . . . . . . . . . . . . . . . . . . 114

5.12 Top: Lower half-plane sheet II poles. Red ellipses reflect the statistical uncertainties, oriented to account for correlations between the real and imaginary parts, for poles from all the parameterisations shown in Table $5.15 \mid$

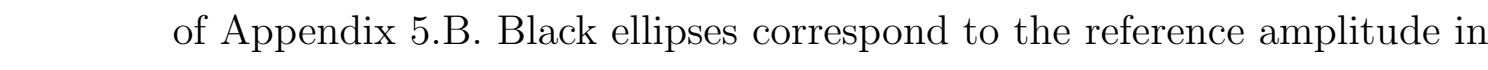
\begin{tabular}{|r|l|l|l|}
\hline Eq. 5.16 & reflecting the statistical (inner) plus systematic (outer) uncertain-
\end{tabular} ties. Bottom: As top but for the corresponding couplings, $c\left(\pi \omega\left\{{ }^{3} S_{1}\right\}\right)_{\mathrm{II}}$ (blue), $c\left(\pi \omega\left\{{ }^{3} D_{1}\right\}\right)_{\text {II }}$ (purple) and $c\left(\pi \phi\left\{{ }^{3} S_{1}\right\}\right)$ ॥ (green). Black ellipses again correspond to the couplings of the reference amplitude in Eq. $5.16 \mid$ where

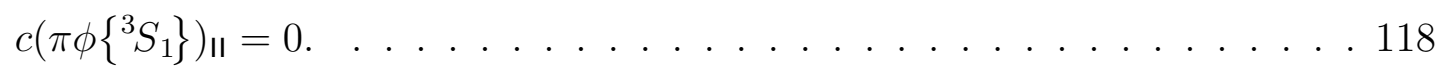

5.13 Sensitivity of the finite-volume spectra to $g_{\pi \omega\left\{{ }^{3} D_{1}\right\}}$. Lighter to darker red curves reflect smaller to larger values of $g_{\pi \omega\left\{{ }^{3} D_{1}\right\}}$ as shown in the key. The central curves correspond to $g_{\pi \omega\left\{{ }^{3} D_{1}\right\}}=1.08$, i.e. the mean value in the reference amplitude in Eq. 5.16. The grey bands reflect the combined statistical and systematic uncertainties of Eq. 5.16 . The horizontal axes are

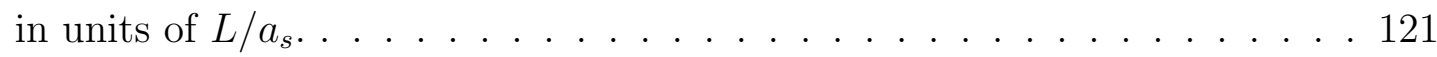

5.14 As Figure $5.13 \mid$ but for varying $\gamma_{\pi \omega\left\{{ }^{3} P_{0}\right\}, \pi \omega\left\{{ }^{3} P_{0}\right\}}^{(0)}$. The central curves corresponds to $\gamma_{\left.\pi \omega{ }^{3} P_{0}\right\}, \pi \omega\left\{{ }^{3} P_{0}\right\}}^{(0)}=0$. The phase-shifts on the left reflect the strengths of

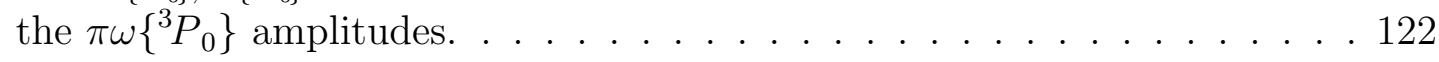

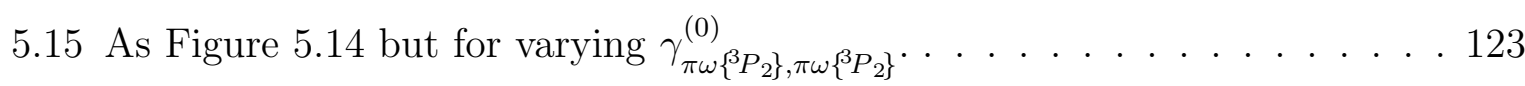

5.16 As Figure 5.11 but for the amplitude in Eq. 5.21. Orange bands reflect only the statistical uncertainty on the scattering parameters. The grey bands are transcribed from Figure $\mid 5.11$. . . . . . . . . . . . . . . . . . . . . . . . 124 
5.17 Upper: As in Figure 5.9 but for the $\pi \omega\left\{{ }^{3} S_{1}\right\}$ (blue), $\pi \omega\left\{{ }^{3} D_{1}\right\}$ (purple), $\pi \phi\left\{{ }^{3} S_{1}\right\}$ (green), $\rho \eta\left\{{ }^{3} S_{1}\right\}$ (orange) and $K^{*} \bar{K}\left\{{ }^{3} S_{1}\right\}$ (red) phase-shifts for the reference amplitude in Eq. 5.21. The faded error bands reflect the statistical uncertainty on the scattering parameters. The $\rho \eta$ and $K^{*} \bar{K}$ "thresholds" are calculated using the $\rho$ and $K^{*}$ masses given above. Lower: As upper but for the mixing-angles $\bar{\epsilon}\left(\pi \omega\left\{{ }^{3} S_{1}\right\} \mid \pi \omega\left\{{ }^{3} D_{1}\right\}\right)$ (blue), $\bar{\epsilon}\left(\pi \omega\left\{{ }^{3} S_{1}\right\} \mid K^{*} \bar{K}\left\{{ }^{3} S_{1}\right\}\right)$ (grey) and $\bar{\epsilon}\left(\pi \omega\left\{{ }^{3} D_{1}\right\} \mid K^{*} \bar{K}\left\{{ }^{3} S_{1}\right\}\right)$ (brown). All other mixing-angles are extremely small and consistent with zero as discussed in the text. . . . . . 126

5.18 Top: The scattering amplitudes-squared, $\rho_{a} \rho_{b}\left|t_{\ell J a, \ell^{\prime} J b}\right|^{2}$, transcribed from \begin{tabular}{|r|r|r|r|}
\hline Figure & 5.10 & with the energy axis converted to physical units. Below the
\end{tabular} amplitudes are the energy levels used to constrain the amplitudes (black points). Bottom: The best estimate of the resonant pole position, where uncertainties combine statistical and systematic uncertainties with variations across parameterisations. The histograms show the best estimate of the magnitude of each coupling with the lightly-shaded region reflecting the combined uncertainties. The $\pi \phi\left\{{ }^{3} S_{1}\right\}$ coupling is an estimate of the upper bound. . . . . . . . . . . . . . . . . . . . . . . 129

5.19 The $b_{1}$ pole position for various pion masses. Blue shows the groundstate mass of the axial-vector octet from a lattice calculation with $m_{\pi} \approx$ $700 \mathrm{MeV}[4]$, red shows the estimate from this work with $m_{\pi} \approx 391 \mathrm{MeV}$ and black is the experimentally determined mass and width of the $b_{1}$ resonance $[5 \mid .130$

6.1 Momentum dependence of the $(\boldsymbol{\eta})_{1}$ energies and energies of the $|\lambda|=0,1$

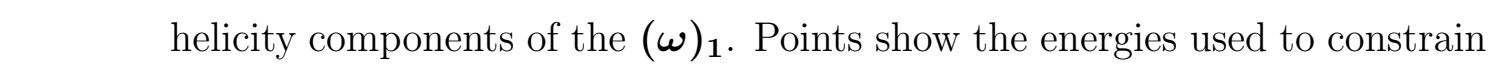
the fits, as discussed in the text, with the errorbars reflecting the statistical uncertainties. Lines and numerical values show results of fits to determine $\xi$ using Eq. 3.10 . . . . . . . . . . . . . . . . . . . . . . . . . . . . . 148 
6.2 Finite-volume spectrum in the $[000] T_{1}^{-}$irrep on four lattice volumes. Points give the energy levels, including statistical uncertainties, from a varia-

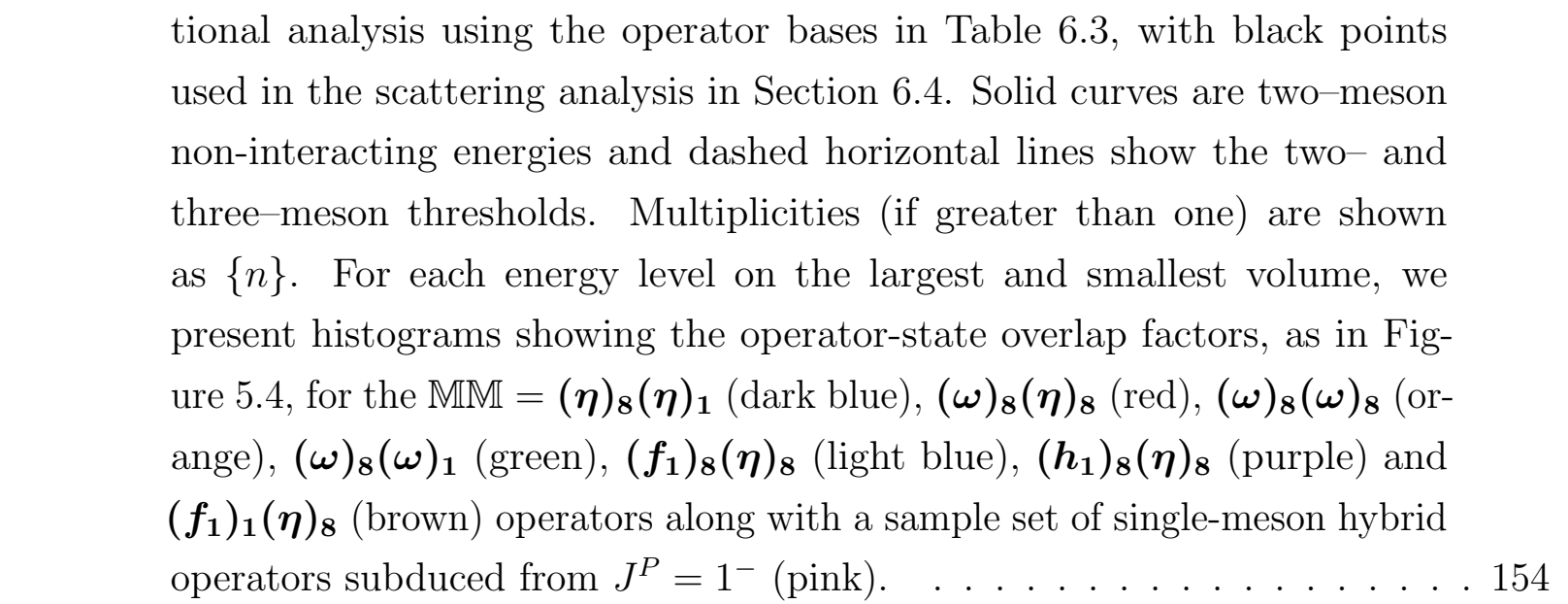

6.3 Principal correlators, $\lambda_{\mathfrak{n}}\left(t, t_{0}=7\right)$, plotted as $e^{E_{\mathfrak{n}}\left(t-t_{0}\right)} \lambda_{\mathfrak{n}}\left(t, t_{0}\right)$, from a variational analysis of the $[000] T_{1}^{-}$irrep on the lattice with $L / a_{s}=24$. Curves show the results of fits described in Section $\mid 2.6$. The horizontal axes are in units of $t / a_{t}$. The lettering corresponds to the lettering in Figure 6.2 . 155

6.4 Continuation of Figure $6.3 . \ldots \ldots \ldots \ldots$

6.5 Continuation of Figure $6.3 \mid \ldots \ldots \ldots$. . . . . . . . . . . . . 157

6.6 Upper: $(\boldsymbol{\eta})_{\mathbf{8}}(\boldsymbol{\eta})_{\mathbf{1}}\left\{{ }^{1} P_{1}\right\}$ (dark blue) and $(\boldsymbol{\omega})_{\mathbf{8}}(\boldsymbol{\eta})_{\mathbf{8}}\left\{{ }^{3} P_{1}\right\}$ (red) phase-shifts. Lower: $(\omega)_{8}(\omega)_{8}\left\{{ }^{3} P_{1}\right\}$ (orange) and $(\omega)_{8}(\omega)_{1}\left\{{ }^{X} P_{1}\right\}$ (green) phase-shifts. \begin{tabular}{|c|c|c|}
\hline The solid curves show the reference amplitude given in Eq. 6.9 & with the \\
\hline
\end{tabular} coloured bands reflecting the statistical (inner) plus systematic (outer) uncertainty. Dashed curves and grey bands correspond to all successful \begin{tabular}{|l|l|l|l|l|}
\hline parameterisations presented in Table & 6.5 & of Appendix & $6 . C$ & with only the \\
\hline
\end{tabular} - statistical uncertainties shown. . . . . . . . . . . . . . . 163

\begin{tabular}{|ll|l|l|l}
\hline 6.7 & As in Figure & 6.6 & but for $\left(\boldsymbol{f}_{\mathbf{1}}\right)_{\mathbf{8}}(\boldsymbol{\eta})_{\mathbf{8}}\left\{{ }^{3} S_{1}\right\}$ (light blue) and $\left(\boldsymbol{h}_{\mathbf{1}}\right)_{\mathbf{8}}(\boldsymbol{\eta})_{\mathbf{8}}\left\{{ }^{3} S_{1}\right\}$ \\
\hline
\end{tabular} (purple) phase-shifts (upper), and for $(\boldsymbol{\omega})_{\mathbf{8}}(\boldsymbol{\eta})_{\mathbf{8}}\left\{{ }^{3} F_{3}\right\}$ (red) and $(\boldsymbol{\omega})_{\mathbf{8}}(\boldsymbol{\omega})_{\mathbf{1}}\left\{{ }^{5} P_{3}\right\}$ (green) phase-shifts (lower). . . . . . . . . . . . . . . . . . 164 
6.8 Upper: the inelasticities $\cos 2 \bar{\epsilon}\left((\boldsymbol{\eta})_{\mathbf{8}}(\boldsymbol{\eta})_{\mathbf{1}}\left\{{ }^{1} P_{1}\right\} \mid \ldots\right)$ Lower: inelasticities \begin{tabular}{|c|c|c|c|}
\hline $\cos 2 \bar{\epsilon}\left((\boldsymbol{\omega})_{\mathbf{8}}(\boldsymbol{\eta})_{\mathbf{8}}\left\{{ }^{3} P_{1}\right\} \mid \ldots\right)$. The reference amplitude given in Eq. 6.9 & is
\end{tabular} plotted with coloured bands reflecting the statistical (inner) plus systematic

\begin{tabular}{|c|}
\hline (outer) uncertainty. The solid curves show the inelasticities for all parame- \\
\hline
\end{tabular}
terisations presented in Table 6.5 in Appendix 6 6.C. . Statistical uncertainties on these parameterisations are omitted for clarity in the plot. . . . . . . . 165

6.9 As in Figure $|6.8|$ but for inelasticities $\cos 2 \bar{\epsilon}\left((\omega)_{8}(\omega)_{8}\left\{{ }^{3} P_{1}\right\} \mid \ldots\right)$ (upper), and for inelasticities $\cos 2 \bar{\epsilon}\left((\omega)_{8}(\omega)_{1}\left\{{ }^{X} P_{1}\right\} \mid \ldots\right)$ (lower). . . . . . . . 166

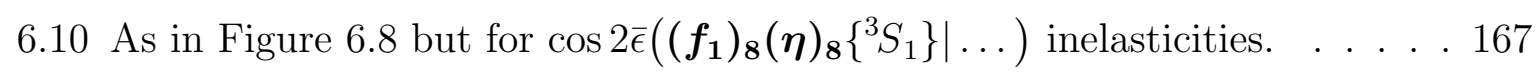

\begin{tabular}{|ll|l|l|l}
6.11 & As Figure & 6.2 & but including, as pink curves and points, the energy levels
\end{tabular}

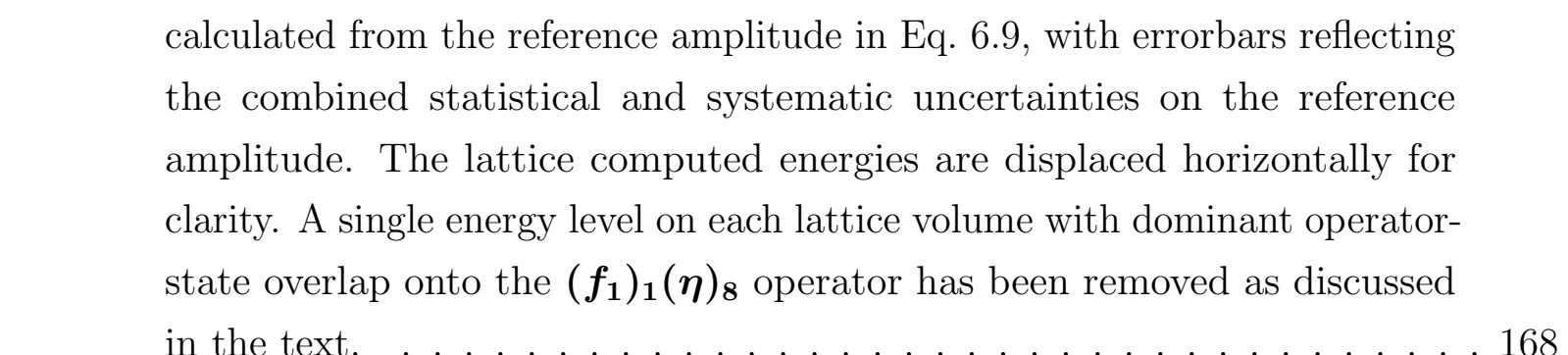

6.12 Lower half-plane sheet II poles. The black point corresponds to the reference

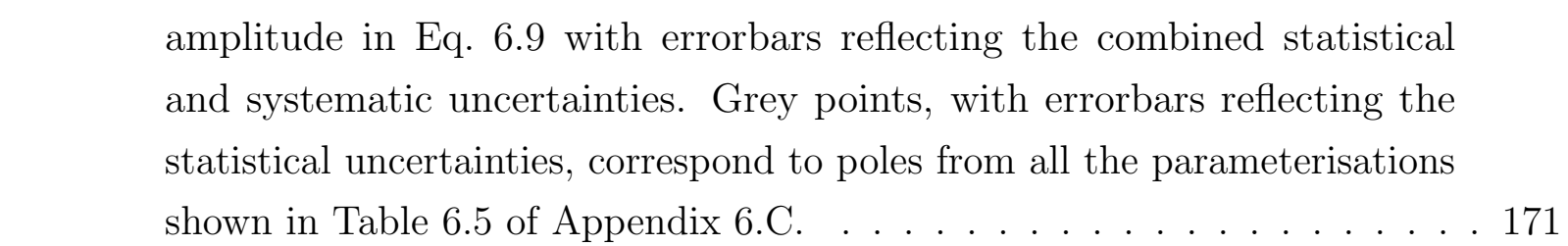

6.13 Couplings of the sheet II poles, shown in Figure 6.12 , to hadron-hadron

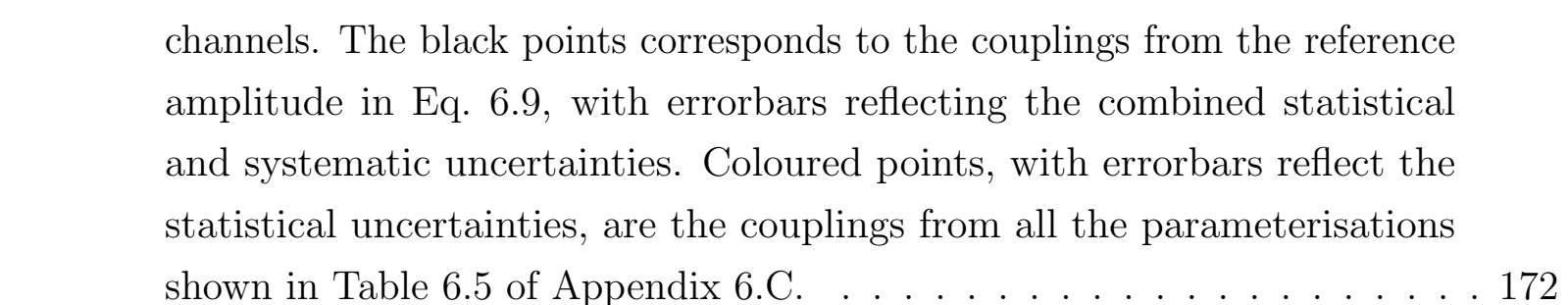

6.14 Points are the best estimates of each coupling $-(\boldsymbol{\eta})_{\mathbf{8}}(\boldsymbol{\eta})_{\mathbf{1}}\left\{{ }^{1} P_{1}\right\}$ (dark blue), \begin{tabular}{|c|}
\hline$(\boldsymbol{\omega})_{\mathbf{8}}(\boldsymbol{\eta})_{\mathbf{8}}\left\{{ }^{3} P_{1}\right\}$ (red), $(\boldsymbol{\omega})_{\mathbf{8}}(\boldsymbol{\omega})_{\mathbf{8}}\left\{{ }^{3} P_{1}\right\}$ (orange), $(\boldsymbol{\omega})_{\mathbf{8}}(\boldsymbol{\omega})_{\mathbf{1}}\left\{{ }^{X} P_{1}\right\}$ (green), \\
\hline \hline$\left(\boldsymbol{f}_{\mathbf{1}}\right)_{\mathbf{8}}(\boldsymbol{\eta})_{\mathbf{8}}\left\{{ }^{3} S_{1}\right\}$ (light blue), $\left(\boldsymbol{h}_{\mathbf{1}}\right)_{\mathbf{8}}(\boldsymbol{\eta})_{\mathbf{8}}\left\{{ }^{3} S_{1}\right\}$ (purple) - where the error \\
\hline bars reflect a combination of statistical and systematic uncertainties and \\
\hline encompass variation over the range of parameterisations. . . . . . . . . . 174
\end{tabular} 
6.15 The $\pi_{1}$ pole. Pink shows the estimate from this work with $m_{\pi} \approx 700 \mathrm{MeV}$ and black is the experimentally determined mass and width of the $\pi_{1}$ resonance 6 6] . . . . . . . . . . . . . . . . . . . . 175

$6.16 \mathrm{SU}(2)_{\mathrm{F}}$ flavour content of the scalar, axial-vector and tensor octets. The $\left(I^{G}\right) J^{P C}$ quantum numbers for the isovector elements are given in the sub-captions. . . . . . . . . . . . . . . . . . . . . . 179

$7.1 \quad$ Phase-shifts, $\delta_{i}$ (solid curves), and diagonal phases, $\psi_{i}$ (dashed curves), for the reference parameterisation quoted in Ref. $|7|-\pi \pi$ (blue), $K \bar{K}$ (red), $\eta \eta$ (green). Coloured bands reflect the uncertainties $\delta_{i}$ while the grey bands give the uncertainties on $\psi_{i}$. . . . . . . . . . . . . . . . . . . . . . . . . . . 191

7.2 Inelasticities, $\cos 2 \bar{\epsilon}_{i j}$, for the reference parameterisation quoted in Ref. $|7|$ $-\pi \pi \mid K \bar{K}$ (orange), $\pi \pi \mid \eta \eta$ (blue), $K \bar{K} \mid \eta \eta$ (purple). Coloured bands reflect the uncertainties on the reference amplitude. . . . . . . . . . . . . . . . . . 192 


\section{List of Tables}

3.1 Subduction of partial-waves, ${ }^{3} \ell_{J}$, for $J \leq 4$ into the irreps, $\Lambda^{P}$, of the

\begin{tabular}{|c|}
\hline octahedral group, $\mathrm{O}_{h}$, relevant for systems overall at rest. The notation \\
\hline \hline$J^{P}\left({ }^{3} \ell_{J}\right)$ denotes the partial-wave content for a given $J^{P}$, with multiple \\
\hline${ }^{3} \ell_{J}$ entries indicating partial-waves which mix dynamically. This table is \\
\hline derived from Table 2 of $[8]$.
\end{tabular}

3.2 Number of distillation vectors $\left(N_{\text {vecs }}\right)$, gauge configurations $\left(N_{\text {cfgs }}\right)$ and time-

\begin{tabular}{|l|}
\hline sources $\left(N_{\text {tsrcs }}\right)$ used to compute correlation functions on the two lattice \\
\hline volumes, as described in the text, for (left) $\rho$ and $\pi$ correlation functions \\
\hline
\end{tabular}
$(\boldsymbol{F}=\mathbf{8})$ and (right) $\rho \pi$ correlation functions $(\boldsymbol{F}=\mathbf{2 7}) . \ldots \ldots 39$

3.3 Meson-meson operators in the $\mathbf{2 7}$ of SU(3) F $_{\mathrm{F}}$ flavour, ordered by increasing

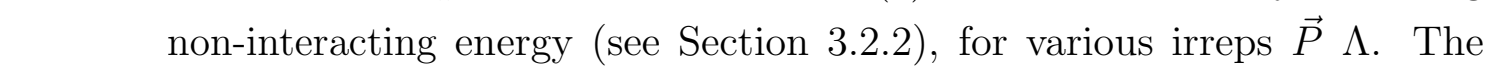
operators, $\rho_{\vec{p}_{1}} \pi_{\vec{p}_{2}}$, are constructed from optimised $\rho$ and $\pi$ operators with

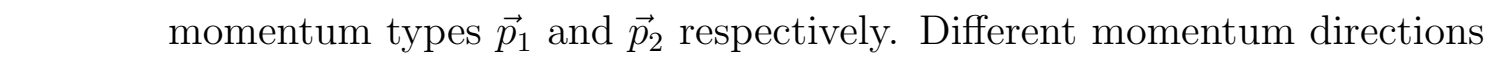
\begin{tabular}{|c|c|c|}
\hline are summed over as in Eq. & 3.8 & $\left\{N_{\text {lin }}\right\}$ denotes the number of linearly- \\
\hline
\end{tabular} independent meson-meson operators at the corresponding non-interacting energy when there is more than one. All operators with corresponding non-interacting energies $a_{t} E_{\mathrm{cm}} \leq 0.455$ for $L / a_{s}=24$ are displayed. Those

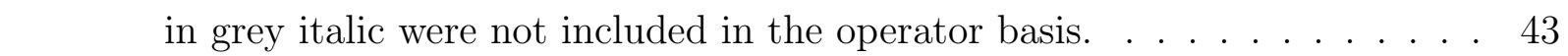

$3.4 \quad$ A reference fit as described in the text with $\chi^{2} / N_{\text {dof }}=1.42$. The first uncertainty in each case is statistical and the second is an estimate of the systematic uncertainty as described in the text. Correlations between the $\boldsymbol{K}$-matrix parameters are displayed on the right. Parameters not shown were fixed to zero. . . . . . . . . . . . . . . . . . . . . . 52 
3.5 Partial-wave $J^{P}\left({ }^{3} \ell_{J}\right)$ subductions for $\ell \leq 3$ at $\vec{P}=[00 n]$ into irreps $\Lambda$ of the

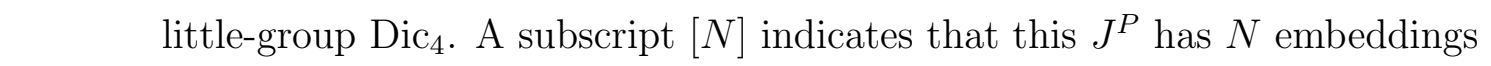
in the irrep $\Lambda$. Partial-waves with $\ell>3$ that couple dynamically to partialwaves with $\ell \leq 3$ are shown in grey italic. This table is derived using the results presented in Refs. $|9|$ and $|10| . \ldots$. . . . . . . . . . . . . . . . . 62

3.6 As Table 3.5 but for $\vec{P}=[0 n n]$ with little-group Dic Dic $_{2} \ldots$. . . . . . . . . 63

3.7 As Table 3.5 , but for $\vec{P}=[n n n]$ with little-group Dic $\mathrm{Dic}_{3}$. . . . . . . . . . . 64

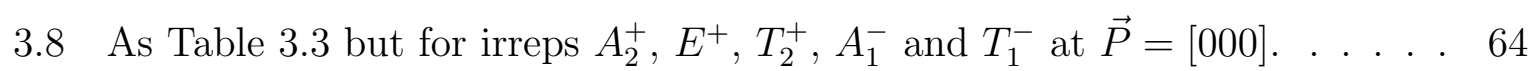

\begin{tabular}{|l|l|l|l|}
\hline 3.9 & As Table & 3.3 & but for irreps $E^{-}$and $T_{2}^{-}$at $\vec{P}=[000]$ and $A_{1}, B_{1}$ and $B_{2}$ at \\
\hline
\end{tabular}

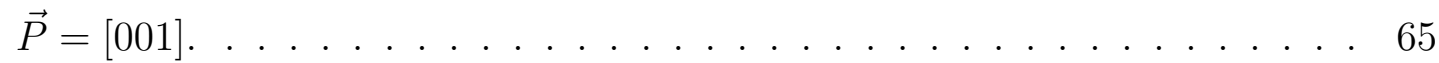

\begin{tabular}{|l|l|l|l|}
\hline 3.10 As Table & 3.3 & but for irreps $E_{2}$ at $\vec{P}=[001] ; A_{1}, B_{1}$ and $B_{2}$ at $\vec{P}=[011]$ \\
\hline
\end{tabular}

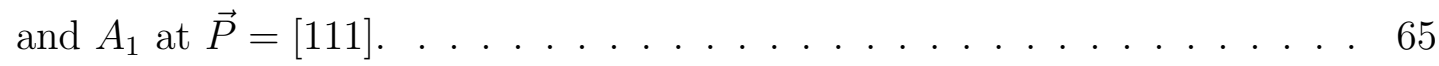

\begin{tabular}{|l|l|l|l|}
\hline 3.11 As Table & 3.3 & but for irreps $E_{2}$ at $\vec{P}=[111]$ and $A_{1}, B_{1}, B_{2}$ and $E_{2}$ at \\
\hline
\end{tabular}

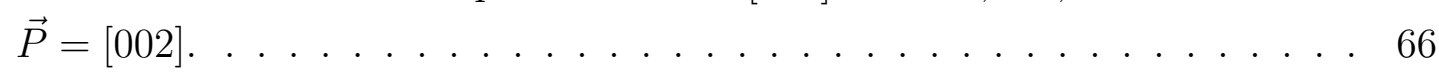

3.12 Polynomial parameterisations of the $\boldsymbol{K}$-matrix as defined in Eq. 3.14 . Each entry in the table indicates the order of the polynomial, $N\left(\left.{ }^{3} \ell_{J}\right|^{3} \ell_{J}^{\prime}\right)$, for the relevant matrix element and "-" denotes a zero entry in the $\boldsymbol{K}$-matrix. The $\chi^{2} / N_{\text {dof }}$ for each fit, describing the lowest 141 energy levels, is given in the final column. The reference fit, whose parameter values are presented in Table 3.4 , is displayed in bold in the first row of this table. . . . . . . . . . 72

4.1 Partial-wave content $(\ell \leq 4)$ of multiplets $\mathbf{8}_{\mathbf{1}}$ and $\mathbf{8}_{\mathbf{2}}$, from a product of two identical octets, $\mathbf{8}_{a} \otimes \mathbf{8}_{a}$, with intrinsic spins $S_{a}$. . . . . . . . . . . 76

4.2 (Left): relevant stable hadron masses. (Right): thresholds relevant for multi-meson levels appearing in $J^{P}=1^{+} . \ldots \ldots \ldots$. . . . . . . . 78

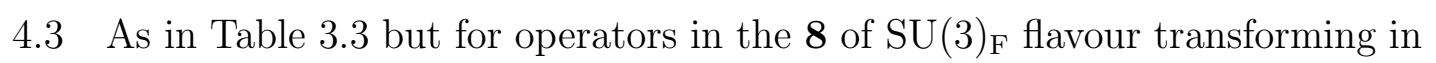
$[000] T_{1}^{+}$. Meson-meson operators are ordered by increasing non-interacting energy (see Section 3.2 .2 ). The operators, $\mathbb{M}_{1\left[\vec{p}_{1}\right]} \mathbb{M}_{2\left[\vec{p}_{2}\right]}$, are constructed from optimised $\mathbb{M}$ operators with momentum types $\vec{p}_{1}$ and $\vec{p}_{2}$ respectively. Different momentum directions are summed over as in Eq. 3.8. Single-meson operators are denoted $\bar{\psi} \bar{\Gamma} \psi$. . . . . . . . . . . . . . . . . . . . . . . . . . 79 
5.1 Number of distillation vectors $N_{\text {vecs }}$, gauge configurations $N_{\text {cfgs }}$, and timesources $N_{\text {tsrcs }}$ used in the computation of correlation functions. . . . . . . . 92

5.2 Left: The masses of relevant stable hadrons with uncertainties. Right:

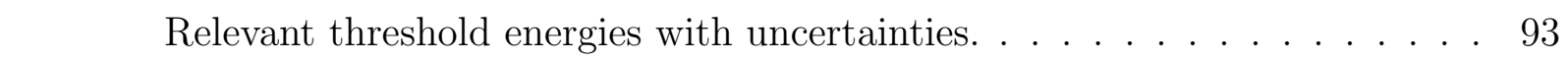

$5.3[000] T_{1}^{+}$operator basis for each lattice volume, with operators ordered by \begin{tabular}{|c|}
\hline increasing $E_{\text {n.i. }}$. The maximum number of single-meson operators, $N$, is \\
\hline denoted by $N \times \bar{\psi} \boldsymbol{\Gamma} \psi$; various subsets of these were considered to obtain \\
\hline robust fits. The number in braces, $\left\{N_{\text {mult }}\right\}$, denotes the multiplicity of \\
\hline
\end{tabular}

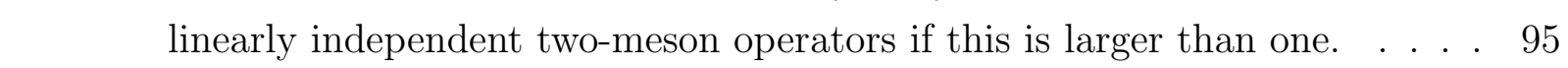

5.4 Sheet labelling as a function of the sign of the imaginary components of $k_{\mathrm{cm}}^{(a)} .116$

5.5 Single-meson and two-meson operators used to compute optimised $\rho$ operators in the [000] $T_{1}^{-}$irrep and $\vec{P} A_{1}$ irreps at various overall momenta on the \begin{tabular}{|c|}
\hline three volumes. Momentum labels on the $\pi$ 's that form the $\pi \pi$ operators \\
\hline
\end{tabular} are omitted for brevity. . . . . . . . . . . . . . . . . . 133

5.6 As Table 5.5 but for optimised $a_{0}$ operators. . . . . . . . . . . . . . 134

5.7 As Table 5.5 but for optimised $K^{*}$ operators. . . . . . . . . . . . . . . . . 134

5.8 As in Table 5.3 but for irrep [001] $A_{2}$. For operators $\mathcal{O}_{\mathbb{R} \mathbb{M}}^{\dagger}$, the superscript $\mathfrak{n}$ \begin{tabular}{|c|}
\hline on $\mathbb{R}^{\mathfrak{n}}$ denotes the $\mathfrak{n}^{\text {th }}$ excited state when $\mathfrak{n} \geq 1$. All $\rho$ and $K^{*}$ operators \\
\hline \hline transform in $[000] T_{1}^{-}$at $\vec{p}=\overrightarrow{0}$ and all $\rho, a_{0}$ and $K^{*}$ operators transform in \\
\hline$\vec{P} A_{1}$ for $\vec{p} \neq \overrightarrow{0}$. Operators shown in gray correspond to $E_{\text {n.i. }}^{(2+1)}$ greater than \\
\hline
\end{tabular} the $E_{\text {n.i. }}^{(2+1)}$ or $E_{\text {n.i. }}^{(3)}$ of operators that have not been included in the basis. . . 135

5.9 As in Table 5.8 but for irrep $[011] A_{2} . \ldots \ldots \ldots$. . . . . . . . 136

5.10 As in Table 5.8 but for irrep $[111] A_{2} . \ldots \ldots \ldots \ldots$. . . . . . . . 136

5.11 As in Table 5.8 but for irrep $[002] A_{2} . \ldots \ldots$. . . . . . . . 137

\begin{tabular}{|l|l|l|l|}
5.12 As Table & 5.8 & for irreps $[000] T_{2}^{+},[000] E^{-},[001] B_{1}$ and $[001] B_{2}$ on the \\
\hline
\end{tabular}

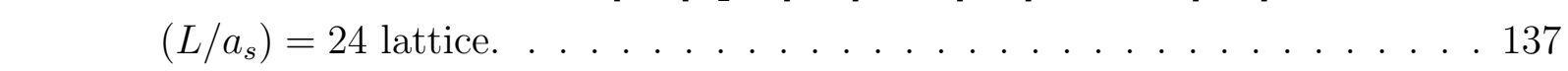

5.13 Parameterisations of elastic $\pi \omega\left\{{ }^{3} S_{1}\right\}$ scattering amplitudes with $N_{\text {pars }}$ free parameters. Fits used 20 energy levels below $\pi \phi$ threshold, as described in the text. The reference amplitude, Eq. 5.12 , is in bold. 'CM' denotes that the Chew-Mandelstam prescription was employed with subtraction at energy $m$ or at threshold $s^{\text {thr }}=\left(m_{\pi}+m_{\omega}\right)^{2}$. Otherwise, we set $I(s)=-i \rho(s) .138$ 
5.14 Parameterisations of dynamically-coupled $\pi \omega\left\{{ }^{3} S_{1}\right\}$ and $\pi \omega\left\{{ }^{3} D_{1}\right\}$ scattering amplitudes. Fits were determined using 20 energy levels below $\pi \phi$ threshold as described in the text. Displayed in bold is the reference amplitude

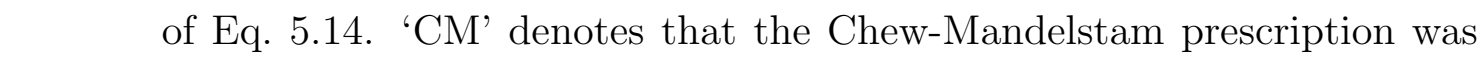
employed with subtraction at energy $m$, the 'pole' parameter in the $K$-matrix. 139

5.15 Parameterisations of coupled $\pi \omega\left\{{ }^{3} S_{1}\right\}, \pi \omega\left\{{ }^{3} D_{1}\right\}$ and $\pi \phi\left\{{ }^{3} S_{1}\right\}$ scattering amplitudes. Fits used 36 energy levels below $\pi \pi \pi \pi$ threshold, as described \begin{tabular}{|c|c|c|}
\hline in the text. Displayed in bold is the reference amplitude of Eq. 5.16.
\end{tabular} 'CM' denotes that the Chew-Mandelstam prescription was employed with subtraction at energy $m$ or at threshold $s_{a}^{\text {thr }}$ where $s_{a}^{\text {thr }}=\left(m_{1}^{(a)}+m_{2}^{(a)}\right)^{2}$. Otherwise, we set $I_{a}(s)=-i \rho_{a}(s) . \ldots \ldots \ldots \ldots \ldots$

5.16 As Table 5.15 but for amplitudes that resulted in poles on the physical sheet off the real axis and were subsequently rejected as discussed in the text. 141

6.1 Number of distillation vectors $\left(N_{\text {vecs }}\right)$, gauge configurations $\left(N_{\text {cfgs }}\right)$ and \begin{tabular}{|c|}
\hline time-sources $\left(N_{\text {tsrcs }}\right)$ used to compute the $\left(\boldsymbol{\pi}_{1}\right)_{8}$ correlation functions on \\
\hline
\end{tabular} each lattice volume, as described in the text. . . . . . . . . . . . . . 146

6.2 (Left): relevant stable hadron masses. (Right): multi-hadron thresholds relevant for $J^{P}=1^{-}$up to $a_{t} E_{\mathrm{cm}} \approx 0.485 . \ldots \ldots$. . . . . . . . 147

$6.3[000] T_{1}^{-}$operator basis for each lattice volume, with two-meson operators \begin{tabular}{|cc|c|}
\hline ordered by increasing $E_{\text {n.i. }}$. As in table 5.3 , the maximum number of \\
\hline
\end{tabular} single-meson operators, $N$, is denoted by $N \times \bar{\psi} \mathbf{\Gamma} \psi$ and various subsets of these were considered to obtain robust fits. The number in braces, $\left\{N_{\text {mult }}\right\}$, denotes the multiplicity of linearly independent two-meson operators if this is larger than one. . . . . . . . . . . . . . . . . . . 150

6.4 Couplings extrapolated to the physical quark mass limit, as described in the text . . . . . . . . . . . . . . . . . . . . 177 
6.5 Parameterisations of the $\boldsymbol{K}$-matrix, as described in the text. Each checkmark ' $\checkmark$ ' in the table indicates freedom in that scattering parameter and a dash '-' denotes a zero entry in the $\boldsymbol{K}$-matrix. The $\chi^{2} / N_{\text {dof }}$ for each fit, describing the 38 energy levels, is given in the final column for different phase-space prescriptions: Chew-Mandelstam (CM) with threshold (thr.) or pole (pole) subtraction or the simple phase-space, ' $I(s)=-i \rho(s)$ '. The reference fit, whose parameter values are presented in Eq. 6.9, is displayed in bold in the first 'row' of this table, i.e. the first block of four rows divided by horizontal dashed lines. . . . . . . . . . . . . . . . . . . . . . . 182

6.6 The correlation matrix for scattering parameters in the reference amplitude given in Eq. 6.9| . . . . . . . . . . . . . . . . . . . . . . . . . . . . . 184 


\section{Introduction}

Quantum chromodynamics (QCD) is the theory of the strong interaction that confines quarks and gluons to hadrons. The quantum field theory has a non-abelian SU(3) gauge symmetry which gives rise to eight gauge fields, gluons, transforming in the adjoint representation of $\mathrm{SU}(3)$. (Anti)quarks are charged under $\mathrm{SU}(3)$, transforming in the (anti)fundamental representation and we attribute (anti)red, (anti)green and (anti)blue to distinguish the three distinct colour charges carried. Overwhelming experimental evidence suggests QCD is a confining theory in which observables must be colourless, meaning eigenstates of the QCD Hamiltonian, hadrons, are forbidden to have a net colour charge. This restricts the allowed quark and gluon constituents of hadrons - certainly a naked quark is prohibited for example. For two quarks, hadrons can consist of a quark-antiquark pair, a meson $(q \bar{q})$ such as the pion. For three quarks, an antisymmetric contraction of the colour charges yields a colour neutral state, a baryon ( $q q q)$ such as the proton, and similarly an anti-baryon where the quarks are replaced with anti-quarks. More elaborate combinations of larger numbers of quarks give rise to a plethora of possible hadronic states, for example tetraquarks and pentaquarks. Furthermore, excitations of the gluonic fields coupled with quarks gives rise to so called hybrids and in the absence of any quark fields at all, glueballs. Although these states are allowed within the QCD framework, they do not necessarily exist.

The vast majority of hadrons appearing in nature are unstable, resonances, and are observed through decays via the strong interaction to, typically, a multitude of stable hadrons in various partial-waves. Experimentally, long-lived (narrow) isolated resonances are observed as 'bumplike' enhancements in the cross-sections of scattering amplitudes of the relevant decay modes, with masses and life times related to the position and width of the enhancement region. Short-lived (broad) resonances are much more difficult to see from cross-sections alone.

The quark model, proposed independently by Gell-Mann [11] and Zweig [12], is a means 
of classifying hadrons based on their constituent quarks. This scheme offered a natural way to group together the light hadrons that were being discovered in the 1950s and 1960s but as more experimental evidence emerged, verifying the success of the quark model, it became a powerful tool within its own right. The majority of resonances are accounted for by the quark model, including broad resonances hard to detect in experiment, however, experimental evidence suggests the existence of hadronic resonances with exotic quantum numbers that are not accounted for - one example being the $\pi_{1}$ resonance.

Contemporary studies of hadron spectroscopy seek to relate the spectrum of hadronic resonances, including their decay properties, to the theory of QCD. Lattice QCD, which considers the theory on a discretised spacetime grid of finite size and numerically calculates correlation functions using Monte-Carlo generated gauge field configurations, has proven to be a hugely successful approach. The discrete spectrum in a finite volume, corresponding to a particular choice of quantum numbers, can be extracted from a matrix of correlation functions, computed using a diverse basis of operators which resemble the hadronic system being studied. An approach introduced by Lüscher utilises the finite volume to relate the discrete spectrum of states to infinite-volume continuum hadron-hadron scattering amplitudes. The corresponding pole singularities and residues of the scattering amplitudes analytically continued into the complex energy plane, give the masses, widths and couplings of the resonances. In this thesis, I will calculate, from first principles QCD, scattering amplitudes of hadrons with non-zero intrinsic spins before determining the pole singularities and interpreting these as resonances. 


\section{Background}

In this chapter, we set out the background necessary to perform calculations of lowenergy observables from first principles QCD. In Section 2.1, we give a brief discussion of continuum QCD with particular attention to the symmetries of the theory and the path integral formulation. The lattice QCD (LQCD) framework will be introduced in Section 2.2, and numerical techniques required to calculate QCD observables nonperturbatively will be given in Section 2.3. In Section 2.4, we detail the implementation of the distillation framework, used in the computation of correlation functions, and discuss the significant advantages it offers. In Section 2.5, we explore the consequences of working in a reduced symmetry, owing to the finite-volume and discretisation of spacetime, and give the construction of operators respecting said symmetries. We follow up in Section 2.6, with the techniques used to extract the discrete spectrum of states from correlation functions. Lastly, in Section 2.7, we present the formalism necessary to calculate infinite-volume continuum QCD scattering amplitudes from the energy eigenstates of the discretised QCD Hamiltonian.

\section{$2.1 \quad$ QCD}

The QCD action can be written,

$$
\mathrm{S}_{\mathrm{QCD}}=\int d^{4} x\{\underbrace{-\frac{1}{4} \sum_{a=1}^{8} \mathrm{~F}_{\mu \nu}^{a} \mathrm{~F}^{a \mu \nu}}_{S_{G}[A]}+\underbrace{\sum_{f} \sum_{i, j=1}^{3} \sum_{\alpha, \beta=1}^{4} \bar{q}_{i, \alpha}^{f}(x)\left[i\left(\gamma^{\mu}\right)_{\alpha \beta}\left(\mathcal{D}_{\mu}\right)_{i j}-m_{f} \delta_{i j} \delta_{\alpha \beta}\right] q_{j, \beta}^{f}(x)}_{S_{F}[A, \bar{q}, q]}\}
$$

where, for the gauge part of the action $S_{G}[A], \mathrm{F}_{\mu \nu}^{a}=\partial_{\mu} \mathrm{A}_{\nu}^{a}-\partial_{\nu} \mathrm{A}_{\mu}^{a}-g_{s} f^{a b c} \mathrm{~A}_{\mu}^{b} \mathrm{~A}_{\nu}^{c}$ is the field strength tensor, $f^{a b c}$ are $\mathrm{SU}(3)$ structure constants, $g_{s}$ is the strong coupling constant and $\mathrm{A}_{\mu}^{a}$ are the gauge fields with $a=1, \ldots, 8$ indexing the adjoint representation of $\mathrm{SU}(3)$. 
In the fermionic part of the action $S_{F}[A, \bar{q}, q],\left(\gamma^{\mu}\right)_{\alpha \beta}$ are the usual Dirac- $\gamma$ matrices and $\left(\mathcal{D}_{\mu}\right)_{i j}=\delta_{i j} \partial_{\mu}+i g_{s} \mathrm{~A}_{\mu}^{a} \lambda_{i j}^{a} / 2$ is the gauge covariant derivative where $\lambda_{i j}^{a}$ are the Gell-Mann matrices and $(i) j=1, \ldots, 3$ is the colour index in the (anti)fundamental representation. For the quark fields $q_{j, \beta}^{f}, \beta$ is the spinor index and $f$ is flavour of which there are six: up, down, strange, charm, bottom and top. $m_{f}$ is a bare quark mass. Since we are interested in light hadron spectroscopy, we restrict to the lightest three flavours, up, down and strange, for the remainder of this thesis.

In QCD there are a number of global, approximate and discrete symmetries that enable us to simultaneously diagonalise the QCD Hilbert space and label hadronic states according to the eigenvalues of the corresponding generators. In particular, it is often convenient to label hadrons $\left(I^{G}\right) J^{P C}$, following the conventions of the PDG 5 .

The Poincaré group, $\mathcal{P}$, which is the semi-direct product of translations and Lorentz transformations, $\mathcal{P}=\mathcal{T}^{1,3} \rtimes \mathrm{O}(1,3)$, is a global symmetry of QCD. The irreducible representations of the subgroup of rotations $\mathrm{SO}(3) \subset \mathrm{O}(1,3)$ that leave massive states invariant are labelled by total angular momentum $J$.

Total isospin, $I$, is the result of an approximate $\mathrm{SU}(2)_{\mathrm{F}}$ flavour symmetry in which the up and down quark masses are assumed to be identical and the quarks are indistinguishable in strong interactions. When isospin symmetry is assumed, the up and down quarks are collectively referred to as the 'light' quarks. Often, a larger $\mathrm{SU}(3)_{\mathrm{F}}$ flavour symmetry, extending the approximate indistinguishability of the up and down quarks to include the strange, is assumed - this is badly broken in reality. Isospin alone is not sufficient to label the irreducible flavour representations of $\mathrm{SU}(3)_{\mathrm{F}}$, owing to the two distinct Casimir invariants, and we will discuss $\mathrm{SU}(3)_{\mathrm{F}}$ flavour symmetry in more detail in Chapters 3,4 and 6 .

Regarding the discrete symmetries, $P$ labels parity and $C$ corresponds to the chargeconjugation parity, defined for neutral hadrons. For hadrons with hypercharge zero but $z$-component of isospin $I_{z} \neq 0$, the $C$-parity operator can be augmented through a prior rotation of $\pi$ about the $y$-component of isospin to give the $G$-parity operator, $\hat{G}=\hat{C} e^{i \pi \hat{I}_{y}}$. This gives a multiplicative quantum number, $G$, that is conserved in strong interactions, assuming isospin is a good symmetry!

\footnotetext{
${ }^{1}$ Hypercharge, $Y$, is defined as $Y=2\left(Q-I_{z}\right)$ where $Q$ is the electric charge.
} 
In the path integral formulation of QCD, correlation functions are given by,

$$
\begin{aligned}
\langle\mathcal{O}\rangle & =\frac{1}{\mathcal{Z}} \int \mathcal{D}[A] \mathcal{D}[\bar{q}, q] \mathcal{O} e^{i \mathrm{~S}_{\mathrm{QCD}}[A, \bar{q}, q]} \\
\mathcal{Z} & =\int \mathcal{D}[A] \mathcal{D}[\bar{q}, q] e^{i \mathrm{~S}_{\mathrm{QCD}}[A, \bar{q}, q]}
\end{aligned}
$$

where $\mathcal{Z}$ is the partition function, $\mathcal{D}[A]$ and $\mathcal{D}[\bar{q}, q]$ are the gauge and fermion integration measures respectively and $\mathcal{O}$ is some gauge-invariant operator, in general consisting of fermion and gauge fields. Typically, an operator is constructed that respects the symmetries of QCD in order to interpolate hadronic states of specific $\left(I^{G}\right) J^{P C}$ quantum numbers and subsequently calculate their properties. As QCD is asymptotically free at high energies, techniques in perturbation theory enable the calculation of these correlation functions to some order in $g_{s}$. However, at the low-energies relevant for hadron spectroscopy, perturbation theory breaks down due to the large coupling constant. One very successful non-perturbative approach in computing correlation functions is lattice QCD, where, by performing a Wick rotation in time $t \rightarrow-i t$ and regularising the theory on a discrete finite grid of Euclidean spacetime points, the path integral can be calculated numerically. The Euclidean action is given by,

$$
\mathrm{S}_{\mathrm{QCD}}^{\mathrm{E}}=\int d^{4} x\{\underbrace{\frac{1}{4} \sum_{a=1}^{8} \mathrm{~F}_{\mu \nu}^{a} \mathrm{~F}^{a \mu \nu}}_{S_{G}^{\mathrm{E}}[A]}+\underbrace{\sum_{f} \sum_{i, j=1}^{3} \sum_{\alpha, \beta=1}^{4} \bar{q}_{i, \alpha}^{f}(x)\left[\left(\gamma_{\mathrm{E}}^{\mu}\right)_{\alpha \beta}\left(\mathcal{D}_{\mu}\right)_{i j}+m_{f} \delta_{i j} \delta_{\alpha \beta}\right] q_{j, \beta}^{f}(x)}_{S_{\mathrm{F}}^{\mathrm{E}}[A, \bar{q}, q]}\}
$$

where $\gamma_{\mathrm{E}}$ are Euclidean $\gamma$-matrices, i.e. $\gamma_{\mathrm{E}}^{\mu}=\left(\gamma_{\mathrm{E}}^{\mu}\right)^{\dagger}$ and $\left\{\gamma_{\mathrm{E}}^{\mu}, \gamma_{\mathrm{E}}^{\nu}\right\}=2 \delta_{\mu \nu}$. It follows that the Euclidean correlation functions are given by,

$$
\begin{aligned}
\langle\mathcal{O}\rangle_{\mathrm{E}} & =\frac{1}{\mathcal{Z}_{\mathrm{E}}} \int \mathcal{D}[A] \mathcal{D}[\bar{q}, q] \mathcal{O}_{\mathrm{E}} e^{-\mathrm{S}_{\mathrm{QCD}}^{\mathrm{E}}[A, \bar{q}, q]} \\
\mathcal{Z}_{\mathrm{E}} & =\int \mathcal{D}[A] \mathcal{D}[\bar{q}, q] e^{-\mathrm{S}_{\mathrm{Q} \mathrm{ED}}^{\mathrm{E}}[A, \bar{q}, q]}
\end{aligned}
$$

For brevity, we drop the 'E' label as we will only consider the Euclidean action and correlation functions for the remainder of this work. 


\subsection{Lattice QCD}

The purpose of LQCD is to provide a framework in which to calculate QCD numerically in a manner in which finite-volume and discretisation effects are under control and, in the limit that the discretisation spacing is taken to zero and the volume of the lattice taken to infinity, infinite-volume continuum QCD is recovered. In this section, we will present the general approach for discretising the QCD action, considering separately the fermionic $S_{\mathrm{F}}[A, \bar{\psi}, \psi]$ and gauge $S_{\mathrm{G}}[A]$ actions, before discussing in more detail the specific improvements and adaptations relevant for generating the gauge fields used in our calculations.

To begin, we define an isotropic hypercubic grid of Euclidean spacetime points $\Sigma$,

$$
\Sigma=\left\{x=\text { an } \mid n \in \mathbb{Z}_{L / a_{s}} \times \mathbb{Z}_{L / a_{s}} \times \mathbb{Z}_{L / a_{s}} \times \mathbb{Z}_{T / a_{t}}\right\}
$$

where $a=a_{s}=a_{t}$ is the lattice spacing, equal in the spatial $a_{s}$ and temporal $a_{t}$ directions, and where $L$ and $T$ are the spatial and temporal extents respectively. Elements $x \in \Sigma$ are referred to as lattice sites and the edges which connect neighbouring sites are referred to as lattice links.

\subsubsection{Discretising the Fermion Action}

Consider first $S_{\mathrm{F}}[A, \bar{\psi}, \psi]$. Restricting the fermion fields to the lattice sites, the $O\left(a^{2}\right)$ discretised partial derivative is given by,

$$
\partial_{\mu} \psi(x)=\frac{\psi(x+a \hat{\mu})-\psi(x-a \hat{\mu})}{2 a}
$$

where $\hat{\mu}$ is a unit vector in the direction $\mu$. In the action, this gives rise to terms that are not gauge invariant, for example under a gauge transformation, the term $\bar{\psi}(x) \psi(x+a \hat{\mu})$ becomes $\bar{\psi}(x) \Omega^{\dagger}(x) \Omega(x+a \hat{\mu}) \psi(x+a \hat{\mu})$ where $\Omega(x) \in \mathrm{SU}(3)$ and $\Omega^{\dagger}(x) \Omega(y) \neq \mathbb{I}$ for $x \neq y$ in general.

To reinstate gauge invariance, link variables, $U_{\mu}(x)$, are introduced, directed along the links from site $x$ to $x+a \hat{\mu}$. Link variables orientated in the opposite direction from site $x+a \hat{\mu}$ to $x$ are defined through the hermitian conjugate, $U_{-\mu}(x+a \hat{\mu})=U_{\mu}(x)^{\dagger}$. Formulated in this way, defining the gauge transformation on $U_{\mu}(x)$ as,

$$
U_{\mu}(x) \rightarrow \Omega(x) U_{\mu}(x) \Omega^{\dagger}(x+a \hat{\mu})
$$


the action can be augmented to recover gauge invariance by appropriately inserting ordered products of $U_{\mu}(x)$ between fermion fields to connect the sites, for instance $\bar{\psi}(x) U_{\mu}(x) \psi(x+$ $a \hat{\mu})$ pertaining to the example above.

Link variables are in fact fundamentally related to the gauge fields in the continuum. They obey the same transformation properties as the continuum gauge transporter, the Wilson line, and as such are interpreted as the gauge transporters on the lattice. The relation with the gauge fields $A_{\mu}(x)$ is given by $U_{\mu}(x)=\exp \left(\operatorname{iag}_{s} A_{\mu}(x)\right)$.

Taking the fermionic part of the QCD action from the expression in Eq. 2.3, and discretising according to the constructions given above, appropriately inserting the link variables to preserve gauge invariance, the discretised fermion action reads,

$$
S_{\mathrm{F}}[U, \bar{\psi}, \psi]=a^{4} \sum_{x \in \Sigma} \sum_{f \in\{u, d, s\}} \sum_{i, j=1}^{3} \sum_{\alpha, \beta=1}^{4} \bar{\psi}_{i, \alpha}^{f}(x)\left[\left(\gamma^{\mu}\right)_{\alpha \beta}\left(\nabla_{\mu}\right)_{i j}+m_{f} \delta_{i j} \delta_{\alpha \beta}\right] \psi_{j, \beta}^{f}(x)
$$

where

$$
\left(\nabla_{\mu}\right)_{i j} \psi_{j, \beta}^{f}(x)=\frac{1}{2 a}\left(U_{\mu}(x)_{i j} \psi_{j, \beta}^{f}(x+a \hat{\mu})-U_{\mu}(x-a \hat{\mu})_{i j}^{\dagger} \psi_{j, \beta}^{f}(x-a \hat{\mu})\right)
$$

and where the $\int d^{4} x$ has been replaced by the sum $a^{4} \sum$. Expanding the link variables in powers $a$, it is straightforward to show that $S_{\mathrm{F}}[U, \bar{\psi}, \psi]=S_{\mathrm{F}}[A, \bar{\psi}, \psi]+O\left(a^{2}\right)$.

A fundamental problem that arises from this naïve discretisation of the fermion action in Eq. 2.8 is 'fermion doubling'. This can be seen most easily in the inversion of the free discretised Dirac operator that appears in the fermion propagator. In momentum space, this is given by 13$]$,

$$
\left(\gamma^{\mu} \nabla_{\mu}+m_{f}\right)^{-1}(p)=\frac{m_{f} \mathbb{I}-i a^{-1} \sum_{\mu} \gamma^{\mu} \sin \left(p_{\mu} a\right)}{m_{f}^{2}+a^{-2} \sum_{\mu} \sin ^{2}\left(p_{\mu} a\right)}
$$

and as $a \rightarrow 0,\left(\gamma^{\mu} \nabla_{\mu}+m_{f}\right)^{-1}(p)$ has the correct pole at $p^{2}=-m_{f}^{2}$. However, shifting the momenta within the Brillouin zone, $p_{\mu} \rightarrow p_{\mu}+\frac{\pi}{a} \hat{\mu}$, gives an additional pole for each unique translation to the vertices of the hypercube, bringing the total number to sixteen.

There are many modifications to the action one could choose to ameliorate the problem of fermion doublers and we introduce a Wilson term, resembling a second order gaugecovariant derivative, that adjusts the mass of the doublers to $O(1 / a)$. In the continuum limit, these become infinitely massive and decouple from the theory. This modifies Eq. 2.8 
giving the Wilson fermion action,

$$
S_{\mathrm{F}}^{\mathrm{W}}[U, \bar{\psi}, \psi]=a^{4} \sum_{x \in \Sigma} \sum_{f \in\{u, d, s\}} \sum_{i, j=1}^{3} \bar{\psi}_{i}^{f}(x)[\gamma^{\mu} \underbrace{\left\{\left(\nabla_{\mu}\right)_{i j}-\frac{a}{2} \gamma_{\mu}\left(\Delta_{\mu}\right)_{i j}\right\}}_{\left(W_{\mu}\right)_{i j}}+m_{f} \delta_{i j}] \psi_{j}^{f}(x)
$$

where

$$
\left(\Delta_{\mu}\right)_{i j} \psi_{j}^{f}(x)=\frac{1}{a^{2}}\left(U_{\mu}(x)_{i j} \psi_{j}^{f}(x+a \hat{\mu})+U_{\mu}(x-a \hat{\mu})_{i j}^{\dagger} \psi_{j}^{f}(x-a \hat{\mu})-2 \psi_{i}^{f}(x)\right) .
$$

Spinor indices have been dropped for brevity. Correcting for the fermion doublers by adding the Wilson term reintroduces discretisation errors at $O(a)$ thus $S_{\mathrm{F}}^{\mathrm{W}}[U, \bar{\psi}, \psi]=$ $S_{\mathrm{F}}[A, \bar{\psi}, \psi]+O(a)$.

\subsubsection{Discretising the Gauge Action}

Now we are in a position to consider $S_{\mathrm{G}}[A]$. The cyclic property of the trace ensures that the trace of any closed Wilson line, i.e. a Wilson loop, is gauge-invariant. Within the lattice framework, Wilson loops are expressed as an ordered finite product of link variables that form a closed boundary to some surface, the simplest being the plaquette consisting of four link variables bounding a planar square,

$$
U_{\mu \nu}(x)=U_{\mu}(x) U_{\nu}(x+a \hat{\mu}) U_{\mu}(x+a \hat{\nu})^{\dagger} U_{\nu}(x)^{\dagger} .
$$

Combining the product of exponentials using the Baker-Campbell-Hausdorff (BCH) formula, $U_{\mu \nu}(x)=\exp \left[i a^{2} g_{s} F_{\mu \nu}(x)+O\left(a^{3}\right)\right]$ and hence,

$$
\begin{aligned}
S_{\mathrm{G}}[U] & =\beta \sum_{x \in \Sigma} \sum_{\mu<\nu} \operatorname{Re} \operatorname{tr}\left[\mathbb{I}-U_{\mu \nu}(x)\right] \\
& =\frac{\beta}{2} \sum_{x \in \Sigma} \sum_{\mu, \nu} \frac{1}{2} \operatorname{tr}\left[\mathbb{I}+U_{\mu \nu}(x) U_{\mu \nu}^{\dagger}(x)\right]-\frac{1}{2} \operatorname{tr}\left[U_{\mu \nu}(x)+U_{\mu \nu}^{\dagger}(x)\right] \\
& =\frac{\beta}{4} \sum_{x \in \Sigma} \sum_{\mu, \nu} \operatorname{tr}\left[\left(\mathbb{I}-U_{\mu \nu}(x)\right)\left(\mathbb{I}-U_{\mu \nu}(x)\right)^{\dagger}\right] \\
& =a^{4} \sum_{x \in \Sigma} \sum_{\mu, \nu} \sum_{a=1}^{8}\left[\frac{1}{4} F_{\mu \nu}^{a}(x) F_{\mu \nu}^{a}(x)+O\left(a^{2}\right)\right]
\end{aligned}
$$

where $\beta=2 / g_{s}^{2}$ and $S_{\mathrm{G}}[U]=S_{\mathrm{G}}[A]+O\left(a^{2}\right)$. 
The Wilson fermion action and gauge action, given above, have discretisation errors at $O(a)$ and $O\left(a^{2}\right)$. In the following sections, we present a summary of the Symanzik improvement program [14] which we use to systematically improve the discretisation errors to $O\left(a^{2}\right)$ and $O\left(a^{4}\right)$ respectively.

Furthermore, we ultimately work with anisotropic lattices, where the temporal lattice spacing is finer than the spatial one, $a_{t}<a_{s}$. This improves the resolution of time-dependent correlation functions that encode the energies of the finite-volume QCD Hamiltonian, while avoiding the large computational cost of a lattice finer in all directions. The modifications to the Symanzik improved fermionic and gauge action for an anisotropic lattice are also detailed below.

\subsubsection{Symanzik-Improved Anisotropic Wilson Fermion Action}

The Symanzik improvement scheme offers a systematic way of introducing irrelevant operators into the lattice action that remove the undesirable discretisation artefacts to some order. Sheikholeslami and Wohlert [15] showed for the Wilson fermion action that $O(a)$ and $O\left(a^{2}\right)$ improvement, restricted to on-shell quantities such as hadron masses and scattering amplitudes, can be achieved by appropriately adding irrelevant operators with mass dimension-5 and 6 respectively.

For the Wilson fermion action in Eq. 2.10, the $O(a)$ discretisation errors can be removed by adding a single dimension-5 operator, referred to as the 'clover term 2 , of the form, $-\frac{a}{2} C_{\mathrm{SW}} \bar{\psi} \sigma^{\mu \nu} F_{\mu \nu} \psi$, to the fermion action. Explicitly,

$$
\begin{aligned}
F_{\mu \nu}(x) & =-\frac{i}{2 a^{2}}\left(Q_{\mu \nu}(x)-Q_{\mu \nu}^{\dagger}(x)\right) \\
4 Q_{\mu \nu}(x) & =U_{\mu}(x) U_{\nu}(x+a \hat{\mu}) U_{\mu}^{\dagger}(x+a \hat{\nu}) U_{\nu}^{\dagger}(x) \\
& +U_{\nu}(x) U_{\mu}^{\dagger}(x-a \hat{\mu}+a \hat{\nu}) U_{\nu}^{\dagger}(x-a \hat{\mu}) U_{\mu}(x-a \hat{\mu}) \\
& +U_{\mu}^{\dagger}(x-a \hat{\mu}) U_{\nu}^{\dagger}(x-a \hat{\mu}-a \hat{\nu}) U_{\mu}(x-a \hat{\mu}-a \hat{\nu}) U_{\nu}(x-a \hat{\nu}) \\
& +U_{\nu}^{\dagger}(x-a \hat{\nu}) U_{\mu}(x-a \hat{\nu}) U_{\nu}(x+a \hat{\mu}-a \hat{\nu}) U_{\mu}^{\dagger}(x)
\end{aligned}
$$

\footnotetext{
${ }^{2}$ The layout of the link variables resembles a four-leaf clover.
} 
where $Q_{\mu \nu}=Q_{\nu \mu}^{\dagger}$. To obtain the correct $O(a)$ improvement, Sheikholeslami and Wohlert 15 showed $C_{\mathrm{SW}}=1$. For $O\left(a^{2}\right)$ improvement, there are significantly more dimension- 6 irrelevant operators required which introduce next-to-nearest neighbour interactions and feature fermionic contact terms that are considerably more complicated to incorporate in numerical calculations. The resulting $O(a)$ Symanzik improved Wilson fermion action is,

$$
S_{\text {impr. F }}^{\mathrm{W}}[U, \bar{\psi}, \psi]=a^{4} \sum_{x \in \Sigma} \sum_{f \in\{u, d, s\}} \sum_{i, j=1}^{3} \bar{\psi}_{i}^{f}(x)\left[\gamma^{\mu}\left(W_{\mu}\right)_{i j}+m_{f} \delta_{i j}-\frac{a}{2} \sum_{\mu>\nu} \sigma^{\mu \nu}\left(F_{\mu \nu}\right)_{i j}\right] \psi_{j}^{f}(x) .
$$

For an anisotropic lattice, the fermionic action is given by splitting the isotropic action into spatial and temporal components as follows [16,

$$
\begin{aligned}
S_{\mathrm{F}}^{\xi}[U, \hat{\bar{\psi}}, \hat{\psi}]=\sum_{x \in \Sigma} \sum_{f \in\{u, d, s\}} \sum_{i, j=1}^{3} \hat{\bar{\psi}}_{i}^{f}(x)\left[v_{t} \gamma_{t}\left(\hat{W}_{t}\right)_{i j}+\frac{v_{s}}{\xi} \sum_{s} \gamma_{s}\left(\hat{W}_{s}\right)_{i j}+\hat{m}_{f} \delta_{i j}\right. \\
\left.-\frac{1}{2}\left(C_{\mathrm{SW}}^{t} \sum_{s} \sigma_{t s}\left(\hat{F}_{t s}\right)_{i j}+\frac{C_{\mathrm{SW}}^{s}}{\xi} \sum_{s>s^{\prime}} \sigma_{s s^{\prime}}\left(\hat{F}_{s s^{\prime}}\right)_{i j}\right)\right] \hat{\psi}_{j}^{f}(x)
\end{aligned}
$$

where hats denote dimensionless quantities, $\hat{\psi}=a_{s}^{3 / 2} \psi, \hat{\bar{\psi}}=a_{s}^{3 / 2} \bar{\psi}, \hat{W}_{\mu}=a_{\mu} W_{\mu}, \hat{\nabla}_{\mu}=$ $a_{\mu} \nabla_{\mu}, \hat{\Delta}_{\mu}=a_{\mu}^{2} \Delta_{\mu}, \hat{F}_{\mu \nu}=a_{\mu} a_{\nu} F_{\mu \nu}$ and where $\xi$ is the desired renormalised anisotropy, $\xi=a_{s} / a_{t}$.

In order to determine coefficients $v_{t}, v_{s}, C_{\mathrm{SW}}^{t}$ and $C_{\mathrm{SW}}^{s}$ to recover $O(a)$ improvement, the method of $v_{s}$-tuning or $v_{t}$-tuning is employed $[16]$. For the lattice actions used in this thesis, $v_{s}$-tuning is used in which $v_{t}=1$ and the clover parameters are found to be $C_{\mathrm{SW}}^{s}=v_{s}$ and $C_{\mathrm{SW}}^{t}=\frac{1}{2}\left(v_{s}+\frac{1}{\xi}\right)$.

As a final modification, three-dimensional stout-smeared [17] and tadpole-improved [18 gauge links are incorporated into the fermion action. Stout-smearing suppresses the mixing with high energy gluon modes in an analytic fashion by repeatedly smoothening the gauge fields using perpendicular staples, products of three link variables connecting sites $x$ and $x+a \hat{\mu}$. Importantly, the stout-smearing is restricted to the spatial staples, with no smearing in time, ensuring the transfer matrix remains physical. Tadpole improvement is a procedure that removes UV divergences that can appear in the gauge links by dividing out the mean field value $\tilde{u}_{\mu}, \tilde{U}_{\mu}(x) \rightarrow \tilde{U}_{\mu}(x) / \tilde{u}_{\mu}$ (no sum on $\mu$ ). The tilde denotes that 
the links are stout-smeared as described above. The choice we take for $\tilde{u}_{\mu}$ is to set $\tilde{u}_{t}=1$, a good estimate when $a_{t} \leq a_{s} / 2$ [19], and calculate $\tilde{u}_{s}$ by taking the fourth root of the spatial plaquette [19] finding $\tilde{u}_{s}=0.9267$ [20,21].

Empirical evidence suggests that the ratio $u_{t} / u_{s}$ provides a tree-level estimate of the renormalisation of the anisotropy [16], thus by defining $\xi=\frac{u_{t}}{u_{s}} \xi_{0}$ and then stout-smearing the tadpole improved gauge-links, the resulting fermion action can be written,

$$
\begin{aligned}
S_{\mathrm{F}}^{\xi}[U, \hat{\bar{\psi}}, \hat{\psi}]=\sum_{x \in \Sigma} & \sum_{f \in\{u, d, s\}} \sum_{i, j=1}^{3} \hat{\bar{\psi}}_{i}^{f}(x) \frac{1}{\tilde{u}_{t}}\left[\gamma_{t}\left(\hat{W}_{t}\right)_{i j}+\frac{v_{s}}{\xi_{0}} \sum_{s} \gamma_{s}\left(\hat{W}_{s}\right)_{i j}+\tilde{u}_{t} \hat{m}_{f} \delta_{i j}\right. \\
& \left.-\frac{1}{2}\left(\frac{1}{2}\left(v_{s}+\frac{1}{\xi}\right) \frac{1}{\tilde{u}_{s}^{2} \tilde{u}_{t}} \sum_{s} \sigma_{t s}\left(\hat{F}_{t s}\right)_{i j}+\frac{v_{s}}{\xi_{0}} \frac{1}{\tilde{u}_{s}^{3}} \sum_{s>s^{\prime}} \sigma_{s s^{\prime}}\left(\hat{F}_{s s^{\prime}}\right)_{i j}\right)\right] \hat{\psi}_{j}^{f}(x)
\end{aligned}
$$

consistent with Eq. (8) in Ref. [20], where the bare fermion anisotropy is defined as $\gamma_{f}=\xi_{0} / v_{s}$ and the bare gauge anisotropy $\gamma_{g}=\xi_{0}$. The tuning of the parameters $\gamma_{f}$ and $\gamma_{g}$ is given in Ref. [20] and is such that the renormalised gauge and fermion anisotropies, $\xi_{g} \approx \xi_{f} \sim 3.5$. We will show explicit calculations of $\xi_{f}$ in later chapters using the relativistic dispersion relation for stable hadrons.

By systematically constructing the anisotropic fermion action in this way, it is straightforward to see that in the limit that $a_{t} \rightarrow a_{s}$, where $\xi_{0}=\xi=v_{s}=1$, the isotropic action given in Eq. 2.14 is recovered.

\subsubsection{Symanzik-Improved Anisotropic Gauge Action}

The gauge action given in Eq. 2.12 has discretisation errors of $O\left(a^{2}\right)$. Following a similar approach to the fermion action, the Symanzik improvement program adds irrelevant operators of dimension- 6 to correct the $O\left(a^{2}\right)$ errors at tree-level where the dimension- 6 operators are configurations of Wilson loops with 6 link variables. Lüscher and Weisz characterise all such operators in Fig. 1b-1d of Ref. [22] and derive a set of constraints on the tree-level coefficients that must be satisfied to attain $O\left(a^{2}\right)$ improvement - see Eq. (37) and (38) of Ref. 22. In addition, to ensure the classical vacuum $U_{\mu}(x)=\mathbb{I}$ for all $x, \mu$ is indeed the true vacuum, $S_{\mathrm{G}}[U]>0$ for all gauge configurations $U$ and this gives further constraints on the coefficients in the form of inequalities as discussed in Appendix A of Ref. [22]. 
One convenient choice of coefficients that we adopt, yielding $O\left(a^{2}\right)$ improvement and maintaining positivity of $S_{\mathrm{G}}[U]$, gives the action,

$$
S_{\mathrm{G}}[U]=\beta \sum_{x \in \Sigma} \sum_{\mu<\nu}\left(\frac{5}{3} \mathcal{P}_{\mu \nu}(x)-\frac{1}{12} \mathcal{R}_{\mu \nu}(x)-\frac{1}{12} \mathcal{R}_{\nu \mu}(x)\right)
$$

where $\mathcal{P}_{\mu \nu}(x)=\operatorname{Re} \operatorname{tr}\left(1-U_{\mu \nu}(x)\right)$ and $\mathcal{R}_{\mu \nu}(x)=\operatorname{Re} \operatorname{tr}\left(1-R_{\mu \nu}(x)\right)$ with $R_{\mu \nu}(x)$ denoting $1 \times 2$ planar rectangular Wilson loops.

Moving to an anisotropic lattice, we again repeat the action in the temporal and spatial components, analogous to the fermion action. Transcribed from Eq. (84) in Ref. [19], this gives,

$$
\begin{aligned}
S_{\mathrm{G}}^{\xi}[U]=\beta \sum_{x \in \Sigma}\left[\frac{1}{\xi_{0}} \sum_{s<s^{\prime}}\right. & \left(\frac{5}{3} \frac{1}{u_{s}^{4}} \mathcal{P}_{s s^{\prime}}(x)-\frac{1}{12} \frac{1}{u_{s}^{6}} \mathcal{R}_{s s^{\prime}}(x)-\frac{1}{12} \frac{1}{u_{s}^{6}} \mathcal{R}_{s^{\prime} s}(x)\right) \\
& \left.+\xi_{0} \sum_{s}\left(\frac{5}{3} \frac{1}{u_{s}^{2} u_{t}^{2}} \mathcal{P}_{s t}(x)-\frac{1}{12} \frac{1}{u_{s}^{2} u_{t}^{4}} \mathcal{R}_{s t}(x)-\frac{1}{12} \frac{1}{u_{s}^{4} u_{t}^{2}} \mathcal{R}_{t s}(x)\right)\right]
\end{aligned}
$$

where the coefficients are chosen such that this action has discretisation errors at $O\left(a_{s}^{4}, a_{t}^{4}\right)$. The gauge links have been tadpole-improved (but not stout-smeared) and the tadpole factors, calculated in Ref. [20], are $u_{t}=1$ and $u_{s}=0.7336$. The two-length rectangular Wilson loops in time modify the transfer matrix making it non-hermitian, leading to problems in the spectral decomposition of two-point correlations functions [23]. This is potentially problematic when we using the variational method, discussed later, to extract energies as the sum of decaying exponentials.

To circumvent this and recover a physical transfer matrix, these length-two temporal Wilson loops in time are removed from the action and the coefficients slightly modified to ensure $O\left(a_{s}^{4}\right)$ improvement, resulting in 20,

$$
\begin{aligned}
S_{\mathrm{G}}^{\xi}[U]=\beta \sum_{x \in \Sigma}\left[\frac{1}{\xi_{0}} \sum_{s<s^{\prime}}\right. & \left(\frac{5}{3} \frac{1}{u_{s}^{4}} \mathcal{P}_{s s^{\prime}}(x)-\frac{1}{12} \frac{1}{u_{s}^{6}} \mathcal{R}_{s s^{\prime}}(x)-\frac{1}{12} \frac{1}{u_{s}^{6}} \mathcal{R}_{s^{\prime} s}(x)\right) \\
& \left.+\xi_{0} \sum_{s}\left(\frac{4}{3} \frac{1}{u_{s}^{2} u_{t}^{2}} \mathcal{P}_{s t}(x)-\frac{1}{12} \frac{1}{u_{s}^{4} u_{t}^{2}} \mathcal{R}_{t s}(x)\right)\right]
\end{aligned}
$$

This action has discretisation errors of $O\left(a_{s}^{4}, a_{t}^{2}\right)$, which is reasonable when $a_{t} \ll a_{s}$, the case for the anisotropic lattices we use. 


\section{$2.3 \quad$ Numerical Simulation}

Having appropriately discretised the QCD action, the correlation function given in Eq. 2.4 becomes,

$$
\begin{aligned}
\langle\mathcal{O}\rangle & =\frac{1}{\mathcal{Z}} \int \prod_{x, \mu} d U_{\mu}(x) \prod_{i, \alpha, f} d \bar{\psi}_{i}^{f}(x) d \psi_{\alpha}^{f}(x) \mathcal{O}[U, \bar{\psi}, \psi] e^{-S_{\mathrm{F}}^{\xi}[U, \bar{\psi}, \psi]-S_{\mathrm{G}}^{\xi}[U]} \\
\mathcal{Z} & =\int \prod_{x, \mu} d U_{\mu}(x) \prod_{i, \alpha, f} d \bar{\psi}_{\alpha}^{f}(x) d \psi_{i}^{f}(x) e^{-S_{\mathrm{F}}^{\xi}[U, \bar{\psi}, \psi]-S_{\mathrm{G}}^{\xi}[U]}
\end{aligned}
$$

where for clarity, colour $(i)$, spin $(\alpha)$ and flavour $(f)$ indices have been written explicitly, $(x)$ labels the spacetime sites and $d U$ is the Haar measure on $\mathrm{SU}(3)$.

The fermionic integral can be performed analytically observing that the discretised fermion action is a quadratic form in $\bar{\psi}, \psi$,

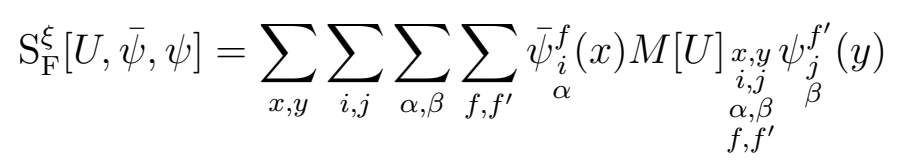

where $M[U]$ is the matrix in spacetime, colour, spin and (diagonal) in flavour, corresponding to the expression in Eq. 2.16. Grassmannian integration gives,

$$
\int \prod_{\boldsymbol{r}} d \bar{\psi}_{\boldsymbol{r}} d \psi_{\boldsymbol{r}} e^{-\bar{\psi} M[U] \psi}=\operatorname{det}_{\boldsymbol{r}} M[U]
$$

where the bold index $\boldsymbol{r}=(x, i, \alpha, f)$ is a multi-index for spacetime, colour, spin and flavour and the sums and indices in the exponential have been suppressed for brevity. Introducing products of the form $\psi_{\boldsymbol{n}} \bar{\psi}_{\boldsymbol{m}}$ in the integral,

$$
\int \prod_{\boldsymbol{r}} d \bar{\psi}_{\boldsymbol{r}} d \psi_{\boldsymbol{r}} \psi_{\boldsymbol{n}} \bar{\psi}_{\boldsymbol{m}} e^{-\bar{\psi} M[U] \psi}=\operatorname{det}_{\boldsymbol{r}} M[U]\left(M[U]^{-1}\right)_{\boldsymbol{n} \boldsymbol{m}}
$$

results in quark propagators $\left(M[U]^{-1}\right)_{n m}$.

After integrating out the fermionic degrees of freedom, the correlation and partition functions become,

$$
\begin{aligned}
\langle\mathcal{O}\rangle & =\frac{1}{\mathcal{Z}} \int \prod_{x, \mu} d U_{\mu}(x) \operatorname{det} M[U] e^{-S_{G}^{\xi}[U]} \mathcal{O}\left[U, f\left(M^{-1}[U]\right)\right] \\
\mathcal{Z} & =\int \prod_{x, \mu} d U_{\mu}(x) \operatorname{det} M[U] e^{-S_{\mathrm{G}}^{\xi}[U]}
\end{aligned}
$$


respectively, where $f\left(M^{-1}[U]\right)$ denotes some function of fermionic propagators. An example for a two-point correlation function is given in Eq. 2.32 in Section 2.4.

The gauge action we constructed is positive for all gauge configurations, as discussed in Section 2.2.4, and as such the integrands in Eq. 2.24 are peaked around configurations maximising $\exp \left\{-S_{\mathrm{G}}^{\xi}[U]\right\}$, i.e. $U=\mathbb{I}$, and exponentially suppressed for configurations away from the identity. We can exploit this exponential weight factor to numerically approximate the integrals using Monte Carlo integration through importance sampling. We calculate the average of the observable $\mathcal{O}\left[U, f\left(M^{-1}[U]\right)\right]$ on a set of gauge configurations $\left\{U_{n}\right\}_{n=1}^{N_{\text {cfgs }}}$,

$$
\langle\mathcal{O}\rangle=\frac{1}{N_{\text {cfgs }}} \sum_{n=1}^{N_{\text {cfgs }}} \mathcal{O}\left[U_{n}, f\left(M^{-1}\left[U_{n}\right]\right)\right]+O\left(\frac{1}{\sqrt{N_{\text {cfgs }}}}\right)
$$

by drawing $U_{n}$ from the probability distribution $\mathcal{P}[U]$ with density,

$$
d \mathcal{P}[U]=\frac{\prod_{x, \mu} d U_{\mu}(x) \operatorname{det} M[U] e^{-S_{\mathrm{G}}^{\xi}[U]}}{\int \prod_{x, \mu} d U_{\mu}(x) \operatorname{det} M[U] e^{-S_{\mathrm{G}}^{\xi}[U]}}
$$

One can systematically improve the statistical precision of LQCD calculations by increasing the number of independent gauge configurations in the sample. We will give the explicit $N_{\text {cfgs }}$ used when discussing the computational details in the relevant chapters. The statistical error on $\langle\mathcal{O}\rangle$ is calculated using jackknife resampling,

$$
\sigma^{2}=\frac{N_{\text {cfgs }}-1}{N_{\text {cfgs }}} \sum_{i=1}^{N_{\text {cfgs }}}\left(\hat{\mathcal{O}}_{i}-\langle\mathcal{O}\rangle\right)^{2}
$$

where $\hat{\mathcal{O}}_{i}=\frac{1}{N_{\text {cfgs }}-1} \sum_{j=1, i \neq j}^{N_{\text {cfgs }}} \mathcal{O}\left[U_{j}, f\left(M^{-1}\left[U_{j}\right]\right)\right]$. All statistical uncertainties are calculated through jackknife resampling unless otherwise stated.

Suitable sets of gauge field configurations for use in this thesis have been constructed using a Rational Hybrid Monte Carlo (RHMC) algorithm, by the Hadron Spectrum Collaboration, for $N_{f}=3$ and $N_{f}=2+1$ flavours of dynamical quarks. The implementations are given in Refs. 20] and 21] respectively.

\subsection{Distillation Smearing}

The primary aim of hadron spectroscopy is to study the low-energy degrees of freedom within QCD. Extracting the low-lying energies from correlation functions computed using 
hadron operators built directly from the fermion fields is difficult, as the correlation function has significant contributions from many excited states. By first smearing the fermion fields on each time-slice, a technique widely used within LQCD calculations in which some linear operator is first applied to the fermion fields with the effect of suppressing the high-energy modes, the calculation of the low-lying spectrum is significantly improved as the asymptotic behaviour of the correlation functions is realised at earlier times.

A commonly used smearing technique, preserving all the symmetries of the lattice, is the Jacobi smearing method [24], in which the gauge-covariant Laplacian is iteratively applied to the fermion fields. The Jacobi smearing operator can be written [25],

$$
J_{\sigma, n_{\sigma}}(t)=\left(1+\frac{\sigma \nabla^{2}(t)}{n_{\sigma}}\right)^{n_{\sigma}}
$$

where $\sigma \geq 0$ and $n_{\sigma}>0$ are tunable parameters and the lattice gauge-covariant Laplacian is

$$
-\nabla_{\boldsymbol{x} \boldsymbol{y}}^{2}(t)=6 \delta_{\boldsymbol{x} \boldsymbol{y}}-\sum_{j=1}^{3}\left(\tilde{U}_{j}(\vec{x}, t)_{a b} \delta_{\vec{x}+\hat{j}, \vec{y}}+\tilde{U}_{j}^{\dagger}(\vec{x}-\hat{j}, t)_{a b} \delta_{\vec{x}-\hat{j}, \vec{y}}\right)
$$

where the multi-index $\boldsymbol{x}=(\vec{x}, a)$ runs over space and colour and $\tilde{U}_{j}(\vec{x}, t)_{a b}$ are the stoutsmeared, tadpole-improved gauge links discussed in Section 2.2.3.

In the limit that $n_{\sigma} \rightarrow \infty$, the Jacobi smearing operator in Eq. 2.28 becomes $J_{\sigma, n_{\sigma}}(t)=$ $\exp \left(\sigma \nabla^{2}(t)\right)$. As $\nabla_{\boldsymbol{x} \boldsymbol{y}}^{2}(t)$ is negative semi-definite, it is clear that the smearing operator exponentially suppresses the high-energy modes. As such, an approximation to the smearing operator can be made by forming an eigenvector representation of $\nabla_{\boldsymbol{x} \boldsymbol{y}}^{2}(t)$, truncated to the lowest few eigenmodes, $\left\{\xi_{x}^{(k)}(t)\right\}_{k=1}^{N_{\text {vecs }}}$ and ordered by magnitude of eigenvalue 25]. The subspace spanned by these lowest modes is referred to as the distillation space with rank $N_{\text {vecs }}$. The distillation operator, $\square_{x y}(t)$, projecting the spatial and colour degrees of freedom of the fermion fields into distillation space is,

$$
\square_{\boldsymbol{x} \boldsymbol{y}}(t)=\sum_{k=1}^{N} \xi_{\boldsymbol{x}}^{(k)}(t) \xi_{\boldsymbol{y}}^{(k) \dagger}(t)
$$

No smearing in time ensures quarks are localised on a single time-slice as necessary for the variational analysis of correlation functions (see Section 2.6). 
In this thesis, all correlation functions are computed within the distillation framework. There are many advantages of utilising distillation smearing and we now provide an example to illustrate some of these.

Consider a two-point correlation function computed on a single gauge configuration, constructed from a fermion bilinear creation operator $(\bar{\psi} \Gamma \psi)^{\dagger}$ at time $t_{\mathrm{src}}$ and annihilated at later time $t$. Furthermore, the operator is projected onto zero-momentum at both source and sink, giving

$$
C\left(t, t_{\mathrm{src}}\right)=\left\langle\sum_{\vec{x}}\{\bar{\psi} \Gamma \psi\}(\vec{x}, t) \cdot \sum_{\vec{y}}\{\bar{\psi} \Gamma \psi\}^{\dagger}\left(\vec{y}, t_{\mathrm{src}}\right)\right\rangle .
$$

Integrating out the fermion fields according to Eq. 2.23, we find,

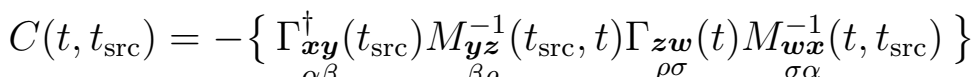

$$
\begin{aligned}
& \underbrace{\alpha \beta \quad \beta \sigma \quad \sigma \alpha}_{\text {connected }} \\
& +\{\underbrace{\left.\Gamma_{\boldsymbol{\alpha} \boldsymbol{y}}^{\dagger}\left(t_{\mathrm{src}}\right) M_{\boldsymbol{y} \boldsymbol{x}}^{-1}\left(t_{\mathrm{src}}, t_{\mathrm{src}}\right)\right\}\left\{\underset{\alpha \beta}{\Gamma_{\boldsymbol{x} \boldsymbol{y}}(t) M_{\boldsymbol{\beta} \boldsymbol{x}}^{-1}(t, t)}\right\}}_{\text {disconnected }}
\end{aligned}
$$

where the trace is over space and colour $(\boldsymbol{x})$ and spin $(\alpha)$. After projecting the quark fields into distillation space, the two-point correlation function becomes,

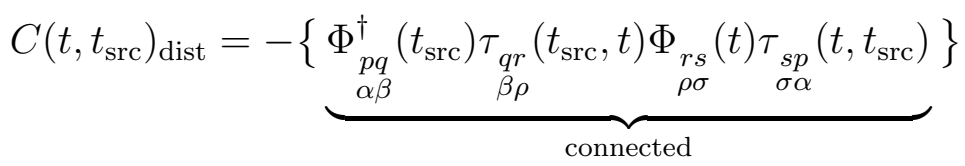

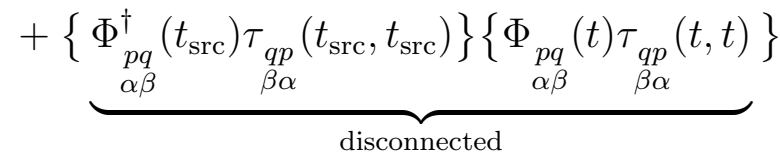

where

$$
\Phi_{p q}(t)=\sum_{\boldsymbol{x}, \boldsymbol{y}} \xi_{\boldsymbol{x}}^{\dagger(p)}(t) \Gamma_{\boldsymbol{\alpha} \boldsymbol{y}}(t) \xi_{\boldsymbol{y}}^{(q)}(t)
$$

are referred to as elementals and

$$
\tau_{p q}\left(t, t_{\mathrm{src}}\right)=\sum_{\boldsymbol{x}, \boldsymbol{y}} \xi_{\boldsymbol{x}}^{\dagger(p)}(t) M_{\alpha \beta}^{-1}\left(t, t_{\mathrm{src}}\right) \xi_{\boldsymbol{y}}^{(q)}\left(t_{\mathrm{src}}\right)
$$

as perambulators and the trace is now over the much smaller distillation space $(p)$ and spin $(\alpha)$. These elementals and perambulators can be stored on disk and reused for subsequent calculations. 


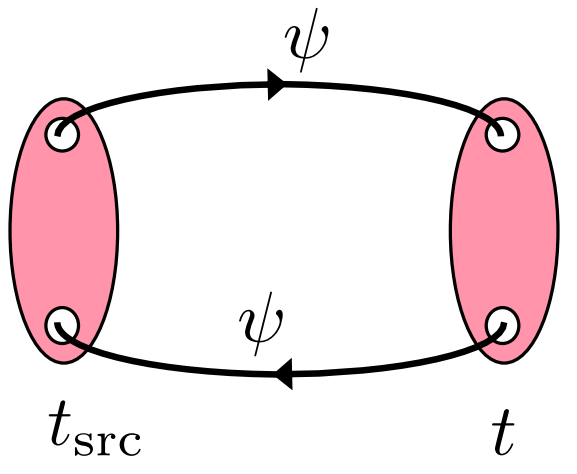

(a) Connected, $\mathcal{C}\left(t, t_{\text {src }}\right)$
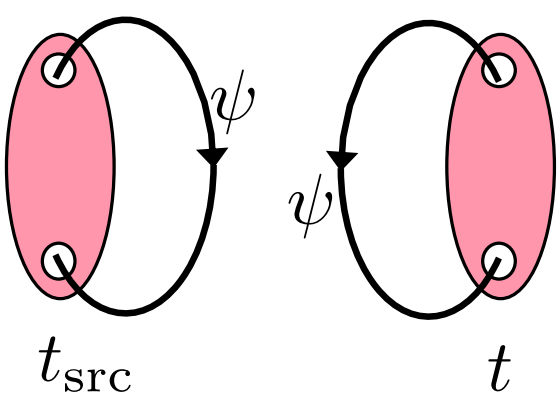

(b) Disconnected, $\mathcal{D}\left(t, t_{\mathrm{src}}\right)$

Figure 2.1: Quark propagation relevant for the two-point correlation function constructed using a fermion bilinear operator, $\bar{\psi} \Gamma \psi$, at source and sink. Shown separately are the 'connected' and 'disconnected' diagrams.

Constructing the perambulators requires inversions of the Dirac matrix. The connected contributions, corresponding to Wick contractions that feature quark propagation from $t_{\text {src }}$ to $t$ and vice-versa, as shown in Figure 2.1a, with definite momentum projection at both source and sink require the inverse of the Dirac matrix, $M^{-1}\left(t_{\mathrm{src}}, t\right)$. We compute the 'backwards' propagator, $M^{-1}\left(t, t_{\mathrm{src}}\right)$ using $\gamma_{5}$-hermiticity of the propagators, $M^{-1}\left(t, t_{\mathrm{src}}\right)=$ $\gamma_{5}\left\{M^{-1}\left(t_{\mathrm{src}}, t\right)\right\}^{\dagger} \gamma_{5}$. The disconnected contributions, Wick contractions that feature no quark propagation between source and sink as shown in Figure 2.1b, require the evaluation of $M^{-1}\left(t_{\mathrm{src}}, t_{\mathrm{src}}\right)$ and $M^{-1}(t, t)$. In our implementation, in order to maximise the signal-tonoise of the disconnected contributions, $\mathcal{D}\left(t, t_{\mathrm{src}}\right)$, for a given time separation, $t-t_{\mathrm{src}}$, we average over all source time slices for the entire temporal extent of the lattice [26], i.e.

$$
\frac{1}{N_{t}} \sum_{t^{\prime}=0}^{N_{t}-1} \mathcal{D}\left(t+t^{\prime}, t_{\mathrm{src}}+t^{\prime}\right)
$$

where $N_{t}=T / a_{t}$. The connected contributions, $\mathcal{C}\left(t, t_{\mathrm{src}}\right)$, are less noisy and are averaged, typically, over a smaller number of time-sources, $N_{\text {tsrcs }}$, equispaced around the lattice 3 Together, the connected and disconnected contributions necessitate the computation of all-to-all propagators, i.e. the full inverse of the Dirac matrix.

Operationally, computing correlation functions featuring disconnected contributions in distillation space significantly reduces the computational costs. For a typical moderate sized

\footnotetext{
${ }^{3}$ We give the $N_{\text {tsrcs }}$ used in the calculations in the following chapters.
} 
lattice used in this thesis, inverting the full Dirac matrix results in $M=\left(L / a_{s}\right)^{3} \times N_{c} \times N_{s}=$ $20^{3} \times 3 \times 4 \sim 10^{5}$ inversions per time-slice compared with $N_{\text {vecs }} \times N_{s}=128 \times 4 \ll M$ inversions required in distillation space, as we need only to invert off the lowest $N_{\text {vecs }}$ vectors.

Previous calculations have set a benchmark for the rank, $N_{\text {vecs }}$, of the distillation space sufficient to reliably extract the low-lying spectrum on a given lattice volume, see Figure 3 of Ref. 25]. It is also shown in Figure 5 of the same reference that the rank of the distillation space scales like the spatial volume. We use these observations to choose an appropriate $N_{\text {vecs }}$ for each lattice volume in our calculations and give this rank explicitly in the following chapters.

\subsection{Symmetries of the Lattice}

Performing calculations in a finite-volume on a discrete grid of points breaks the Poincaré symmetry of the infinite-volume continuum. In particular, for an anisotropic lattice with a cubic spatial volume and periodic boundary conditions, the subgroup $\mathcal{T}^{3} \rtimes \mathrm{O}(3) \subset \mathcal{P}$ of spatial translations, rotations and reflections is broken to that of $\mathcal{T}_{\text {latt }}^{3} \rtimes \mathrm{O}_{h}^{\mathrm{D}}$ 27 where $\mathcal{T}_{\text {latt }}^{3}$ is the group of discrete translational symmetries and $\mathrm{O}_{h}^{\mathrm{D}}$ is the double cover of the octahedral group. In this work, we will consider only integer-spin, as relevant for mesons, and restrict to the single cover $\mathrm{O}_{h}$. The spatially periodic boundary conditions quantise the momenta, restricting it to values $\vec{P}=(2 \pi / L)\left(n_{x}, n_{y}, n_{z}\right)$ where $n_{i} \in \mathbb{Z}$. We use a shorthand notation when labelling momenta in which the $2 \pi / L$ factor is omitted, e.g. $\vec{P}=\left[n_{x}, n_{y}, n_{z}\right]$ or $\left[n_{x} n_{y} n_{z}\right]$.

For lattice frames at non-zero overall momenta, $\vec{P} \neq \overrightarrow{0}$, the octahedral group is further broken to the little groups of symmetries, i.e. subgroups of $\mathrm{O}_{h}$ denoted $\operatorname{LG}(\vec{P})$, that preserve the momentum $\vec{P}$. For $|\vec{P}|^{2} \leq 4$, the little groups $\operatorname{LG}(\vec{P}) \subset \mathrm{O}_{h}$ are the point groups, $\mathrm{C}_{n v}$ : cyclic groups of order $n$ with the inclusion of $n$ reflection planes containing the principal axis of rotation. $]^{4}$

The infinite-volume irreducible representations, irreps, of $\mathrm{O}(3)$, labelled $\left(J^{P}, m\right)$ where $m$ is the projection of $J$ along the $z$-axis, are not irreps of $\mathrm{O}_{h}$, of which there are a finite number [8]. Subsequently, the infinite tower of irreps $\left(J^{P}, m\right)$ are distributed across the

\footnotetext{
${ }^{4}$ In the case of $\mathrm{O}_{h}^{D}$, point groups $\mathrm{C}_{n v}$ are replaced with $\mathrm{Dic}_{n}$ : dicyclic groups of order $4 n$.
} 
finite number of irreps of $\mathrm{O}_{h}$, which we label $\left(\Lambda^{P}, \mu\right)$ with $\Lambda$ the irrep and $\mu$ the row within that irrep $5^{5}$

For lattice frames in-flight, in which the hadronic system has non-zero overall momentum with respect to the lattice, parity is no longer a good quantum number and continuum irreps, labelled $(J, \lambda)$ where the helicity $\lambda$ is the projection of angular momentum along $\vec{P}$, are distributed across the finite number of irreps, $\vec{P} \Lambda$, of $\operatorname{LG}(\vec{P})$. The reduced symmetry and mixing of parities in these in-flight systems leads to a distribution pattern in $J$ that is typically more dense than for irreps at rest.

The distribution of total angular momentum $J$ amongst the lattice irreps is commonly referred to as subduction. The subduction coefficients, $\mathcal{S}_{\Lambda, \mu}^{J, m}$, are defined as the inner product of lattice and continuum states,

$$
\mathcal{S}_{\Lambda, \mu}^{J, m}=\langle J, m \mid \Lambda, \mu\rangle
$$

and are used to give the linear combination of continuum states that transform irreducibly on the lattice, i.e. $|\Lambda, \mu\rangle=\sum_{m} \mathcal{S}_{\Lambda, \mu}^{J, m}|J, m\rangle$. Acting on lattice states by elements of $\mathrm{O}_{h}$, or the relevant little group of symmetries $\operatorname{LG}(\vec{P})$ for non-zero momentum, simply mixes the rows of the irrep, much like $\mathrm{SO}(3)$ rotations mix the $z$-components of spin, $m$, or helicities, $\lambda$, in the infinite-volume continuum.

To construct two-particle states that transform irreducibly, appropriate linear combinations of products of single-particle states are required. For continuum states, these linear combinations are given through $\mathrm{SO}(3)$ Clebsch-Gordan coefficients $\mathcal{C}(\ldots)$ for $J_{1} \otimes J_{2} \rightarrow J$,

$$
|J, m\rangle=\sum_{m_{1}, m_{2}} \mathcal{C}\left(\begin{array}{ccc}
J_{1} & J_{2} & J \\
m_{1} & m_{2} & m
\end{array}\right)\left|J_{1}, m_{1}\right\rangle\left|J_{2}, m_{2}\right\rangle
$$

whereas for lattice states at rest,

$$
|\Lambda, \mu\rangle=\sum_{\mu_{1}, \mu_{2}} \mathbb{C}\left(\begin{array}{lll}
\Lambda_{1} & \Lambda_{2} & \Lambda \\
\mu_{1} & \mu_{2} & \mu
\end{array}\right)\left|\Lambda_{1}, \mu_{1}\right\rangle\left|\Lambda_{2}, \mu_{2}\right\rangle
$$

where $\mathbb{C}(\ldots)$ are lattice Clebsch-Gordan coefficients for $\Lambda_{1} \otimes \Lambda_{2} \rightarrow \Lambda$ [28]. The subduction coefficients and lattice Clebsch-Gordan coefficients we use in this thesis follow from the conventions set out in Refs. [10,29,30].

\footnotetext{
${ }^{5}$ The rows, $\mu$, are analogous to the $z$-component of angular momentum, $m$.
} 
Having established the relationship between the irreducible representations of $\mathrm{O}(3)$ and $\mathrm{O}_{h}$, it is straightforward to construct operators that transform irreducibly on the lattice. For a continuum operator, $\mathcal{O}^{J, m}(\vec{P}, t)$, with definite quantum numbers, constructed on timeslice $t$ and projected onto definite momentum $\vec{P}$, the lattice operator, transforming irreducibly under the symmetry of the lattice grid and boundary, $\mathcal{O}^{\dagger \Lambda \mu}(\vec{P}, t)$, is given by,

$$
\mathcal{O}^{\dagger \Lambda \mu}(\vec{P}, t)=\sum_{m} \mathcal{S}_{\Lambda \mu}^{J m} \mathcal{O}^{\dagger J m}(\vec{P}, t)
$$

where $m$ is replaced by helicity $\lambda$ for $\vec{P} \neq \overrightarrow{0}$. We will discuss in detail the operator constructions in the subsequent chapters.

\subsection{Extracting Energies and Spin Identification}

In order to robustly determine the discrete finite-volume energy eigenstates in each irrep, we first compute a large matrix of two-point correlation functions,

$$
C_{i j}(t)=\left\langle 0\left|\mathcal{O}_{i}(t) \mathcal{O}_{j}^{\dagger}(0)\right| 0\right\rangle
$$

by employing a diverse basis of operators $\mathcal{O}_{i}$, subduced into the relevant lattice irrep and constructed with the desired quantum numbers. The spectral decomposition, obtained by inserting a complete set of eigenstates of the finite-volume QCD Hamiltonian $|\mathfrak{n}\rangle$, is given as the sum of decaying exponentials,

$$
C_{i j}(t)=\sum_{\mathfrak{n}} \frac{1}{2 E_{\mathfrak{n}}}\left\langle 0\left|\mathcal{O}_{i}\right| \mathfrak{n}\right\rangle\left\langle\mathfrak{n}\left|\mathcal{O}_{j}^{\dagger}\right| 0\right\rangle e^{-E_{\mathfrak{n}} t}
$$

where $E_{\mathfrak{n}}$ is the energy of the $\mathfrak{n}^{\text {th }}$ eigenstate and $\left\langle\mathfrak{n}\left|\mathcal{O}_{i}^{\dagger}\right| 0\right\rangle \equiv Z_{i}^{\mathfrak{n}}$ are operator-state matrix elements, commonly referred to as overlap factors. As discussed in Section 2.5, continuum spins mix within lattice irreps and as such one cannot rigorously identify the spins of finite-volume eigenstates. However, if rotational symmetry is restored, lattice operators recover the continuum operator from which they were subduced. In this sense, for a lattice close to restoring rotational symmetry, lattice operators are expected to have relatively large operator-state overlaps onto the eigenstates with continuum spins from which they were subduced [29]. We will make use of these operator-state overlap factors in the following chapters. 
We extract the spectra in each irrep by applying the variational method [31], which solves the generalised eigenvalue problem,

$$
C_{i j}(t) v_{j}^{\mathfrak{n}}(t)=\lambda_{\mathfrak{n}}\left(t, t_{0}\right) C_{i j}\left(t_{0}\right) v_{j}^{\mathfrak{n}}(t)
$$

and we determine the energy levels $E_{\mathfrak{n}}$ in each irrep by fitting principal correlators $\lambda_{\mathfrak{n}}\left(t, t_{0}\right)$ to the form,

$$
\lambda_{\mathfrak{n}}\left(t, t_{0}\right)=\left(1-A_{\mathfrak{n}}\right) e^{-E_{\mathfrak{n}}\left(t-t_{0}\right)}+A_{\mathfrak{n}} e^{-E_{\mathfrak{n}}^{\prime}\left(t-t_{0}\right)} .
$$

The constants $A_{\mathfrak{n}}$ and $E_{\mathfrak{n}}^{\prime}$ soak up any residual excited state contamination and $t_{0}$ is appropriately chosen such that both $C\left(t_{0}\right)$ is saturated by the lowest $\operatorname{dim}(C)$ eigenstates and signal-to-noise is sufficiently large to extract $E_{\mathfrak{n}}$ from $\lambda_{\mathfrak{n}}\left(t, t_{0}\right)[32$. The advantage of working on an anisotropic lattice becomes clear here: a finer temporal spacing improves the resolution of the principal correlators and enables efficient disentangling of excited state energies.

By construction, $v_{i}^{\mathfrak{m} \dagger}(t) C_{i j}\left(t_{0}\right) v_{j}^{\mathfrak{n}}(t)=\delta_{\mathfrak{m} \mathfrak{n}}$ for all $t$ and the operator-state overlap factors relate to the eigenvectors via $Z_{j}^{\mathfrak{n}}(t)=v_{i}^{\mathfrak{n} \dagger}(t) C_{i j}\left(t_{0}\right) e^{E_{\mathfrak{n}} t_{0} / 2}$. In practice there is little variation in $Z_{i}^{\mathfrak{n}}(t)$, and subsequently $v_{i}^{\mathfrak{n}}(t)$, as a function of time for reasonable $t>t_{0}$. By choosing a suitable reference timeslice, $t_{Z}>t_{0}$, in which $C(t)$ is optimally reconstructed for $\operatorname{dim} C$ states via Eq. 2.41, we construct a variationally optimised operator, $\Omega_{\mathfrak{n}}^{\dagger}$, efficient at interpolating the $\mathfrak{n}^{\text {th }}$ eigenstate in the spectrum and given by $\Omega_{\mathfrak{n}}^{\dagger}=\sum_{i} v_{i}^{\mathfrak{n}}\left(t_{Z}\right) \mathcal{O}_{i}^{\dagger}$. These are used extensively as the building blocks of multi-meson operators as we will discuss in the following chapters.

Although $Z_{i}^{\mathfrak{n}}\left(t_{Z}\right)$ is perfectly suitable to quote as an operator-state overlaps factor, in practice, we fit $Z_{i}^{\mathfrak{n}}(t)$ to both a constant form, $Z_{i}^{\mathfrak{n}}(t)=A_{i}^{\mathfrak{n}}$, and to a constant plus a decaying exponential in time of the form,

$$
Z_{i}^{\mathfrak{n}}(t)=A_{i}^{\mathfrak{n}}+B_{i}^{\mathfrak{n}} e^{-C_{i}^{\mathfrak{n}}\left(t-t_{0}\right)}
$$

where $B_{i}^{\mathfrak{n}}$ and $C_{i}^{\mathfrak{n}}$ soak up residual excited state contamination. We take $A_{i}^{\mathfrak{n}}$ of the form that provides the best fit as the value of $Z_{i}^{\mathfrak{n}}(t)$.

\section{7 $\quad$ Scattering Formalism}

Within the lattice QCD framework, the periodic finite-volume means there are no free asymptotic states that are required in order to define a scattering system. Furthermore, 
any finite-volume eigenstate appears as a pole of the correlation function at a real value of energy, however, in the infinite-volume continuum any pole above the first multi-particle threshold is necessarily a resonance and must appear at complex energies. As such, above the first multi-particle threshold, there is no direct relation between energy eigenstates of the finite-volume QCD Hamiltonian and infinite-volume continuum states. This is commonly attributed to Maiani and Testa's no-go theorem [33].

Seminal work by Lüscher [34 36] in the mid 1980's, pre-dating the work of Maiani and Testa, related the infinite-volume scattering amplitudes of two identical scalar particles to finite-volume energy levels through a quantisation condition that has since been extended by many others 3745 to accommodate the most general two particle scattering systems. We describe the formalism below.

It is instructive to begin by introducing the $\boldsymbol{t}$-matrix for general two-to-two scattering processes, related to the unitary symmetric $\boldsymbol{S}$-matrix via $\boldsymbol{S}=\mathbf{1}+2 i \sqrt{\boldsymbol{\rho}} \cdot \boldsymbol{t} \cdot \sqrt{\boldsymbol{\rho}}$. Unitarity ensures conservation of probability and time-reversal invariance forces $\boldsymbol{S}$ to be symmetric. Here, $\boldsymbol{\rho}$ is a matrix of phase-space factors that we will return to later. The $\boldsymbol{t}$-matrix can be expressed as a function of the standard three Mandelstam variables $\boldsymbol{t}(s, t, u)$ where conservation of momentum imposes the relation $s+t+u=\sum_{i=1}^{4} m_{i}^{2}$ with $m_{1,2}$ and $m_{3,4}$ masses of the initial and final states respectively. For scattering particles with zero intrinsic spin, we can partial-wave project in the $s$-channel the components $t_{a b}(s, t, u)$, where $a, b$ label hadron-hadron channel, by expanding in a basis of Legendre polynomials $P_{\ell}(x)$,

$$
t_{a b}^{\ell}(s)=\frac{1}{2} \int_{-1}^{1} d x P_{\ell}(x) t_{a b}(s, t(x), u(x))
$$

where $t_{a b}^{\ell}(s)$ is the scattering amplitude in partial-wave $\ell$ and $x=\cos \left(\theta_{a b}\right)_{\mathrm{cm}}$ with $\left(\theta_{a b}\right)_{\mathrm{cm}}$ the scattering angle in the $\mathrm{cm}$-frame. In this case, as the scattering particles have zero intrinsic spin, $\ell$ and total angular momenta $J$ coincide and as such orbital angular momentum is conserved in the interaction. In general, non-zero intrinsic spin $S$ allows partial-waves of different $\ell$ to mix: for example, a vector-pseudoscalar pair such as $\rho \pi$ appears in $J^{P}=1^{+}$ in either a ${ }^{3} S_{1}$ - or ${ }^{3} D_{1}$-wave. We express $\boldsymbol{t}$ in the canonical $\ell S$ basis with components $t_{\ell S J m a, \ell^{\prime} S^{\prime} J^{\prime} m^{\prime} b}(s)=\delta_{J J^{\prime}} \delta_{m m^{\prime}} t_{\ell S a, \ell^{\prime} S^{\prime} b}(s)$. In this basis $\boldsymbol{\rho}$ is a diagonal matrix of phase-space factors with components,

$$
\rho_{\ell S J m a, \ell^{\prime} S^{\prime} J^{\prime} m^{\prime} b}=\delta_{\ell \ell^{\prime}} \delta_{S S^{\prime}} \delta_{J J^{\prime}} \delta_{m m^{\prime}} \delta_{a b} \frac{2 k_{\mathrm{cm}}^{(a)}}{\sqrt{s}}
$$


and the $\mathrm{cm}$-frame momentum $k_{\mathrm{cm}}^{(a)}$ for hadron-hadron channel $a$ is given by,

$$
k_{\mathrm{cm}}^{(a)}=\frac{1}{2 \sqrt{s}}\left(s-\left(m_{1}^{(a)}+m_{2}^{(a)}\right)^{2}\right)^{\frac{1}{2}}\left(s-\left(m_{1}^{(a)}-m_{2}^{(a)}\right)^{2}\right)^{\frac{1}{2}} .
$$

The quantisation condition relating infinite-volume scattering amplitudes to the finitevolume spectrum in a periodic $L \times L \times L$ box is best summarised by Eq. 22 of Ref. [44]. For the most general two-particle scattering systems, it can be written in the $\ell S$ basis as

$$
\underset{\ell S J m a}{\operatorname{det}}[\mathbf{1}+i \boldsymbol{\rho} \cdot \boldsymbol{t} \cdot(\mathbf{1}+i \overline{\mathbf{M}})]=0
$$

where $(\mathcal{M})^{44}=16 \pi \boldsymbol{t}$ and $\left(\delta G^{V}\right)^{[4]}=\frac{i}{16 \pi} \boldsymbol{\rho} \cdot(\mathbf{1}+i \overline{\mathcal{M}})$ relates this equation to the notation of Ref. [44] and 1 represents $\delta_{\ell \ell^{\prime}} \delta_{S S^{\prime}} \delta_{J J^{\prime}} \delta_{m m^{\prime}} \delta_{a b}$. The matrix of functions encapsulating the kinematics in a finite-volume is

$$
\begin{array}{rl}
\overline{\mathcal{M}}_{\ell S J m a, \ell^{\prime} S^{\prime} J^{\prime} m^{\prime} b}=\delta_{a b} \delta_{S S^{\prime}} \sum_{m_{\ell}, m_{\ell}^{\prime}, m_{S}} & \mathcal{C}\left(\begin{array}{ccc}
\ell & S & J \\
m_{\ell} & m_{S} & m
\end{array}\right) \mathcal{C}\left(\begin{array}{ccc}
\ell^{\prime} & S & J^{\prime} \\
m_{\ell}^{\prime} & m_{S} & m^{\prime}
\end{array}\right) \\
& \times \sum_{\bar{\ell}, \bar{m}_{\ell}} \frac{(4 \pi)^{3 / 2}}{\left[k_{\mathrm{cm}}^{(a)}\right]^{\bar{\ell}+1}} c_{\bar{\ell}, \bar{m}_{\ell}}^{\vec{n}}\left(\left[k_{\mathrm{cm}}^{(a)}\right]^{2} ; L\right) \int d \Omega Y_{\ell m_{\ell}}^{*} Y_{\bar{\ell} \bar{m}_{\ell}}^{*} Y_{\ell^{\prime} m_{\ell}^{\prime}},
\end{array}
$$

where $\overline{\mathcal{M}}$ is diagonal in intrinsic spin $S$ and hadron-hadron channel $a$. The volume dependence is encoded in the functions $c_{\ell, m_{\ell}}^{\vec{n}}\left(k_{\mathrm{cm}}^{2} ; L\right)$ which are defined as follows,

$$
c_{\ell, m_{\ell}}^{\vec{n}}\left(k_{\mathrm{cm}}^{2} ; L\right)=\frac{\sqrt{4 \pi}}{\gamma L^{3}}\left(\frac{2 \pi}{L}\right)^{\ell-2} Z_{\ell, m_{\ell}}^{\vec{n}}\left[1 ;\left(\frac{k_{\mathrm{cm}} L}{2 \pi}\right)^{2}\right], \quad Z_{\ell, m_{\ell}}^{\vec{n}}\left[d ; x^{2}\right]=\sum_{\vec{r} \in \mathcal{P}_{\vec{n}}} \frac{|\vec{r}|^{\ell} Y_{\ell, m_{\ell}}(\vec{r})}{\left.\left.|| \vec{r}\right|^{2}-x^{2}\right)^{d}},
$$

where the sum is over the elements of the set $\mathcal{P}_{\vec{n}}=\left\{\vec{r} \in \mathbb{R}^{3} \mid \vec{r}=\hat{\gamma}^{-1}(\vec{m}-\alpha \vec{n})\right\}, \vec{m}$ is an integer triplet and $\vec{n}$ is the normalised vector $\vec{n}=\frac{L}{2 \pi} \vec{P}$. The scale factor $\alpha=\frac{1}{2}\left[1+\frac{m_{1}^{2}-m_{2}^{2}}{\mathrm{~s}}\right]$ reflects the asymmetry for unequal masses of scattering particles. $\hat{\gamma}^{-1}$ denotes the Lorentz boost to the centre of momentum frame with $\hat{\gamma}^{-1} \vec{x} \equiv \gamma^{-1} \vec{x}_{\|}+\vec{x}_{\perp}$, where $\gamma=E_{\mathrm{lab}} / E_{\mathrm{cm}}$ and $\vec{x}_{\|}$and $\vec{x}_{\perp}$ are the components of $\vec{x}$ parallel and perpendicular respectively to the total momentum $\vec{P}$.

The product $\int d \Omega Y_{\ell m_{\ell}}^{*} Y_{\bar{\ell} \bar{m}_{\ell}}^{*} Y_{\ell^{\prime} m_{\ell}^{\prime}}$ can be written as a product of $\mathrm{SO}(3)$ Clebsch-Gordan 
coefficients,

$$
\begin{aligned}
\sqrt{4 \pi} \int d \Omega Y_{\ell m_{\ell}}^{*} Y_{\bar{\ell} \bar{m}_{\ell}}^{*} Y_{\ell^{\prime} m_{\ell}^{\prime}}=(-1)^{m_{\ell}} \sqrt{\frac{(2 \ell+1)\left(2 \ell^{\prime}+1\right)}{2 \bar{\ell}+1}} \\
\times \mathcal{C}\left(\begin{array}{ccc}
\ell^{\prime} & \ell & \bar{\ell} \\
0 & 0 & 0
\end{array}\right) \mathcal{C}\left(\begin{array}{ccc}
\ell^{\prime} & \ell & \bar{\ell} \\
m_{\ell}^{\prime} & -m_{\ell} & \bar{m}_{\ell}
\end{array}\right)
\end{aligned}
$$

which gives a much more numerically tractable form with obvious symmetries that can be readily exploited.

The quantisation condition for a given lattice irrep can be obtained by subducing the $(J, m)$ components into the irrep $\Lambda$, in the manner described in Section 2.5. For non-zero momentum, this can be implemented by first rotating to a helicity basis, and then using the helicity-based subduction coefficients presented in Table II of [10]. The subduction of $\overline{\mathcal{M}}$ takes the form,

$$
\overline{\mathcal{M}}_{\ell S J n a, \ell^{\prime} S^{\prime} J^{\prime} n^{\prime} b}^{\vec{n}, \Lambda} \delta_{\Lambda, \Lambda^{\prime}} \delta_{\mu, \mu^{\prime}}=\sum_{\substack{m, \lambda \\ m^{\prime}, \lambda^{\prime}}} \mathcal{S}_{\Lambda \mu n}^{J \lambda *} \mathcal{D}_{m \lambda}^{(J) *}(R) \overline{\mathcal{M}}_{\ell S J m a, \ell^{\prime} S^{\prime} J^{\prime} m^{\prime} b}^{\vec{n}} \mathcal{S}_{\Lambda^{\prime} \mu^{\prime} n^{\prime}}^{J^{\prime} \lambda^{\prime}} \mathcal{D}_{m^{\prime} \lambda^{\prime}}^{\left(J^{\prime}\right)}(R)
$$

where $R$ is the rotation, given explicitly in Table VI of [10], which takes the $m$ quantisation axis into the direction of $\vec{n}$. The subduction coefficients include the embedding index $n$, in the case that a partial-wave can appear more than once in an irrep. This will be discussed in more detail in the later chapters. Finally, we arrive at the subduced quantisation condition,

$$
\underset{\ell S J n a}{\operatorname{det}}\left[\mathbf{1}+i \boldsymbol{\rho} \cdot \boldsymbol{t} \cdot\left(\mathbf{1}+i \overline{\mathcal{M}}^{\vec{n}, \Lambda}\right)\right]=0,
$$

where 1 represents $\delta_{\ell \ell^{\prime}} \delta_{S S^{\prime}} \delta_{J J^{\prime}} \delta_{n n^{\prime}} \delta_{a b}$, and where the interpretation of multiple embeddings is that if a partial-wave appears $N$ times in irrep $\Lambda$, the $\boldsymbol{t}$-matrix for that partial-wave appears identically as $N$ block diagonal entries in $\boldsymbol{t}$.

In order to calculate the scattering amplitudes using this quantisation condition, we take the finite-volume energy levels computed in irrep $\Lambda$ and use them to determine the behaviour of $\boldsymbol{t}$ as a function of energy. As a simple example, take elastic scattering, where a single hadron-hadron channel appears in a single partial-wave. Unitarity of the 
$S$-matrix means the $t$-matrix, a complex scalar function, can be expressed in terms of a real energy-dependent phase-shift $\delta(s)$, where

$$
t(s)=\frac{1}{\rho(s)} e^{i \delta(s)} \sin \delta(s)=\frac{1}{\rho(s)} \frac{1}{\cot \delta(s)-i} .
$$

We can subsequently invert Eq. 2.53, with the definition of $t(s)$ given above, and obtain a one-to-one relation between $s$ and $\delta(s)$. This is used to calculate a discrete set of phase-shift points: one point at each determined finite-volume energy level in irrep $\Lambda$, below the inelastic threshold.

To interpolate $t(s)$ in the entire energy region of interest, we take a suitable parameterisation of $t(s)$ and, using Eq. 2.53, calculate the spectrum in each irrep $\Lambda$. We then vary the parameters until the resulting finite-volume spectrum optimally agrees with the lattice computed energies by minimising an appropriate $\chi^{2}$. As a test of robustness, we then repeat this procedure for many different forms of parameterisation. This approach generalises to inelastic scattering where a one-to-one relation between finite-volume energy levels and infinite-volume scattering amplitudes does not exist and we must parameterise the energy dependence of the $\boldsymbol{t}$-matrix.

The implementation and methodology will be described in much greater detail and developed throughout this thesis in the relevant sections. We will discuss, for example, the forms of the parameterisations, systematic tests of robustness and analysis of the amplitudes in the relevant chapters. 


\section{Scattering of $\rho \pi$ in isospin-2 at $m_{\pi} \sim 700 \mathrm{MeV}$}

Hadron spectroscopy is predominantly the investigation of resonances which decay strongly into hadrons, such as the pion, which are stable under the strong interaction. Many resonances which decay into multi-hadron final states do so through intermediate states featuring resonances of non-zero intrinsic spin. For example, the $J^{P C}=1^{++}$axial-vector $a_{1}(1260)$ meson dominantly decays into a $\pi \pi \pi$ final state through $\rho(770) \pi$, where the vector $\rho(770)$ decays into $\pi \pi$. Similarly, its $C$-parity counter-part, the $J^{P C}=1^{+-} b_{1}(1235)$, features dominant decays into $\pi \pi \pi \pi$ final states with an intermediate $\omega(782) \pi$. Once an intermediate hadron has non-zero intrinsic spin, it becomes possible for more than one partial-wave to be present for a given $J^{P}$ through the coupling of the orbital angular momentum $\ell$ to the intrinsic spin $S$. For example, in the case of both the $J^{P}=1^{+}$ axial-vectors, $a_{1}$ decaying to $\rho \pi$ and $b_{1}$ decaying to $\omega \pi$, the $\rho$ and $\omega$ have intrinsic spin $S=1$ and both $S$ and $D$-waves can contribute. Indeed it is possible to measure the relative decay amplitudes [46, 47].

While significant progress has been made studying meson-meson scattering using lattice QCD [48], calculations have not before accounted for the effects of dynamically-coupled partial-waves when processes feature scattering hadrons with non-zero intrinsic spin 1

An attempt was made in nucleon-nucleon scattering in the spin-triplet channel which has the same partial-wave decomposition as $\rho \pi$ scattering, and a closely related quantisation condition in finite-volume. $2^{2}$ A non-relativistic quantisation condition for $N N$ was presented

\footnotetext{
${ }^{1}$ Some recent work 49 , which has considered vector-pseudoscalar scattering in the light sector, makes brief comment on the possibility of contributions from dynamically-coupled partial-waves, but does not incorporate this in the analysis.

${ }^{2}$ There is a slightly smaller symmetry in $\rho \pi$ owing to the unequal masses of the $\rho$ and the $\pi$-altering the $\alpha$ in Eq. 2.50 .
} 
in Ref. [50], and an attempt to determine the ${ }^{3} S_{1} /{ }^{3} D_{1}$-wave mixing appeared in Ref. [51]. In this chapter, we report on the first calculation of the energy dependence of partial-wave scattering amplitudes for $\rho \pi$ in isospin-2, including the coupled $S$ and $D$-wave system with $J^{P}=1^{+}$. In this exploratory study, we work with heavier-than-physical light quarks, so the $\rho$ becomes stable against decay to $\pi \pi$. Specifically, we work at the $\mathrm{SU}(3)_{\mathrm{F}}$ flavour symmetric point with three degenerate flavours of quark $(u, d, s)$ tuned to have mass approximately equal to the physical strange quark mass, giving a pion mass $\sim 700 \mathrm{MeV}$. In this way we are justified in considering elastic $\rho \pi$ scattering provided we stay below the $\pi \pi \pi$ threshold $!^{3}$ Furthermore, the exotic isospin considered her $\biguplus^{4}$ leads us to expect that the $\rho \pi$ scattering amplitudes will be non-resonant and, based upon isospin- $2 \pi \pi$ scattering [28], they are likely to be relatively weak. A study of $\rho \pi$ scattering within a non-relativistic quark model 61] found weak, mainly repulsive scattering, with the ${ }^{3} S_{1}$ phase-shift being largest, but not exceeding $-35^{\circ}$, and a rather small mixing between the ${ }^{3} S_{1}$ and ${ }^{3} D_{1}$ partial-waves. As such, $\rho \pi$ scattering in isospin-2 makes for an ideal testing ground for which to develop the analysis tools and intuition for vector-pseudoscalar scattering in a lattice calculation.

The suspected weakness of the $\rho \pi$ interactions in isospin-2 will likely lead to small energy shifts in the finite-volume spectrum with respect to the 'non-interacting' energies expected were $\rho$ and $\pi$ to have no residual hadron-hadron interactions. It is therefore vital that the spectra be accurately and reliably calculated. This can be achieved by employing a large basis of interpolating operators, $\mathcal{O}_{i}$, constructed with the quantum numbers of isospin- $2 \rho \pi$, in order to compute a matrix of correlation functions and solve a generalised eigenvalue problem, as discussed in Section 2.6, to reliably extract the energy spectrum.

Specifically, for the case of vector-pseudoscalar scattering, the total intrinsic spin $S=1$ can couple with the orbital angular momentum $\ell$ to give three distinct total angular momenta $J$ for $\ell \geq 1$. In the absence of interactions, this gives rise to many degenerate energy levels - these may only be split slightly in the interacting case or not at all. A large operator basis containing appropriate operator structures is essential in order to disentangle these near-degenerate states.

\footnotetext{
${ }^{3}$ No suitably mature formalism and practical implementation for relating finite-volume spectra to three-body scattering amplitudes yet exists, but see $52,60$. for progress.

${ }^{4} \mathrm{~A}$ hadron of total isospin-2 must contain at least four light quarks and no substantial experimental evidence suggests such a resonant four-quark state exists in this energy range.
} 
We utilise the relevant symmetries of the finite volume when calculating correlation functions, as discussed in Section 2.5, which allows us to identify which partial-waves are contributing to each energy level. In a limited number of cases, an energy level is dominantly affected by a single partial-wave, elastic scattering, and here a value of the phase-shift for that partial-wave, at that energy, can be determined via a one-to-one mapping. More generally, an energy level is affected by multiple partial-waves and a more sophisticated analysis technique is required - the energy dependence of partial-wave amplitudes is parameterised and multiple energy levels are considered simultaneously. This approach is similar to that used in coupled-channel scattering [4, 62,64]. Significant constraints on scattering amplitudes come from spectra computed for systems with overall non-zero momentum with respect to the lattice, and indeed we find that the sign of the off-diagonal coupling between $S$-wave and $D$-wave can only be obtained from such 'in-flight' cases. We begin by examining the features of vector-pseudoscalar scattering in an infinite volume.

\subsection{Vector-Pseudoscalar Scattering}

In this section, we discuss the features of a scattering process that involves one or more hadrons with non-zero intrinsic spin. We explore the consequences for hadron-hadron scattering in an infinite volume and distinguish these from features that are purely a consequence of the finite volume. The results are illustrated through a discussion of vector-pseudoscalar scattering.

\subsubsection{Infinite Volume}

In an infinite-volume continuum, total angular momentum $J$ is a good quantum number and can be constructed by taking a tensor product of the orbital angular momentum $\ell$ with the total intrinsic spin $S$ (itself constructed via a tensor product of the intrinsic spins of the two scattering hadrons), i.e. $\ell \otimes S=|\ell-S| \oplus \ldots \oplus \ell+S$. Parity, $P$, is another good quantum number and is given by $P=\eta_{1} \eta_{2}(-1)^{\ell}$, where $\eta_{1}$ and $\eta_{2}$ are the intrinsic parities of the hadrons. It follows that, in some cases, hadron-hadron states with a particular $J^{P}$ 
can be formed from multiple $\ell S$ combinations.5

For the case of vector-pseudoscalar scattering, $S=1$, and thus, for $\ell \geq 1, J$ can take one of a triplet of values $J=\{\ell-1, \ell, \ell+1\}$. The intrinsic parities of vector and pseudoscalar mesons are each negative and it follows that each $J^{P}$ in the sequence, $1^{+}, 2^{-}, 3^{+}, \ldots$, can be formed from two distinct $\ell S$ combinations. In spectroscopic notation, ${ }^{2 S+1} \ell_{J}$, these are $\left\{{ }^{3} S_{1},{ }^{3} D_{1}\right\},\left\{{ }^{3} P_{2},{ }^{3} F_{2}\right\},\left\{{ }^{3} D_{3},{ }^{3} G_{3}\right\}, \ldots$ and the pattern continues indefinitely. For these $J^{P}$ values, even though the scattering process may only have a single hadron-hadron channel kinematically open, there are two partial-wave channels which can couple dynamically. For example, considering $J^{P}=1^{+}$, the $\boldsymbol{t}$-matrix, introduced in Section 2.7, can be written as,

$$
\begin{aligned}
\boldsymbol{t} & =\left[\begin{array}{cc}
t\left({ }^{3} S_{1} \mid{ }^{3} S_{1}\right) & t\left({ }^{3} S_{1} \mid{ }^{3} D_{1}\right) \\
t\left({ }^{3} S_{1} \mid{ }^{3} D_{1}\right) & t\left({ }^{3} D_{1} \mid{ }^{3} D_{1}\right)
\end{array}\right] \\
& =\frac{1}{2 i \rho}\left[\begin{array}{cc}
\cos (2 \bar{\epsilon}) \exp \left[2 i \delta^{3} S_{1}\right]-1 & i \sin (2 \bar{\epsilon}) \exp \left[i\left(\delta_{{ }^{3} S_{1}}+\delta^{3} D_{1}\right)\right] \\
i \sin (2 \bar{\epsilon}) \exp \left[i\left(\delta^{3} S_{1}+\delta^{3} D_{1}\right)\right] & \cos (2 \bar{\epsilon}) \exp \left[2 i \delta^{3} D_{1}\right]-1
\end{array}\right],
\end{aligned}
$$

where $\rho\left(E_{\mathrm{cm}}\right)=2 k_{\mathrm{cm}} / E_{\mathrm{cm}}$ is the phase-space factor and the second line presents the common Stapp-parameterisation [65] in terms of two phase-shifts, $\delta_{{ }^{3} S_{1}}\left(E_{\mathrm{cm}}\right), \delta_{{ }^{3} D_{1}}\left(E_{\mathrm{cm}}\right)$, and a mixing-angle, $\bar{\epsilon}\left(E_{\mathrm{cm}}\right)$, describing the dynamical coupling between the two channels ${ }^{6}$ The symmetric nature of the $\boldsymbol{t}$-matrix resulting from the time-reversal symmetry of QCD is seen explicitly.

This parameterisation automatically respects coupled-channel unitarity, expressed in this context as $\operatorname{Im}\left[t^{-1}\left(\left.{ }^{3} \ell_{J}\right|^{3} \ell_{J}^{\prime}\right)\right]=-\rho \delta_{\ell \ell^{\prime}}$ for energies above the threshold, where the phase-space is the same for both the ${ }^{3} S_{1}$ and ${ }^{3} D_{1}$ channels.7 Within the $\ell S$ basis, it can be shown through the partial-wave expansion in Eq. 2.45 that, in the absence of

${ }^{5}$ The choice of the $\ell S$ basis as opposed to, say, a helicity basis is one made for later convenience: it has the advantage that the threshold behaviour of $\ell S$ basis states is given in terms of the value of $\ell$.

${ }^{6}$ The sign of the off-diagonal entries, and hence the sign of $\bar{\epsilon}$, is physically relevant and impacts the spin and angular dependence of the scattering amplitudes. This is in contrast to the case where different hadronic channels are coupled - there the sign cannot be measured and it is usual to parameterise in terms of an inelasticity parameter which discards this sign information.

${ }^{7}$ When there are additional coupled channels featuring different scattering hadrons, $\rho\left(E_{\mathrm{cm}}\right)$ is diagonal in the channel space but no longer proportional to the identity as $k_{\mathrm{cm}}$ depends on the scattering hadron masses. 
any resonant enhancements, the threshold behaviour of the $\boldsymbol{t}$-matrix elements is simply $t\left(\left.{ }^{3} \ell_{J}\right|^{3} \ell_{J}^{\prime}\right) \propto\left(k_{\mathrm{cm}}\right)^{\ell+\ell^{\prime}}$.

\subsubsection{Finite Volume}

We perform calculations in a finite periodic cubic volume and this causes there to be 'mixing' between partial-waves of distinct $J^{P}$. We summarise the subduction of low-lying partial-waves for a vector-pseudoscalar system at $\vec{P}=\overrightarrow{0}$ in Table 3.1. The subduction is controlled only by values of $J^{P}$, but recall that in some cases multiple ${ }^{3} \ell_{J}$ constructions can give the same $J^{P}$. The table distinguishes these two possible types of 'mixing'. For systems with non-zero overall momentum $\vec{P}$, the partial-wave subductions for a vector-pseudoscalar system are presented in Tables 3.5-3.7 in Appendix 3.A.

In order to determine infinite-volume scattering amplitudes, we calculate finite-volume energy levels and utilise the quantisation condition, given in Eq. 2.53, which relates the two quantities. If, in a certain energy region, only one partial-wave has a non-negligible value, the relation takes the commonly-used form,

$$
\cot \delta\left(E_{\mathrm{cm}}\right)=-\cot \phi\left(E_{\mathrm{cm}}, L\right)
$$

where $\cot \phi\left(E_{\mathrm{cm}}, L\right)=-\overline{\mathcal{M}}\left(E_{\mathrm{cm}}, L\right)$ is a complex function, encoding the kinematical and symmetry-breaking effects of the finite volume. In this case, each finite-volume energy level can be used to determine the value of the partial-wave phase-shift at that particular energy. In the case of vector-pseudoscalar scattering, an example might be the rest-frame $E^{+}$irrep at energies near threshold. Here the ${ }^{3} D_{2}$ wave is expected to be much larger than the ${ }^{3} G_{4}$ wave, or any wave of still higher $\ell$, owing to the effect of the centrifugal barrier which ensures that $t\left(\left.{ }^{3} D_{2}\right|^{3} D_{2}\right) \sim\left(k_{\mathrm{cm}}\right)^{4} \gg t\left(\left.{ }^{3} G_{4}\right|^{3} G_{4}\right) \sim\left(k_{\mathrm{cm}}\right)^{8}$. If multiple energy levels can be obtained, from calculations on one or more volumes at rest and in-flight, repeated use of Eq. 3.2 will yield the energy-dependence of the phase-shift. A demonstration of this can be seen in $\pi \pi$ isospin-1 scattering in ${ }^{1} P_{1}$-wave - see Figure 10 in Ref. 30].

Where multiple partial-waves are present, but still only a single hadron-hadron channel is kinematically accessible, the quantisation condition for a given irrep is the determinant of a matrix equation. This encodes both the dynamical mixing of partial-waves (present even in an infinite volume), through $\boldsymbol{t}$, and the 'mixing' of partial-waves due to the finite volume, through $\overline{\mathcal{M}}$. For example, in the rest-frame $T_{1}^{+}$irrep, considering the partial-wave 


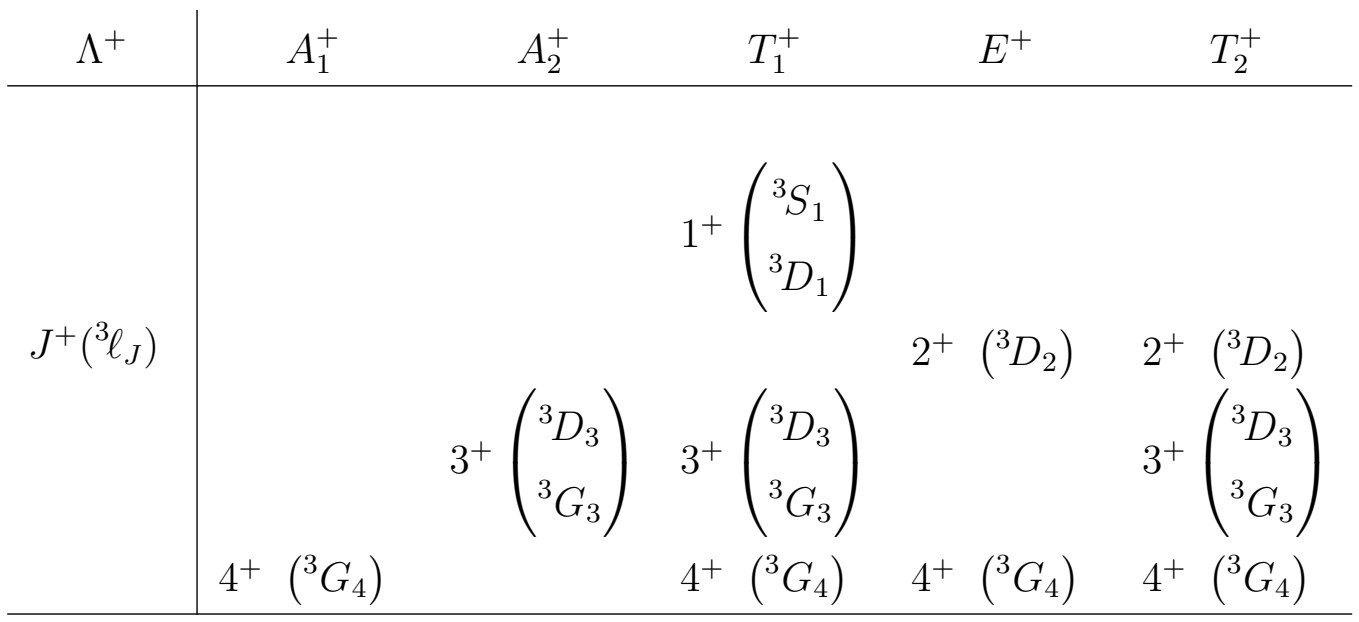

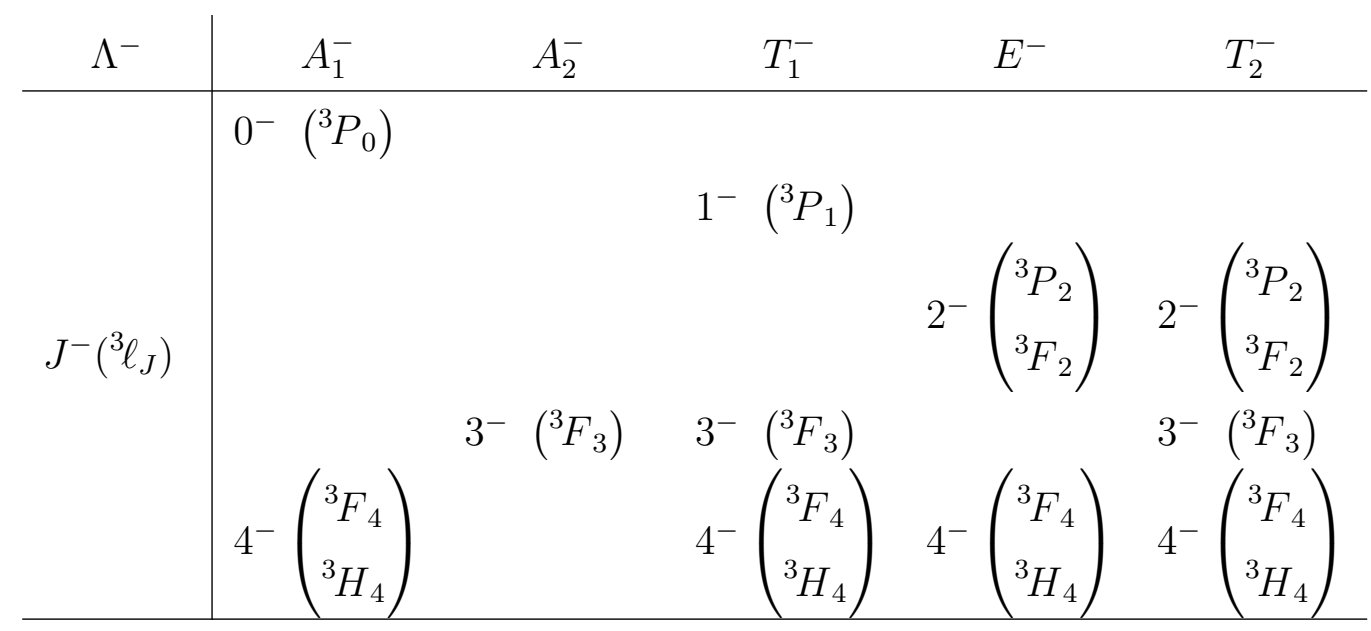

Table 3.1: Subduction of partial-waves, ${ }^{3} \ell_{J}$, for $J \leq 4$ into the irreps, $\Lambda^{P}$, of the octahedral group, $\mathrm{O}_{h}$, relevant for systems overall at rest. The notation $J^{P}\left({ }^{3} \ell_{J}\right)$ denotes the partial-wave content for a given $J^{P}$, with multiple ${ }^{3} \ell_{J}$ entries indicating partial-waves which mix dynamically. This table is derived from Table 2 of [8].

content with $\ell \leq 2$, we have dynamical mixing between the ${ }^{3} S_{1}$ and ${ }^{3} D_{1}$-waves with $J^{P}=1^{+}$. The $J^{P}=3^{+}$wave ${ }^{3} D_{3}$ 'mixes' with $1^{+}$only because of the reduced symmetry of the finite volume. The $\boldsymbol{t}$-matrix is,

$$
\boldsymbol{t}=\left[\begin{array}{ccc}
t\left({ }^{3} S_{1} \mid{ }^{3} S_{1}\right) & t\left({ }^{3} S_{1} \mid{ }^{3} D_{1}\right) & 0 \\
t\left({ }^{3} S_{1} \mid{ }^{3} D_{1}\right) & t\left({ }^{3} D_{1} \mid{ }^{3} D_{1}\right) & 0 \\
0 & 0 & t\left({ }^{3} D_{3} \mid{ }^{3} D_{3}\right)
\end{array}\right]
$$


where the off-diagonal contributions dynamically couple ${ }^{3} S_{1}$ and ${ }^{3} D_{1}$. The non-vanishing elements of $\overline{\mathcal{M}}$ in this $3 \times 3$ space ensure that all three waves contribute to the finite-volume spectrum. To accommodate additional partial-waves this $\boldsymbol{t}$-matrix is augmented by inserting diagonal blocks of $J^{P}$, where off-diagonal degrees of freedom within each block allow dynamical mixing between partial-waves of common $J^{P}$.

In the case of multiple partial-waves, coupled either dynamically or due to the finite volume, each energy level provides a constraint on the $\boldsymbol{t}$-matrix at that energy, through Eq. 2.53, but is not sufficient to determine the multiple (energy dependent) unknowns in $\boldsymbol{t}$. A number of such constraints, each coming from a different finite-volume energy level, are required to determine $\boldsymbol{t}\left(E_{\mathrm{cm}}\right)$. Considering systems with overall non-zero momentum is one way to obtain many energy levels 8 - the moving frame changes the spatial boundary conditions, which in turn modifies the quantisation condition giving a different set of functions in $\overline{\mathcal{M}}$. This is discussed in detail in Ref. [37, 39, 40] and has been successfully applied in determinations of coupled-channel $\boldsymbol{t}$-matrices in Refs. 4, 7, 62, 64, 66]. We will present the details of the approach, relevant to the current case of vector-pseudoscalar scattering, in Section 3.6 .

\subsection{Operator Construction}

To make a robust determination of the finite-volume energy spectrum in each irrep, we compute an $N \times N$ matrix of two-point correlation functions using $N$ independent interpolating operators with appropriate quantum numbers, $C_{i j}(t)=\left\langle 0\left|\mathcal{O}_{i}\left(t+t_{\mathrm{src}}\right) \mathcal{O}_{j}^{\dagger}\left(t_{\mathrm{src}}\right)\right| 0\right\rangle$.

In order to investigate meson-meson scattering, we need to construct an appropriate set of operator structures which overlap strongly onto the eigenstates of QCD in a finite volume with the quantum numbers of the meson-meson scattering problem. Operators which resemble meson-meson states, constructed as products of operators which resemble single mesons of definite momentum, prove to be very effective - see e.g. Figure 6 of Ref. [4]. We describe how to construct these meson-meson operators in the sections to follow, with a particular focus, relevant to this calculation, on operators that respect $\mathrm{SU}(3)_{\mathrm{F}}$ flavour symmetry and which resemble vector-pseudoscalar states.

\footnotetext{
${ }^{8}$ One could also use energy levels calculated on elongated lattices and/or lattices with twisted boundary conditions to provide additional independent constraints 44 .
} 


\subsubsection{Single-Meson Operators in $\mathrm{SU}(3)_{\mathrm{F}}$ Flavour Representa- tions}

Following Refs. 10, 29, we construct single-meson operators from fermion bilinears. These have a spin and spatial structure built from Dirac $\gamma$-matrices and gauge-covariant derivatives, are projected onto overall momentum $\vec{p}$, and have a flavour structure that transforms in a particular $\mathrm{SU}(3)_{\mathrm{F}}$ multiplet. Explicitly, the construction is,

$$
\mathcal{O}_{\boldsymbol{F} \nu}^{\dagger J m}(\vec{p}, t)=\sum_{\vec{x}} e^{i \vec{p} \cdot \vec{x}} \sum_{\nu_{1}, \nu_{2}} \mathcal{C}_{\mathrm{SU}(3)}\left(\begin{array}{ccc}
\overline{\mathbf{3}} & \mathbf{3} & \boldsymbol{F} \\
\nu_{1} & \nu_{2} & \nu
\end{array}\right) \bar{q}_{\nu_{1}}(\vec{x}, t) \boldsymbol{\Gamma}_{t} q_{\nu_{2}}(\vec{x}, t)
$$

Here $\boldsymbol{\Gamma}_{t}$ denotes a product of $\gamma$-matrices and up to 3 gauge-covariant derivatives acting in position, colour and Dirac spin-space on time-slice $t$. The constructions are engineered to have definite continuum $J^{P}$ and $m$ where, for $\vec{p}=\overrightarrow{0}, m$ is the projection of $J$ along the $z$-axis and, for $\vec{p} \neq \overrightarrow{0}, m$ is replaced by the helicity, $\lambda$ - see Ref. [10. This is done by taking a circular basis of vector-like $\gamma$-matrices, $\Gamma_{i}$, and gauge-covariant derivatives, $\overleftrightarrow{D}_{i} \equiv \overleftarrow{D}_{i}-\vec{D}_{i}$, expressed in the Cartesian basis as

$$
\begin{array}{lll}
\Gamma_{ \pm 1}=\mp \frac{i}{\sqrt{2}}\left(\Gamma_{x} \mp i \Gamma_{y}\right) & \overleftrightarrow{D}_{ \pm 1}=\mp \frac{i}{\sqrt{2}}\left(\overleftrightarrow{D}_{x} \mp i \overleftrightarrow{D}_{y}\right) \\
\Gamma_{0}=i \Gamma_{z} & \overleftrightarrow{D}_{0}=i \overleftrightarrow{D}_{z}
\end{array}
$$

These spin-1 derivatives and Dirac $\gamma$-matrices are then combined using $\mathrm{SO}(3)$ ClebschGordan coefficients, to give $\boldsymbol{\Gamma}$ with definite $J^{P}$ and $m$. For example, a single derivative operator coupled to a spin-1 product of $\gamma$-matrices is given by,

$$
\boldsymbol{\Gamma}=\sum_{m_{1}, m_{2}} \mathcal{C}\left(\begin{array}{ccc}
1 & 1 & J \\
m_{1} & m_{2} & m
\end{array}\right) \Gamma_{m_{1}} \overleftrightarrow{D}_{m_{2}}
$$

and similarly for a two derivative operator, we first couple the derivatives to some total angular momentum, $J_{D}$, and then couple this with the $\gamma$-matrices to acquire our desired $(J, m)$,

$$
\boldsymbol{\Gamma}=\sum_{\substack{m_{1}, m_{2} \\
m_{3}, m_{D}}} \mathcal{C}\left(\begin{array}{ccc}
1 & J_{D} & J \\
m_{1} & m_{D} & m
\end{array}\right) \mathcal{C}\left(\begin{array}{ccc}
1 & 1 & J_{D} \\
m_{2} & m_{3} & m_{D}
\end{array}\right) \Gamma_{m_{1}} \overleftrightarrow{D}_{m_{2}} \overleftrightarrow{D}_{m_{3}}
$$

The quark fields, $q_{\nu}(\vec{x}, t)$, corresponding to the up, down and strange quarks $(u, d, s)$, are in the 3 multiplet of $\mathrm{SU}(3)_{\mathrm{F}}$, illustrated in Figure 3.1. The elements can be uniquely 

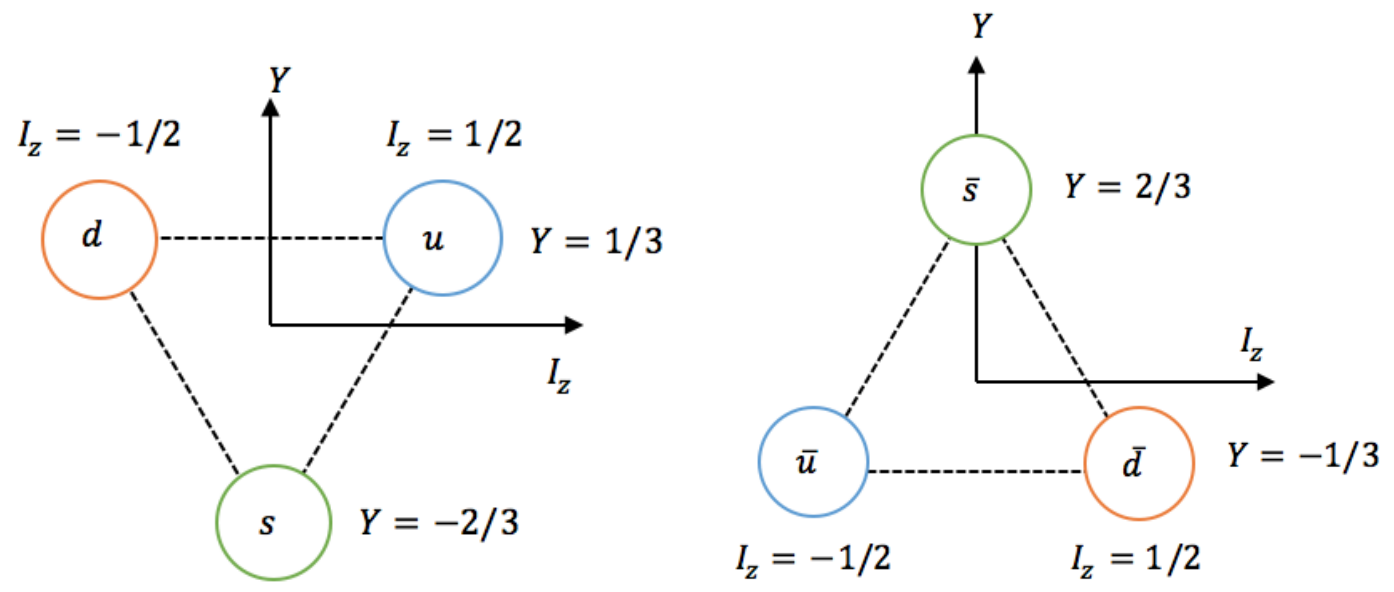

Figure 3.1: (Left): quark content of flavour irrep 3. (Right): anti-quark content of $\overline{3}$.

labelled by $\nu=\left(I, Y, I_{z}\right)$, where we recall $I$ is the isospin, $Y$ is the hypercharge and $I_{z}$ is the $z$-component of isospin. The $\mathcal{C}_{\mathrm{SU}(3)}(\ldots)$ are $\mathrm{SU}(3)_{\mathrm{F}}$ Clebsch-Gordan coefficients and we follow the conventions given in De Swart 67. The sum over $\mathrm{SU}(3)_{\mathrm{F}}$ components projects the quark-bilinear onto a definite $\mathrm{SU}(3)_{\mathrm{F}}$ flavour multiplet $\boldsymbol{F}$, which can be either $\mathbf{1}$ or $\mathbf{8}$.

These operators of definite $J^{P}$ and $m$ are subduced into the appropriate lattice irreps of $\mathrm{O}_{h}$ or $\mathrm{LG}(\vec{p})$ as discussed in Section 2.5. The subduction does not impact the flavour representation and the result is an operator, $\mathcal{O}_{\boldsymbol{F} \nu}^{\dagger \mu}(\vec{p}, t)=\sum_{m} \mathcal{S}_{\Lambda \mu}^{J m} \mathcal{O}_{\boldsymbol{F} \nu}^{\dagger J}(\vec{p}, t)$, in a particular irrep. As an example, consider a pseudoscalar $\mathrm{SU}(3)_{\mathrm{F}}$ singlet, $\boldsymbol{F}=\mathbf{1}, \nu=$ $(0,0,0), \boldsymbol{\Gamma}_{t}=\gamma_{5}$ and $\vec{p}=\overrightarrow{0}$. Subducing gives the lattice operator,

$$
\mathcal{O}_{\mathbf{1}(0,0,0)}^{\dagger A_{1}^{-} 1}=\frac{1}{\sqrt{3}}\left(\bar{u} \gamma_{5} u+\bar{d} \gamma_{5} d+\bar{s} \gamma_{5} s\right)
$$

\subsubsection{Two-Meson Operators in $\mathrm{SU}(3)_{\mathrm{F}}$ Flavour Representations}

Operators which resemble a pair of mesons can be constructed from a product of two single-meson operators. We follow the approach presented in Refs. [28, 30] and in this section concentrate on constructing operators in definite $\mathrm{SU}(3)_{\mathrm{F}}$ multiplets. Writing out 
the flavour structure explicitly, the meson-meson operator takes the form,

$$
\begin{aligned}
& \mathcal{O}_{\boldsymbol{F} \nu}^{\dagger \mu}\left(\begin{array}{l}
\boldsymbol{F}_{1}\left[\vec{p}_{1}\right] \Lambda_{1} \\
\boldsymbol{F}_{2}\left[\vec{p}_{2}\right] \Lambda_{2}
\end{array} \mid \vec{p}_{12}\right)=\sum_{\nu_{1}, \nu_{2}} \mathcal{C}_{\mathrm{SU}(3)}\left(\begin{array}{ccc}
\boldsymbol{F}_{\mathbf{1}} & \boldsymbol{F}_{\mathbf{2}} & \boldsymbol{F} \\
\nu_{1} & \nu_{2} & \nu
\end{array}\right) \sum_{\mu_{1}, \mu_{2}} \mathbb{C}\left(\begin{array}{ccc}
{\left[\vec{p}_{1}\right] \Lambda_{1}} & {\left[\vec{p}_{2}\right] \Lambda_{2}} & {\left[\vec{p}_{12}\right] \Lambda} \\
\mu_{1} & \mu_{2} & \mu
\end{array}\right) \\
& \times \sum_{\substack{\vec{p}_{i} \in\left\{\vec{p}_{i}\right\}^{*} \\
\vec{p}_{1}+\vec{p}_{2}=\vec{p}_{12}}} \Omega_{\boldsymbol{F}_{1} \nu_{1}}^{\dagger \Lambda_{1} \mu_{1}}\left(\vec{p}_{1}\right) \Omega_{\boldsymbol{F}_{2} \nu_{2}}^{\dagger \Lambda_{2} \mu_{2}}\left(\vec{p}_{2}\right),
\end{aligned}
$$

where the optimised operator $\Omega^{\dagger}{ }_{\boldsymbol{F}_{i} \nu_{i}}^{\Lambda_{i} \mu_{i}}\left(\vec{p}_{i}\right)$ interpolates a meson of momentum $\vec{p}_{i}$ in the $\boldsymbol{F}_{\boldsymbol{i}}$ flavour multiplet with component $\nu_{i}$. The lattice Clebsch-Gordan coefficients, $\mathbb{C}(\ldots)$, are required to couple irreps $\left[\vec{p}_{1}\right] \Lambda_{1} \otimes\left[\vec{p}_{2}\right] \Lambda_{2} \rightarrow\left[\vec{p}_{12}\right] \Lambda$, and the momentum sum runs over all momenta related to $\vec{p}_{i}$ by an allowed lattice rotation, $\vec{p}_{i} \in\left\{\vec{p}_{i}\right\}^{*}$, such that $\vec{p}_{1}+\vec{p}_{2}=\vec{p}_{12}-$ see Ref. 28 for details.

Since single-meson operators are restricted to the $\mathrm{SU}(3)_{\mathrm{F}}$ octet, 8, and singlet, $\mathbf{1}$, meson-meson operators are restricted to the $\mathbf{2 7}, \mathbf{1 0}, \overline{\mathbf{1 0}}, \mathbf{8}$ and $\mathbf{1}$ multiplets. In this work, we will perform calculations with exact $\mathrm{SU}(3)_{\mathrm{F}}$ symmetry and focus on $\rho \pi$ scattering in isospin-2, which lies in the $\mathbf{2 7}$ multiplet. We are at liberty to choose any component of the 27 multiplet when we calculate the energy spectra, as they are all equivalent, and we choose $\nu=(2,0,2)$. The $\mathrm{SU}(3)_{\mathrm{F}}$ Clebsch-Gordan coefficients in Eq. 3.8 ensure that the relevant meson-meson operators come from products of single-meson operators with flavour structure $\boldsymbol{F}=\mathbf{8}$ and $\nu=(1,0,1)$. The meson content for the pseudoscalar and vector octets is shown in Figure 3.2. As $G$-parity is negative, there are no pseudoscalar-pseudoscalar or vector-vector channels which can mix with isospin- $2 \rho \pi$.

As a simple example, consider the meson-meson operator transforming in $T_{1}^{+}$, row 2 , constructed from optimised operators transforming in $T_{1}^{-}$and $A_{1}^{-}$according to Eq. 3.8 . The Clebsch-Gordan coefficients give,

$$
\begin{aligned}
\mathcal{O}_{\mathbf{2 7}(2,0,2)}^{\dagger T_{1}^{+} 2} & =\Omega_{\mathbf{8}(1,0,1)}^{\dagger T_{1}^{-} 2} \Omega_{\mathbf{8}(1,0,1)}^{\dagger A_{1}^{-} 1} \\
& =\left(\rho^{+}\right)^{\dagger}\left(\pi^{+}\right)^{\dagger},
\end{aligned}
$$

where the corresponding $\mathrm{SU}(2)_{\mathrm{F}}$ flavour mesons are given explicitly in the last equality for clarity. 

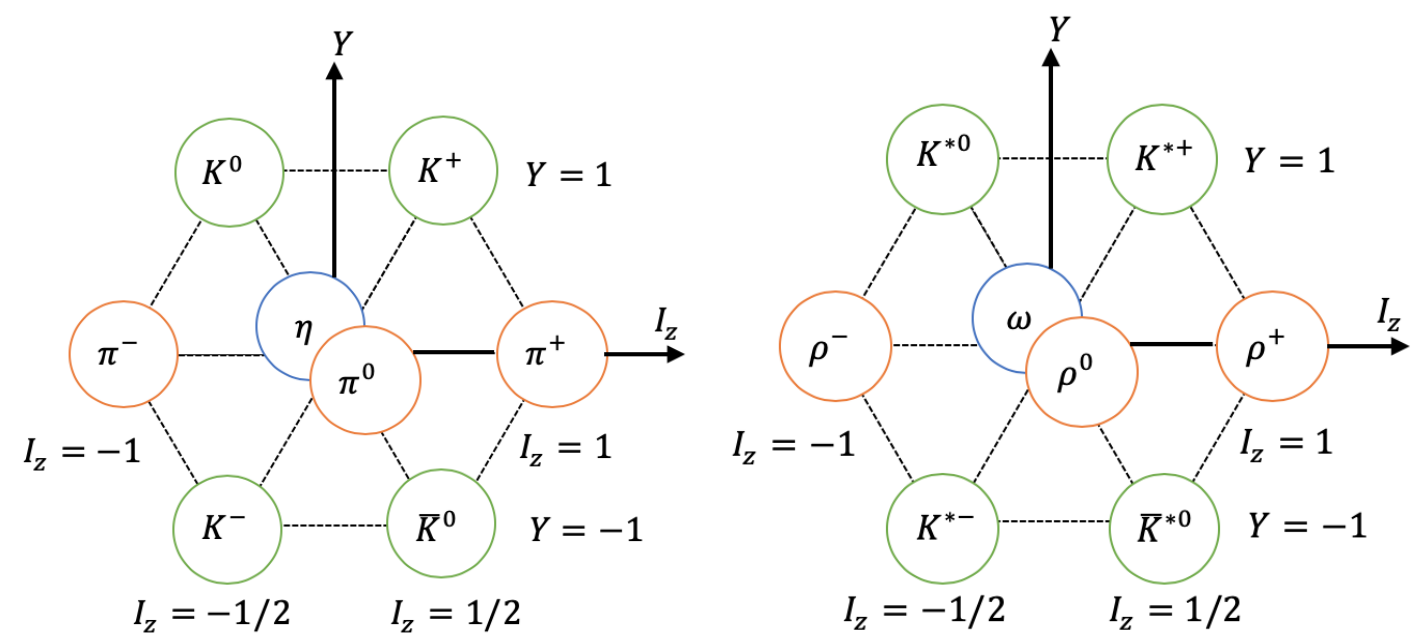

Figure 3.2: Meson content of the $J^{P}=0^{-}$pseudoscalar octet (left) and the $J^{P}=1^{-}$ vector octet (right). Orange corresponds to $I=1$, green to $I=1 / 2$ and blue to $I=0$.

The basis of meson-meson operators used to form the matrix $C_{i j}(t)$ can be constructed using different magnitudes of momentum, ${ }^{9}\left|\vec{p}_{1}\right|,\left|\vec{p}_{2}\right|$, where directions of the momenta are summed over in Eq. 3.8 subject to $\vec{p}_{1}+\vec{p}_{2}=\vec{P}$. There is a close association between the finite-volume energy-levels when mesons have no meson-meson interactions, $E_{\text {n.i. }}^{(2)}=\sqrt{m_{\pi}^{2}+\left|\vec{p}_{1}\right|^{2}}+\sqrt{m_{\rho}^{2}+\left|\vec{p}_{2}\right|^{2}}$, which we refer to as 'non-interacting' energies, and these operators. Earlier studies have found that meson-meson operators, which closely resemble the non-interacting states in the energy range of interest, are efficient at interpolating the corresponding finite-volume energies [28, 30]. This suggests that, if we are interested in only a certain energy range, operators which correspond to a non-interacting energy which lies far above this energy region do not need to be included in the basis.

When a single-meson operator for a vector meson has non-zero momentum, the reduced symmetry of the lattice means that the different helicity components subduce into $N_{\lambda}$ different irreps of $\operatorname{LG}\left(\vec{p}_{1}\right)$. Each of these vector operators can be combined, via Eq. 3.8 , with a pseudoscalar operator transforming in some irrep of $\operatorname{LG}\left(\vec{p}_{2}\right)$, to form a set of linearly-independent vector-pseudoscalar operators at some overall momentum $\vec{P}$ in some

\footnotetext{
${ }^{9}$ Strictly speaking, this should be $\left\{\vec{p}_{i}\right\}^{*}$ as indicated in Eq. 3.8 , rather than magnitude, but for $|\vec{p}|^{2}<9$, $\left\{\vec{p}_{i}\right\}^{*}$ is uniquely identified by its magnitude.
} 
irrep $\Lambda$. Furthermore, each vector operator when combined with a pseudoscalar operator may appear numerous times within a single irrep, e.g. [001] $E_{2} \otimes[011] A_{2} \rightarrow 2 \times[001] E_{2}$, and form multiple linearly-independent vector-pseudoscalar operators. We refer to this number as the multiplicity (which could be zero). Together, this means that there can be many linearly-independent vector-pseudoscalar operators, transforming within some irrep $\Lambda$, which correspond to the same non-interacting energy and we denote the total number of such operators as $N_{\text {lin }}$. It is important to emphasise that $N_{\text {lin }}$ is the sum of the multiplicities for each of the $N_{\lambda}$ vector operators combined with the appropriate pseudoscalar operator.

For example, consider vector-pseudoscalar operators overall at rest, $\vec{P}=\overrightarrow{0}$, in the $T_{1}^{+}$ irrep, which we write as $[000] T_{1}^{+}$. The operator corresponding to lowest non-interacting energy features a vector meson at rest (in the $T_{1}^{-}$irrep) coupled to a pseudoscalar at rest (in the $A_{1}^{-}$irrep). In this case, $N_{\lambda}=1$, and there is only one operator corresponding to the one way of coupling $[000] T_{1}^{-} \otimes[000] A_{1}^{-} \rightarrow[000] T_{1}^{+}\left(N_{\text {lin }}=1\right)$. Of course, there are still three equivalent rows of the $T_{1}$ irrep.

On the other hand, for a vector meson with momentum $\vec{p}=[001]$, the helicity 0 and \pm 1 components subduce into the [001] $A_{1}$ and [001] $E_{2}$ irreps respectively $\left(N_{\lambda}=2\right)$. Combining the vector with a pseudoscalar so that the vector-pseudoscalar operator is overall at rest, there are two linearly independent operators transforming in $[000] T_{1}^{+}$from $[001] A_{1} \otimes[001] A_{2} \rightarrow[000] T_{1}^{+}$and $[001] E_{2} \otimes[001] A_{2} \rightarrow[000] T_{1}^{+}\left(N_{\text {lin }}=2\right)$.

If the vector meson has momentum $\vec{p}=[011]$, the three helicities subduce into three different irreps, [011] $A_{1},[011] B_{1}$ and $[011] B_{2}\left(N_{\lambda}=3\right)$. When combined appropriately with the pseudoscalar, this gives three linearly-independent vector-pseudoscalar operators transforming in [000] $T_{1}^{+}$from [011] $A_{1} \otimes[011] A_{2} \rightarrow[000] T_{1}^{+},[011] B_{1} \otimes[011] A_{2} \rightarrow[000] T_{1}^{+}$ and $[011] B_{2} \otimes[011] A_{2} \rightarrow[000] T_{1}^{+}\left(N_{\text {lin }}=3\right)$.

While we have illustrated how multiple meson-meson operators with the same associated non-interacting energies can arise by considering a vector-pseudoscalar operator overall at rest, this situation also occurs when there is an overall non-zero momentum. For example, with $\vec{P}=[001],[001] A_{1} \otimes[011] A_{2} \rightarrow[001] E_{2}$ and [001] $E_{2} \otimes[011] A_{2} \rightarrow 2 \times[001] E_{2}$ giving $N_{\lambda}=2$ and $N_{\text {lin }}=3$ (as [001] $E_{2} \otimes[011] A_{2}$ into [001] $E_{2}$ has a multiplicity of two). In all cases, the non-interacting meson-meson spectrum will feature degeneracies: for each non-interacting energy, the degeneracy is equal to $N_{\text {lin }}$ of the corresponding meson-meson 
operator. As one might anticipate, failing to include all the $N_{\text {lin }}$ meson-meson operators at each corresponding non-interacting energy in a given energy region can lead to an incomplete spectrum. This is demonstrated clearly in Figure 8 of [68] and will be crucial in the scattering calculations in the later chapters.

\subsection{Computational Details}

Calculations of correlation functions were performed on two anisotropic lattices of volumes $\left(L / a_{s}\right)^{3} \times\left(T / a_{t}\right)=20^{3} \times 128$ and $24^{3} \times 128$, with spatial lattice spacing $a_{s} \sim 0.12 \mathrm{fm}$ and temporal lattice spacing $a_{t}=a_{s} / \xi \sim(4.7 \mathrm{GeV})^{-1}$ where $\xi \sim 3.5$ is the anisotropy. $L$ and $T$ are the spatial and temporal extents of the lattice respectively. We use gauge fields generated from the improved anisotropic gauge and fermion actions in Section 2.2 with $N_{f}=3$ degenerate flavours of dynamical quarks [20]. The quark masses are tuned to approximately equal to the physical strange quark mass, giving exact $\mathrm{SU}(3)_{\mathrm{F}}$ symmetry. The flavour octet of pseudoscalars is found to have a mass $\sim 700 \mathrm{MeV}$, while the vector octet has a mass $\sim 1020 \mathrm{MeV}$. With these heavy masses, exponentially suppressed finite-volume and temporal effects are negligible as $m_{\pi} L \gtrsim 10$ and $m_{\pi} T \gtrsim 18$.

Correlation functions are computed in a distillation space of rank $N_{\text {vecs }}$. Statistical precision is increased by averaging correlation functions over a number, $N_{\text {tsrcs }}$, of independent time-sources, $t_{\text {src }}$. To reduce statistical correlations between the energy levels for different moving frames, we averaged over a different set of time-sources for each non-zero momentum. The rank of the distillation space, number of gauge configurations, $N_{\text {cfgs }}$, and number of time-sources used for the computations of $\rho, \pi$ and $\rho \pi$ correlation functions, on each lattice, are shown in Table 3.2 .

When quoting results in physical units, we set the scale using the $\Omega$-baryon mass. From the value obtained on a lattice of spatial volume $\left(L / a_{s}\right)^{3} \times\left(T / a_{t}\right)=16^{3} \times 128$, $a_{t} m_{\Omega}^{\text {latt. }}=0.3593(7)\left[69\right.$, and the experimental mass, $m_{\Omega}^{\text {exp. }}=1672.45(29) \mathrm{MeV}$ [5], we obtain the inverse temporal spacing via $a_{t}^{-1}=m_{\Omega}^{\text {exp. }} / a_{t} m_{\Omega}^{\text {latt. }}$, giving $a_{t}^{-1}=4655 \mathrm{MeV}$. 


\begin{tabular}{c|ccccc|ccc}
$\left(L / a_{s}\right)^{3} \times\left(T / a_{t}\right)$ & $N_{\text {vecs }}$ & $N_{\text {cfgs }}$ & $N_{\text {tsrcs }}$ & & $\left(L / a_{s}\right)^{3} \times\left(T / a_{t}\right)$ & $N_{\text {vecs }}$ & $N_{\text {cfgs }}$ & $N_{\text {tsrcs }}$ \\
\hline $20^{3} \times 128$ & 128 & 197 & 8 & & $20^{3} \times 128$ & 128 & 502 & $1-3$ \\
$24^{3} \times 128$ & 160 & 499 & 1 & & $24^{3} \times 128$ & 160 & 607 & $1-3$
\end{tabular}

Table 3.2: Number of distillation vectors $\left(N_{\text {vecs }}\right)$, gauge configurations $\left(N_{\text {cfgs }}\right)$ and time-sources $\left(N_{\text {tsrcs }}\right)$ used to compute correlation functions on the two lattice volumes, as described in the text, for (left) $\rho$ and $\pi$ correlation functions $(\boldsymbol{F}=\mathbf{8})$ and (right) $\rho \pi$ correlation functions $(\boldsymbol{F}=\mathbf{2 7})$.

\subsection{Dispersion Relation}

In order to study $\rho \pi$ scattering, we must compute the momentum dependence of the relevant stable mesons' energies and check that they satisfy the relativistic dispersion relations by determining the anisotropy, $\xi \equiv a_{s} / a_{t}$. The relativistic dispersion relation for a stable hadron is, up to discretisation effects,

$$
\left(a_{t} E_{\vec{n}}\right)^{2}=\left(a_{t} m\right)^{2}+\frac{1}{\xi^{2}}\left(\frac{2 \pi}{L / a_{s}}\right)^{2}|\vec{n}|^{2},
$$

where $m$ is the mass of the hadron and $E_{\vec{n}}$ is its energy with momentum $\vec{p}=\frac{2 \pi}{L} \vec{n}$. Differences between the values of $\xi$ measured from different hadrons are due to discretisation, finitevolume and temporal effects, with the latter two expected to be negligible given the values of $m_{\pi} L$ and $m_{\pi} T$. The energies of the ground-state flavour octet vector and pseudoscalar mesons, hereafter referred to as $\rho$ and $\pi$, with momentum $|\vec{n}|^{2} \leq 4$ were calculated from a variational analysis of matrices of correlation functions involving bases of single-meson operators. The analyses also gave the optimised operators for interpolating the $\rho$ and $\pi$ with the various momenta. These are used in the construction of vector-pseudoscalar operators, as discussed in Section 3.2 .

The extracted energies are shown in Figure 3.3 along with the results of fits using Eq. 3.10. For the $\rho$, the energies of the different helicity components were calculated independently from each relevant irrep of $\operatorname{LG}(\vec{p})$, e.g. at $\vec{p}=[001]$ the $\lambda=0$ energies were calculated from the $[001] A_{1}$ irrep and $|\lambda|=1$ from [001] $E_{2}$. From the figure, it can be seen that the $\xi$ values extracted from the $\pi$ and the $|\lambda|=1 \rho$ are in reasonable agreement, but the value from the $\lambda=0 \rho$ differs from the $\pi$ at the $2 \%$ level 10 This discrepancy is

\footnotetext{
${ }^{10}$ The energy splitting between different helicity components of the vector can be seen for calculations
} 
dominated by discretisation effects and we propagate a conservative estimate of systematic uncertainty by using a value of $\xi=3.486(43)$, derived by considering the smallest and largest values within one standard deviation of the mean from the fits in Figure 3.3 . As the meson-meson interactions in isospin- $2 \rho \pi$ scattering are weak and the corresponding energy shifts small, the uncertainty on $\xi$ is found to be the largest source of systematic uncertainty on the scattering amplitudes. On the contrary, should interactions be more significant the relative contribution of the uncertainty on the anisotropy to the total uncertainty on the amplitudes ought to be smaller and indeed this is seen later in Chapter 5 .

on a $16^{3} \times 128$ lattice with the same lattice action in previous works - see Figures 12 and 13 in Ref. 10 and Figure 4 in Ref. 70]. 

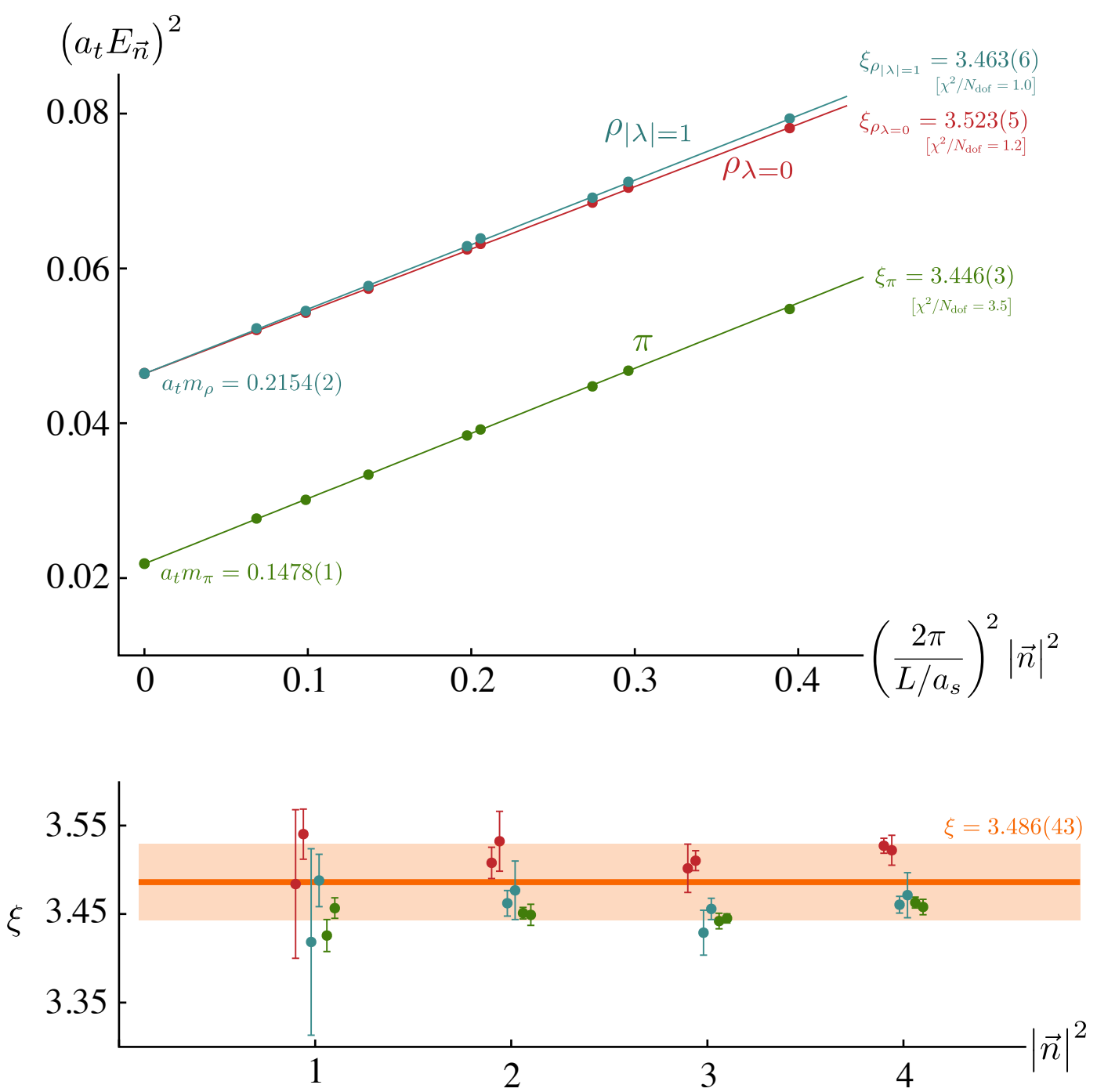

Figure 3.3: Upper panel: Momentum dependence of the $\pi$ and $\rho$ energies where both $|\lambda|=0,1$ helicity components of the $\rho$ are considered. The statistical errors on the energies are smaller than the points. Lines and numerical values show results of fits to determine $\xi$ using Eq. 3.10. Lower panel: points show the effective momentum-dependent $\xi$ obtained via $\left[\left(\frac{2 \pi}{L / a_{s}}\right)^{2}|\vec{n}|^{2} /\left(\left(a_{t} E_{\vec{n}}\right)^{2}-\left(a_{t} m\right)^{2}\right)\right]^{1 / 2}$, with the two volumes $\left(L / a_{s}=20,24\right)$ and the different mesons offset slightly for clarity. The orange line and band indicate, respectively, the value and uncertainty on $\xi$ we use when investigating $\rho \pi$ scattering as described in the text. 


\subsection{Finite-Volume Spectra}

To determine finite-volume energy spectra for isospin- $2 \rho \pi$, matrices of correlation functions were calculated, using bases of meson-meson operators as outlined in Section 3.2, for all irreps $\vec{P} \Lambda$ where $|\vec{P}|^{2} \leq 4\left(\frac{2 \pi}{L}\right)^{2}$. Table 3.3 shows the operators used in the $T_{1}^{+}$irrep at rest and the $A_{2}$ irreps in-flight (operator lists for the other irreps are shown in Tables 3.8 - 3.11 of Appendix 3.B - note the multiple linearly-independent operators appearing at many of the non-interacting energies as discussed in Section 3.2. For each irrep, the finite-volume spectrum was extracted by application of the variational method. As an example, in Figure 3.5, we show the lowest eight principal correlators for the $T_{1}^{+}$irrep in Figure 3.4 along with the corresponding spectrum and operator-state matrix elements, $Z_{i}^{\mathfrak{n}} \equiv\left\langle\mathfrak{n}\left|\mathcal{O}_{i}^{\dagger}(0)\right| 0\right\rangle$. From the figure, it can be seen that the matrix of correlation functions is nearly block diagonal in the momentum-based operator construction with respect to operators with the same $E_{\mathrm{n} . \mathrm{i}}$, and that different linear combinations of the multiple meson-meson operators, corresponding to the same $E_{\text {n.i }}$, are distinguishing the $N_{\text {lin }}$ nearly degenerate energy levels.

In Figures 3.6 and 3.7, we show the volume dependence of the extracted energies for all irreps at rest and $A_{2}$ irreps in-flight. Spectra for other in-flight irreps can be found in Figure 3.13 in Appendix 3.C. Figure 3.7 illustrates the dense distribution of energy levels, typical of the reduced symmetry for in-flight irreps, and the multiple energy levels which would be degenerate in the absence of interactions. Nevertheless, it can be seen that all the energy levels can be extracted with good statistical precision. Since we choose to restrict our two-meson operators to be constructed from only single-meson operators with momentum $|\vec{n}|^{2} \leq 4$, we will only extract scattering amplitudes for $a_{t} E_{\mathrm{cm}} \leq 0.41$, below the non-interacting energy corresponding to the lowest excluded operator ${ }^{11}$ No other meson-meson scattering channels have thresholds below the $\pi \pi \pi$ threshold which opens at $a_{t} E_{\mathrm{cm}}=0.443$.

Some qualitative expectations for the behaviour of scattering amplitudes can be inferred from the spectra presented in Figures 3.6 and 3.7. For example, we expect the lowest energy-level in both the $E^{-}$and $T_{2}^{-}$irreps to have a dominant contribution from the ${ }^{3} P_{2^{-}}$

\footnotetext{
${ }^{11}$ The lowest-lying excluded operator, across all irreps and volumes, is $\rho_{[012]} \pi_{[0-10]}$, which corresponds to a non-interacting energy of $a_{t} E_{\mathrm{cm}}=0.4124$ on the lattice with $L / a_{s}=24$.
} 


\begin{tabular}{|c|c|c|c|c|}
\hline$[000] T_{1}^{+}$ & {$[001] A_{2}$} & {$[011] A_{2}$} & {$[111] A_{2}$} & {$[002] A_{2}$} \\
\hline$\rho_{[000]} \pi_{[000]}$ & $\rho_{[001]} \pi_{[000]}$ & $\rho_{[011]} \pi_{[000]}$ & $\rho_{[111]} \pi_{[000]}$ & $\rho_{[001]} \pi_{[001]}$ \\
\hline$\{2\} \rho_{[001]} \pi_{[00-1]}$ & $\rho_{[000]} \pi_{[001]}$ & $\{2\} \rho_{[001]} \pi_{[010]}$ & $\{2\} \rho_{[011]} \pi_{[100]}$ & $\rho_{[002]} \pi_{[000]}$ \\
\hline$\{3\} \rho_{[011]} \pi_{[0-1-1]}$ & $\{2\} \rho_{[011]} \pi_{[0-10]}$ & $\rho_{[000]} \pi_{[011]}$ & $\{2\} \rho_{[100]} \pi_{[011]}$ & $\{2\} \rho_{[011]} \pi_{[0-11]}$ \\
\hline \multirow[t]{8}{*}{2}$\rho_{[111]} \pi_{[-1-1-1]}$ & $\{2\} \rho_{[010]} \pi_{[0-11]}$ & $\{2\} \rho_{[111]} \pi_{[-100]}$ & $\rho_{[000]} \pi_{[111]}$ & $\rho_{[000]} \pi_{[002]}$ \\
\hline & $\rho_{[002]} \pi_{[00-1]}$ & $\{3\} \rho_{[110]} \pi_{[-101]}$ & $\{2\} \rho_{[112]} \pi_{[00-1]}$ & $\{2\} \rho_{[012]} \pi_{[0-10]}$ \\
\hline & $\{2\} \rho_{[111]} \pi_{[-1-10]}$ & $\{2\} \rho_{[100]} \pi_{[-111]}$ & $\{3\} \rho_{[012]} \pi_{[10-1]}$ & $\{2\} \rho_{[111]} \pi_{[-1-11]}$ \\
\hline & $\{2\} \rho_{[110]} \pi_{[-1-11]}$ & $\{2\} \rho_{[012]} \pi_{[00-1]}$ & $\{2\} \rho_{[002]} \pi_{[11-1]}$ & $\{2\} \rho_{[010]} \pi_{[0-12]}$ \\
\hline & $\rho_{[00-1]} \pi_{[002]}$ & $\{2\} \rho_{[002]} \pi_{[01-1]}$ & $\{2\} \rho_{[11-1]} \pi_{[002]}$ & $\{2\} \rho_{[112]} \pi_{[-1-10]}$ \\
\hline & $\{2\} \rho_{[012]} \pi_{[0-1-1]}$ & $\{2\} \rho_{[01-1]} \pi_{[002]}$ & $\{3\} \rho_{[01-1]} \pi_{[102]}$ & $\{2\} \rho_{[-1-10]} \pi_{[112]}$ \\
\hline & & $\{2\} \rho_{[00-1]} \pi_{[012]}$ & $\{2\} \rho_{[00-1]} \pi_{[112]}$ & \\
\hline & & $\{3\} \rho_{[112]} \pi_{[-10-1]}$ & & \\
\hline 8 ops. & 12 ops. & 15 ops. & 10 ops. & 7 ops. \\
\hline
\end{tabular}

Table 3.3: Meson-meson operators in the $\mathbf{2 7}$ of SU $(3)_{\mathrm{F}}$ flavour, ordered by increasing non-interacting energy (see Section 3.2.2), for various irreps $\vec{P} \Lambda$. The operators, $\rho_{\vec{p} 1} \pi_{\vec{p}_{2}}$, are constructed from optimised $\rho$ and $\pi$ operators with momentum types $\vec{p}_{1}$ and $\vec{p}_{2}$ respectively. Different momentum directions are summed over as in Eq. 3.8. $\left\{N_{\text {lin }}\right\}$ denotes the number of linearly-independent meson-meson operators at the corresponding non-interacting energy when there is more than one. All operators with corresponding non-interacting energies $a_{t} E_{\mathrm{cm}} \leq 0.455$ for $L / a_{s}=24$ are displayed. Those in grey italic were not included in the operator basis.

wave, see Table 3.1, owing to the centrifugal barrier suppressing the higher partial-waves appearing in these irreps. These energy-levels are found just below the corresponding non-interacting energies suggesting the ${ }^{3} P_{2}$-wave is weakly attractive. Across all irreps, there are clearly no large departures from the non-interacting spectra, the number of energy levels is the same as the number expected in the absence of interactions, and no energy levels lie systematically below the $\rho \pi$ threshold. These observations likely indicate the absence of narrow resonances or bound-states, and suggest that only a relatively weak interaction is present. In order to get a quantitative understanding we proceed to calculate the scattering amplitudes. 

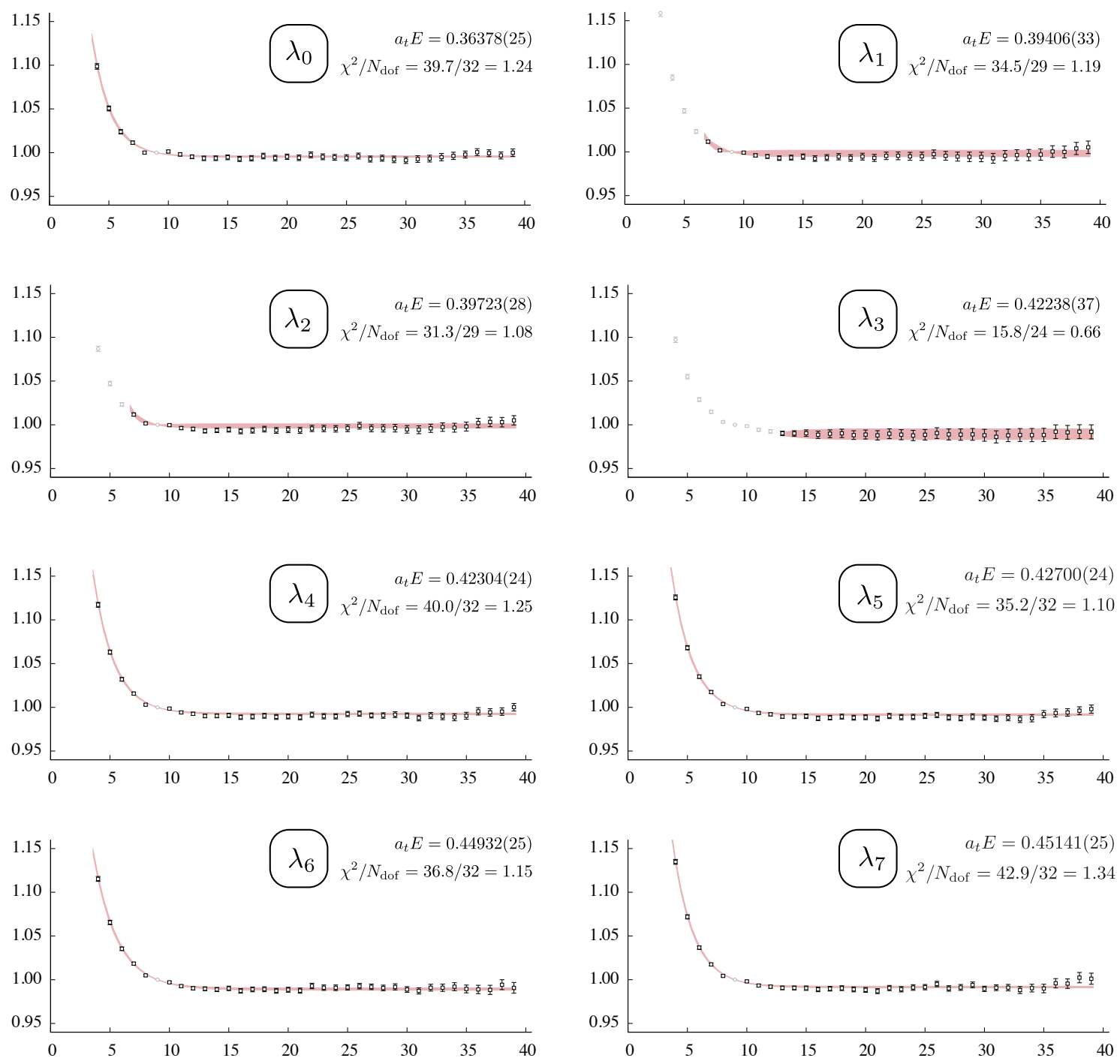

Figure 3.4: Principal correlators, $\lambda_{\mathfrak{n}}\left(t, t_{0}=9\right)$, plotted as $e^{E_{\mathfrak{n}}\left(t-t_{0}\right)} \lambda_{\mathfrak{n}}\left(t, t_{0}\right)$, from a variational analysis of the $[000] T_{1}^{+}$irrep on the lattice with $L / a_{s}=24$. Curves show the results of fits described in Section 2.6. The horizontal axes are in units of $t / a_{t}$. 


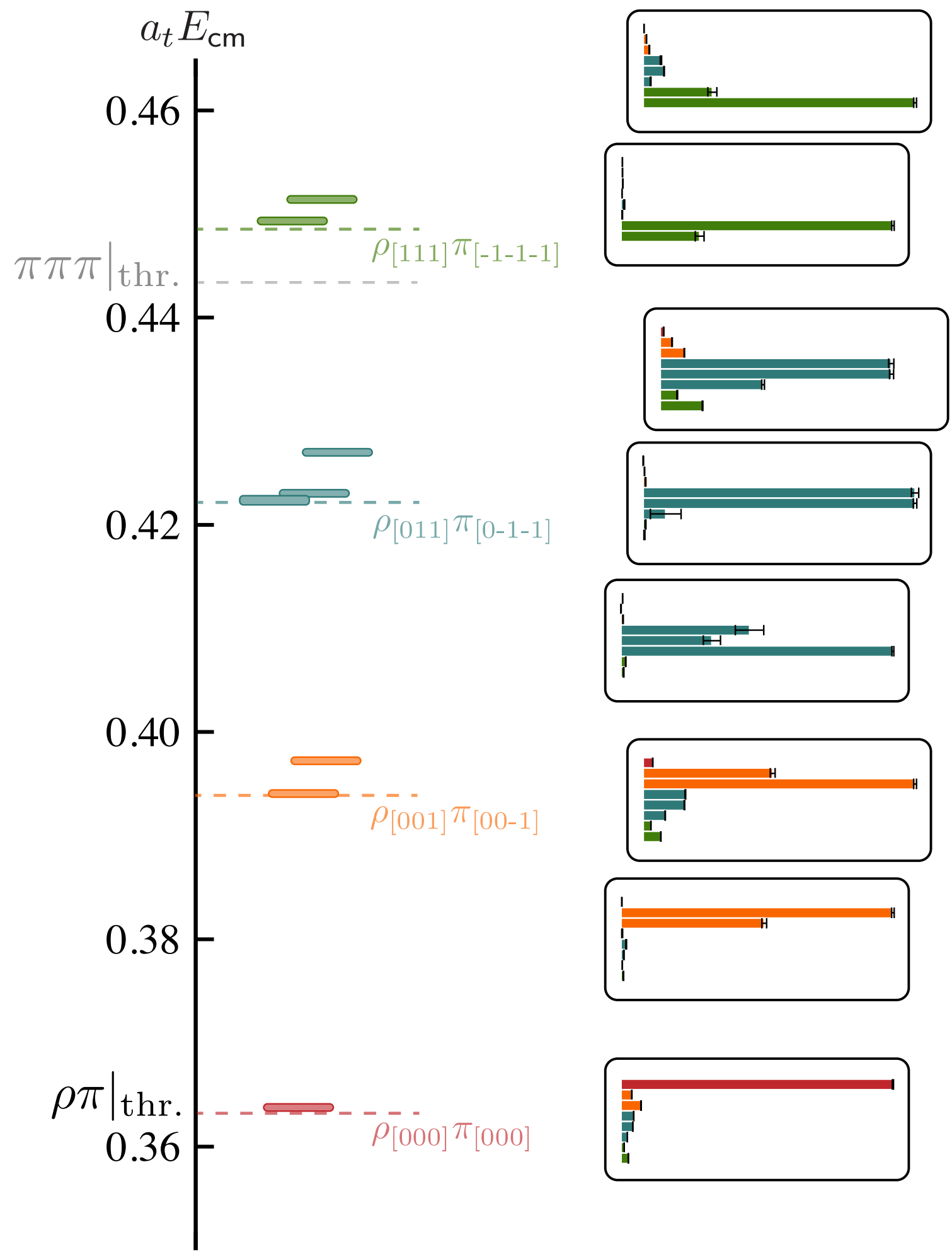

Figure 3.5: Left: finite-volume energy levels in the $[000] T_{1}^{+}$irrep on the lattice with $L / a_{s}=24$. Dashed lines indicate the location of non-interacting energies. Right: histograms showing the corresponding operator-state overlaps, $Z_{i}^{\mathfrak{n}}=\left\langle\mathfrak{n}\left|\mathcal{O}_{i}^{\dagger}(0)\right| 0\right\rangle$, for the operators ordered as in Table 3.3. The colours reflect the non-interacting energies associated with each operator. The overlaps are normalised such that the largest value for a given operator across all energy levels is equal to one. 

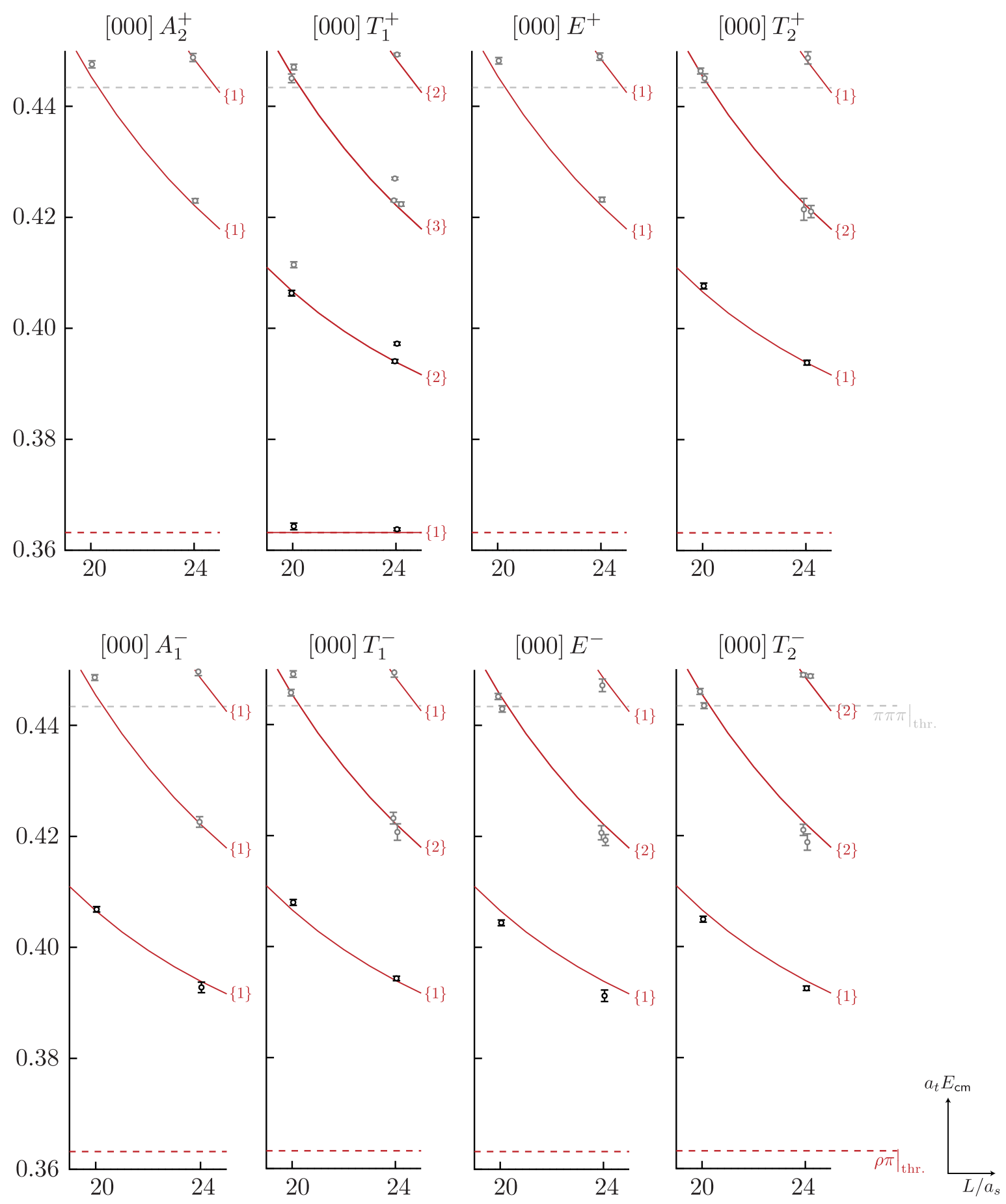

Figure 3.6: Energy spectra in irreps at rest. Black and grey points, slightly displaced in $L / a_{s}$ for clarity, show the extracted energy levels below and above $a_{t} E_{\mathrm{cm}}=0.41$ respectively. Errorbars reflect the statistical uncertainties. Points in grey are not used in the subsequent analysis in Section 3.6. Dashed lines show the $\rho \pi$ and $\pi \pi \pi$ thresholds. Solid red curves indicate non-interacting meson-meson energies, labelled with their degeneracies. 

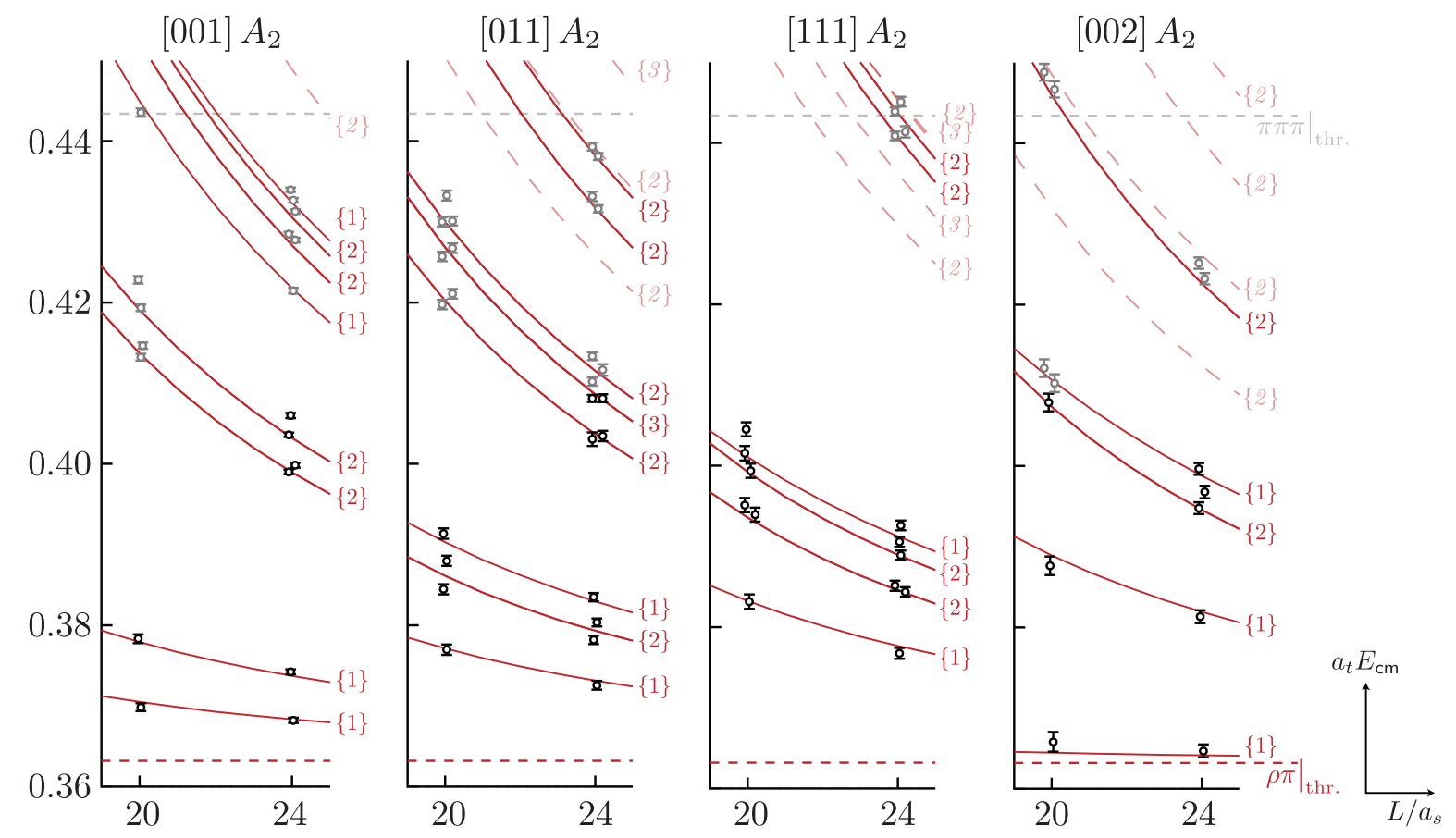

Figure 3.7: As Figure 3.6 but for $A_{2}$ irreps with $\vec{P} \neq \overrightarrow{0}$. Dashed red curves indicate non-interacting meson-meson energies corresponding to operators not included in the basis. Errors on the points show the statistical uncertainty added in quadrature to the systematic uncertainty from the uncertainty placed on $\xi$. 


\subsection{Scattering Amplitudes}

The general relationship between infinite-volume scattering amplitudes and finite-volume energy levels is presented in Section 2.7 and specific details for vector-pseudoscalar scattering are discussed in Section 3.1. In the case that no partial-waves are coupled dynamically, the $\boldsymbol{t}$-matrix is diagonal in $\ell$ and infinite-volume scattering in each partial-wave, ${ }^{3} \ell_{J}$, can be described by a single real-valued energy-dependent parameter called the phase-shift, $\delta_{{ }^{\ell_{J}}}\left(E_{\mathrm{cm}}\right)$. This appears in the scattering $\boldsymbol{t}$-matrix as $t_{\ell J n, \ell J n}=\frac{1}{\rho} \exp \left[i \delta_{3_{\ell_{J}}}\right] \sin \left(\delta_{3_{\ell_{J}}}\right)$. Recall that in an irrep where just a single partial-wave makes a non-negligible contribution, the quantisation condition reduces to the form shown in Eq. 3.2 - this can be evaluated to give a phase-shift point, $\delta_{3 \ell_{J}}\left(E_{\mathrm{cm}}^{(k)}\right)$, at each finite-volume energy level, $E_{\mathrm{cm}}^{(k)}$.

Formally, the infinite number of partial-waves which subduce into the irrep $\Lambda$ appear in the quantisation condition. Even though the angular-momentum barrier suppresses the contributions of partial-waves of higher $\ell$ at low energies, for vector-pseudoscalar scattering multiple partial-waves with the same threshold behaviour can appear in a single irrep. For example, the ${ }^{3} P_{1}$ and ${ }^{3} P_{2}$ partial-waves both appear in [011] $A_{1}$. This prevents the use of a one-to-one mapping between energy levels and phase-shift points of the type given in Eq. 3.2. Furthermore, when two partial-waves are dynamically coupled, the scattering $\boldsymbol{t}$-matrix is no longer diagonal in $\ell$ and is described by three real energydependent parameters ${ }^{12}$ These can be expressed as two phase-shifts and an angle, as in Eq. 3.1. In this case, again, there is no one-to-one mapping between energy levels and phase-shift points.

The approach we take to determine scattering amplitudes when the energy spectrum is dependent on more than a single energy-dependent scattering parameter, is to, as in Refs. [4, 7, 28, 62, 64, 66, 71, parameterise the energy-dependence of the $\boldsymbol{t}$-matrix. In this way, for any given set of parameter values, a finite-volume spectrum is calculated in each irrep by solving Eq. 2.53. We follow the approach of Ref. [64 where this calculated

\footnotetext{
${ }^{12}$ Given the constraints from unitarity of the $\boldsymbol{S}$-matrix and the time-reversal symmetry of QCD.
} 
spectrum is compared to the computed lattice spectrum using the following $\chi^{2}[30]$,

$$
\begin{aligned}
\chi^{2}\left(\left\{a_{i}\right\}\right)=\sum_{L} \sum_{\vec{P} \Lambda, \vec{P}^{\prime} \Lambda^{\prime}} \sum_{\mathfrak{n}, \mathfrak{n}^{\prime}}\left[E_{\mathrm{cm}}(L ; \vec{P} \Lambda ; \mathfrak{n})-\right. & \left.E_{\mathrm{cm}}^{\mathrm{par} .}\left(L ; \vec{P} \Lambda ; \mathfrak{n} ;\left\{a_{i}\right\}\right)\right] C^{-1}\left(L ; \vec{P} \Lambda, \mathfrak{n} ; \vec{P}^{\prime} \Lambda^{\prime}, \mathfrak{n}^{\prime}\right) \\
& \times\left[E_{\mathrm{cm}}\left(L ; \vec{P}^{\prime} \Lambda^{\prime} ; \mathfrak{n}^{\prime}\right)-E_{\mathrm{cm}}^{\mathrm{par} .}\left(L ; \vec{P}^{\prime} \Lambda^{\prime} ; \mathfrak{n}^{\prime} ;\left\{a_{i}\right\}\right)\right]
\end{aligned}
$$

where $E_{\mathrm{cm}}^{\text {par. }}\left(L ; \vec{P} \Lambda ; \mathfrak{n} ;\left\{a_{i}\right\}\right)$ is the $\mathfrak{n}^{\text {th }}$ energy-level satisfying Eq. 2.53 for a $\boldsymbol{t}$-matrix parameterised by $\left\{a_{i}\right\}$. The data covariance matrix, $C$, gives the correlations between energy levels on the same lattice volume. By minimising the $\chi^{2}$ with respect to the free parameters $\left\{a_{i}\right\}$, the best description of the spectrum may be obtained. The sensitivity to the choice of scattering-amplitude parameterisation can be tested by using a variety of different parameterisations.

In the case of a single partial-wave, not dynamically coupled to any others, a convenient parameterisation of,

$$
t\left(E_{\mathrm{cm}}\right)=\frac{1}{\rho\left(E_{\mathrm{cm}}\right)} \exp \left\{i \delta_{{ } \ell_{J}}\left(E_{\mathrm{cm}}\right)\right\} \sin \left\{\delta_{\beta_{\ell_{J}}}\left(E_{\mathrm{cm}}\right)\right\}=\frac{E_{\mathrm{cm}} / 2}{k_{\mathrm{cm}} \cot \left\{\delta_{3 \ell_{J}}\left(E_{\mathrm{cm}}\right)\right\}-i k_{\mathrm{cm}}},
$$

is the effective range expansion,

$$
k_{\mathrm{cm}}^{2 \ell+1} \cot \left\{\delta_{{ }^{3} \ell_{J}}\left(E_{\mathrm{cm}}\right)\right\}=\frac{1}{a\left(\left.{ }^{3} \ell_{J}\right|^{3} \ell_{J}\right)}+\frac{1}{2} r\left(\left.{ }^{3} \ell_{J}\right|^{3} \ell_{J}\right) k_{\mathrm{cm}}^{2}+O\left(k_{\mathrm{cm}}^{4}\right),
$$

where the constants $a\left(\left.{ }^{3} \ell_{J}\right|^{3} \ell_{J}\right)$ and $r\left(\left.{ }^{3} \ell_{J}\right|^{3} \ell_{J}\right)$ are respectively the scattering length and effective range of the partial-wave ${ }^{3} \ell_{J}$, and the threshold behaviour of the amplitude, controlled by the value of $\ell$, is explicitly included by construction.

For partial-waves of equal $J^{P}$ but different $\ell$ that can couple dynamically, the $\boldsymbol{K}$-matrix formalism is a useful way of expressing the unitarity of the $\boldsymbol{S}$-matrix in terms of a real symmetric matrix, $\boldsymbol{K}(s){ }_{13}^{13}$ The inverse of the $\boldsymbol{K}$-matrix is related to the inverse of the $\boldsymbol{t}$-matrix by,

$$
\left[t^{-1}(s)\right]_{\ell J, \ell^{\prime} J}=\frac{1}{\left(2 k_{\mathrm{cm}}\right)^{\ell}}\left[K^{-1}(s)\right]_{\ell J, \ell^{\prime} J} \frac{1}{\left(2 k_{\mathrm{cm}}\right)^{\ell^{\prime}}}+\delta_{\ell \ell^{\prime}} I(s),
$$

where we recall $s=E_{\mathrm{cm}}^{2}$. The powers of $k_{\mathrm{cm}}$ ensure the correct behaviour at threshold. Unitarity of the S-matrix is guaranteed provided that $\operatorname{Im} I(s)=-\rho(s)$ for energies

\footnotetext{
${ }^{13}$ Previous lattice QCD calculations $4,62,64$ have demonstrated the effectiveness of the $\boldsymbol{K}$-matrix formalism in describing many resonant and non-resonant features of coupled-channel scattering.
} 
above the vector-pseudoscalar threshold and $\operatorname{Im} I(s)=0$ below threshold. The real part of $I(s)$ is arbitrary. A simple choice we take is $I(s)=-i \rho(s)$. An alternative which utilises the analytic properties of the amplitude, known as the Chew-Mandelstam prescription [72], constructs Re $I(s)$ using a dispersive integral of $\rho(s)$. The implementation of this prescription used here mirrors that in Ref. [64], and we choose to subtract such that $\operatorname{Re} I\left(s=\left(m_{\pi}+m_{\rho}\right)^{2}\right)=0$. A discussion of the Chew-Mandelstam phase-space is given in Appendix 3.D.

The $\boldsymbol{K}$-matrix can handle the case relevant to the finite volume where different $J^{P}$ values, which are uncoupled in an infinite volume, become coupled in the determinant of Eq. 2.53. This is achieved by forming a block-diagonal matrix out of the $\boldsymbol{K}$-matrices for each $J$. For example, the $\boldsymbol{t}$-matrix described in Eq. 3.3 will feature the $\boldsymbol{K}$-matrix,

$$
\mathbf{K}=\left[\begin{array}{ccc}
K\left({ }^{3} S_{1} \mid{ }^{3} S_{1}\right)(s) & K\left({ }^{3} S_{1} \mid{ }^{3} D_{1}\right)(s) & 0 \\
K\left({ }^{3} S_{1} \mid{ }^{3} D_{1}\right)(s) & K\left({ }^{3} D_{1} \mid{ }^{3} D_{1}\right)(s) & 0 \\
0 & 0 & K\left({ }^{3} D_{3} \mid{ }^{3} D_{3}\right)(s)
\end{array}\right]
$$

where $K\left(\left.{ }^{3} \ell_{J}\right|^{3} \ell_{J^{\prime}}^{\prime}\right)(s) \equiv K_{\ell J, \ell^{\prime} J^{\prime}}(s)$ is a real function of $s$. A simple choice of parameterisation for the $\boldsymbol{K}$-matrix is to express each element as a finite-order polynomial in $s$,

$$
K_{\ell J, \ell^{\prime} J}(s)=\sum_{n \geq 0}^{N\left(\left.{ }^{3} \ell_{J}\right|^{3} \ell_{J}^{\prime} J\right)} c_{n}\left(\left.{ }^{3} \ell_{J}\right|^{3} \ell_{J}^{\prime}\right) s^{n}
$$

where the coefficients $c_{n}\left(\left.{ }^{3} \ell_{J}\right|^{3} \ell_{J}^{\prime}\right)$ are real parameters. Other forms are explored in later chapters.

\subsubsection{Uncoupled $P$-wave Scattering}

As discussed, when only a single partial-wave makes a non-negligible contribution, the finite-volume quantisation condition reduces to a one-to-one mapping from finite-volume energy levels to phase-shift values at those energies. For isospin- $2 \rho \pi$ scattering, we initially assume that the ${ }^{3} P_{0},{ }^{3} P_{1},{ }^{3} P_{2}$ partial-waves dominate respectively the [000] $A_{1}^{-}$, $T_{1}^{-},\left(E^{-}, T_{2}^{-}\right)$irreps at low energy, proposing that the $F$-wave contributions can be neglected (see Table 3.1 for the partial-waves subduced into these irreps). Using the energy levels presented in Figure 3.6, we obtain two phase-shift points from each irrep. These are shown in Figure 3.8 where the inner errorbars show the statistical uncertainty on 
$E_{\mathrm{cm}}$ and $\delta_{3} P_{J}\left(E_{\mathrm{cm}}\right)$, while the outer errorbars on $\delta_{3} P_{J}\left(E_{\mathrm{cm}}\right)$ also include a conservative estimate of the systematic error which was obtained by varying the hadron masses and, more importantly, the anisotropy within their uncertainties. We find the largest systematic variations occur when $a_{t} m_{\rho}, a_{t} m_{\pi}$ are large and $\xi$ is small, and vice-versa, ${ }^{14}$ consistent with the observation that this causes the largest changes in the non-interacting energies, $E_{\text {n.i. }}$

To interpolate the scattering amplitudes in the energy range being considered, we parameterise the energy dependence of the $\boldsymbol{t}$-matrix using an effective range expansion, Eq. 3.13, truncated at the scattering length, $k_{\mathrm{cm}}^{2 \ell+1} \cot \left(\delta_{{ }^{3} \ell_{J}}\right)=a\left(\left.{ }^{3} \ell_{J}\right|^{3} \ell_{J}\right)^{-1}$, and minimise a $\chi^{2}$ with respect to $a\left(\left.{ }^{3} \ell_{J}\right|^{3} \ell_{J}\right)$. We fit independently for each partial-wave obtaining,

$$
\begin{array}{ll}
a\left({ }^{3} P_{0} \mid{ }^{3} P_{0}\right)=(-21 \pm 53 \pm 145) \cdot a_{t}^{3} & \chi^{2} / N_{\text {dof }}=0.37 /(2-1)=0.37 \\
a\left({ }^{3} P_{1} \mid{ }^{3} P_{1}\right)=(-133 \pm 49 \pm 172) \cdot a_{t}^{3} & \chi^{2} / N_{\text {dof }}=0.20 /(2-1)=0.20 \\
a\left({ }^{3} P_{2} \mid{ }^{3} P_{2}\right)=(+273 \pm 58 \pm 184) \cdot a_{t}^{3} & \chi^{2} / N_{\text {dof }}=6.57 /(4-1)=2.19
\end{array}
$$

where again the first error reflects the statistical uncertainty and the second error is an estimate of the systematic uncertainty.

The energy dependencies of the phase-shifts corresponding to these scattering-length descriptions are displayed in Figure 3.8. It is clear that the systematic uncertainties are dominating the uncertainties - this is a consequence of the relatively large uncertainty assigned to $\xi \sqrt{15}$ coupled with the rather weak interaction in this scattering channel which leads to small shifts of energies from their non-interacting values.

\subsubsection{Coupled $S, P, D$-wave Scattering}

In general, irreps feature a number of non-negligible partial-waves contributing to every energy level in the spectra and there is no longer a one-to-one mapping between energies and scattering amplitudes. To use the information from the energy levels across all the irreps, we perform a global analysis of the finite-volume spectra presented in Figures 3.6, 3.7 and 3.13. Each energy level provides a constraint on a combination of partial-wave amplitudes at that energy. To do this, as described above, we parameterise the energy-dependence of

\footnotetext{
${ }^{14}$ For $a_{t} m_{\rho}, a_{t} m_{\pi}$ small and $\xi$ large we find a compatible order of magnitude of variation in the parameters but of opposite sign. We therefore quote the systematic error as symmetric about the mean.

${ }^{15}$ Because of the slightly different $\xi$ obtained from the helicity 0 and \pm 1 components of the $\rho$.
} 


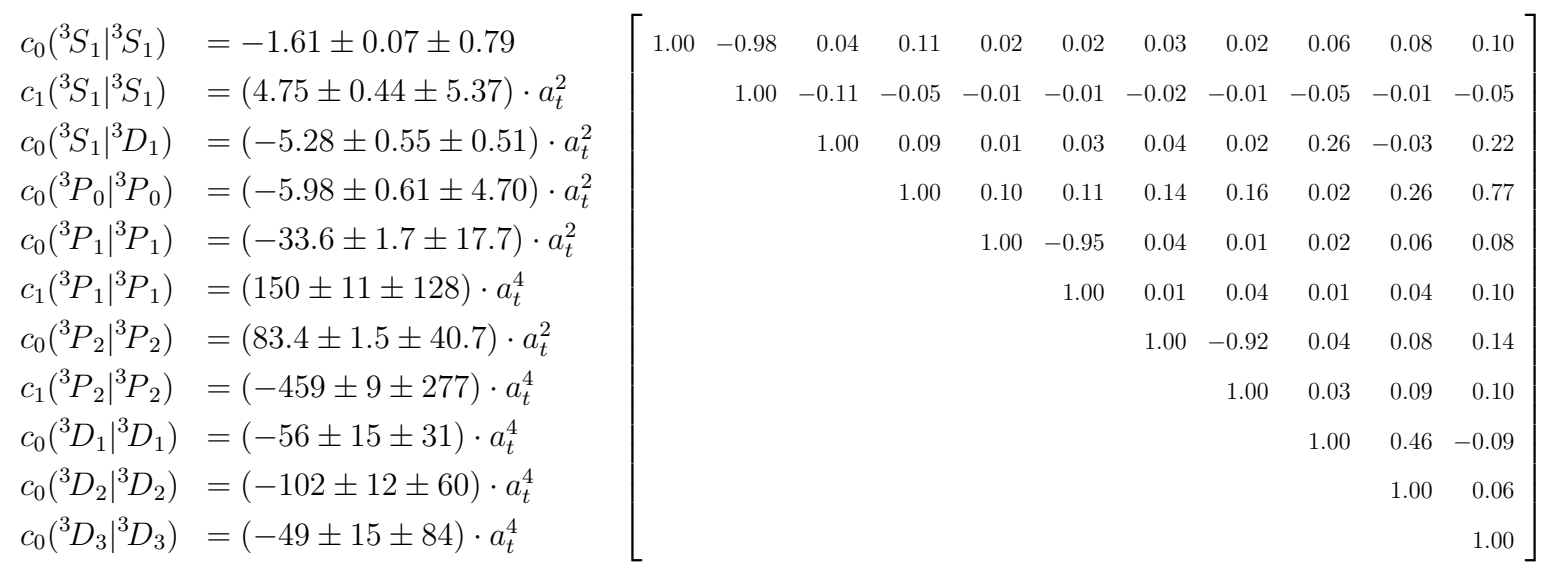

Table 3.4: A reference fit as described in the text with $\chi^{2} / N_{\text {dof }}=1.42$. The first uncertainty in each case is statistical and the second is an estimate of the systematic uncertainty as described in the text. Correlations between the $\boldsymbol{K}$-matrix parameters are displayed on the right. Parameters not shown were fixed to zero.

the block-diagonal $\boldsymbol{t}$-matrix and vary the parameters to give the best description of the finite-volume spectra by minimising the $\chi^{2}$ given in Eq. 3.11. We allow for non-negligible $\rho \pi$ isospin-2 amplitudes in the ${ }^{3} S_{1},{ }^{3} P_{0},{ }^{3} P_{1},{ }^{3} P_{2},{ }^{3} D_{1},{ }^{3} D_{2}$ and ${ }^{3} D_{3}$ partial-waves, including the dynamical couplings between the ${ }^{3} S_{1}$ and ${ }^{3} D_{1}$ waves and the ${ }^{3} P_{2}$ and ${ }^{3} F_{2}$ waves.

A number of polynomial parameterisations of the $\boldsymbol{K}$-matrix were considered and one example giving a good description of the 141 energy levels below $a_{t} E_{\mathrm{cm}}=0.41$ is provided by the fit shown in Table 3.4 where a $\boldsymbol{K}$-matrix parameterisation with 11 parameters was used. There are linear plus constant terms in $K\left({ }^{3} S_{1} \mid{ }^{3} S_{1}\right), K\left({ }^{3} P_{1} \mid{ }^{3} P_{1}\right)$ and $K\left({ }^{3} P_{2} \mid{ }^{3} P_{2}\right)$, and constant terms for all other relevant $K\left({ }^{3} \ell_{J} \mid{ }^{3} \ell_{J}^{\prime}\right)$ except $K\left({ }^{3} P_{2} \mid{ }^{3} F_{2}\right)=0$. The table also gives statistical uncertainties, estimates of systematic uncertainties from varying $a_{t} m_{\pi}$, $a_{t} m_{\rho}$ and $\xi$, and correlations between the parameters. We refer to this parameterisation and set of fit values as the reference amplitude.

Presented in Figures 3.9 and 3.10 are the finite-volume spectra determined on each irrep by solving Eq. 2.53 for the reference amplitude. The lattice computed energies, previously plotted in Figures 3.6 and 3.7, are also shown on the figure and we observe very good agreement between the two sets of energy levels (as expected from the $\chi^{2}$ ). The reference amplitude successfully predicts the location of levels which were not used to constrain the parameterisation (grey points), but a couple of features should be noted. Firstly, in 
Figure 3.9 some levels are apparently missed by the scattering parameterisation in the the $E^{-}, T_{1}^{-}$and $T_{2}^{-}$irreps around $a_{t} E_{\mathrm{cm}}=0.42$. The presence of these levels relies upon the inclusion of $F$-wave scattering amplitudes, which are neglected in the reference amplitude. Secondly, in Figure 3.10 the $A_{2}$ irreps with $\vec{P}=[011]$ and $\vec{P}=[002]$ appear to have energy levels missing in the lattice QCD calculation around $a_{t} E_{\mathrm{cm}}=0.425$ and $a_{t} E_{\mathrm{cm}}=0.415$ respectively. This is expected because the corresponding vector-pseudoscalar operators were not included in the bases used (see Section 3.5 - Table 3.3 and Figure 3.7.

A wide range of possible parameterisations that allow non-zero values for all constants $c_{n}\left(\left.{ }^{3} \ell_{J}\right|^{3} \ell_{J}^{\prime}\right)$ provided $\ell+\ell^{\prime}+2 n \leq 4$ were considered. This ensures the $\boldsymbol{K}$-matrix has parameter freedom in all terms up to order $a_{t}^{4}{ }^{16}$ Table 3.12 in Appendix 3.12 shows a selection of these fits along with the corresponding $\chi^{2} / N_{\text {dof }}$. Parameterisations without freedom in the $K\left(\left.{ }^{3} S_{1}\right|^{3} D_{1}\right)(s)$ polynomial are not able to give a good description of the finite-volume spectra, a point we return to in Section 3.6.3. However, a $K\left(\left.{ }^{3} P_{2}\right|^{3} F_{2}\right)(s)$ term does not appear to be required - this is consistent with expectations that the dynamical mixing between ${ }^{3} P_{2}$ and ${ }^{3} F_{2}$ is suppressed by the angular momentum barrier at these relatively low energies just above threshold.

$\boldsymbol{K}$-matrix parameterisations which include pole terms, efficient at describing resonant behaviour and bound states, did not give a good description of the finite-volume spectra and we do not include such parameterisations in Table 3.12. This is consistent with our qualitative observations on the spectra in Section 3.5 .

For all the parameterisations in Table 3.12 with $\chi^{2} / N_{\text {dof }} \leq 1.5$, Figure 3.11 shows the two phase-shifts and mixing-angle in the Stapp parameterisation, Eq. 3.1, for the dynamically-coupled ${ }^{3} S_{1}$ and ${ }^{3} D_{1}$ partial-waves, and the phase-shifts for the ${ }^{3} P_{0},{ }^{3} P_{1}$, ${ }^{3} P_{2},{ }^{3} D_{2}$ and ${ }^{3} D_{3}$ partial-waves. It can be seen that the scattering amplitudes are robust under parameterisation variations and the phase-shifts are consistent within statistical uncertainties. As expected, the systematic uncertainty on each parameterisation is largely due to $\xi$ and hence discretisation effects dominate the uncertainties.

We conclude that $\rho \pi$ in isospin-2 is weakly repulsive in ${ }^{3} S_{1}$. The other phase-shifts are consistent with zero within the systematic uncertainties, though there are hints of weak attraction in ${ }^{3} P_{2}$ and weak repulsion in ${ }^{3} P_{0},{ }^{3} P_{1}$ and ${ }^{3} D_{J}$. The dynamical mixing between the ${ }^{3} S_{1}$ and ${ }^{3} D_{1}$-waves is small but significantly non-zero within the systematic

\footnotetext{
${ }^{16}$ Including terms with higher powers of $a_{t}$ did not significantly improve the quality of fit.
} 
uncertainties and across all parameterisations. In the following section we investigate in more detail how the spectra depend on the mixing angle $\bar{\epsilon}$.

\subsubsection{Constraints on the ${ }^{3} S_{1}-{ }^{3} D_{1}$ Mixing-Angle}

To demonstrate that the ${ }^{3} S_{1}-{ }^{3} D_{1}$ mixing angle, $\bar{\epsilon}$, is being robustly constrained in the energy range considered, we investigate which energy levels are providing the most stringent constraints on it. If we neglect $\ell \geq 4$, the quantisation conditions for irreps at rest admitting ${ }^{3} S_{1},{ }^{3} D_{1}$-waves are independent of the sign of $\bar{\epsilon}$, whereas the quantisation conditions for irreps in-flight depend on the sign of $\bar{\epsilon}$. This means that for spatially periodic boundary conditions in a cubic box, ignoring contributions from $\ell \geq 4$, in-flight irreps must be considered in order to uniquely determine $\bar{\epsilon}$ from finite-volume spectra 17

Figure 3.12 shows finite-volume spectra in the [000] $T_{1}^{+}$irrep and the $\vec{P} A_{2}$ irreps, $\vec{P} \neq \overrightarrow{0}$, as a function of the $\boldsymbol{K}$-matrix parameter, $c_{0}\left({ }^{3} S_{1} \mid{ }^{3} D_{1}\right)$, along with the corresponding phase-shifts $\delta_{{ }^{3} S_{1}}, \delta_{{ }^{3} D_{1}}$ and mixing angle $\bar{\epsilon}{ }^{18}$ The reference parameterisation in Table 3.4 has been used, varying $c_{0}\left({ }^{3} S_{1} \mid{ }^{3} D_{1}\right)$ while keeping all other parameters fixed. The symmetry of the finite-volume spectrum in $[000] T_{1}^{+}$about $c_{0}\left(\left.{ }^{3} S_{1}\right|^{3} D_{1}\right)=0$ illustrates the expected sign independence at rest. For the $A_{2}$ irreps in-flight, the finite-volume spectra are clearly asymmetric about $c_{0}\left({ }^{3} S_{1} \mid{ }^{3} D_{1}\right)=0$ and energy levels have a varying degree of dependence on $\bar{\epsilon}$. Furthermore, the phase-shifts vary only within their systematic uncertainties for $-20 \leq c_{0}\left(\left.{ }^{3} S_{1}\right|^{3} D_{1}\right) \leq 20$, in stark contrast to $\bar{\epsilon}$. This suggests that the constraints placed on $c_{0}\left({ }^{3} S_{1} \mid{ }^{3} D_{1}\right)$ by the finite-volume spectra are the most significant in determining $\bar{\epsilon}$ and Figure 3.12 illustrates the numerous energy levels in the region $a_{t} E_{\mathrm{cm}} \leq 0.41$ which provide these constraints, e.g. the splitting between the $4^{\text {th }}$ and $5^{\text {th }}$ energy levels in the [002] $A_{2}$ irrep is strongly dependent on $c_{0}\left(\left.{ }^{3} S_{1}\right|^{3} D_{1}\right)$ in the small range we consider. Other irreps in-flight admitting the dynamically coupled ${ }^{3} S_{1}$ and ${ }^{3} D_{1}$ partial-waves provide additional constraints on $c_{0}\left(\left.{ }^{3} S_{1}\right|^{3} D_{1}\right)$ and subsequently $\bar{\epsilon}$. We conclude that these finite-volume calculations robustly determine the magnitude and sign of $\bar{\epsilon}$.

\footnotetext{
${ }^{17}$ If contributions of partial-waves with $\ell \geq 4$ are included for irreps overall at rest, then in general the finite-volume spectra are no longer independent of the sign of $\bar{\epsilon}$.

${ }^{18}$ The relations in Eq. 3.1 and Eq. 3.14 can be manipulated to show that the sign of $c_{0}\left(\left.{ }^{3} S_{1}\right|^{3} D_{1}\right)$ is dependent on the sign of $\bar{\epsilon}$ whereas the phase-shifts are independent of the sign of $c_{0}\left(\left.{ }^{3} S_{1}\right|^{3} D_{1}\right)$.
} 

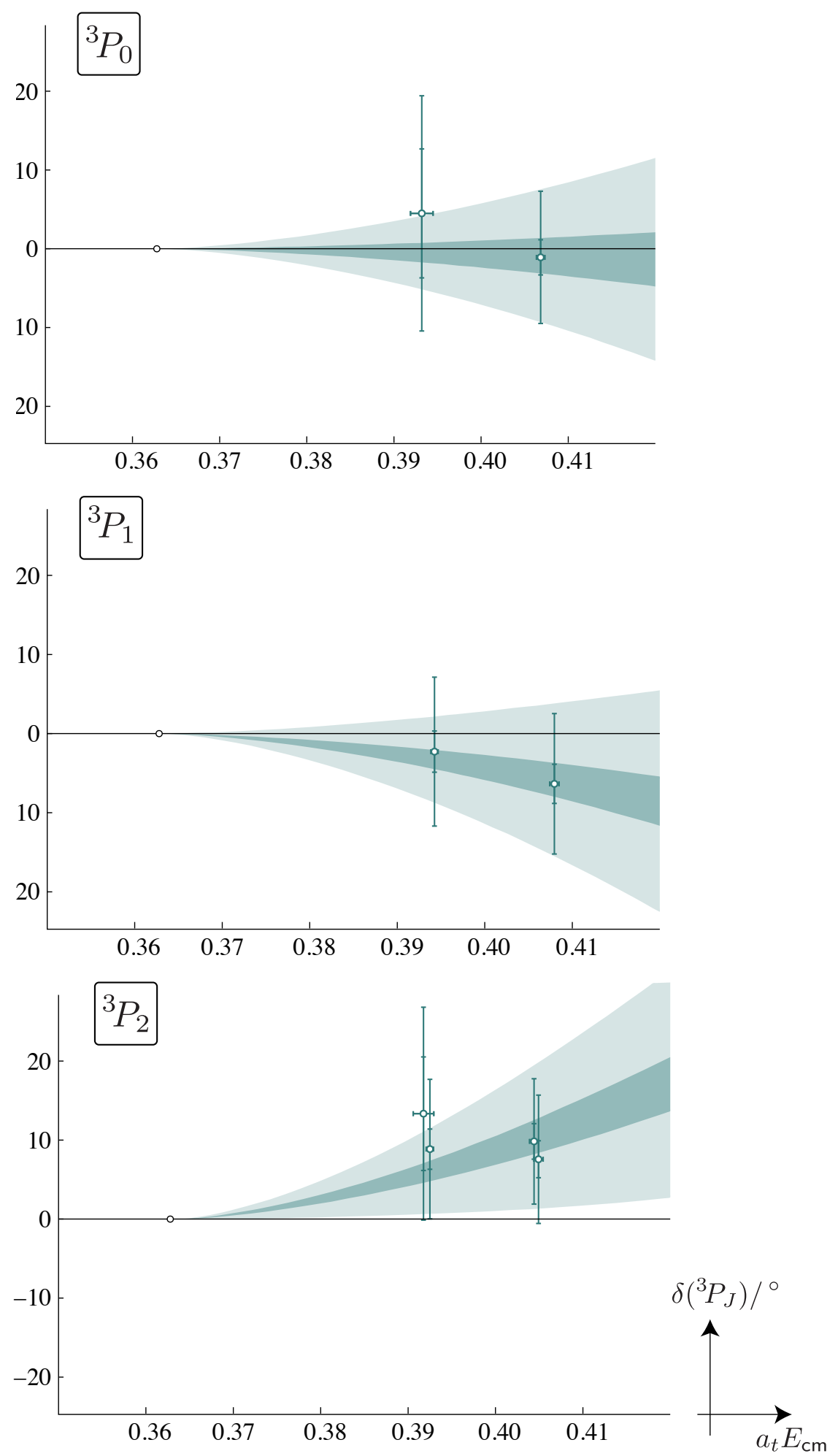

Figure 3.8: Phase-shifts for the ${ }^{3} P_{0},{ }^{3} P_{1}$ and ${ }^{3} P_{2}$ partial-waves. The points are as described in the text. Inner bands reflect the statistical uncertainties on the phase-shifts from the fits in Eq. 3.17 and outer bands reflect the combined statistical and systematic uncertainties. 

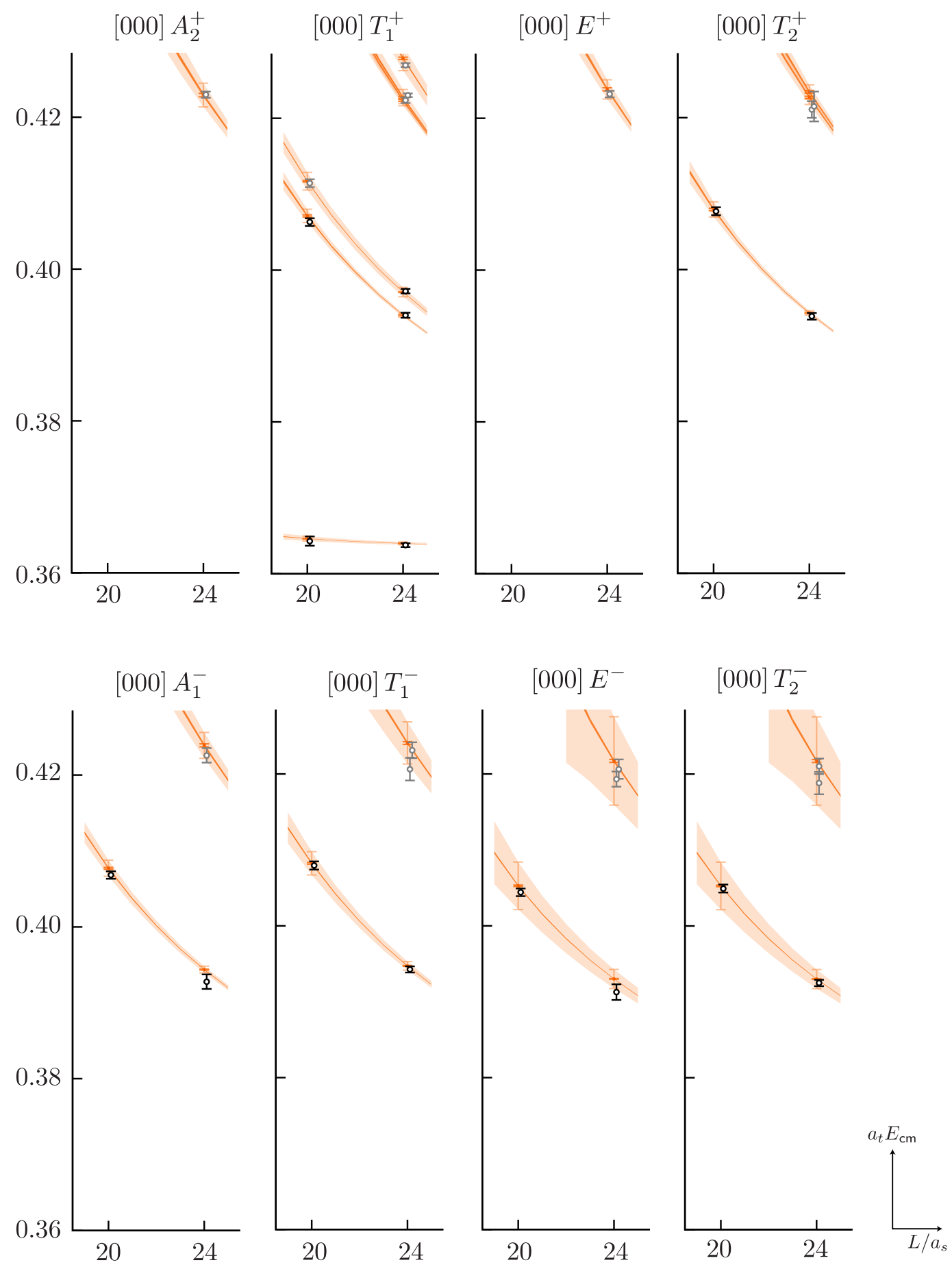

Figure 3.9: Volume-dependent spectra for irreps with $\vec{P}=\overrightarrow{0}$. Black and grey points, slightly displaced in $L / a_{s}$ for clarity, are, as in Figure 3.6, energy levels extracted from analyses of correlation functions. Orange points and bands show solutions to Eq. 2.53 for the reference $\boldsymbol{K}$-matrix parameterisation in Table 3.4. The inner dark orange error bars/error bands reflect the statistical uncertainties and the outer lighter orange error bars/error bands also include systematic uncertainties. 

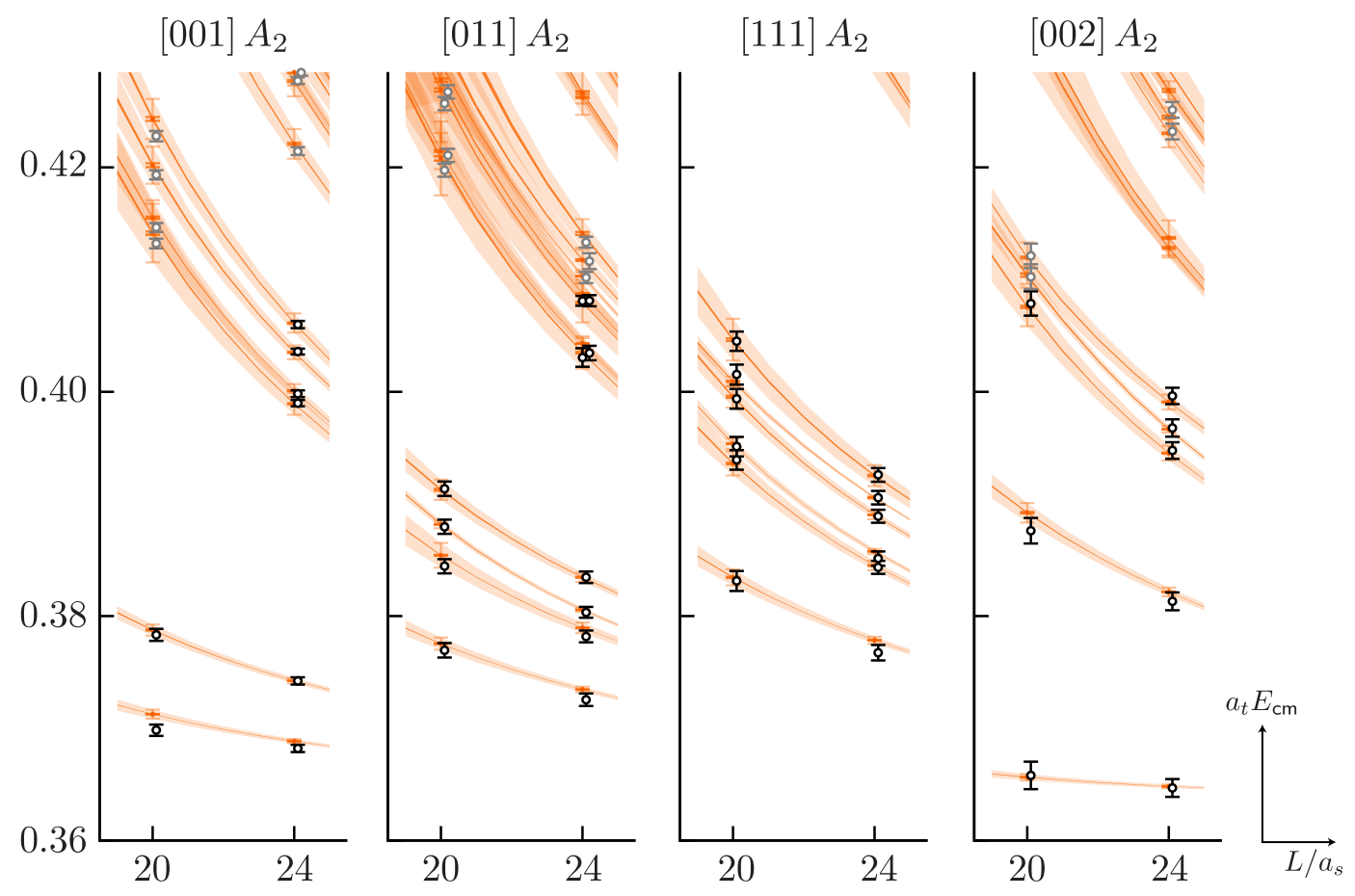

Figure 3.10: As Figure 3.9 but for $A_{2}$ irreps with $\vec{P} \neq \overrightarrow{0}$. 


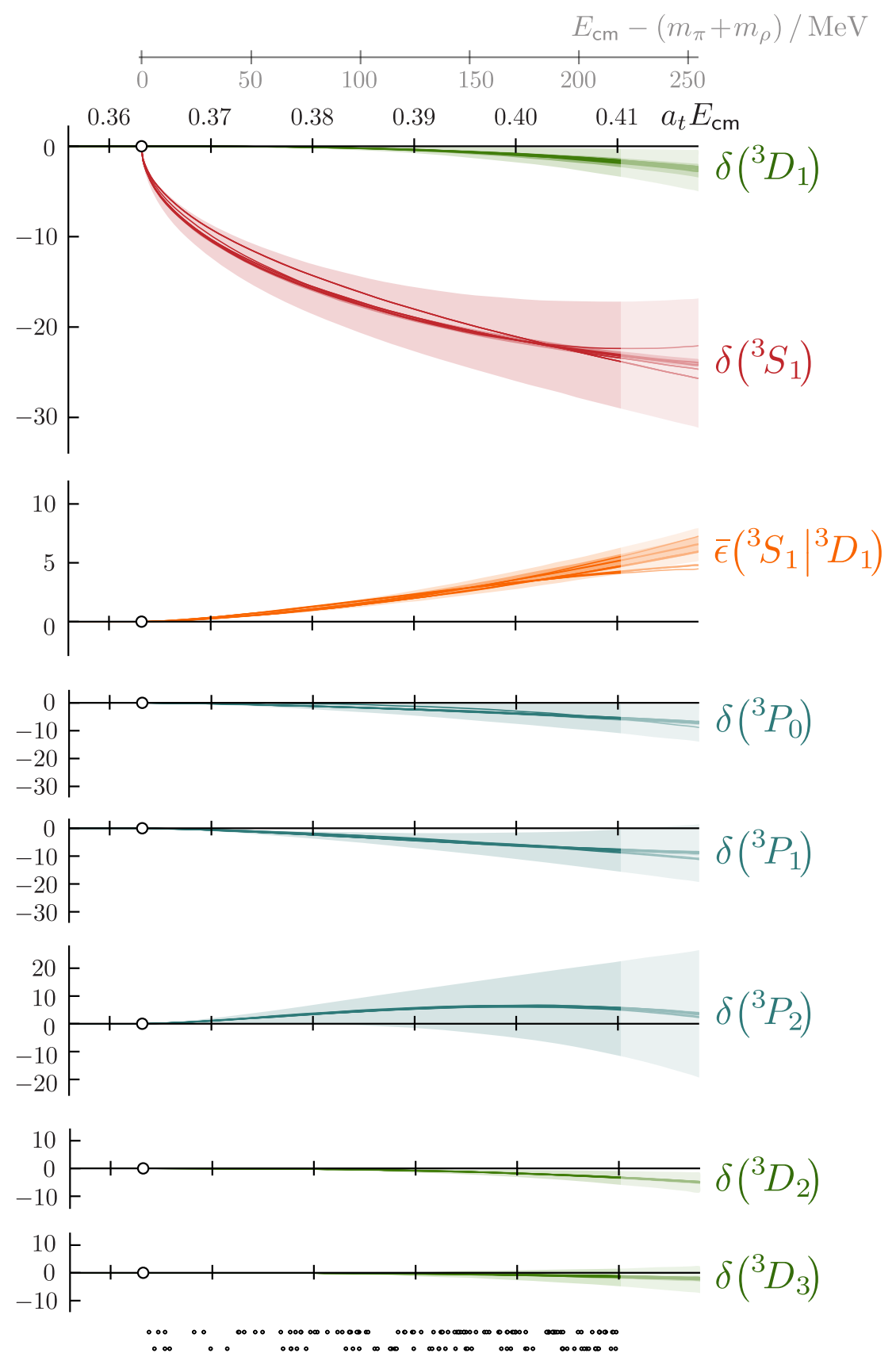

Figure 3.11: Phase-shifts for partial-waves, ${ }^{3} \ell_{J}$, and ${ }^{3} S_{1}-{ }^{3} D_{1}$ mixing angle, $\bar{\epsilon}$, as described in the text. Each curve corresponds to a parameterisation in Table 3.12 with $\chi^{2} / N_{\text {dof }} \leq 1.5$. The darker inner band (typically thinner than the width of the curves) reflects the statistical uncertainty on the reference parameterisation in Table 3.4 and the lighter outer bands correspond to the combined statistical and systematic uncertainties on this parameterisation. Faded regions highlight that no energy levels have been used to constrain the phase-shifts and mixing angle when $a_{t} E_{\mathrm{cm}} \geq 0.41$. The discrete energy levels used as constraints are shown as small dots at the bottom of the figure with the top and bottom rows for $L / a_{s}=24$ and 20 respectively. An axis reflecting energy above threshold in physical units is displayed at the top of the figure. 

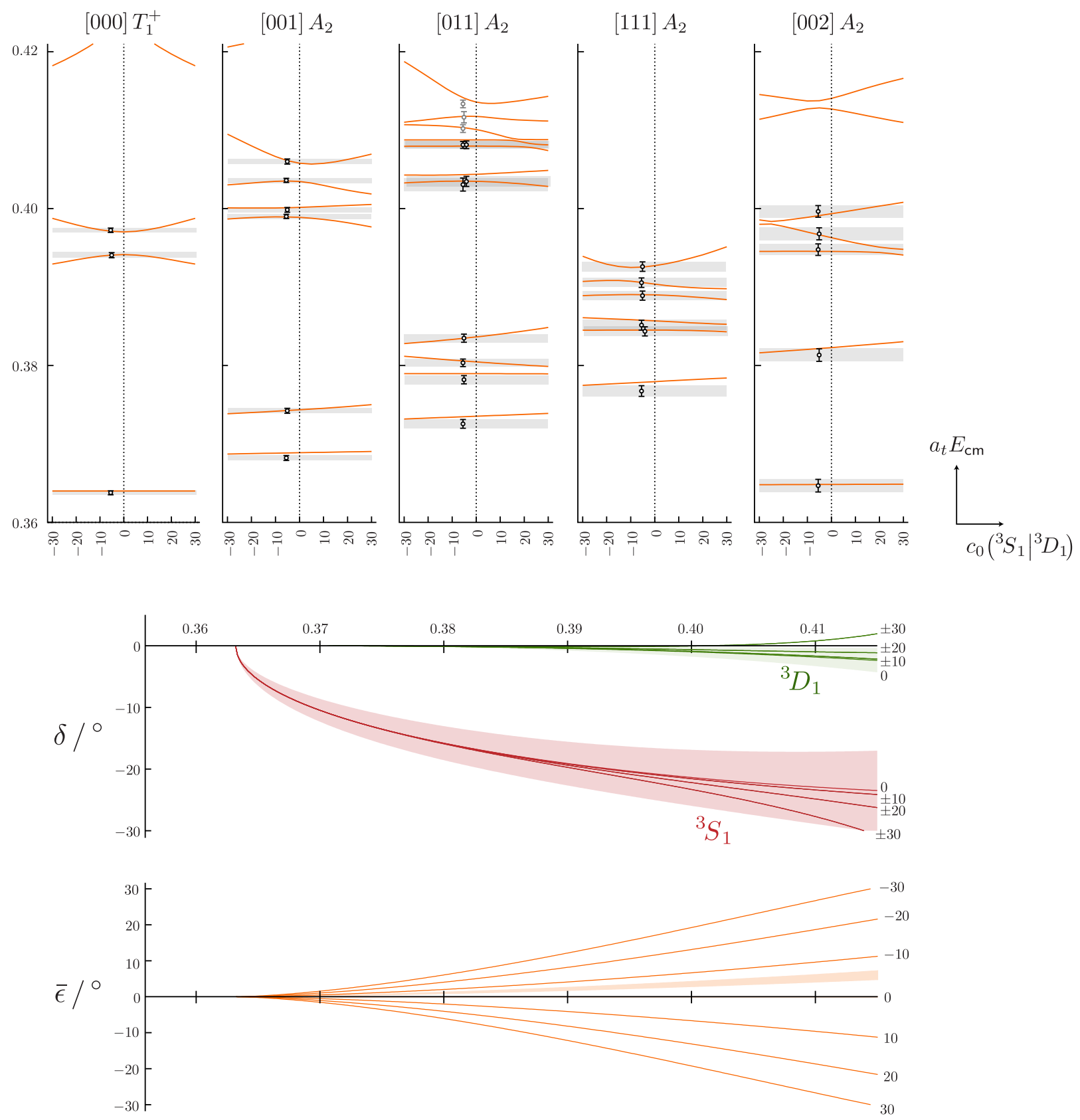

Figure 3.12: Upper: Finite-volume spectra in the [000] $T_{1}^{+}$and $\vec{P} \neq \overrightarrow{0} A_{2}$ irreps on the $L / a_{s}=24$ lattice as a function of $c_{0}\left(\left.{ }^{3} S_{1}\right|^{3} D_{1}\right)$ as described in the text. Black and grey points are, as in Figure 3.6, energy levels extracted from correlation functions, plotted at $c_{0}\left(\left.{ }^{3} S_{1}\right|^{3} D_{1}\right)=-5.28$ the value in the reference amplitude parameterisation in Table 3.4 . Grey bands are to guide the eye and show the combined statistical and systematic uncertainties on the black points. Orange curves show the finite-volume spectra from the reference amplitude when $c_{0}\left(\left.{ }^{3} S_{1}\right|^{3} D_{1}\right)$ is varied with the other parameters fixed. Lower: ${ }^{\delta_{S_{1}}}\left(E_{\mathrm{cm}}\right), \delta_{{ }^{3} D_{1}}\left(E_{\mathrm{cm}}\right)$ and $\bar{\epsilon}\left(E_{\mathrm{cm}}\right)$ for the reference amplitude with a selection of values for $c_{0}\left(\left.{ }^{3} S_{1}\right|^{3} D_{1}\right)$. The shaded bands shows the combined statistical and systematic uncertainties of the reference amplitudes, i.e. when $c_{0}\left({ }^{3} S_{1} \mid{ }^{3} D_{1}\right)=-5.28$. 


\subsection{Summary}

In this chapter, we have reported on the first calculation of $\rho \pi$ scattering using lattice QCD, focusing on the isospin-2 channel. As expected for an exotic isospin, the hadron-hadron interactions are found to be relatively weak. The angular momentum barrier at low energy provides a natural hierarchy in $\ell$, and the coupling of $\ell$ with the intrinsic spin of the $\rho$ leads to a number of partial-waves for a given $J^{P}$. The possibility of 'spin-orbit' forces in QCD allows amplitudes of common $\ell$, but distinct $J$, to differ. For each of $J^{P}=1^{+}, 2^{-}, 3^{+}, \ldots$, there are two dynamically-coupled partial-waves, and for $1^{+}$we are able to determine the ${ }^{3} S_{1}$ and ${ }^{3} D_{1}$ amplitudes along with the coupling between them. We are also able to determine the scattering phase-shifts for all partial-waves of $\ell \leq 2$.

Our results followed from application of the formalism relating scattering amplitudes in an infinite volume to the discrete spectrum of QCD in a finite periodic volume defined by the lattice. We computed this spectrum in two spatial volumes in a version of QCD where the degenerate $u, d$ quarks are heavier than in experiment, such that they are degenerate with the strange quark and the theory has an exact $\mathrm{SU}(3)_{\mathrm{F}}$ flavour symmetry. The resulting theory has octet pseudoscalar mesons ( such as the $\pi$ ) of mass $\sim 700 \mathrm{MeV}$ and stable octet vectors mesons (such as the $\rho$ ) of mass $\sim 1020 \mathrm{MeV}$.

Spectra were obtained by variational analysis of matrices of two-point correlation functions computed using bases of operators resembling $\rho \pi$. The large number of partialwaves contributing, together with the weakness of the interactions, leads to spectra which feature many nearly-degenerate states. The use of bases of operators featuring all relevant 'meson-meson' constructions in the energy region of interest leads to a robust determination, where the nearly degenerate states are resolved in the variational solution by virtue of their orthogonal overlap structures in the space of operators.

The spectra obtained in the two volumes, featuring 141 energy levels, were used to constrain the energy dependence of multiple partial-waves. Amplitudes were parameterised and the parameters adjusted so that the predicted finite-volume spectra matched the calculated spectra, as quantified by a correlated $\chi^{2}$. The dependence on the particular form of parameterisations used was explored and found to be rather modest. The largest single source of systematic uncertainty in the calculation was due to the difference in the lattice anisotropy for the $\pi$ and the various helicity components of the $\rho$. This is a relatively 
small discretisation effect, but its impact in this particular calculation is amplified by the weakness of the interactions. This causes the finite-volume energy levels to be shifted relatively little from their non-interacting values.

The resulting scattering amplitudes presented in Figure 3.11 show a phase-shift in the ${ }^{3} S_{1}$ channel which is clearly non-zero and repulsive. Phase-shifts for the other extracted partial waves are found to be compatible with zero within their systematic error. The mixing between ${ }^{3} S_{1}$ and ${ }^{3} D_{1}$ in $J^{P}=1^{+}$, as quantified by a mixing angle $\bar{\epsilon}$ in the Stapp parameterisation, is determined and found to be small but significantly non-zero. We are able to determine its sign by considering spectra where the $\rho \pi$ has overall non-zero momentum with respect to the lattice.

The low energy (near threshold) behaviour of the scattering amplitudes can be summarised in terms of the corresponding scattering lengths. Using the definition, $\lim _{k_{\mathrm{cm}} \rightarrow 0} k_{\mathrm{cm}}^{2 \ell+1} \cot \left[\delta^{3} \ell_{J}\right]=a\left(\left.{ }^{3} \ell_{J}\right|^{3} \ell_{J}\right)^{-1}$, we find for the reference amplitude 19

$$
\begin{array}{ll}
a\left({ }^{3} S_{1} \mid{ }^{3} S_{1}\right)=(-5.44 \pm 0.10 \pm 0.88) \cdot a_{t} & m_{\pi} a\left({ }^{3} S_{1} \mid{ }^{3} S_{1}\right)=(-0.80 \pm 0.01 \pm 0.13) \\
a\left({ }^{3} P_{0} \mid{ }^{3} P_{0}\right)=(-132 \pm 14 \pm 104) \cdot a_{t}^{3} & m_{\pi}^{3} a\left({ }^{3} P_{0} \mid{ }^{3} P_{0}\right)=(-0.43 \pm 0.05 \pm 0.34) \\
a\left({ }^{3} P_{1} \mid{ }^{3} P_{1}\right)=(-303 \pm 12 \pm 114) \cdot a_{t}^{3} & m_{\pi}^{3} a\left({ }^{3} P_{1} \mid{ }^{3} P_{1}\right)=(-0.98 \pm 0.04 \pm 0.37) \\
a\left({ }^{3} P_{2} \mid{ }^{3} P_{2}\right)=(502 \pm 14 \pm 362) \cdot a_{t}^{3} & m_{\pi}^{3} a\left({ }^{3} P_{2} \mid{ }^{3} P_{2}\right)=(1.62 \pm 0.05 \pm 1.17) \\
a\left({ }^{3} D_{2} \mid{ }^{3} D_{2}\right)=(-8950 \pm 1050 \pm 5330) \cdot a_{t}^{5} & m_{\pi}^{5} a\left({ }^{3} D_{2} \mid{ }^{3} D_{2}\right)=(-0.63 \pm 0.07 \pm 0.38) \\
a\left({ }^{3} D_{3} \mid{ }^{3} D_{3}\right)=(-4320 \pm 1310 \pm 7270) \cdot a_{t}^{5} & m_{\pi}^{5} a\left({ }^{3} D_{3} \mid{ }^{3} D_{3}\right)=(-0.30 \pm 0.09 \pm 0.51) .
\end{array}
$$

The qualitative behaviour of the ${ }^{3} P_{J}$-waves is the same as that found in Section 3.6.1 where only irreps with a single non-negligible partial wave were considered and each of the ${ }^{3} P_{J}$ scattering lengths given above is consistent within errors with those found in that section.

In conclusion, we have demonstrated how scattering amplitudes involving hadrons with non-zero spin can be computed using lattice QCD. Further applications of the approach presented in this study include the isospin-1 $\rho \pi$ and $\omega \pi$ systems. In $J^{P}=1^{+}$, experiments observe low-lying resonances, the $a_{1}$ and $b_{1}$ axial-vectors respectively, both of which have been measured to have significant coupling to both ${ }^{3} S_{1}$ and ${ }^{3} D_{1}$ partial-waves 47,73 . We will examine these two systems in the following two chapters. Furthermore, experiments

\footnotetext{
${ }^{19}$ We do not quote a ${ }^{3} D_{1}$ scattering length because $t\left({ }^{3} D_{1} \mid{ }^{3} D_{1}\right) \sim\left(k_{\mathrm{cm}}\right)^{4} \ll t\left({ }^{3} S_{1} \mid{ }^{3} D_{1}\right) \sim\left(k_{\mathrm{cm}}\right)^{2}$ at threshold and as such the contribution of $\bar{\epsilon}$ cannot be neglected, unlike in the ${ }^{3} S_{1}$ case.
} 
in the charmonium sector appear to show resonant behaviour in the exotic-flavour $J / \psi \pi$ channel. First attempts to determine lattice QCD spectra here have appeared [68, 74], but as yet, there has been no determination of the scattering amplitudes.

\section{APPENDICES}

\section{A Subduction Tables}

Tables 3.5, 3.6 and 3.7 present the subduction patterns for vector-pseudoscalar partialwaves with $\ell \leq 3$ for momenta of type $[00 n],[0 n n]$ and $[n n n]$ respectively for integer $n$.

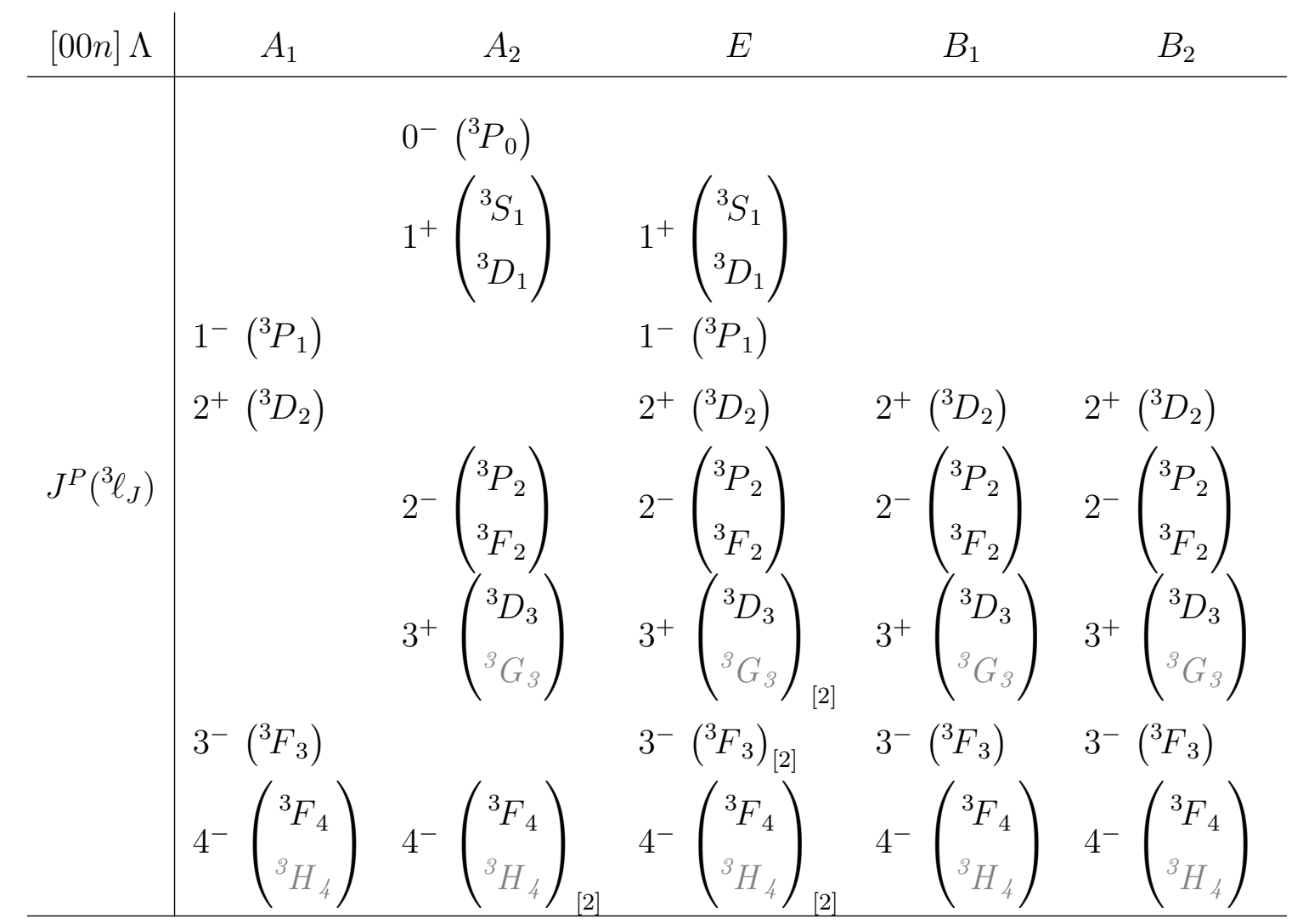

Table 3.5: Partial-wave $J^{P}\left({ }^{3} \ell_{J}\right)$ subductions for $\ell \leq 3$ at $\vec{P}=[00 n]$ into irreps $\Lambda$ of the little-group Dic 4 . A subscript $[N]$ indicates that this $J^{P}$ has $N$ embeddings in the irrep $\Lambda$. Partial-waves with $\ell>3$ that couple dynamically to partial-waves with $\ell \leq 3$ are shown in grey italic. This table is derived using the results presented in Refs. [9] and [10]. 


\begin{tabular}{|c|c|c|c|c|}
\hline$[0 n n] \Lambda$ & $A_{1}$ & $A_{2}$ & $B_{1}$ & $B_{2}$ \\
\hline$J^{P}\left({ }^{3} \ell_{J}\right)$ & $\begin{array}{l}1^{-}\left({ }^{3} P_{1}\right) \\
2^{+}\left({ }^{3} D_{2}\right)_{[2]} \\
2^{-}\left(\begin{array}{c}{ }^{3} P_{2} \\
{ }^{3} F_{2}\end{array}\right) \\
3^{+}\left(\begin{array}{c}{ }^{3} D_{3} \\
{ }^{3} G_{3}\end{array}\right) \\
3^{-}\left({ }^{3} F_{3}\right)_{[2]} \\
4^{-}\left(\begin{array}{c}{ }^{3} F_{4} \\
{ }^{3} H_{1}\end{array}\right)\end{array}$ & $\begin{array}{l}0^{-}\left({ }^{3} P_{0}\right) \\
1^{+}\left(\begin{array}{c}{ }^{3} S_{1} \\
{ }^{3} D_{1}\end{array}\right) \\
2^{+}\left({ }^{3} D_{2}\right) \\
2^{-}\left(\begin{array}{l}{ }^{3} P_{2} \\
{ }^{3} F_{2}\end{array}\right) \\
3^{+}\left(\begin{array}{l}{ }^{3} D_{3} \\
{ }^{3} G_{3} \\
3^{-}\left({ }^{3} F_{3}\right) \\
4^{-}\left({ }^{3} F_{4}\right. \\
{ }^{3} H_{4}\end{array}\right.\end{array}$ & $\begin{array}{l}1^{+}\left(\begin{array}{c}{ }^{S_{1}} \\
{ }^{3} D_{1}\end{array}\right) \\
1^{-}\left({ }^{3} P_{1}\right) \\
2^{+}\left({ }^{3} D_{2}\right) \\
2^{-}\left(\begin{array}{c}{ }^{3} P_{2} \\
{ }^{3} F_{2}\end{array}\right) \\
3^{+}\left({ }^{3} D_{3}\right. \\
3^{3} G_{3} \\
\left.4^{3} F_{3}\right) \\
4^{-}\left({ }^{3} F_{4}\right. \\
{ }^{3} H_{4}\end{array}$ & $\begin{array}{l}1^{+}\left(\begin{array}{c}{ }^{3}{ }^{3} D_{1} \\
1^{-}\end{array}\left({ }^{3} P_{1}\right)\right. \\
2^{+}\left({ }^{3} D_{2}\right) \\
2^{-}\left({ }^{3} P_{2}\right) \\
\left.{ }^{3} F_{2}\right) \\
3^{+}\left({ }^{3} D_{3}\right. \\
3^{3} G_{3} \\
\left.4^{-}{ }^{3} F_{3}\right) \\
\left({ }^{3} F_{4}\right. \\
{ }^{3} H_{4}\end{array}$ \\
\hline
\end{tabular}

Table 3.6: As Table 3.5. but for $\vec{P}=[0 n n]$ with little-group $\mathrm{Dic}_{2}$. 


\begin{tabular}{|c|c|c|c|}
\hline$[n n n] \Lambda$ & $A_{1}$ & $A_{2}$ & $E$ \\
\hline$J^{P}\left({ }^{3} \ell_{J}\right)$ & $\begin{array}{l}1^{-}\left({ }^{3} P_{1}\right) \\
2^{+}\left({ }^{3} D_{2}\right) \\
3^{+}\left({ }^{3} D_{3}\right. \\
3^{-}\left({ }^{3} F_{3}\right) \\
4^{-}\left({ }^{3} F_{4}\right. \\
{ }^{3} H_{3}\end{array}$ & 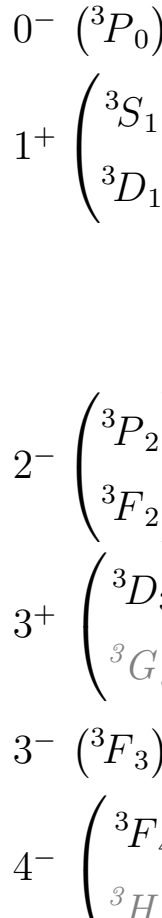 & $\begin{array}{l}1^{+}\left(\begin{array}{c}{ }^{3} S_{1} \\
{ }^{3} D_{1}\end{array}\right. \\
1^{-}\left({ }^{3} P_{1}\right) \\
2^{+}\left({ }^{3} D_{2}\right) \\
2^{-}\left({ }^{3} P_{2}\right. \\
\left.{ }^{3} F_{2}\right) \\
3^{+}\left({ }^{3} D_{3}\right. \\
3^{3} G_{3} \\
\left({ }^{3} F_{3}\right) \\
4^{-}\left({ }^{3} F_{4}\right. \\
{ }^{3} H_{1}\end{array}$ \\
\hline
\end{tabular}

Table 3.7: As Table 3.5, but for $\vec{P}=[n n n]$ with little-group $\mathrm{Dic}_{3}$.

\section{B Operator Bases}

We show the operator basis in Tables 3.8-3.11 for all irreps considered in Figures 3.6, 3.7 and 3.13 that were not shown in Table 3.3 .

\begin{tabular}{r:rrrrr}
{$[000] A_{2}^{+}$} & {$[000] E^{+}$} & {$[000] T_{2}^{+}$} & {$[000] A_{1}^{-}$} & {$[000] T_{1}^{-}$} \\
\hline$\rho_{[011]} \pi_{[0-1-1]}$ & $\rho_{[011]} \pi_{[0-1-1]}$ & $\rho_{[001]} \pi_{[00-1]}$ & $\rho_{[001]} \pi_{[00-1]}$ & $\rho_{[001]} \pi_{[00-1]}$ \\
$\rho_{[111]} \pi_{[-1-1-1]}$ & $\rho_{[111]} \pi_{[-1-1-1]}$ & $\{2\} \rho_{[011]} \pi_{[0-1-1]}$ & $\rho_{[011]} \pi_{[0-1-1]}$ & $\{2\} \rho_{[011]} \pi_{[0-1-1]}$ \\
& & $\rho_{[111]} \pi_{[-1-1-1]}$ & $\rho_{[111]} \pi_{[-1-1-1]}$ & $\rho_{[111]} \pi_{[-1-1-1]}$ \\
\hline 2 ops. & 2 ops. & 4 ops. & 3 ops. & 4 ops.
\end{tabular}

Table 3.8: As Table 3.3 but for irreps $A_{2}^{+}, E^{+}, T_{2}^{+}, A_{1}^{-}$and $T_{1}^{-}$at $\vec{P}=[000]$. 


\begin{tabular}{r:c:ccrc}
{$[000] E^{-}$} & {$[000] T_{2}^{-}$} & {$[001] A_{1}$} & {$[001] B_{1}$} & \multicolumn{1}{c}{$[001] B_{2}$} \\
\hline$\rho_{[001]} \pi_{[00-1]}$ & $\rho_{[001]} \pi_{[00-1]}$ & $\rho_{[011]} \pi_{[0-10]}$ & $\rho_{[011]} \pi_{[0-10]}$ & $\{2\} \rho_{[011]} \pi_{[0-10]}$ \\
$\{2\} \rho_{[011]} \pi_{[0-1-1]}$ & $\{2\} \rho_{[011]} \pi_{[0-1-1]}$ & $\rho_{[010]} \pi_{[0-11]}$ & $\rho_{[010]} \pi_{[0-11]}$ & $\{2\} \rho_{[010]} \pi_{[0-11]}$ \\
$\rho_{[111]} \pi_{[-1-1-1]}$ & $\{2\} \rho_{[111]} \pi_{[-1-1-1]}$ & $\rho_{[111]} \pi_{[-1-10]}$ & $\{2\} \rho_{[111]} \pi_{[-1-10]}$ & $\rho_{[111]} \pi_{[-1-10]}$ \\
& & & $\rho_{[110]} \pi_{[-1-11]}$ & $\{2\} \rho_{[110]} \pi_{[-1-11]}$ & $\rho_{[110]} \pi_{[-1-11]}$ \\
& & & $\rho_{[012]} \pi_{[0-1-1]}$ & $\rho_{[012]} \pi_{[0-1-1]}$ & $\{2\} \rho_{[012]} \pi_{[0-1-1]}$ \\
\hline 4 ops. & 5 ops. & & 4 ops. & 6 ops. & 6 ops.
\end{tabular}

Table 3.9: As Table 3.3 but for irreps $E^{-}$and $T_{2}^{-}$at $\vec{P}=[000]$ and $A_{1}, B_{1}$ and $B_{2}$ at $\vec{P}=[001]$.

\begin{tabular}{|c|c|c|c|}
\hline$[001] E_{2}$ & {$[011] A_{1}$} & {$[011] B_{1}$} & {$[011] B_{2}$} \\
\hline$\rho_{[001]} \pi_{[000]}$ & $\rho_{[001]} \pi_{[010]}$ & $\rho_{[011]} \pi_{[000]}$ & $\rho_{[011]} \pi_{[000]}$ \\
\hline$\rho_{[000]} \pi_{[001]}$ & $\rho_{[111]} \pi_{[-100]}$ & $\rho_{[001]} \pi_{[010]}$ & $\{2\} \rho_{[001]} \pi_{[010]}$ \\
\hline$\{3\} \rho_{[011]} \pi_{[0-10]}$ & $\{3\} \rho_{[110]} \pi_{[-101]}$ & $\rho_{[000]} \pi_{[011]}$ & $\rho_{[000]} \pi_{[011]}$ \\
\hline$\{3\} \rho_{[010]} \pi_{[0-11]}$ & $\rho_{[100]} \pi_{[-111]}$ & $\{2\} \rho_{[111]} \pi_{[-100]}$ & $\rho_{[111]} \pi_{[-100]}$ \\
\hline$\rho_{[002]} \pi_{[00-1]}$ & $\rho_{[012]} \pi_{[00-1]}$ & $\{3\} \rho_{[110]} \pi_{[-101]}$ & $\{3\} \rho_{[110]} \pi_{[-101]}$ \\
\hline$\{3\} \rho_{[111]} \pi_{[-1-10]}$ & $\rho_{[002]} \pi_{[01-1]}$ & $\{2\} \rho_{[100]} \pi_{[-111]}$ & $\rho_{[100]} \pi_{[-111]}$ \\
\hline$\{3\} \rho_{[110]} \pi_{[-1-11]}$ & $\rho_{[01-1]} \pi_{[002]}$ & $\rho_{[012]} \pi_{[00-1]}$ & $\{2\} \rho_{[012]} \pi_{[00-1]}$ \\
\hline$\rho_{[00-1]} \pi_{[002]}$ & $\rho_{[00-1]} \pi_{[012]}$ & $\rho_{[002]} \pi_{[01-1]}$ & $\{2\} \rho_{[002]} \pi_{[01-1]}$ \\
\hline$\{3\} \rho_{[012]} \pi_{[0-1-1]}$ & $\{3\} \rho_{[-10-1]} \pi_{[112]}$ & 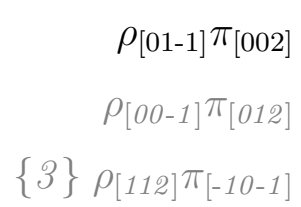 & $\begin{array}{r}\{2\} \rho_{[01-1]} \pi_{[002]} \\
\{2\} \rho_{[00-1]} \pi_{[012]} \\
\{3\} \rho_{[112]} \pi_{[-10-1]}\end{array}$ \\
\hline & ops. & & 3 ops. \\
\hline
\end{tabular}

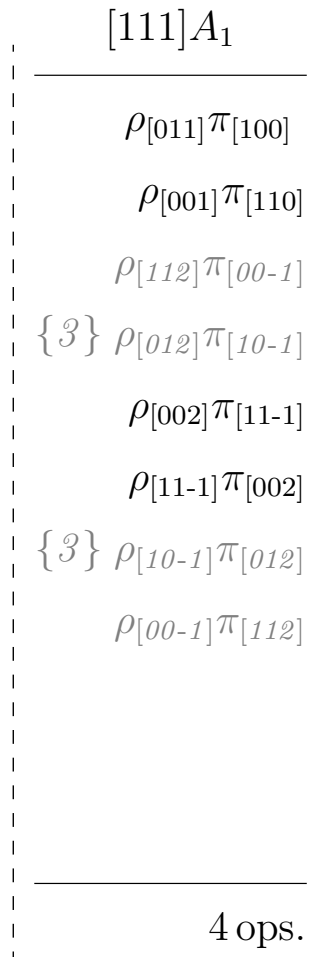

Table 3.10: As Table 3.3 but for irreps $E_{2}$ at $\vec{P}=[001] ; A_{1}, B_{1}$ and $B_{2}$ at $\vec{P}=[011]$ and $A_{1}$ at $\vec{P}=[111]$. 


\begin{tabular}{|c|c|c|c|c|}
\hline$[111] E_{2}$ & {$[002] A_{1}$} & {$[002] B_{1}$} & {$[002] B_{2}$} & {$[002] E_{2}$} \\
\hline$\rho_{[111]} \pi_{[000]}$ & $\rho_{[011]} \pi_{[0-11]}$ & $\rho_{[011]} \pi_{[0-11]}$ & $\{2\} \rho_{[011]} \pi_{[0-11]}$ & $\rho_{[001]} \pi_{[001]}$ \\
\hline$\{3\} \rho_{[011]} \pi_{[100]}$ & $\rho_{[012]} \pi_{[0-10]}$ & $\rho_{[012]} \pi_{[0-10]}$ & $\{2\} \rho_{[012]} \pi_{[0-10]}$ & $\rho_{[002]} \pi_{[000]}$ \\
\hline$\{3\} \rho_{[100]} \pi_{[011]}$ & $\rho_{[111]} \pi_{[-1-11]}$ & $\{2\} \rho_{[111]} \pi_{[-1-11]}$ & $\rho_{[111]} \pi_{[-1-11]}$ & $\{3\} \rho_{[011]} \pi_{[0-11]}$ \\
\hline$\rho_{[000]} \pi_{[111]}$ & $\rho_{[0-10]} \pi_{[012]}$ & $\rho_{[0-10]} \pi_{[012]}$ & $\{2\} \rho_{[0-10]} \pi_{[012]}$ & $\rho_{[000]} \pi_{[002]}$ \\
\hline$\{3\} \rho_{[112]} \pi_{[00-1]}$ & $\rho_{[112]} \pi_{[-1-10]}$ & $\{2\} \rho_{[112]} \pi_{[-1-10]}$ & $\rho_{[112]} \pi_{[-1-10]}$ & $\{3\} \rho_{[012]} \pi_{[0-10]}$ \\
\hline$\{6\} \rho_{[012]} \pi_{[10-1]}$ & $\rho_{[110]} \pi_{[-1-12]}$ & $\{2\} \rho_{[110]} \pi_{[-1-12]}$ & $\rho_{[110]} \pi_{[-1-12]}$ & $\{3\} \rho_{[111]} \pi_{[-1-11]}$ \\
\hline$\{3\} \rho_{[002]} \pi_{[11-1]}$ & & & & $\{3\} \rho_{[0-10]} \pi_{[012]}$ \\
\hline$\{3\} \rho_{[11-1]} \pi_{[002]}$ & & & & $\{3\} \rho_{[112]} \pi_{[-1-10]}$ \\
\hline$\{6\} \rho_{[01-1]} \pi_{[102]}$ & & & & $\{3\} \rho_{[110]} \pi_{[-1-12]}$ \\
\hline$\{3\} \rho_{[00-1]} \pi_{[112]}$ & & & & \\
\hline $140 \mathrm{ps}$. & 2 ops. & 3 ops. & 3 ops. & 9 ops \\
\hline
\end{tabular}

Table 3.11: As Table 3.3 but for irreps $E_{2}$ at $\vec{P}=[111]$ and $A_{1}, B_{1}, B_{2}$ and $E_{2}$ at $\vec{P}=[002]$. 


\section{C Additional Spectra}

We provide here the finite-volume spectra plots for irreps at non-zero momenta, not shown in Figures 3.6 and 3.7, in Figure 3.13. 

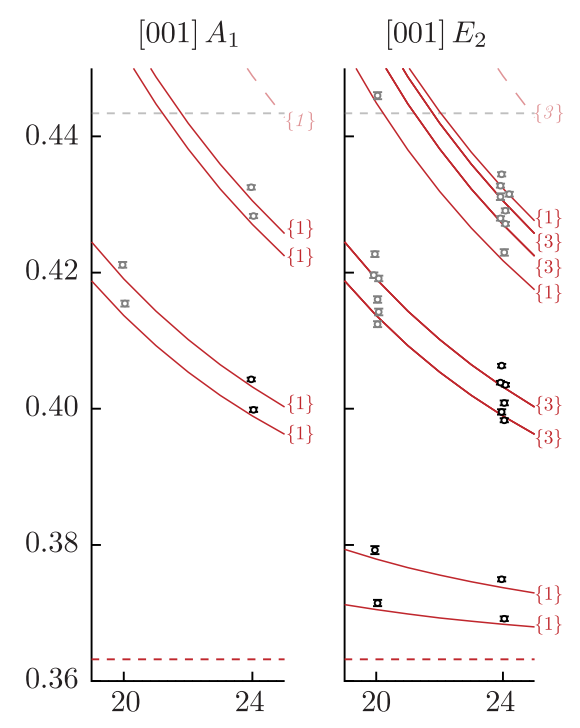

[001] $B_{1}$

[001] $B_{2}$
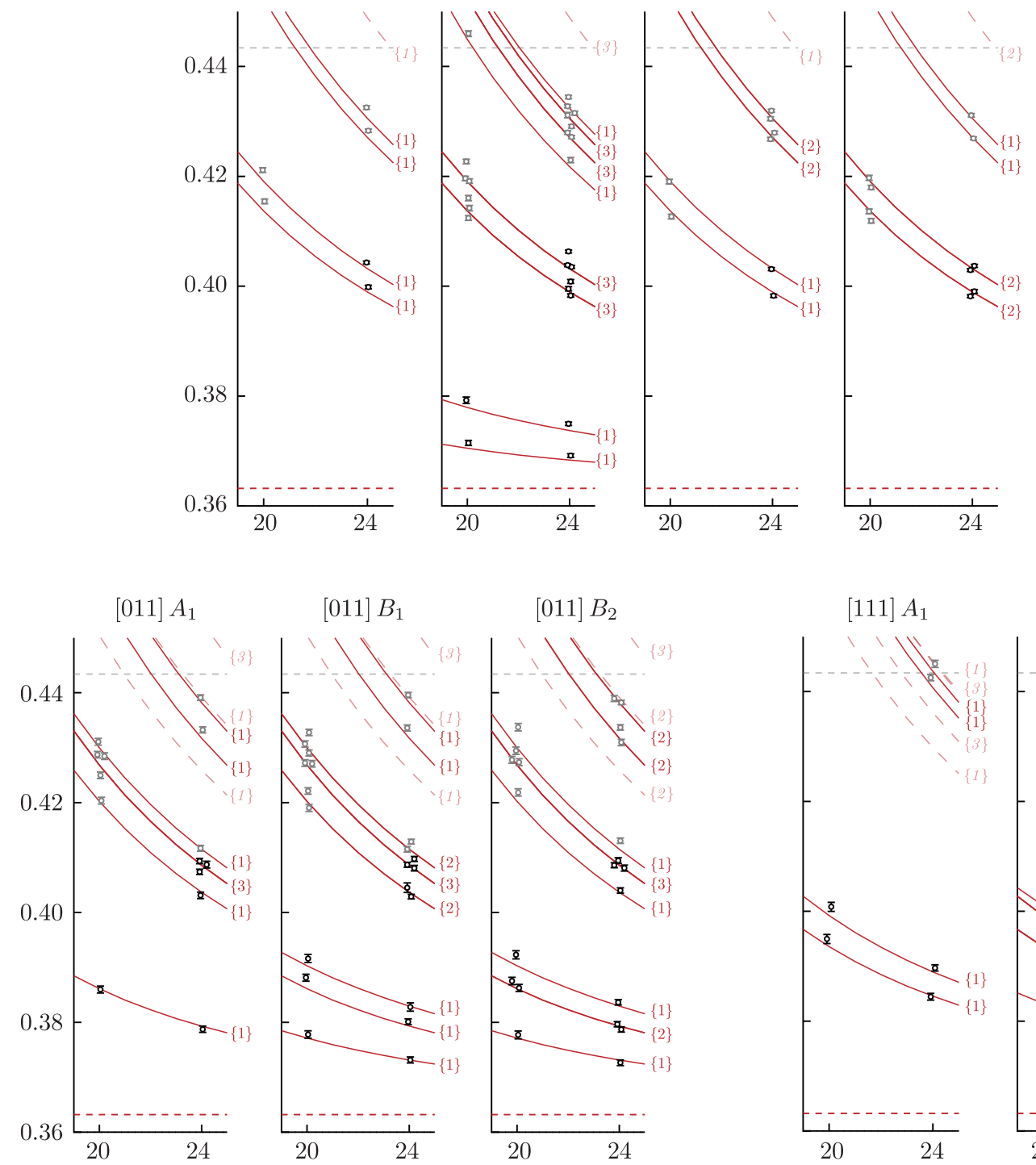

[011] $B_{1}$

[011] $B_{2}$

[111] $A_{1}$

[111] $E_{2}$
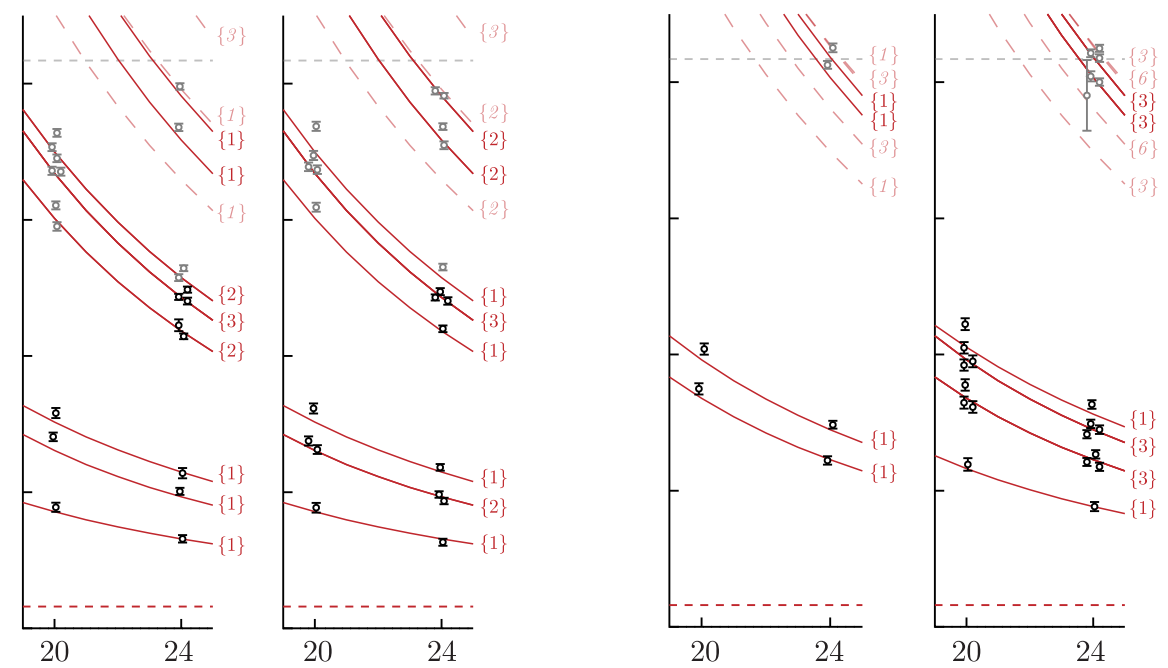

[002] $A_{1}$

[002] $E_{2}$

$[002] B_{1}$

$[002] B_{2}$
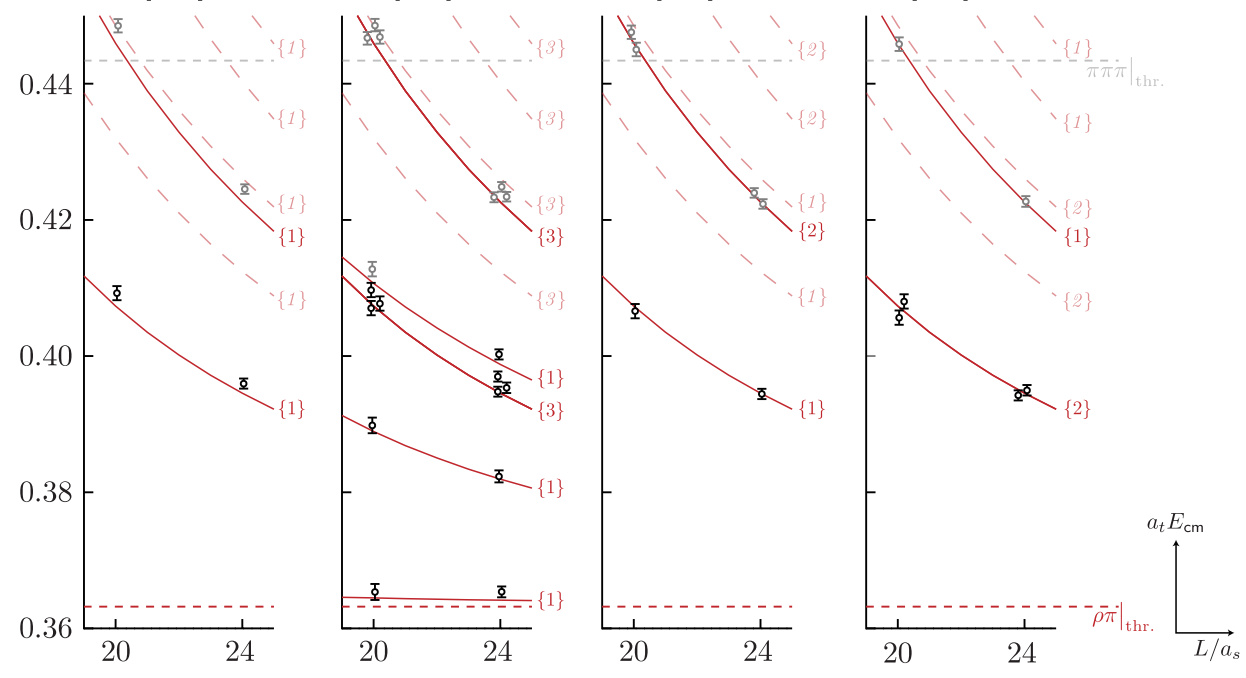

Figure 3.13: As Figure 3.7 but for all other irreps with $|\vec{P}|^{2} \leq 4$. 


\section{D Chew-Mandelstam Phase-Space}

The $\boldsymbol{t}$-matrix can be conveniently expressed in terms of a real symmetric $\boldsymbol{K}$-matrix and a diagonal matrix $\boldsymbol{I}$ as shown in Eq. 3.14. Unitarity of the $\boldsymbol{S}$-matrix for real $s$ is guaranteed provided,

$$
\operatorname{Im} I_{a}(s)= \begin{cases}-\rho_{a}(s) & \text { if } s \geq s_{\mathrm{thr}}^{(a)} \\ 0 & \text { if } s<s_{\mathrm{thr}}^{(a)}\end{cases}
$$

leaving the real part of $I_{a}(s)$ arbitrary. Here, we have introduced an index $a$ labelling the hadron-hadron channel to generalise the discussion. Of course, in the context of $\rho \pi$ scattering presented in this chapter, there is only one channel.

A simple choice for $I_{a}(s)$ immediately follows from the constraints imposed by unitary: choosing $I_{a}(s)=-i \rho_{a}(s)$. Although a perfectly good choice, this form of $I_{a}(s)$ does not make use of the analytic properties of the amplitude. Furthermore, this choice does not give any useful behaviour of the amplitude near thresholds or any resonant pole masses.

An alternative is constructed through the Chew-Mandelstam function [72] where the real part is related to the imaginary part through a dispersive integral. The physical $\boldsymbol{S}$-matrix amplitudes are assumed to be analytic functions of complex $s$, and it follows from Eq. 3.14 that $I_{a}(s)$ are analytic functions of complex $s$ on a domain which excludes the branch cut originating from the square root in $\rho_{a}(s)$ at threshold, $s_{\text {thr. }}^{(a)}=\left(m_{1}^{(a)}+m_{2}^{(a)}\right)^{2}$. Using the Cauchy integral formula, one can write,

$$
I_{a}(s)=\frac{1}{2 \pi i} \int_{\mathcal{C}_{a}} d s^{\prime} \frac{I_{a}\left(s^{\prime}\right)}{s^{\prime}-s}
$$

where $\mathcal{C}_{a}$ is the boundary of the open set $\mathcal{U}_{a} \subset \mathbb{C}$ where $I_{a}(s)$ is analytic, shown in Figure 3.14.

By deforming the contour $\mathcal{C}_{a}$ such that the boundary goes out toward infinity and assuming $I_{a}(s)$ has compact support for $|s| \rightarrow \infty$, the contour integral in Eq. 3.18 can be expressed as an integral along the real axis from $s_{\text {thr. }}^{(a)}$ to infinity,

$$
\begin{aligned}
I_{a}(s) & =\frac{1}{2 \pi i} \lim _{\epsilon \rightarrow 0} \int_{s_{\text {thr. }}^{(a)}}^{\infty} d s^{\prime} \frac{I_{a}\left(s^{\prime}+i \epsilon\right)-I_{a}\left(s^{\prime}-i \epsilon\right)}{s^{\prime}-s} \\
& =-\frac{1}{\pi} \int_{s_{\text {thr. }}^{(a)}}^{\infty} d s^{\prime} \frac{\rho_{a}\left(s^{\prime}\right)}{s^{\prime}-s}
\end{aligned}
$$




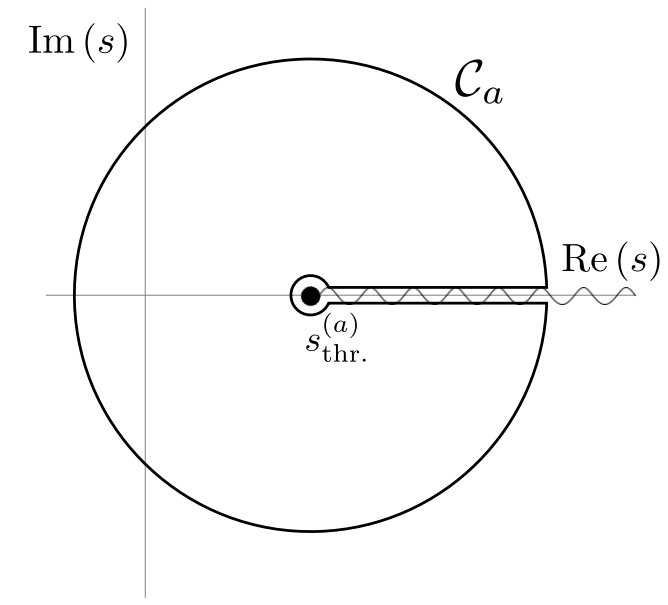

Figure 3.14: A sketch of the contour $\mathcal{C}_{a}$ in the complex $s$-plane. The conventional choice of branch cut from $s_{\text {thr. }}^{(a)}=\left(m_{1}^{(a)}+m_{2}^{(a)}\right)^{2}$ towards infinity along the positive real axis is shown.

where the second equality follows from the 'Real analyticity' [75 of $\boldsymbol{t}(s): \boldsymbol{t}(s)=\boldsymbol{t}\left(s^{*}\right)^{*}$ and so $I_{a}\left(s^{*}\right)=I_{a}(s)^{*}$, giving $I_{a}(s+i \epsilon)-I_{a}(s-i \epsilon)=2 \operatorname{Im} I_{a}(s+i \epsilon)=-2 \rho_{a}(s+i \epsilon)$. This integral is however divergent and needs regularising. This can be done through a 'subtraction', where the regularised integral can be written as,

$$
I_{a}(s)=I_{a}\left(s_{\text {thr. }}^{(a)}\right)-\frac{s-s_{\text {thr. }}^{(a)}}{\pi} \int_{s_{\text {thr. }}^{(a)}}^{\infty} d s^{\prime} \frac{\rho_{a}\left(s^{\prime}\right)}{\left(s^{\prime}-s\right)\left(s^{\prime}-s_{\text {thr. }}^{(a)}\right)}
$$

which integrated explicitly gives [64,

$$
I_{a}(s)=I_{a}\left(s_{\mathrm{thr} .}^{(a)}\right)+\frac{\rho_{a}(s)}{\pi} \log \left[\frac{\xi_{a}(s)+\rho_{a}(s)}{\xi_{a}(s)-\rho_{a}(s)}\right]-\frac{\xi_{a}(s)}{\pi} \frac{m_{2}^{(a)}-m_{1}^{(a)}}{m_{2}^{(a)}+m_{1}^{(a)}} \log \frac{m_{2}}{m_{1}},
$$

where $\xi_{a}(s)=1-\left(m_{2}^{(a)}+m_{1}^{(a)}\right)^{2} / s$.

The expression in Eq. 3.21 has a residual arbitrariness in the real part of $I_{a}\left(s_{\mathrm{thr}}^{(a)}\right)$ for which one choice we employ is to set this to zero, i.e. $\operatorname{Re} I_{a}\left(s=s_{\text {thr. }}^{(a)}\right)=0$, and we refer to this as 'threshold subtraction'. Another convenient choice, when the $\boldsymbol{K}$-matrix has a 'pole term' with mass parameter $m$, is to set $I_{a}\left(s_{\text {thr. }}^{(a)}\right)$ such that $\operatorname{Re} I_{a}\left(s=m^{2}\right)=0$, which we refer to as 'pole subtraction'. With this choice, in the energy region around $s=m^{2}$, the $\boldsymbol{t}$-matrix resembles a Breit-Wigner form and the mass $m$ and Breit-Wigner mass $m_{R}$ coincide [64]. 


\section{E Scattering Parameterisations}

Table 3.12 shows the different parameterisations of the $\boldsymbol{K}$-matrix considered in the parameterisation variation as discussed in detail in Section 3.6.2. 


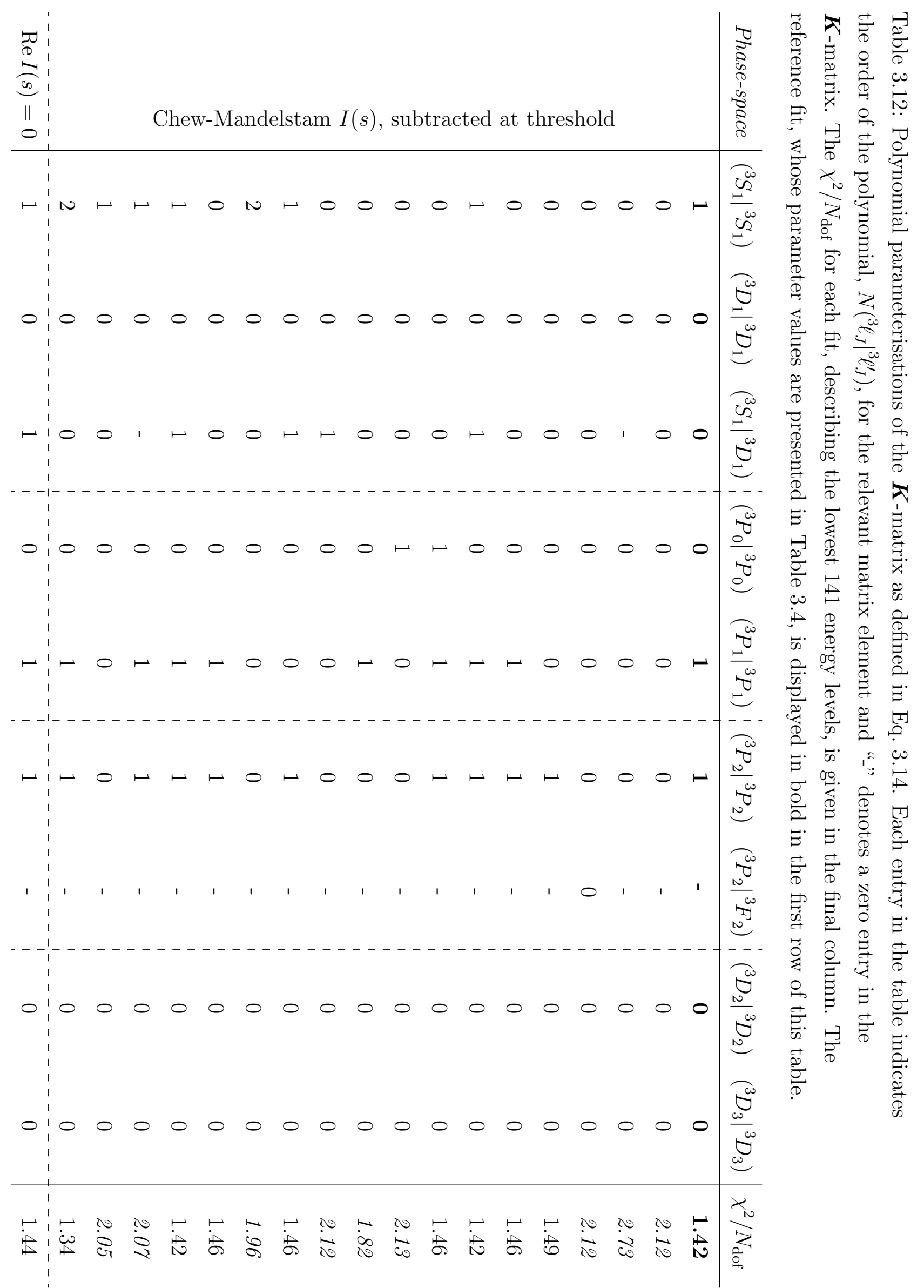




\section{Investigation of $\rho \pi$ in isospin-1 at $m_{\pi} \sim 700 \mathrm{MeV}$}

The scattering of $\rho \pi$ with exotic isospin- 2 was found, as expected, to have weak interactions in all partial-wave amplitudes up to the $\pi \pi \pi$ threshold. Naturally, one may want to consider $\rho \pi$ scattering in quantum numbers where more significant interactions are expected, e.g. $\rho \pi$ in isospin- 1 where an $a_{1}$ axial-vector resonance features. In this short chapter, we examine the isospin-1 $\rho \pi$ system in the $\mathrm{SU}(3)_{\mathrm{F}}$ flavour limit and find evidence that, at this pion mass, the $a_{1}$ axial-vector is a bound-state. We begin with a discussion on $G$-parity and Bose-symmetry at the $\mathrm{SU}(3)_{\mathrm{F}}$ point and then present the finite-volume spectra.

\subsection{G-Parity in $\mathrm{SU}(3)_{\mathrm{F}}$}

Within the $\mathrm{SU}(3)_{\mathrm{F}}$ flavour framework, the $a_{1}$ meson, with $\left(I^{G}\right) J^{P C}=\left(1^{-}\right) 1^{++}$, forms part of an axial-vector octet. We label the $\mathrm{SU}(3)_{\mathrm{F}}$ multiplets by the isoscalar component and carry a subscript denoting the irrep, for example, $\left(\boldsymbol{f}_{\mathbf{1}}\right)_{\mathbf{8}}$ labels the axial-vector octet hosting the $a_{1}$. We will adopt this labelling convention from here-on-in unless otherwise specified, so the $\pi$ and $\rho$ mesons belong to the $(\boldsymbol{\eta})_{\mathbf{8}}$ (pseudoscalar) and $(\boldsymbol{\omega})_{\mathbf{8}}$ (vector) octets respectively and the corresponding singlets, $(\boldsymbol{\eta})_{1}$ and $(\boldsymbol{\omega})_{1}$, contain admixtures of the $\eta, \eta^{\prime}$ and $\omega, \phi$ mesons respectively. Unlike in the isospin-2 case, where the corresponding $\mathrm{SU}(3)_{\mathrm{F}}$ representation 27 can be formed only from products of two-mesons in octet multiplets, $\mathbf{8} \otimes \mathbf{8}$, the $\mathrm{SU}(3)_{\mathrm{F}}$ representation $\mathbf{8}$ and can be formed from both $\mathbf{8} \otimes \mathbf{1}$ and $\mathbf{8} \otimes \mathbf{8}$.

A subtlety that arises when considering the product of two $\mathrm{SU}(3)_{\mathrm{F}}$ representations, that does not occur in $\mathrm{SU}(2)_{\mathrm{F}}$, is the possibility of multiple embeddings within some target flavour representation. In $\mathrm{SU}(2)_{\mathrm{F}}$, the product of two isospins, $I_{1} \otimes I_{2}=\left|I_{1}-I_{2}\right| \oplus \cdots \oplus I_{1}+I_{2}$, gives a single embedding in each isospin $\left|I_{1}-I_{2}\right|,\left|I_{1}-I_{2}\right|+1, \cdots, I_{1}+I_{2}$. However, in 
$\mathrm{SU}(3)_{\mathrm{F}}$, the tensor product of two octets for example, $\mathbf{8} \otimes \mathbf{8}$, gives rise to the two linearly independent octet embeddings $\mathbf{8}_{\mathbf{1}}$ and $\mathbf{8}_{\mathbf{2}}$.

Following the conventions given in DeSwart's [67], the $\mathrm{SU}(3)_{\mathrm{F}}$ Clebsch-Gordan coefficients for $\mathbf{8} \otimes \mathbf{8} \rightarrow \mathbf{8}_{1} \oplus \mathbf{8}_{2}$ are symmetric and anti-symmetric in $\mathbf{8}_{\mathbf{1}}$ and $\mathbf{8}_{\mathbf{2}}$ respectively,

$$
\left(\begin{array}{ccc}
8 & 8 & 8_{i} \\
\nu_{1} & \nu_{2} & \nu
\end{array}\right)=\xi_{i}\left(\begin{array}{ccc}
8 & 8 & 8_{i} \\
\nu_{2} & \nu_{1} & \nu
\end{array}\right)
$$

where $\xi_{1}=1$ and $\xi_{2}=-1$. It is useful at this point to write out the non-zero $\mathrm{SU}(3)_{\mathrm{F}}$ Clebsch-Gordan coefficients for the two embeddings explicitly. As we are at liberty to work with any member of the octet, we choose $\nu=\left(I, Y, I_{z}\right)=(1,0,1)$. We label the initial octets $\mathbf{8}_{a}$ and $\boldsymbol{8}_{b}$ in order to distinguish them. The states $\left|\boldsymbol{F} ; I, Y, I_{z}\right\rangle$ are then given by,

$$
\begin{array}{r}
\left|\mathbf{8}_{1} ; 1,0,1\right\rangle=-\sqrt{\frac{3}{10}}\left|\mathbf{8}_{a} ; \frac{1}{2}, 1, \frac{1}{2}\right\rangle\left|\mathbf{8}_{b} ; \frac{1}{2},-1, \frac{1}{2}\right\rangle-\sqrt{\frac{3}{10}}\left|\mathbf{8}_{a} ; \frac{1}{2},-1, \frac{1}{2}\right\rangle\left|\mathbf{8}_{b} ; \frac{1}{2}, 1, \frac{1}{2}\right\rangle \\
+\frac{1}{\sqrt{5}}\left|\mathbf{8}_{a} ; 1,0,1\right\rangle\left|\mathbf{8}_{b} ; 0,0,0\right\rangle+\frac{1}{\sqrt{5}}\left|\mathbf{8}_{a} ; 0,0,0\right\rangle\left|\mathbf{8}_{b} ; 1,0,1\right\rangle \\
\left|\mathbf{8}_{2} ; 1,0,1\right\rangle=\frac{1}{\sqrt{6}}\left|\mathbf{8}_{a} ; \frac{1}{2}, 1, \frac{1}{2}\right\rangle\left|\mathbf{8}_{b} ; \frac{1}{2},-1, \frac{1}{2}\right\rangle-\frac{1}{\sqrt{6}}\left|\mathbf{8}_{a} ; \frac{1}{2},-1, \frac{1}{2}\right\rangle\left|\mathbf{8}_{b} ; \frac{1}{2}, 1, \frac{1}{2}\right\rangle \\
+\frac{1}{\sqrt{3}}\left|\mathbf{8}_{a} ; 1,0,1\right\rangle\left|\mathbf{8}_{b} ; 1,0,0\right\rangle-\frac{1}{\sqrt{3}}\left|\mathbf{8}_{a} ; 1,0,0\right\rangle\left|\mathbf{8}_{b} ; 1,0,1\right\rangle .
\end{array}
$$

These isovector components of the octets $\mathbf{8}_{\mathbf{1}}$ and $\mathbf{8}_{\mathbf{2}}$ have definite opposite $G$-parities. This can be readily seen by acting with the $G$-parity operator on the states $\left|\boldsymbol{8}_{i} ; 1,0,1\right\rangle$ written above. It is convenient to consider $\hat{G}$ as $\hat{C}$ followed by a $\pi$-rotation about the $y$-component of isospin, $\hat{R}$, as it is then straightforward to show [76],

$$
\begin{aligned}
& \hat{C}\left|\mathbf{8}_{a} ; I, Y, I_{z}\right\rangle=C_{a}(-1)^{\frac{Y}{2}+I_{z}}\left|\mathbf{8}_{a} ; I,-Y,-I_{z}\right\rangle \\
& \hat{R}\left|\mathbf{8}_{a} ; I, Y, I_{z}\right\rangle=(-1)^{I-I_{z}}\left|\mathbf{8}_{a} ; I, Y,-I_{z}\right\rangle \\
& \hat{G}\left|\mathbf{8}_{a} ; I, Y, I_{z}\right\rangle=C_{a}(-1)^{\frac{Y}{2}+I}\left|\mathbf{8}_{a} ; I,-Y, I_{z}\right\rangle,
\end{aligned}
$$

where $C_{a}$ is the intrinsic $C$-parity of the neutral element of the octet, for example, $C_{a}=+1$ for $(\boldsymbol{\eta})_{8}$ and $C_{a}=-1$ for $(\boldsymbol{\omega})_{8}$. It immediately follows that,

$$
\begin{aligned}
& \hat{G}\left|\mathbf{8}_{1} ; 1,0,1\right\rangle=-C_{a} C_{b}\left|\mathbf{8}_{1} ; 1,0,1\right\rangle \\
& \hat{G}\left|\mathbf{8}_{2} ; 1,0,1\right\rangle=+C_{a} C_{b}\left|\mathbf{8}_{2} ; 1,0,1\right\rangle .
\end{aligned}
$$


As an example, if $\boldsymbol{8}_{a}$ denotes the pseudoscalar octet $(\boldsymbol{\eta})_{\mathbf{8}}\left(C_{a}=+1\right)$ and $\boldsymbol{8}_{b}$ the vector octet $(\omega)_{\mathbf{8}}\left(C_{b}=-1\right)$, then $\left|\mathbf{8}_{1} ; 1,0,1\right\rangle$ will have positive $G$-parity and $\left|\mathbf{8}_{2} ; 1,0,1\right\rangle$ negative.

In the case of $\mathbf{8} \otimes \mathbf{1} \rightarrow \mathbf{8}$, the $G$-parity is much simpler. For $\nu=(1,0,1)$, the two-meson state transforming in $\mathbf{8}$ is given trivially by,

$$
|\mathbf{8} ; 1,0,1\rangle=\left|\mathbf{8}_{a} ; 1,0,1\right\rangle\left|\mathbf{1}_{b} ; 0,0,0\right\rangle
$$

and the $G$-parity is $G=-C_{a} C_{b}$.

One could of course have chosen a different component of the octet with hypercharge zero with which to work, for example the neutral element $\nu=(0,0,0)$, and a similar argument would follow. For the remainder of this thesis, we always consider a target component of $\nu=(1,0,1)$ and use the $G$-parity conventions laid out here.

These considerations are important when constructing meson-meson operators in order to ensure they have the desired $G$-parity. Had we chosen different SU(3)F Clebsch-Gordan coefficients that did not project the flavour embeddings onto definite $G$-parity, mesonmeson operators constructed in both octet multiplets would need to be included and states of both $G$-parities would feature in the spectrum. This would significantly increase the size of the operator basis and subsequently the computational cost. Having flavour representations of definite symmetry also enables us to take advantage of Bose-symmetry when considering products of identical meson octets which we discuss below.

\subsection{Bose-Symmetry in $\mathrm{SU}(3)_{\mathrm{F}}$}

It is instructive to first think about Bose-symmetry in $\mathrm{SU}(2)_{\mathrm{F}}$. For a pair of identical mesons $\mathbb{M}_{1} \mathbb{M}_{2}$, the total wavefunction, comprised of a product of flavour $(\chi)$, spin $(Q)$ and spatial $(R)$ wavefunctions, must be symmetric under the interchange $\mathbb{M}_{1} \leftrightarrow \mathbb{M}_{2}$ and this places constraints on the symmetries of each wavefunction! For example, $\pi \pi$ in isospin- 1 is symmetric in spin $\left(Q_{S}\right)$, antisymmetric in isospin $\left(\chi_{A}\right)$ and therefore must be antisymmetric in the spatial wavefunction $\left(R_{A}\right)$. This forbids isospin- $1 \pi \pi$ in an even partial-wave.

In $\mathrm{SU}(3)_{\mathrm{F}}$, using the symmetries defined by the choice of $\mathrm{SU}(3)_{\mathrm{F}}$ Clebsch-Gordan coefficients in Eq. 4.1, analogous restrictions can be deduced. Consider first identical

\footnotetext{
${ }^{1}$ For a product of mesons, the colour wavefunction is always symmetric.
} 


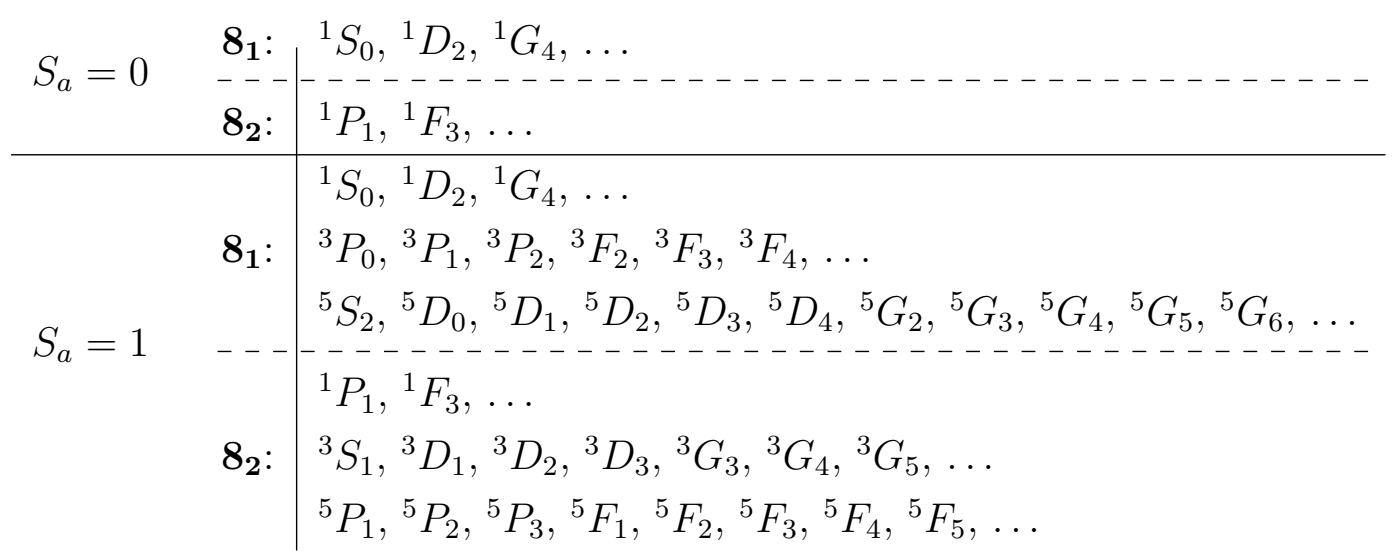

Table 4.1: Partial-wave content $(\ell \leq 4)$ of multiplets $\mathbf{8}_{\mathbf{1}}$ and $\mathbf{8}_{\mathbf{2}}$, from a product of two identical octets, $\boldsymbol{8}_{a} \otimes \mathbf{8}_{a}$, with intrinsic spins $S_{a}$.

octets of intrinsic spin-0. Total spin $S$ is zero and the spin wavefunction, $Q_{S}$, is symmetric meaning the product of flavour and spatial wavefunctions must be overall symmetric. This leaves two possible combinations, $\chi_{A} R_{A}$ or $\chi_{S} R_{S}$. As $\mathbf{8}_{\mathbf{1}}$ and $\mathbf{8}_{\mathbf{2}}$ are symmetric and anti-symmetric embeddings in flavour respectively, we deduce only partial-waves of even $\ell$ are permitted in $\mathbf{8}_{\mathbf{1}}$ and odd $\ell$ in $\mathbf{8}_{\mathbf{2}}$.

For identical octets with intrinsic spin-1, the symmetry of the spin wavefunction, depending on the total spin of the meson-meson pair, is symmetric for $S=0,2$ and anti-symmetric for $S=1$. It follows that when the spin wavefunction is symmetric, the product of flavour and spatial wavefunctions must be totally symmetric, so either $\chi_{A} R_{A}$ or $\chi_{S} R_{S}$, and when anti-symmetric, the same product must be totally anti-symmetric, either $\chi_{S} R_{A}$ or $\chi_{A} R_{S}$. This restricts partial-waves with even $S+\ell$ to the multiplet $\mathbf{8}_{\mathbf{1}}$ and odd $S+\ell$ to $\mathbf{8}_{\mathbf{2}}$.

To summarise, for the product of two identical $\mathrm{SU}(3)_{\mathrm{F}}$ octet mesons transforming in $\boldsymbol{8}_{i}$, partial-waves are permitted where $(-1)^{\ell+S}=\xi_{i}$. Table 4.1 summarises the partial-wave contents of $\mathbf{8}_{\mathbf{1}}$ and $\mathbf{8}_{\mathbf{2}}$ for $\ell \leq 4$.

\subsection{Operator Constructions in $[000] T_{1}^{+}$}

Single-meson operators transforming in the axial-vector octet $\left(\boldsymbol{f}_{\mathbf{1}}\right)_{\mathbf{8}}$ are constructed according to Eq. 3.4. As discussed previously, we choose $\nu=(1,0,1)$, corresponding to the 
$a_{1}^{+}$meson, with $\left(I^{G}\right) J^{P C}=\left(1^{-}\right) 1^{++}$. At rest, $J^{P}=1^{+}$subduces into the $[000] T_{1}^{+}$irrep and we construct a total of 8 single-meson-like operators, using up to two gauge-covariant derivatives, as described in Section 3.2.1.

To determine a sufficient basis of meson-meson operators, it is instructive to consider all relevant two-meson thresholds below the first three-meson threshold, $(\boldsymbol{\eta})_{\mathbf{8}}(\boldsymbol{\eta})_{\mathbf{8}}(\boldsymbol{\eta})_{\mathbf{8}}$, at $E_{\mathrm{cm}}=0.4434$. We first consider two-meson thresholds formed from the product $\mathbf{8} \otimes \mathbf{8}$ of which there are three: $(\eta)_{\mathbf{8}}(\eta)_{\mathbf{8}},(\omega)_{\mathbf{8}}(\eta)_{\mathbf{8}}$ and $(\omega)_{\mathbf{8}}(\omega)_{\mathbf{8}}$. As in the isospin-2 study, all meson-meson operators with corresponding non-interacting energies up to, and a modest distance above, the cut-off are included in the operator basis for a robust determination of the spectra.

Consider first $(\boldsymbol{\eta})_{\mathbf{8}}(\boldsymbol{\eta})_{\mathbf{8}}$-like operators. Using Eq. 4.5, negative $G$-parity constructions appear in the $\mathbf{8}_{\mathbf{1}}$ embedding and Bose-symmetry constraints restrict $(\boldsymbol{\eta})_{\mathbf{8}}(\boldsymbol{\eta})_{\mathbf{8}}$ to even partial-waves, of which only $\ell \geq 4$ appear in $[000] T_{1}^{+}$. Subsequently, there are no $(\boldsymbol{\eta})_{\mathbf{8}}(\boldsymbol{\eta})_{\mathbf{8}}$ operators with non-interacting energies below the cut-off and as such none are included in the basis.2

For $(\omega)_{\mathbf{8}}(\omega)_{\mathbf{8}}$, negative $G$-parity again occurs for operators constructed in the flavour symmetric embedding, $\mathbf{8}_{\mathbf{1}}$. The allowed $S$-waves for identical vector-vector multiplets, shown in Table 4.1, are ${ }^{1} S_{0}$ and ${ }^{5} S_{2}$ in $\mathbf{8}_{\mathbf{1}}$ and ${ }^{3} S_{1}$ in $\mathbf{8}_{\mathbf{2}}$. As neither ${ }^{1} S_{0}$ nor ${ }^{5} S_{2}$ subduce into $[000] T_{1}^{+}$, there are no $(\omega)_{8}(\omega)_{8}$ operators at threshold. Corresponding non-interacting energies for $(\omega)_{8}(\omega)_{8}$ operators with non-zero relative momenta are above the cut-off and such operators are excluded from the basis.

For $(\boldsymbol{\omega})_{\mathbf{8}}(\boldsymbol{\eta})_{\mathbf{8}}$, negative $G$-parity comes through the $\mathbf{8}_{\mathbf{2}}$ embedding. No Bose-symmetry constraints apply here as the multiplets are not identical. Meson-meson operators are constructed according to Eq. 3.2 .2 and, as an explicit example, we give $\mathcal{O}_{\mathbf{8}_{\mathbf{2}}(1,0,1)}^{\dagger T_{1}^{+} \text {constructed }}$ from optimised operators transforming in $[000] T_{1}^{-}$and $[000] A_{1}^{-}$,

$$
\begin{aligned}
& \mathcal{O}_{\mathbf{8}_{\mathbf{2}}(1,0,1)}^{\dagger T_{1}^{+} 2}= {\left[\frac{1}{\sqrt{6}}\left(\Omega_{\mathbf{8}\left(\frac{1}{2}, 1, \frac{1}{2}\right)}^{\dagger T_{2}^{-} 2} \Omega_{\mathbf{8}\left(\frac{1}{2},-1, \frac{1}{2}\right)}^{\dagger A_{-}^{-} 1}-\Omega_{\mathbf{8}\left(\frac{1}{2},-1, \frac{1}{2}\right)}^{\dagger T_{\mathbf{8}\left(\frac{1}{2}, 1, \frac{1}{2}\right)}^{-} 2} \Omega^{\dagger A_{-}^{-} 1}\right)\right.} \\
&\left.+\frac{1}{\sqrt{3}}\left(\Omega_{\mathbf{8}(1,0,1)}^{\dagger T_{1}^{-} 2} \Omega_{\mathbf{8}(1,0,0)}^{\dagger A_{1}^{-1}}-\Omega_{\mathbf{8}(1,0,0)}^{\dagger T_{1}^{-} 2} \Omega_{\mathbf{8}(1,0,1)}^{\dagger A_{1}^{-} 1}\right)\right] \\
&=\frac{1}{\sqrt{6}}\left[\left(K^{*+}\right)^{\dagger}\left(\bar{K}^{0}\right)^{\dagger}-\left(\bar{K}^{* 0}\right)^{\dagger}\left(K^{+}\right)^{\dagger}\right]+\frac{1}{\sqrt{3}}\left[\left(\rho^{+}\right)^{\dagger}\left(\pi^{0}\right)^{\dagger}-\left(\rho^{0}\right)^{\dagger}\left(\pi^{+}\right)^{\dagger}\right] .
\end{aligned}
$$

where in the last equality we give the $\mathrm{SU}(2)_{\mathrm{F}}$ operators explicitly for reference.

\footnotetext{
${ }^{2}$ The smallest momenta required occurs at $|\vec{p}|^{2}=5$ with $[210] A_{2} \otimes[210] A_{2} \rightarrow[000] T_{1}^{+}$.
} 


\begin{tabular}{cl} 
mesons & $a_{t} E_{\mathrm{cm}}$ \\
\hline$(\boldsymbol{\eta})_{\mathbf{8}}$ & $0.1478(1)$ \\
$\left(\boldsymbol{f}_{\mathbf{0}}\right)_{\mathbf{1}}$ & $0.2007(18)$ \\
$(\boldsymbol{\omega})_{\mathbf{8}}$ & $0.2154(2)$ \\
$(\boldsymbol{\omega})_{\mathbf{1}}$ & $0.2174(3)$
\end{tabular}

\begin{tabular}{cl} 
threshold & $a_{t} E_{\mathrm{cm}}$ \\
\hline $\boldsymbol{( \boldsymbol { \eta } ) _ { \mathbf { 8 } } ( \boldsymbol { f } _ { \mathbf { 0 } } ) _ { \mathbf { 1 } }}$ & $0.3485(18)$ \\
$(\boldsymbol{\omega})_{\mathbf{8}}(\boldsymbol{\eta})_{\mathbf{8}}$ & $0.3632(2)$ \\
$(\boldsymbol{\omega})_{\mathbf{8}}(\boldsymbol{\omega})_{\mathbf{8}}$ & $0.4308(3)$ \\
$(\boldsymbol{\omega})_{\mathbf{8}}(\boldsymbol{\omega})_{\mathbf{1}}$ & $0.4328(4)$ \\
$(\boldsymbol{\eta})_{\mathbf{8}}(\boldsymbol{\eta})_{\mathbf{8}}(\boldsymbol{\eta})_{\mathbf{8}}$ & $0.4434(2)$
\end{tabular}

Table 4.2: (Left): relevant stable hadron masses. (Right): thresholds relevant for multi-meson levels appearing in $J^{P}=1^{+}$.

The two-meson thresholds formed from the product $\mathbf{8} \otimes \mathbf{1}$ that lie below the cutoff and have a negative $G$-parity are $(\boldsymbol{\eta})_{\mathbf{8}}\left(\boldsymbol{f}_{\mathbf{0}}\right)_{\mathbf{1}}$ and $(\boldsymbol{\omega})_{\mathbf{8}}(\boldsymbol{\omega})_{\mathbf{1}}$. Operators resembling both these pairs of mesons are included in the basis.

For reference, relevant stable hadron masses and thresholds relevant for $J^{P}=1^{+}$are recorded in Table 4.2. The complete list of operators used to compute the matrix of correlation functions in $[000] T_{1}^{+}$is given in Table 4.3 . 


\begin{tabular}{|c|c|c|}
\hline$L / a_{s}=16$ & $L / a_{s}=20$ & $L / a_{s}=24$ \\
\hline $8 \times \bar{\psi} \boldsymbol{\Gamma} \psi$ & $8 \times \bar{\psi} \boldsymbol{\Gamma} \psi$ & $8 \times \bar{\psi} \boldsymbol{\Gamma} \psi$ \\
\hline$(\omega)_{8_{[000]}}(\eta)_{8_{[000]}}$ & $(\omega)_{8[000]}(\eta)_{8[000]}$ & $(\boldsymbol{\omega})_{\mathbf{8}_{[000]}}(\boldsymbol{\eta})_{\mathbf{8}_{[000]}}$ \\
\hline$\left(f_{0}\right)_{1_{[001]}}(\eta)_{8[00-1]}$ & $\left(f_{0}\right)_{1[001]}(\eta)_{8[00-1]}$ & $\left(f_{0}\right)_{1_{[001]}}(\eta)_{8[00-1]}$ \\
\hline$\{2\}(\boldsymbol{\omega})_{\mathbf{8}_{[001]}}(\boldsymbol{\eta})_{\mathbf{8}_{[00-1]}}$ & $\{2\}(\boldsymbol{\omega})_{8_{[001]}}(\boldsymbol{\eta})_{8_{[00-1]}}$ & $\{2\}(\boldsymbol{\omega})_{\mathbf{8}_{[001]}}(\boldsymbol{\eta})_{\mathbf{8}_{[00-1]}}$ \\
\hline$(\omega)_{\mathbf{1}_{[000]}}(\omega)_{8_{[000]}}$ & $(\omega)_{1_{[000]}}(\omega)_{8_{[000]}}$ & $\left(f_{0}\right)_{1_{[011]}}(\eta)_{8[0-1-1]}$ \\
\hline & $\left(f_{0}\right)_{1_{[011]}}(\eta)_{8_{[0-1-1]}}$ & $\{3\}(\omega)_{\mathbf{8}_{[011]}}(\boldsymbol{\eta})_{\mathbf{8}_{[0-1-1]}}$ \\
\hline & $\{3\}(\boldsymbol{\omega})_{8_{[011]}}(\boldsymbol{\eta})_{8_{[0-1-1]}}$ & $(\omega)_{1_{[000]}}(\omega)_{8_{[000]}}$ \\
\hline & & $\left(f_{0}\right)_{1_{[111]}}(\eta)_{8_{[-1-1-1]}}$ \\
\hline & & $\{2\}(\boldsymbol{\omega})_{\mathbf{8}_{[111]}}(\boldsymbol{\eta})_{\mathbf{8 [ - 1 - 1 - 1 ]}}$ \\
\hline & & \\
\hline 13 ops. & 17 ops. & 20 ops. \\
\hline
\end{tabular}

Table 4.3: As in Table 3.3 but for operators in the 8 of SU(3) $)_{F}$ flavour transforming in $[000] T_{1}^{+}$. Meson-meson operators are ordered by increasing non-interacting energy (see Section 3.2.2). The operators, $\mathbb{M}_{1\left[\vec{p}_{1}\right]} \mathbb{M}_{2\left[\vec{p}_{2}\right]}$, are constructed from optimised $\mathbb{M}$ operators with momentum types $\vec{p}_{1}$ and $\vec{p}_{2}$ respectively. Different momentum directions are summed over as in Eq. 3.8. Single-meson operators are denoted $\bar{\psi} \boldsymbol{\Gamma} \psi$.

\subsection{Finite-Volume Spectra}

Matrices of correlation functions were calculated on the lattice described in Section 3.3. In addition, we also used a smaller volume, $\left(L / a_{s}\right)^{3} \times\left(T / a_{t}\right)=16^{3} \times 128$ for which $N_{\text {vecs }}=64$, $N_{\text {cfgs }}=532$ and $N_{\text {tsrcs }}=2$. The finite-volume spectra were determined using a variational analysis and we present the energy levels in Figure 4.1.

We make some qualitative observations from the spectra. Firstly, we see an energy level far below the lowest threshold which appears to be volume independent, characteristic of a bound-state. This is in fact unsurprising as a previous study on these lattices of the isovector spectrum [29] finds, using a basis of single-meson-like operators only, a ground state in the $\left(\boldsymbol{f}_{\mathbf{1}}\right)_{\mathbf{8}}$ multiplet around the same energy.

Examining the spectra above the lowest energy level, we observe that the levels with largest operator-state overlaps onto $(\omega)_{\mathbf{8}}(\boldsymbol{\eta})_{\mathbf{8}^{-}}$-like operators appear to be consistent with or displaced a small distance above each of the non-interacting $(\boldsymbol{\omega})_{\mathbf{8}}(\boldsymbol{\eta})_{\mathbf{8}}$ energies. Analogous 
behaviour was seen in the $\rho \pi$ isospin-2 spectra (Figure 3.5) where weak ${ }^{3} S_{1}$-wave and negligibly small ${ }^{3} D_{1}$ - and ${ }^{3} D_{3}$-wave amplitudes were determined.

An energy level with dominant operator-state overlap onto the $(\omega)_{\mathbf{8}}(\boldsymbol{\omega})_{\mathbf{1}}$ operator is found to be roughly consistent with threshold on each volume, indicative of weak interaction in ${ }^{3} S_{1}$-wave $(\omega)_{8}(\omega)_{1}$ in this energy region.

Regarding the $(\boldsymbol{\eta})_{\mathbf{8}}\left(\boldsymbol{f}_{\mathbf{0}}\right)_{1}$-like energy levels, these are found to be poorly determined and carry large uncertainties, owing to the noisy $\left(\boldsymbol{f}_{\mathbf{0}}\right)_{\mathbf{1}}$ with vacuum quantum numbers. However, we find the levels are broadly consistent with the corresponding non-interacting energies.

As a final comment, we find the next excited state in the single-meson-like spectrum in Ref. [29] was found to be above $(\boldsymbol{\eta})_{\mathbf{8}}(\boldsymbol{\eta})_{\mathbf{8}}(\boldsymbol{\eta})_{\mathbf{8}}$ threshold. This is consistent with our observations in Figure 4.1 that we do not observe any additional levels or resonant like features below the three-body cutoff. 


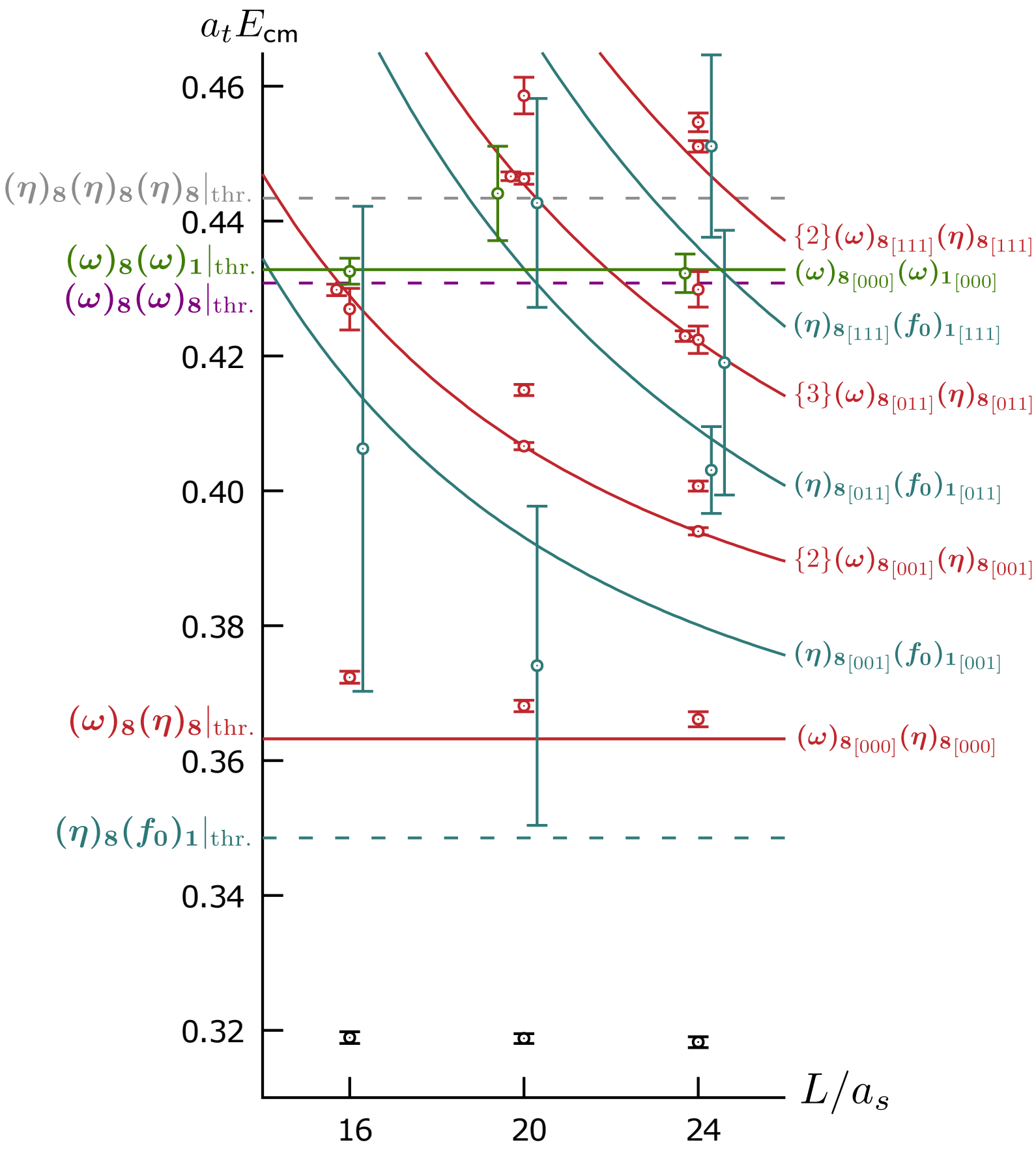

Figure 4.1: Finite-volume spectrum for the $\left(\boldsymbol{f}_{\mathbf{1}}\right)_{\mathbf{8}}$ flavour multiplet in the [000] $T_{1}^{+}$irrep on each lattice volume. Energy levels are coloured according to the largest operator-state overlap factors. Black points correspond to energy levels with dominant operator-state overlap factors onto $q \bar{q}$-like operators, red are $(\boldsymbol{\omega})_{\mathbf{8}}(\boldsymbol{\eta})_{\mathbf{8}}$-like, blue are $(\boldsymbol{\eta})_{\mathbf{8}}\left(\boldsymbol{f}_{\mathbf{0}}\right)_{\mathbf{1}}$-like and green are $(\omega)_{8}(\omega)_{1}$-like. Errorbars reflect statistical uncertainties only. Points are slightly displaced horizontally for clarity. Solid curves reflect non-interacting energies corresponding to meson-meson operators included in the basis, as shown in Table 4.3, and dashed lines are thresholds. 


\subsection{Summary}

To conclude, the $\left(\boldsymbol{f}_{\mathbf{1}}\right)_{\mathbf{8}}$ multiplet appears to be a bound-state and interactions in the ${ }^{3} S_{1} /{ }^{3} D_{1}, \rho \pi$ partial-waves appear to be weak and similar in characteristic to the $\rho \pi$ isospin2 study, with no evidence of resonant behaviour in an energy region below the three-body cutoff. A rigorous scattering analysis would require significantly improved statistical precision on the poorly determined $(\boldsymbol{\eta})_{\mathbf{8}}\left(f_{0}\right)_{1}$ levels and many moving frame spectra, as in the isospin-2 study, to constrain the large number of coupled channels and partial-waves in this scattering system. Furthermore, given that the qualitative observations suggest amplitudes are similar in characteristic to the isospin-2 calculation, we do not pursue this analysis further.

At a lighter pion mass, we may expect to observe resonant behaviour in isospin-1 $\rho \pi$. For narrow resonances, the spectrum calculated using a basis of single-meson operators appears to give energy levels near the corresponding resonant mass determined from an amplitude analysis - for example the $\rho$ in Ref. [30]. This makes the spectra figures in Ref. [29], calculated using a basis of single-meson-like operators, a particularly useful guide for identifying potentially resonant channels. As shown in Figure 14 of Ref. [29], the $\rho \pi$ threshold first appears to be below the $a_{1}$ energy on a lattice where $m_{\pi} \sim 391 \mathrm{MeV}$. At this pion mass however, previous lattice calculations 30 find the $\rho$ meson to be an unstable narrow resonance and $\rho \pi$ subsequently becomes the three-body $\pi \pi \pi$ channel. Although significant recent progress has seen development in a quantisation condition for three-body scattering [52 60], the formalism has not matured to the level where we could apply it to the $a_{1}$.

As mentioned at the end of Chapter 3 , the $b_{1}$ axial-vector, the $C$-parity partner of the $a_{1}$, has experimentally observed decays to $\pi \omega$, dynamically-coupled in ${ }^{3} S_{1^{-}},{ }^{3} D_{1}$-wave. At $m_{\pi} \sim 391 \mathrm{MeV}$ the $\omega$ is stable against strong decay. The negative $G$-parity of the $\omega$ forbids the decay to $\pi \pi$ and the lowest relevant threshold, $\pi \pi \pi$, is closed. Considering the $q \bar{q}$-like spectra with $b_{1}$ quantum numbers, shown in Figure 15 of Ref. [29], we observe an energy level a modest distance above $\pi \omega$ threshold. This makes for an ideal candidate with which to examine the effects of dynamically-coupled partial-waves in a resonant system. With the framework and formalism in place, we proceed with a study of $\pi \omega$ scattering in Chapter 5 . 


\section{A $b_{1}$ resonance at $m_{\pi} \sim 391 \mathrm{MeV}$}

In Chapter 3, we presented a first calculation of $\rho \pi$ scattering in isospin-2, determining the ${ }^{3} S_{1}$ - and ${ }^{3} D_{1}$-wave $J^{P}=1^{+}$amplitudes and their dynamical mixing, finding relatively weak effects as expected in this exotic isospin channel. This was deliberately chosen as a testing ground where resonant physics is not expected to feature and enabled the development of the necessary tools and techniques for performing scattering calculations of hadrons with non-zero spin. In Chapter 4, we examined $\rho \pi$ in isospin-1 at the same heavy pion mass and found evidence of a bound $a_{1}$ and weak interactions in $\rho \pi$. In this chapter, we explore the scattering of a vector-pseudoscalar pair in a system where resonance physics is expected.

The experimentally-observed $b_{1}(1235)[5]$ isovector with $J^{P C}=1^{+-}$is dominantly seen through its decay to the $\pi \omega$ final state, where the $\omega$ is the lightest isoscalar vector meson and has a very small decay width to three pions. As in the case of $\rho \pi$, we can have the $\pi$ and $\omega$ in a relative ${ }^{3} S_{1}$ - or ${ }^{3} D_{1}$-wave - indeed, by studying the angular distribution in the decay of the $b_{1}$, experiments have estimated the amplitudes of these two partial-waves [47].

In this chapter, we will report on a study of the isovector $J^{P C}=1^{+-}$channel, in which we expect to see a $b_{1}$ resonance decaying to $\pi \omega$. In light of the discussion in the summary of Chapter 4, we make use of $N_{f}=2+1$ lattice configurations generated with a light-quark mass such that the pion has a mass around $391 \mathrm{MeV}$. With this light-quark mass, the $\omega$ meson is found to have a mass around $881 \mathrm{MeV}$ [26, 77], and hence is stable against decay to three pions whilst the $b_{1}$ is expected to resonate, just above $\pi \omega$ threshold.

To study the $b_{1}$ we compute matrices of correlation functions in three lattice volumes, in a number of irreps at several overall momenta, employing a wide range of operators resembling both single-hadron and multi-hadron structures. In addition to $\pi \omega$, the $\pi \phi$ channel becomes kinematically open in the energy region of interest. Although the $\phi$ is heavier than the $\omega$, we find that it is also stable against decay to $K \bar{K}$ and $\pi \pi \pi$ at the 
light-quark mass considered here. Furthermore, we pay attention to the fact that threebody channels, $\pi \pi \eta$ and $\pi K \bar{K}$, which have relatively low thresholds even for $m_{\pi} \approx 391$ $\mathrm{MeV}$, can, in principle, play a role. Experimentally, three-body decays of resonances are found to be dominated by two-body isobar resonances. For example, in a $\pi \pi \eta$ final state at relatively small total energy, the Dalitz plot will be expected to have the bulk of the events in narrow horizontal and vertical bands around $m_{\pi \pi} \sim m_{\rho}$ and $m_{\pi \eta} \sim m_{a_{0}}$. 1. The extensive use of three-meson operators, resembling $\pi \pi \eta$ and $\pi K \bar{K}$, are employed in this study.

In this calculation, we determine a large number of finite-volume energy levels in multiple volumes and moving frames. To constrain the $J^{P}=1^{+}$amplitudes, up to 36 energy levels, each typically having statistical uncertainty at the tenth of a percent level, are utilised. In addition, systematic tests exploring the role of the three-body channels are performed. As discussed in previous chapters, the three-body scattering formalism has not reached a maturity needed for a rigorous calculation, nevertheless we find evidence that these three-body channels have negligible effect in this particular case involving a low-lying $b_{1}$ resonance.

A previous lattice QCD study [49] of the $b_{1}$ limited itself to the rest-frame in one rather small volume. By considering only two degenerate flavours of light quarks and no strange quarks, any physics associated with the $\pi \phi$ channel was disallowed. A very small operator basis was used, such that only one usable energy level was obtained and this had a statistical uncertainty at the percent level. Enforcing elastic ${ }^{3} S_{1}$-wave scattering only, ignoring any effect from the ${ }^{3} D_{1}$-wave, and fixing the decay coupling of an assumed $b_{1}$ resonance at a value equal to that extracted from experimental measurements, a crude estimate of the $b_{1}$ mass was made in the case that the pion mass is $266 \mathrm{MeV}$. An earlier study 78 used a different approach in which the light-quark mass was tuned such that the $b_{1}$ decay to $\pi \omega$ is exactly at kinematic threshold. From the time-dependence of a single correlation function, an estimate of the decay coupling was inferred.

It has been suggested $\left[79\right.$ that the $\pi \phi$ channel, coupled to $\pi \omega$, may feature a $Z_{s}$ resonance analogous to the $Z_{c}$ enhancement that has been seen in the $\pi J / \psi$ final state [80,81. We will find no evidence of a $Z_{s}$ resonance in this work.

We begin by examining the operator constructions relevant for this calculation.

\footnotetext{
${ }^{1}$ There will also be a diagonal 'reflection' of the $a_{0}$ band due to the symmetry of exchanging the pions.
} 


\subsection{Operator Constructions}

The region we study includes the opening of several multi-hadron thresholds: $\pi \omega, \pi \phi$, $\pi \pi \eta$ and $\pi K \bar{K}$, and we find that this necessitates the inclusion of two-meson-like and three-meson-like operators in our basis, as well as single-meson operators of fermion-bilinear form which we expect to have good overlap with any bound state or relatively-narrow resonance present. Four-meson thresholds lie beyond the energy region we consider, and previous calculations suggest that local tetraquark-like operators have little effect on the spectra [68, 82], so neither of these types of operators are included in the basis. The construction of interpolating operators resembling single-meson, two-meson and three-meson structures is discussed in the subsections which follow.

\subsubsection{Single-Meson Operators}

The construction of 'single-meson-like' operators follows the procedure detailed in Section 3.2.1, with $\mathrm{SU}(2)_{\mathrm{F}}$ Clebsch-Gordan coefficients replacing $\mathrm{SU}(3)_{\mathrm{F}}$ in Eq. 3.4. Singlemeson operators are written as $\bar{\psi} \mathbf{\Gamma} \psi$ for the remainder of this chapter.

In the construction of optimised operators for the stable $\omega\left(\Omega_{\omega}^{\dagger}\right)$ and $\phi\left(\Omega_{\phi}^{\dagger}\right)$, in each relevant irrep, a variational analysis is performed on a matrix of correlation functions calculated from a basis of hidden-light $(\bar{u} \boldsymbol{\Gamma} u+\bar{d} \boldsymbol{\Gamma} d)$ and hidden-strange $(\bar{s} \boldsymbol{\Gamma} s)$ flavour structure, i.e. isospin-0. All relevant disconnected contributions that arise from selfannihilations of quarks at source and sink respectively are computed but are found to be small as shown in Figures 4 and 5 of Ref. [26]. This is consistent with the experimentally motivated 'OZI' rule which postulates that diagrams with disconnected quark lines are suppressed relative to connected ones - an implication being that $q \bar{q}$ pairs in isoscalar mesons prefer not to annihilate. Within each irrep, the ground-state and first excited-state are projected out, found to have dominant operator-state overlaps with hidden-light and hidden-strange operators respectively, and correspond to the $\omega$ and $\phi$ mesons.

We use the same flavour basis, but with pseudoscalar bilinear structures, for determining the optimum $\eta$ operator $\left(\Omega_{\eta}^{\dagger}\right)$ in each irrep. Unlike in the vector case, there is significant mixing between the hidden-light and hidden-strange flavour structures as is observed through the disconnected diagrams (significant off-diagonal flavour contributions, see Figures 2 and 3 in Ref. [26]), indicating, as is well known, that the 'OZI' rule does not apply in the pseudoscalar channel. 


\subsubsection{Two-Meson Operators}

Our approach to constructing operators which resemble a two-meson structure has been discussed in Section 3.2.2, where we again replace $\mathrm{SU}(3)_{\mathrm{F}}$ Clebsch-Gordan coefficients with those for $\mathrm{SU}(2)_{\mathrm{F}}$.

For energies below three-meson thresholds, previous calculations have suggested that a sufficient set of operators for a reliable calculation of the spectra consists of singlemeson and two-meson operators. Recalling the discussion in Section 3.2.2, two-meson operators $\mathcal{O}_{\mathbb{M}_{1} \mathbb{M}_{2}}^{\dagger \Lambda \mu}\left(\vec{p}_{12}\right)$ are efficient at interpolating the finite-volume energy levels near to the associated non-interacting energies,

$$
E_{\text {n.i. }}^{(2)}=\sqrt{m_{1}^{2}+\left|\vec{p}_{1}\right|^{2}}+\sqrt{m_{2}^{2}+\left|\vec{p}_{2}\right|^{2}}
$$

and truncating the two-meson operator bases when the corresponding non-interacting energies are beyond the energy region of interest has been demonstrated to be sufficient for a robust determination of the spectra. In this chapter, two-meson operators are written $\mathbb{M}_{1\left[\vec{p}_{1}\right]} \mathbb{M}_{2\left[\vec{p}_{2}\right]}$ in all tables and figures, with $\mathbb{M}$ labelling the meson and $\vec{p}$ the momentum type.

Correlation functions with $\mathbb{M M}$ operators at the source and/or sink feature Wick contractions in which quarks annihilate either within an isoscalar meson or between two mesons. Considering a basis with overall isospin-1 as relevant here, with $\mathbb{M}=\bar{u} \boldsymbol{\Gamma} d$ and $\mathbb{M M}=\{\pi \omega, \pi \phi\}$, we need to evaluate diagrams whose structure is similar to those shown in Figure 1 of $64 \cdot{ }^{2}$

\subsubsection{Three-Meson Operators}

Three-meson operators, and operators with a structure resembling more than three mesons, can be constructed by iteratively applying the two-meson operator construction. Schematically,

$$
\mathcal{O}_{\mathbb{M}_{1} \mathbb{M}_{2} \mathbb{M}_{3}}^{\dagger \Lambda \mu}\left(\vec{p}_{123}\right)=\sum_{\substack{\mu_{12}, \mu_{3} \\
\vec{p}_{\vec{p}_{12} \in\left\{\vec{p}_{12}\right\}^{*}} \\
\vec{p}_{12} \in\left\{\vec{p}_{3}\right\}^{*}}} \mathbb{C}\left(\begin{array}{ccc}
{\left[\vec{p}_{12}\right] \Lambda_{12}} & {\left[\vec{p}_{3}\right] \Lambda_{3}} & {\left[\vec{p}_{123}\right] \Lambda} \\
\mu_{12} & \mu_{3} & \mu
\end{array}\right) \mathcal{O}_{\mathbb{M}_{1} \mathbb{M}_{2}}^{\dagger \Lambda_{12} \mu_{12}}\left(\vec{p}_{12}\right) \Omega_{\mathbb{M}_{3}}^{\dagger \Lambda_{3} \mu_{3}}\left(\vec{p}_{3}\right)
$$

\footnotetext{
${ }^{2}$ This figure refers to $\mathbb{M M}=\{\pi K, \eta K\}$ in isospin- $1 / 2$.
} 
where $\mathcal{O}_{\mathbb{M}_{1} \mathbb{M}_{2}}^{\dagger \mu}$ is a two-meson operator constructed from a product of optimised singlemeson operators. We have dropped the flavour labels here for brevity. Note that it does not matter with which optimised single-mesons we formed the intermediate two-meson operator, i.e. $\mathcal{O}_{\mathbb{M}_{1} \mathbb{M}_{2}}^{\dagger \Lambda \mu}\left(\vec{p}_{12}\right), \mathcal{O}_{\mathbb{M}_{2} \mathbb{M}_{3}}^{\dagger \Lambda \mu}\left(\vec{p}_{23}\right)$ or $\mathcal{O}_{\mathbb{M}_{1} \mathbb{M}_{3}}^{\dagger \mu}\left(\vec{p}_{13}\right)$, as the tensor product is associative. An argument for determining a sufficient set of three-meson operators, analogous to that presented previously, would suggest calculating the corresponding non-interacting energies,

$$
E_{\text {n.i. }}^{(3)}=\sqrt{m_{1}^{2}+\left|\vec{p}_{1}\right|^{2}}+\sqrt{m_{2}^{2}+\left|\vec{p}_{2}\right|^{2}}+\sqrt{m_{3}^{2}+\left|\vec{p}_{3}\right|^{2}}
$$

and enforcing a similar truncation on the basis. While this approach has the advantage of being straightforward, it pays no attention to the fact that we expect certain twomeson pairs to feature resonating behaviour, the finite-volume analogue of the Dalitz-plot enhancements, or that they may form bound-states.

Consider the example of $\pi \pi \pi$ in isospin-2. Following the construction above, we would be attempting to describe energy eigenstates of the $\pi \pi$ isospin-1 subsystem using $\mathcal{O}_{\mathbb{M}_{1} \mathbb{M}_{2}}^{\dagger \mu}$ constructed using only ' $\pi \pi^{\prime}$ '-like operators. To reliably determine the isovector $\pi \pi$ spectra, i.e. the $\rho$ spectra, an operator basis including both $\bar{\psi} \mathbf{\Gamma} \psi$ and $\pi \pi$-like operators is needed as shown in Figure 1 of Ref. [30]. An alternative approach, based upon this observation and used in Ref. [68], utilises an optimised two-meson operator which will be a linear combination of $\bar{\psi} \mathbf{\Gamma} \psi$ and $\pi \pi$-like operators. We denote such an optimised operator $\Omega_{\mathbb{R}}^{\dagger}$, where $\mathbb{R}$ indicates the meson with the corresponding quantum numbers, i.e. $\Omega_{\rho}^{\dagger}$ for the example above $3^{3}$ In general, multiple optimised operators may be relevant $-\Omega_{\mathbb{R}^{n}}^{\dagger}$ denotes the optimal interpolating operator for the $\mathfrak{n}^{\text {th }}$ excited state in the relevant meson-meson subsystem.

Combining these operators with an optimised single-meson operator yields an alternative set of three-meson operators, given schematically by,

$$
\mathcal{O}_{\mathbb{R}_{12} \mathbb{M}_{3}}^{\dagger \Lambda \mu}\left(\vec{p}_{123}\right)=\sum_{\mu_{12}, \mu_{3}} \sum_{\substack{\vec{p}_{12} \in\left\{\vec{p}_{12}\right\}^{*} \\
\vec{p}_{12} \in\left\{\vec{p}_{3}\right\}^{*} \\
\vec{p}_{12}+\vec{p}_{3}=\vec{p}_{123}}} \mathbb{C}\left(\begin{array}{ccc}
{\left[\vec{p}_{12}\right] \Lambda_{12}} & {\left[\vec{p}_{3}\right] \Lambda_{3}} & {\left[\vec{p}_{123}\right] \Lambda} \\
\mu_{12} & \mu_{3} & \mu
\end{array}\right) \Omega_{\mathbb{R}_{12}}^{\dagger \Lambda_{12} \mu_{12}}\left(\vec{p}_{12}\right) \Omega_{\mathbb{M}_{3}}^{\dagger \Lambda_{3} \mu_{3}}\left(\vec{p}_{3}\right) .
$$

\footnotetext{
${ }^{3}$ Lattice irreps contain more than one spin but for convenience we choose the label $\mathbb{R}$ corresponding to the lightest such meson, e.g. in $[000] T_{1}^{-}$we choose $\rho$.
} 
By design, we anticipate that these three-meson operators will efficiently interpolate finite-volume levels in the region of an energy value,

$$
E_{\mathrm{n} . \mathrm{i} .}^{(2+1)}=E_{\mathbb{R}_{12}^{\mathrm{n}}}^{\Lambda_{12}}\left(\vec{p}_{12}\right)+\sqrt{m_{3}^{2}+\left|\vec{p}_{3}\right|^{2}}
$$

where $E_{\mathbb{R}_{12}^{n}}^{\Lambda_{12}}\left(\vec{p}_{12}\right)$ are finite-volume energies calculated in the two-meson subsystem in irrep $\left[\vec{p}_{12}\right] \Lambda_{12}$, i.e. they will efficiently capture interaction in the two-meson subsystem assuming weak residual interaction with the third meson. Calculating $E_{\text {n.i. }}^{(2+1)}$ energies, for all possible combinations of two-meson subsystems that together with the third meson give the desired quantum numbers, and truncating at a desired energy, provides a procedure for selecting which of these three-meson operators to include in the basis.

To illustrate the construction presented above, consider the example of a three-meson operator resembling $\pi \pi \eta$ in the irrep [000] $T_{1}^{+}$with $I^{G}=1^{+}$. We begin with the construction shown in Eq. 5.1. For $\vec{p}_{1}=\vec{p}_{2}=\vec{p}_{3}=\overrightarrow{0}$, there is only one possible irrep,

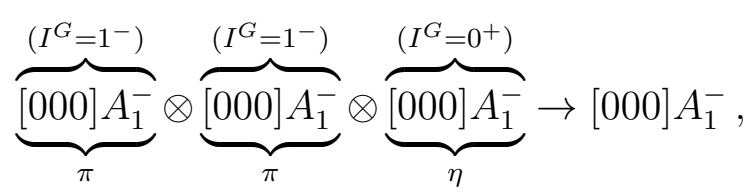

so no non-interacting $\pi \pi \eta$ level, or corresponding operator, appears in [000] $T_{1}^{+}$at threshold. If the pions are both given one unit of momentum, $\vec{p}_{1}=\vec{p}_{2}=[001]$ and $\vec{p}_{3}=\overrightarrow{0}$ (recalling that directions of momenta $\vec{p}_{i}$ are summed over), the product,

$$
\overbrace{\underbrace{[001] A_{2}}_{\pi}}^{\left(I^{G}=1^{-}\right)} \otimes \overbrace{[\underbrace{(001] A_{2}}_{\pi}}^{\left(I^{G}=1^{-}\right)} \otimes \overbrace{[\underbrace{(000] A_{1}^{-}}_{\eta}}^{\left(I^{G}=0^{+}\right)} \rightarrow \overbrace{[000] T_{1}^{+}}^{\left(I^{G}=1^{+}\right)} \oplus \ldots
$$

appears once in $[000] T_{1}^{+}$with $I^{G}=1^{+}$. Following the construction outlined in Eq. 5.1 yields one operator of the form $\mathcal{O}_{\pi \pi \eta}^{\dagger}$ with corresponding non-interacting energy,

$$
E_{\text {n.i. }}^{(3)}=2 \sqrt{m_{\pi}^{2}+\left(\frac{2 \pi}{L}\right)^{2}}+m_{\eta} .
$$


Now, we consider bound-states and resonances in the $\pi \pi$ and $\pi \eta$ two-meson subsystems and construct operators according to Eq. 5.2. Unlike in the previous construction, the order in which we combine the single-meson operators does matter as the intermediate $\Omega_{\mathbb{R}}^{\dagger}$ depends on the flavour structure of the two-meson subsystem. As before, for $\vec{p}_{1}=\vec{p}_{2}=\vec{p}_{3}=\overrightarrow{0}$ there is no embedding in [000] $T_{1}^{+}$. For $\vec{p}_{1}=\vec{p}_{2}=[001]$ and $\vec{p}_{3}=\overrightarrow{0}$, there are two possible distinct two-meson subsystems to consider.

First, for the $\pi \pi$ subsystem, there are three possible flavour combinations, $I^{G}=$ $0^{+}, 1^{+}, 2^{+}$, and three possible irreps with momentum $\vec{p}_{12}=\overrightarrow{0}$, namely [000] $A_{1}^{+},[000] T_{1}^{-}$and [000] $E^{+}$. When combined with the $\eta$, only the $\pi \pi$ subsystem with $I^{G}=1^{+}$transforming in $[000] T_{1}^{-}$gives the desired overall flavour and irrep. This $\pi \pi$ subsystem contains quantum numbers corresponding to the $\rho$ and the construction is, schematically,

$$
\begin{gathered}
(\overbrace{\pi}^{(\overbrace{}^{[001] A_{2}}} \otimes \overbrace{\pi}^{\left(I^{G}=1^{-}\right)} \overbrace{[001] A_{2}}^{\left(I^{G}=1^{-}\right)}) \otimes \overbrace{\underbrace{[000] A_{1}^{-}}_{\eta}}^{\left(I^{G}=0^{+}\right)} \rightarrow \overbrace{[000] T_{1}^{+}}^{\left(I^{G}=1^{+}\right)} \\
\underbrace{[000] T_{1}^{-}}_{\rho} \otimes \overbrace{\overbrace{[000] A_{1}^{-}}^{\left(I^{G}=1^{+}\right)}}^{\left[I^{G}=0^{+}\right)} \rightarrow \overbrace{[000] T_{1}^{+}}^{\left(I^{G}=1^{+}\right)} .
\end{gathered}
$$

Calculating the $E_{\text {n.i. }}^{(2+1)}$ energies amounts to determining the $\rho$-like energy eigenstates in [000] $T_{1}^{-}$with $I^{G}=1^{+}$and adding these to the $\eta$ energy according to Eq. 5.3 ,

$$
E_{\text {n.i. }}^{(2+1)}=E_{\rho^{\mathfrak{n}}}^{T_{1}^{-}}([000])+m_{\eta},
$$

where we recall that $\rho^{\mathfrak{n}}$ denotes the $\mathfrak{n}^{\text {th }}$ energy eigenstate within the irrep. In many cases, including here, only the lowest energy two-meson state $(\mathfrak{n}=0)$ yields an operator below the energy cut-off.

The second possible construction considers the $\pi \eta$ subsystem where there is only one flavour combination, $I^{G}=1^{-}$, and one possible irrep, [001] $A_{1}$. These quantum numbers correspond to the $a_{0}$ meson. Schematically,

$$
\begin{gathered}
(\overbrace{\pi}^{(\overbrace{}^{[001] A_{2}}} \otimes \overbrace{\eta}^{\left.I^{G}=1^{-}\right)} \underbrace{\left(I^{G}=0^{+}\right)}_{[000] A_{1}^{-}}) \otimes \overbrace{[001] A_{2}}^{\left(I^{G}=1^{-}\right)} \rightarrow \overbrace{[000] T_{1}^{+}}^{\left(I^{G}=1^{+}\right)} \\
\overbrace{\underbrace{(001] A_{1}}_{a_{0}}}^{\left(I^{G}=1^{-}\right)} \otimes \overbrace{\overbrace{[001] A_{2}}^{\left(I^{G}=1^{-}\right)}}^{\underbrace{\left(I^{G}=1^{+}\right)}_{\pi}} \rightarrow \overbrace{[000] T_{1}^{+}},
\end{gathered}
$$


and, as before, we determine the $E_{\text {n.i. }}^{(2+1)}$ energies by calculating the $a_{0}$-like energy eigenstates in [001] $A_{1}$ with $I^{G}=1^{-}$and add these to the $\pi$ energy according to Eq. 5.3 ,

$$
E_{\text {n.i. }}^{(2+1)}=E_{a_{0} \mathfrak{n}}^{A_{1}}([001])+\sqrt{m_{\pi}^{2}+\left(\frac{2 \pi}{L}\right)^{2}}
$$

For each $E_{\text {n.i. }}^{(2+1)}$ below some energy cut-off, we can construct operators of the form $\mathcal{O}_{a_{0} \pi}^{\dagger}$ via Eq. 5.2. The $E_{a_{0}}^{A_{1}}([001])$ energies are an example of a case where it may be prudent to consider multiple states $(\mathfrak{n} \geq 0)$ in the two-body sector. Figure 4 of Ref. 62] shows the $[001] A_{1}$ spectra corresponding to the $E_{a_{0}}^{A_{1}}([001])$ energies - there are many nearby low-lying energy levels on each volume. Following the construction given in Eq. 5.2 leads to multiple operators of the form $\mathcal{O}_{a_{0} \pi}^{\dagger}$ corresponding to similar $E_{\text {n.i. }}^{(2+1)}$.

As an example of the large number of diagrams needed to evaluate the $\mathbb{R M}$ operators, necessary to efficiently interpolate both $\pi \pi \eta$ - and $\pi K \bar{K}$-like states, we consider the case of an $a_{0} \pi$ operator at the sink and a $q \bar{q}$-like operator at the source. Here, the optimised $a_{0}$ operators are formed from a linear superposition of $q \bar{q}, \pi \eta$ and $K \bar{K}$ constructions, listed in Table 5.6. This leads to the diagrammatic components shown in Figure 5.1 which need to be connected to the quark lines from the $\pi$ and the source operator to form complete Wick contractions. It follows that, even in this simple case of a $b_{1}-a_{0} \pi$ correlator, we would have diagrams with the structures shown in Figure 5.2 . 


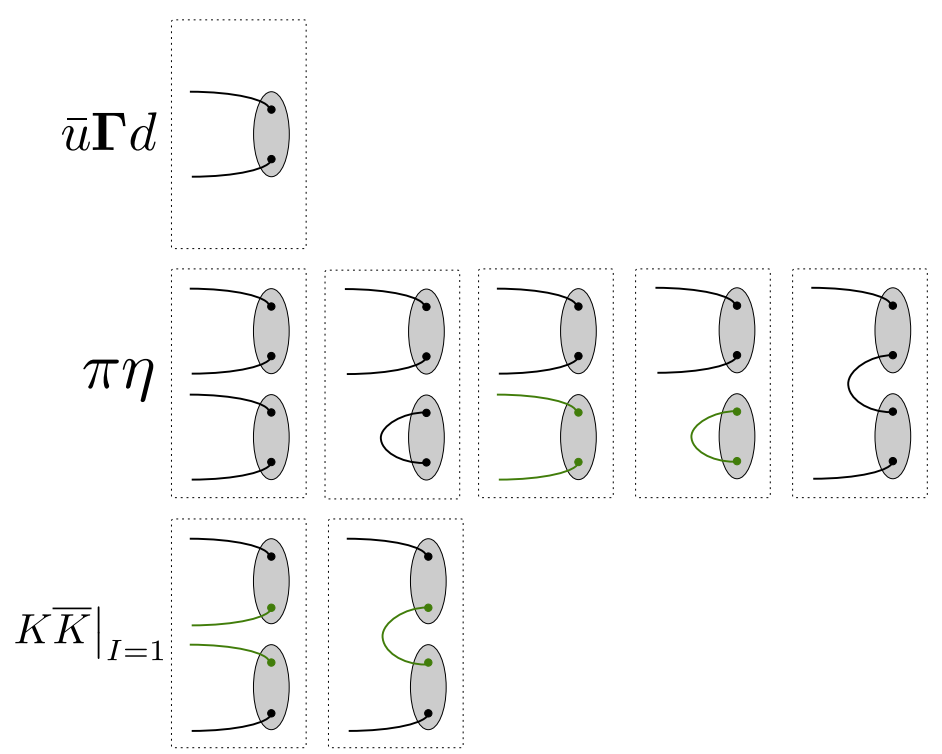

Figure 5.1: Quark propagation lines (black are light quarks, green are strange quarks) from operator constructions featuring in an optimised $a_{0}$-like operator.

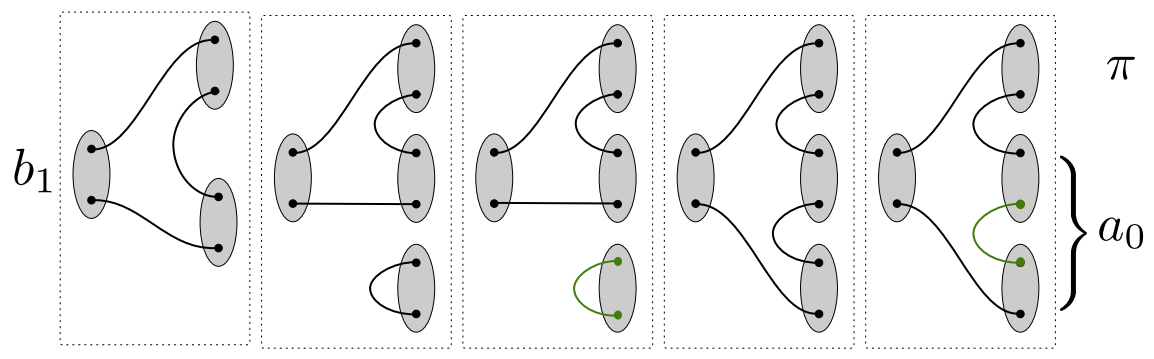

Figure 5.2: Wick contraction topologies for $b_{1}-a_{0} \pi$. Left meson resembles the $b_{1}$, upper right meson the $\pi$ and the remaining one or two mesons the $a_{0}$ (only a subset of the topologies in Figure 5.1 is relevant here).

\subsection{Lattice Details}

Correlation functions were computed on anisotropic lattices of spatial volumes $\left(L / a_{s}\right)^{3}=$ $16^{3}, 20^{3}$ and $24^{3}$ each having temporal extent $T / a_{t}=128$, where the temporal lattice spacing, $a_{t}$, is finer than the spatial lattice spacing, $a_{s} \sim 0.12 \mathrm{fm}$, with an anisotropy $\xi=a_{s} / a_{t} \sim 3.5$. Gauge fields were generated from the improved gauge and fermion action discussed in Section 2.2 , with $N_{f}=2+1$ flavours of dynamical quarks where the strange 


\begin{tabular}{c|ccc}
$\left(L / a_{s}\right)^{3} \times\left(T / a_{t}\right)$ & $N_{\text {vecs }}$ & $N_{\text {cfgs }}$ & $N_{\text {tsrcs }}$ \\
\hline $16^{3} \times 128$ & 64 & 479 & $8-32$ \\
$20^{3} \times 128$ & 128 & $452-603$ & 4 \\
$24^{3} \times 128$ & 160 & 553 & 4
\end{tabular}

Table 5.1: Number of distillation vectors $N_{\text {vecs }}$, gauge configurations $N_{\text {cfgs }}$, and time-sources $N_{\text {tsrcs }}$ used in the computation of correlation functions.

quark is tuned to approximately its physical mass and the degenerate light quarks are such that $m_{\pi} \sim 391 \mathrm{MeV}$ [21. All relevant Wick contractions were calculated without requiring additional propagator inversions beyond the basic set of $t_{\mathrm{src}}-t$ and $t-t$ perambulators for light and strange quarks, which were computed for use in previous calculations on these lattices. The very large number of diagrams incurs only a combinatoric cost associated with the contraction of the perambulators with the operator constructions.

Correlation functions were computed using the number of distillation vectors, gauge configurations and time-sources shown in Table 5.1. Typically, we calculated all the elements of the matrix of correlation functions, including the transposes, $C_{i j}$ and $C_{j i}$, which are related by hermiticity. In a few cases where there are a particularly large number of diagrams contributing, we made use of hermiticity to infer $C_{j i}$ from the computed $C_{i j}$. Masses of relevant stable hadrons are shown in Table 5.2 , where $\pi, K, \eta^{(\prime)}$ and $\sigma$ masses are taken from Refs. 28], 64], 62 and [7] respectively. Using energy levels on three lattice volumes, we determine the masses and anisotropies of the $\omega$ and $\phi$ mesons and show the results in Figure 5.3 .

The same characteristic splitting between the $|\lambda|=0,1$ components, as for the stable $\rho$ meson in Section 3.4 at the larger quark mass, is found, and we attribute this splitting to discretisation effects given that $m_{\pi} L \gtrsim 4$ and $m_{\pi} T \gtrsim 9$, meaning the finite-volume effects here are also small. As in Section 3.4, the values of $a_{t} m_{\omega}, a_{t} m_{\phi}$ and $\xi$ used are obtained by taking the largest variations within one standard deviation of the means across the different helicities. This yields the masses given in Table 5.2 and an anisotropy $\xi=3.443(48)$ which is consistent with the anisotropies previously determined for $\pi, K$ and $\eta 28,62,64$. 


\begin{tabular}{rlrrc} 
meson $\left(J^{P}\right)$ & \multicolumn{1}{c}{$a_{t} m$} & & threshold & $a_{t} E_{\mathrm{cm}}$ \\
\cline { 5 - 6 }$\pi\left(0^{-}\right)$ & $0.06906(13)$ & & $\pi \omega$ & $0.22447(32)$ \\
$K\left(0^{-}\right)$ & $0.09698(9)$ & & $\pi \pi \eta$ & $0.24176(26)$ \\
$\eta\left(0^{-}\right)$ & $0.10364(19)$ & & $\pi \phi$ & $0.24855(25)$ \\
$\sigma\left(0^{+}\right)$ & $0.1316(9)$ & & $\pi \bar{K}$ & $0.26302(18)$ \\
$\omega\left(1^{-}\right)$ & $0.15541(29)$ & & $\pi \pi \sigma \pi$ & $0.26972(92)$ \\
$\eta^{\prime}\left(0^{-}\right)$ & $0.1641(10)$ & & $\pi \pi \eta^{\prime}$ & $0.30222(102)$
\end{tabular}

Table 5.2: Left: The masses of relevant stable hadrons with uncertainties. Right: Relevant threshold energies with uncertainties.

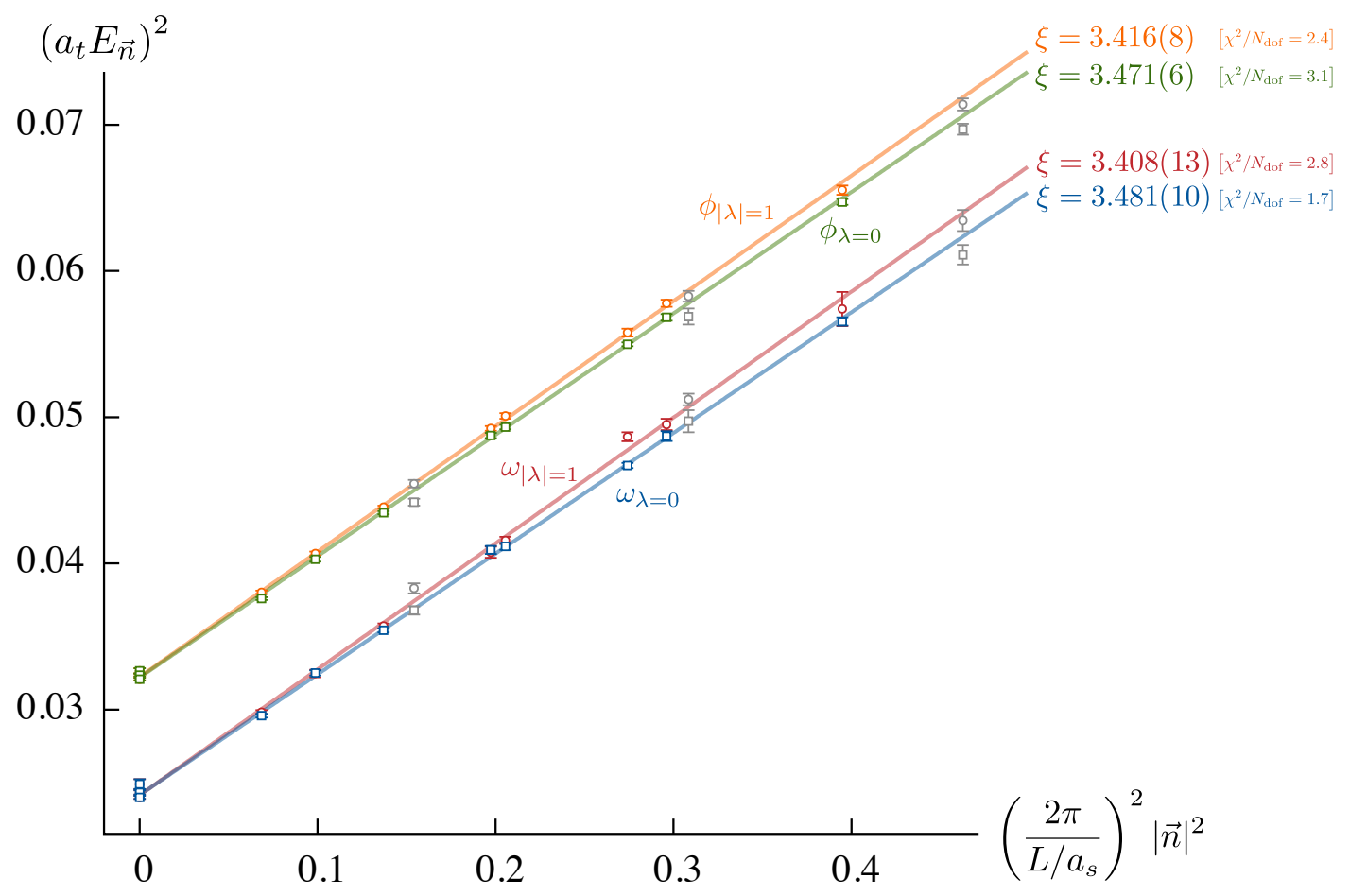

Figure 5.3: Momentum dependence of $\omega$ and $\phi$ energies and fits to Eq. 3.10. Blue and red lines correspond to the $\omega$ meson with $|\lambda|=0$ and 1 respectively. Similarly, green and orange lines correspond to the $\phi$ meson with $|\lambda|=0$ and 1. Points are shown with statistical uncertainties and grey points show the $\left(L / a_{s}\right)=16$ in-flight energies which are not included in the fit. 


\subsection{Operator Bases}

In this study, we are principally interested in irreps that contain $J^{P}=1^{+}$. For irreps at rest, $J^{P}=1^{+}$subduces only into $T_{1}^{+}$. However, for in-flight irreps, different helicity components of $J^{P}=1^{+}$are subduced across multiple irreps, as shown in Tables 3.5 - 3.7. For example, $\lambda=0$ and \pm 1 subduce into $A_{2}$ and $E_{2}$ respectively for overall momentum $\vec{P}=[001]$. Furthermore, at non-zero momentum, parity is no longer a good quantum number and so many irreps contain both $J^{+}$and $J^{-}$, e.g. $1^{+}$and $1^{-}$.

For irreps in flight, we will restrict our attention to $\vec{P} A_{2}$. These contain subductions of the $\lambda=0$ part of $J^{P}=1^{+}$but, because reflection parity $\tilde{\eta}=P(-1)^{J}$ is a good quantum number for $\lambda=0$, they do not contain $J^{P}=1^{-}$. In contrast, [001] $E_{2}$ contains $J^{P}=1^{-}$ as well as $J^{P}=1^{+}$. The latter gives comparatively lower-lying $J^{P}=1^{-}$levels, as seen in Ref. [30], and so will lead to a dense spectrum of mixed $J^{P}=1^{+}$and $1^{-}$energy eigenstates. Considering only $\vec{P} A_{2}$ allows us to avoid the complication of disentangling the $J^{P}=1^{+}$ and $1^{-}$scattering amplitudes.

The relevant thresholds for the isovector sector with positive $G$-parity are shown in Table 5.2. In the construction of correlation matrices we utilise two-meson operators resembling $\pi \omega$ and $\pi \phi$ and three-meson operators resembling $\pi \pi \eta$ and $\pi K \bar{K}$. All threemeson operators are of the form $\mathcal{O}_{\mathbb{R M}}^{\dagger}$ corresponding to $\rho \eta$ and $a_{0} \pi$ for $\pi \pi \eta$-like operators and $a_{0} \pi$ and $K^{*} \bar{K}$ for $\pi K \bar{K}$-like operators 4 As the $\pi \pi \sigma$-threshold also opens in our energy region, three-meson operators resembling $\rho \sigma$ and $a_{1} \pi$ were considered for inclusion. These appear in a relative $P$-wave in the $[000] T_{1}^{+}$and $\vec{P} A_{2}$ irreps at values of $E_{\text {n.i. }}^{(2+1)}$ that lie far above $\pi \pi \pi \pi$-threshold. Similarly, relevant $\pi \pi \sigma$ non-interacting energies, $E_{\text {n.i. }}^{(3)}$, are far above $\pi \pi \pi \pi$-threshold. Although the construction of operators resembling four-mesons could be done analogously to the three-meson operator construction described above, we do not include these in our basis and choose to restrict to energies below the $\pi \pi \pi \pi$-threshold.

The operator basis used for the $[000] T_{1}^{+}$irrep on each lattice volume is presented in Table 5.3. Included are all two-meson and three-meson operators corresponding to $E_{\text {n.i. }}^{(2)}$ and $E_{\text {n.i. }}^{(2+1)}$ below $\pi \pi \pi \pi$-threshold $!^{5}$ The operator lists for $\vec{P} A_{2}$ irreps with $\vec{P} \neq \overrightarrow{0}$ are recorded

\footnotetext{
${ }^{4}$ The optimised operators $\Omega_{\mathbb{R}}^{\dagger}$ for $\rho, a_{0}$ and $K^{*}$ used in $\mathbb{R M}$ operator constructions are determined independently in each relevant irrep using variational analysis with the operator bases that are presented in Appendix 5.A.

${ }^{5}$ There are no $E_{\text {n.i. }}^{(3)}$ below $4 m_{\pi}$ in $[000] T_{1}^{+}$.
} 


\begin{tabular}{ccc}
\hline$L / a_{s}=16$ & $L / a_{s}=20$ & $L / a_{s}=24$ \\
\hline $22 \times \bar{\psi} \boldsymbol{\Gamma} \psi$ & $22 \times \bar{\psi} \boldsymbol{\Gamma} \psi$ & $22 \times \bar{\psi} \boldsymbol{\Gamma} \psi$ \\
\hline$\pi_{[000]} \omega_{[000]}$ & $\pi_{[000]} \omega_{[000]}$ & $\pi_{[000]} \omega_{[000]}$ \\
$\pi_{[000]} \phi_{[000]}$ & $\pi_{[000]} \phi_{[000]}$ & $\pi_{[000]} \phi_{[000]}$ \\
$\rho_{[000]} \eta_{[000]}$ & $\rho_{[000]} \eta_{[000]}$ & $\rho_{[000]} \eta_{[000]}$ \\
$K_{[000]}^{*} \bar{K}_{[000]}$ & $K_{[000]}^{*} \bar{K}_{[000]}$ & $K_{[000]}^{*} \bar{K}_{[000]}$ \\
& & $\{2\} \pi_{[001]} \omega_{[001]}$
\end{tabular}

Table 5.3: [000] $T_{1}^{+}$operator basis for each lattice volume, with operators ordered by increasing $E_{\text {n.i. }}$. The maximum number of single-meson operators, $N$, is denoted by $N \times \bar{\psi} \boldsymbol{\Gamma} \psi$; various subsets of these were considered to obtain robust fits. The number in braces, $\left\{N_{\text {mult }}\right\}$, denotes the multiplicity of linearly independent two-meson operators if this is larger than one.

in Appendix 5.A. We include, as well as all low-lying two-meson operators, also the lowest three-meson $(\mathbb{R M})$ operator in each irrep, with the intention of robustly determining the spectra up to the lowest $E_{\text {n.i. }}^{(2+1)}$ or $E_{\text {n.i. }}^{(3)}$ energy. Moving frames were found to be essential in determining the sign of the off-diagonal entry in the $\boldsymbol{t}$-matrix between dynamically-coupled partial-waves in vector-pseudoscalar scattering, as shown in Section 3.6.3, and provide more energy levels with which to constrain the amplitudes.

In order to estimate the strength of partial-waves with $J \geq 2$ that feature in $[000] T_{1}^{+}$and $\vec{P} A_{2}$, on the largest volume, we also computed spectra in irreps $[000] E^{-},[000] T_{2}^{+},[001] B_{1}$ and [001] $B_{2}$, whose partial-wave content are presented in Tables 3.1 and 3.5. In addition to the vector-pseudoscalar partial-waves presented in the tables, the [001] $B_{1}$ and $[001] B_{2}$ irreps also feature pseudoscalar-pseudoscalar $J^{P}=3^{-}\left({ }^{1} F_{3}\right)$ partial-waves: $\pi \pi\left\{{ }^{1} F_{3}\right\}$ and $K \bar{K}\left\{{ }^{1} F_{3}\right\}$. The operator bases used for these irreps are presented in Appendix 5.A.

As we are considering the $G$-parity positive isovector sector, the neutral channels have charge-conjugation $C=-$. The contributing $J^{P C}$ includes our target $1^{+-}$where we expect a low-lying $b_{1}$ resonance, which in the quark model would be a $q \bar{q}$ spin-singlet in a $P$-wave. The $2^{--}$and $3^{--}$channels are expected to resonate at a higher energy, corresponding to the $\rho_{2}, \rho_{3}$ resonances, which would be spin-triplet $D$-waves in the quark model. Still higher, we might have a $3^{+-}$resonance, $b_{3}$, as a spin-singlet $F$-wave $q \bar{q}$. In addition, we 
also have contributions from the exotic $0^{--}$and $2^{+-}$channels - they do not appear in the $q \bar{q}$ quark model and previous lattice calculations [29] suggest that they may resonate in the form of hybrid mesons at much higher energy. As such, because they do not resonate in this energy region and feature at least a $P$-wave threshold suppression, it follows that we expect all partial waves except $J^{P}=1^{+}$to be small at low energies, and indeed we will find this to be the case.

\subsection{Finite-Volume Spectra}

The finite-volume spectra, extracted from a variational analysis of the matrix of correlation functions computed from the bases of operators given in Table 5.3, are presented in Figure 5.4. On the largest volume $\left(L / a_{s}=24\right)$, the principal correlators illustrate the quality of the signal extraction for each energy level and the operator-state overlap factors reflect the contributions from operators in the basis. It can be seen from the overlap factors that the two-meson and, in particular, the three-meson operator constructions efficiently interpolate the associated states. In some cases, an eigenstate has a dominant overlap with only one operator, suggesting that the state closely resembles that particular operator structure.

Consider first the number of energy levels expected below $a_{t} E_{\mathrm{cm}} \approx 0.27$ on each volume. In the absence of residual meson-meson interactions, we would expect four on each lattice volume: one at each of the two $E_{\text {n.i. }}^{(2)}$ corresponding to $\pi_{000} \omega_{000}$ and $\pi_{000} \phi_{000}$, shown as solid horizontal lines in the figure, and one at each of the $E_{\text {n.i. }}^{(2+1)}$ corresponding to $\rho_{000} \eta_{000}$ and $K_{000}^{*} \bar{K}_{000}$, shown as short dotted horizontal lines. Counting the number of energy levels actually extracted, we find five, with an 'additional' level appearing near $\pi \phi$ threshold. This may suggest the existence of a narrow resonance, as seen in a calculation of the $\rho$ 30 and $K^{*}\left[83\right.$ resonances, with a mass close to $\pi \phi$ threshold $\left.\right|^{6}$ On the largest volume, the consequence of $\pi_{001} \omega_{001}$ having a multiplicity of two is clear: two energy levels are found, one very close to the non-interacting energy and one somewhat higher in energy.

In Figure 5.4, we also present an investigation of the importance of including $\mathbb{R M}$ operators in the basis. The rightmost panel shows the spectrum extracted when $\rho \eta$ and

\footnotetext{
${ }^{6}$ We will see that the proximity of the resonance to $\pi \phi$ threshold is purely coincidental as hinted at by the operator overlaps in Figure 5.4 as discussed below.
} 
$K^{*} \bar{K}$ operators are excluded, compared to the spectrum extracted with the full basis. With the smaller basis, we see that typically the levels close to the $\rho \eta$ and $K^{*} \bar{K}$ 'noninteracting' energies are no longer found. The spectrum at lower energies shows only modest discrepancies, except on the smallest lattice volume $\left(L / a_{s}=16\right)$ where we might indeed expect the finite-volume effects associated with $\rho \eta$ and $K^{*} \bar{K}$ to be largest. Finding 'incorrect' spectra due to 'incomplete' operator bases has been demonstrated in previous works. One example can be seen in Figure 1 of Ref. [30] where including both $\bar{\psi} \boldsymbol{\Gamma} \psi$ and $\pi \pi$ operators is shown to be essential in order to robustly determine the $\rho$ spectrum. Figure 5.4 demonstrates an analogue of this for the case of three-meson operators.

Qualitative observations about the spectrum can be ascertained from a consideration of the operator-state overlap factors shown in Figure 5.4. The energy level just below $\pi \omega$ threshold on all volumes has significant overlap onto both $\pi_{000} \omega_{000}$ and $\bar{\psi} \boldsymbol{\Gamma} \psi$ operators. This characteristic might be expected if a $q \bar{q}$-like resonance lies nearby [4, 30]. For the two levels in close proximity to $\pi \phi$ threshold, one appears dominated by $\bar{\psi} \boldsymbol{\Gamma} \psi$ operators with some overlap onto $\pi \omega, \rho \eta$ and $K^{*} \bar{K}$ operators, while the other is completely dominated by $\pi_{000} \phi_{000}$. Furthermore, we observe that all other levels have very small overlaps with the $\pi_{000} \phi_{000}$ operator, reflecting the fact that the matrix of correlation functions is approximately block diagonal with respect to $\pi_{000} \phi_{000}$. This suggests that $\pi \phi$ is essentially 'decoupled', as might be expected from the 'OZI rule'. The states close to the $\rho \eta$ and $K^{*} \bar{K}$ 'non-interacting' energies are observed to have large overlap with $\rho \eta$ and $K^{*} \bar{K}$ operators respectively. The highest two states shown, near to the $\pi_{001} \omega_{001}$ two-fold degenerate noninteracting energy, differ somewhat in their overlaps. The level shifted up has overlap with both the $\pi_{001} \omega_{001}$ and $\bar{\psi} \boldsymbol{\Gamma} \psi$ operators, while the other, which lies on the non-interacting energy, has significant overlap only with the $\pi_{001} \omega_{001}$ operators.

In Figure 5.5, we present the cm-frame finite-volume spectra for irreps $[000] T_{1}^{+}$and $\vec{P} A_{2}$ on the three volumes, with only those levels found below the lowest $E_{\text {n.i. }}^{(2+1)}$ or $E_{\text {n.i. }}^{(3)}$ shown. $7^{7}$ Points in grey are levels that prove to be sensitive to the presence of $\rho \eta, K^{*} \bar{K}$ and $a_{0} \pi$ operators in the basis, or which are very close to the energy cut-off, and these levels are excluded from the main scattering analysis in Section 5.5. Although we take a conservative approach and exclude these levels, we will find in Section 5.5 that they

\footnotetext{
${ }^{7}$ Errorbars on the energy levels include estimates of systematic uncertainly coming from varying $t_{0}$ and fitting time ranges, and reasonable variations of the operator basis. Also included is the effect of the uncertainty on the anisotropy which appears when we boost back from the 'lab' energy to the $\mathrm{cm}$ frame.
} 
are mainly well described by the scattering amplitudes, and we re-examine these levels in Section 5.7 .

For irreps $\vec{P} A_{2}$ with $\vec{P} \neq \overrightarrow{0}$, the density of energy levels is much higher than in irreps at rest - more momentum combinations for two- and three-mesons with associated $E_{\text {n.i. }}^{(2)}$, $E_{\text {n.i. }}^{(2+1)}$ and $E_{\text {n.i. }}^{(3)}$ lying below the $\pi \pi \pi \pi$-threshold are possible. This can make identifying an 'additional' level more challenging in these irreps. However, in the [111] $A_{2}$ irrep we can clearly see an additional energy level on each volume relative to the number expected from counting the non-interacting two-meson energies. We also observe an 'avoided level crossing' where the $\pi_{000} \omega_{111}$ non-interacting energy crosses $a_{t} E_{\mathrm{cm}} \sim 0.25$, another signature characteristic of a narrow resonance in this energy region.

In Figure 5.6, the finite-volume spectra on the $L / a_{s}=24$ lattice volume for irreps $[000] T_{2}^{+},[000] E^{-},[001] B_{1}$ and [001] $B_{2}$ is presented. We observe very little deviation of the extracted energy levels from non-interacting $\pi \omega$ energies, suggesting that the $\pi \omega$ scattering amplitudes in $J \geq 2$ partial-waves are very small in this energy region. We also find levels in [001] $B_{1}$ and [001] $B_{2}$ consistent with non-interacting $\pi \pi$ energies and with dominant overlaps onto $\pi \pi$ operators. This is in line with the results of Ref. [30] where the $\pi \pi\left\{{ }^{1} F_{3}\right\}$ amplitude $\left(J^{P}=3^{-}\right)$was found to be consistent with zero in this energy region. We also find a level in [001] $B_{1}$ consistent with the non-interacting $K \bar{K}$ energy and with dominant overlap onto $K \bar{K}$ operators, suggesting that the opening of the $K \bar{K}$ threshold does not enhance the scattering in $J^{P}=3^{-}$. 

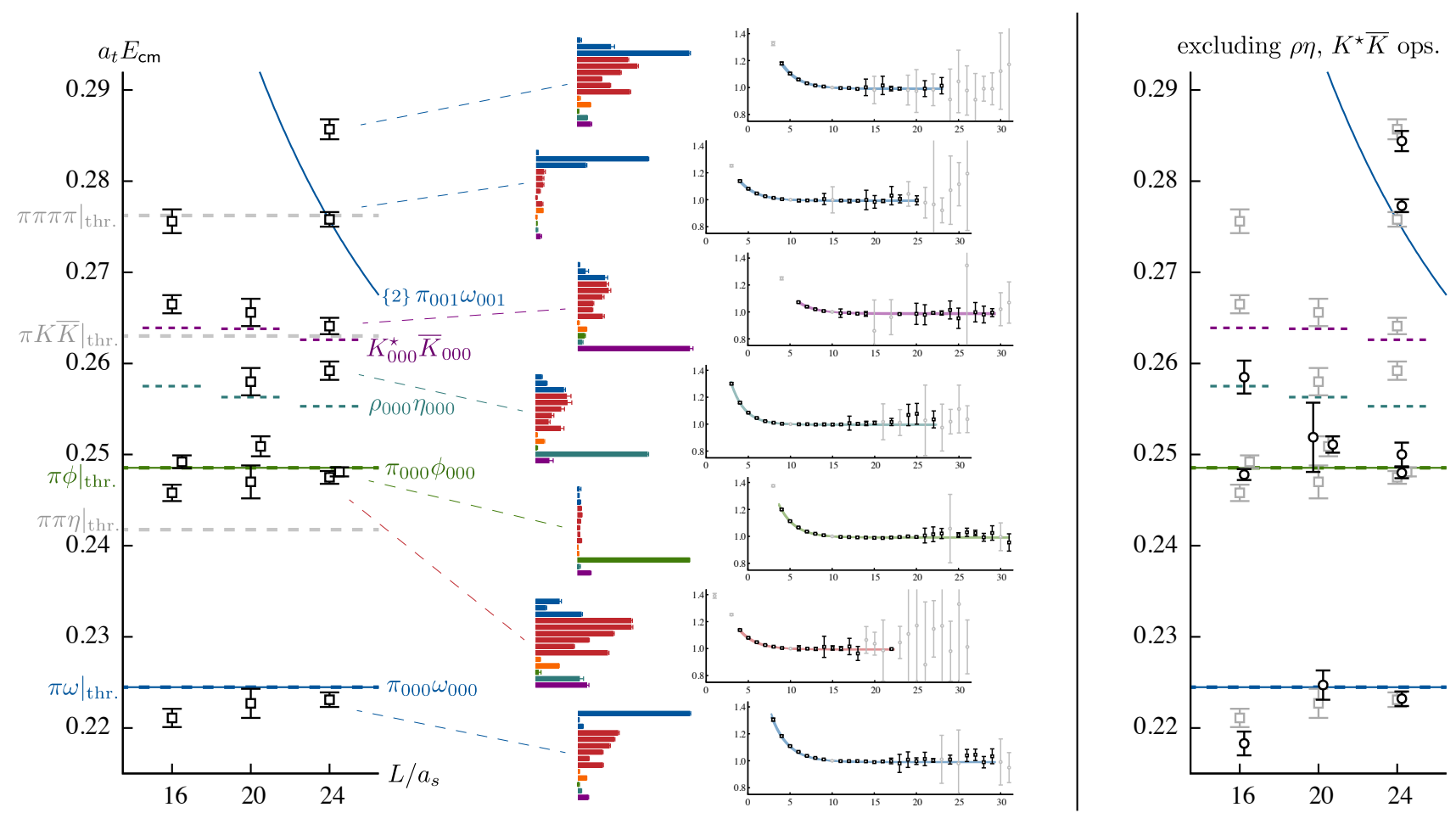

Figure 5.4: Left: Finite-volume spectrum in the $[000] T_{1}^{+}$irrep on three lattice volumes. Black points give the energy levels, including statistical uncertainties, from a variational analysis using the operator bases in Table 5.3. Solid curves are two-meson non-interacting energies, $a_{t} E_{\text {n.i. }}^{(2)}$, short dashed horizontal lines are $a_{t} E_{\text {n.i. }}^{(2+1)}$, and long dashed horizontal lines show the two-, three-, and four-meson thresholds. Multiplicities (if greater than one) are shown as $\{n\}$. For each energy level on the largest volume, we show the principal correlators, plotted as $\lambda_{\mathfrak{n}}\left(t, t_{0}\right) e^{E_{\mathfrak{n}}\left(t-t_{0}\right)}$ for $t_{0}=10 a_{t}$ so that a horizontal line is observed when a single exponential dominates. Points show $\lambda_{\mathfrak{n}}(t, 10)$ and error bars correspond to the one-sigma statistical uncertainty. Curves show fits to the form described in the text; the curves show the fit range and grey points are not included in the fit. The histograms show the operator-state overlap factors, $Z_{i}^{\mathfrak{n}}=\left\langle\mathfrak{n}\left|\mathcal{O}_{i}^{\dagger}(0)\right| 0\right\rangle$, for each energy level on the largest volume for the $\mathbb{M M}=\pi \omega$ (dark blue), $\pi \phi$ (green) and $\mathbb{R M}=\rho \eta$ (blue-green), $K^{*} \bar{K}$ (purple) operators along with a sample set of single-meson operators subduced from $J^{P}=1^{+}$(red) and $J^{P}=3^{+}$(orange). The overlaps are normalised such that the largest value for any given operator across all energy levels is equal to one. Right: The spectrum extracted when $\rho \eta$ and $K^{*} \bar{K}$ operators are excluded from the basis (black) compared with the complete spectrum (grey). 

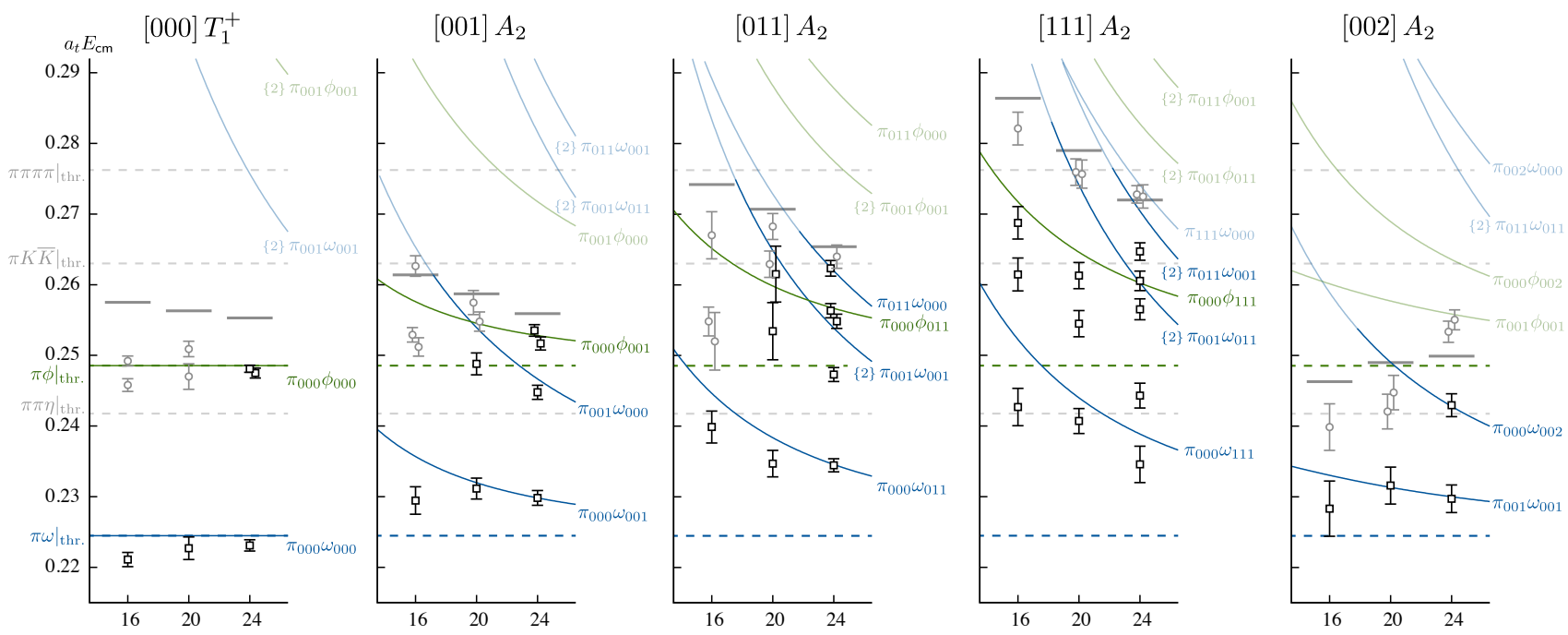

Figure 5.5: Finite-volume energy levels in the cm-frame for [000] $T_{1}^{+}$and $\vec{P} A_{2}$ below the lowest $E_{\text {n.i. }}^{(2+1)}$ or $E_{\text {n.i. }}^{(3)}$. Black points are used in the scattering analysis in Section 5.5 while grey points are excluded from the main analysis as discussed in the text. Solid curves are two-meson non-interacting energies, $a_{t} E_{\text {n.i. }}^{(2)}$, short solid grey horizontal lines show the lowest $E_{\text {n.i. }}^{(2+1)}$ or $E_{\text {n.i. }}^{(3)}$, and long dashed horizontal lines show the two-, three-, and four-meson thresholds. Multiplicities (if greater than one) are shown as $\{n\}$. The horizontal axes are in units of $L / a_{s}$. 


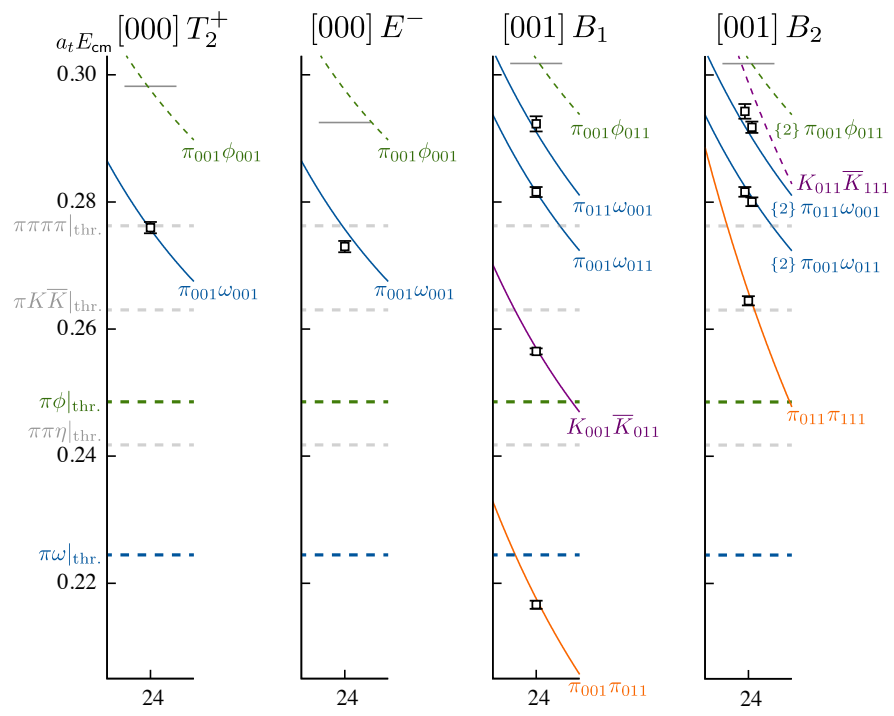

Figure 5.6: As Figure 5.5 but for irreps [000] $T_{2}^{+},[000] E^{-},[001] B_{1}$ and [001] $B_{2}$ on the largest lattice volume. Dashed curves show non-interacting two-meson energies where the corresponding operator was not included in the basis. 


\subsection{Scattering Analysis}

Scattering amplitudes are calculated following the procedure described in Section 3.6, utilising the general two-body formalism set out in Section 2.7. We therefore mainly restrict our attention to the two-body channels, $\pi \omega$ and $\pi \phi$, and in Section 5.7 we estimate the systematic effects of neglecting the three-body channels $\pi \pi \eta$ and $\pi K \bar{K}$ in the energy region considered, finding them to be small.

In the case of a vector-pseudoscalar channel in $J^{P}=1^{+}$, there is never rigorously elastic scattering - as soon as the $\pi \omega$ threshold opens, there are always two coupled partial-waves, ${ }^{3} S_{1}$ and ${ }^{3} D_{1}$. However, at low energies the angular momentum suppression of the $D$-wave may make the system effectively elastic in $S$-wave and we examine this case in Section 5.5.1.

Scattering with more than one partial-wave was discussed in detail in Chapter 3 and it is straightforward to incorporate multiple hadron-hadron channels. We parameterise the energy dependence of $\boldsymbol{t}(s)$ which, for more than one partial-wave and hadron-hadron channel, can be expressed in terms of a real symmetric $K$-matrix, $\boldsymbol{K}(s)$, where

$$
\left[t^{-1}(s)\right]_{\ell J a, \ell^{\prime} J b}=\frac{1}{\left(2 k^{(a)}\right)^{\ell}}\left[K^{-1}(s)\right]_{\ell J a, \ell^{\prime} J b} \frac{1}{\left(2 k^{(b)}\right)^{\ell^{\prime}}}+\delta_{\ell \ell^{\prime}} I_{a b}(s),
$$

is an augmented version of $\boldsymbol{K}(s)$ given in Eq. 3.14 accommodating a hadron-hadron channel index $a$. It follows that $I_{a b}(s)=I_{a}(s) \delta_{a b}$ is a matrix diagonal in hadron-hadron channel where unitarity constrains the imaginary part of $I_{a}(s)$ in each hadron-hadron channel. As presented in Appendix 3.D, we can take a number of different forms of $I_{a}(s)$, all subject to the constraints imposed by unitarity, to diversify the parameterisations.

One parameterisation we utilise expresses the components of $\boldsymbol{K}^{-1}(s)$ as polynomials in $s$,

$$
\left[K^{-1}(s)\right]_{\ell J a, \ell^{\prime} J b}=\sum_{n=0}^{N} c_{\ell J a, \ell^{\prime} J b}^{(n)} s^{n},
$$

where $\boldsymbol{c}^{(n)}$ is a real symmetric matrix. Flexibility in this form comes from varying $N$ and allowing parameter freedom in different combinations of $c_{\ell J a, \ell^{\prime} J b}^{(n)}$ coefficients.

An alternative is to parameterise the components of $\boldsymbol{K}(s)$ directly, using a parameterisation of the form,

$$
K_{\ell J a, \ell^{\prime} J b}(s)=\frac{g_{\ell J a}(s) g_{\ell^{\prime} J b}(s)}{m^{2}-s}+\sum_{n=0}^{N} \gamma_{\ell J a, \ell^{\prime} J b}^{(n)} s^{n}
$$


where $m$ is a real parameter, $g_{\ell J a}(s)$ is some real polynomial in $s$, and $\gamma^{(n)}$ is a symmetric matrix of real parameters. These forms assume nothing about a nearby resonance or bound state but the inclusion of a pole term can efficiently describe such behaviour where it is present. These and similar $K$-matrix parameterisations have been successfully used in previous lattice QCD calculations of three coupled-channel resonant scattering 7,62 and the non-resonant vector-pseudoscalar scattering in Chapter 3 (Ref. [84]).

As an explicit example, one that we will make use of later, consider a $\boldsymbol{K}$-matrix parameterisation suitable for describing the dynamically-coupled $J^{P}=1^{+}$channels $\pi \omega\left\{{ }^{3} S_{1}\right\}, \pi \omega\left\{{ }^{3} D_{1}\right\}$ and $\pi \phi\left\{{ }^{3} S_{1}\right\},{ }^{8}$ One possible choice, with 7 free parameters, is,

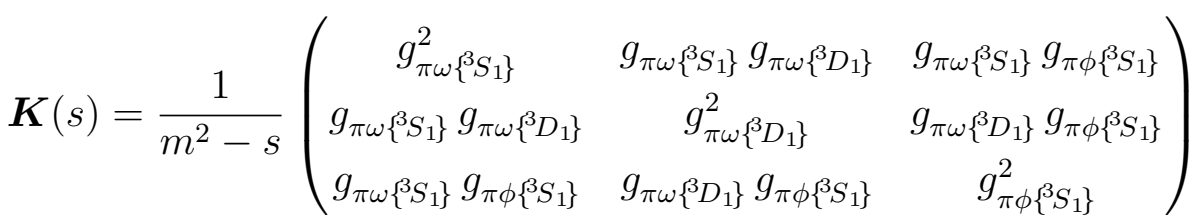

$$
\begin{aligned}
& +\left(\begin{array}{ccc}
\gamma_{\pi \omega\left\{{ }^{3} S_{1}\right\}, \pi \omega\left\{{ }^{3} S_{1}\right\}}^{(0)} & \gamma_{\pi \omega\left\{{ }^{3} S_{1}\right\}, \pi \omega\left\{{ }^{3} D_{1}\right\}}^{(0)} & 0 \\
\gamma_{\pi \omega\left\{{ }^{3} S_{1}\right\}, \pi \omega\left\{{ }^{3} D_{1}\right\}}^{(0)} & 0 & 0 \\
0 & 0 & \gamma_{\pi \phi\left\{{ }^{3} S_{1}\right\}, \pi \phi\left\{{ }^{3} S_{1}\right\}}^{(0)}
\end{array}\right)
\end{aligned}
$$

where this form allows mixing between $\pi \omega$ and $\pi \phi$ channels only through $g_{\pi \phi\left\{{ }^{3} S_{1}\right\}}$.

To include additional partial-waves that contribute as a consequence of the finitevolume but which do not mix in an infinite-volume, i.e. those with distinct $J^{P}$ as seen in Tables 3.1 and 3.5-3.7, we modify the $\boldsymbol{t}$-matrix by including additional diagonal blocks, each corresponding to a unique $J^{P}$ (recall the example given in Eq. 3.3.).

Statistical uncertainties on the scattering parameters and parameter correlations are determined by calculating the second derivatives of the correlated $\chi^{2}$ at its minimum. We make a conservative estimate of systematic uncertainties on each scattering parameter due to the uncertainties on stable hadron masses and the anisotropy by repeating the $\chi^{2}$ minimisation fitting procedure at all the various combinations of $\xi \pm \delta \xi$ and $m_{i} \pm \delta m_{i} !^{9}$ Unlike in the case of $\rho \pi$ scattering in isospin-2, where the largest systematic variation in the amplitude was observed by decreasing the hadron masses and increasing the anisotropy (and

\footnotetext{
${ }^{8}$ In principle, we should also consider $\pi \phi$ in the ${ }^{3} D_{1}$ partial-wave; however, suppression due to the centrifugal barrier factor, compounded with strong OZI suppression of $\pi \phi$, suggests it will be negligibly small. We will indeed find that the amplitude is consistent with zero in the energy region we consider.

${ }^{9}$ Values of the anisotropy, masses and uncertainties are given in Section 5.2 .
} 
vice-versa), the significant interactions at play here necessitate an evaluation of all possible combinations - as it is less clear how these variations propagate the uncertainty. For each of these minimisations, we keep the finite-volume energies, $E_{\mathrm{cm}}$, their corresponding uncertainties, $\delta E_{\mathrm{cm}}$, and correlations between energy levels fixed, where,

$$
\begin{aligned}
a_{t} E_{\mathrm{cm}} & =f\left(a_{t} E_{\mathrm{lat}}, \xi\right)=\sqrt{\left(a_{t} E_{\mathrm{lat}}\right)^{2}-\frac{1}{\xi^{2}}\left(\frac{2 \pi}{L / a_{s}}\right)^{2}|\vec{n}|^{2}} \\
a_{t} \delta E_{\mathrm{cm}} & =\sqrt{\left(\frac{\partial f}{\partial\left(a_{t} E_{\mathrm{lat}}\right)}\right)^{2}\left(a_{t} \delta E_{\mathrm{lat}}\right)^{2}+\left(\frac{\partial f}{\partial \xi}\right)^{2} \delta \xi^{2}},
\end{aligned}
$$

and $E_{\text {lat }}$ is the energy in the lattice frame. For each scattering parameter, the largest change in the central value is quoted as its systematic uncertainty.

\subsubsection{Elastic $\pi \omega\left\{{ }^{3} S_{1}\right\}$ Scattering}

Below $\pi \phi$ threshold, the kinematically-open hadron channels are the two-body $\pi \omega$ and three-body $\pi \pi \eta$. We expect $\pi \pi \eta$ to become an important channel near the lowest $E_{\text {n.i. }}^{(2+1)}$ where the $\rho$ and $a_{0}$ resonances enhance the $\pi \pi$ and $\pi \eta$ subsystems respectively. The lowest $E_{\text {n.i. }}^{(2+1)}$ (and $E_{\text {n.i. }}^{(3)}$ ) in each of the irreps we consider is typically much higher in energy than the $\pi \phi$ threshold. For a first analysis, we therefore ignore $\pi \pi \eta$ and return to this in Section 5.7 .

In this energy region only slightly above $\pi \omega$ threshold, the centrifugal barrier suppresses contributions of higher-partial waves, $t_{\ell J, \ell^{\prime} J} \sim k_{\mathrm{cm}}^{\ell+\ell^{\prime}}$, such that we expect the ${ }^{3} D_{1}$ contributions to the coupled $\left\{{ }^{3} S_{1},{ }^{3} D_{1}\right\}$ partial-waves to be rather small, assuming the threshold suppression is not overridden by a resonant enhancement in ${ }^{3} D_{1}$. Similarly, following the discussion in Section 5.3, $\pi \omega$ scattering amplitudes in other partial-waves that appear in these irreps due to the finite-volume are expected to be suppressed relative to the ${ }^{3} S_{1}$ amplitude and to have no significant resonant enhancement below $\pi \phi$ threshold. As such, we can attempt an elastic analysis of $\pi \omega\left\{{ }^{3} S_{1}\right\}$ scattering at low energy.

We use 20 levels, all at least one standard deviation below the $\pi \phi$ threshold. Specifically, for each irrep, these correspond to the lowest level on each of the $\left(L / a_{s}\right)=16$ and 20 volumes and the lowest two levels on the $\left(L / a_{s}\right)=24$ volume ${ }^{10}$ shown as the black points below $\pi \phi$ threshold in Figure 5.5. The discrete phase-shift points, calculated through the

\footnotetext{
${ }^{10}$ On the $\left(L / a_{s}\right)=24$ volume, of the two levels close to $\pi \phi$ threshold, the slightly lower level is included
} 
one-to-one mapping described in Section 3.2, are determined and are plotted in Figure 5.7. There is a clear trend for them to increase, as energy increases from $\pi \omega$ threshold, to a value of around $90^{\circ}$ as they approach the energy cut-off at $\pi \phi$ threshold. This is indicative of a narrow resonance with mass located somewhere near $\pi \phi$ threshold.

In addition to extracting discrete phase-shift points, we also fit the spectrum using energy-dependent parameterisations of elastic scattering. A selection of choices which describe the finite-volume spectra well are included as grey curves in Figure 5.7 with the details of the parameterisations presented in Table 5.13 of Appendix 5.B. As an example of a parameterisation which can describe the data, we choose as a reference amplitude, plotted as the blue curve in Figure 5.7 ,

$$
K(s)=\frac{g_{\pi \omega\left\{S_{1} S_{1}\right\}}^{2}}{m^{2}-s}
$$

using the Chew-Mandelstam prescription for $I(s)$ with $\operatorname{Re} I\left(s=m^{2}\right)=0$. We find the best fit description of the finite-volume spectrum is,

$$
\begin{array}{cc}
m= & (0.2472 \pm 0.0007 \pm 0.0003) \cdot a_{t}^{-1} \\
g_{\pi \omega\left\{{ }_{1} S_{1}\right\}}= & (0.068 \pm 0.009 \pm 0.010) \cdot a_{t}^{-1}
\end{array}\left[\begin{array}{cc}
1 & -0.04 \\
& 1
\end{array}\right]
$$

where the first uncertainty is statistical and the second is systematic as discussed above, and where the matrix shows the correlations between the parameters.

\subsubsection{Dynamically-Coupled $\pi \omega\left\{{ }^{3} S_{1}\right\}, \pi \omega\left\{{ }^{3} D_{1}\right\}$ Scattering}

Here we relax the assumption of negligible $\pi \omega\left\{{ }^{3} D_{1}\right\}$ contributions and perform a coupledchannel analysis on the dynamically-coupled $\pi \omega\left\{{ }^{3} S_{1}\right\}$ and $\pi \omega\left\{{ }^{3} D_{1}\right\}$ system, restricted to the same low energy region below $\pi \phi$ threshold as in Section 5.5.1. Motivated by the apparent resonant behaviour in the $\pi \omega\left\{{ }^{3} S_{1}\right\}$ phase-shift in Figure 5.7, we should allow for a resonance to have a $\pi \omega\left\{{ }^{3} D_{1}\right\}$ coupling, as this could significantly enhance the $\pi \omega\left\{{ }^{3} D_{1}\right\}$ contribution above the suppression imparted by the centrifugal barrier.

but the slightly higher level, essentially a decoupled $\pi \phi$ energy level as indicated by the histograms in Figure 5.4. is excluded. 


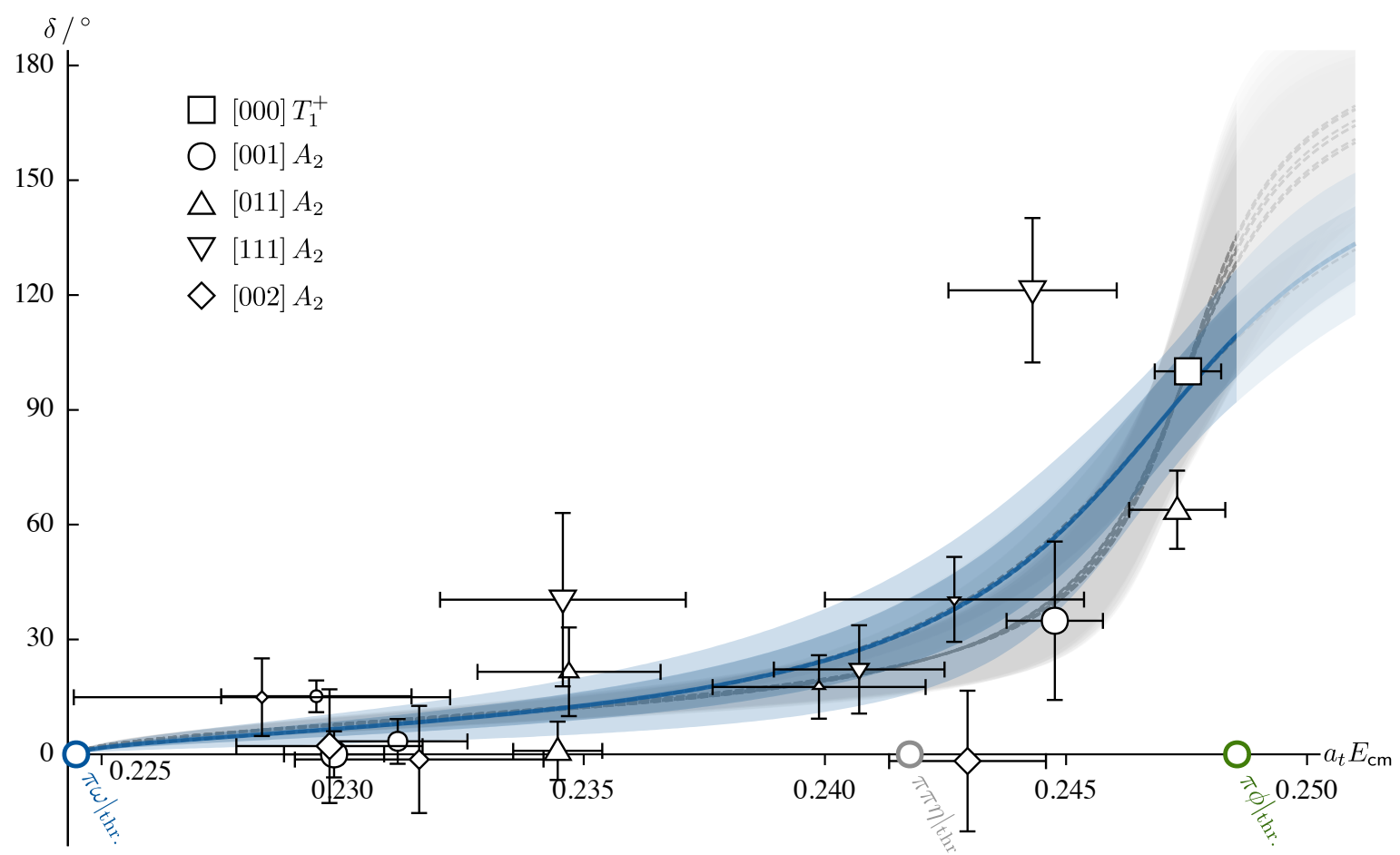

Figure 5.7: $\pi \omega\left\{{ }^{3} S_{1}\right\}$ elastic phase-shift assuming no ${ }^{3} D_{1}$ amplitude. The blue line shows the reference amplitude given in Eq. 5.12 with the blue bands reflecting the statistical (inner) plus systematic (outer) uncertainty. Grey lines and bands correspond to a range parameterisations presented in Table 5.13 of Appendix 5.B with only the statistical uncertainties shown. The point size (small to large) of the discrete phase-shift point encodes the lattice volume (small to large).

A choice of reference amplitude capable of describing the finite-volume spectra is the three-parameter,

$$
\boldsymbol{K}(s)=\frac{1}{m^{2}-s}\left(\begin{array}{cc}
g_{\pi \omega\left\{{ }^{\beta} S_{1}\right\}}^{2} & g_{\pi \omega\left\{{ }^{\beta} S_{1}\right\}} g_{\pi \omega\left\{{ }^{\beta} D_{1}\right\}} \\
g_{\pi \omega\left\{{ }^{\beta} S_{1}\right\}} g_{\pi \omega\left\{{ }^{\beta} D_{1}\right\}} & g_{\pi \omega\left\{{ }^{3} D_{1}\right\}}^{2}
\end{array}\right),
$$

again using the Chew-Mandelstam prescription for $I(s)$ with $\operatorname{Re} I\left(s=m^{2}\right)=0$. The resulting best-fit parameters are found to be, 


$$
\left.\begin{array}{rl}
m= & (0.2471 \pm 0.0007 \pm 0.0004) \cdot a_{t}^{-1} \\
g_{\pi \omega\left\{{ }^{3} S_{1}\right\}}= & (0.071 \pm 0.011 \pm 0.010) \cdot a_{t}^{-1} \\
g_{\pi \omega\left\{{ }^{3} D_{1}\right\}}= & (0.45 \pm 0.91 \pm 0.28) \cdot a_{t} \\
\chi^{2} / N_{\text {dof }}=\frac{14.9}{20-3}=0.87 .
\end{array}\right]
$$

The parameters $m$ and $g_{\pi \omega\left\{{ }^{3} S_{1}\right\}}$ are compatible with those of the reference amplitude in Eq. 5.12 and we find $g_{\pi \omega\left\{{ }^{3} D_{1}\right\}}$ to be consistent with zero within uncertainties. In Figure 5.8, we present the $\pi \omega\left\{{ }^{3} S_{1}\right\}$ and $\pi \omega\left\{{ }^{3} D_{1}\right\}$ phase-shifts and the $\bar{\epsilon}\left(\pi \omega\left\{{ }^{3} S_{1}\right\} \mid \pi \omega\left\{{ }^{3} D_{1}\right\}\right)$ mixingangle as defined in the Stapp-parameterisation [65] and given in Eq. 3.1. A number of different $\boldsymbol{K}$-matrix parameterisations were explored and are plotted as the grey curves in Figure 5.8 and listed in Table 5.14 of Appendix 5.B. We observe that all descriptions exhibit a $\pi \omega\left\{{ }^{3} S_{1}\right\}$ phase-shift compatible with the behaviour seen in Section 5.5.1, a $\pi \omega\left\{{ }^{3} D_{1}\right\}$ phase-shift that is very small, and a mixing-angle that is consistent with zero within a modest uncertainty over this energy range.

\subsubsection{Coupled $\pi \omega\left\{{ }^{3} S_{1}\right\}, \pi \omega\left\{{ }^{3} D_{1}\right\}$ and $\pi \phi\left\{{ }^{3} S_{1}\right\}$ Scattering}

We now consider scattering amplitudes in an energy region up to the $\pi \pi \pi \pi$-threshold. In this region, $\pi \omega, \pi \pi \eta, \pi \phi, \pi K \bar{K}$ and $\pi \pi \sigma$ are all kinematically open, however, by using only energy levels below the lowest $E_{\text {n.i. }}^{(2+1)}$ or $E_{\text {n.i. }}^{(3)}$ in each irrep, and excluding any energy levels which show significant sensitivity to the presence of $\rho \eta, K^{*} \bar{K}$ and $a_{0} \pi$ operators, we propose that we can effectively neglect the effect of three-body channels. This provides a total of 36 energy levels - all the black points shown in Figure 5.5.

Both $\pi \omega$ and $\pi \phi$ are vector-pseudoscalar channels dynamically-coupled in ${ }^{3} S_{1}$ and ${ }^{3} D_{1}$ partial-waves. However, considering the angular momentum suppression for the heavier threshold and the lack of mixing observed in the histograms presented in Figure 5.4, we assume that $\pi \phi\left\{{ }^{3} D_{1}\right\}$ will have negligible impact at low energies. We will in fact show that this is the case in Section 5.7. Subsequently, we are left with a system of three coupled channels: $\pi \omega\left\{{ }^{3} S_{1}\right\}, \pi \omega\left\{{ }^{3} D_{1}\right\}$ and $\pi \phi\left\{{ }^{3} S_{1}\right\}$. Many other partial-waves can contribute to the finite-volume spectra as can be seen from Tables 3.1 and 3.5- 3.7, but, as discussed in Section 5.3, we expect these to be negligibly small and will show this explicitly in Section 5.7 
To parameterise the energy dependence of the three-channel $\boldsymbol{t}$-matrix, we use $\boldsymbol{K}$ matrices of the form in Eq. 5.8 restricted to linear expansions in $g_{\ell J a}(s)$ and $\gamma(s)$. Many parameterisations are used and successful fits are recorded in Table 5.15 of Appendix 5.B. It should be noted that, while use of the $\boldsymbol{K}$-matrix guarantees unitarity, it does not guarantee good analytic properties. Indeed, we found that some parameterisations, which successfully describe the finite-volume spectra, have $\boldsymbol{t}$-matrix pole singularities at complex energies on the physical sheet. Such poles are forbidden by causality, and these parameterisations must be rejected as giving rise to unphysical solutions. A list of such parameterisations is given in Table 5.16 of Appendix 5.B and the resulting amplitudes are omitted from any subsequent figures.

As a reference amplitude, the five parameter $\boldsymbol{K}$-matrix,

$\boldsymbol{K}(s)=\frac{1}{m^{2}-s}\left(\begin{array}{ccc}g_{\pi \omega\left\{{ }^{\beta} S_{1}\right\}}^{2} & g_{\pi \omega\left\{{ }^{\beta} S_{1}\right\}} g_{\pi \omega\left\{{ }^{\beta} D_{1}\right\}} & 0 \\ g_{\pi \omega\left\{{ }^{3} S_{1}\right\}} g_{\pi \omega\left\{{ }^{3} D_{1}\right\}} & g_{\pi \omega\left\{{ }^{3} D_{1}\right\}}^{2} & 0 \\ 0 & 0 & 0\end{array}\right)+\left(\begin{array}{ccc}\gamma_{\pi \omega\left\{{ }_{1} S_{1}\right\}, \pi \omega\left\{{ }^{3} S_{1}\right\}}^{(0)} & 0 & 0 \\ 0 & 0 & 0 \\ 0 & 0 & \gamma_{\pi \phi\left\{{ }^{3} S_{1}\right\}, \pi \phi\left\{{ }_{1} S_{1}\right\}}^{(0)}\end{array}\right)$,

provides a good description of the finite-volume spectra with fewest free parameters, used with the Chew-Mandelstam prescription with $\operatorname{Re} I_{a}\left(s=m^{2}\right)=0$. The best-fit parameters are,

$$
\begin{aligned}
& m=(0.2465 \pm 0.0007 \pm 0.0001) \cdot a_{t}^{-1} \quad\left[\begin{array}{lllll}
1 & -0.05 & 0.05 & -0.01 & -0.23
\end{array}\right] \\
& g_{\pi \omega\left\{S_{1}\right\}}=(0.106 \pm 0.007 \pm 0.007) \cdot a_{t}^{-1} \quad \begin{array}{lllll}
1 & 0.70 & -0.54 & -0.06
\end{array} \\
& g_{\pi \omega\left\{D_{1}\right\}}=(1.08 \pm 0.47 \pm 0.28) \cdot a_{t} \\
& \gamma_{\pi \omega\left\{{ }^{\beta} S_{1}\right\}, \pi \omega\left\{{ }^{\beta} S_{1}\right\}}^{(0)}=-0.35 \pm 0.19 \pm 0.18 \\
& \gamma_{\pi \phi\left\{{ }^{3} S_{1}\right\}, \pi \phi\left\{{ }^{3} S_{1}\right\}}^{(0)}=0.90 \pm 0.24 \pm 0.27 \\
& \chi^{2} / N_{\text {dof }}=\frac{36.8}{36-5}=1.19 . \\
& 1-0.39-0.06 \\
& 1 \quad 0.22
\end{aligned}
$$

No improvement in the description of the finite-volume spectra was found by including freedom in $g_{\pi \phi\left\{{ }_{1}\right\}}$. Subsequently, we fixed this parameter to be zero in the reference amplitude. 
There is no established method in the literature to minimally display the 6 real degrees of freedom of the $\boldsymbol{S}$-matrix in three-channel scattering. For the two-channel case, the Stapp-parameterisation is minimal with regard to unitarity and reduces to single-channel phase-shifts when the channels decouple. No generalisation to more channels that naturally reduces to the two-channel Stapp-parameterisation appears in the literature. In Chapter 7 we provide such a generalisation to $n$-channels where, if $k$ are decoupled, the scattering $S$-matrix naturally block diagonalises into an $(n-k)$ coupled-channel block and a diagonal block containing $k$ decoupled phase-shifts. This definition of phase-shift and mixing-angles presented in Chapter 7 is used whenever we present the $\boldsymbol{S}$-matrix for more than two coupled-channels.

The phase-shifts and mixing-angles, explicitly defined for three coupled-channel scattering in Eq. 7.8 of Chapter 7, are plotted in Figure 5.9 for the reference amplitude in Eq. 5.16 (coloured curves) and for other successful parameterisations listed in Table 5.15 of Appendix 5.B (grey curves). We observe that the behaviour of the $\pi \omega\left\{{ }^{3} S_{1}\right\}$ phase-shift is in close agreement with the results of Section 5.5.2. and the $\pi \omega\left\{{ }^{3} D_{1}\right\}$ phase-shift is once more very small and compatible with zero. The $\pi \phi\left\{{ }^{3} S_{1}\right\}$ phase-shift shows a small positive tendency indicative of a weak attraction. The mixing-angle $\bar{\epsilon}\left(\pi \omega\left\{{ }^{3} S_{1}\right\} \mid \pi \omega\left\{{ }^{3} D_{1}\right\}\right)$ is small and positive, while the mixing angles $\bar{\epsilon}\left(\pi \omega\left\{{ }^{3} S_{1}\right\} \mid \pi \phi\left\{{ }^{3} S_{1}\right\}\right)$ and $\bar{\epsilon}\left(\pi \omega\left\{{ }^{3} D_{1}\right\} \mid \pi \phi\left\{{ }^{3} S_{1}\right\}\right)$ are around two orders of magnitude smaller and statistically consistent with zero across all parameterisations.

To complement the phase-shifts and mixing-angles, the same amplitudes are plotted as $\rho_{a} \rho_{b}\left|t_{\ell J a, \ell^{\prime} J b}\right|^{2}$ in Figure 5.10. A significant bump-like enhancement in the $\pi \omega\left\{{ }^{3} S_{1}\right\} \rightarrow$ $\pi \omega\left\{{ }^{3} S_{1}\right\}$ element is observed - a canonical indication for a resonance in a scattering cross-section measurement.

In Figure 5.11, we present the finite-volume energies calculated using the reference amplitude of Eq. 5.16, which are found to be in good agreement with the lattice computed energies, as suggested by the small $\chi^{2}$. Notably, for levels not included in the fits, shown in grey, the predicted spectra on the $\left(L / a_{s}\right)=20,24$ volumes appear to be mainly in reasonable agreement, whereas on the $\left(L / a_{s}\right)=16$ volume there is much more discrepancy. This may be attributed to more significant contributions from three-meson amplitudes on smaller volumes, further supported by the observation that there is a much larger variation in the spectrum in the $[000] T_{1}^{+}$irrep on the smaller volume when three-meson 
like operators are removed - see Figure 5.4. We re-examine these levels in Section 5.7 where we estimate the effects of the three-body channels.

A final comment concerns the systematic uncertainties on the scattering amplitudes due to the uncertainty placed on the anisotropy. Unlike in the $\rho \pi$ isospin- 2 case, where the weak nature of the interactions meant the uncertainty on the anisotropy dominated the systematic effects, here the interactions are strong and this uncertainty contributes relatively little. This can be seen from the relative sizes of the inner and outer bands in Figures 5.9 and 5.10 .

To summarise, the characteristic 'bump' we found in the scattering magnitudes in Figure 5.10 and the observed avoided level crossing in the [111] $A_{2}$ spectrum, seen in Figure 5.5, suggests a resonance. To demonstrate this rigorously, we proceed to determine the pole singularities of our scattering amplitudes. 


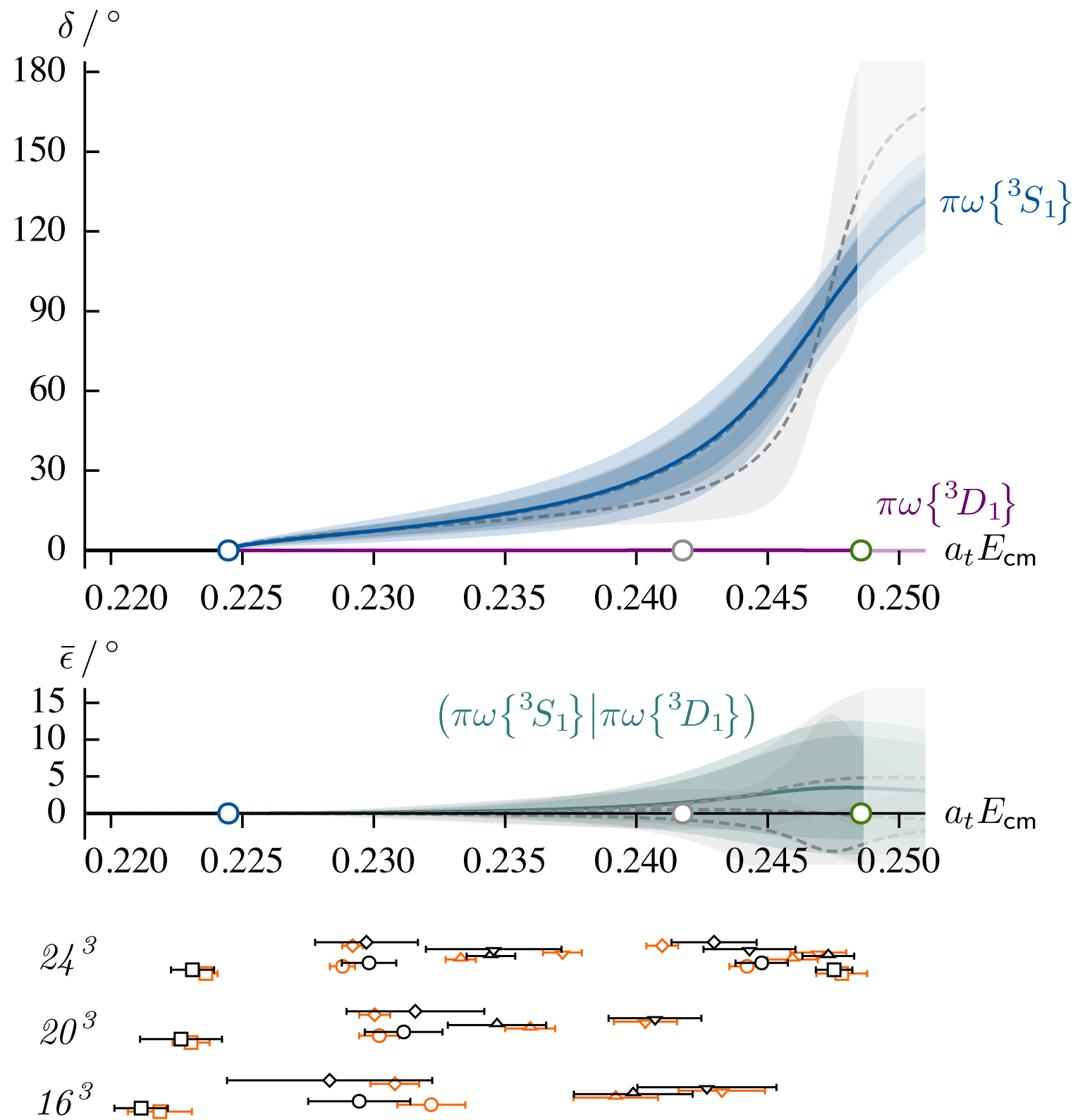

Figure 5.8: Upper: $\pi \omega\left\{{ }^{3} S_{1}\right\}$ (blue) and $\pi \omega\left\{{ }^{3} D_{1}\right\}$ (purple) phase-shifts for the reference amplitude in Eq. 5.14 with the bands reflecting the statistical (inner) plus systematic (outer) uncertainties. In grey are parameterisations given in Table 5.14 of Appendix $5 . \mathrm{B}$ with only statistical uncertainties shown. Middle: As upper but for the mixing-angle, $\bar{\epsilon}\left(\pi \omega\left\{{ }^{3} S_{1}\right\} \mid \pi \omega\left\{{ }^{3} D_{1}\right\}\right)$. Lower: Black points are the finite-volume energy levels used to constrain the fit and orange points are the energy levels calculated using Eq. 2.53 for the reference amplitude in Eq. 5.14 . 

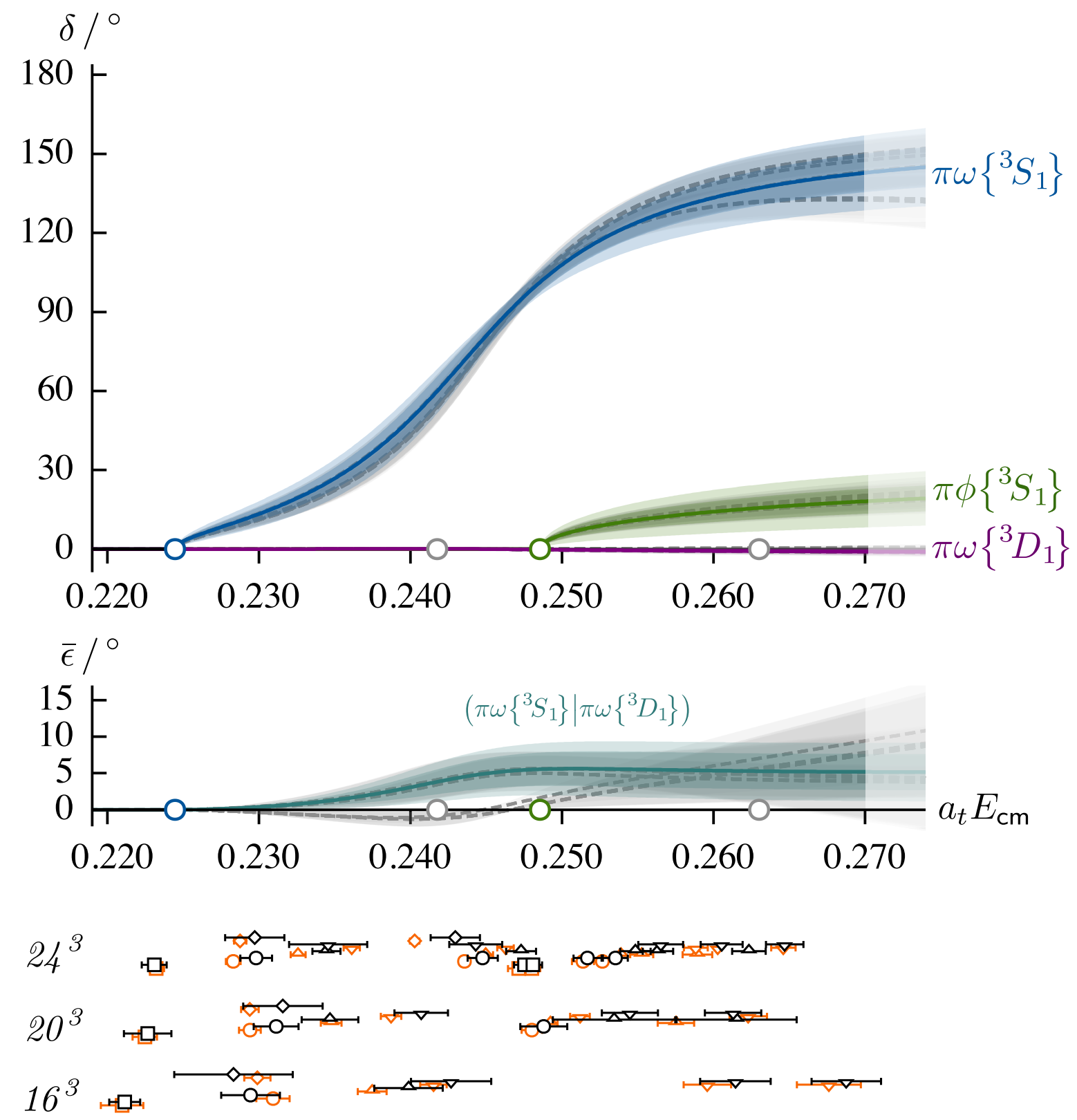

Figure 5.9: Upper: As in Figure 5.8 but for the $\pi \omega\left\{{ }^{3} S_{1}\right\}$ (blue), $\pi \omega\left\{{ }^{3} D_{1}\right\}$ (purple) and $\pi \phi\left\{{ }^{3} S_{1}\right\}$ (green) phase-shifts for the reference amplitude in Eq. 5.16 and for other parameterisations presented in Table 5.15 of Appendix 5.B (grey). Middle: As upper but for the mixing-angle $\bar{\epsilon}\left(\pi \omega\left\{{ }^{3} S_{1}\right\} \mid \pi \omega\left\{{ }^{3} D_{1}\right\}\right)$. The other mixing-angles, $\bar{\epsilon}\left(\pi \omega\left\{{ }^{3} S_{1}\right\} \mid \pi \phi\left\{{ }^{3} S_{1}\right\}\right)$ and $\bar{\epsilon}\left(\pi \omega\left\{{ }^{3} D_{1}\right\} \mid \pi \phi\left\{{ }^{3} S_{1}\right\}\right)$, are extremely small and consistent with zero for all parameterisations and are not plotted. Lower: The energy levels used to constrain the scattering amplitude (black) and their corresponding description by the reference amplitude in Eq. 5.16 (orange). 

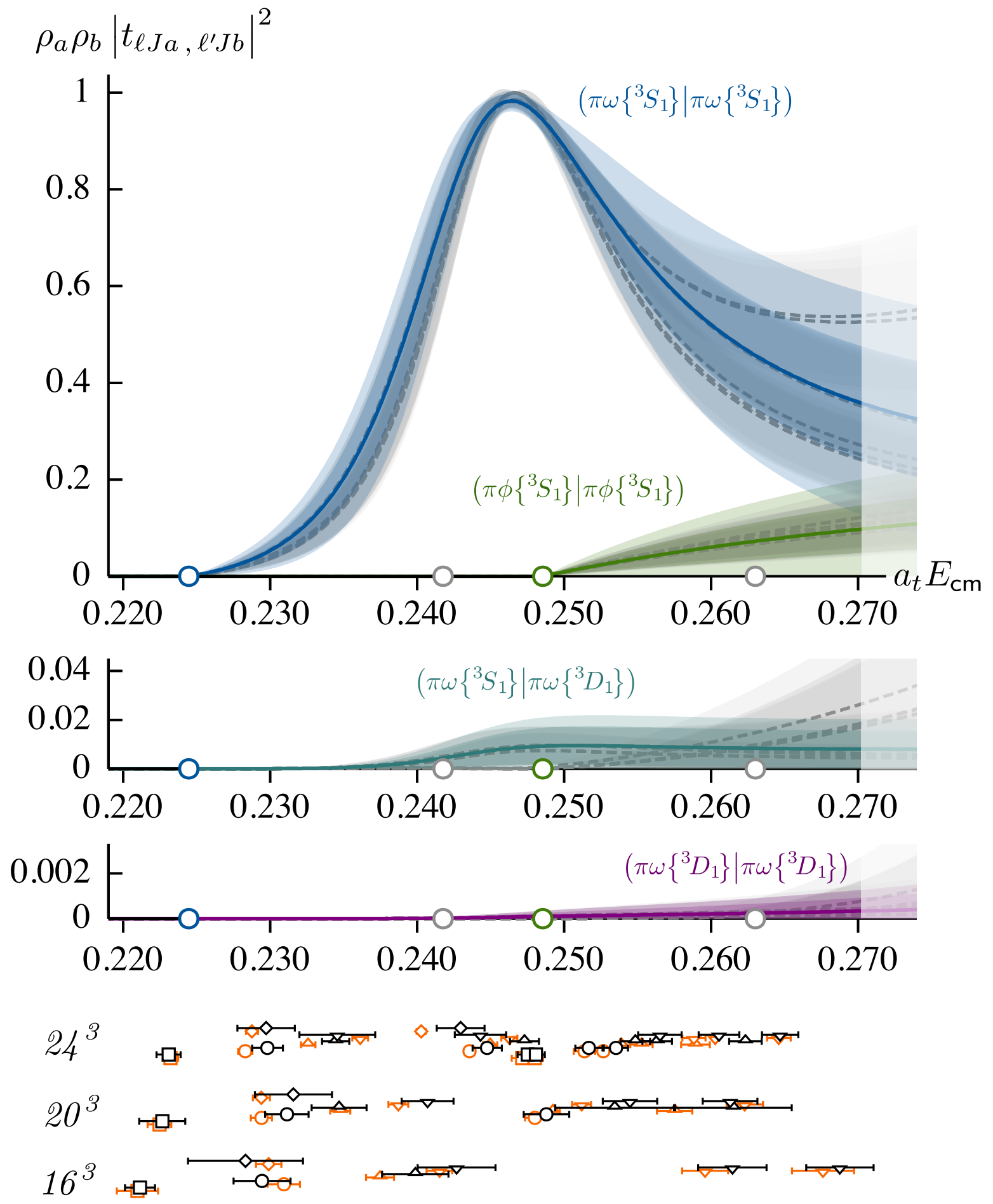

Figure 5.10: As Figure 5.9 but for $\rho_{a} \rho_{b}\left|t_{\ell J a, \ell^{\prime} J b}\right|^{2}$. Coloured curves illustrate the reference amplitude in Eq. 5.16 with bands reflecting the statistical (inner) plus systematic (outer) uncertainty. Other parameterisations presented in Table 5.15 of Appendix 5.B are in grey with bands reflecting only the statistical uncertainties. $\rho_{a} \rho_{b}\left|t_{\ell J a, \ell^{\prime} J b}\right|^{2}$ not plotted are significantly smaller than those shown and are consistent with zero. 

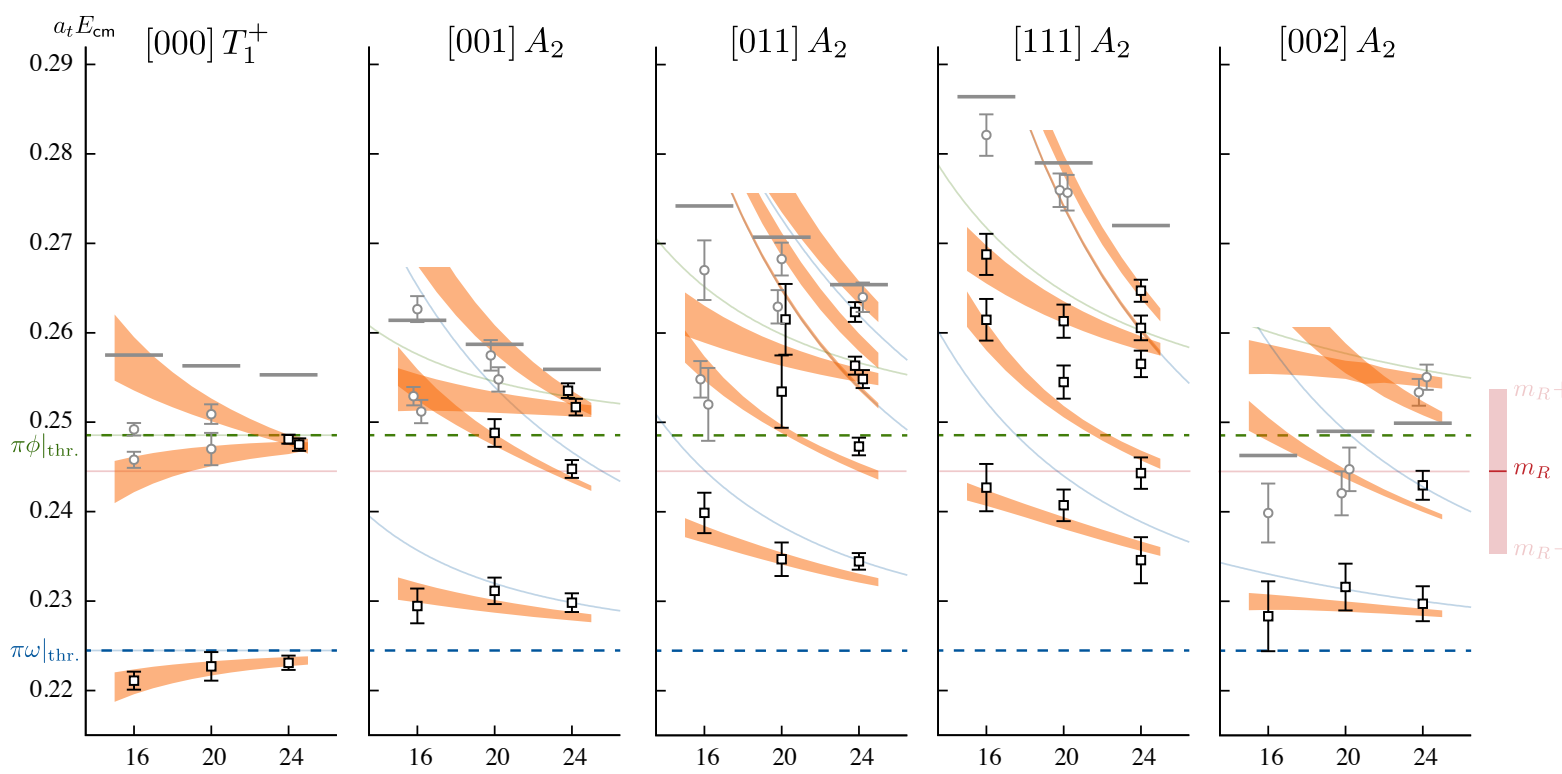

Figure 5.11: As Figure 5.5 but including, as orange bands, the energy levels calculated from the reference amplitude in Eq. 5.16 using Eq. 2.53 as a function $L / a_{s}$. The thickness of the bands reflect the combined statistical and systematic uncertainties. The vertical red band on the right of the figure indicates the position of the resonant pole of $m_{R}$ and width $\Gamma_{R}$ as determined in Section 5.6. The red horizontal line at the resonant mass is shown in each irrep to guide the eye. 


\subsection{Pole Analysis for Coupled-Channel Amplitudes}

At each threshold, unitary of the $S$-matrix necessitates a branch point singularity and the corresponding branch cut divides the complex s-plane into two Riemann sheets so that for $n$ open thresholds there are $2^{n}$ sheets. Riemann sheets can be labelled by the sign of the imaginary component of the $\mathrm{cm}$-frame momentum $k_{\mathrm{cm}}^{(a)}$ in each hadron channel $a$ and we identify the physical sheet, where physical scattering occurs, just above real energy axis, as having $\operatorname{Im}\left(k_{\mathrm{cm}}^{(a)}\right)>0$ for all $a$. On the physical sheet, causality forbids complex-valued poles and restricts any singularities to lie on the real axis below threshold - these correspond to bound-states 11 Amplitudes that permit complex-valued poles on the physical sheet give an unacceptable description of the scattering process. Sheets with other sign combinations are referred to as unphysical and complex-valued poles, found in complex-conjugate pairs, on these sheets correspond to resonances.

For poles away from the real axis, we define the real and imaginary parts of the pole singularity at $s=s_{0}$ as the mass $m_{R}$ and the width $\Gamma_{R}$ of a resonance respectively, with $\sqrt{s_{0}}=m_{R} \pm \frac{i}{2} \Gamma_{R}$. For narrow resonances, with a single dominant decay mode, this definition of the resonance mass and width agree well with the location of the peak and full-width at half-maximum of the 'bump' respectively, observed in Figure 5.10. It is much more difficult to see resonant behaviour in strongly coupled-channel scattering or for broader resonances, observed in the resonant $a_{0}[62]$ and $f_{0}[7]$, which do not have the distinctive characteristic bump of a narrow resonance, however, the determined pole singularity is free from such ambiguities.

For the three channels $\pi \omega\left\{{ }^{3} S_{1}\right\}, \pi \omega\left\{{ }^{3} D_{1}\right\}$ and $\pi \phi\left\{{ }^{3} S_{1}\right\}$ considered here, there are four Riemann sheets owing to the two distinct hadron-hadron thresholds, $\pi \omega$ and $\pi \phi$. The labelling conventions are summarised in Table 5.4 and agree with those in Ref. 62. For physical scattering close to $\pi \phi$ threshold, the lower-half plane of sheet II and III and the upper-half plane of sheet IV are close to physical scattering 62]. Depending on the strength of the couplings to the hadron-hadron channels, a resonance can appear in slightly different positions across the sheets. A discussion is presented for the strongly coupled $a_{0}[62$ where toy amplitudes are used to illustrate this behaviour.

At complex energies close to a pole singularity at $s_{0}$, the scattering $\boldsymbol{t}$-matrix can be

\footnotetext{
${ }^{11}$ Real-valued poles below threshold on unphysical sheets are referred to as virtual bound-states.
} 


\begin{tabular}{rcc} 
Sheet & $\operatorname{Im} k_{\pi \omega}$ & $\operatorname{Im} k_{\pi \phi}$ \\
\hline I & + & + \\
II & - & + \\
III & - & - \\
IV & + & -
\end{tabular}

Table 5.4: Sheet labelling as a function of the sign of the imaginary components of $k_{\mathrm{cm}}^{(a)}$.

written in the factorised form,

$$
t_{\ell J a, \ell^{\prime} J b}\left(s \sim s_{0}\right) \sim \frac{c_{\ell J a} c_{\ell^{\prime} J b}}{s_{0}-s}
$$

where the complex valued couplings $c_{\ell J a}$ reflect the strength of the resonance coupling to channel $a\left\{{ }^{3} \ell_{J}\right\}$. For each coupled hadron-hadron channel, the coupling is determined only up to a sign. This leads to a sign ambiguity here between the $\pi \omega$ and $\pi \phi$ couplings. Conversely, the relative sign between dynamically-coupled partial-waves, in this case $\pi \omega\left\{{ }^{3} S_{1}\right\}$ and $\pi \omega\left\{{ }^{3} D_{1}\right\}$, can be determined due to the aforementioned unique determination of $t\left(\pi \omega\left\{{ }^{3} S_{1}\right\} \mid \pi \omega\left\{{ }^{3} D_{1}\right\}\right)$. We showed for the vector-pseudoscalar $\rho \pi$ that the sign of $t\left(\rho \pi\left\{{ }^{3} S_{1}\right\} \mid \rho \pi\left\{{ }^{3} D_{1}\right\}\right)$ was uniquely determined using moving frame irreps in Figure 3.12 .

For each parameterisation successfully describing the finite-volume spectra we perform a search across all Riemann sheets for a large complex $s$-rang ${ }^{12}$ and determine any pole singularities appearing in the amplitudes and corresponding couplings by factorising the $\boldsymbol{t}$-matrix according to Eq. 5.17. Uncertainties on the pole and couplings in each case are estimated by appropriately propagating through the uncertainties and correlations on the fit parameters. For the reference amplitude given in Eq. 5.16, pole singularities were found in complex conjugate pairs on sheet II at,

$$
a_{t}{\sqrt{s_{0}}}_{\mathrm{II}}=0.2435(13)(10) \pm \frac{i}{2} 0.0175(20)(19)
$$

where the first uncertainty is statistical and the second is systematic. A complex conjugate pair of poles was also found on sheet III in the same location up to the precision shown in

\footnotetext{
${ }^{12}$ We search approximately $\operatorname{Re} a_{t} \sqrt{s} \in[0.21,0.28]$ and $2 \operatorname{Im} a_{t} \sqrt{s} \in[-0.2,0.2]$. Any pole outside this real energy region would not be well constrained by our data.
} 
Eq. 5.18. The corresponding couplings for the pole in the lower-half plane are,

$$
\begin{aligned}
& a_{t} c\left(\pi \omega\left\{{ }^{3} S_{1}\right\}\right)_{\mathrm{II}}=0.106(6)(6) \exp [-i \pi 0.078(28)(26)] \\
& a_{t} c\left(\pi \omega\left\{{ }^{3} D_{1}\right\}\right)_{\mathrm{II}}=0.010(4)(3) \exp [-i \pi 0.181(26)(24)],
\end{aligned}
$$

and $c\left(\pi \phi\left\{{ }^{3} S_{1}\right\}\right)_{\|}$is exactly zero, a result of the choice of reference amplitude. Considered as a ratio we have,

$$
\begin{aligned}
\left|c\left(\pi \omega\left\{{ }^{3} D_{1}\right\}\right)_{\mathrm{II}} / c\left(\pi \omega\left\{{ }^{3} S_{1}\right\}\right)_{\mathrm{II}}\right| & =0.091(37)(20) \\
\arg \left[c\left(\pi \omega\left\{{ }^{3} D_{1}\right\}\right)_{\mathrm{II}} / c\left(\pi \omega\left\{{ }^{3} S_{1}\right\}\right)_{\mathrm{II}}\right] & =-\pi 0.103(26)(24) .
\end{aligned}
$$

The fact that the poles on sheets II and III are in essentially the same position is a consequence of the $\pi \phi$ channel being almost completely decoupled from the $\pi \omega$ channel as discussed in Section 5.4 .

For each successful three-channel parameterisation, recorded in Table 5.15 of Appendix $5 . \mathrm{B}$, we found poles and couplings broadly consistent with those given above. We show these in Figure 5.12 , observing that the scatter over different parameterisations is not significantly larger than the uncertainty on the reference amplitude. The calculation of the error ellipses, presented in the figure, are detailed in Appendix 5.C. 

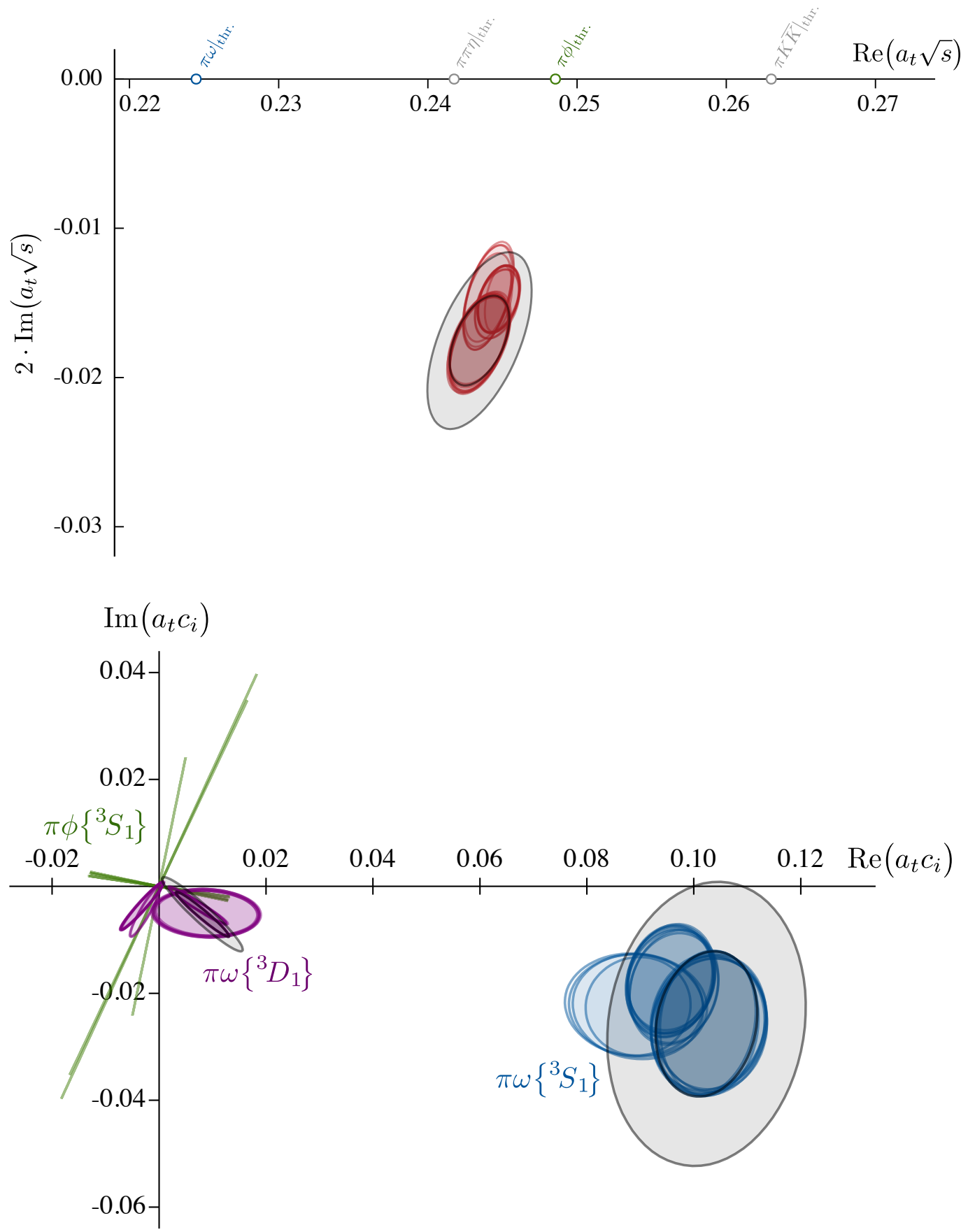

Figure 5.12: Top: Lower half-plane sheet II poles. Red ellipses reflect the statistical uncertainties, oriented to account for correlations between the real and imaginary parts, for poles from all the parameterisations shown in Table 5.15 of Appendix 5.B. Black ellipses correspond to the reference amplitude in Eq. 5.16 reflecting the statistical (inner) plus systematic (outer) uncertainties. Bottom: As top but for the corresponding couplings, $c\left(\pi \omega\left\{{ }^{3} S_{1}\right\}\right)$ ॥ (blue), $c\left(\pi \omega\left\{{ }^{3} D_{1}\right\}\right)_{\|}$(purple) and $c\left(\pi \phi\left\{{ }^{3} S_{1}\right\}\right)_{\|}$(green). Black ellipses again correspond to the couplings of the reference amplitude in Eq. 5.16 where $c\left(\pi \phi\left\{{ }^{3} S_{1}\right\}\right)$ ॥l $=0$. 


\subsection{Systematic Tests}

To test the robustness of the extracted scattering amplitudes and the determination of the resonant pole and couplings, we consider three sources of potential systematic uncertainties due to possibilities we have so far neglected. First, we examine the partial-waves that mix as a consequence of the finite-volume, which we neglected based on observations discussed in Section 5.4, and the $\pi \phi\left\{{ }^{3} D_{1}\right\}$ amplitude which we asserted was negligible. Second, we examine the dependence of the energy levels on the $\pi \omega\left\{{ }^{3} D_{1}\right\}, \pi \omega\left\{{ }^{3} P_{0}\right\}$ and $\pi \omega\left\{{ }^{3} P_{2}\right\}$ parameters to demonstrate that we are able to constrain these amplitudes. Third, we make an estimate of the possible size of effects due to the neglected three-body channels that are not currently possible to rigorously incorporate into the analysis.

\subsubsection{Additional Partial-Waves}

We first consider the $\pi \omega\left\{{ }^{3} P_{0}\right\}$ and $\pi \omega\left\{{ }^{3} P_{2}\right\}$ amplitudes that enter in the $\vec{P} A_{2}$ irreps as shown in Tables 3.5-3.7. Since a $P$-wave has less threshold suppression than a $D$-wave, we might expect these waves to be at least as important as $\pi \omega\left\{{ }^{3} D_{1}\right\}$, though they are not expected to be resonant at such low energies. Augmenting the reference amplitude as defined in Eq. 5.16, we allow a non-zero amplitude in the $\pi \omega\left\{{ }^{3} P_{0}\right\}$ and $\pi \omega\left\{{ }^{3} P_{2}\right\}$ channels by including a constant $\gamma$-term for each in the $\boldsymbol{K}$-matrix and for these additional channels we set $\operatorname{Re} I_{a}\left(s=\left(m_{\pi}+m_{\omega}\right)^{2}\right)=0$ in the Chew-Mandelstam phase-space. The resulting $\boldsymbol{t}$-matrix is block diagonal in $J^{P}$, reflecting the fact that this mixing is a result of the reduced symmetry on the lattice. We fit to the same 36 energy levels as in Section 5.5.3 and, allowing all parameters to vary, find,

$$
\begin{aligned}
m & =(0.2466 \pm 0.0007) \cdot a_{t}^{-1} & & \gamma_{\pi \omega\left\{S_{1}\right\}, \pi \phi\left\{{ }^{3} S_{1}\right\}}^{(0)}=0.79 \pm 0.25 \\
g_{\pi \omega\left\{S_{1}\right\}} & =(0.105 \pm 0.007) \cdot a_{t}^{-1} & \gamma_{\pi \omega\left\{{ }^{3} P_{0}\right\}, \pi \omega\left\{{ }^{3} P_{0}\right\}}^{(0)} & =(-8 \pm 21) \cdot a_{t}^{2} \\
g_{\pi \omega\left\{{ }^{3} D_{1}\right\}} & =(1.12 \pm 0.46) \cdot a_{t} & \gamma_{\pi \omega\left\{P_{2}\right\}, \pi \omega\left\{{ }^{3} P_{2}\right\}}^{(0)} & =(-10 \pm 12) \cdot a_{t}^{2} \\
\gamma_{\pi \omega\left\{{ }_{1} S_{1}\right\}, \pi \omega\left\{{ }^{3} S_{1}\right\}}^{(0)} & =-0.34 \pm 0.19 & \chi^{2} / N_{\text {dof }} & =\frac{34.4}{36-7}=1.19,
\end{aligned}
$$

where correlations between the $\pi \omega\left\{{ }^{3} S_{1}\right\}, \pi \omega\left\{{ }^{3} D_{1}\right\}$ and $\pi \phi\left\{{ }^{3} S_{1}\right\}$ parameters are compatible with those shown in Eq. 5.16, and correlations between these and $\pi \omega\left\{{ }^{3} P_{0}\right\}$ and $\pi \omega\left\{{ }^{3} P_{2}\right\}$ parameters are small. We observe that the amplitudes in both $\pi \omega\left\{{ }^{3} P_{0}\right\}$ and $\pi \omega\left\{{ }^{3} P_{2}\right\}$ are 
consistent with zero. A similar approach allowing for $\pi \omega\left\{{ }^{3} D_{2}\right\}$ and $\pi \omega\left\{{ }^{3} D_{3}\right\}$ parameter freedom finds no evidence for large amplitudes as one would expect given the larger angular momentum suppression and lack of low-energy resonances with $J^{P C}=2^{+-}$and $3^{+-}$.

In order to investigate the possible effect of the previously excluded $\pi \phi\left\{{ }^{3} D_{1}\right\}$, we take the reference amplitude in Eq. 5.16 and extend it to include a constant diagonal $\gamma$-term in $\pi \phi\left\{{ }^{3} D_{1}\right\}$ in the $\boldsymbol{K}$-matrix. Once again fitting to the 36 energy levels and allowing all parameters to vary, we find the $\pi \phi\left\{{ }^{3} D_{1}\right\}$ parameter to be consistent with zero, as expected, with all other parameters compatible with those presented in Eq. 5.16.

\subsubsection{Spectrum dependence on $\pi \omega\left\{{ }^{3} P_{0}\right\}, \pi \omega\left\{{ }^{3} P_{2}\right\}$ and $\pi \omega\left\{{ }^{3} D_{1}\right\}$}

To examine how the $\pi \omega\left\{{ }^{3} P_{0}\right\}, \pi \omega\left\{{ }^{3} P_{2}\right\}$ and $\pi \omega\left\{{ }^{3} D_{1}\right\}$ amplitudes are constrained in our analysis, we examine how the particular energy levels in the finite-volume spectra depend upon the strength of these partial-waves. For $\pi \omega\left\{{ }^{3} D_{1}\right\}$ this is shown in Figure 5.13 , where the curves present the finite-volume energy spectrum for the reference amplitude in Eq. 5.16, varying the value of $g_{\pi \omega\left\{{ }^{3} D_{1}\right\}}$ while keeping all other parameters fixed. In each irrep, we see a level near the lowest $\pi \phi$ non-interacting energy which appears to be independent of the value of $g_{\pi \omega\left\{{ }^{3} D_{1}\right\}}$, as expected given the complete decoupling of $\pi \phi$ in the reference amplitude. The figure shows that most other levels have significant dependence on $g_{\pi \omega\left\{{ }^{3} D_{1}\right\}}$, in particular those low down in the spectra, indicating that the lattice computed levels are providing constraint on the ${ }^{3} D_{1}$-wave strength, but there are some notable exceptions. In irreps [011] $A_{2}$ and [111] $A_{2}$, there are levels observed to be consistent with the two-fold degenerate non-interacting $\pi \omega$ energies, which show no visible dependence on $g_{\pi \omega\left\{{ }^{3} D_{1}\right\}}$.

Interestingly, the position of these same levels proves to be strongly dependent on the amplitude strength in the $\pi \omega\left\{{ }^{3} P_{0}\right\}$ and $\pi \omega\left\{{ }^{3} P_{2}\right\}$ partial-waves, so the lattice computed energies allow us to confidently limit the amplitude of these $P$-waves to be very small in this energy region. This is expected given the $P$-wave threshold suppression and lack of $J^{P C}=0^{+-}, 2^{+-}$resonances in this energy region. Figures 5.14 and 5.15 show the analogue of Figure 5.13 but for varying $\pi \omega\left\{{ }^{3} P_{0}\right\}$ and $\pi \omega\left\{{ }^{3} P_{2}\right\}$ channel parameters respectively. In these two cases, the reference amplitude in Eq. 5.16 is modified, as described in Section 5.7.1, to include a constant $\gamma$-term in the $K$-matrix for channels $\pi \omega\left\{{ }^{3} P_{0}\right\}$ and $\pi \omega\left\{{ }^{3} P_{2}\right\}$. 

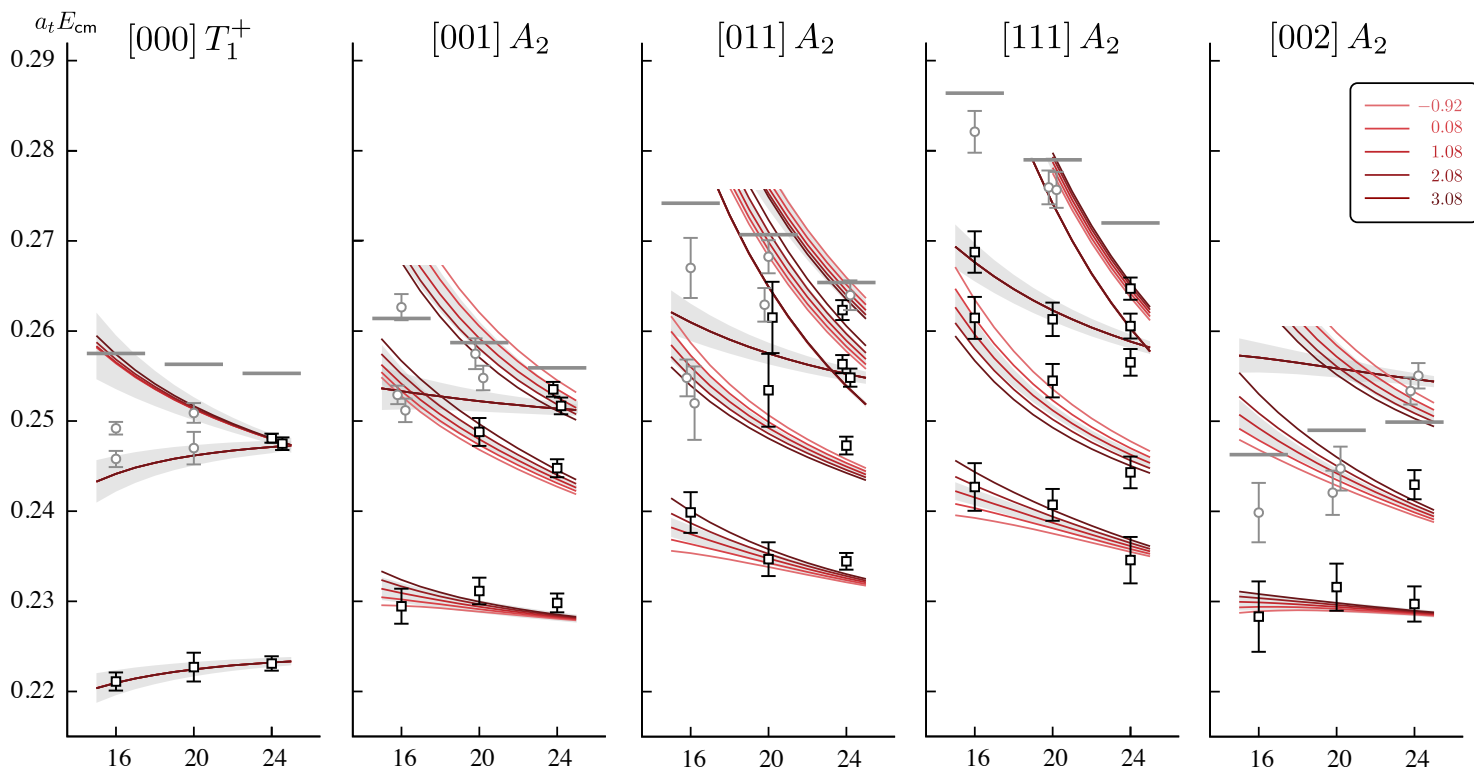

Figure 5.13: Sensitivity of the finite-volume spectra to $g_{\pi \omega\left\{{ }^{3} D_{1}\right\}}$. Lighter to darker red curves reflect smaller to larger values of $g_{\pi \omega\left\{{ }^{3} D_{1}\right\}}$ as shown in the key. The central curves correspond to $g_{\pi \omega\left\{{ }^{3} D_{1}\right\}}=1.08$, i.e. the mean value in the reference amplitude in Eq. 5.16 . The grey bands reflect the combined statistical and systematic uncertainties of Eq. 5.16 . The horizontal axes are in units of $L / a_{s}$.

\subsubsection{Three-body channels}

For the light-quark masses used in this calculation, the resonant behaviour is found to occur between the relatively low-lying $\pi \pi \eta$ threshold and the somewhat higher-lying $\pi K \bar{K}$ threshold. As such, we might worry that these channels could have a significant impact on the physics in this region. We previously saw that in Figure 5.4 there appeared to be deviations in the finite-volume spectra depending on whether or not three-meson operators were included in the bases, most notably on the smallest volume. As a precaution, we ensured that we only made use of those energy levels which lie below the lowest $E_{\text {n.i. }}^{(2+1)}$ value and which show no significant dependence on the presence/absence of $\rho \eta, K^{*} \bar{K}$ or $a_{0} \pi$ operators.

In this section, we attempt to quantify the size of possible contributions from the threebody sector on our scattering amplitudes and resonance pole by treating the scattering system as though $\pi \pi$ in $\pi \pi \eta$ can be completely replaced by a stable $\rho$ with a fixed mass 

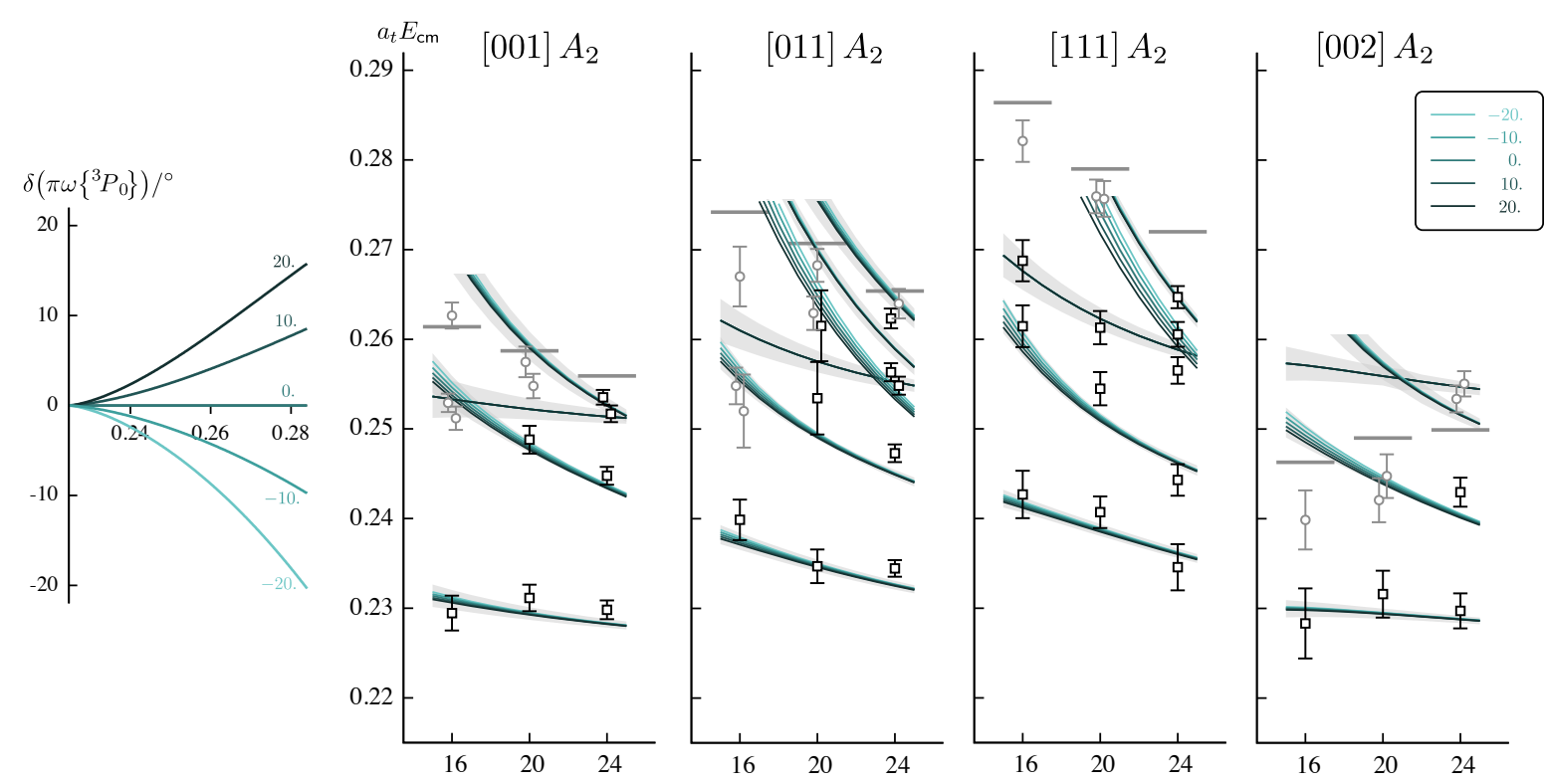

Figure 5.14: As Figure 5.13 but for varying $\gamma_{\pi \omega\left\{{ }^{3} P_{0}\right\}, \pi \omega\left\{{ }^{3} P_{0}\right\}}^{(0)}$. The central curves corresponds to $\gamma_{\pi \omega\left\{{ }^{3} P_{0}\right\}, \pi \omega\left\{{ }^{3} P_{0}\right\}}^{(0)}=0$. The phase-shifts on the left reflect the strengths of the $\pi \omega\left\{{ }^{3} P_{0}\right\}$ amplitudes.

$a_{t} m_{\rho}=0.1509(2)$, (the pole mass obtained from the $\pi \pi$ isospin- 1 scattering amplitudes in Ref. [30]) and $\pi K$ in $\pi K \bar{K}$ can be completely replaced by a stable $K^{*}$ with $a_{t} m_{K^{*}}=$ $0.1648(1)$ (the bound-state pole mass in the $K^{*}$ scattering analysis presented in Ref. [66]). In this way we augment our scattering matrix with two extra channels $\rho \eta\left\{{ }^{3} S_{1}\right\}$ and $K^{*} \bar{K}\left\{{ }^{3} S_{1}\right\}$.

This approach cannot be expected to completely describe the finite-volume spectra because, for example, whenever the $\rho$ has non-zero momentum, we expect there to be more than one corresponding energy level, as indicated by Figure 1 in Ref. [30]. A model where the $\rho$ is treated as a stable particle cannot capture this and will not even give the right number of energy levels in the 'three-body' spectrum. However, at the light-quark masses and lattice volumes used in this calculation, the nearest non-interacting $\pi \pi$ energy in the rest-frame $\rho$ spectra is much higher than $a_{t} E_{\mathrm{cm}}=0.1509$, as shown in Figure 1 of Ref. [30], and there is effectively only one finite-volume level which lies very close to the $\rho$ resonance mass. In this case, provided we only approximate $\pi \pi \eta$-like levels where the corresponding $\pi \pi$ subsystem is overall at rest, the stable $\rho$ may be a reasonable first 

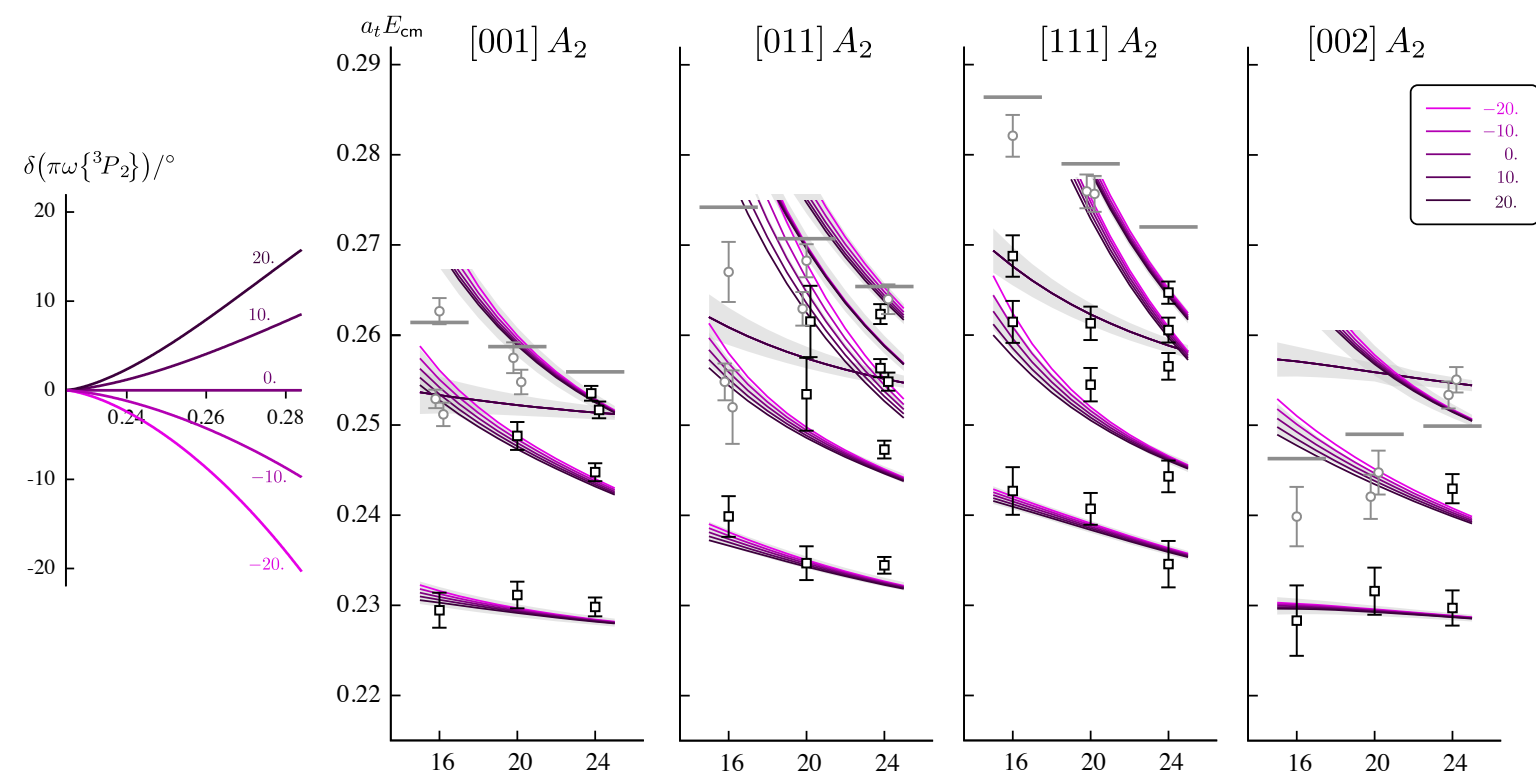

Figure 5.15: As Figure 5.14 but for varying $\gamma_{\pi \omega\left\{{ }^{3} P_{2}\right\}, \pi \omega\left\{{ }^{3} P_{2}\right\}}^{(0)}$.

approximation to the true three-body physics.

For $[000] T_{1}^{+}$, the relevant low-lying three-meson like operators are of the form $\rho_{000} \eta_{000}$ and $K_{000}^{*} \bar{K}_{000}$ as shown in Table 5.3 . We will therefore restrict our analysis to include the 36 energy levels with which we used to constrain the amplitudes in Section 5.5.3 in addition to the remaining energies in $[000] T_{1}^{+}$shown in Figure 5.4 , giving a total of 48 levels to constrain five coupled channels. Taking the reference amplitude in Eq. 5.16, modified to include a 'pole plus constant' term in $\rho \eta\left\{{ }^{3} S_{1}\right\}$ and $K^{*} \bar{K}\left\{{ }^{3} S_{1}\right\}$, we find best-fit parameters,

$$
\begin{aligned}
& m=(0.2485 \pm 0.0008) \cdot a_{t}^{-1} \\
& \gamma_{\pi \omega\left\{{ }^{\beta} S_{1}\right\}, \pi \omega\left\{{ }^{3} S_{1}\right\}}^{(0)}=-0.52 \pm 0.16 \\
& g_{\pi \omega\left\{{ }^{3} S_{1}\right\}}=(0.14 \pm 0.01) \cdot a_{t}^{-1} \\
& \gamma_{\left.\left.\pi \phi{ }^{3} S_{1}\right\}, \pi \phi{ }^{3} S_{1}\right\}}^{(0)}=0.64 \pm 0.17 \\
& g_{\pi \omega\left\{{ }^{\beta} D_{1}\right\}}=(1.8 \pm 0.5) \cdot a_{t} \\
& \gamma_{\rho \eta\left\{{ }^{3} S_{1}\right\}, \rho \eta\left\{{ }^{3} S_{1}\right\}}^{(0)}=-1.82 \pm 0.13 \\
& g_{\rho \eta\left\{S_{1}\right\}}=(0.0 \pm 0.1) \cdot a_{t}^{-1} \\
& \gamma_{K^{*} \bar{K}\left\{{ }^{\beta} S_{1}\right\}, K^{*} \bar{K}\left\{{ }^{3} S_{1}\right\}}^{(0)}=1.27 \pm 0.52 \\
& g_{K^{*} \bar{K}\left\{{ }^{3} S_{1}\right\}}=(0.20 \pm 0.01) \cdot a_{t}^{-1} \\
& \chi^{2} / N_{\text {dof }}=\frac{46.6}{48-9}=1.19 .
\end{aligned}
$$

The $\pi \omega\left\{{ }^{3} S_{1}\right\}, \pi \omega\left\{{ }^{3} D_{1}\right\}$ and $\pi \phi\left\{{ }^{3} S_{1}\right\}$ parameters are in reasonable agreement with those found for the reference amplitude in Eq. 5.16. We show in Figure 5.16 the finite-volume 

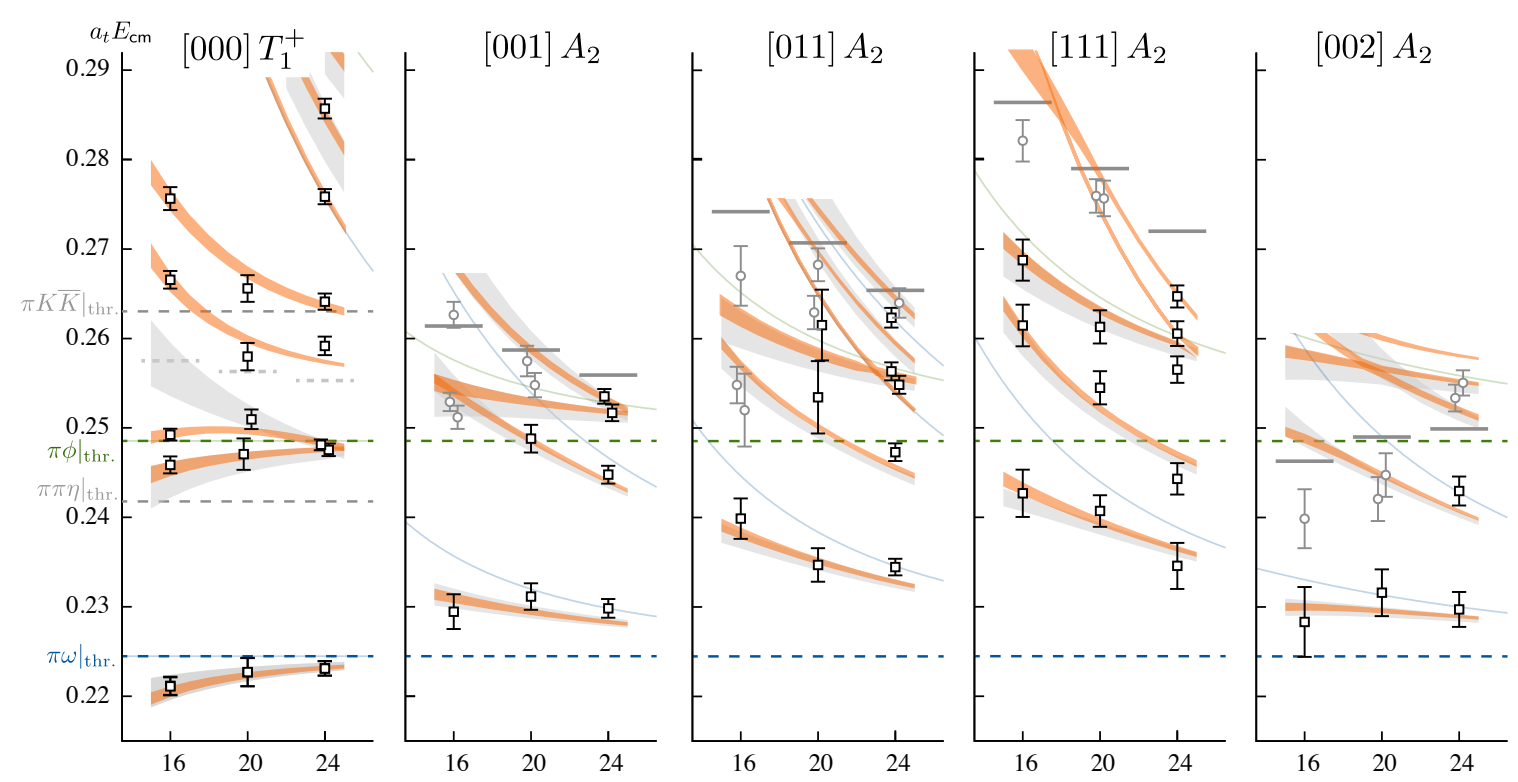

Figure 5.16: As Figure 5.11 but for the amplitude in Eq. 5.21. Orange bands reflect only the statistical uncertainty on the scattering parameters. The grey bands are transcribed from Figure 5.11.

spectra calculated through Eq. 2.53, analogous to Figure 5.11. We observe that the dependence of the finite-volume energy levels in moving-frame irreps, lying below the lowest $E_{\text {n.i. }}^{(2+1)}$, on the new 'three-body' part of the amplitude is very slight. However, there is improved agreement in [000] $T_{1}^{+}$where the previously excluded levels, in particular on the $\left(L / a_{s}\right)=16$ volume, are now described quite well. We argue that this shows our original selection criteria, giving the 36 energy levels across all irreps, is sound and leads to a robust determination of the scattering $\boldsymbol{t}$-matrix.

For this limited five coupled-channel analysis of $\pi \omega\left\{{ }^{3} S_{1}\right\}, \pi \omega\left\{{ }^{3} D_{1}\right\}, \pi \phi\left\{{ }^{3} S_{1}\right\}, \rho \eta\left\{{ }^{3} S_{1}\right\}$ and $K^{*} \bar{K}\left\{{ }^{3} S_{1}\right\}$, utilising the generalised $n$-channel Stapp-parameterisation presented in Chapter 7, the five phase-shifts and three non-zero mixing-angles are presented in Figure 5.17. We find that seven of the mixing-angles, all featuring either $\pi \phi\left\{{ }^{3} S_{1}\right\}$ and/or $\rho \eta\left\{{ }^{3} S_{1}\right\}$, are extremely small and consistent with zero. This illustrates the natural reduction from the five-channel parameterisation to the three-channel parameterisation in the case that two channels decouple.

As a final test of the effects of the $\rho \eta\left\{{ }^{3} S_{1}\right\}$ and $K^{*} \bar{K}\left\{{ }^{3} S_{1}\right\}$ channels, we find the resonance 
pole and corresponding couplings. There are 16 Riemann sheets and several 'mirror poles', but the closest pole is located at,

$$
a_{t} \sqrt{s_{0}}=0.2448(12)-\frac{i}{2} 0.0215(21)
$$

which agrees within uncertainties with the pole position found in Section 5.6. The corresponding couplings are,

$$
\begin{aligned}
a_{t} c\left(\pi \omega\left\{{ }^{3} S_{1}\right\}\right)_{\|} & =0.117(7) \exp [-i \pi 0.084(20)] \\
a_{t} c\left(\pi \omega\left\{{ }^{3} D_{1}\right\}\right)_{\|} & =0.016(4) \exp [-i \pi 0.182(22)] \\
a_{t} c\left(\rho \eta\left\{{ }^{3} S_{1}\right\}\right)_{\|} & =0.003(52) \\
a_{t} c\left(K^{*} \bar{K}\left\{{ }^{3} S_{1}\right\}\right)_{\|} & =0.166(8) \exp [-i \pi 0.043(12)],
\end{aligned}
$$

where we exclude the meaningless phase on $a_{t} c\left(\rho \eta\left\{{ }^{3} S_{1}\right\}\right)_{\text {II }}$ as the magnitude is consistent with zero and where $c\left(\pi \phi\left\{{ }^{3} S_{1}\right\}\right)_{\|}=0$ by choice of amplitude. This decoupling of the $\rho \eta\left\{{ }^{3} S_{1}\right\}$ and $\pi \phi\left\{{ }^{3} S_{1}\right\}$ channels was evident in the corresponding mixing-angles which were all found to be negligible and consistent with zero as discussed above. The coupling to $\rho \eta\left\{{ }^{3} S_{1}\right\}$ is small but has a large uncertainty, while the coupling to $K^{*} \bar{K}\left\{{ }^{3} S_{1}\right\}$ is larger. We might expect the $K^{*} \bar{K}$ coupling to be comparable to the $\pi \omega$ coupling because in an 'OZI rule' obeying framework they differ only in the flavour of $q \bar{q}$ pair creation needed to allow the resonance to decay.

We conclude that although we cannot currently rigorously handle three-body contributions due to $\pi \pi \eta$ and $\pi K \bar{K}$, we do not see any evidence to suggest that they significantly affect the results reported here. 

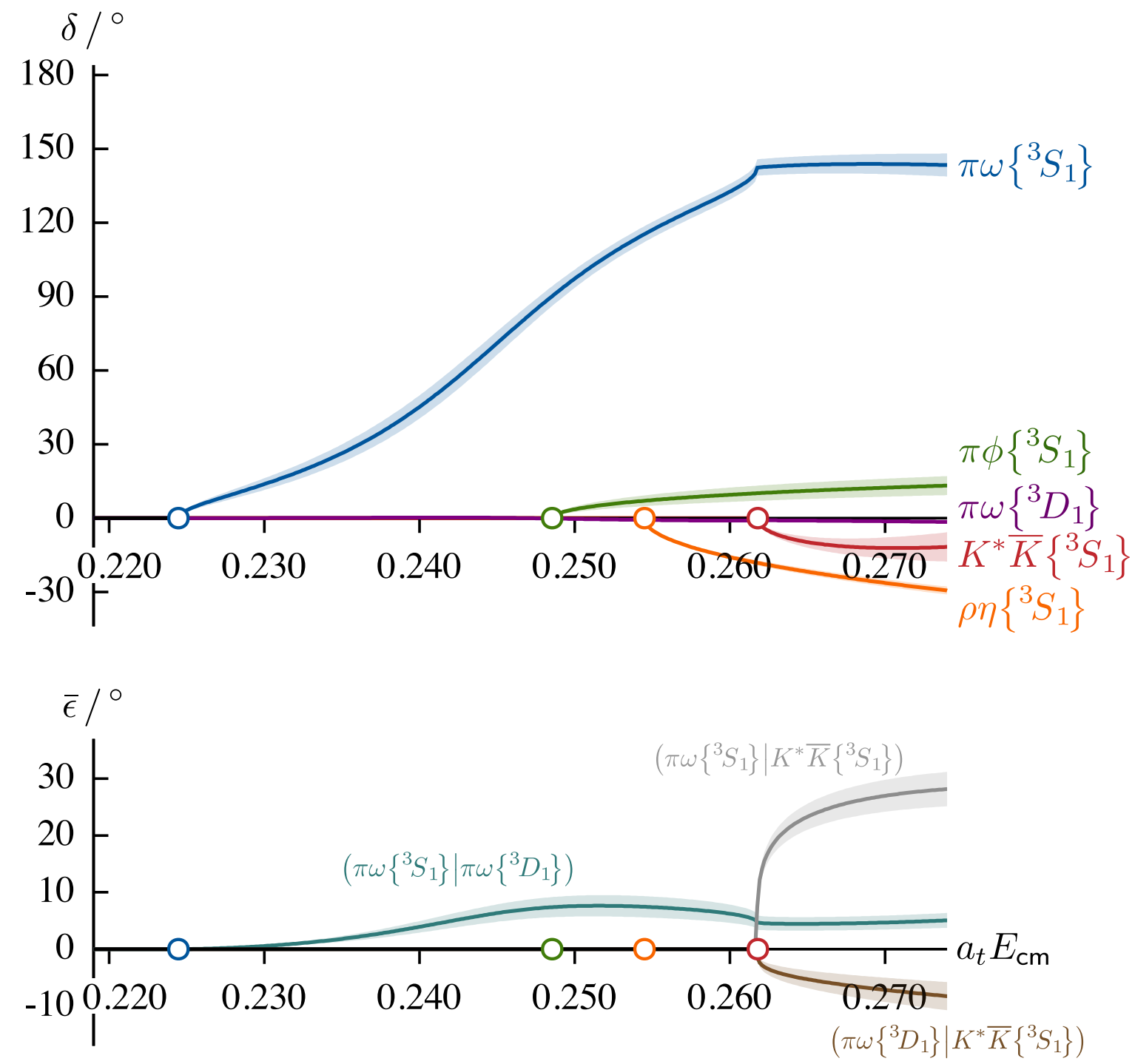

Figure 5.17: Upper: As in Figure 5.9 but for the $\pi \omega\left\{{ }^{3} S_{1}\right\}$ (blue), $\pi \omega\left\{{ }^{3} D_{1}\right\}$ (purple), $\pi \phi\left\{{ }^{3} S_{1}\right\}$ (green), $\rho \eta\left\{{ }^{3} S_{1}\right\}$ (orange) and $K^{*} \bar{K}\left\{{ }^{3} S_{1}\right\}$ (red) phase-shifts for the reference amplitude in Eq. 5.21. The faded error bands reflect the statistical uncertainty on the scattering parameters. The $\rho \eta$ and $K^{*} \bar{K}$ "thresholds" are calculated using the $\rho$ and $K^{*}$ masses given above. Lower: As upper but for the mixing-angles $\bar{\epsilon}\left(\pi \omega\left\{{ }^{3} S_{1}\right\} \mid \pi \omega\left\{{ }^{3} D_{1}\right\}\right)$ (blue), $\bar{\epsilon}\left(\pi \omega\left\{{ }^{3} S_{1}\right\} \mid K^{*} \bar{K}\left\{{ }^{3} S_{1}\right\}\right)$ (grey) and $\bar{\epsilon}\left(\pi \omega\left\{{ }^{3} D_{1}\right\} \mid K^{*} \bar{K}\left\{{ }^{3} S_{1}\right\}\right)$ (brown). All other mixing-angles are extremely small and consistent with zero as discussed in the text. 


\subsection{Interpretation}

All $J^{P}=1^{+}$amplitude parameterisations used that prove to be capable of describing the finite-volume spectra in the energy region we are considering, had the same characteristic resonant bump in the $\pi \omega\left\{{ }^{3} S_{1}\right\}$ to $\pi \omega\left\{{ }^{3} S_{1}\right\}$ amplitude squared, with relatively small enhancements in the diagonal $\pi \omega\left\{{ }^{3} D_{1}\right\}$ and $\pi \phi\left\{{ }^{3} S_{1}\right\}$ elements. In the off-diagonal amplitudes, the $\pi \omega\left\{{ }^{3} S_{1}\right\}$ to $\pi \omega\left\{{ }^{3} D_{1}\right\}$ amplitude was found to be small but positive. The remaining off-diagonal elements were all found to be extremely small and consistent with zero. In each of the $J^{P}=1^{+}$amplitudes, we found a complex conjugate pair of poles on sheets II and III, which we interpret as the effect of a single resonance.

To quote results in physical units, we choose to set the scale using the $\Omega$-baryon mass measured on these lattices, $a_{t} m_{\Omega}=0.2951$ [85], and the physical $\Omega$-baryon mass, $m_{\Omega}^{\text {phys }}=1672.45(29) \mathrm{MeV}[5]$. This gives $a_{t}^{-1}=m_{\Omega}^{\text {phys }} /\left(a_{t} m_{\Omega}\right)=5666 \mathrm{MeV}$ and stable hadron masses $m_{\pi} \approx 391 \mathrm{MeV}, m_{K} \approx 549 \mathrm{MeV}, m_{\eta} \approx 587 \mathrm{MeV}, m_{\omega} \approx 881 \mathrm{MeV}$ and $m_{\phi} \approx 1017 \mathrm{MeV}$.

Using this scale setting, we summarise the scattering amplitudes resulting from the $J^{P}=1^{+}$amplitude analysis in Figure 5.18, expressing all quantities in physical units. We find a $b_{1}$ resonant pole of mass $m_{R}=1382(15) \mathrm{MeV}$ and width $\Gamma_{R}=91(31) \mathrm{MeV}$, where the uncertainties are a conservative estimate from a combination of statistical and systematic uncertainties and encompass variation over different parameterisations. Similarly, we find for the couplings,

$$
\begin{aligned}
\left|c_{\pi \omega\left\{S_{1}\right\}}\right| & =564(114) \mathrm{MeV} \\
\left|c_{\pi \omega\left\{D_{1}\right\}}\right| & =81(56) \mathrm{MeV} \\
\left|c_{\pi \phi\left\{S_{1}\right\}}\right| & =59(41) \mathrm{MeV} .
\end{aligned}
$$

In Figure 5.19, we plot the position of the pole found in this calculation compared to the experimental $b_{1}$ resonance, with mass $m_{b_{1}}=1230(3) \mathrm{MeV}$ and width $\Gamma_{b_{1}}=142(9) \mathrm{MeV}[5]$, and a lattice calculation at the $\mathrm{SU}(3)_{\mathrm{F}}$ point with $m_{\pi} \approx 700 \mathrm{MeV}[29]$.

In the latter calculation, the $b_{1}$ forms part of an axial-vector octet, the $\left(\boldsymbol{h}_{\mathbf{1}}\right)_{\mathbf{8}}$ following the notation in Chapter 3, with a mass around $1525 \mathrm{MeV}$. The pseudoscalar-vector threshold $(\boldsymbol{\eta})_{\mathbf{8}}(\boldsymbol{\omega})_{\mathbf{8}}$, corresponding to $\pi \omega$, is at roughly $1695 \mathrm{MeV}$, and thus the $b_{1}$ is 
stable at this pion mass. We observe that the trajectory of the pole with varying pion mass appears to be similar to that of the $\rho$ and $K^{*}$ mesons shown in Ref. [4] and [83] respectively, as may be expected for a reasonably narrow resonance.

Since we find the $b_{1}$ to be a narrow resonance a moderate distance above $\pi \omega$ threshold, it is reasonable to compute theoretical 'branching fractions' for its decay to $\pi \omega$. For channels $a\left\{{ }^{3} \ell_{J}\right\}$ these are given by [5],

$$
\operatorname{Br}\left(R \rightarrow a\left\{{ }^{3} \ell_{J}\right\}\right) \equiv \frac{1}{\Gamma_{R}} \cdot \frac{\left|c_{\ell J a}\right|^{2}}{m_{R}} \rho_{a}\left(m_{R}\right)
$$

As mentioned in Ref. [7], the sum of these partial branching fractions does not necessarily give unity. Using this definition, we obtain,

$$
\begin{aligned}
\operatorname{Br}\left(b_{1} \rightarrow \pi \omega\left\{{ }^{3} S_{1}\right\}\right) & \sim 93 \% \\
\operatorname{Br}\left(b_{1} \rightarrow \pi \omega\left\{{ }^{3} D_{1}\right\}\right) & \sim 2 \%,
\end{aligned}
$$

and observe the $\pi \phi\left\{{ }^{3} S_{1}\right\}$ branching fraction is zero as the channel is kinematically closed $\left(m_{R}<m_{\pi}+m_{\phi}\right)$.

A crude extrapolation of the couplings to the physical value of the light quark masses comes if we assume them to be independent of the light quark masses once the threshold behaviour is removed. This is not guaranteed, but has been observed in lattice calculations of the $\rho$ [4, 86 95$]$ and $K^{*}$ 83, 89, 96, 98] couplings at various values of $m_{\pi}$. Considering the ratio,

$$
\left|\frac{c_{\pi \omega\left\{{ }^{3} \ell_{J}\right\}}^{\text {phys. }}}{\left(k_{\pi \omega}^{\text {phys. }}\right)^{\ell}}\right|=\left|\frac{c_{\pi \omega\left\{{ }^{3} \ell_{J}\right\}}}{\left(k_{\pi \omega}\right)^{\ell}}\right|,
$$

where the $\mathrm{cm}$-frame momentum is evaluated at the resonance pole position, and where we use the values presented above on the right-hand side, and the experimental $b_{1}$ mass to compute $k_{\pi \omega}^{\text {phys. }}$, gives a prediction of $\left|c_{\pi \omega\left\{{ }^{3} D_{1}\right\}}^{\text {phys. }}\right|=146(101) \mathrm{MeV}$. Subsequently, we obtain an estimate for the ratio of couplings at the physical pion mass of,

$$
\left|\frac{c_{\pi \omega\left\{{ }^{3} D_{1}\right\}}^{\text {phys. }}}{c_{\pi \omega\left\{{ }^{3} S_{1}\right\}}^{\text {phys. }}}\right|=0.27(20) .
$$

The PDG [5] reports a ratio of $D$-wave to $S$-wave amplitudes for the $b_{1}$ resonance of magnitude $0.277(27)$, which is not computed at the complex pole position and therefore not precisely the same quantity as we quote. 

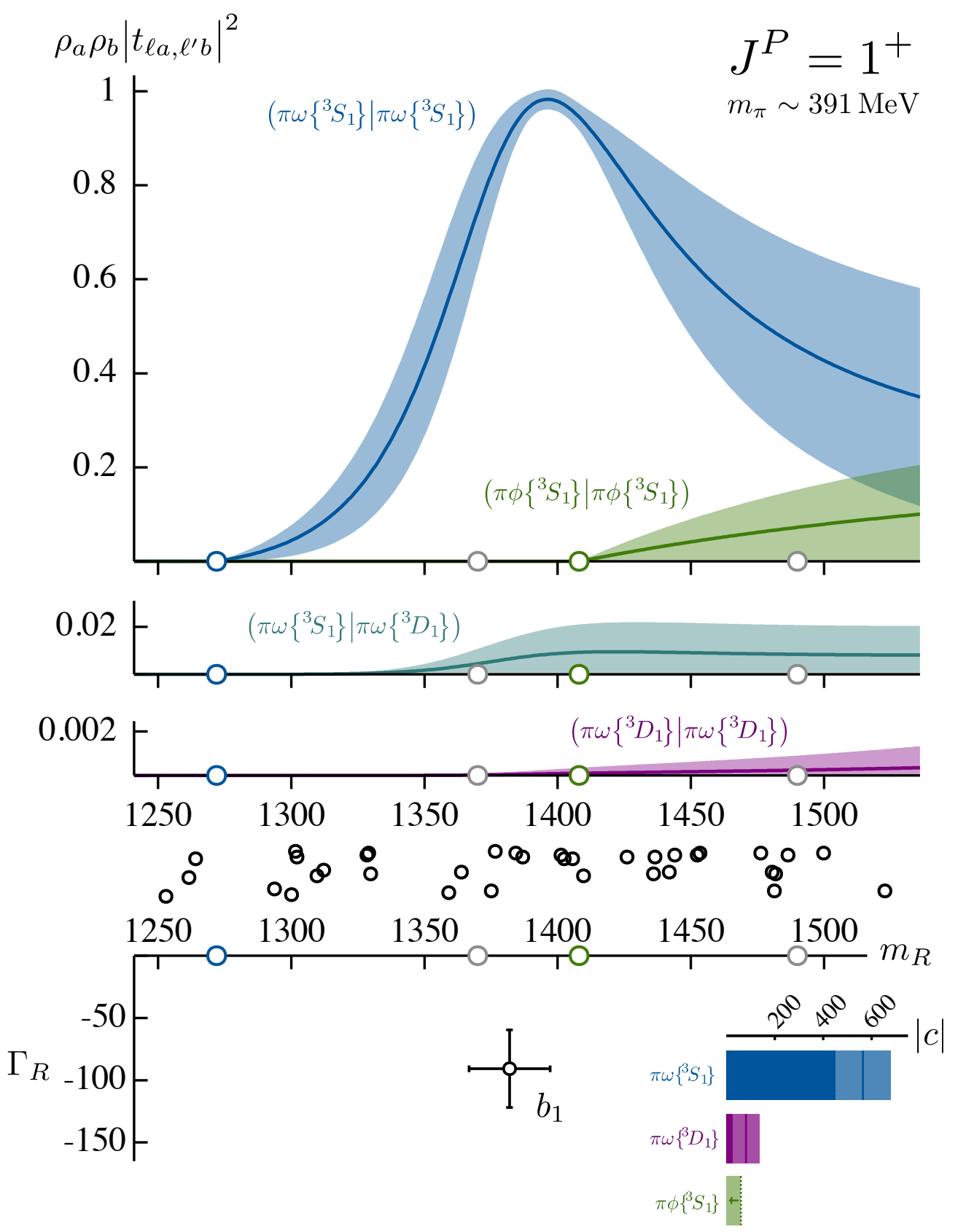

Figure 5.18: Top: The scattering amplitudes-squared, $\rho_{a} \rho_{b}\left|t_{\ell J a, \ell^{\prime} J b}\right|^{2}$, transcribed from Figure 5.10 with the energy axis converted to physical units. Below the amplitudes are the energy levels used to constrain the amplitudes (black points). Bottom: The best estimate of the resonant pole position, where uncertainties combine statistical and systematic uncertainties with variations across parameterisations. The histograms show the best estimate of the magnitude of each coupling with the lightly-shaded region reflecting the combined uncertainties. The $\pi \phi\left\{{ }^{3} S_{1}\right\}$ coupling is an estimate of the upper bound. 


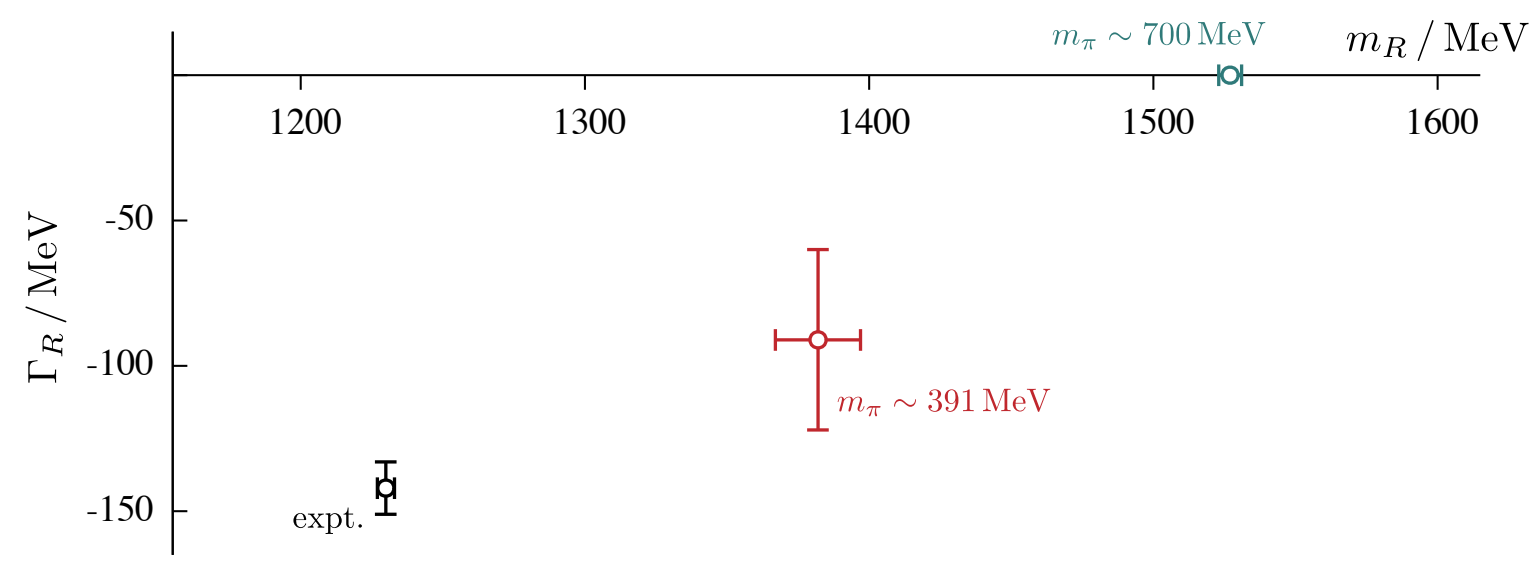

Figure 5.19: The $b_{1}$ pole position for various pion masses. Blue shows the ground-state mass of the axial-vector octet from a lattice calculation with $m_{\pi} \approx 700 \mathrm{MeV}$ [4], red shows the estimate from this work with $m_{\pi} \approx 391 \mathrm{MeV}$ and black is the experimentally determined mass and width of the $b_{1}$ resonance [5]. 


\subsection{Summary}

In this chapter we have presented, for the first time in lattice QCD, a calculation of coupled $\pi \omega, \pi \phi$ scattering. Analysis of the obtained finite-volume spectra allowed us to calculate the scattering amplitudes, including the effects of dynamically-coupled $\pi \omega\left\{{ }^{3} S_{1}\right\}$ and $\pi \omega\left\{{ }^{3} D_{1}\right\}$ and resolve the mixing-angle between these two channels. A clear $b_{1}$ resonance was observed, visible as a rapid increase in the $\pi \omega\left\{{ }^{3} S_{1}\right\}$ phase-shift through $90^{\circ}$ or correspondingly as a bump in the magnitude of the $\pi \omega\left\{{ }^{3} S_{1}\right\} \rightarrow \pi \omega\left\{{ }^{3} S_{1}\right\} \boldsymbol{t}$-matrix element. More rigorously, we found pole singularities on unphysical Riemann sheets relatively close to the real energy axis with couplings that are large for the $\pi \omega\left\{{ }^{3} S_{1}\right\}$ final state, significantly smaller for $\pi \omega\left\{{ }^{3} D_{1}\right\}$ and compatible with zero for $\pi \phi$. The mass and width of the $b_{1}$ resonance found in this calculation, with light-quark masses such that $m_{\pi} \approx 391 \mathrm{MeV}$, appear to be compatible with a smooth interpolation between a stable state for much larger quark mass, and the experimental resonance at lower quark mass.

We have demonstrated the effectiveness of three-meson operators, constructed by utilising earlier calculations of meson-meson scattering channels [30,62, 66], in interpolating energy levels closely associated with isobar enhancements in the three-meson system. For the first time in a lattice calculation, we have demonstrated how a failure to incorporate such operators into the basis leads to incorrect finite-volume spectra. We explored the role of three-body channels in the scattering system, circumventing the current lack of a complete three-body formalism by treating the $\rho$ and $K^{*}$ as stable in a limited study, and found they had a negligible effect in this particular case of a low-lying $b_{1}$ resonance.

In performing this systematic test, we performed for the first time in a lattice QCD calculation a five coupled-channel scattering analysis, $\pi \omega\left\{{ }^{3} S_{1}\right\}, \pi \omega\left\{{ }^{3} D_{1}\right\}, \pi \phi\left\{{ }^{3} S_{1}\right\}, \rho \eta\left\{{ }^{3} S_{1}\right\}$, $K^{*} \bar{K}\left\{{ }^{3} S_{1}\right\}$, presenting the phase-shifts and mixing-angles using a parameterisation that extends that of the conventional two-channel Stapp-parameterisation to accommodate any number of channels.

Furthermore, observations were made of how particular finite-volume energy levels depend upon the various partial-waves which 'mix' due to the cubic nature of the lattice boundary and also upon the strength of the ${ }^{3} D_{1}$-wave coupling to the resonance pole both demonstrating our ability to constrain the amplitudes as claimed and as a useful reference for future calculations. 
As expected, no $I^{G}=1^{+}$resonances are observed with a mass comparable to the $b_{1}$ in $J^{P}=0^{-}, 2^{-}$channels. Notably, no resonating behaviour is observed in the largely decoupled $\pi \phi$ channel, suggesting the absence of a $Z_{s}$ which might be proposed as an analogue of the $Z_{c}$ seen in $\pi J / \psi$.

This work has advanced lattice techniques for studying coupled-channel scattering involving hadrons with non-zero spin and operators which effectively interpolate three hadrons. Looking forward, once a three-hadron scattering formalism is practical to use, a future calculation would enable the rigorous determination of the $\pi \pi \eta$ and $\pi K \bar{K}$ scattering amplitudes. Furthermore, utilising such a formalism would allow the calculation of the $G$-parity-negative axial-vector, the $a_{1}$, which would make for an interesting comparison. Moving on from the simplest low-lying resonances, and as the light-quark mass approaches its physical value, it becomes more important to reliably determine such three-hadron scattering processes. 


\section{APPENDICES}

\section{A Operator Tables}

We present here tables of operators as referred to in the text.

\begin{tabular}{|c|c|c|c|}
\hline & $L / a_{s}=16$ & $L / a_{s}=20$ & $L / a_{s}=24$ \\
\hline \multirow{2}{*}{$\rho_{[000], T_{1}^{-}}$} & $26 \times \bar{\psi} \boldsymbol{\Gamma} \psi$ & $26 \times \bar{\psi} \boldsymbol{\Gamma} \psi$ & $12 \times \bar{\psi} \boldsymbol{\Gamma} \psi$ \\
\hline & $3 \times \pi \pi$ & $2 \times \pi \pi$ & \\
\hline \multirow{2}{*}{$\rho_{[001], A_{1}}$} & $8 \times \bar{\psi} \boldsymbol{\Gamma} \psi$ & $18 \times \bar{\psi} \boldsymbol{\Gamma} \psi$ & $18 \times \bar{\psi} \boldsymbol{\Gamma} \psi$ \\
\hline & $4 \times \pi \pi$ & $4 \times \pi \pi$ & $4 \times \pi \pi$ \\
\hline \multirow{2}{*}{$\rho_{[011], A_{1}}$} & $27 \times \bar{\psi} \boldsymbol{\Gamma} \psi$ & $\overline{27 \times \bar{\psi} \boldsymbol{\Gamma} \psi}$ & $27 \times \bar{\psi} \boldsymbol{\Gamma} \psi$ \\
\hline & $3 \times \pi \pi$ & $3 \times \pi \pi$ & $3 \times \pi \pi$ \\
\hline \multirow{2}{*}{$\rho_{[111], A_{1}}$} & $8 \times \bar{\psi} \boldsymbol{\Gamma} \psi$ & $21 \times \bar{\psi} \boldsymbol{\Gamma} \psi$ & $\begin{array}{l}21 \times \bar{\psi} \boldsymbol{\Gamma} \psi\end{array}$ \\
\hline & $3 \times \pi \pi$ & $3 \times \pi \pi$ & $3 \times \pi \pi$ \\
\hline
\end{tabular}

Table 5.5: Single-meson and two-meson operators used to compute optimised $\rho$ operators in the $[000] T_{1}^{-}$irrep and $\vec{P} A_{1}$ irreps at various overall momenta on the three volumes. Momentum labels on the $\pi$ 's that form the $\pi \pi$ operators are omitted for brevity. 


\begin{tabular}{|c|c|c|}
\hline & $L / a_{s}=16$ & $L / a_{s}=20$ \\
\hline \multirow{3}{*}{$a_{0[001], A_{1}}$} & $14 \times \bar{\psi} \boldsymbol{\Gamma} \psi$ & $14 \times \bar{\psi} \boldsymbol{\Gamma} \psi$ \\
\hline & $4 \times \pi \eta$ & $4 \times \pi \eta$ \\
\hline & $2 \times \bar{K} K$ & $2 \times \bar{K} K$ \\
\hline \multirow[b]{2}{*}{$a_{0[011], A_{1}}$} & $18 \times \bar{\psi} \boldsymbol{\Gamma} \psi$ & $18 \times \bar{\psi} \boldsymbol{\Gamma} \psi$ \\
\hline & $4 \times \pi \eta$ & $4 \times \pi \eta$ \\
\hline \multicolumn{3}{|c|}{ 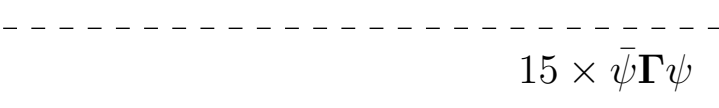 } \\
\hline \multirow[t]{2}{*}{$a_{0[111], A_{1}}$} & & $4 \times \pi \eta$ \\
\hline & & $2 \times \bar{K} K$ \\
\hline
\end{tabular}

Table 5.6: As Table 5.5 but for optimised $a_{0}$ operators.

\begin{tabular}{|c|c|c|c|}
\hline & $L / a_{s}=16$ & $L / a_{s}=20$ & $L / a_{s}=24$ \\
\hline$K_{[000], T_{1}^{-}}^{*}$ & $6 \times \bar{\psi} \boldsymbol{\Gamma} \psi$ & $16 \times \bar{\psi} \boldsymbol{\Gamma} \psi$ & $9 \times \bar{\psi} \boldsymbol{\Gamma} \psi$ \\
\hline$K_{[001], A_{1}}^{*}$ & $\begin{array}{r}8 \times \bar{\psi} \boldsymbol{\Gamma} \psi \\
2 \times \pi K\end{array}$ & $\begin{array}{r}16 \times \bar{\psi} \boldsymbol{\Gamma} \psi \\
6 \times \pi K\end{array}$ & $8 \times \bar{\psi} \mathbf{\Gamma} \psi$ \\
\hline$K_{[011], A_{1}}^{*}$ & $\begin{array}{r}8 \times \bar{\psi} \boldsymbol{\Gamma} \psi \\
3 \times \pi K\end{array}$ & $\begin{array}{r}26 \times \bar{\psi} \boldsymbol{\Gamma} \psi \\
\quad 6 \times \pi K\end{array}$ & \\
\hline$K_{[111], A_{1}}^{*}$ & $\begin{array}{r}8 \times \bar{\psi} \boldsymbol{\Gamma} \psi \\
4 \times \pi K\end{array}$ & $\begin{array}{c}9 \times \bar{\psi} \boldsymbol{\Gamma} \psi \\
4 \times \pi K\end{array}$ & $9 \times \bar{\psi} \boldsymbol{\Gamma} \psi$ \\
\hline
\end{tabular}

Table 5.7: As Table 5.5 but for optimised $K^{*}$ operators. 


\begin{tabular}{ccc}
\hline$L / a_{s}=16$ & $L / a_{s}=20$ & $L / a_{s}=24$ \\
\hline $12 \times \bar{\psi} \boldsymbol{\Gamma} \psi$ & $12 \times \bar{\psi} \boldsymbol{\Gamma} \psi$ & $12 \times \bar{\psi} \boldsymbol{\Gamma} \psi$ \\
$-\pi_{[000]} \omega_{[001]}$ & $\pi_{[000]} \omega_{[001]}$ & $\pi_{[000]} \omega_{[001]}$ \\
$\pi_{[000]} \phi_{[001]}$ & $\pi_{[001]} \omega_{[000]}$ & $\pi_{[001]} \omega_{[000]}$ \\
$\rho_{[001]} \eta_{[000]}$ & $\pi_{[000]} \phi_{[001]}$ & $\pi_{[000]} \phi_{[001]}$ \\
$a_{0[001]} \pi_{[000]}$ & $\rho_{[001]} \eta_{[000]}$ & $\rho_{[001]} \eta_{[000]}$ \\
$\pi_{[001]} \omega_{[000]}$ & $a_{0[001]} \pi_{[000]}$ & $K_{[001]}^{*} \bar{K}_{[000]}$ \\
$K_{[001]}^{*} \bar{K}_{[000]}$ & $K_{[001]}^{*} \bar{K}_{[000]}$ & $\rho_{[001]}^{1} \eta_{[000]}$ \\
$\rho_{[000]} \eta_{[001]}$ & & $\rho_{[000]} \eta_{[001]}$ \\
$\pi_{[001]} \phi_{[000]}$ & & $\pi_{[001]} \phi_{[000]}$ \\
& & $K_{[000]}^{*} \bar{K}_{[001]}$ \\
& & $\{2\} \pi_{[001]} \omega_{[011]}$ \\
& & $\{2\} \pi_{[011]} \omega_{[001]}$ \\
\hline
\end{tabular}

Table 5.8: As in Table 5.3 but for irrep [001] $A_{2}$. For operators $\mathcal{O}_{\mathbb{R M}}^{\dagger}$, the superscript $\mathfrak{n}$ on $\mathbb{R}^{\mathfrak{n}}$ denotes the $\mathfrak{n}^{\text {th }}$ excited state when $\mathfrak{n} \geq 1$. All $\rho$ and $K^{*}$ operators transform in $[000] T_{1}^{-}$at $\vec{p}=\overrightarrow{0}$ and all $\rho, a_{0}$ and $K^{*}$ operators transform in $\vec{P} A_{1}$ for $\vec{p} \neq \overrightarrow{0}$. Operators shown in gray correspond to $E_{\text {n.i. }}^{(2+1)}$ greater than the $E_{\text {n.i. }}^{(2+1)}$ or $E_{\text {n.i. }}^{(3)}$ of operators that have not been included in the basis. 


\begin{tabular}{|c|c|c|}
\hline$L / a_{s}=16$ & $L / a_{s}=20$ & $L / a_{s}=24$ \\
\hline \multirow{2}{*}{\multicolumn{3}{|c|}{$\begin{array}{c}21 \times \bar{\psi} \boldsymbol{\Gamma} \psi \\
------\end{array}$}} \\
\hline & & \\
\hline$\pi_{[000]} \omega_{[011]}$ & $\pi_{[000]} \omega_{[011]}$ & $\pi_{[000]} \omega_{[011]}$ \\
\hline$\pi_{[000]} \phi_{[011]}$ & $\pi_{[000]} \phi_{[011]}$ & $\{2\} \pi_{[001]} \omega_{[001]}$ \\
\hline$\rho_{[011]} \eta_{[000]}$ & $\{2\} \pi_{[001]} \omega_{[001]}$ & $\pi_{[000]} \phi_{[011]}$ \\
\hline$K_{[011]}^{*} \bar{K}_{[000]}$ & $\rho_{[011]} \eta_{[000]}$ & $\pi_{[011]} \omega_{[000]}$ \\
\hline$\{2\} \pi_{[001]} \omega_{[001]}$ & $a_{0[011]} \pi_{[000]}$ & $\rho_{[011]} \eta_{[000]}$ \\
\hline$a_{0[011]} \pi_{[000]}$ & $K_{[011]}^{*} \bar{K}_{[000]}$ & \\
\hline$\pi_{[011]} \omega_{[000]}$ & & \\
\hline
\end{tabular}

Table 5.9: As in Table 5.8 but for irrep [011] $A_{2}$.

\begin{tabular}{rcc}
\hline$L / a_{s}=16$ & $L / a_{s}=20$ & $L / a_{s}=24$ \\
\hline $15 \times \bar{\psi} \boldsymbol{\Gamma} \psi$ & $15 \times \bar{\psi} \boldsymbol{\Gamma} \psi$ & $15 \times \bar{\psi} \boldsymbol{\Gamma} \psi$ \\
$\pi_{[000]} \omega_{[111]}$ & $\pi_{[000]} \omega_{[111]}$ & $\pi_{[000]} \omega_{[111]}$ \\
$\pi_{[000]} \phi_{[111]}$ & $\pi_{[000]} \phi_{[111]}$ & $\pi_{[000]} \phi_{[111]}$ \\
$\rho_{[111]} \eta_{[000]}$ & $\{2\} \pi_{[001]} \omega_{[011]}$ & $\{2\} \pi_{[001]} \omega_{[011]}$ \\
$K_{[111]}^{*} \bar{K}_{[000]}$ & $\rho_{[111]} \eta_{[000]}$ & $\{2\} \pi_{[011]} \omega_{[001]}$ \\
$\{2\} \pi_{[001]} \omega_{[011]}$ & $K_{[111]}^{*} \bar{K}_{[000]}$ & $\rho_{[111]} \eta_{[000]}$ \\
$\pi_{[111]} \omega_{[000]}$ & $a_{0[111]} \pi_{[000]}$ & $\pi_{[111]} \omega_{[000]}$ \\
$\{2\} \pi_{[011]} \omega_{[001]}$ & & $K_{[111]}^{*} \bar{K}_{[000]}$ \\
\hline
\end{tabular}

Table 5.10: As in Table 5.8 but for irrep [111] $A_{2}$. 


\begin{tabular}{ccc}
\hline$L / a_{s}=16$ & $L / a_{s}=20$ & $L / a_{s}=24$ \\
\hline $20 \times \bar{\psi} \boldsymbol{\Gamma} \psi$ & $20 \times \bar{\psi} \boldsymbol{\Gamma} \psi$ & $20 \times \bar{\psi} \boldsymbol{\Gamma} \psi$ \\
\hdashline$\pi_{[001]} \omega_{[001]}$ & $\pi_{[001]} \omega_{[001]}$ & $\pi_{[001]} \omega_{[001]}$ \\
$\rho_{[001]} \eta_{[001]}$ & $\rho_{[001]} \eta_{[001]}$ & $\pi_{[000]} \omega_{[002]}$ \\
$K_{[001]}^{*} \bar{K}_{[001]}$ & $\pi_{[000]} \omega_{[002]}$ & $\rho_{[001]} \eta_{[001]}$ \\
$\pi_{[000]} \omega_{[002]}$ & $\pi_{[001]} \phi_{[001]}$ & $\pi_{[001]} \phi_{[001]}$ \\
$\pi_{[001]} \phi_{[001]}$ & $K_{[001]}^{*} \bar{K}_{[001]}$ & $K_{[001]}^{*} \bar{K}_{[001]}$ \\
$\rho_{[001]}^{\perp} \eta_{[001]}$ & $a_{0[001]} \pi_{[001]}$ & $\pi_{[000]} \phi_{[002]}$ \\
\hline
\end{tabular}

Table 5.11: As in Table 5.8 but for irrep [002] $A_{2}$.

\begin{tabular}{cccc}
\hline$[000] T_{2}^{+}$ & {$[000] E^{-}$} & {$[001] B_{1}$} & {$[001] B_{2}$} \\
\hline $14 \times \bar{\psi} \boldsymbol{\Gamma} \psi$ & $12 \times \bar{\psi} \boldsymbol{\Gamma} \psi$ & $9 \times \bar{\psi} \boldsymbol{\Gamma} \psi$ & $9 \times \bar{\psi} \boldsymbol{\Gamma} \psi$ \\
\hline$\pi_{[001]} \omega_{[001]}$ & $\pi_{[001]} \omega_{[001]}$ & $\pi_{[011]} \pi_{[001]}$ & $\pi_{[111]} \pi_{[011]}$ \\
& $\bar{K}_{[011]} K_{[001]}$ & $\{2\} \pi_{[001]} \omega_{[011]}$ \\
& $\pi_{[001]} \omega_{[011]}$ & $\{2\} \pi_{[011]} \omega_{[001]}$ \\
& $\pi_{[011]} \omega_{[001]}$ \\
\hline
\end{tabular}

Table 5.12: As Table 5.8 for irreps $[000] T_{2}^{+},[000] E^{-},[001] B_{1}$ and $[001] B_{2}$ on the $\left(L / a_{s}\right)=24$ lattice. 


\section{B Scattering Parameterisations}

We present here tables of scattering parameterisations as referred to in Section 5.5 .

\begin{tabular}{|c|c|c|c|}
\hline Parameterisation & Further Restrictions & $N_{\text {pars }}$ & $\chi^{2} / N_{\text {dof }}$ \\
\hline Breit-Wigner & - & 2 & 0.84 \\
\hline $\begin{array}{l}\text { Effective Range } \\
k_{c m} \cot (\delta)=a^{-1}+\frac{1}{2} r k_{c m}^{2}\end{array}$ & $\begin{array}{l}- \\
----------- \\
-\end{array}$ & $\begin{array}{c}2 \\
--- \\
4\end{array}$ & $\begin{array}{c}0.86 \\
----- \\
0.80\end{array}$ \\
\hline $\begin{array}{l}K=\frac{g^{2}}{m^{2}-s}+\gamma^{(0)}+\gamma^{(1)} s \\
I(s)=-i \rho(s)\end{array}$ & 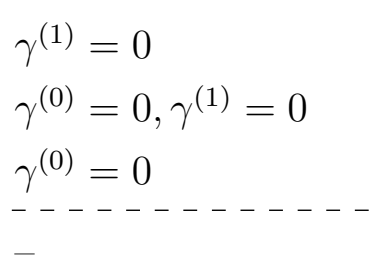 & $\begin{array}{c}3 \\
2 \\
3 \\
-- \\
4\end{array}$ & $\begin{array}{c}0.76 \\
0.84 \\
0.75 \\
---- \\
0.80\end{array}$ \\
\hline$K=\frac{g^{2}}{m^{2}-s}+\gamma^{(0)}+\gamma^{(1)} s$ & $\gamma^{(1)}=0$ & 3 & 0.76 \\
\hline $\mathrm{CM} \operatorname{Re}\left\{I\left(s=s^{\mathrm{thr}}\right)=0\right\}$ & $\begin{array}{l}\gamma^{(0)}=0, \gamma^{(1)}=0 \\
\gamma^{(0)}=0 \\
-\end{array}$ & $\begin{array}{c}2 \\
3 \\
-- \\
4\end{array}$ & $\begin{array}{r}0.84 \\
0.76 \\
---- \\
0.80\end{array}$ \\
\hline $\begin{array}{l}K=\frac{g^{2}}{m^{2}-s}+\gamma^{(0)}+\gamma^{(1)} s \\
\mathrm{CM} \operatorname{Re}\left\{I\left(s=m^{2}\right)=0\right\}\end{array}$ & $\begin{array}{l}\gamma^{(1)}=0 \\
\gamma^{(0)}=\mathbf{0}, \gamma^{(1)}=\mathbf{0} \\
\gamma^{(0)}=0\end{array}$ & $\begin{array}{l}3 \\
2 \\
3 \\
--\end{array}$ & $\begin{array}{c}0.76 \\
\mathbf{0 . 8 4} \\
0.76 \\
-----\end{array}$ \\
\hline $\begin{array}{l}K^{-1}=c^{(0)}+c^{(1)} s \\
I(s)=-i \rho(s)\end{array}$ & - & 2 & 0.84 \\
\hline $\begin{array}{l}K^{-1}=c^{(0)}+c^{(1)} s \\
\mathrm{CM} \operatorname{Re}\left\{I\left(s=s^{\mathrm{thr}}\right)=0\right\}\end{array}$ & - & 2 & 0.84 \\
\hline
\end{tabular}

Table 5.13: Parameterisations of elastic $\pi \omega\left\{{ }^{3} S_{1}\right\}$ scattering amplitudes with $N_{\text {pars }}$ free parameters. Fits used 20 energy levels below $\pi \phi$ threshold, as described in the text. The reference amplitude, Eq. 5.12, is in bold. 'CM' denotes that the Chew-Mandelstam prescription was employed with subtraction at energy $m$ or at threshold $s^{\text {thr }}=\left(m_{\pi}+m_{\omega}\right)^{2}$. Otherwise, we set $I(s)=-i \rho(s)$. 


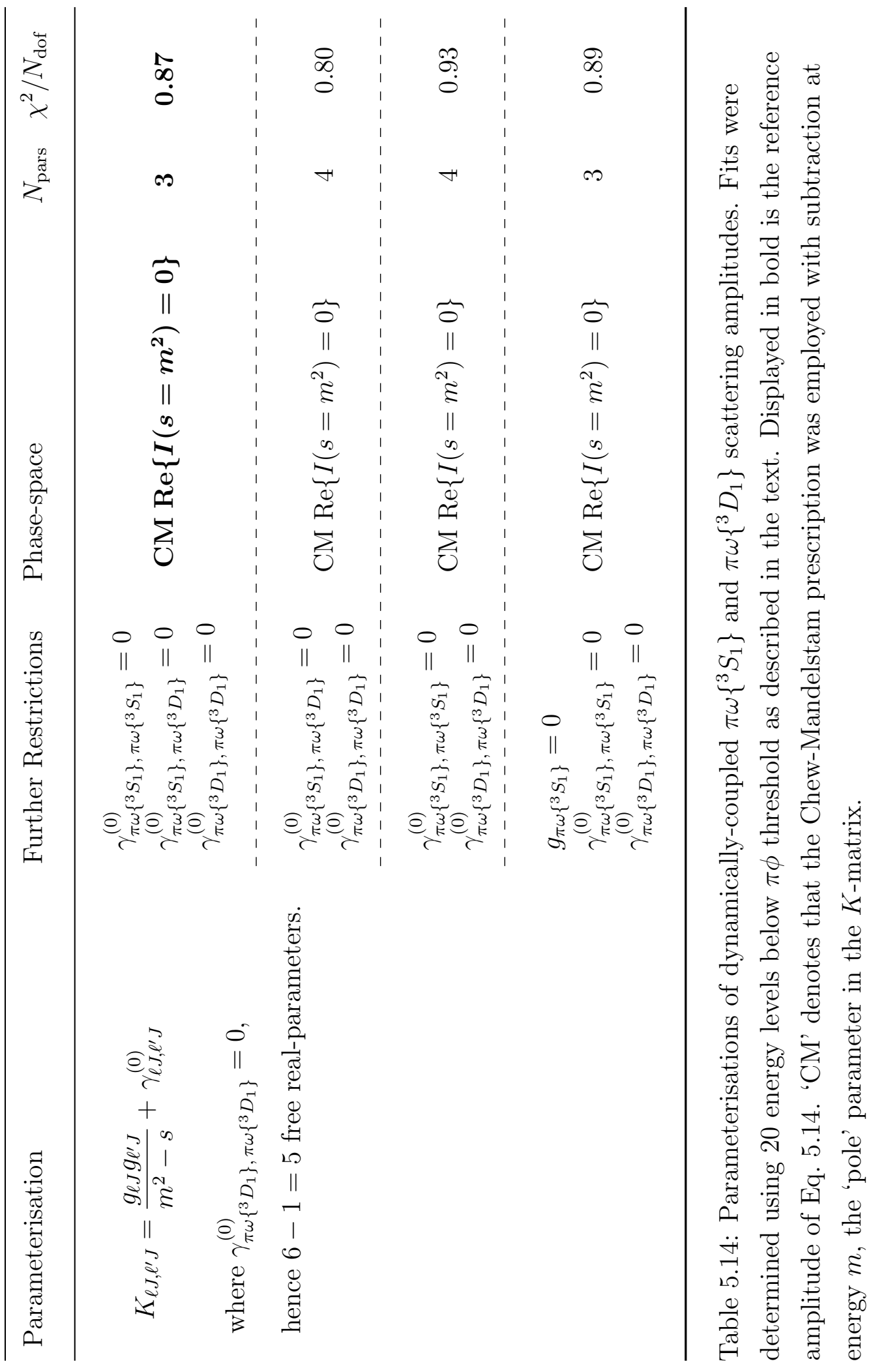




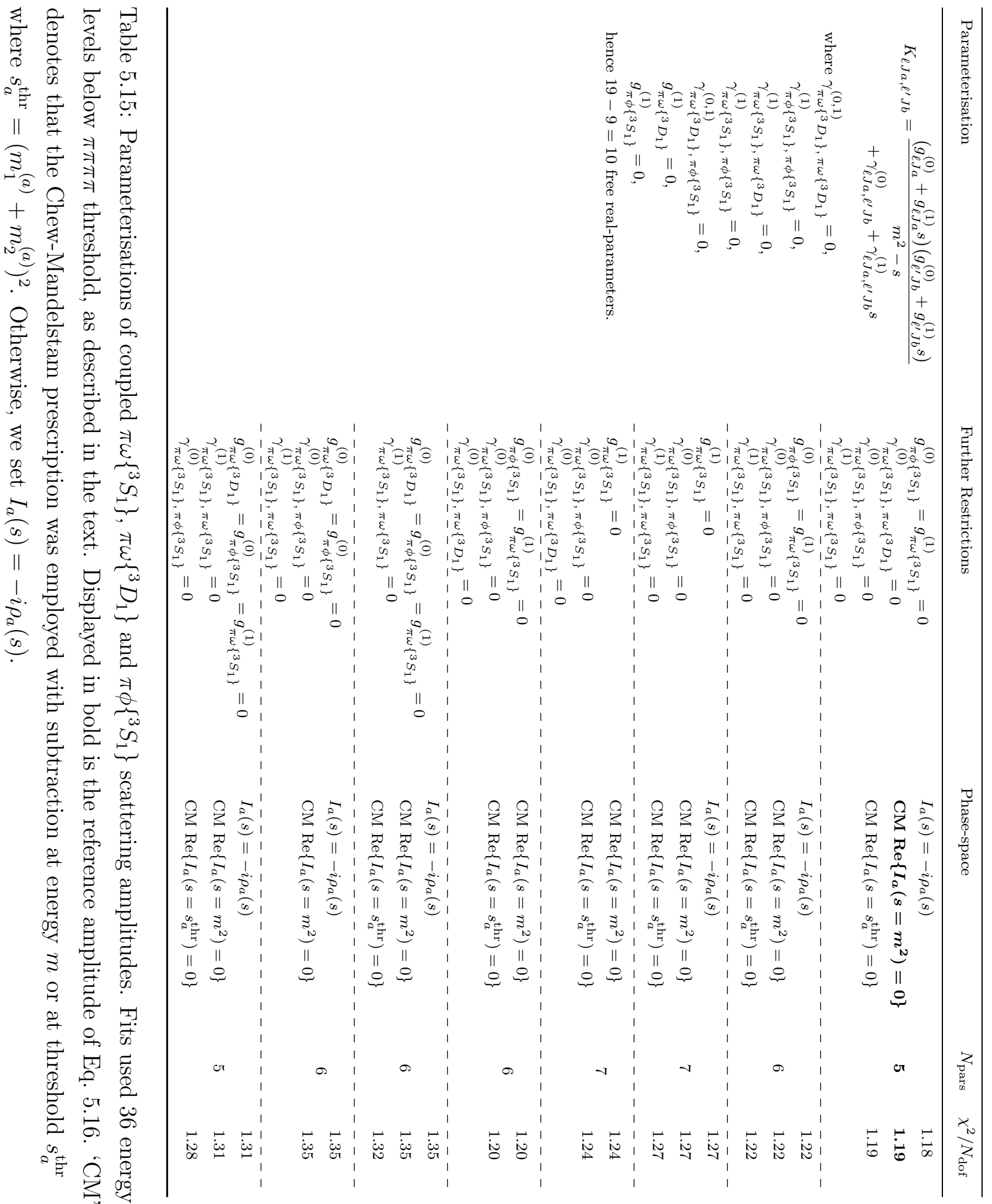




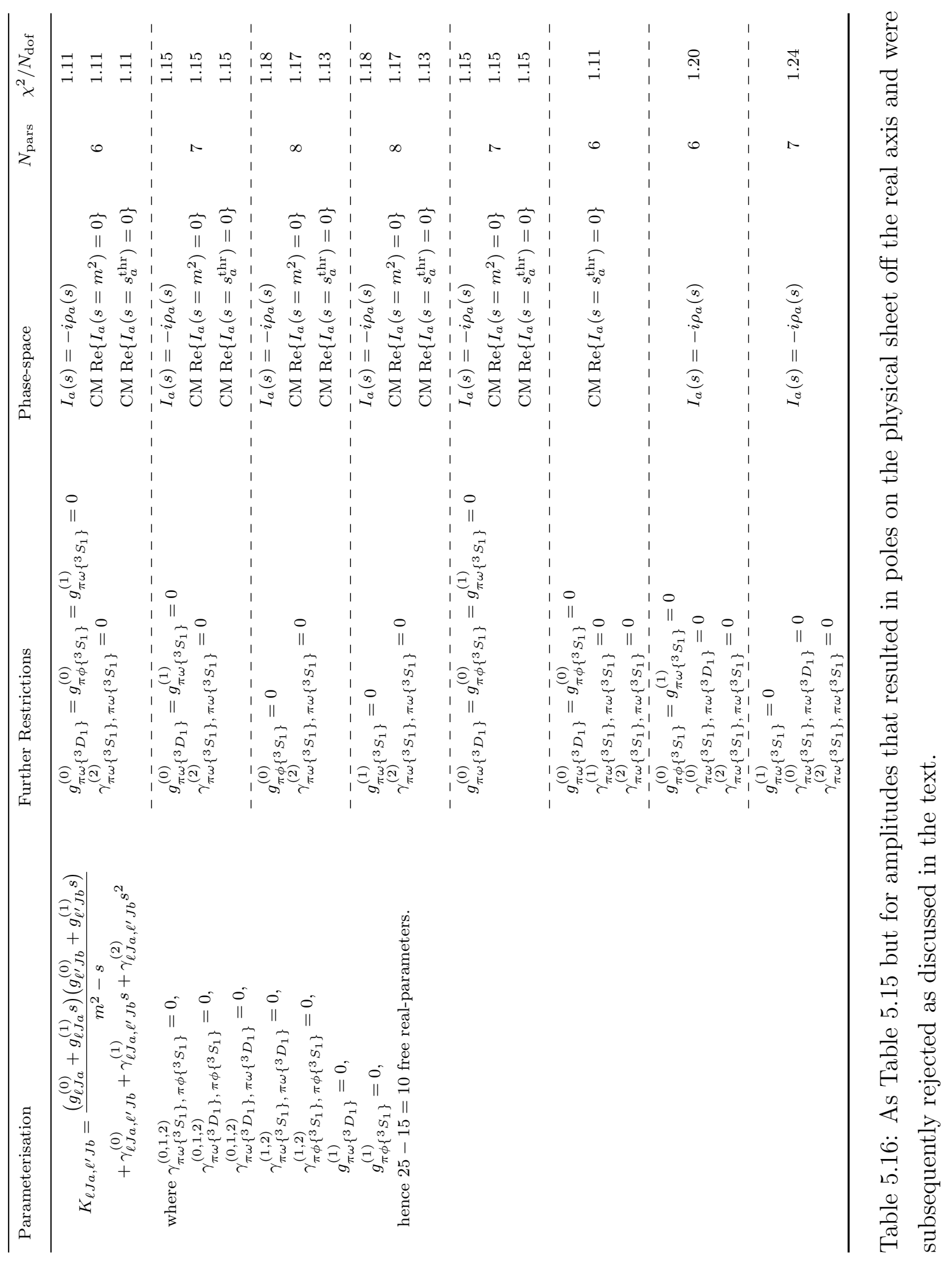




\section{C Error Ellipses}

The error ellipses plotted in Figure 5.12 show the one standard deviation confidence intervals for the correlated real and imaginary parts of the pole locations and corresponding couplings. In this appendix, we present the details of how these ellipses are determined.

Consider two normally distributed random variables $X_{i} \sim \mathcal{N}\left(0, \sigma_{X_{i}}\right), i=1,2$ with covariance matrix $\mathcal{C}=\operatorname{cov}\left[X_{i}, X_{j}\right]=\mathbb{E}\left[\left(X_{i}-\mathbb{E}\left[X_{i}\right]\right)\left(X_{j}-\mathbb{E}\left[X_{j}\right]\right)\right]$. The equation of an ellipse is given by the quadratic form,

$$
\boldsymbol{x}^{T} \mathcal{C}^{-1} \boldsymbol{x}=s
$$

where $\boldsymbol{x}=\left(x_{1}, x_{2}\right)$ and $s>0$ is the scale. As $\mathcal{C}$ is a real (positive semi-definite) symmetric matrix, a rotation diagonalises the quadratic form,

$$
\boldsymbol{x}^{T} \Lambda \boldsymbol{x}^{\prime}=s,
$$

where $\Lambda=\operatorname{diag}\left(\lambda_{1}^{-1}, \lambda_{2}^{-1}\right)$ with $\lambda_{1} \geq \lambda_{2} \geq 0$ eigenvalues ${ }^{13}$ of $\mathcal{C}$ and $\boldsymbol{x}=Q \boldsymbol{x}^{\prime}$ with $Q=\left(\boldsymbol{v}_{1}, \boldsymbol{v}_{2}\right)$ the orthogonal matrix of eigenvectors. The resulting ellipse is tilted, with the major axis rotated to align with the eigenvector corresponding to the largest eigenvalue. Explicitly written,

$$
\left(\frac{x_{1}^{\prime}}{\sqrt{\lambda_{1}}}\right)^{2}+\left(\frac{x_{2}^{\prime}}{\sqrt{\lambda_{2}}}\right)^{2}=s .
$$

In this basis, $X_{i}^{\prime} \sim \mathcal{N}\left(0, \lambda_{i}\right)$ are independent normal distributions and as such the left hand side of Eq. 5.29 represents the sum of squared standard normal distributions hence giving a $\chi^{2}$ probability distribution for the scale, $s \sim \chi^{2}(2)$. For the usual one standard deviation $(68 \%)$ confidence interval, we seek $k$ such that $P(s<k)=0.68$, finding $k=2.28$. This gives the one standard deviation error ellipse,

$$
\left(\frac{x_{1}^{\prime}}{\sqrt{\lambda_{1}}}\right)^{2}+\left(\frac{x_{2}^{\prime}}{\sqrt{\lambda_{2}}}\right)^{2}=2.28 .
$$

In the basis of $X_{i}$, the error ellipse has major axis length $2 \sqrt{2.28 \lambda_{1}}$, the minor axis has length $2 \sqrt{2.28 \lambda_{2}}$ and angle the major axis makes with the horizontal is $\alpha=\arctan \frac{\left(v_{1}\right)_{2}}{\left(v_{1}\right)_{1}}$. As $X_{1}, X_{2}$ represent the real and imaginary parts respectively of the poles or couplings, they do not have mean zero in general and we simply translate the ellipse to $\left(\mu_{X_{1}}, \mu_{X_{2}}\right)$, giving $X_{i} \sim \mathcal{N}\left(\mu_{X_{i}}, \sigma_{X_{i}}\right)$, with covariance matrix $\mathcal{C}$ as before.

\footnotetext{
${ }^{13}$ If $\lambda_{i}=0$, the ellipse is not well-defined.
} 


\section{A $\pi_{1}$ exotic resonance at $m_{\pi} \sim 700 \mathrm{MeV}$}

In the quark model, only a subset of all possible $J^{P C}$ are attainable from a quark-antiquark pair. The total intrinsic spin $S$ is either 0 or 1 and therefore, coupled with orbital angular momentum $\ell$, the total angular momentum $J \in\{|\ell-1|, \ell, \ell+1\}$. It follows that parity is given by $P=(-1)^{\ell+1}$ and charge conjugation $C=(-1)^{\ell+S}$ for flavourless mesons. These quantum numbers define the non-exotic sequence, i.e. $0^{-+}, 0^{++}, 1^{--}, 1^{+-}, 1^{++}, \ldots$ and other quantum numbers not permitted within this framework form the exotic sequence 1 , $0^{+-}, 0^{--}, 1^{-+}, 2^{+-}, \ldots$ These are attainable, for example, by coupling the quark-antiquark pair with excited gluonic degrees of freedom, a hybrid meson, or additional quark-antiquark pairs, such as a tetraquark.

Several experiments have reported an isovector $1^{-+}$state, the $\pi_{1}$, ranging in mass from $1.4-2 \mathrm{GeV}$, however there are experimental and interpretational issues surrounding each of them - in particular the existence of two states, the $\pi_{1}(1400)$ and $\pi_{1}(1600)$, is contested. A comprehensive summary of the theoretical and experimental status is presented in Ref. [99].

A recent reanalysis of the $\pi \eta^{(\prime)}$ data measured at COMPASS 100 was carried out using coupled-channel amplitudes that enforce the unitarity and analyticity of the $S$-matrix [6]. The pole singularities were calculated by analytically continuing the amplitudes into the complex $s$-plane and a single $1^{-+}$pole, with mass $\sim 1564 \mathrm{MeV}$ and width $\sim 492 \mathrm{MeV}$, was unambiguously determined, with no evidence for a second pole that could be identified with another $\pi_{1}$ resonance.

Lattice QCD calculations have also found evidence for a single isovector $1^{-+}$state in this energy region. A fairly recent calculation determines the isovector spectrum 29] for a

\footnotetext{
${ }^{1}$ Not to be confused with the flavour exotics, such as $\rho \pi$ in isospin- 2 .
} 
number of exotic $J^{P C}$ multiplets, at four pion masses, the lightest being $m_{\pi} \sim 391 \mathrm{MeV}$ (computed on the same lattices as those used in Chapter 5) and heaviest $m_{\pi} \sim 700 \mathrm{MeV}$ (computed on the same lattices as those used in Chapters 3 and 4 ). In each case, evidence of a single isovector $1^{-+}$state in an energy region around $\sim 2 \mathrm{GeV}$ was found. Although this lattice calculation utilises a large, diverse basis of hybrid-like fermion bilinear operators to determine the spectra, no operators resembling multi-hadron states are incorporated and as such the spectra is not robust. Furthermore, many two-hadron thresholds are kinematically open in a region much lower than the observed $\pi_{1}$ like state and to rigorously calculate the $\pi_{1}$ necessitates the determination of pole singularities of the scattering amplitudes. It is to this problem we turn here.

Once more we make use of the $N_{f}=3$ lattices used in Chapters 3 and 4 with $m_{\pi} \sim$ $700 \mathrm{MeV}$. Throughout this chapter, we use the notation for $\mathrm{SU}(3)_{\mathrm{F}}$ multiplets set out in Chapter 4, where we recall that flavour multiplets are labelled by the isospin-0 component. As such, the relevant flavour octets are labelled as follows: pseudoscalar $(\boldsymbol{\eta})_{\mathbf{8}}$, vector $(\boldsymbol{\omega})_{\mathbf{8}}$, scalar $\left(\boldsymbol{f}_{\mathbf{0}}\right)_{\mathbf{8}}$, axial-vectors $\left(\boldsymbol{f}_{\mathbf{1}}\right)_{\mathbf{8}}$ and $\left(\boldsymbol{h}_{\mathbf{1}}\right)_{\mathbf{8}}$, tensor $\left(\boldsymbol{f}_{\mathbf{2}}\right)_{\mathbf{8}}$. The $\mathrm{SU}(2)_{\mathrm{F}}$ components of the pseudoscalar and vector octets are presented in Figure 3.2 and the others, listed above, in Figure 6.16 of Appendix 6.A. We remind the reader that for the corresponding flavour singlets, the subscript $\mathbf{8}$ is replaced with $\mathbf{1}$. For the exotic octet featuring the isovector $1^{-+}$meson, we label the octet $\left(\boldsymbol{\pi}_{1}\right)_{8}$ rather than $\left(\boldsymbol{\eta}_{1}\right)_{8}$ for clarity.

The manifestation of $\mathrm{SU}(3)_{\mathrm{F}}$ significantly reduces the number of relevant two-meson channels. As we are at liberty to pick any component of $\left(\boldsymbol{\pi}_{\mathbf{1}}\right)_{\mathbf{8}}$, we choose $\nu=(1,0,1)$, which has a negative $G$-parity. Therefore, when combining two flavour octet mesons into a single octet, $\mathbf{8} \otimes \mathbf{8} \rightarrow \mathbf{8}_{1} \oplus \mathbf{8}_{2}$, only one of these two possible flavour embeddings has an isovector component with negative $G$-parity, as discussed in Section 4.1. Furthermore, Bose-symmetry restricts the allowed partial-wave configurations of two identical mesons. This plays an important role here, as, for example, two identical pseudoscalar octets, $(\boldsymbol{\eta})_{\mathbf{8}}(\boldsymbol{\eta})_{\mathbf{8}}$, are forbidden ${ }^{1} P_{1}$-wave and similarly, identical vector octets, $(\boldsymbol{\omega})_{\mathbf{8}}(\boldsymbol{\omega})_{\mathbf{8}}$, are forbidden in ${ }^{1} P_{1^{-}}$and ${ }^{5} P_{1}$-wave (but are permitted in ${ }^{3} P_{1}$-wave).

Despite these restrictions, there remain a large number of two-meson channels transforming in $\left(\boldsymbol{\pi}_{1}\right)_{\mathbf{8}}$. Those relevant, appearing in the energy range we consider, include a single pseudoscalar-pseudoscalar $(\boldsymbol{\eta})_{8}(\boldsymbol{\eta})_{1}\left\{{ }^{1} P_{1}\right\}$ channel, a single vector-pseudoscalar channel, $(\boldsymbol{\omega})_{\mathbf{8}}(\boldsymbol{\eta})_{\mathbf{8}}\left\{{ }^{3} P_{1}\right\}$ and two axial-vector-pseudoscalar channels, $\left(\boldsymbol{f}_{\mathbf{1}}\right)_{\mathbf{8}}(\boldsymbol{\eta})_{\mathbf{8}}\left\{{ }^{3} S_{1}\right\}$ and 
$\left(\boldsymbol{h}_{\mathbf{1}}\right)_{\mathbf{8}}(\boldsymbol{\eta})_{\mathbf{8}}\left\{{ }^{3} S_{1}\right\}$. Furthermore, two vector-vector thresholds open, $(\boldsymbol{\omega})_{\mathbf{8}}(\boldsymbol{\omega})_{\mathbf{8}}$ and $(\boldsymbol{\omega})_{\mathbf{8}}(\boldsymbol{\omega})_{\mathbf{1}}$, resulting in four vector-vector channels, $(\omega)_{\mathbf{8}}(\omega)_{8}\left\{{ }^{3} P_{1}\right\},(\omega)_{8}(\omega)_{1}\left\{{ }^{1} P_{1}\right\},(\omega)_{8}(\omega)_{1}\left\{{ }^{3} P_{1}\right\}$ and $(\boldsymbol{\omega})_{\mathbf{8}}(\boldsymbol{\omega})_{\mathbf{1}}\left\{{ }^{5} P_{1}\right\}$, completing an eight coupled-channel system.

Unphysically heavy pions help circumvent the need to incorporate three-meson scattering amplitudes. At rest, we will find all $E_{\text {n.i. }}^{(2+1)}$ and $E_{\text {n.i. }}^{(3)}$ energies on these lattices are above the energy region of interest. For moving-frames, the $E_{\text {n.i. }}^{(2+1)}$ and $E_{\text {n.i. }}^{(3)}$ energies are lower-lying, found to be near the anticipated resonant region. Moreover, the parity mixing at non-zero momentum means low-lying resonances, such as the $\left(f_{2}\right)_{8}$, will also feature, significantly complicating this scattering calculation of the $\left(\boldsymbol{\pi}_{1}\right)_{8}$. In this study, we therefore restrict to rest-frame spectra only.

At a lighter pion mass, away from the $\mathrm{SU}(3)_{\mathrm{F}}$ point, the two-meson channels separate into their distinguishable $\mathrm{SU}(2)_{\mathrm{F}}$ components. For the relevant two-meson channels listed above, recalling that we take the $\nu=(1,0,1)$ component of the $\left(\boldsymbol{\pi}_{1}\right)_{8}$, these are as follows: pseudoscalar-pseudoscalar $(\boldsymbol{\eta})_{\mathbf{8}}(\boldsymbol{\eta})_{\mathbf{1}} \rightarrow \pi \eta, \pi \eta^{\prime}$, vector-pseudoscalar $(\omega)_{\mathbf{8}}(\boldsymbol{\eta})_{\mathbf{8}} \rightarrow$ $\rho \pi, K^{*} \bar{K}$, vector-vector $(\boldsymbol{\omega})_{\mathbf{8}}(\boldsymbol{\omega})_{\mathbf{8}} \rightarrow \rho \omega, \rho \phi, K^{*} \bar{K}^{*}$ and $(\boldsymbol{\omega})_{\mathbf{8}}(\boldsymbol{\omega})_{1} \rightarrow \rho \omega, \rho \phi$, axial-vectorpseudoscalar $\left(\boldsymbol{f}_{\mathbf{1}}\right)_{\mathbf{8}}(\boldsymbol{\eta})_{\mathbf{8}} \rightarrow a_{1} \eta, a_{1} \eta^{\prime}, f_{1} \pi, f_{1}^{\prime} \pi, K_{1} \bar{K}$ and $\left(\boldsymbol{h}_{\mathbf{1}}\right)_{\mathbf{8}}(\boldsymbol{\eta})_{\mathbf{8}} \rightarrow b_{1} \pi, K_{1} \bar{K}$. We will discuss couplings in these $\mathrm{SU}(2)_{\mathrm{F}}$ channels when we interpret the results.

\subsection{Computational Details}

Calculations of correlation functions were performed on four anisotropic lattices. In addition to the $\left(L / a_{s}\right)^{3} \times\left(T / a_{t}\right)=20^{3} \times 128$ and $24^{3} \times 128$ lattices used in Chapter 3 , and $\left(L / a_{s}\right)^{3} \times\left(T / a_{t}\right)=16^{3} \times 128$ used in Chapter 4, we also make use of a smaller volumes, $\left(L / a_{s}\right)^{3} \times\left(T / a_{t}\right)=12^{3} \times 96$, generated from the same action. On all volumes, exponentially suppressed finite-volume and temporal effects remain negligible with $m_{\pi} L \gtrsim 6$ and $m_{\pi} T \gtrsim 14$ on the smallest volume.

Correlation functions were computed using the number of distillation vectors, gauge configurations and time-sources shown in Table 6.1. As in previous calculations, we typically compute all the elements of the matrix of correlation functions, however, in a few cases we made use of hermiticity to infer $C_{j i}$ from the computed $C_{i j}$.

To ensure all relevant two-meson operators are included in the basis, we examine the location of all two-body thresholds by first determining all stable hadron masses. In 


\begin{tabular}{c|ccc}
$\left(L / a_{s}\right)^{3} \times\left(T / a_{t}\right)$ & $N_{\text {vecs }}$ & $N_{\text {cfgs }}$ & $N_{\text {tsrcs }}$ \\
\hline $12^{3} \times 96$ & 48 & 219 & 24 \\
$16^{3} \times 128$ & 64 & 529 & 4 \\
$20^{3} \times 128$ & 128 & 501 & 4 \\
$24^{3} \times 128$ & 160 & 607 & 4
\end{tabular}

Table 6.1: Number of distillation vectors $\left(N_{\text {vecs }}\right)$, gauge configurations $\left(N_{\text {cfgs }}\right)$ and time-sources $\left(N_{\text {tsrcs }}\right)$ used to compute the $\left(\boldsymbol{\pi}_{\mathbf{1}}\right)_{\mathbf{8}}$ correlation functions on each lattice volume, as described in the text.

addition to the stable pseudoscalar $(\boldsymbol{\eta})_{8}$ and vector $(\boldsymbol{\omega})_{8}$ octets, we consider the scalar, axial-vector and tensor octets. Both axial-vector octets are stable, the $\left(\boldsymbol{f}_{\mathbf{1}}\right)_{\mathbf{8}}$ appearing far below $(\boldsymbol{\omega})_{\mathbf{8}}(\boldsymbol{\eta})_{\mathbf{8}}$ threshold as shown in Figure 4.1 in Section 4.4 . For the tensor octet, the $(\boldsymbol{\eta})_{\mathbf{8}}(\boldsymbol{\eta})_{\mathbf{8}}$ threshold lies far below the calculated ' $q \bar{q}$ ' mass, as shown in Figure 13 of Ref. [26], and as such the tensor is unstable. Regarding the scalar octet, the determined ' $q \bar{q}$ ' mass is found to coincide with the $(\boldsymbol{\eta})_{\mathbf{8}}(\boldsymbol{\eta})_{\mathbf{8}}$ threshold. For the purposes of this study, we shall be conservative and treat the scalar octet as unstable.

Paired with each octet is a corresponding $\mathrm{SU}(3)_{\mathrm{F}}$ singlet. The pseudoscalar singlet is found to be much heavier than its octet counterpart. The vector, axial-vector and tensor singlets are all approximately degenerate 2 with the corresponding octet masses, shown in Figure 13 of Ref. [26], and as a result the axial-vector singlets are also stable. The tensor singlet, as is the case for the tensor octet, is unstable and decays in $D$-wave to $(\boldsymbol{\eta})_{\mathbf{8}}(\boldsymbol{\eta})_{\mathbf{8}}$. The scalar singlet with vacuum quantum numbers is found to be much lower in mass relative to the octet and is stable.

To summarise, we present the stable hadron masses in Table 6.2. Also shown are the relevant multi-hadron thresholds transforming in the $\left(\boldsymbol{\pi}_{1}\right)_{8}$ multiplet where we have truncated at the $\left(\boldsymbol{f}_{\mathbf{1}}\right)_{\mathbf{1}}(\boldsymbol{\eta})_{\mathbf{8}}$ threshold. This is a moderate distance above the anticipated resonant region of $a_{t} E_{\mathrm{cm}} \sim 0.46$, guided by the exotic ' $q \bar{q}$ ' spectrum in Figure 13 of Ref. [26].

For the scattering calculation of the hybrid $\left(\boldsymbol{\pi}_{\mathbf{1}}\right)_{\mathbf{8}}$, two-meson operators featuring $(\boldsymbol{\eta})_{\mathbf{8}}$, $(\omega)_{8},(\eta)_{1}$ and $(\omega)_{1}$ at non-zero overall momentum are required. We check the momentum

\footnotetext{
${ }^{2}$ The $\left(f_{1}\right)_{\mathbf{8}}$ and $\left(f_{1}\right)_{1}$ are slightly split with the singlet marginally heavier.
} 


\begin{tabular}{ll|cl} 
octet & $a_{t} E_{\mathrm{cm}}$ & singlet & $a_{t} E_{\mathrm{cm}}$ \\
\hline$(\boldsymbol{\eta})_{\mathbf{8}}$ & $0.1478(1)$ & $(\boldsymbol{\eta})_{\mathbf{1}}$ & $0.2017(11)$ \\
$(\boldsymbol{\omega})_{\mathbf{8}}$ & $0.2154(2)$ & $(\boldsymbol{\omega})_{\mathbf{1}}$ & $0.2174(3)$ \\
$\left(\boldsymbol{f}_{\mathbf{1}}\right)_{\mathbf{8}}$ & $0.3203(6)$ & $\left(\boldsymbol{f}_{\mathbf{0}}\right)_{\mathbf{1}}$ & $0.2007(18)$ \\
$\left(\boldsymbol{h}_{\mathbf{1}}\right)_{\mathbf{8}}$ & $0.3272(6)$ & $\left(\boldsymbol{f}_{\mathbf{1}}\right)_{\mathbf{1}}$ & $0.3364(14)$ \\
& & $\left(\boldsymbol{h}_{\mathbf{1}}\right)_{\mathbf{1}}$ & $0.3288(17)$
\end{tabular}

\begin{tabular}{cl} 
threshold & $a_{t} E_{\mathrm{cm}}$ \\
\hline$(\boldsymbol{\eta})_{\mathbf{8}}(\boldsymbol{\eta})_{\mathbf{1}}$ & $0.3495(11)$ \\
$(\boldsymbol{\omega})_{\mathbf{8}}(\boldsymbol{\eta})_{\mathbf{8}}$ & $0.3632(2)$ \\
$(\boldsymbol{\omega})_{\mathbf{8}}(\boldsymbol{\omega})_{\mathbf{8}}$ & $0.4308(3)$ \\
$(\boldsymbol{\omega})_{\mathbf{8}}(\boldsymbol{\omega})_{\mathbf{1}}$ & $0.4324(7)$ \\
$(\boldsymbol{\eta})_{\mathbf{8}}(\boldsymbol{\eta})_{\mathbf{8}}(\boldsymbol{\eta})_{\mathbf{8}}$ & $0.4434(2)$ \\
$\left(\boldsymbol{f}_{\mathbf{1}}\right)_{\mathbf{8}}(\boldsymbol{\eta})_{\mathbf{8}}$ & $0.4681(6)$ \\
$\left(\boldsymbol{h}_{\mathbf{1}}\right)_{\mathbf{8}}(\boldsymbol{\eta})_{\mathbf{8}}$ & $0.4750(6)$ \\
$\left(\boldsymbol{f}_{\mathbf{1}}\right)_{\mathbf{1}}(\boldsymbol{\eta})_{\mathbf{8}}$ & $0.4842(14)$
\end{tabular}

Table 6.2: (Left): relevant stable hadron masses. (Right): multi-hadron thresholds relevant for $J^{P}=1^{-}$up to $a_{t} E_{\mathrm{cm}} \approx 0.485$.

dependence of the $(\boldsymbol{\eta})_{1}$ and $(\boldsymbol{\omega})_{1}$ energies satisfy the relativistic dispersion relation and give a consistent value of the anisotropy compared to the value obtained in Section 3.4 for the $(\boldsymbol{\eta})_{8}$ and $(\boldsymbol{\omega})_{8}$ mesons.

The energies of the ground-state $(\boldsymbol{\eta})_{1}$ and $(\boldsymbol{\omega})_{1}$ mesons were calculated from a variational analysis of matrices of correlation functions involving bases of flavour singlet single-meson operators. The analyses also gave the optimised operators for interpolating the $(\boldsymbol{\eta})_{1}$ and $(\omega)_{1}$ with the various momenta used in the construction of two-meson operators. The fits to the dispersion relation given in Eq. 3.10 for $(\boldsymbol{\eta})_{1}$ and $(\boldsymbol{\omega})_{1}$ are shown in Figure 6.1. We used energies on two volumes, $L / a_{s}=20,24$, to constrain the fits. For the $(\boldsymbol{\eta})_{\mathbf{1}}$, energies calculated in lattice irreps with $|\vec{p}|^{2} \leq 4$ were used. Regarding the $(\boldsymbol{\omega})_{1}$, we used energies calculated in lattice irreps with $|\vec{p}|^{2} \leq 4$ on the larger volume and $|\vec{p}|^{2} \leq 2$ on the smaller ${ }^{3}$

We find the anisotropies for the helicity $|\lambda|=0,1$ components of the $(\omega)_{1}$ are comparable to those found for $(\boldsymbol{\omega})_{8}$ and similarly the anisotropy calculated for the $(\boldsymbol{\eta})_{1}$ agrees with that of $(\boldsymbol{\eta})_{\mathbf{8}}$. We therefore take as the value of the anisotropy, $\xi=3.486(43)$, the determined value used in Chapter 3 , for the remainder of this work.

\footnotetext{
${ }^{3}(\omega)_{1}$ energies computed on the $L / a_{s}=20$ volume used $N_{\text {cfgs }}=197$.
} 


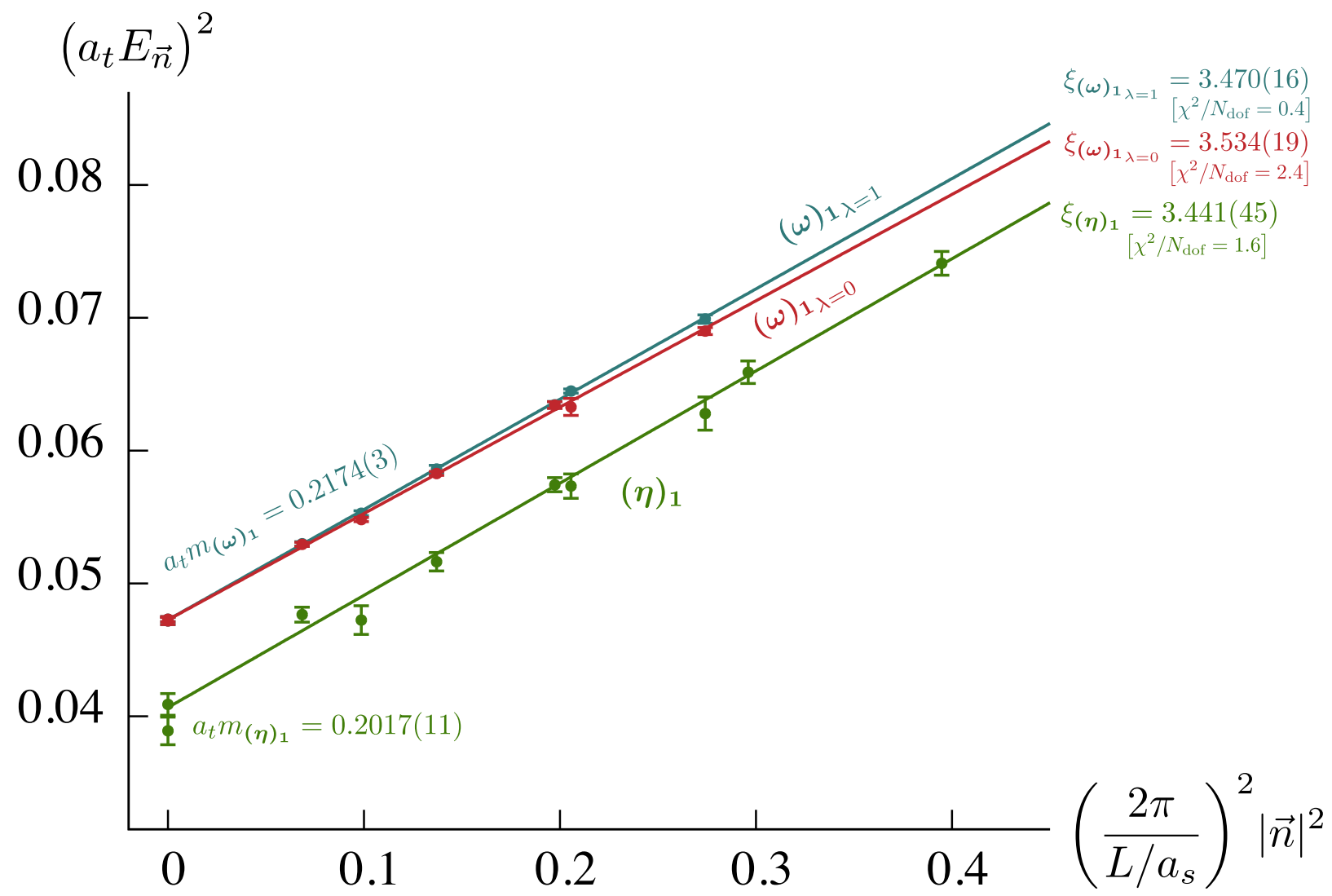

Figure 6.1: Momentum dependence of the $(\boldsymbol{\eta})_{1}$ energies and energies of the $|\lambda|=0,1$ helicity components of the $(\omega)_{1}$. Points show the energies used to constrain the fits, as discussed in the text, with the errorbars reflecting the statistical uncertainties. Lines and numerical values show results of fits to determine $\xi$ using Eq. 3.10.

\subsection{Operator Bases}

At rest, $J^{P}=1^{-}$subduces into the $[000] T_{1}^{-}$irrep and the energy region we consider includes the opening of several multi-hadron thresholds. This necessitates the inclusion of a large number of two-meson-like operators in the basis, however, we will show that three-meson-like operators are expected to interpolate levels above the energy cut-off and are subsequently not included. A large number of single-meson-like operators transforming in $\left(\pi_{1}\right)_{8}$ are incorporated, resembling hybrid mesons. Four-meson thresholds lie beyond the energy region we consider and previous calculations [68,82] suggest local tetraquark-like operators have negligible effect on the spectrum, so neither of these types of operators are 
included in the basis.

\subsubsection{Single-Meson Operators}

Single-meson operators transforming in $\left(\boldsymbol{\pi}_{1}\right)_{\mathbf{8}}$ are constructed according to Eq. 3.4. Notably, there are no single-meson operators formed simply from a product of $\gamma$-matrices in a fermion bilinear that have the appropriate quantum numbers. Within the operator constructions, gluonic degrees of freedom enter through covariant derivatives. For example, the $J^{P C}=1^{-+}$fermion bilinear hybrid operator, $\bar{\psi} \mathbf{\Gamma} \psi$, constructed using the commutator of two-derivatives, is given by,

$$
\boldsymbol{\Gamma}=\sum_{\substack{m_{1}, m_{2} \\
m_{3}, m_{D}}} \mathcal{C}\left(\begin{array}{ccc}
1 & 1 & 1 \\
m_{1} & m_{D} & m
\end{array}\right) \mathcal{C}\left(\begin{array}{ccc}
1 & 1 & 1 \\
m_{2} & m_{3} & m_{D}
\end{array}\right) \gamma_{m_{1}} \overleftrightarrow{D}_{m_{2}} \overleftrightarrow{D}_{m_{3}}
$$

which is proportional to the field strength tensor. For the $[000] T_{1}^{-}$irrep in the $\left(\boldsymbol{\pi}_{1}\right)_{8}$ flavour multiplet, we construct all possible single-meson-like hybrid operators using up to three gauge-covariant derivatives. This gives a total of 18 operators with continuum spins of $J=1,3$ or 4 .

\subsubsection{Two-Meson Operators}

The construction of operators resembling a pair of mesons follows the approach detailed in Chapter 3. To ensure all relevant two-meson operators are included in the bases, we calculate the non-interacting energies for each two-meson threshold listed in Table 6.2. We include the corresponding two-meson operators for all those non-interacting energies below $a_{t} E_{\mathrm{cm}}=0.485$. We present the final operator basis used for the [000] $T_{1}^{-}$irrep on each lattice volume in Table 6.3.

\subsubsection{Three-Meson Operators}

Included in Table 6.2 are three-body thresholds below $\left(\boldsymbol{f}_{\mathbf{1}}\right)_{\mathbf{1}}(\boldsymbol{\eta})_{\mathbf{8}}$, for which the only one is $(\boldsymbol{\eta})_{\mathbf{8}}(\boldsymbol{\eta})_{\mathbf{8}}(\boldsymbol{\eta})_{\mathbf{8}}$. We consider any $E_{\text {n.i. }}^{(3)}$ or $E_{\text {n.i. }}^{(2+1)}$ appearing below the energy cut-off, analogous to the exercise done in Chapter 5 , ensuring that all relevant operators are included in the basis in order to determine a robust spectra. 


\begin{tabular}{|c|c|c|c|}
\hline$L / a_{s}=12$ & $L / a_{s}=16$ & $L / a_{s}=20$ & $L / a_{s}=24$ \\
\hline $18 \times \bar{\psi} \mathbf{\Gamma} \psi$ & $18 \times \bar{\psi} \boldsymbol{\Gamma} \psi$ & $18 \times \bar{\psi} \mathbf{\Gamma} \psi$ & $18 \times \bar{\psi} \boldsymbol{\Gamma} \psi$ \\
\hline$(\eta)_{8[001]}(\eta)_{1[001]}$ & $(\eta)_{8[001]}(\eta)_{1[001]}$ & $(\eta)_{8[001]}(\eta)_{1[001]}$ & $(\eta)_{8_{[001]}}(\eta)_{1[001]}$ \\
\hline$\left(f_{1}\right)_{8_{[000]}}(\eta)_{8_{[000]}}$ & $(\omega)_{8[001]}(\eta)_{8[001]}$ & $(\omega)_{8[001]}(\eta)_{8[001]}$ & $(\omega)_{8_{[001]}}(\eta)_{8[001]}$ \\
\hline$\left(h_{1}\right)_{8_{[000]}}(\eta)_{8[000]}$ & $\left(f_{1}\right)_{8_{[000]}}(\eta)_{8[000]}$ & $(\eta)_{8[011]}(\eta)_{1[011]}$ & $(\boldsymbol{\eta})_{\mathbf{8}_{[011]}}(\boldsymbol{\eta})_{1_{[011]}}$ \\
\hline$(\omega)_{8[001]}(\eta)_{8[001]}$ & $\left(h_{1}\right)_{8_{[000]}}(\eta)_{8[000]}$ & $\{2\}(\boldsymbol{\omega})_{\mathbf{8}_{[011]}}(\boldsymbol{\eta})_{\mathbf{8}_{[011]}}$ & $\{2\}(\boldsymbol{\omega})_{\mathbf{8}_{[011]}}(\boldsymbol{\eta})_{\mathbf{8}_{[011]}}$ \\
\hline \multirow[t]{9}{*}{$\left(f_{1}\right)_{1_{[000]}}(\eta)_{8_{[000]}}$} & $(\boldsymbol{\eta})_{\mathbf{8}_{[011]}}(\boldsymbol{\eta})_{\mathbf{1}_{[011]}}$ & $(\omega)_{\mathbf{8}_{[001]}}(\boldsymbol{\omega})_{\mathbf{8}_{[001]}}$ & $(\boldsymbol{\eta})_{\mathbf{8}_{[111]}}(\boldsymbol{\eta})_{\mathbf{1}_{[111]}}$ \\
\hline & $\left(f_{1}\right)_{1[000]}(\eta)_{8[000]}$ & $\{4\}(\boldsymbol{\omega})_{\mathbf{8}_{[001]}}(\boldsymbol{\omega})_{\mathbf{1}_{[001]}}$ & $(\omega)_{8_{[111]}}(\eta)_{8_{[111]}}$ \\
\hline & $(\omega)_{8[001]}(\omega)_{8[001]}$ & $\left(f_{1}\right)_{8_{[000]}}(\eta)_{8[000]}$ & $(\omega)_{\mathbf{8}_{[001]}}(\omega)_{\mathbf{8}_{[001]}}$ \\
\hline & $\{4\}(\boldsymbol{\omega})_{\mathbf{8}_{[001]}}(\boldsymbol{\omega})_{\mathbf{1}_{[001]}}$ & $(\eta)_{8_{[111]}}(\eta)_{1_{[111]}}$ & $\{4\}(\omega)_{8_{[001]}}(\omega)_{1[001]}$ \\
\hline & $\{2\}(\boldsymbol{\omega})_{\mathbf{8}_{[011]}}(\boldsymbol{\eta})_{\mathbf{8}_{[011]}}$ & $\left(h_{1}\right)_{8_{[000]}}(\eta)_{8[000]}$ & $(\boldsymbol{\eta})_{\mathbf{8}_{[002]}}(\boldsymbol{\eta})_{\mathbf{1}_{[002]}}$ \\
\hline & & $(\omega)_{8[111]}(\eta)_{8[111]}$ & $\left(f_{1}\right)_{8[000]}(\eta)_{8[000]}$ \\
\hline & & $\left(f_{1}\right)_{1[000]}(\eta)_{8[000]}$ & $\left(h_{1}\right)_{8[000]}(\eta)_{8[000]}$ \\
\hline & & $(\omega)_{8[002]}(\eta)_{8[002]}$ & $(\boldsymbol{\omega})_{\mathbf{8}_{[002]}}(\boldsymbol{\eta})_{\mathbf{8}_{[002]}}$ \\
\hline & & & $\left(f_{1}\right)_{1_{[000]}}(\eta)_{8[000]}$ \\
\hline
\end{tabular}

Table 6.3: [000] $T_{1}^{-}$operator basis for each lattice volume, with two-meson operators ordered by increasing $E_{\text {n.i. }}$. As in table 5.3 , the maximum number of single-meson operators, $N$, is denoted by $N \times \bar{\psi} \boldsymbol{\Gamma} \psi$ and various subsets of these were considered to obtain robust fits. The number in braces, $\left\{N_{\text {mult }}\right\}$, denotes the multiplicity of linearly independent two-meson operators if this is larger than one.

At threshold, each $(\boldsymbol{\eta})_{\mathbf{8}}$ meson is at zero momenta, analogous to the $\pi \pi \eta$ case in Section 5.1.3, and the product of [000] $A_{1}^{-}$irreps does not appear in [000] $T_{1}^{-}$. Considering various combinations of $\vec{p}_{1}, \vec{p}_{2}$ and $\vec{p}_{3}$, we find the lowest $E_{\text {n.i. }}^{(3)}$ appearing in $[000] T_{1}^{-}$ corresponds to the following product,

$$
\underbrace{[011] A_{2}}_{(\boldsymbol{\eta})_{8}} \otimes \underbrace{[001] A_{2}}_{(\boldsymbol{\eta})_{8}} \otimes \underbrace{[001] A_{2}}_{(\boldsymbol{\eta})_{8}} \rightarrow 2 \times \underbrace{[000] T_{1}^{-}}_{\left(\pi_{1}\right)_{8}} \oplus \ldots
$$

where the two embeddings in $[000] T_{1}^{-}$arise from two distinct ways of combining the 
momenta shown below, $4^{4}$

$$
\underbrace{[001] E_{2}}_{(\boldsymbol{\eta})_{8}(\eta)_{8}} \otimes \underbrace{[001] A_{2}}_{(\eta)_{8}} \rightarrow \underbrace{[000] T_{1}^{-}}_{\left(\pi_{1}\right)_{8}},
$$

and

$$
\underbrace{[011] A_{2}}_{(\boldsymbol{\eta})_{8}} \otimes \underbrace{[011] B_{1}}_{(\boldsymbol{\eta})_{8}(\boldsymbol{\eta})_{8}} \rightarrow \underbrace{[000] T_{1}^{-}}_{\left(\boldsymbol{\pi}_{1}\right)_{8}} .
$$

For the lattice volumes in this study, the corresponding $E_{\text {n.i. }}^{(3)}$ is far above the energy cut-off - on the largest volume $E_{\text {n.i. }}^{(3)}=0.5152$.

Regarding the $E_{\text {n.i. }}^{(2+1)}$ energies, we consider the finite-volume spectra in the $(\boldsymbol{\eta})_{\mathbf{8}}(\boldsymbol{\eta})_{\mathbf{8}}$ subsystem appearing in $[001] E_{2}$ and $[011] B_{1}$. The lowest-lying energy in each case corresponds to the stable $\left(\boldsymbol{f}_{\mathbf{1}}\right)_{\mathbf{8}}$ state with $E_{\mathrm{cm}} \approx 0.32$ and thus any excitations must appear at a cm-energy greater than this. As such, we can immediately infer a conservative lower bound of $E_{\text {n.i. }}^{(2+1)} \approx 0.495$ using the largest volume, beyond the energy cut-off of $a_{t} E_{\mathrm{cm}}=0.485$. Subsequently, no three-meson operators are included in the basis.

\subsection{Finite-Volume Spectra}

The finite-volume spectrum on each lattice volume was calculated using a variational analysis of the matrix of correlation functions computed using the operator basis given in Table 6.3. The extracted energies are presented in Figure 6.2. On the smallest and largest volumes $\left(L / a_{s}=12\right.$ and 24$)$ we also present the operator-state overlap factors.

The quality of the principal correlator fits on all volumes was good for the vast majority of the levels, as reflected in the small statistical uncertainties in Figure 6.2, and we show the principal correlator fits on the $L / a_{s}=24$ volume in Figures 6.3 -6.5 as an illustration. Little systematic variation was observed on all volumes under a reasonable range of $t_{0}$ values with two notable exceptions.

The first is a noisy energy level on the $L / a_{s}=20$ volume at $a_{t} E_{\mathrm{cm}} \approx 0.48$, found to have large operator-state overlap onto the $\left(\boldsymbol{f}_{\mathbf{1}}\right)_{\mathbf{8}}(\boldsymbol{\eta})_{\mathbf{8}}$-like operator. A large systematic

\footnotetext{
${ }^{4}$ We are not considering here multiple flavour embeddings that could arise in the product of three $\mathrm{SU}(3)_{\mathrm{F}}$ octets.
} 
uncertainty was added to reflect the variation for different choices of $t_{0}$. The second is a level on the $L / a_{s}=24$ volume close to the $(\boldsymbol{\eta})_{\mathbf{8}_{[002]}}(\boldsymbol{\eta})_{\mathbf{1}_{[002]}}$ non-interacting energy, found with significant operator-state overlap onto the associated operator - shown in the histogram labelled ' $\mathcal{N}$ ' in Figure 6.2. The corresponding principal correlator shown in Figure 6.4 appears to have residual excited-state contamination. This was refitted for a number of different $t$-ranges and a systematic error was added reflecting this - the final value being $a_{t} E_{\mathrm{cm}}=0.4640(57)$.

Before we proceed to a scattering analysis, we make some qualitative observations on the spectra shown in Figure 6.2. Consider first the number of energy levels expected on each volume, beginning with the largest, $L / a_{s}=24$. In the absence of any residual meson-meson interactions, we would expect one level at each corresponding meson-meson non-interacting energies, with the appropriate number of degenerate levels where the multiplicity is greater than one. This results in seventeen levels. We find however a total of eighteen, with an 'extra level', labelled ' $\mathcal{M}$ ', appearing in the cluster of energies at $a_{t} E_{\mathrm{cm}} \sim 0.46$. This extra level can be clearly seen on the $L / a_{s}=16$ and 20 volumes, away from any nearby non-interacting energies, also around $a_{t} E_{\mathrm{cm}} \sim 0.46$. On the smallest volume, $L / a_{s}=12$, the shifts from the non-interacting energies are more pronounced but a total of six levels are found, compared with the five anticipated in the non-interacting regime. Collectively, these observations are similar to those made in the $b_{1}$ study in Chapter 5 , albeit on a spectra far more dense with more coupled channels. Nevertheless, this suggests that a narrow resonance is present.

The operator-state overlap factors shown for the smallest and largest volumes in Figure 6.2 allow us to make further qualitative observations. For the largest volume, the lowest six levels, ' $\mathcal{A}-\mathcal{F}$ ', are all consistent with the corresponding non-interacting energies and have dominant overlap with the associated operators, either $(\boldsymbol{\eta})_{\mathbf{8}}(\boldsymbol{\eta})_{\mathbf{1}^{-}}$or $(\boldsymbol{\omega})_{\mathbf{8}}(\boldsymbol{\eta})_{\mathbf{8}}$-like, with negligible overlap onto any others. This suggests no resonant behaviour in this energy region and fairly weak interactions.

Around $a_{t} E_{\mathrm{cm}} \sim 0.45$, a level (' $\mathcal{G}$ ') consistent with the non-interacting $(\boldsymbol{\omega})_{\mathbf{8}_{[111]}}(\boldsymbol{\eta})_{\mathbf{8}_{[111]}}$ energy is seen with dominant overlap onto the $(\boldsymbol{\omega})_{\mathbf{8}_{[111]}}(\boldsymbol{\eta})_{\mathbf{8}_{[111]}}$ operator but also with moderate mixing with hybrid $q \bar{q}$-like operators. Above this, five vector-vector levels can be clearly identified, ' $\mathcal{H}-\mathcal{L}$ ', of which modest mixing with the hybrid single-meson operators is seen for a couple. Located on the top of this cluster is a level with dominant overlap 
onto the hybrid fermion bilinear operators - ' $\mathcal{M}$ '. A large amount of mixing is seen with $\left(\boldsymbol{h}_{\mathbf{1}}\right)_{\mathbf{8}_{[000]}}(\boldsymbol{\eta})_{\mathbf{8}_{[000]}}$ and $\left(\boldsymbol{f}_{\mathbf{1}}\right)_{\mathbf{8}_{[000]}}(\boldsymbol{\eta})_{\mathbf{8}_{[000]}}$ and also with the $(\boldsymbol{\omega})_{\mathbf{8}}(\boldsymbol{\eta})_{\mathbf{8}^{-}}$-like operators, peaked at the $(\boldsymbol{\omega})_{\mathbf{8}_{[111]}}(\boldsymbol{\eta})_{\mathbf{8}_{[111]}}$ momenta combination. One might expect this given the level observed to have dominant overlap onto $(\boldsymbol{\omega})_{\mathbf{8}_{[111]}}(\boldsymbol{\eta})_{\mathbf{8}_{[111]}}$ also had significant contributions from $q \bar{q}$-like operators. A small amount of mixing is seen with the other two-meson operators. Next highest in energy is the refitted level, ' $\mathcal{N}$ ', dominated by $(\boldsymbol{\eta})_{\mathbf{8}_{[002]}}(\boldsymbol{\eta})_{\mathbf{1}[002]}$ with small overlap across all other operators.

Above the $\left(f_{1}\right)_{\mathbf{8}}(\boldsymbol{\eta})_{\mathbf{8}}$ threshold are four levels, the lowest three, ' $\mathcal{O}-\mathcal{Q}$ ', showing vary-

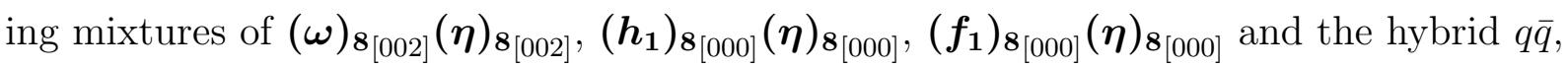
suggesting significant interactions between these channels around this energy region. The final level (' $\mathcal{R}$ ') is consistent with the $\left(\boldsymbol{f}_{1}\right)_{1}(\boldsymbol{\eta})_{\mathbf{8}}$ threshold and appears decoupled, with dominant overlap onto $\left(\boldsymbol{f}_{\mathbf{1}}\right)_{\mathbf{1}_{[000]}}(\boldsymbol{\eta})_{\mathbf{8}_{[000]}}$ and negligible overlap for all other operators.

As a comparison, we examine the operator-state overlap factors on the smallest volume. Here, we expect any interactions to yield more pronounced shifts in the energy spectra away from the corresponding non-interacting energies $5^{5}$ This appears to be the case and all levels show a large amount of mixing in the overlaps factors, with the exception of $\left(\boldsymbol{f}_{\mathbf{1}}\right)_{\mathbf{1}_{[000]}}(\boldsymbol{\eta})_{\mathbf{8}_{[000]}}$ which again appears to be totally decoupled.

The $\left(\boldsymbol{f}_{\mathbf{1}}\right)_{\mathbf{1}}(\boldsymbol{\eta})_{\mathbf{8}}$ threshold is taken as a cut-off and all levels below $a_{t} E_{\mathrm{cm}}=0.48$ are utilised in the scattering analysis (the black points in Figure 6.2). In addition, the black level just above the $\left(\boldsymbol{f}_{\mathbf{1}}\right)_{\mathbf{1}}(\boldsymbol{\eta})_{\mathbf{8}}$ threshold on the $L / a_{s}=12$ is included - anticipated to provide good constraints on mixing with the $\left(\boldsymbol{f}_{\mathbf{1}}\right)_{\mathbf{8}}(\boldsymbol{\eta})_{\mathbf{8}}$ and $\left(\boldsymbol{h}_{\mathbf{1}}\right)_{\mathbf{8}}(\boldsymbol{\eta})_{\mathbf{8}}$ channels, as evident from the operator-state overlaps ${ }^{6}$

\footnotetext{
${ }^{5} \mathrm{~A}$ consequence of the smaller polynomial-suppressed volume factors, relative to the largest lattice, appearing in the quantisation condition.

${ }^{6}$ Since $\left(f_{1}\right)_{1}(\eta)_{\mathbf{8}}$ appears to be totally decoupled, we include this level above $\left(f_{1}\right)_{\mathbf{1}}(\eta)_{\mathbf{8}}$-threshold.
} 


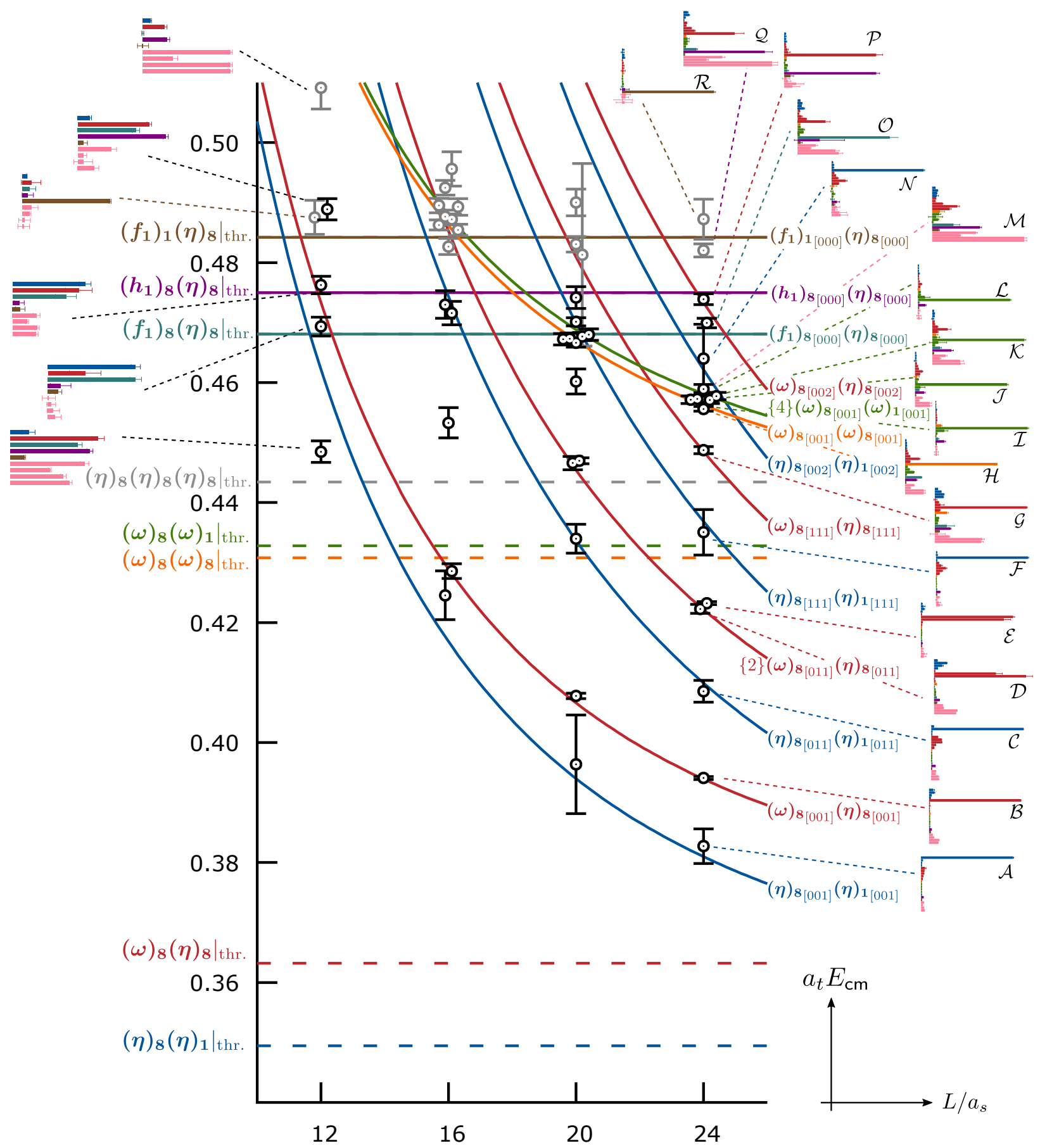

Figure 6.2: Finite-volume spectrum in the $[000] T_{1}^{-}$irrep on four lattice volumes. Points give the energy levels, including statistical uncertainties, from a variational analysis using the operator bases in Table 6.3, with black points used in the scattering analysis in Section 6.4. Solid curves are two-meson non-interacting energies and dashed horizontal lines show the two- and three-meson thresholds. Multiplicities (if greater than one) are shown as $\{n\}$. For each energy level on the largest and smallest volume, we present histograms showing the operator-state overlap factors, as in Figure 5.4, for the $\mathbb{M M}=(\eta)_{8}(\eta)_{1}$ (dark blue), $(\omega)_{8}(\eta)_{8}$ (red), $(\omega)_{8}(\omega)_{8}$ (orange), $(\omega)_{8}(\omega)_{1}$ (green), $\left(f_{1}\right)_{8}(\eta)_{8}$ (light blue), $\left(h_{1}\right)_{8}(\eta)_{8}$ (purple) and $\left(f_{1}\right)_{1}(\eta)_{8}$ (brown) operators along with a sample set of single-meson hybrid operators subduced from $J^{P}=1^{-}$(pink). 

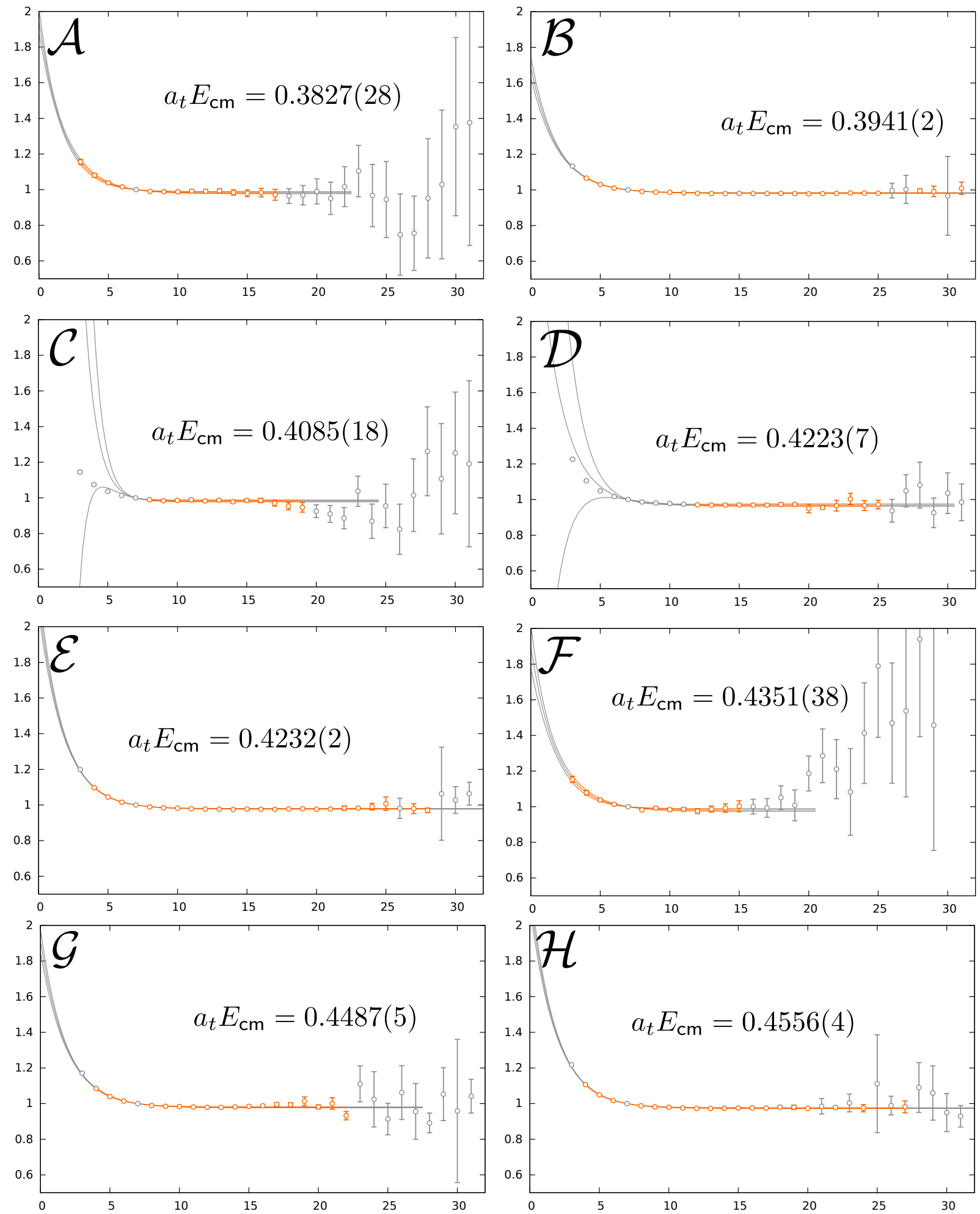

Figure 6.3: Principal correlators, $\lambda_{\mathfrak{n}}\left(t, t_{0}=7\right)$, plotted as $e^{E_{\mathfrak{n}}\left(t-t_{0}\right)} \lambda_{\mathfrak{n}}\left(t, t_{0}\right)$, from a variational analysis of the $[000] T_{1}^{-}$irrep on the lattice with $L / a_{s}=24$. Curves show the results of fits described in Section 2.6. The horizontal axes are in units of $t / a_{t}$. The lettering corresponds to the lettering in Figure 6.2 . 

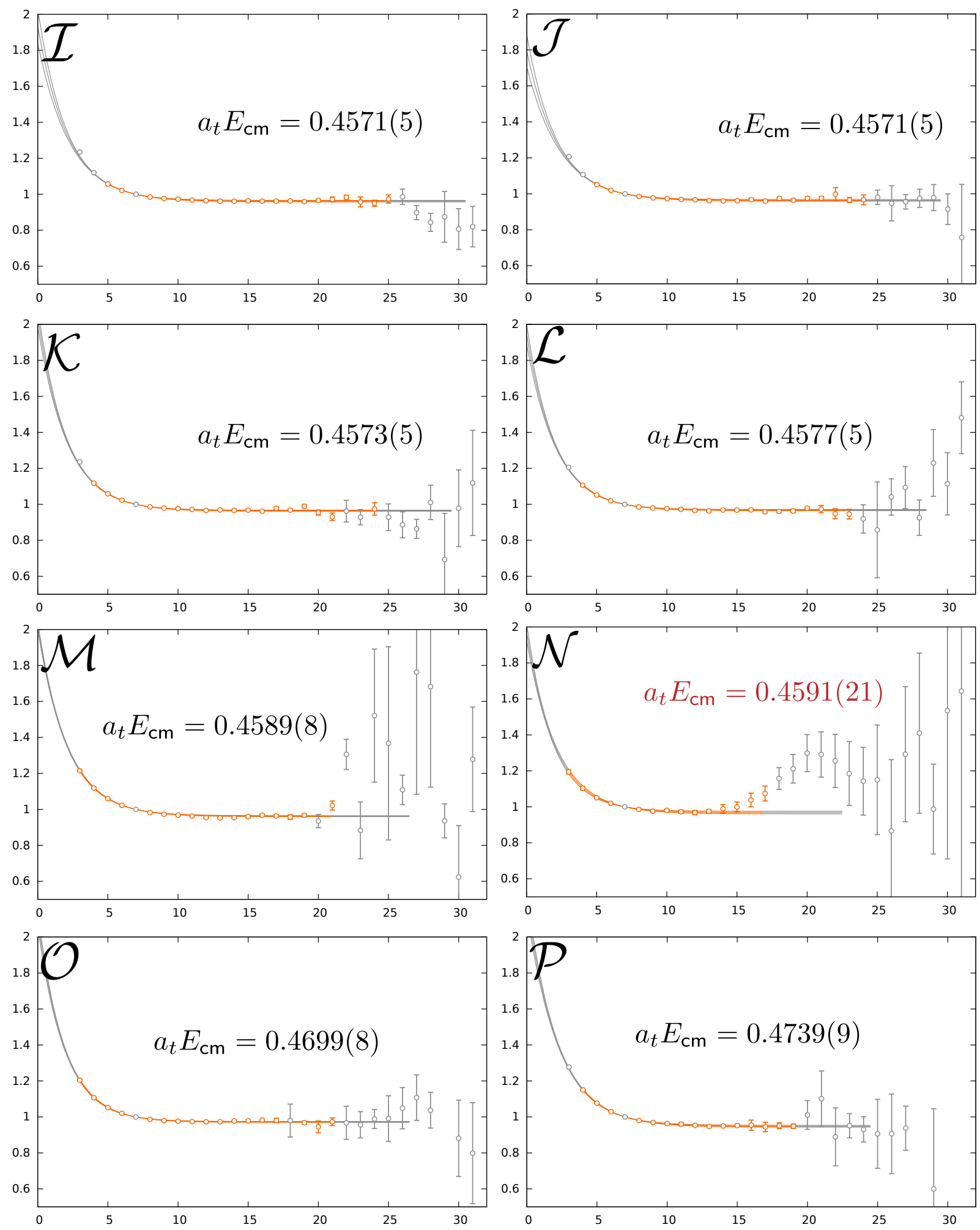

Figure 6.4: Continuation of Figure 6.3. 

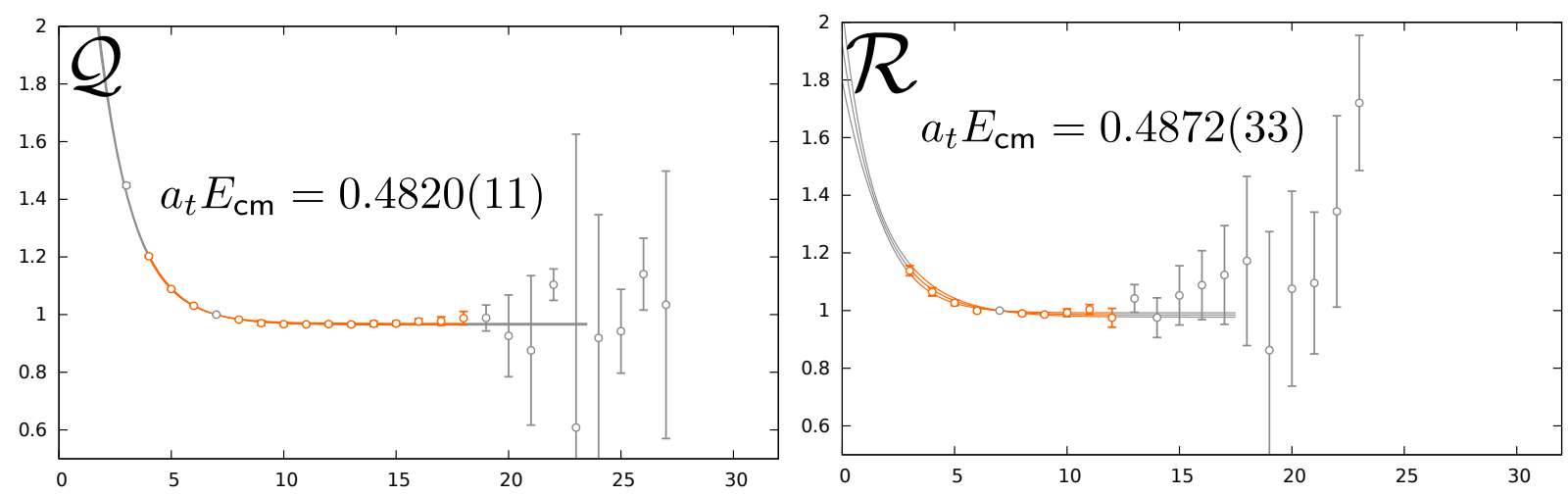

Figure 6.5: Continuation of Figure 6.3 . 


\subsection{Scattering Analysis}

In this section, we present the results of the scattering analysis, following the procedure described in Section 3.6, and once again use the general two-body formalism set out in Section 2.7.

Recalling the discussion in the introduction, we analyse eight coupled $J^{P}=1^{-}$channels. We have a single pseudoscalar-pseudoscalar channel, $(\boldsymbol{\eta})_{\mathbf{8}}(\boldsymbol{\eta})_{\mathbf{1}}\left\{{ }^{1} P_{1}\right\}$, and three (axial)vector-pseudoscalar channels: $(\boldsymbol{\omega})_{\mathbf{8}}(\boldsymbol{\eta})_{\mathbf{8}}\left\{{ }^{3} P_{1}\right\},\left(\boldsymbol{f}_{\mathbf{1}}\right)_{\mathbf{8}}(\boldsymbol{\eta})_{\mathbf{8}}\left\{{ }^{3} S_{1}\right\}$ and $\left(\boldsymbol{h}_{\mathbf{1}}\right)_{\mathbf{8}}(\boldsymbol{\eta})_{\mathbf{8}}\left\{{ }^{3} S_{1}\right\}$. In addition to these vector-pseudoscalar like channels, where only one total intrinsic spin of $S=1$ is possible, we also incorporate vector-vector channels. The three distinct spin-spin combinations, $S=0,1,2$, give rise here to three dynamically-coupled $J^{P}=1^{-}$partialwaves, namely $(\omega)_{\mathbf{8}}(\omega)_{1}\left\{{ }^{1} P_{1}\right\},(\omega)_{\mathbf{8}}(\omega)_{1}\left\{{ }^{3} P_{1}\right\}$ and $(\omega)_{\mathbf{8}}(\omega)_{1}\left\{{ }^{5} P_{1}\right\}$, distinguished by the 'spin-spin' couplings. Bose-symmetry forbids the same three partial-wave configurations for the $(\omega)_{\mathbf{8}}(\omega)_{\mathbf{8}}$ meson-meson channel - only the ${ }^{3} P_{1}$-wave is non-vanishing.

As well as the eight $J^{P}=1^{-}$coupled-channels, we must also incorporate a further two $J^{P}=3^{-}$channels that mix in $[000] T_{1}^{-}$due to the reduced symmetry of the lattice. The first is $(\boldsymbol{\omega})_{\mathbf{8}}(\boldsymbol{\eta})_{\mathbf{8}}\left\{{ }^{3} F_{3}\right\}$, contributing at the next leading order in threshold suppression at the two-fold degenerate $(\boldsymbol{\omega})_{\mathbf{8}_{[011]}}(\boldsymbol{\eta})_{\mathbf{8}_{[011]}}$ non-interacting energy. Second is the $(\boldsymbol{\omega})_{\mathbf{8}}(\boldsymbol{\omega})_{1}\left\{{ }^{5} P_{3}\right\}$ channel, contributing at the four-fold degenerate $(\omega)_{\mathbf{8}_{[001]}}(\boldsymbol{\omega})_{\mathbf{1}_{[001]}}$ non-interacting energy.

Before presenting the results of the scattering analysis, we make an important observation having utilised the rest-frame spectra only. The quantisation condition subduced in the $[000] T_{1}^{-}$irrep is invariant under the interchange of the $(\omega)_{8}(\omega)_{1}\left\{{ }^{1} P_{1}\right\},(\omega)_{8}(\omega)_{1}\left\{{ }^{3} P_{1}\right\}$ and $(\boldsymbol{\omega})_{\mathbf{8}}(\boldsymbol{\omega})_{\mathbf{1}}\left\{{ }^{5} P_{1}\right\} \boldsymbol{t}$-matrix scattering parameters. Therefore, as these amplitudes cannot be uniquely determined, we refer to them collectively as $(\boldsymbol{\omega})_{\mathbf{8}}(\boldsymbol{\omega})_{\mathbf{1}}\left\{{ }^{X} P_{1}\right\}$ for subsequent phase-shifts, mixing-angles and resonant couplings. A proof of this observation is presented in Appendix 6.B.

In order to parameterise the energy dependence of the ten-channel $\boldsymbol{t}$-matrix, we use a $\boldsymbol{K}$-matrix formulation, recalling the relationship between the $\boldsymbol{t}$-matrix and $\boldsymbol{K}$-matrix given in Eq. 5.6. This is block diagonal with respect to $J^{P}$ and we write,

$$
\boldsymbol{K}(s)=\left(\begin{array}{cc}
\boldsymbol{K}_{\mathbf{1}^{-}}(s) & 0 \\
0 & \boldsymbol{K}_{\mathbf{3}^{-}}(s)
\end{array}\right) .
$$


For the eight-by-eight $\boldsymbol{K}_{1^{-}}(s)$ matrix, hosting the $J^{P}=1^{-}$amplitudes, we adopt the 'pole + constant' form of parameterisation,

$$
\boldsymbol{K}_{\mathbf{1}^{-}}(s)=\frac{\boldsymbol{g}^{\mathbf{2}}(s)}{m^{2}-s}+\boldsymbol{\gamma}_{\mathbf{1}^{-}}^{(\mathbf{0})},
$$

where $\boldsymbol{g}^{\mathbf{2}}(s)$ is the matrix with components $g_{S \ell J a}(s) g_{S^{\prime} \ell^{\prime} J b}(s)$, recalling $a, b$ are hadron channel labels, and $\gamma_{1^{-}}^{(0)}$ is a real constant symmetric matrix. There are many degrees of freedom in this form and we make two further reasonable restrictions. First, shown to be sufficient in the $b_{1}$ analysis in Chapter 5, we take $g_{S \ell J a}(s)$ to be constant. The second follows from observations of the finite-volume spectra in Figure 6.2. Energy levels with dominant operator-state overlap factors onto vector-vector channels appear very near the corresponding non-interacting energies and have small operator-state overlap factors with all other operators. We anticipate that these are weakly coupled and, as such, block diagonalise the constant $\gamma_{1^{-}}^{(\mathbf{0})}$ matrix with respect to the vector-vector channels,

$$
\gamma_{1^{-}}^{(0)}=\left(\begin{array}{cc}
\gamma_{1^{-}}^{(0)}{ }^{\mathrm{nVV}} . & 0 \\
0 & \gamma_{1^{-}}^{(0) \mathrm{VV}}
\end{array}\right)
$$

and allow parameter freedom in the diagonal components only of the four-by-four vectorvector block, $\gamma_{1^{-}}^{(\mathbf{0}) \mathrm{VV}}$. We impose no restrictions on the four-by-four non vector-vector block, $\gamma_{1^{-}}^{(\mathbf{0}) \mathrm{nVV}}$. Note that mixing amongst the vector-vector channels and between other $J^{P}=1^{-}$channels is still permitted through the matrix of couplings, $\boldsymbol{g}^{\mathbf{2}}(s)$.

We utilise three phase-space prescriptions: simple phase-space, where $I_{1^{-}}(s)=-i \rho_{a}(s)$, and the Chew-Mandelstam phase-space, with $\operatorname{Re} I_{1^{-} a}\left(s=m^{2}\right)=0$ or $\operatorname{Re} I_{1^{-} a}\left(s=s_{\text {thr. }}\right)=$ 0 .

Regarding the two-by-two matrix of $J^{P}=3^{-}$amplitudes, we set $\boldsymbol{K}_{\mathbf{3}^{-}}(s)=\boldsymbol{\gamma}_{\mathbf{3}^{-}}^{(\mathbf{0})}$ where,

$$
\gamma_{\mathbf{3}^{-}}^{(0)}=\left(\begin{array}{cc}
\gamma_{(\omega)_{\mathbf{8}}(\eta)_{\mathbf{8}}\left\{{ }^{3} F_{3}\right\},(\omega)_{\mathbf{8}}(\eta)_{\mathbf{8}}\left\{{ }^{3} F_{3}\right\}}^{(0)} & 0 \\
0 & \gamma_{(\omega)_{\mathbf{8}}(\omega)_{1}\left\{{ }^{5} P_{3}\right\},(\omega)_{\mathbf{8}}(\omega)_{1}\left\{{ }^{5} P_{3}\right\}}^{(0)}
\end{array}\right)
$$

No exotic $J^{P C}=3^{-+}$resonances are expected in this energy region and we will show that a constant matrix of this form proves to be sufficient to describe the spectra. We use a Chew-Mandelstam prescription with $\operatorname{Re} I_{3^{-}}{ }_{a}\left(s=s_{\text {thr. }}\right)=0$ in all parameterisations.

We find parameterisations of the form given in Eq. 6.5, subject to these additional reasonable constraints discussed above, feature 25 independent free parameters and are sufficiently flexible to test the robustness of the scattering amplitudes. 
Scattering amplitudes are determined following the procedure detailed in the previous chapters. We fit many parameterisations to the 38 energy levels shown as black points in Figure 6.2 and record successful fits, with $\chi^{2} / N_{\text {dof }}<1.2$, in Table 6.5 of Appendix 6.C.

Analogous to the scattering analysis in the previous two chapters, we choose a reference amplitude, representative of all other parameterisations. Here, we take the reference parameterisation to be diagonal in $\gamma_{1^{-}}^{(0)}$ with freedom in each of the eight diagonal components and take all but $g_{(\boldsymbol{\omega})_{\mathbf{8}}(\boldsymbol{\omega})_{1}\left\{{ }^{1} P_{1}\right\}}$ and $g_{(\boldsymbol{\omega})_{8}(\boldsymbol{\omega})_{1}\left\{{ }^{5} P_{1}\right\}}$ to be non-zero in $\boldsymbol{g}^{2}$, giving a total of seventeen free parameters. The Chew-Mandelstam prescription with pole subtraction, Re $I_{1^{-}}{ }_{a}\left(s=m^{2}\right)=0$, is taken for $\boldsymbol{K}_{\mathbf{1}^{-}}(s)$. This parameterisation is shown in bold in Table 6.5 and the resulting fit to the lattice data yields the following parameters,

$$
\begin{aligned}
& m=(0.4622 \pm 0.0028 \pm 0.0005) \cdot a_{t}^{-1} \gamma_{(\eta)_{8}(\eta)_{1}\left\{{ }^{1} P_{1}\right\}}^{(0)}=(-1.9 \pm 1.2 \pm 3.0) \cdot a_{t}^{2} \\
& g_{(\eta)_{8}(\eta)_{1}\left\{P_{1}\right\}}=(0.40 \pm 0.13 \pm 0.10) \\
& g_{(\omega)_{8}(\eta)_{8}\left\{{ }^{3} P_{1}\right\}}=(0.40 \pm 0.12 \pm 0.02) \\
& g_{\left(\boldsymbol{f}_{1}\right)_{\mathbf{8}}(\boldsymbol{\eta})_{\mathbf{8}}\left\{{ }^{3} S_{1}\right\}}=(0.31 \pm 0.13 \pm 0.10) \cdot a_{t}^{-1} \\
& g_{\left(\boldsymbol{h}_{\mathbf{1}}\right)_{\mathbf{8}}(\boldsymbol{\eta})_{\mathbf{8}}\left\{{ }^{3} S_{1}\right\}}=(-0.29 \pm 0.21 \pm 0.09) \cdot a_{t}^{-1} \\
& g_{(\omega)_{8}(\omega)_{8}\left\{{ }^{3} P_{1}\right\}}=(-0.23 \pm 0.15 \pm 0.02) \\
& g_{(\omega)_{8}(\omega)_{1}\left\{{ }^{3} P_{1}\right\}}=(0.20 \pm 0.25 \pm 0.04) \\
& \gamma_{(\omega)_{8}(\eta)_{8}\left\{{ }^{3} F_{3}\right\}}^{(0)}=(-340 \pm 130 \pm 270) \cdot a_{t}^{6} \\
& \gamma_{(\omega)_{8}(\eta)_{8}\left\{{ }^{3} P_{1}\right\}}^{(0)}=(-6.5 \pm 1.2 \pm 4.8) \cdot a_{t}^{2} \\
& \gamma_{\left(f_{1}\right)_{8}(\eta)_{8}\left\{{ }^{3} S_{1}\right\}}^{(0)}=(-2.2 \pm 1.1 \pm 1.0) \\
& \gamma_{\left(\boldsymbol{h}_{\mathbf{1}}\right)_{\mathbf{8}}(\boldsymbol{\eta})_{\mathbf{8}}\left\{{ }^{3} S_{1}\right\}}^{(0)}=(0.3 \pm 1.0 \pm 0.2) \\
& \gamma_{(\omega)_{8}(\omega)_{8}\left\{{ }^{3} P_{1}\right\}}^{(0)}=(2.9 \pm 4.3 \pm 4.1) \cdot a_{t}^{2} \\
& \gamma_{(\omega)_{8}(\omega)_{1}\left\{{ }^{1} P_{1}\right\}}^{(0)}=(12 \pm 13 \pm 7) \cdot a_{t}^{2} \\
& \gamma_{(\omega)_{8}(\omega)_{1}\left\{{ }^{3} P_{1}\right\}}^{(0)}=(9.2 \pm 6.8 \pm 5.7) \cdot a_{t}^{2} \\
& \gamma_{(\omega)_{8}(\omega)_{1}\left\{{ }^{5} P_{1}\right\}}^{(0)}=(12 \pm 13 \pm 7) \cdot a_{t}^{2} \\
& \gamma_{(\omega)_{8}(\omega)_{1}\left\{{ }^{5} P_{3}\right\}}^{(0)}=(3.7 \pm 9.6 \pm 5.4) \cdot a_{t}^{2} \\
& \chi^{2} / N_{\text {dof }}=\frac{15.1}{38-17}=0.72 \text {, }
\end{aligned}
$$

where the first uncertainty is statistical and the second reflects the systematic uncertainties obtained from considering variations in the stable hadron masses and anisotropy. The systematic uncertainties we quote are calculated as the largest shift on the central values of each parameter, taken from successful fits of the scattering amplitudes where the anisotropy and stable hadron masses are varied by $\pm 1 \sigma .7$ The correlation matrix for the reference amplitude scattering parameters is given in Table 6.6 in Appendix 6.C.

\footnotetext{
${ }^{7}$ We vary all the stable hadron masses in the same way, i.e. all $+1 \sigma$ or all $-1 \sigma$.
} 
The $J^{P}=1^{-}$phase-shifts, presented using the 8-channel Stapp-parameterisation (detailed in Chapter 7), and the two $J^{P}=3^{-}$phase-shifts are plotted in Figures 6.6-6.7. The solid coloured curves and error bands show the reference amplitude and all other successful parameterisations, listed in Table 6.5, are shown as dashed coloured curves with grey error bands.

We are not able to uniquely determine the sign of the off-diagonal elements of the $\boldsymbol{t}$-matrix between the dynamically-coupled ${ }^{1} P_{1^{-}},{ }^{3} P_{1^{-}}$and ${ }^{5} P_{1^{-}}(\boldsymbol{\omega})_{\mathbf{8}}(\boldsymbol{\omega})_{\mathbf{1}}$ in the rest frame irrep. This is because the $\overline{\mathcal{M}}$-matrix is diagonal, as shown in Appendix 6.B, and the determinant is subsequently independent on the sign of these off-diagonal $\boldsymbol{t}$-matrix terms. In addition, all other off-diagonal $\boldsymbol{t}$-matrix components also have a sign ambiguity, as each hadron channel can be rephased independently, subject to the $\boldsymbol{S}$-matrix being symmetric.

Because of this, all the mixing-angles are presented as sign-independent inelasticities, defined as $\cos 2 \bar{\epsilon}_{i j}$. With this definition, an inelasticity of unity means no mixing, and of zero means maximally mixed. The $J^{P}=1^{-}$inelasticities are displayed in Figures $6.8-6.10$. The $J^{P}=3^{-}$amplitudes have a mixing-angle fixed to be zero in all parameterisations.

For all parameterisations presented in Table 6.5, the phase-shifts and inelasticities are in very good agreement. The statistical and combined statistical and systematic uncertainties of the reference amplitude are shown as the inner and outer coloured bands respectively, in each of the figures. We see the systematic uncertainty on each phase-shift and inelasticity is roughly comparable to the size of statistical uncertainties.

We make some qualitative observations from Figures 6.6 - 6.10. Firstly, the $(\boldsymbol{\eta})_{\mathbf{8}}(\boldsymbol{\eta})_{1}\left\{{ }^{1} P_{1}\right\}$ phase-shift has a sharp rise through $90^{\circ}$ at $a_{t} E_{\mathrm{cm}} \sim 0.46$, characteristic of a narrow resonance located in this region. The $(\boldsymbol{\omega})_{\mathbf{8}}(\boldsymbol{\eta})_{\mathbf{8}}\left\{{ }^{3} P_{1}\right\}$ phase-shift turns very sharply around this energy and the $(\boldsymbol{\eta})_{\mathbf{8}}(\boldsymbol{\eta})_{\mathbf{1}}\left\{{ }^{1} P_{1}\right\} \mid(\boldsymbol{\omega})_{\mathbf{8}}(\boldsymbol{\eta})_{\mathbf{8}}\left\{{ }^{3} P_{1}\right\}$ inelasticity approaches zero. This would suggest a resonance strongly coupled to both $(\boldsymbol{\eta})_{\mathbf{8}}(\boldsymbol{\eta})_{\mathbf{1}}\left\{{ }^{1} P_{1}\right\}$ and $(\boldsymbol{\omega})_{\mathbf{8}}(\boldsymbol{\eta})_{\mathbf{8}}\left\{{ }^{3} P_{1}\right\}$. The large uncertainties in the reference amplitude for the $(\boldsymbol{\eta})_{\mathbf{8}}(\boldsymbol{\eta})_{\mathbf{1}}\left\{{ }^{1} P_{1}\right\}$ and $(\boldsymbol{\omega})_{\mathbf{8}}(\boldsymbol{\eta})_{\mathbf{8}}\left\{{ }^{3} P_{1}\right\}$ phase-shifts at $a_{t} E_{\mathrm{cm}} \sim 0.46$, are an artefact of the phase-shifts becoming extremely steep in this neighbourhood. Therefore, a small change in $a_{t} E_{\mathrm{cm}}$ around $a_{t} E_{\mathrm{cm}} \sim 0.46$ gives a large change in the $\boldsymbol{S}$-matrix and as such these phase-shifts have large uncertainties here. Other parameterisations, with less steep phase-shifts in this energy region, can be seen that do not exhibit this behaviour.

Phase-shifts in the vector-vector channels, $(\boldsymbol{\omega})_{\mathbf{8}}(\boldsymbol{\omega})_{\mathbf{8}}\left\{{ }^{3} P_{1}\right\}$ and $(\boldsymbol{\omega})_{\mathbf{8}}(\boldsymbol{\omega})_{\mathbf{1}}\left\{{ }^{X} P_{1}\right\}$, are 
relatively small and appear to be consistent with zero within uncertainty. Similarly, inelasticities with respect to any vector-vector channel are found to be near unity, although non-trivial behaviour around $a_{t} E_{\mathrm{cm}} \sim 0.46$ in the inelasticities with respect to both $(\boldsymbol{\eta})_{\mathbf{8}}(\boldsymbol{\eta})_{\mathbf{1}}\left\{{ }^{1} P_{1}\right\}$ and $(\boldsymbol{\omega})_{\mathbf{8}}(\boldsymbol{\eta})_{\mathbf{8}}\left\{{ }^{3} P_{1}\right\}$ suggests some sensitivity to the resonance-like behaviour.

The phase-shifts of the axial-vector-pseudoscalar channels, $\left(\boldsymbol{f}_{\mathbf{1}}\right)_{\mathbf{8}}(\boldsymbol{\eta})_{\mathbf{8}}\left\{{ }^{3} S_{1}\right\}$ and $\left(\boldsymbol{h}_{\mathbf{1}}\right)_{\mathbf{8}}(\boldsymbol{\eta})_{\mathbf{8}}\left\{{ }^{3} S_{1}\right\}$, appear to turn on rapidly at threshold, as do the inelasticities featuring either of these channels. This behaviour in the inelasticities is somewhat unsurprising given the operator-state overlap factors shown in Figure 6.2, which show a large degree of mixing between operators corresponding to these channels - in particular on the $L / a_{s}=12$ lattice.

Finally, we comment on the $J^{P}=3^{-}$amplitudes presented in Figure 6.7. These appear to be very small in the energy range, with no signs of any resonant enhancement, as expected in this low-energy region.

To demonstrate the quality of the scattering amplitudes at reproducing the lattice spectra, in Figure 6.11, we present the finite-volume spectrum calculated using the reference amplitude. For the lattice computed spectrum on each volume, a single energy level with dominant operator-state overlap onto the $\left(f_{1}\right)_{1}(\boldsymbol{\eta})_{\mathbf{8}}$ operator has been removed for clarity in the figure - for example the level labelled ' $\mathcal{R}$ ' in Figure 6.2. Such levels will not be interpolated by our reference amplitude as it does not incorporate the $\left(\boldsymbol{f}_{\mathbf{1}}\right)_{\mathbf{1}}(\boldsymbol{\eta})_{\mathbf{8}}\left\{{ }^{3} S_{1}\right\}$ channel (this was anticipated to be largely decoupled as discussed in Section 6.3). We observe excellent agreement between each lattice computed energy used as a constraint and those obtained from the reference amplitude. In addition, levels coloured grey, that were excluded from the analysis, are seen to be very well described. Avoided level crossings, characteristic of a narrow resonance, can be clearly seen in an energy region centred around $a_{t} E_{\mathrm{cm}} \sim 0.46$.

To summarise, the observed sharp increase in the $(\boldsymbol{\eta})_{\mathbf{8}}(\boldsymbol{\eta})_{1}\left\{{ }^{1} P_{1}\right\}$ phase-shift and enhancements in a number of inelasticities around $a_{t} E_{\mathrm{cm}} \sim 0.46$, in addition to the "extra level' seen in the finite-volume spectra, strongly suggests the presence of a resonance. We proceed to determine the pole singularities of our scattering amplitudes in order to calculate any such resonances rigorously. 


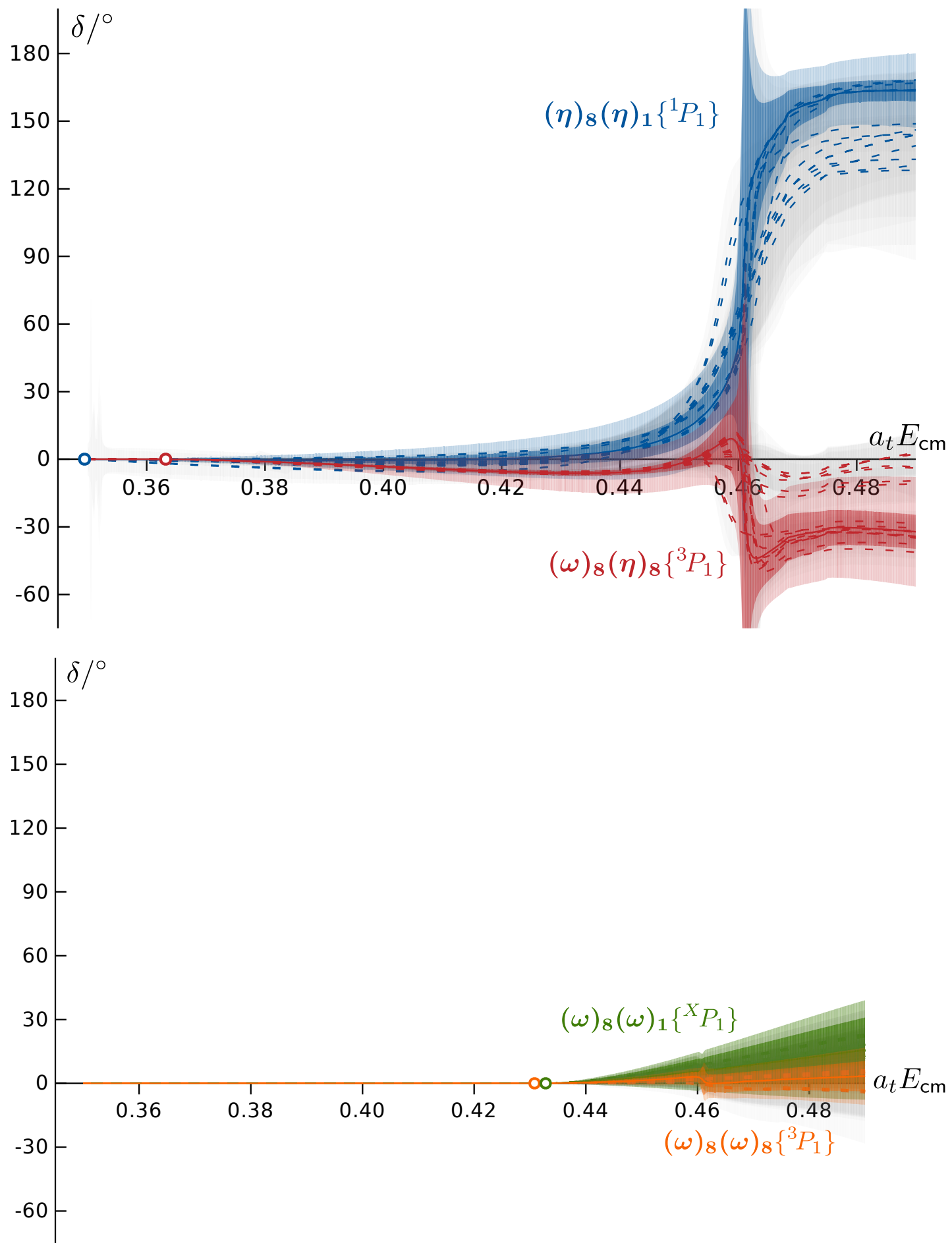

Figure 6.6: Upper: $(\boldsymbol{\eta})_{\mathbf{8}}(\boldsymbol{\eta})_{\mathbf{1}}\left\{{ }^{1} P_{1}\right\}$ (dark blue) and $(\boldsymbol{\omega})_{\mathbf{8}}(\boldsymbol{\eta})_{\mathbf{8}}\left\{{ }^{3} P_{1}\right\}$ (red) phase-shifts. Lower: $(\omega)_{\mathbf{8}}(\omega)_{\mathbf{8}}\left\{{ }^{3} P_{1}\right\}$ (orange) and $(\omega)_{\mathbf{8}}(\boldsymbol{\omega})_{\mathbf{1}}\left\{{ }^{X} P_{1}\right\}$ (green) phase-shifts. The solid curves show the reference amplitude given in Eq. 6.9 with the coloured bands reflecting the statistical (inner) plus systematic (outer) uncertainty. Dashed curves and grey bands correspond to all successful parameterisations presented in Table 6.5 of Appendix 6.C with only the statistical uncertainties shown. 

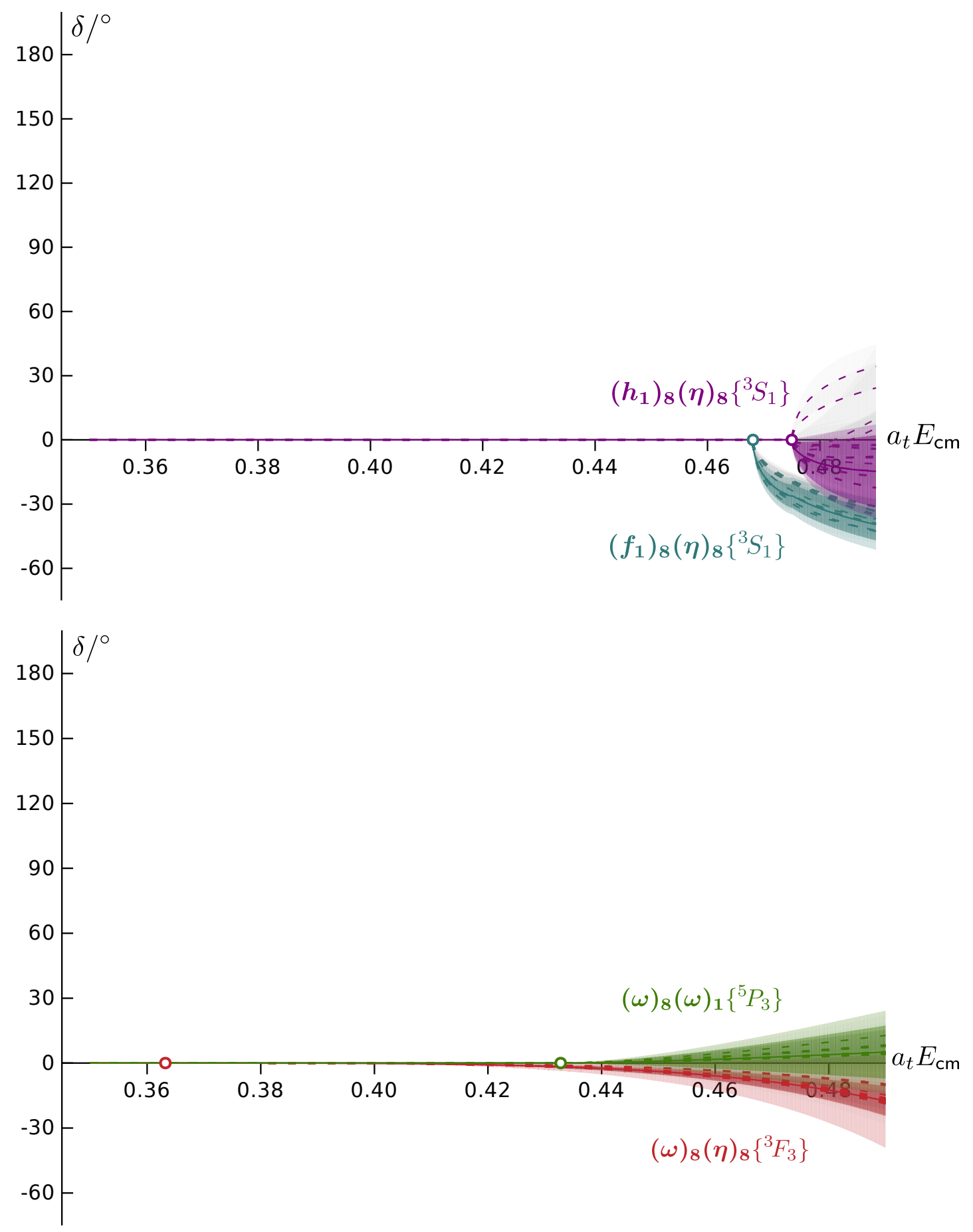

Figure 6.7: As in Figure 6.6 but for $\left(\boldsymbol{f}_{\mathbf{1}}\right)_{\mathbf{8}}(\boldsymbol{\eta})_{\mathbf{8}}\left\{{ }^{3} S_{1}\right\}$ (light blue) and $\left(\boldsymbol{h}_{\mathbf{1}}\right)_{\mathbf{8}}(\boldsymbol{\eta})_{\mathbf{8}}\left\{{ }^{3} S_{1}\right\}$ (purple) phase-shifts (upper), and for $(\boldsymbol{\omega})_{\mathbf{8}}(\boldsymbol{\eta})_{\mathbf{8}}\left\{{ }^{3} F_{3}\right\}$ (red) and $(\boldsymbol{\omega})_{\mathbf{8}}(\boldsymbol{\omega})_{\mathbf{1}}\left\{{ }^{5} P_{3}\right\}$ (green) phase-shifts (lower). 

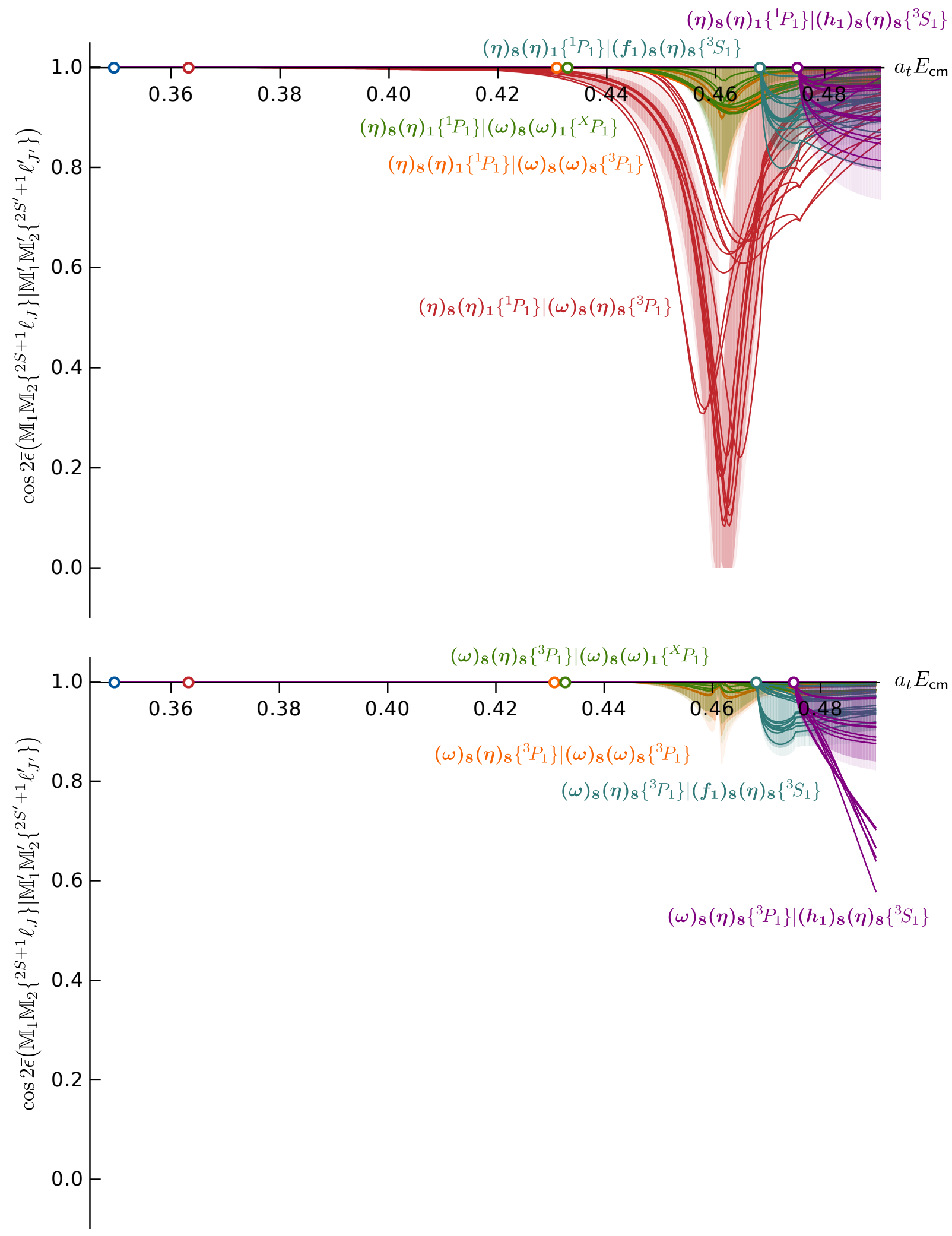

Figure 6.8: Upper: the inelasticities $\cos 2 \bar{\epsilon}\left((\boldsymbol{\eta})_{\mathbf{8}}(\boldsymbol{\eta})_{\mathbf{1}}\left\{{ }^{1} P_{1}\right\} \mid \ldots\right)$ Lower: inelasticities $\cos 2 \bar{\epsilon}\left((\boldsymbol{\omega})_{\mathbf{8}}(\boldsymbol{\eta})_{\mathbf{8}}\left\{{ }^{3} P_{1}\right\} \mid \ldots\right)$. The reference amplitude given in Eq. 6.9 is plotted with coloured bands reflecting the statistical (inner) plus systematic (outer) uncertainty. The solid curves show the inelasticities for all parameterisations presented in Table 6.5 in Appendix 6.C. Statistical uncertainties on these parameterisations are omitted for clarity in the plot. 

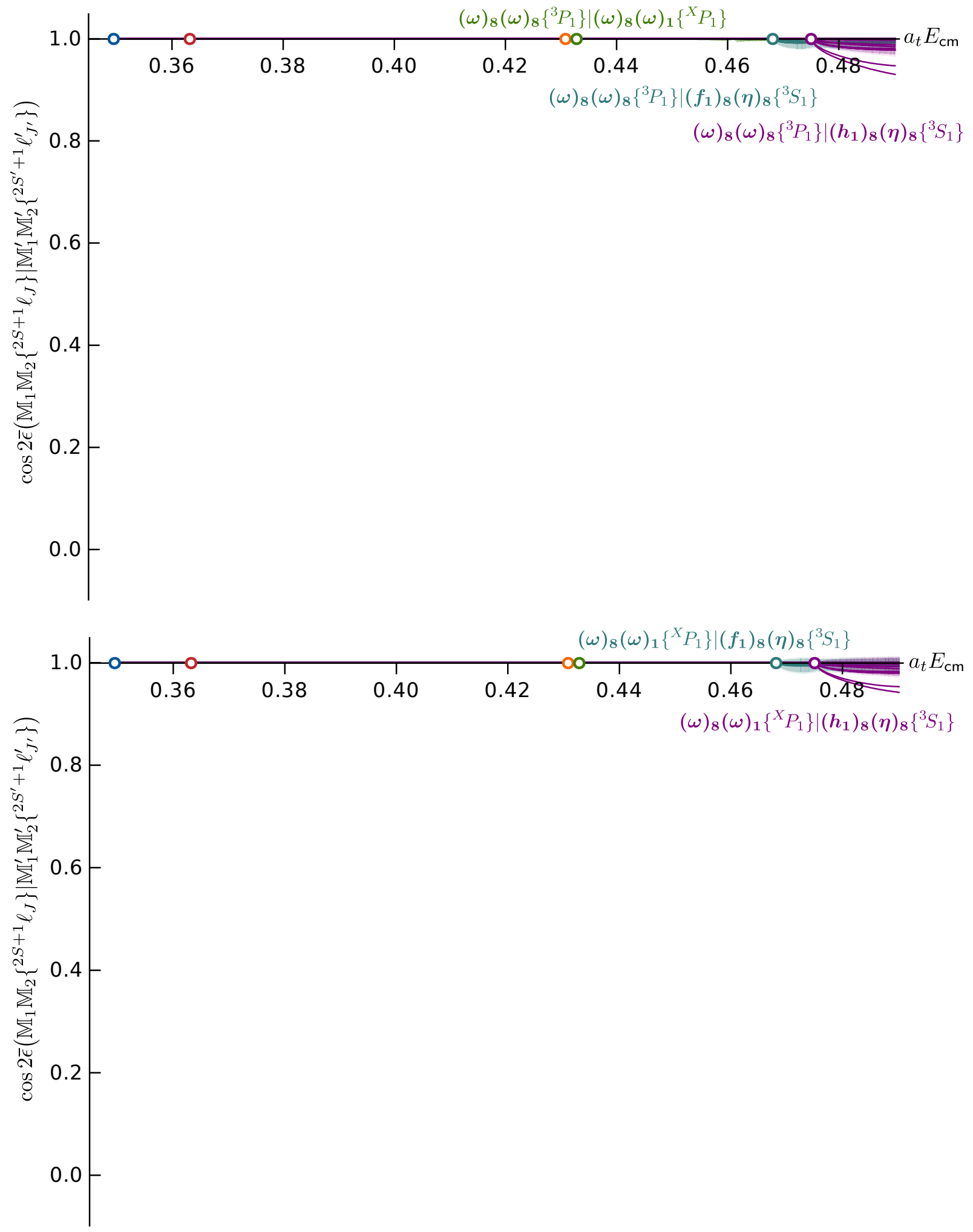

Figure 6.9: As in Figure 6.8 but for inelasticities $\cos 2 \bar{\epsilon}\left((\boldsymbol{\omega})_{8}(\boldsymbol{\omega})_{8}\left\{{ }^{3} P_{1}\right\} \mid \ldots\right)$ (upper), and for inelasticities $\cos 2 \bar{\epsilon}\left((\omega)_{8}(\omega)_{1}\left\{{ }^{X} P_{1}\right\} \mid \ldots\right)$ (lower). 


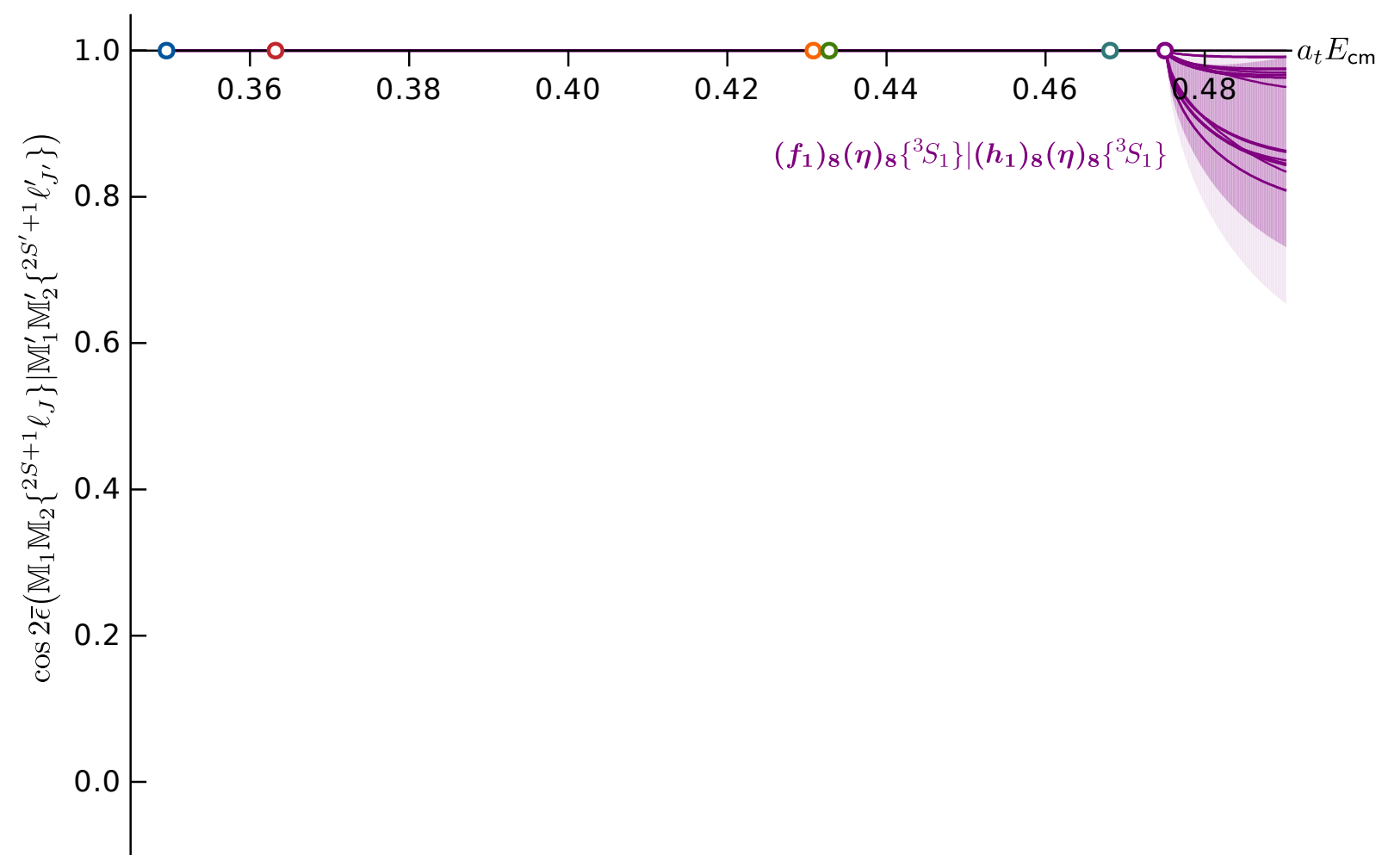

Figure 6.10: As in Figure 6.8 but for $\cos 2 \bar{\epsilon}\left(\left(\boldsymbol{f}_{\mathbf{1}}\right)_{\mathbf{8}}(\boldsymbol{\eta})_{\mathbf{8}}\left\{{ }^{3} S_{1}\right\} \mid \ldots\right)$ inelasticities. 


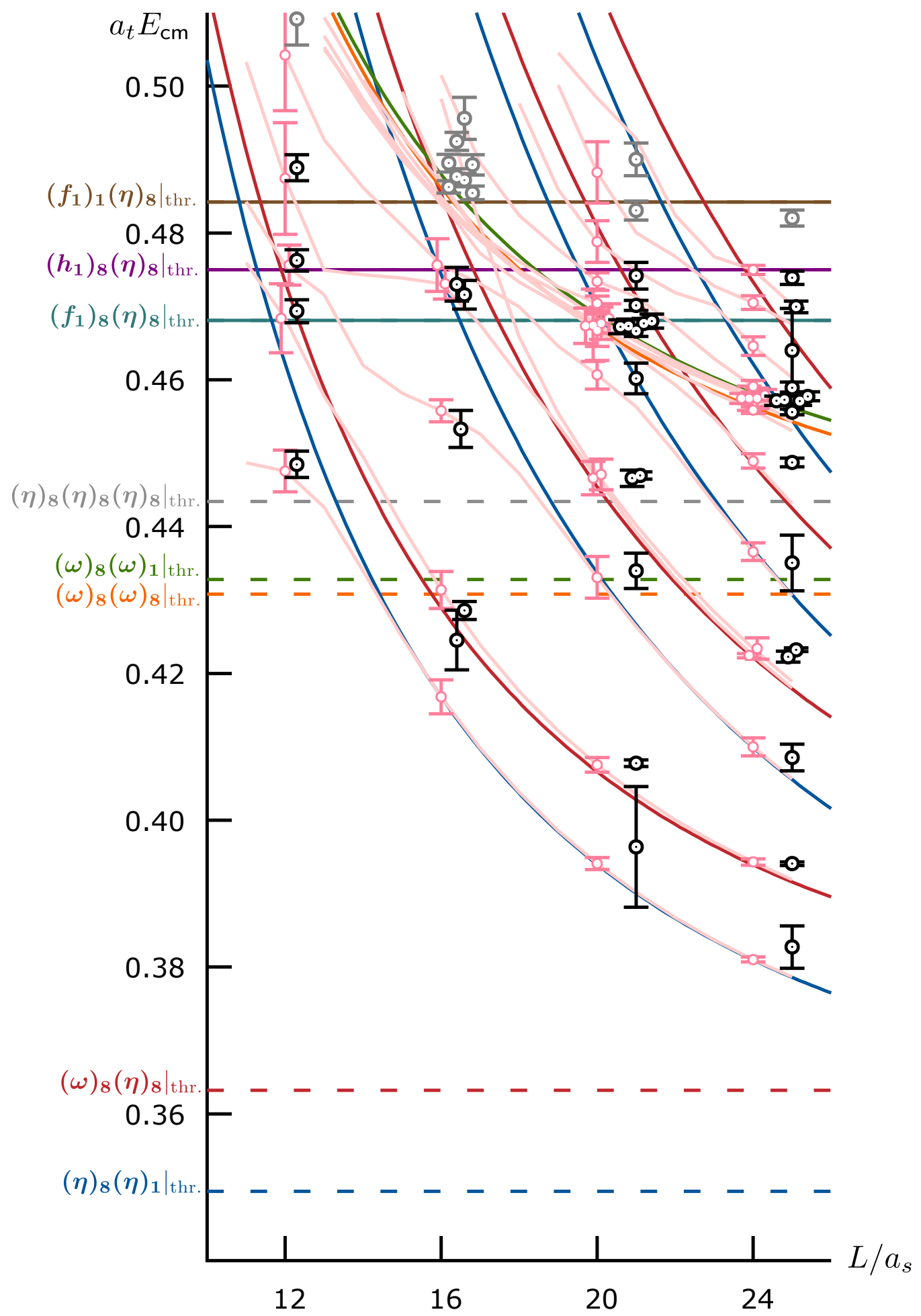

Figure 6.11: As Figure 6.2 but including, as pink curves and points, the energy levels calculated from the reference amplitude in Eq. 6.9, with errorbars reflecting the combined statistical and systematic uncertainties on the reference amplitude. The lattice computed energies are displaced horizontally for clarity. A single energy level on each lattice volume with dominant operator-state overlap onto the $\left(\boldsymbol{f}_{\mathbf{1}}\right)_{\mathbf{1}}(\boldsymbol{\eta})_{\mathbf{8}}$ operator has been removed as discussed in the text. 


\subsection{Pole Analysis}

In Section 5.6, we discussed pole singularities in coupled-channel scattering amplitudes. Here, we have six distinct hadron-hadron thresholds opening in the energy region considered and therefore a total of 64 Riemann sheets. We will label the sheets by the sign of the imaginary part of the momentum in the six channels, ordered according to increasing threshold energies, $\left((\boldsymbol{\eta})_{8}(\boldsymbol{\eta})_{1},(\omega)_{8}(\eta)_{8},(\omega)_{8}(\omega)_{8},(\omega)_{8}(\omega)_{1},\left(f_{1}\right)_{8}(\eta)_{8},\left(h_{1}\right)_{8}(\eta)_{8}\right)$. For a relatively narrow resonance pole located at $a_{t} E_{\mathrm{cm}} \sim 0.46$, the Riemann sheet closest to physical scattering would have a negative imaginary component of $\mathrm{cm}$-momenta in the $(\boldsymbol{\eta})_{\mathbf{8}}(\boldsymbol{\eta})_{\mathbf{1}},(\boldsymbol{\omega})_{\mathbf{8}}(\boldsymbol{\eta})_{\mathbf{8}},(\boldsymbol{\omega})_{\mathbf{8}}(\boldsymbol{\omega})_{\mathbf{8}}$ and $(\boldsymbol{\omega})_{\mathbf{8}}(\boldsymbol{\omega})_{\mathbf{1}}$ channels and a positive imaginary component in $\left(\boldsymbol{f}_{\mathbf{1}}\right)_{\mathbf{8}}(\boldsymbol{\eta})_{\mathbf{8}}$ and $\left(\boldsymbol{h}_{\mathbf{1}}\right)_{\mathbf{8}}(\boldsymbol{\eta})_{\mathbf{8}}$, i.e. $(-,-,-,-,+,+)$. For convenience we shall refer to this as sheet II. Sheet I corresponds to positive imaginary components for all cm-momenta, $(+,+,+,+,+,+)$, referred to as the physical sheet.

For each parameterisation successfully describing the finite-volume spectra, tabulated in Table 6.5, we perform a search on all 64 Riemann sheets for a large complex $s$-rang $€^{8}$ and determine any pole singularities and corresponding couplings appearing in the amplitudes by factorising the $\boldsymbol{t}$-matrix as discussed in Section 5.6. As we cannot uniquely determine the sign in the $\boldsymbol{t}$-matrix for each coupled hadron-hadron channel, and cannot distinguish the $(\boldsymbol{\omega})_{\mathbf{8}}(\boldsymbol{\omega})_{\mathbf{1}} J^{P}=1^{-}$amplitudes in this study, as discussed in the previous section, there is a sign ambiguity on each of the couplings.

For the reference amplitude given in Eq. 6.9, a single complex conjugate pair of pole singularities was found on sheet II at complex energy,

$$
a_{t} \sqrt{s_{0}}=0.4607(26)(4) \pm \frac{i}{2} 0.0144(54)(34)
$$

where the first uncertainty is statistical and the second is systematic from varying the hadron masses and anisotropy, as described previously. The corresponding couplings for

\footnotetext{
${ }^{8}$ We search approximately $\operatorname{Re} a_{t} \sqrt{s} \in[0.35,0.49]$ and $2 \operatorname{Im} a_{t} \sqrt{s} \in[-0.1,0.1]$. Any pole outside this real energy region would not be well constrained by our data.
} 
the lower-half plane pole are,

$$
\begin{aligned}
& a_{t} c\left((\boldsymbol{\eta})_{\mathbf{8}}(\boldsymbol{\eta})_{\mathbf{1}}\left\{{ }^{1} P_{1}\right\}\right)_{\mathrm{II}}=0.076(19)(16) \exp [-i \pi 0.044(20)(46)] \\
& a_{t} c\left((\boldsymbol{\omega})_{\mathbf{8}}(\boldsymbol{\eta})_{\mathbf{8}}\left\{{ }^{3} P_{1}\right\}\right)_{\|}=0.066(14)(1) \exp [-i \pi 0.106(28)(71)] \\
& a_{t} c\left((\boldsymbol{\omega})_{\mathbf{8}}(\boldsymbol{\omega})_{\mathbf{8}}\left\{{ }^{3} P_{1}\right\}\right)_{\|}=0.025(16)(3) \exp [-i \pi 0.028(31)(25)] \\
& a_{t} c\left((\boldsymbol{\omega})_{\mathbf{8}}(\boldsymbol{\omega})_{\mathbf{1}}\left\{{ }^{X} P_{1}\right\}\right)_{\|}=0.021(25)(5) \exp [-i \pi 0.013(34)(27)] \\
& a_{t} c\left(\left(\boldsymbol{f}_{\mathbf{1}}\right)_{\mathbf{8}}(\boldsymbol{\eta})_{\mathbf{8}}\left\{{ }^{3} S_{1}\right\}\right)_{\mathrm{II}}=0.212(38)(30) \exp [i \pi 0.046(31)(15)] \\
& a_{t} c\left(\left(\boldsymbol{h}_{\mathbf{1}}\right)_{\mathbf{8}}(\boldsymbol{\eta})_{\mathbf{8}}\left\{{ }^{3} S_{1}\right\}\right)_{\|}=0.188(81)(33) \exp [-i \pi 0.002(24)(24)] \text {, }
\end{aligned}
$$

where again, the first error is statistical and the second is systematic. In addition, 'mirror poles' were robustly determined on several unphysical sheets, $(-, \pm, \pm, \pm,+,+)$, found at positions similar to that given in Eq. 6.10 with small shifts due to the small couplings to these channels [7]. Mirror poles on several sheets with a negative imaginary component of $\mathrm{cm}$-frame momentum in $\left(\boldsymbol{f}_{\mathbf{1}}\right)_{\mathbf{8}}(\boldsymbol{\eta})_{\mathbf{8}}$ or $\left(\boldsymbol{h}_{\mathbf{1}}\right)_{\mathbf{8}}(\boldsymbol{\eta})_{\mathbf{8}}$ were poorly determined and varied significantly in location relative to the sheet II pole, owing to the relatively large uncertainties on the large couplings in these channels. We found no poles on sheet I, in particular no complex valued poles which indicates good causal behaviour of the reference amplitude in this energy region.

For every parameterisation listed in Table 6.5, we found a single complex conjugate pair of pole singularities on sheet II, compatible with the pair found for the reference amplitude, and a similar distribution of mirror poles on other unphysical sheets. The lower-half plane sheet II pole singularities, closest to the region of physical scattering, are shown in Figure 6.12, We observe a well determined pole in each case with excellent agreement across all parameterisations.

Figure 6.13 shows the couplings for all parameterisations in Table 6.5. We observe little variation in the $(\boldsymbol{\eta})_{\mathbf{8}}(\boldsymbol{\eta})_{\mathbf{1}}\left\{{ }^{1} P_{1}\right\},(\boldsymbol{\omega})_{\mathbf{8}}(\boldsymbol{\eta})_{\mathbf{8}}\left\{{ }^{3} P_{1}\right\},(\boldsymbol{\omega})_{\mathbf{8}}(\boldsymbol{\omega})_{\mathbf{8}}\left\{{ }^{3} P_{1}\right\}$ and $(\boldsymbol{\omega})_{\mathbf{8}}(\boldsymbol{\omega})_{\mathbf{1}}\left\{{ }^{X} P_{1}\right\}$ couplings, with more substantial spread seen in $\left(\boldsymbol{f}_{\mathbf{1}}\right)_{\mathbf{8}}(\boldsymbol{\eta})_{\mathbf{8}}\left\{{ }^{3} S_{1}\right\}$ and $\left(\boldsymbol{h}_{\mathbf{1}}\right)_{\mathbf{8}}(\boldsymbol{\eta})_{\mathbf{8}}\left\{{ }^{3} S_{1}\right\}$. This is to be expected given fewer energy levels used to constrain the amplitude around the $\left(f_{1}\right)_{8}(\eta)_{8}$ and $\left(h_{1}\right)_{8}(\eta)_{8}$ thresholds.

The $(\boldsymbol{\eta})_{\mathbf{8}}(\boldsymbol{\eta})_{\mathbf{1}}\left\{{ }^{1} P_{1}\right\}$ and $(\boldsymbol{\omega})_{\mathbf{8}}(\boldsymbol{\eta})_{\mathbf{8}}\left\{{ }^{3} P_{1}\right\}$ couplings are seen to be very well determined and non-zero on all parameterisations. The $(\omega)_{\mathbf{8}}(\boldsymbol{\omega})_{\mathbf{8}}\left\{{ }^{3} P_{1}\right\}$ and $(\boldsymbol{\omega})_{\mathbf{8}}(\boldsymbol{\omega})_{\mathbf{1}}\left\{{ }^{X} P_{1}\right\}$ are comparatively much smaller and show hints of being non-zero, however, we find them roughly consistent with zero. The $\left(\boldsymbol{f}_{1}\right)_{\mathbf{8}}(\boldsymbol{\eta})_{\mathbf{8}}$ and $\left(\boldsymbol{h}_{\mathbf{1}}\right)_{\mathbf{8}}(\boldsymbol{\eta})_{\mathbf{8}}$ couplings appear to be large, 


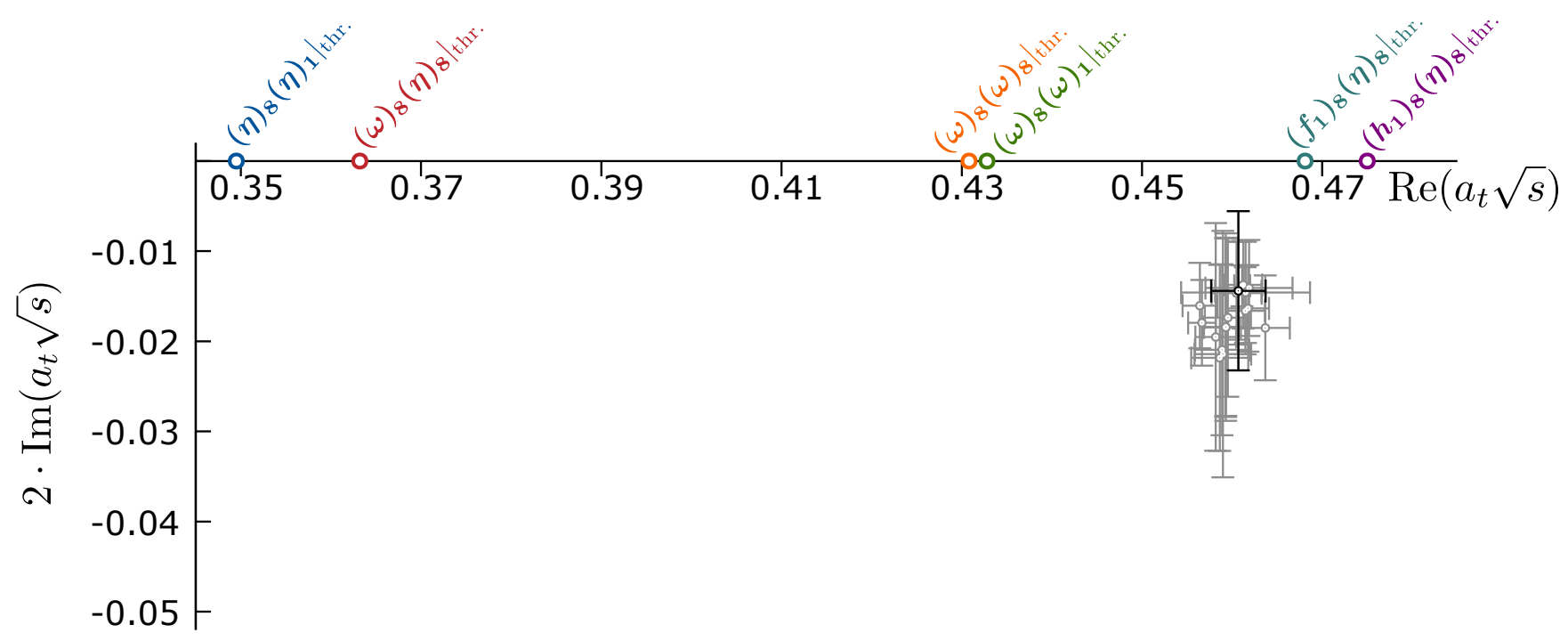

Figure 6.12: Lower half-plane sheet II poles. The black point corresponds to the reference amplitude in Eq. 6.9 with errorbars reflecting the combined statistical and systematic uncertainties. Grey points, with errorbars reflecting the statistical uncertainties, correspond to poles from all the parameterisations shown in Table 6.5 of Appendix 6.C. however, they are poorly determined. 

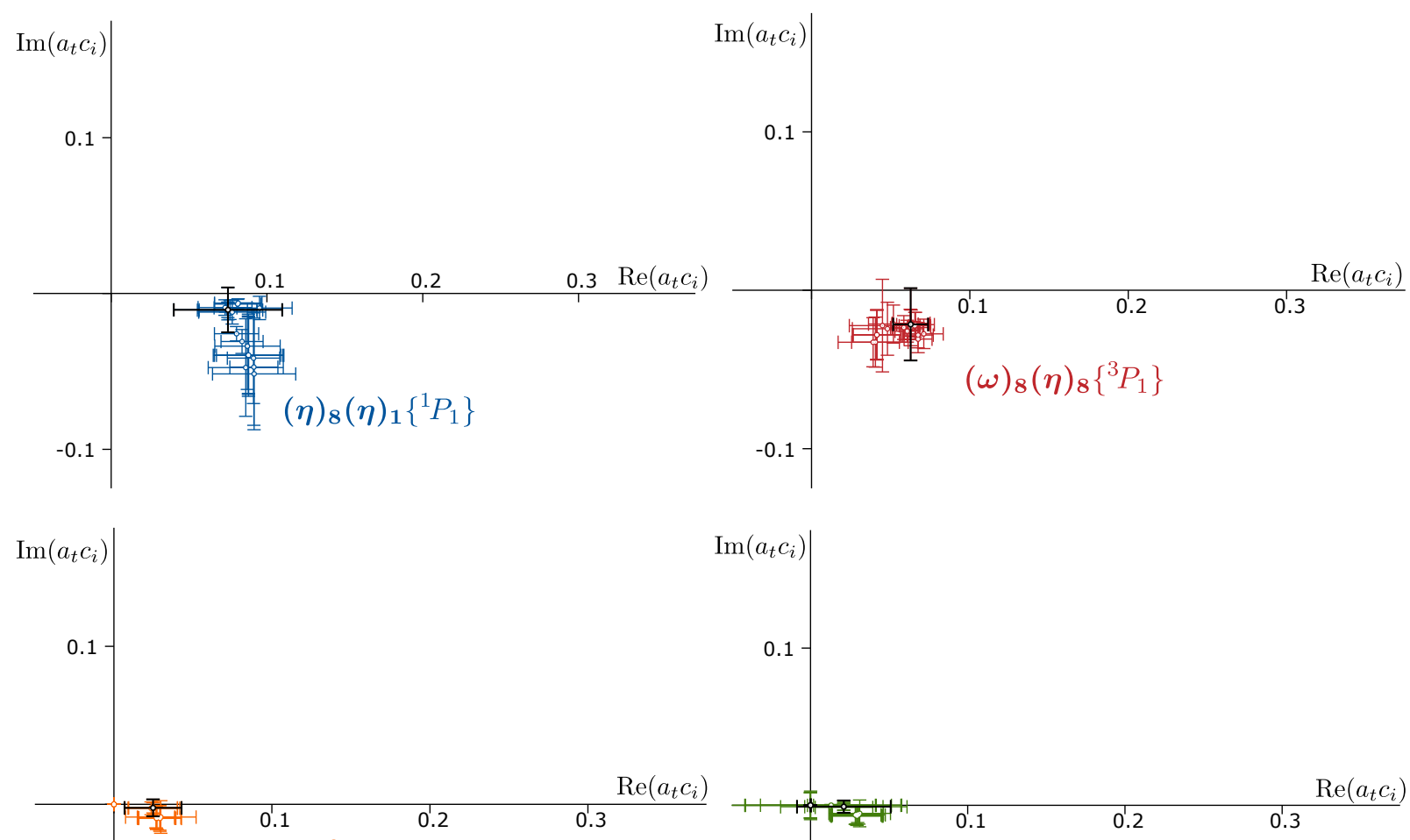

$(\omega)_{8}(\omega)_{8}\left\{{ }^{3} P_{1}\right\}$

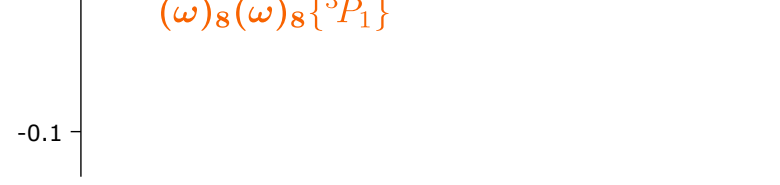

$(\omega)_{8}(\omega)_{1}\left\{{ }^{X} P_{1}\right\}$
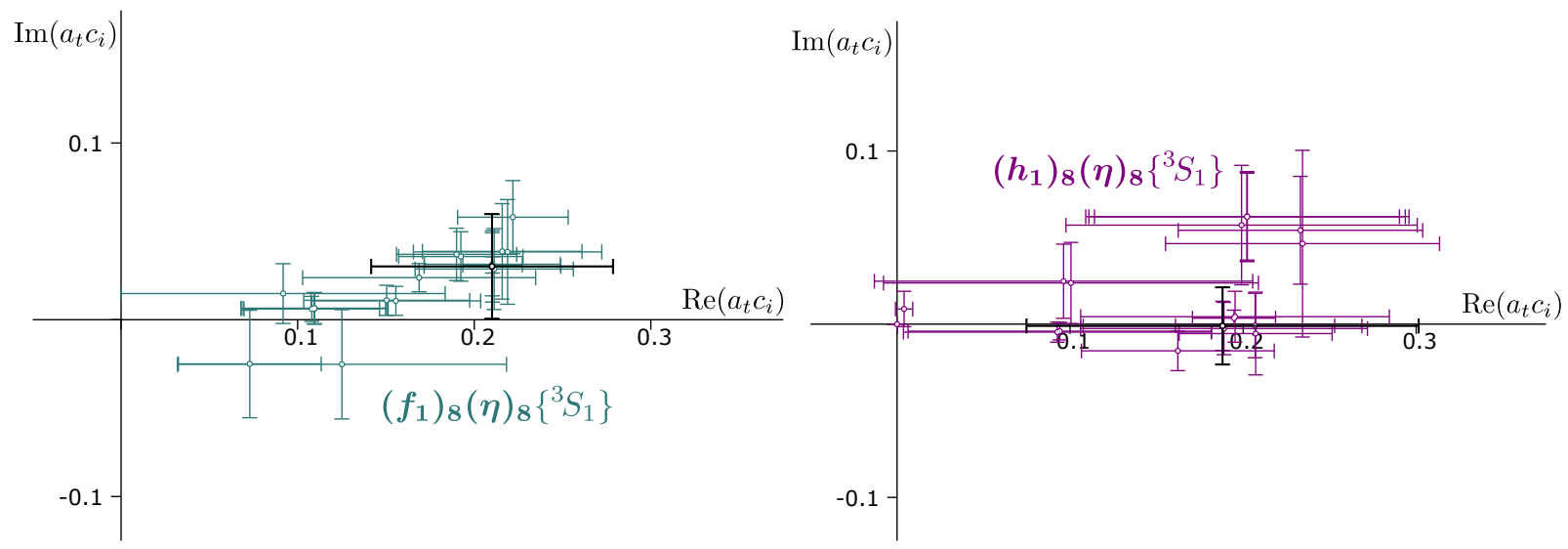

Figure 6.13: Couplings of the sheet II poles, shown in Figure 6.12, to hadron-hadron channels. The black points corresponds to the couplings from the reference amplitude in Eq. 6.9, with errorbars reflecting the combined statistical and systematic uncertainties. Coloured points, with errorbars reflect the statistical uncertainties, are the couplings from all the parameterisations shown in Table 6.5 of Appendix 6.C. 


\subsection{Interpretation}

For every amplitude parameterisation that was capable of describing the finite-volume spectra, we found in the $J^{P}=1^{-}$amplitudes, a pair of complex conjugate poles, located on sheet II, and corresponding mirror poles on other unphysical sheets, which we interpret as a single resonance.

Using the scale setting procedure detailed in Section 3.3 where $a_{t}^{-1}=4655 \mathrm{MeV}$, we summarise the results of the scattering analysis, expressing all quantities in physical units. We find a $\left(\boldsymbol{\pi}_{\boldsymbol{1}}\right)_{\mathbf{8}}$ resonant pole with mass $m_{R}=2148(33) \mathrm{MeV}$ and width $\Gamma_{R}=95(69) \mathrm{MeV}$ where the single uncertainty quoted is a conservative estimate from a combination of statistical and systematic uncertainties and encompasses variation over the range of parameterisations. Similarly, we find for the couplings,

$$
\begin{aligned}
\left|c_{(\boldsymbol{\eta})_{8}(\eta)_{1}\left\{{ }^{1} P_{1}\right\}}\right| & =470(177) \mathrm{MeV} \\
\left|c_{(\boldsymbol{\omega})_{8}(\eta)_{8}\left\{{ }^{3} P_{1}\right\}}\right| & =300(133) \mathrm{MeV} \\
\left|c_{(\boldsymbol{\omega})_{8}(\boldsymbol{\omega})_{8}\left\{{ }^{3} P_{1}\right\}}\right| & =158(90) \mathrm{MeV} \\
\left|c_{(\boldsymbol{\omega})_{8}(\boldsymbol{\omega})_{1}\left\{{ }^{X} P_{1}\right\}}\right| & =191(132) \mathrm{MeV} \\
\left|c_{\left(\boldsymbol{f}_{1}\right)_{8}(\boldsymbol{\eta})_{8}\left\{{ }^{3} S_{1}\right\}}\right| & =841(486) \mathrm{MeV} \\
\left|c_{\left(\boldsymbol{h}_{1}\right)_{8}(\boldsymbol{\eta})_{8}\left\{{ }^{3} S_{1}\right\}}\right| & =936(555) \mathrm{MeV}
\end{aligned}
$$

and present these best estimates in Figure 6.14.

As a comparison, we plot the $\pi_{1}$ hybrid resonance pole found in the reanalysis 6 of the COMPASS $\pi \eta^{(\prime)}$ data [100], along with the resonance pole found in this study, where $m_{\pi} \sim 700 \mathrm{MeV}$, in Figure 6.15. We can see at the physical light-quark mass, the resonance is broader and lighter than that found at the unphysically heavy light-quark mass - behaviour that we have observed in the $b_{1}$ (Figure 5.19), and has been shown for the $\rho[4]$ and $K^{*}[83$.

For the $\rho$ and $K^{*}$ resonances, the couplings to $\pi \pi$ and $\pi K$ respectively appear to be independent of $m_{\pi}$, once the appropriate phase-space factors have been removed. Assuming similar behaviour for the $\pi_{1}$, we can extrapolate the couplings presented in Eq. 6.12 to the physical light-quark mass.

We determine the couplings to each $\mathrm{SU}(2)_{\mathrm{F}}$ channel by weighting the $\mathrm{SU}(3)_{\mathrm{F}}$ coupling 


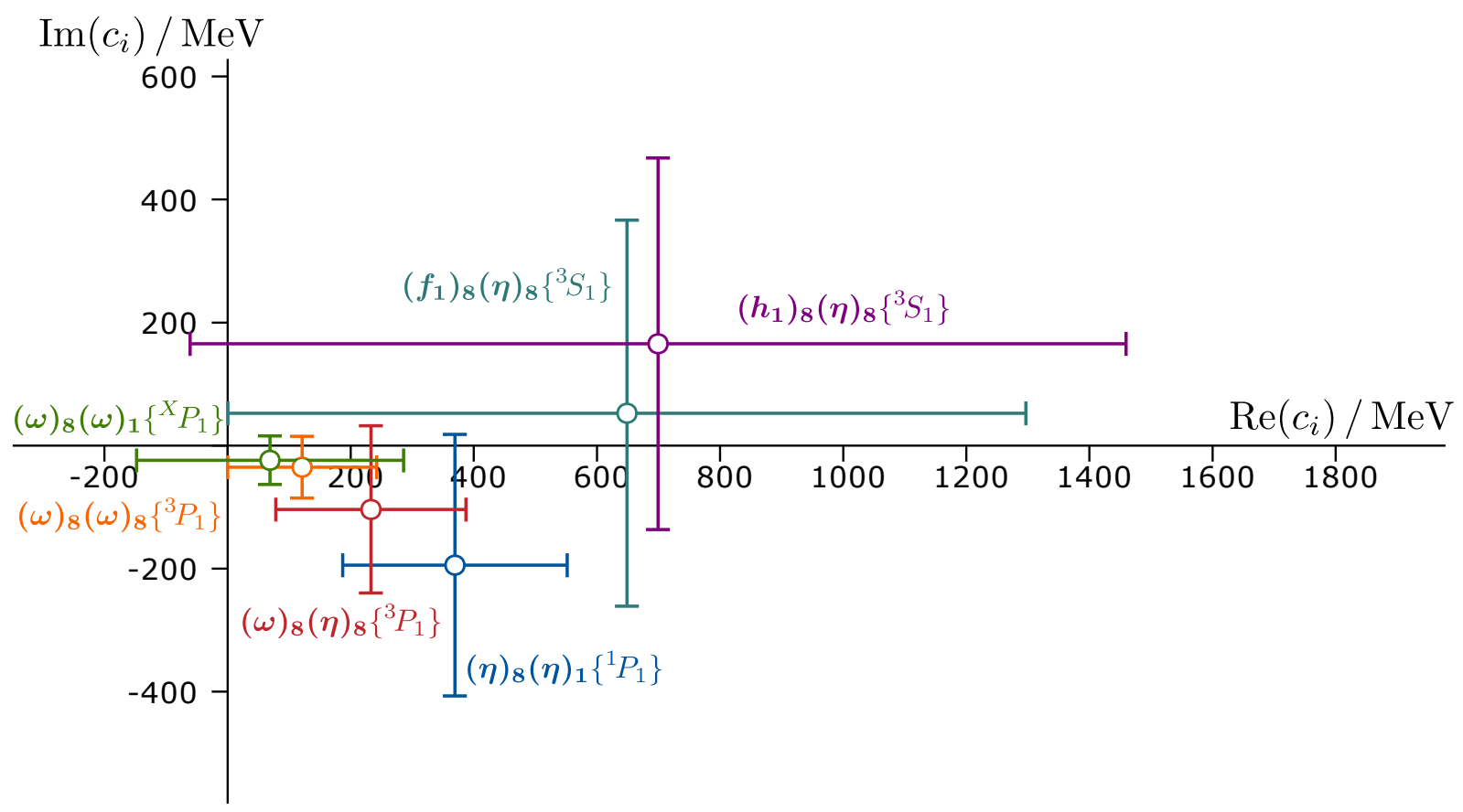

Figure 6.14: Points are the best estimates of each coupling $-(\boldsymbol{\eta})_{\mathbf{8}}(\boldsymbol{\eta})_{\mathbf{1}}\left\{{ }^{1} P_{1}\right\}$ (dark blue), $(\boldsymbol{\omega})_{8}(\boldsymbol{\eta})_{\mathbf{8}}\left\{{ }^{3} P_{1}\right\}$ (red), $(\boldsymbol{\omega})_{\mathbf{8}}(\boldsymbol{\omega})_{\mathbf{8}}\left\{{ }^{3} P_{1}\right\}$ (orange), $(\boldsymbol{\omega})_{\mathbf{8}}(\boldsymbol{\omega})_{\mathbf{1}}\left\{{ }^{X} P_{1}\right\}$ (green), $\left(\boldsymbol{f}_{\mathbf{1}}\right)_{\mathbf{8}}(\boldsymbol{\eta})_{\mathbf{8}}\left\{{ }^{3} S_{1}\right\}$ (light blue), $\left(\boldsymbol{h}_{\mathbf{1}}\right)_{\mathbf{8}}(\boldsymbol{\eta})_{\mathbf{8}}\left\{{ }^{3} S_{1}\right\}$ (purple) - where the error bars reflect a combination of statistical and systematic uncertainties and encompass variation over the range of parameterisations.

by the appropriate $\mathrm{SU}(3)_{\mathrm{F}}$ Clebsch-Gordan coefficients,

$$
\left|c_{\left(\mathbb{M}_{1}\right)_{I_{1}}\left(\mathbb{M}_{2}\right)_{I_{2}}\left\{2 S+1 \ell_{J}\right\}}^{\mathrm{SU}(2)}\right|=\left|c_{\left(\mathbb{M}_{1}\right)_{\boldsymbol{F}_{1}}\left(\mathbb{M}_{\mathbf{2}}\right)_{\boldsymbol{F}_{2}}\left\{2 S+1 \ell_{J}\right\}}^{\mathrm{SU}(3)}\right| \sqrt{\sum_{\substack{Y_{1}, Y_{2} \\
I_{z_{1}}, I_{z_{2}}}}\left[\mathcal{C}_{\mathrm{SU}(3)}\left(\begin{array}{ccc}
\boldsymbol{F}_{1} & \boldsymbol{F}_{2} & \boldsymbol{8}_{i} \\
\nu_{1} & \nu_{2} & \nu
\end{array}\right)\right]^{2}} .
$$

Recall $\nu=\left(I, Y, I_{z}\right)=(1,0,1)$ and that $i=1,2$ depending on which embedding gives a negative $G$-parity. As SU $(2)_{\mathrm{F}}$ flavour irreps are labelled by total isospin, we sum over the different hypercharges and $z$-components of isospin for a given total isospin and normalise such that the sum squared of the magnitudes of the $\mathrm{SU}(2)_{F}$ couplings gives the magnitude squared $\mathrm{SU}(3)_{\mathrm{F}}$ coupling.

The isoscalar components of the $\mathrm{SU}(3)_{\mathrm{F}}$ multiplets are an admixture of two $\mathrm{SU}(2)_{\mathrm{F}}$ mesons. As such, the $\mathrm{SU}(2)_{\mathrm{F}}$ couplings that feature isoscalar components of the $\mathrm{SU}(3)_{\mathrm{F}}$ 


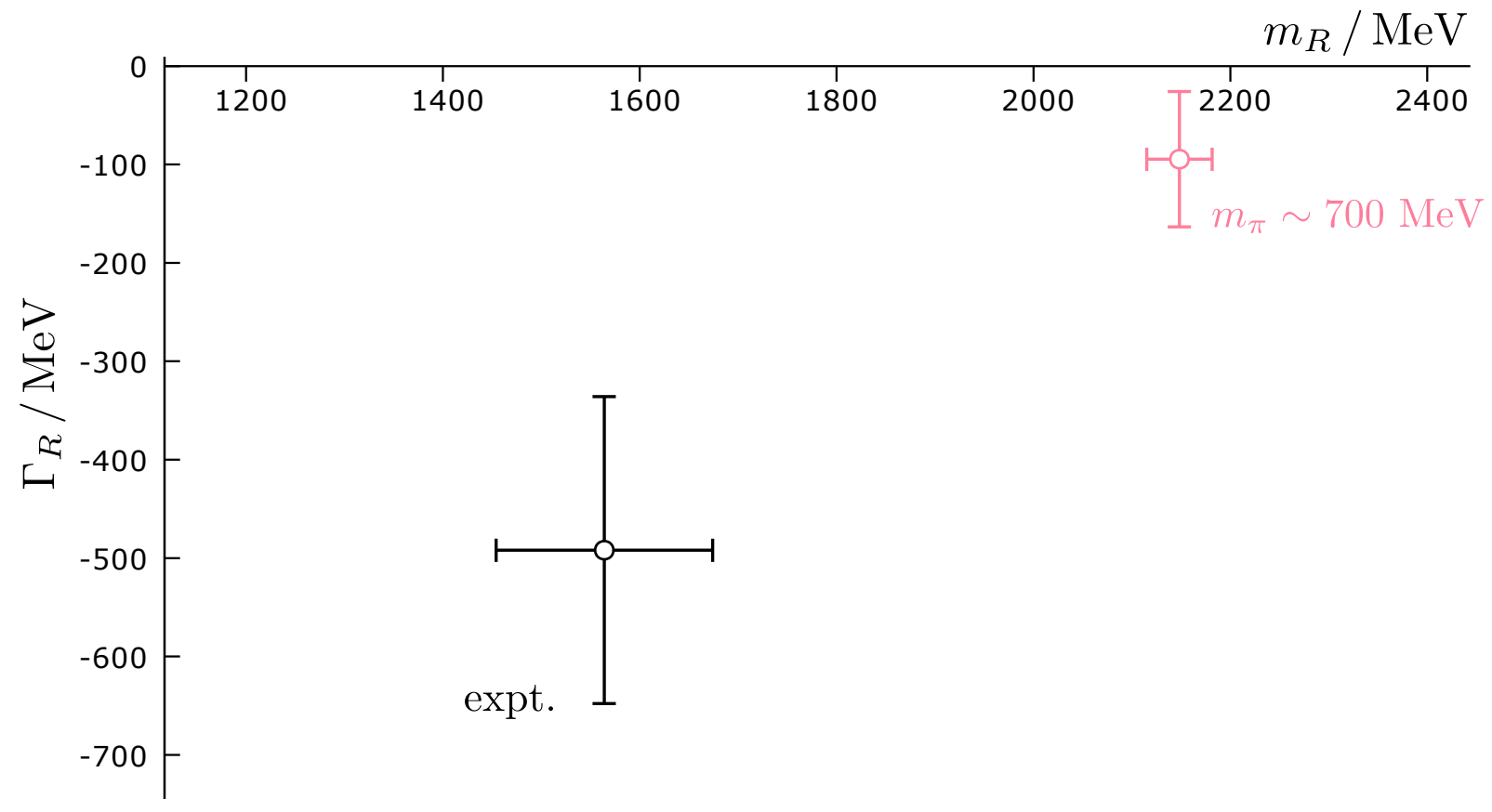

Figure 6.15: The $\pi_{1}$ pole. Pink shows the estimate from this work with $m_{\pi} \approx 700 \mathrm{MeV}$ and black is the experimentally determined mass and width of the $\pi_{1}$ resonance [6].

multiplets, via non-vanishing Clebsch-Gordan coefficients in Eq. 6.13, are appropriately split ${ }^{9}$ between the two $\mathrm{SU}(2)_{\mathrm{F}}$ mesons. For example, for the isoscalar components of the octet and singlet pseudoscalars, $(\boldsymbol{\eta})_{\mathbf{8}}$ and $(\boldsymbol{\eta})_{1}$, we use an octet-singlet basis mixing angle of $\theta_{P}=-10^{\circ}[26]$, such that the isoscalar elements of the $\mathrm{SU}(3)_{\mathrm{F}}$ pseudoscalar octet and singlet, expressed in terms of $\mathrm{SU}(2)_{\mathrm{F}}$ states, $\eta$ and $\eta^{\prime}$, are given by,

$$
\begin{aligned}
& \left|(\boldsymbol{\eta})_{\mathbf{8}} ; 0,0,0\right\rangle=\cos \left(\theta_{P}\right)|\eta\rangle+\sin \left(\theta_{P}\right)\left|\eta^{\prime}\right\rangle \\
& \left|(\boldsymbol{\eta})_{\mathbf{1}} ; 0,0,0\right\rangle=-\sin \left(\theta_{P}\right)|\eta\rangle+\cos \left(\theta_{P}\right)\left|\eta^{\prime}\right\rangle,
\end{aligned}
$$

and therefore, the couplings to the pseudoscalar-pseudoscalar channels are,

$$
\begin{aligned}
\left|c_{\pi \eta\left\{{ }^{1} P_{1}\right\}}^{\mathrm{SU}(2)}\right| & =\sin \left(\theta_{P}\right)\left|c_{(\boldsymbol{\eta})_{8}(\eta)_{1}\left\{{ }^{1} P_{1}\right\}}^{\mathrm{SU}(3)}\right| \\
\left|c_{\pi \eta^{\prime}\left\{{ }^{1} P_{1}\right\}}^{\mathrm{SU}(2)}\right| & =\cos \left(\theta_{P}\right)\left|c_{(\boldsymbol{\eta})_{8}(\boldsymbol{\eta})_{1}\left\{{ }^{1} P_{1}\right\}}^{\mathrm{SU}(3)}\right| .
\end{aligned}
$$

This of course preserves the property that the sum squared magnitude of the $\mathrm{SU}(2)_{\mathrm{F}}$ couplings gives the squared magnitude $\mathrm{SU}(3)_{\mathrm{F}}$ coupling. For the isoscalar components of

\footnotetext{
${ }^{9}$ Experiments have determined the admixtures for a number of isoscalar mesons.
} 
the octet and singlet axial-vectors, $\left(f_{1}\right)_{8}$ and $\left(f_{1}\right)_{1}$, we replace $\eta$ and $\eta^{\prime}$ in Eq. 6.14 with $f_{1}$ and $f_{1}^{\prime}$ respectively ${ }^{10}$ and take $\theta_{P}=-34^{\circ}$ [26]. Similarly, for the isoscalar octet and singlet vectors, $(\omega)_{8}$ and $(\omega)_{1}$, we replace $\eta$ and $\eta^{\prime}$ in Eq. 6.14 with $\omega$ and $\phi$ respectively and set $\theta_{P}=-52^{\circ}[26$.

Lastly, phase-space factors are removed by scaling the $\mathrm{SU}(2)_{\mathrm{F}}$ couplings by the ratio of $\mathrm{cm}$-frame momentum, evaluated at the resonance pole position at each pion mass,

$$
\left|c_{\left(\mathbb{M}_{1}\right)_{I_{1}}\left(\mathbb{M}_{2}\right)_{I_{2}}\left\{2^{2 S+1} \ell_{J}\right\}}^{\text {phs }}\right|=\left.\left|c_{\left(\mathbb{M}_{1}\right)_{I_{1}}\left(\mathbb{M}_{2}\right)_{I_{2}}\left\{{ }^{2 S+1} \ell_{J}\right\}}\right| \frac{k_{\mathrm{cm}}^{\text {phys. }}}{k_{\mathrm{cm}}}\right|^{\ell} .
$$

As an explicit example, consider $(\boldsymbol{\omega})_{\mathbf{8}}(\boldsymbol{\eta})_{\mathbf{8}}\left\{{ }^{3} P_{1}\right\}$. The $\mathrm{SU}(2)_{\mathrm{F}}$ two-meson flavour content was given in Eq. 4.7 which we transcribe below for brevity,

$$
\left|(\boldsymbol{\omega})_{\mathbf{8}}(\boldsymbol{\eta})_{\mathbf{8}} ; 1,0,1\right\rangle=\frac{1}{\sqrt{6}}\left(\left|K^{*+}\right\rangle\left|\bar{K}^{0}\right\rangle-\left|\bar{K}^{* 0}\right\rangle\left|K^{+}\right\rangle\right)+\frac{1}{\sqrt{3}}\left(\left|\rho^{+}\right\rangle\left|\pi^{0}\right\rangle-\left|\rho^{0}\right\rangle\left|\pi^{+}\right\rangle\right) .
$$

Following Eqs. 6.13 and 6.16 , the physical $\pi_{1}$ couplings to $\rho \pi\left\{{ }^{3} P_{1}\right\}$ and $K^{*} \bar{K}\left\{{ }^{3} P_{1}\right\}$ are given by,

$$
\begin{aligned}
\left|c_{\rho \pi\left\{{ }^{3} P_{1}\right\}}^{\text {phys. }}\right| & =\sqrt{\frac{2}{3}}\left|c_{(\omega)_{8}(\eta)_{8}\left\{{ }^{3} P_{1}\right\}}\right|\left|\frac{k_{\rho \pi}^{\text {phys. }}}{k_{\rho \pi}}\right| \\
\left|c_{K^{*} \bar{K}\left\{{ }^{3} P_{1}\right\}}^{\text {phys }}\right| & =\sqrt{\frac{1}{3}}\left|c_{(\omega)_{8}(\eta)_{8}\left\{{ }^{3} P_{1}\right\}}\right|\left|\frac{k_{K^{*} \bar{K}}^{\text {phys. }}}{k_{K^{*} \bar{K}}}\right| .
\end{aligned}
$$

Note that no isoscalar elements of the $\mathrm{SU}(3)_{\mathrm{F}}$ featured in this example.

We present the couplings to each $\mathrm{SU}(2)_{\mathrm{F}}$ meson-meson channel in Table 6.4. Here, the uncertainties reflect those on the couplings in Eq. 6.12 together with the uncertainties on the masses and widths of the resonant poles, propagated through the ratio of $\mathrm{cm}$-frame momenta 11 Masses of the mesons at the physical light-quark limit were taken from the PDG [5] and masses at the $\mathrm{SU}(3)_{\mathrm{F}}$ point with $m_{\pi} \sim 700 \mathrm{MeV}$ were taken from Table 6.2 .

\footnotetext{
${ }^{10}$ We take the $f_{1}$ and $f_{1}^{\prime}$ to correspond to the experimentally observed $f_{1}(1285)$ and $f_{1}(1420)$ respectively 5 .

${ }^{11} \mathrm{We}$ take the physical $\pi_{1}$ mass and width to be $m_{R}^{\text {phys. }}=1564 \pm 110 \mathrm{MeV}$ and $\Gamma_{R}^{\text {phys. }}=492 \pm 156 \mathrm{MeV}$ respectively 6 .
} 


\begin{tabular}{|c|c|c|}
\hline $\begin{array}{c}\mathrm{SU}(3)_{\mathrm{F}} \\
\mathbb{M}_{\mathbf{1}} \mathbb{M}_{\mathbf{2}}\left\{{ }^{2 S+1} \ell_{J}\right\}\end{array}$ & $\begin{array}{c}\mathrm{SU}(2)_{\mathrm{F}} \\
\mathbb{M}_{1} \mathbb{M}_{2}\left\{{ }^{2 S+1} \ell_{J}\right\}\end{array}$ & $\left|c_{\mathbb{M}_{1} \mathbb{M}_{2}\left\{{ }^{2 S+1} \ell_{J}\right\}}^{\text {phys. }}\right| / \mathrm{MeV}$ \\
\hline \multirow{2}{*}{$(\boldsymbol{\eta})_{\mathbf{8}}(\boldsymbol{\eta})_{1}\left\{{ }^{1} P_{1}\right\}$} & $\pi \eta\left\{{ }^{1} P_{1}\right\}$ & $81(32)$ \\
\hline & $\pi \eta^{\prime}\left\{{ }^{1} P_{1}\right\}$ & $343(140)$ \\
\hline \multirow{2}{*}{$(\boldsymbol{\omega})_{8}(\boldsymbol{\eta})_{8}\left\{{ }^{3} P_{1}\right\}$} & $\rho \pi\left\{{ }^{3} P_{1}\right\}$ & $\begin{array}{r}------- \\
227(105)\end{array}$ \\
\hline & $K^{*} \bar{K}\left\{{ }^{3} P_{1}\right\}$ & $124(59)$ \\
\hline \multirow{3}{*}{$(\boldsymbol{\omega})_{\mathbf{8}}(\boldsymbol{\omega})_{\mathbf{8}}\left\{{ }^{3} P_{1}\right\}$} & $\rho \omega\left\{{ }^{3} P_{1}\right\}$ & $71(44)$ \\
\hline & $\rho \phi\left\{{ }^{3} P_{1}\right\}$ & $106(64)$ \\
\hline & $K^{*} \bar{K}^{*}\left\{{ }^{3} P_{1}\right\}$ & $166(100)$ \\
\hline \multirow{2}{*}{$(\boldsymbol{\omega})_{8}(\omega)_{1}\left\{{ }^{X} P_{1}\right\}$} & $\rho \omega\left\{{ }^{X} P_{1}\right\}$ & $179(132)$ \\
\hline & $\rho \phi\left\{{ }^{3} P_{1}\right\}$ & $163(119)$ \\
\hline \multirow{5}{*}{$\left(f_{1}\right)_{8}(\boldsymbol{\eta})_{8}\left\{{ }^{3} S_{1}\right\}$} & $a_{1} \eta\left\{{ }^{3} S_{1}\right\}$ & $371(214)$ \\
\hline & $a_{1} \eta^{\prime}\left\{{ }^{3} S_{1}\right\}$ & $65(38)$ \\
\hline & $f_{1} \pi\left\{{ }^{3} S_{1}\right\}$ & $314(181)$ \\
\hline & $f_{1}^{\prime} \pi\left\{{ }^{3} S_{1}\right\}$ & $207(120)$ \\
\hline & $K_{1} \bar{K}\left\{{ }^{3} S_{1}\right\}$ & $652(376)$ \\
\hline \multirow{2}{*}{$\left(\boldsymbol{h}_{\mathbf{1}}\right)_{\mathbf{8}}(\boldsymbol{\eta})_{\mathbf{8}}\left\{{ }^{3} S_{1}\right\}$} & $b_{1} \pi\left\{{ }^{3} S_{1}\right\}$ & $\begin{array}{l}---\cdots \\
763(453)\end{array}$ \\
\hline & $K_{1} \bar{K}\left\{{ }^{3} S_{1}\right\}$ & $540(320)$ \\
\hline
\end{tabular}

Table 6.4: Couplings extrapolated to the physical quark mass limit, as described in the text.

\subsection{Summary}

In this chapter we have presented, for the first time in lattice QCD, a calculation of the $\pi_{1}$ resonance from coupled-channel scattering. Furthermore, this is the first time vector-vector channels have been incorporated in a lattice QCD scattering calculation.

Utilising the rest-frame finite-volume spectra, scattering amplitudes in $(\boldsymbol{\eta})_{\mathbf{8}}(\boldsymbol{\eta})_{\mathbf{1}}\left\{{ }^{1} P_{1}\right\}$, $(\boldsymbol{\omega})_{\mathbf{8}}(\boldsymbol{\eta})_{\mathbf{8}}\left\{{ }^{3} P_{1}\right\},(\boldsymbol{\omega})_{\mathbf{8}}(\boldsymbol{\omega})_{\mathbf{8}}\left\{{ }^{3} P_{1}\right\},(\boldsymbol{\omega})_{\mathbf{8}}(\boldsymbol{\omega})_{\mathbf{1}}\left\{{ }^{X} P_{1}\right\},\left(\boldsymbol{f}_{\mathbf{1}}\right)_{\mathbf{8}}(\boldsymbol{\eta})_{\mathbf{8}}\left\{{ }^{3} S_{1}\right\}$ and $\left(\boldsymbol{h}_{\mathbf{1}}\right)_{\mathbf{8}}(\boldsymbol{\eta})_{\mathbf{8}}\left\{{ }^{3} S_{1}\right\}$ were determined for a range of parameterisations. In addition, we found small $(\boldsymbol{\omega})_{\mathbf{8}}(\boldsymbol{\eta})_{\mathbf{8}}\left\{{ }^{3} F_{3}\right\}$ and $(\boldsymbol{\omega})_{\mathbf{8}}(\boldsymbol{\omega})_{\mathbf{1}}\left\{{ }^{5} P_{3}\right\}$ scattering amplitudes, with no evidence of resonant 
enhancement in the exotic $J^{P C}=3^{-+}$sector at this energy range.

By analytically continuing the scattering amplitudes into the complex energy plane, we unambiguously found a single resonance pole with mass $m_{R}=2148(33)$, relatively close to the real energy axis with width $\Gamma_{R}=95(69)$, that we interpreted as the $\pi_{1}$ resonance. Of particular note, we found no evidence of a second resonance pole in this energy region. Couplings to $(\boldsymbol{\eta})_{\mathbf{8}}(\boldsymbol{\eta})_{\mathbf{1}}\left\{{ }^{1} P_{1}\right\}$ and $(\boldsymbol{\omega})_{\mathbf{8}}(\boldsymbol{\eta})_{\mathbf{8}}\left\{{ }^{3} P_{1}\right\}$ were robustly determined, found to be non-zero and comparable in magnitude, while $(\boldsymbol{\omega})_{\mathbf{8}}(\boldsymbol{\omega})_{\mathbf{8}}\left\{{ }^{3} P_{1}\right\}$ and $(\omega)_{8}(\omega)_{1}\left\{{ }^{X} P_{1}\right\}$ couplings were smaller and consistent with zero. Hints of large couplings to both $\left(\boldsymbol{f}_{\mathbf{1}}\right)_{\mathbf{8}}(\boldsymbol{\eta})_{\mathbf{8}}\left\{{ }^{3} S_{1}\right\}$ and $\left(\boldsymbol{h}_{\mathbf{1}}\right)_{\mathbf{8}}(\boldsymbol{\eta})_{\mathbf{8}}\left\{{ }^{3} S_{1}\right\}$ were observed but these were poorly determined.

For the first time in a lattice QCD calculation, we performed an eight coupled-channel scattering analysis, presenting the phase-shifts and mixing-angles through the eight-channel generalised Stapp-parameterisation.

This work has demonstrated the advances and capabilities of lattice calculations for studying many coupled-channel systems and in particular, in a system where large experimental efforts towards calculating the lightest exotic meson are being undertaken. It would be desirable to pin down more precisely the couplings to the $\left(\boldsymbol{f}_{\mathbf{1}}\right)_{\mathbf{8}}(\boldsymbol{\eta})_{\mathbf{8}}\left\{{ }^{3} S_{1}\right\}$ and $\left(\boldsymbol{h}_{\mathbf{1}}\right)_{\mathbf{8}}(\boldsymbol{\eta})_{\mathbf{8}}\left\{{ }^{3} S_{1}\right\}$, featuring the $b_{1} \pi$ and $f_{1} \pi$ channels which have been seen experimentally and appear in this calculation to show hints of large couplings. Looking forward, once a three-hadron scattering formalism is practical to use, finite-volume spectra from moving-frame irreps could be incorporated into the analysis as additional constraints, and moreover, a calculation of the $\pi_{1}$ at a lighter pion mass would make for an excellent comparison.

\section{APPENDICES}

\section{A $\mathrm{SU}(3)_{\mathbf{F}}$ Octet Multiplets}

For reference, we present the $\mathrm{SU}(2)_{\mathrm{F}}$ contents of the $\mathrm{SU}(3)_{\mathrm{F}}$ scalar, axial-vector and tensor octets in Figure 6.16. 


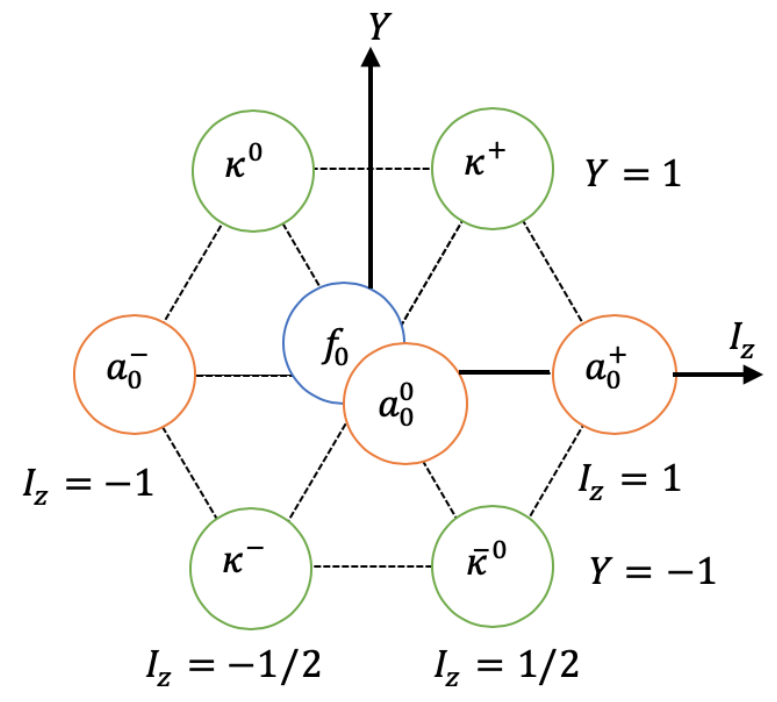

(a) $\left(1^{-}\right) 0^{++}\left(f_{0}\right)_{8}$ octet

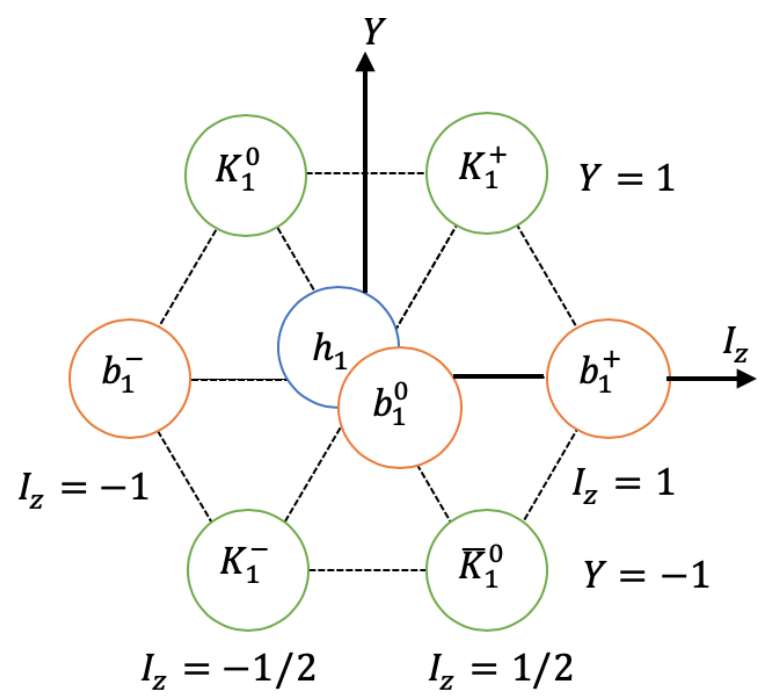

(c) $\left(1^{+}\right) 1^{+-}\left(h_{1}\right)_{8}$ octet

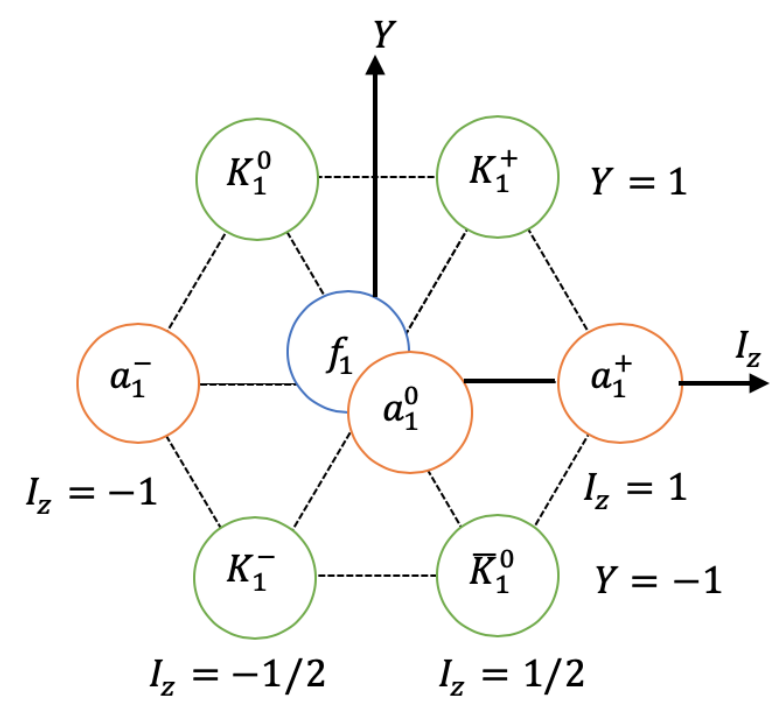

(b) $\left(1^{-}\right) 1^{++}\left(f_{1}\right)_{8}$ octet

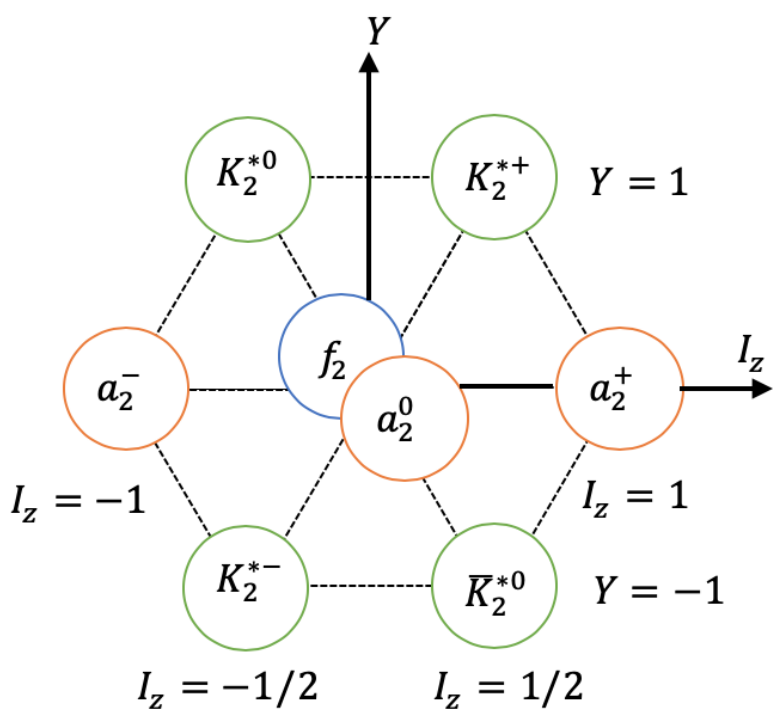

(d) $\left(1^{-}\right) 2^{++}\left(f_{2}\right)_{8}$ octet

Figure 6.16: $\mathrm{SU}(2)_{\mathrm{F}}$ flavour content of the scalar, axial-vector and tensor octets. The $\left(I^{G}\right) J^{P C}$ quantum numbers for the isovector elements are given in the sub-captions. 


\section{B Indistinguishable $(\omega)_{8}(\omega)_{1}$ Amplitudes}

The quantisation condition subduced into the $[000] T_{1}^{-}$irrep cannot distinguish between the three $J^{P}=1^{-}$vector-vector $(\omega)_{8}(\omega)_{1}$-channels. The scattering parameters within the $\boldsymbol{t}$-matrix, parameterising the ${ }^{1} P_{1^{-}},{ }^{3} P_{1^{-}}$and ${ }^{5} P_{1^{-}}$waves, can be freely interchanged while leaving the determinant invariant. As such, we cannot uniquely determine these amplitudes here and must incorporate moving frames in the analysis if we are to make such a distinction. We show this invariance by examining symmetries of the quantisation condition below.

For convenience, we transcribe the quantisation condition subduced into [000] $T_{1}^{-}$,

$$
\underset{\ell S J n a}{\operatorname{det}}\left[\mathbf{1}+i \boldsymbol{\rho} \cdot \boldsymbol{t} \cdot\left(\mathbf{1}+i \overline{\mathcal{M}}^{\overrightarrow{0}, T_{1}^{-}}\right)\right]=0,
$$

and take the second row of the irrep ${ }^{12}$ It follows from Eq. 2.52 that as the only non-zero subduction coefficient is trivially $\mathcal{S}_{T_{1}^{-} 2}^{10}=1$, the quantisation condition can be equivalently written in the $|\ell S J m a\rangle$ basis as,

$$
\operatorname{det}_{\ell S J a}[\mathbf{1}+i \boldsymbol{\rho} \cdot \boldsymbol{t} \cdot(\mathbf{1}+i \overline{\mathcal{M}})]=0
$$

where $\overline{\mathcal{M}}$ is at momentum zero and has components given in Eq. 2.49. It is this matrix we examine now.

From Eq. 2.49, it is immediate that $\overline{\mathcal{M}}$ is diagonal in intrinsic spin and thus no mixing between the three $P$-wave vector-vector channels given above can feature in $\overline{\mathcal{M}}$. We therefore look at the diagonal components in each of these channels. It is useful here to make observation about the $Z$-functions and integral of the product of spherical harmonics expressed in Eqs. 2.50 and 2.51 respectively.

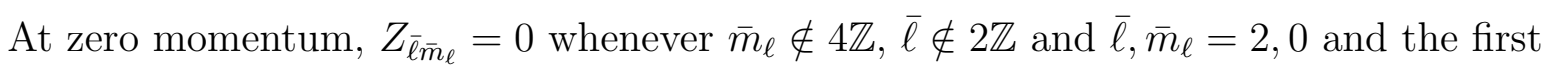
few non-zero $Z_{\bar{\ell} \bar{m}_{\ell}}$ are $Z_{00}, Z_{40}$ and $Z_{4 \pm 4}$. For $\bar{\ell} \geq 4$, it is immediate from Eq. 2.51 that $\int d \Omega Y_{1 m_{\ell}}^{*} Y_{\bar{\ell} \bar{m}_{\ell}}^{*} Y_{1 m_{\ell}^{\prime}}=0$ as the Clebsch-Gordan coefficients,

$$
\mathcal{C}\left(\begin{array}{lll}
1 & 1 & \bar{\ell} \\
0 & 0 & 0
\end{array}\right)=0
$$

\footnotetext{
${ }^{12}$ It does not matter which row we consider, however the second row gives more symmetric ClebschGordan coefficients which will be useful later.
} 
For $\bar{\ell}=\bar{m}=0$, it follows from Eq. 2.51 that $\int d \Omega Y_{1 m_{\ell}}^{*} Y_{00}^{*} Y_{1 m_{\ell}^{\prime}}=\delta_{m_{\ell} m_{\ell}^{\prime}}$ and hence, from Eq. 2.49, that,

$$
\begin{aligned}
\overline{\mathcal{M}}_{1 S J m a, 1 S J^{\prime} m^{\prime} a} & =\sum_{m_{\ell}, m_{\ell}^{\prime}, m_{S}} \mathcal{C}\left(\begin{array}{ccc}
1 & S & J \\
m_{\ell} & m_{S} & m
\end{array}\right) \mathcal{C}\left(\begin{array}{ccc}
1 & S & J^{\prime} \\
m_{\ell}^{\prime} & m_{S} & m^{\prime}
\end{array}\right)\left(\frac{4 \pi}{k_{\mathrm{cm}}^{(a)}}\right) c_{0,0}^{\overrightarrow{0}}\left(\left[k_{\mathrm{cm}}^{(a)}\right]^{2} ; L\right) \delta_{m_{\ell} m_{\ell}^{\prime}} \\
& =\sum_{m_{S}} \mathcal{C}\left(\begin{array}{ccc}
1 & S & J \\
-m_{S} & m_{S} & m
\end{array}\right) \mathcal{C}\left(\begin{array}{ccc}
1 & S & J^{\prime} \\
-m_{S} & m_{S} & m^{\prime}
\end{array}\right)\left(\frac{4 \pi}{k_{\mathrm{cm}}^{(a)}}\right) c_{0,0}^{\overrightarrow{0}}\left(\left[k_{\mathrm{cm}}^{(a)}\right]^{2} ; L\right) \\
& =\delta_{J J^{\prime}} \delta_{m m^{\prime}}\left(\frac{4 \pi}{k_{\mathrm{cm}}^{(a)}}\right) c_{0,0}^{\overrightarrow{0}}\left(\left[k_{\mathrm{cm}}^{(a)}\right]^{2} ; L\right) .
\end{aligned}
$$

As the only non-zero subduction coefficient for $J=3$ in the second row of $T_{1}^{-}$is trivially $\mathcal{S}_{T_{1}^{-} 2}^{30}=1$, we find that the matrix $\overline{\mathcal{M}}$ is invariant under permutations of all the $P$-wave $(\omega)_{8}(\omega)_{1}$-channels.

The matrix of phase-space factors $\boldsymbol{\rho}$ is also invariant under such permutations. The $\boldsymbol{t}$-matrix however forbids mixing between $J=1$ and $J=3$ and subsequently, in general, we cannot interchange the ${ }^{X} P_{1}$ and ${ }^{5} P_{3}$ scattering parameters. Nevertheless, for any $\boldsymbol{t}$ matrix, interchanging the ${ }^{1} P_{1},{ }^{3} P_{1}$ and ${ }^{5} P_{1}$ scattering parameters will leave the determinant invariant and therefore, using the $[000] T_{1}^{-}$energy levels as constraints, we cannot distinguish the ${ }^{1} P_{1^{-}},{ }^{3} P_{1^{-}}$and ${ }^{5} P_{1^{-} \text {wave }}(\omega)_{8}(\omega)_{1}$ scattering amplitudes.

\section{C Scattering Parameterisations}

We present the table of scattering parameterisations that successfully described the finitevolume spectra, as discussed in the text, in Table 6.5. The correlations between the scattering parameters of the reference amplitude (the first parameterisation shown in bold in Table 6.5 are presented in Table 6.6. Entries in the table with a dash '-' in the $\chi^{2} / N_{\text {dof }}$ column correspond to parameterisations that failed to converge to a minimum. 


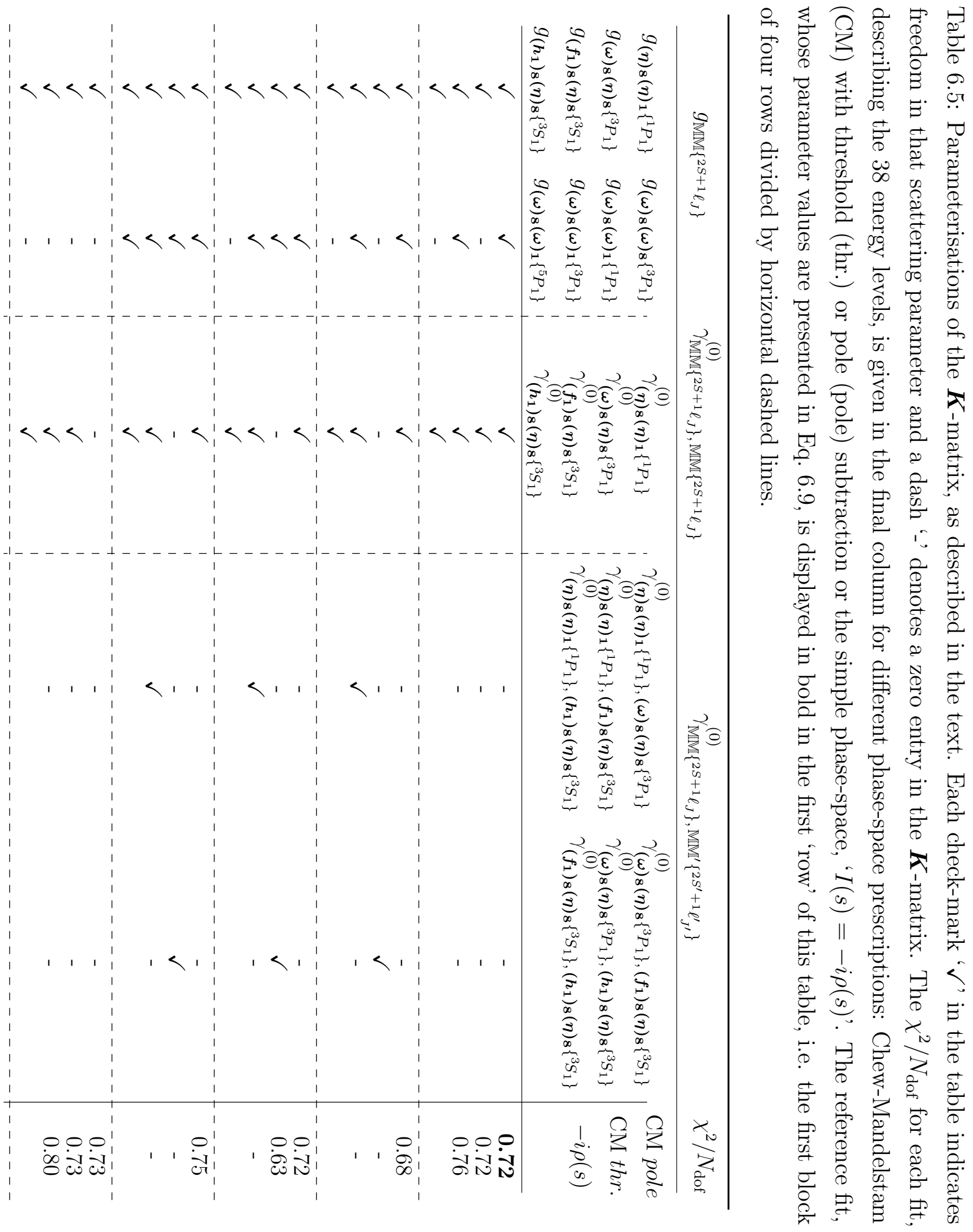




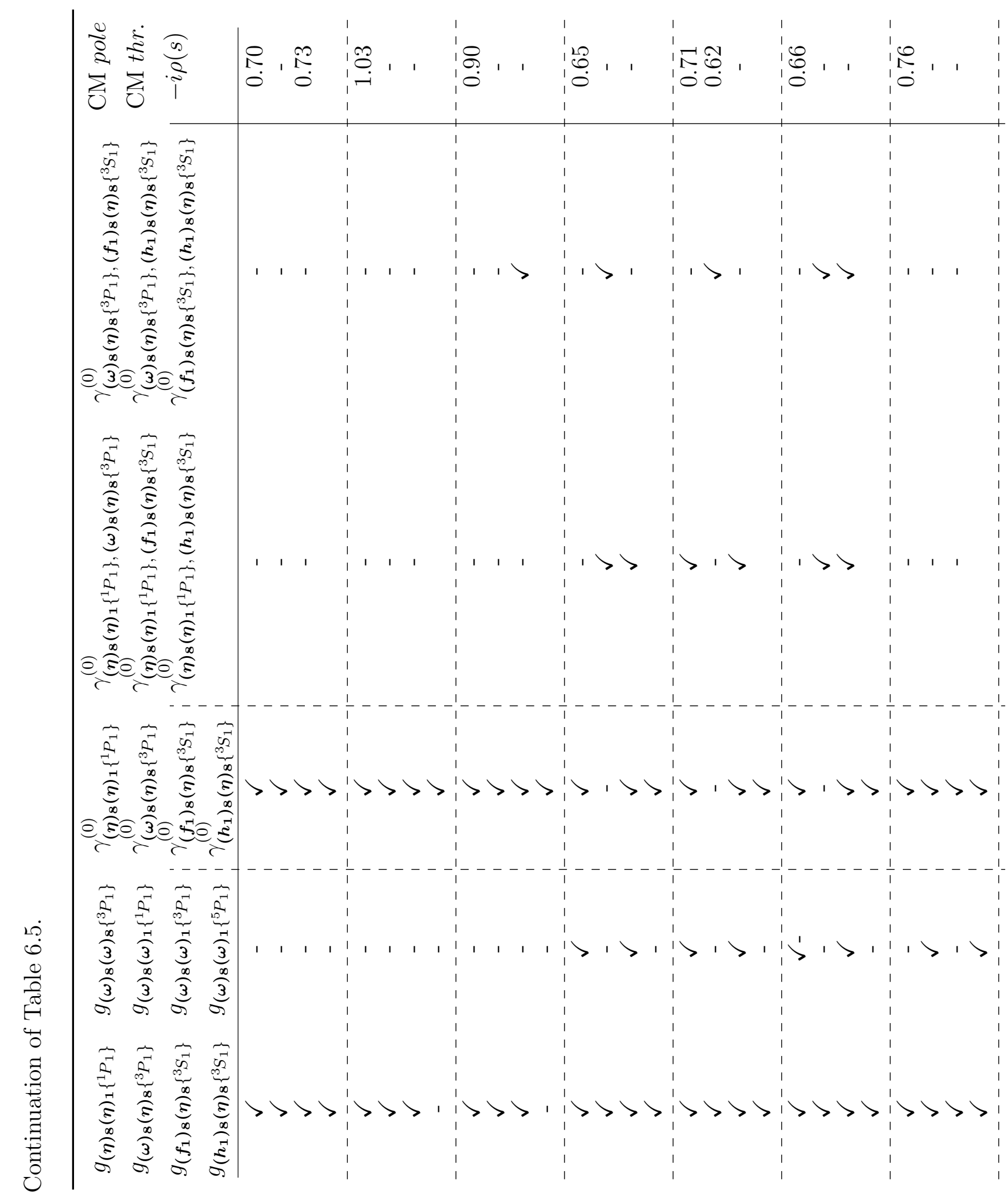




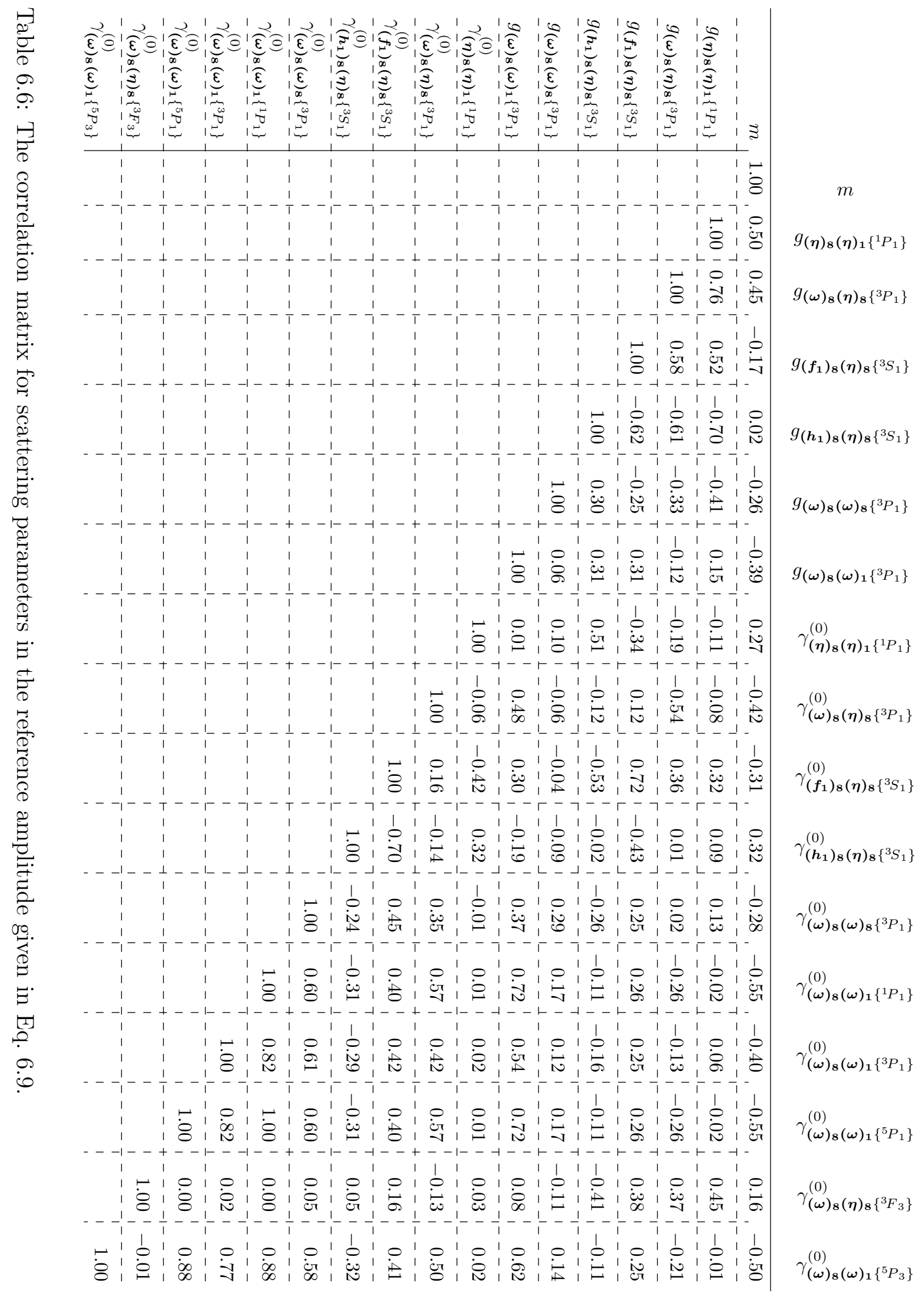




\section{Generalised Stapp-parameterisation}

There is no established method in the literature to minimally display the $S$-matrix in three or more channel scattering. An approach that is often taken involves plotting the real and imaginary parts of each of the elements, however, this contains redundancies as it does not account for the constraints provided by unitarity. Plotting the magnitudes via $\rho_{a} \rho_{b}\left|t_{a b}\right|^{2}$ has the advantage of being closely related to a differential cross-section, but discards important phase information. For the two channel case, the Stapp-parameterisation 65 is minimal with regard to unitarity and reduces to single-channel phase-shifts when the channels decouple. No generalisation to more channels, that naturally reduces to the two-channel Stapp parameterisation, appears in the literature. In this chapter, we provide such a generalisation to $n$-channels, preserving the notion of $n$ phase-shifts and $n(n-1) / 2$ mixing-angles.

We begin by defining the exponential map from the Lie Algebra $\mathrm{LU}(n)$ to the Lie Group $\mathrm{U}(n)$ as,

$$
\text { Exp: } \begin{aligned}
\mathrm{LU}(n) & \rightarrow \mathrm{U}(n) \\
X & \rightarrow \exp (i X) .
\end{aligned}
$$

With this definition, a basis for $\mathrm{LU}(n)$ is given by the set of $n^{2}, n \times n$ Hermitian matrices. A convenient choice are the sets $\left\{\Delta_{i} \mid 1 \leq i \leq n\right\},\left\{\Theta_{i j} \mid 1 \leq i<j \leq n\right\}$ and $\left\{\Psi_{i j} \mid 1 \leq i<\right.$ $j \leq n\}$ where,

$$
\begin{aligned}
\left(\Delta_{i}\right)_{a b} & =\delta_{i a} \delta_{i b} \quad(\text { no sum on } i) \\
\left(\Theta_{i j}\right)_{a b} & =\delta_{i a} \delta_{j b}+\delta_{j a} \delta_{i b}, \quad(i<j) \\
\left(\Psi_{i j}\right)_{a b} & =i \delta_{i a} \delta_{j b}-i \delta_{j a} \delta_{i b} \quad(i<j) .
\end{aligned}
$$

In order to construct a general $n \times n$ symmetric unitary matrix $S$, we exponentiate the 
subset of $n(n+1) / 2$ symmetric matrices, $\left\{\Delta_{i}, \Theta_{j k}\right\}$, and take $S=B B^{T}$ where,

$$
\begin{aligned}
B= & \exp \left(i \delta_{1} \Delta_{1}\right) \exp \left(i \delta_{2} \Delta_{2}\right) \ldots \exp \left(i \delta_{n} \Delta_{n}\right) \\
& \times \exp \left(i \bar{\epsilon}_{n-1 n} \Theta_{n-1 n}\right) \ldots \exp \left(i \bar{\epsilon}_{12} \Theta_{12}\right)
\end{aligned}
$$

Here $B^{T}$ denotes the matrix transpose of $B$ and $\left\{\delta_{i}, \bar{\epsilon}_{j k}\right\}$ are a set of $n(n+1) / 2$ real parameters.

With this choice, for two channels, $\delta_{1}, \delta_{2}$ and $\bar{\epsilon}_{12}$ are exactly the Stapp phase-shifts and mixing-angle of Ref. [65]. If instead we take $S=\tilde{B} \tilde{B}^{T}$, where,

$$
\begin{aligned}
\tilde{B}= & \exp \left(i \theta_{n-1 n} \Psi_{n-1 n}\right) \ldots \exp \left(i \theta_{12} \Psi_{12}\right) \\
& \times \exp \left(i \tilde{\delta}_{1} \Delta_{1}\right) \exp \left(i \tilde{\delta}_{2} \Delta_{2}\right) \ldots \exp \left(i \tilde{\delta}_{n} \Delta_{n}\right),
\end{aligned}
$$

we obtain a parameterisation similar to that of Blatt and Biedenharn [101] where $\tilde{\delta}$ are the eigen-phaseshifts and $\theta$ are some mixing-angles.

We use the indexing $\bar{\epsilon}_{i j}$ and $\Theta_{i j}$ to conveniently label the angle and matrix respectively that mix channels $i$ and $j$. By construction, this parameterisation gives a symmetric unitary matrix with $n(n+1) / 2$ independent free parameters and provides a natural $n$-channel extension of the two-channel Stapp parameterisation.

As an example of this construction, for two-channels, the basis defined above gives the matrices,

$$
\begin{aligned}
& \Delta_{1}=\left(\begin{array}{ll}
1 & 0 \\
0 & 0
\end{array}\right), \quad \Delta_{2}=\left(\begin{array}{ll}
0 & 0 \\
0 & 1
\end{array}\right), \\
& \Theta_{12}=\left(\begin{array}{ll}
0 & 1 \\
1 & 0
\end{array}\right), \quad \Psi_{12}=\left(\begin{array}{cc}
0 & i \\
-i & 0
\end{array}\right) \text {. }
\end{aligned}
$$

It follows that setting $n=2$ in Eq. 7.5 gives,

$$
S=\left(\begin{array}{cc}
\cos \left(2 \bar{\epsilon}_{12}\right) e^{2 i \delta_{1}} & i \sin \left(2 \bar{\epsilon}_{12}\right) e^{i\left(\delta_{1}+\delta_{2}\right)} \\
i \sin \left(2 \bar{\epsilon}_{12}\right) e^{i\left(\delta_{1}+\delta_{2}\right)} & \cos \left(2 \bar{\epsilon}_{12}\right) e^{2 i \delta_{2}}
\end{array}\right)
$$

which is precisely the Stapp-parameterisation. 
The generalised three-channel Stapp-parameterisation has 6 free real-parameters (three phase-shifts and three mixing-angles) and is obtained by taking $n=3$ in Eq. 7.5. Fixing $\bar{\epsilon}_{13}=0$ and $\bar{\epsilon}_{23}=0$ reduces to the two-channel Stapp-parameterisation in channels 1 and 2 , and leaves a single phase-shift in the channel 3. An analogous reduction applies for other appropriate combinations of mixing-angles taken to be zero. Explicitly, the elements of the $S$-matrix are,

$$
\begin{aligned}
& S_{11}=\left(\chi_{12} c_{13}^{2}-s_{13}^{2}\right) e^{2 i \delta_{1}} \\
& S_{12}=c_{13}\left(i \sigma_{12} c_{23}-s_{13} s_{23}\left(1+\chi_{12}\right)\right) e^{i\left(\delta_{1}+\delta_{2}\right)} \\
& S_{13}=c_{13}\left(i c_{23} s_{13}\left(1+\chi_{12}\right)-\sigma_{12} s_{23}\right) e^{i\left(\delta_{1}+\delta_{3}\right)} \\
& S_{22}=\left(\chi_{12} c_{23}^{2}+\chi_{12} s_{13}^{2} s_{23}^{2}-c_{13}^{2} s_{23}^{2}-2 i \sigma_{12} s_{13} s_{23} c_{23}\right) e^{2 i \delta_{2}} \\
& S_{23}=\left(\sigma_{12} s_{13}\left(s_{23}^{2}-c_{23}^{2}\right)+i c_{13}^{2} c_{23} s_{23}\left(1+\chi_{12}\right)\right) e^{i\left(\delta_{2}+\delta_{3}\right)} \\
& S_{33}=\left(c_{13}^{2} c_{23}^{2}-\chi_{12} s_{13}^{2} c_{23}^{2}-\chi_{12} s_{23}^{2}-2 i \sigma_{12} s_{13} s_{23} c_{23}\right) e^{2 i \delta_{3}}
\end{aligned}
$$

where

$$
\begin{aligned}
& \chi_{12}=\cos \left(2 \bar{\epsilon}_{12}\right), c_{13}=\cos \left(\bar{\epsilon}_{13}\right), c_{23}=\cos \left(\bar{\epsilon}_{23}\right) \\
& \sigma_{12}=\sin \left(2 \bar{\epsilon}_{12}\right), s_{13}=\sin \left(\bar{\epsilon}_{13}\right), s_{23}=\sin \left(\bar{\epsilon}_{23}\right) .
\end{aligned}
$$

These conventions mean that $\delta_{1}=\frac{1}{2} \arg \left(S_{11}\right)$, which is in agreement with Refs. 7, 62, 63, where the 'phase-shifts', $\psi_{i}$, are defined as $\psi_{i}=\frac{1}{2} \arg S_{i i}$. However, we see for $\delta_{2}$ and $\delta_{3}$ there are corrections to the phase due to the imaginary components $\propto \sigma_{12} s_{13} s_{23} c_{23}$ in the expressions for $S_{22}$ and $S_{33}$, given in Eq. 7.8. For a very weakly mixed channel these corrections are very small and $\delta_{i} \approx \psi_{i}$ for $i=2,3$.

We make a note here that determining $\left\{\delta_{i}, \bar{\epsilon}_{j k}\right\}$ directly from some $S$-matrix is much less trivial than in determining eigen-phaseshifts and mixing-angles by diagonalising $S$. The relationship between $\left\{\delta_{i}, \bar{\epsilon}_{j k}\right\}$ and $\left\{\tilde{\delta}_{i}, \theta_{j k}\right\}$ is highly non-linear and related through the BCH formula, invoked when equating $S=B B^{T}=\tilde{B} \tilde{B}^{T}$ for $B$ and $\tilde{B}$ defined in Eq. 7.5 and Eq. 7.6 respectively. The method we use to determine $\left\{\delta_{i}, \bar{\epsilon}_{j k}\right\}$ is detailed below. 


\subsection{Determination of $\left\{\delta_{i}, \bar{\epsilon}_{j k}\right\}$}

In order to determine the phase-shifts and mixing-angles as a function of $\mathrm{cm}$-frame energy, we solve $S\left(\delta_{i}, \bar{\epsilon}_{j k} ; E_{\mathrm{cm}}\right)=S^{\text {lat. }}\left(E_{\mathrm{cm}}\right)$ for $\delta_{i}\left(E_{\mathrm{cm}}\right)$ and $\bar{\epsilon}_{j k}\left(E_{\mathrm{cm}}\right)$ where $S^{\text {lat. }}\left(E_{\mathrm{cm}}\right)$ is an $S$-matrix calculated from the energy dependent scattering amplitudes - for example the reference amplitudes presented in the previous chapters. The approach we take here is to construct a differential equation at each $E_{\mathrm{cm}}$ in the space of $\delta_{i}\left(E_{\mathrm{cm}}\right)$ and $\bar{\epsilon}_{j k}\left(E_{\mathrm{cm}}\right)$ with a fixed point corresponding to the phase-shifts and mixing-angles that give $S\left(\delta_{i}, \bar{\epsilon}_{j k} ; E_{\mathrm{cm}}\right)=S^{\text {lat. }}\left(E_{\mathrm{cm}}\right)$. We implement an Euler flow to solve for this fixed point to some specified tolerance. The construction is given below.

Define $f\left(\delta_{i}, \bar{\epsilon}_{j k} ; E_{\mathrm{cm}}\right)$ to be the Frobenius norm of the difference of $S$ and $S^{\text {lat. }}$

$$
f\left(\delta_{i}, \bar{\epsilon}_{j k} ; E_{\mathrm{cm}}\right)=\left\|S\left(\delta_{i}, \bar{\epsilon}_{j k} ; E_{\mathrm{cm}}\right)-S^{\text {lat. }}\left(E_{\mathrm{cm}}\right)\right\|_{\text {fro. }}^{2}
$$

where $\left\|a_{i j}\right\|_{\text {fro. }}=\sqrt{\sum_{i j}\left|a_{i j}\right|^{2}}$ is the sum over the modulus squared of each matrix element. By construction, $f\left(\delta_{i}, \bar{\epsilon}_{j k} ; E_{\mathrm{cm}}\right)$ is a positive semi-definite function, attaining its minimum when $S\left(\delta_{i}, \bar{\epsilon}_{j k} ; E_{\mathrm{cm}}\right)=S^{\text {lat. }}\left(E_{\mathrm{cm}}\right)$. The partial derivative with respect to $\delta_{i}\left(E_{\mathrm{cm}}\right)$ and $\bar{\epsilon}_{j k}\left(E_{\mathrm{cm}}\right)$ is,

$$
\frac{\partial f}{\partial x_{a}}\left(\boldsymbol{x}\left(E_{\mathrm{cm}}\right) ; E_{\mathrm{cm}}\right)=\sum_{i j} 2 \operatorname{Re}\left(\frac{\partial S_{i j}^{*}}{\partial x_{a}}\left(\boldsymbol{x}\left(E_{\mathrm{cm}}\right) ; E_{\mathrm{cm}}\right) \times_{e}\left(S_{i j}\left(\boldsymbol{x} ; E_{\mathrm{cm}}\right)-S_{i j}^{l a t .}\left(E_{\mathrm{cm}}\right)\right)\right),
$$

where $\boldsymbol{x}\left(E_{\mathrm{cm}}\right)$ denotes the vector of phase-shifts and mixing-angles, $\left\{\delta_{i}\left(E_{\mathrm{cm}}\right), \bar{\epsilon}_{j k}\left(E_{\mathrm{cm}}\right)\right\}$, and $\times_{e}$ denotes element-wise matrix multiplication.

To obtain the fixed point, and hence the phase-shift and mixing-angles, the parameters $\boldsymbol{x}$ are updated at each iteration by,

$$
\boldsymbol{x}^{(n+1)}=\boldsymbol{x}^{(n)}-h \frac{\partial f}{\partial \boldsymbol{x}^{(n)}},
$$

where $h$ is some constant step size and the fixed point is given in the limit $n \rightarrow \infty$. In practice, we terminate when both,

$$
\begin{aligned}
& \epsilon>\left|\frac{\left\|S\left(\boldsymbol{x}^{(N+1)} ; E_{\mathrm{cm}}\right)-S^{\text {lat. }}\left(E_{\mathrm{cm}}\right)\right\|_{\text {fro. }}-\left\|S\left(\boldsymbol{x}^{(N)} ; E_{\mathrm{cm}}\right)-S^{\text {lat. }}\left(E_{\mathrm{cm}}\right)\right\|_{\text {fro. }}}{\left\|S\left(\boldsymbol{x}^{(N)} ; E_{\mathrm{cm}}\right)-S^{\text {lat. }}\left(E_{\mathrm{cm}}\right)\right\|_{\text {fro. }}}\right| \\
& \epsilon>\left\|S\left(\boldsymbol{x}^{(N)} ; E_{\mathrm{cm}}\right)-S^{\text {lat. }}\left(E_{\mathrm{cm}}\right)\right\|_{\text {fro. }},
\end{aligned}
$$


where the first condition ensures convergence of the flow and the second gives $\boldsymbol{x}^{(N)} \approx \boldsymbol{x}^{(\infty)}$ for $\epsilon$ small.

We propagate parameter errors and correlations in the scattering amplitudes onto $\boldsymbol{x}^{(N)}\left(E_{\mathrm{cm}}\right)$ by first generating an ensemble of scattering parameter values according to a multivariate Gaussian distribution defined by the parameter covariance matrix. This ensemble is propagated under jackknife to calculate an ensemble of $\boldsymbol{x}^{(N)}\left(E_{\mathrm{cm}}\right)$ and the corresponding means and uncertainties are determined.

As a final comment we point out a useful property of the above construction. The parameters $\delta_{i}\left(E_{\mathrm{cm}}\right), \bar{\epsilon}_{j k}\left(E_{\mathrm{cm}}\right)$ are continuous functions of $E_{\mathrm{cm}}$ and as such, the solution $\boldsymbol{x}^{(N)}\left(E_{\mathrm{cm}}\right)$ to Eq. 7.11 provides an excellent set of priors at energy $E_{\mathrm{cm}}+\Delta E_{\mathrm{cm}}$, provided $\Delta E_{\mathrm{cm}}$ is sufficiently small, speeding up the rate of convergence.

\subsection{Properties of $S\left(\delta_{i}, \bar{\epsilon}_{j k}\right)$}

This $S$-matrix parameterisation has a number of elegant properties. Firstly, ordering the kinematic thresholds by increasing energy, we see that below the $N^{\text {th }}$ threshold, for real $E_{\mathrm{cm}}$, the symmetric $S$-matrix has components $S_{n i}=S_{i n}=\delta_{n i}$ for $n \geq N$ and therefore $\delta_{n}\left(E_{\mathrm{cm}}\right)=\bar{\epsilon}_{i n}\left(E_{\mathrm{cm}}\right)=0$ for all $n \geq N$ and all $i$. As the $N^{\text {th }}$ threshold opens, the phase-shift $\delta_{N}\left(E_{\mathrm{cm}}\right)$ and $N-1$ mixing-angles $\bar{\epsilon}_{i N}\left(E_{\mathrm{cm}}\right)$ for $1 \leq i \leq N-1$ 'turn on'. In this sense, at each kinematic threshold, the parameterisation naturally extends to accommodate the additional channel(s) 1$]$ Furthermore, if $\bar{\epsilon}_{i N}\left(E_{\mathrm{cm}}\right)=0$ for all $1 \leq i \leq N-1$ in a region above the $N^{\text {th }}$-threshold, we see that this channel decouples and the parameterisation appears to be block diagonal with an $(N-1) \times(N-1)$-block and a single decoupled phase-shift $\delta_{N}\left(E_{\mathrm{cm}}\right)$. We observed this in the five coupled-channel $b_{1}$ study in Section 5.7 .3 where two-channels decoupled, illustrated clearly in Figure 5.17 .

\subsection{Examples}

In this section, we apply the parameterisation to the scattering amplitudes determined for the three coupled $S$-wave pseudoscalar-pseudoscalar system, featuring the $f_{0}$ resonance [7]. We will compare the phase-shifts with those reported in the aforementioned reference and

\footnotetext{
${ }^{1}$ Multiple channels if more than one partial-wave features at a single kinematic threshold.
} 
examine any differences. In each of these calculations, the phase-shifts were defined as the overall phase of the diagonal elements of the $S$-matrix, $\psi_{i}=\frac{1}{2} \arg S_{i i}$. We will also present the mixing-angles through inelasticities, $\cos 2 \bar{\epsilon}$, as there is a sign ambiguity in each of the off-diagonal $S$-matrix elements. This arises from a freedom to rephase the hadron states, subject to the $S$-matrix being symmetric, and means in this case the sign is unphysical. 'Inelasticities', $\eta_{i}$, were defined as the magnitudes of the diagonal components of the $S$-matrix, $\eta_{i}=\left|S_{i i}\right|$, in Ref. [7]. Below the third coupled-channel threshold, the single inelasticity $\cos 2 \bar{\epsilon}_{12}$ is equivalent to $\left|S_{11}\right|=\left|S_{22}\right|$ however, above this threshold, there is no meaningful comparison and as such we only plot $\cos 2 \bar{\epsilon}_{i j}$.

\subsection{1 $f_{0}$}

The $f_{0}$ features in a three coupled-channel system of $\pi \pi, K \bar{K}$ and $\eta \eta$ with vacuum quantum numbers, i.e. $\left(I^{G}\right) J^{P C}=\left(0^{+}\right) 0^{++}$. In brief, the results of Ref. [7] show a somewhat decoupled $\eta \eta$ channel and strongly coupled $\pi \pi$ and $K \bar{K}$. In Figure 7.1 we present the phase-shifts $\delta_{i}$ and diagonal phases $\psi_{i}$.

The figure shows that the phase-shifts and diagonal phases agree below the $\eta \eta$-threshold - expected since these are equivalent in this energy region. Above $\eta \eta$-threshold, small deviations between $\delta_{2,3}$ and $\psi_{2,3}$ can be seen due to the differences in the phase of the diagonal entries ${ }^{2}$ of the $S$-matrix, coming from the term $\propto i \sigma_{12} s_{13} s_{23} c_{23}$ in Eq. 7.8. As the $\eta \eta$ is found to be approximately decoupled in Ref. [7], the difference between the phase-shifts and diagonal phases is expected to be modest since the term $\propto i \sigma_{12} s_{13} s_{23} c_{23}$ will be suppressed for small mixing-angles.

Presented in Figure 7.2 are the three inelasticities. We find a large deviation away from unity in the inelasticity between $\pi \pi \mid K \bar{K}$ compared to $K \bar{K} \mid \eta \eta$ and $\pi \pi \mid \eta \eta$. The $\pi \pi \mid \eta \eta$ inelasticity is shown to be consistent with zero outside the energy range $0.21 \lesssim a_{t} E_{\mathrm{cm}} \lesssim 0.23$ and within this interval, the difference from unity is found to be modest. This observation is manifest in the behaviour of the phase-shifts and diagonal phases in Figure 7.1 . In the energy range $0.21 \lesssim a_{t} E_{\mathrm{cm}} \lesssim 0.23$, small discrepancies between $\delta_{2,3}$ and $\psi_{2,3}$ are observed however, outside this interval they are consistent, as $\sigma_{12} s_{13} s_{23} c_{23}=0$ for $\bar{\epsilon}_{13}=0$.

\footnotetext{
${ }^{2}$ Recall that $\delta_{1}$ is identical to $\psi_{1}$ by construction - see Eq. 7.8
} 


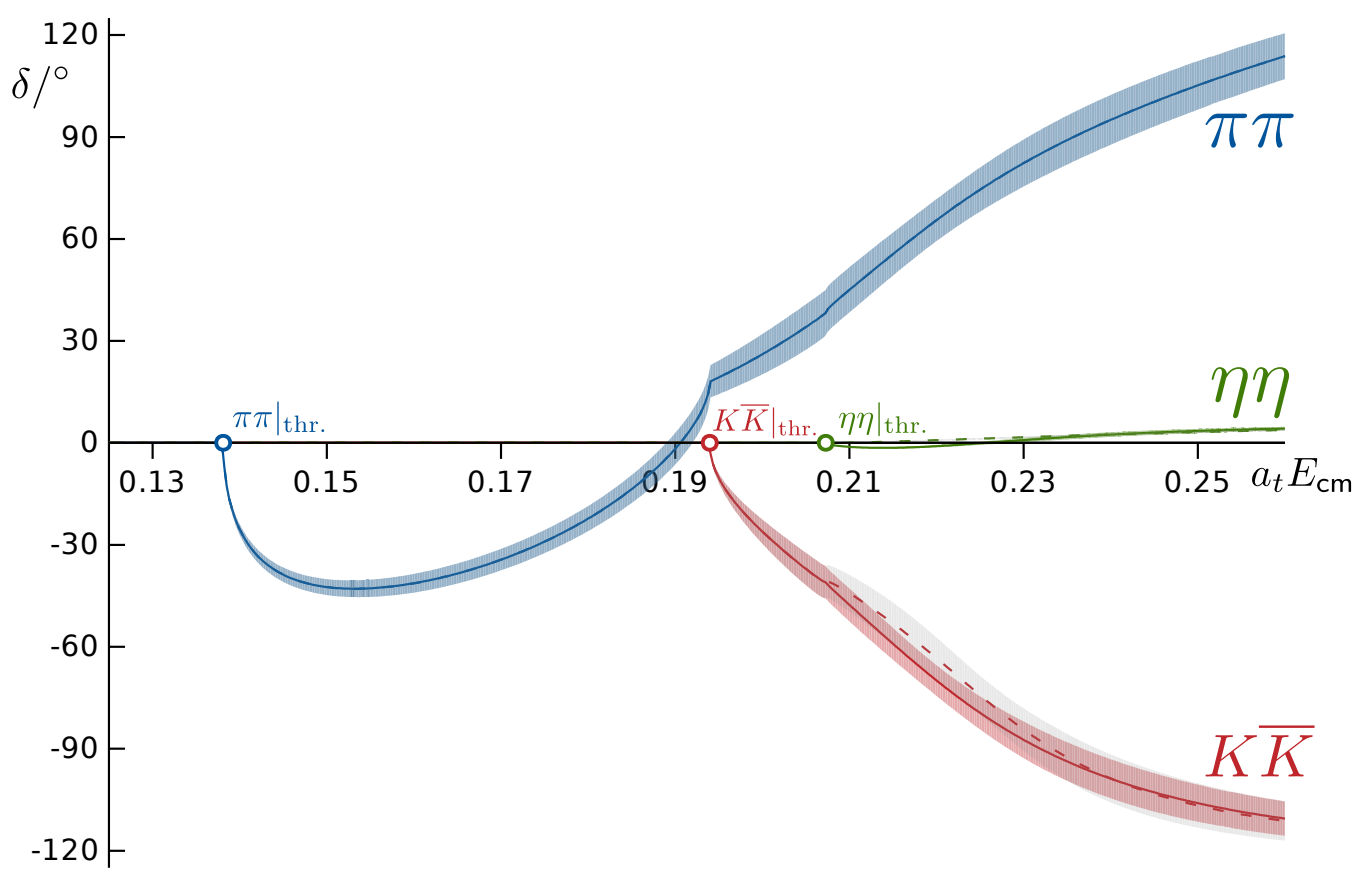

Figure 7.1: Phase-shifts, $\delta_{i}$ (solid curves), and diagonal phases, $\psi_{i}$ (dashed curves), for the reference parameterisation quoted in Ref. $|7|-\pi \pi$ (blue), $K \bar{K}$ (red), $\eta \eta$ (green). Coloured bands reflect the uncertainties $\delta_{i}$ while the grey bands give the uncertainties on $\psi_{i}$. 


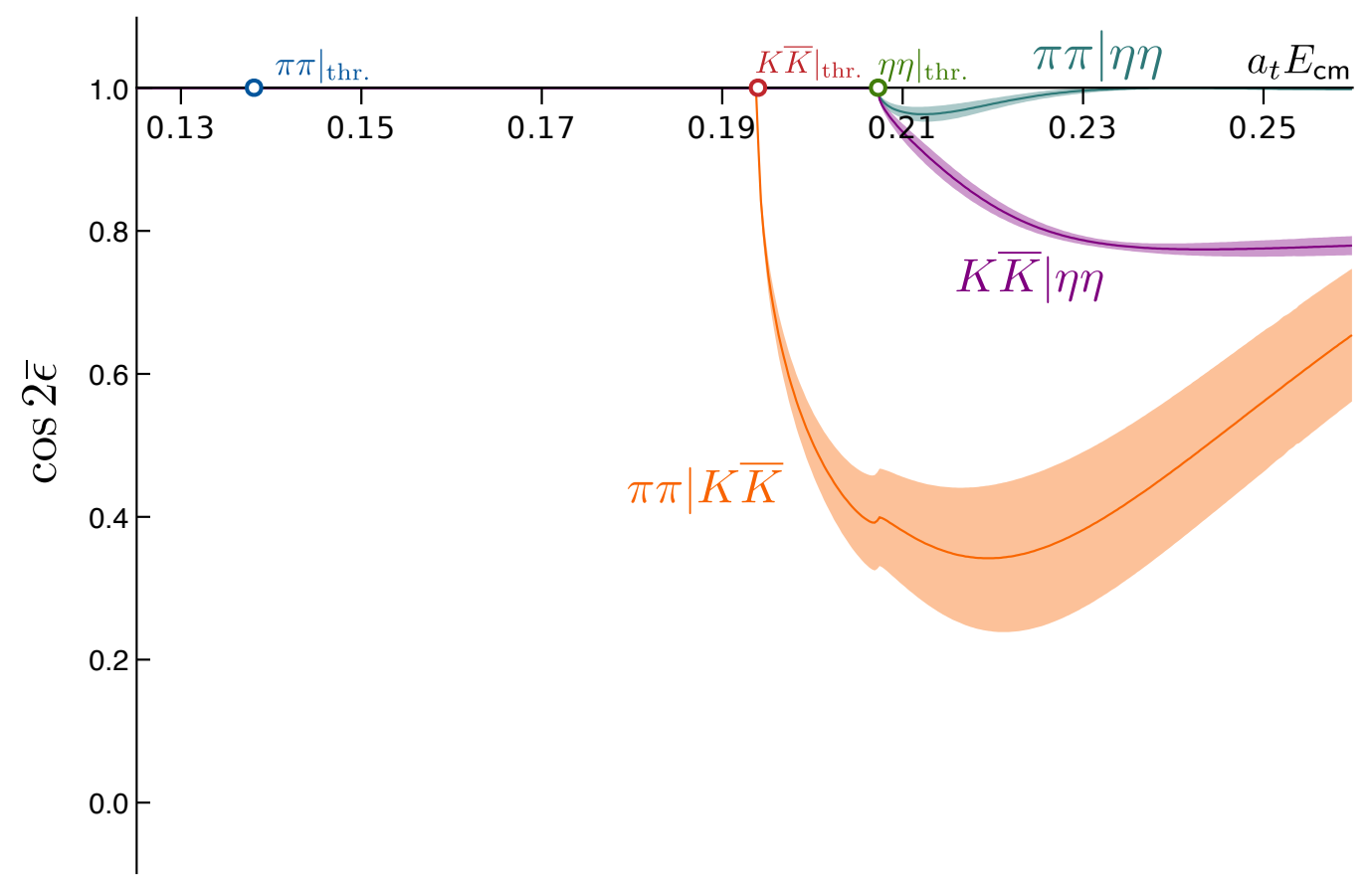

Figure 7.2: Inelasticities, $\cos 2 \bar{\epsilon}_{i j}$, for the reference parameterisation quoted in Ref. [7] $\pi \pi \mid K \bar{K}$ (orange), $\pi \pi \mid \eta \eta$ (blue), $K \bar{K} \mid \eta \eta$ (purple). Coloured bands reflect the uncertainties on the reference amplitude. 


\subsection{Summary}

The parameterisation presented in this chapter is a natural $n$-channel extension of the two-channel Stapp-parameterisation [65], minimally displaying the $n(n+1) / 2$ degrees of freedom of an $n \times n$ symmetric unitary matrix. In the case that $k$-channels decouple, the parameterisation block diagonalises, with an $(n-k) \times(n-k)$ block, governing the remaining coupled $(n-k)$-channels, and the $k$-block a diagonal matrix of phases.

We have presented a method for calculating the phase-shifts and mixing-angles and have presented the results for the three coupled-channel scattering systems featuring the $f_{0}$.

As a final comment, this parameterisation allows relative sign information between dynamically-coupled partial-waves to be captured in the mixing-angles, unlike the $\rho_{a} \rho_{b}\left|t_{a b}\right|^{2}$ magnitude squared where such information is discarded, vital for scattering calculations of hadrons with non-zero intrinsic spin. 


\section{Conclusion}

Over the course of my $\mathrm{PhD}$, my ambition was to perform scattering calculations of hadrons with non-zero intrinsic spin from first principles QCD. Here, I summarise how that goal was achieved.

In Chapter 3, I presented the first lattice calculation of $\rho \pi$ scattering in isospin-2. I showed how a non-zero intrinsic spin leads to dynamically-coupled partial-waves and gave an overview on how the finite-volume energy spectra can be used to determine the scattering amplitudes. A basis of vector-pseudoscalar operators resembling isospin- $2 \rho \pi$-like states was constructed at the $\mathrm{SU}(3)_{\mathrm{F}}$ point. Furthermore, a discussion on how multiple linearly independent vector-pseudoscalar operators can appear at a single non-interacting energy and the importance of including all such operators was discussed. Using an anisotropic lattice, large matrices of two-point correlation functions were computed within the distillation framework, and the energy spectra for $\rho \pi$ in isospin- 2 were determined from a variational analysis. I utilised the Lüscher quantisation condition to determine all $S$-, $P$ - and $D$-wave amplitudes from a global analysis of a large number of finite-volume energies. For the first time in a lattice QCD calculation, the mixing-angle, governing the dynamical mixing between the ${ }^{3} S_{1}$ and ${ }^{3} D_{1}$ partial-waves, was determined and the dependence of the finite-volume spectra on the mixing-angle was given.

In Chapter 4, I examined $\rho \pi$ in isospin-1. A comprehensive discussion of the manifestation of Bose-symmetry and $G$-parity at the $\mathrm{SU}(3)_{\mathrm{F}}$ point was presented and appropriate bases of single-meson and two-meson operators constructed. I gave a qualitative discussion of the rest-frame spectra and concluded that the $a_{1}$ axial-vector meson appeared to feature as a bound-state.

The $b_{1}$ axial-vector, with opposite charge-conjugation to the $a_{1}$, decays dominantly into the $\pi \omega$ vector-pseudoscalar channel, dynamically coupled in ${ }^{3} S_{1}$ - and ${ }^{3} D_{1}$-wave. At light-quark masses resulting in a pion mass $\sim 391 \mathrm{MeV}$, the $\omega$ meson is stable against strong decay to $\pi \pi \pi$ and a first lattice QCD calculation of coupled $\pi \omega$ and $\pi \phi$ scattering, 
incorporating dynamically-coupled ${ }^{3} S_{1^{-}}$and ${ }^{3} D_{1}$-waves in $\pi \omega$, was presented in Chapter 5 . Single-, two- and three-meson operators were constructed and finite-volume spectra were determined via a variational analysis of matrices of two-point correlation functions. Utilising the relationship between the discrete spectrum of finite-volume energies and infinite-volume scattering amplitudes, I found a narrow axial-vector resonance $\left(J^{P C}=1^{+-}\right)$, the analogue of the $b_{1}$ meson, with mass $m_{R} \approx 1380 \mathrm{MeV}$ and width $\Gamma_{R} \approx 91 \mathrm{MeV}$. The resonance was found to couple dominantly to $S$-wave $\pi \omega$, with a much-suppressed coupling to $D$-wave $\pi \omega$, and a negligible coupling to $\pi \phi$ consistent with the 'OZI rule'.

Chapter 6 was dedicated to a first lattice QCD scattering calculation of the exotic $J^{P C}=1^{-+}$sector. To circumvent the need for a three-body scattering formalism, I once again worked at a heavy pion mass $\sim 700 \mathrm{MeV}$. At this pion mass, the exotic $\pi_{1}$ was anticipated to resonate in an energy region featuring eight coupled channels: one pseudoscalar-pseudoscalar, one vector-pseudoscalar, two axial-vector-pseudoscalar and four vector-vector. Finite-volume spectra were calculated in the rest-frame so that the conservation of parity ensured potentially troublesome low-lying $a_{0^{-}}$and $a_{2}$-like resonances and contributions from three-body channels were circumvented. A coupledchannel scattering analysis was performed and a single resonance pole was robustly determined, which I interpreted as the exotic $\pi_{1}$ resonance. Correspondingly, the couplings to all coupled channels were calculated and extrapolated to the physical light-quark mass limit.

The calculations in this thesis demonstrate the capability lattice QCD has in determining the rich spectrum of hadronic resonances. The techniques developed will enable first principles calculations in channels featuring the exotic ' $X, Y, Z$ ' states in the charm and bottom sectors, the vast majority strongly decaying via hadrons with non-zero intrinsic spins. Furthermore, these techniques combined with a fully mature three-body formalism will enable rigorous first principles calculations of hadrons such as the $a_{1}$ meson, which has a dominant decay to the dynamically-coupled ${ }^{3} S_{1}$ - and ${ }^{3} D_{1}$-wave $\rho \pi$ where the unstable $\rho$ decays to $\pi \pi$. The recent progress in both the formalism and tools, required for rigorous calculations of scattering amplitudes and resonances from lattice QCD, makes for an exciting future in hadron spectroscopy. 


\section{Bibliography}

[1] SciDAC, LHPC, UKQCD Collaboration, R. G. Edwards and B. Joo, The Chroma software system for lattice QCD, Nucl. Phys. Proc. Suppl. 140 (2005) 832, hep-lat/0409003.

[2] M. A. Clark, R. Babich, K. Barros, R. C. Brower, and C. Rebbi, Solving Lattice QCD systems of equations using mixed precision solvers on GPUs, Comput. Phys. Commun. 181 (2010) 1517-1528, arXiv:0911.3191.

[3] R. Babich, M. A. Clark, and B. Joo, Parallelizing the QUDA Library for Multi-GPU Calculations in Lattice Quantum Chromodynamics, in SC 10 (Supercomputing 2010) New Orleans, Louisiana, November 13-19, 2010, 2010. arXiv:1011.0024.

[4] D. J. Wilson, R. A. Briceño, J. J. Dudek, R. G. Edwards, and C. E. Thomas, Coupled $\pi \pi, K \bar{K}$ scattering in $P$-wave and the $\rho$ resonance from lattice $Q C D$, Phys. Rev. D92 (2015), no. 9 094502, [arXiv:1507.02599].

[5] Particle Data Group Collaboration, M. T. et al., Review of particle physics, Phys. Rev. D98 (Aug, 2018) 030001.

[6] JPAC Collaboration, A. Rodas et al., Determination of the pole position of the lightest hybrid meson candidate, Phys. Rev. Lett. 122 (2019), no. 4 042002, arXiv:1810.04171.

[7] R. A. Briceño, J. J. Dudek, R. G. Edwards, and D. J. Wilson, Isoscalar $\pi \pi, K \bar{K}, \eta \eta$ scattering and the $\sigma, f_{0}, f_{2}$ mesons from $Q C D$, arXiv:1708.06667.

[8] R. C. Johnson, Angular momentum on a lattice, Phys. Lett. B114 (1982) 147.

[9] D. C. Moore and G. T. Fleming, Multiparticle States and the Hadron Spectrum on the Lattice, Phys. Rev. D74 (2006) 054504, hep-lat/0607004. 
[10] C. E. Thomas, R. G. Edwards, and J. J. Dudek, Helicity operators for mesons in flight on the lattice, Phys. Rev. D85 (2012) 014507, arXiv:1107.1930.

[11] M. Gell-Mann, A schematic model of baryons and mesons, Physics Letters 8 (1964), no. $3214-215$.

[12] G. Zweig, An SU(3) model for strong interaction symmetry and its breaking; Version 2, CERN-TH-412 (Feb, 1964) 80 p.

[13] C. Gattringer and C. B. Lang, Quantum Chromodynamics on the Lattice. Springer, 2010.

[14] K. Symanzik, Continuum limit and improved action in lattice theories: (i). principles and $\phi^{4}$ theory, Nuclear Physics B226 (1983), no. $1187-204$.

[15] B. Sheikholeslami and R. Wohlert, Improved Continuum Limit Lattice Action for QCD with Wilson Fermions, Nucl. Phys. B259 (1985) 572.

[16] P. Chen, Heavy quarks on anisotropic lattices: The Charmonium spectrum, Phys. Rev. D64 (2001) 034509, hep-lat/0006019.

[17] C. Morningstar and M. J. Peardon, Analytic smearing of SU(3) link variables in lattice QCD, Phys. Rev. D69 (2004) 054501, hep-lat/0311018.

[18] G. P. Lepage and P. B. Mackenzie, On the viability of lattice perturbation theory, Phys. Rev. D48 (1993) 2250-2264, hep-lat/9209022.

[19] G. P. Lepage, Redesigning lattice QCD, Lect. Notes Phys. 479 (1997) 1-48, hep-lat/9607076.

[20] R. G. Edwards, B. Joo, and H.-W. Lin, Tuning for Three-flavors of Anisotropic Clover Fermions with Stout-link Smearing, Phys. Rev. D78 (2008) 054501, arXiv:0803.3960.

[21] Hadron Spectrum Collaboration, H.-W. Lin et al., First results from $2+1$ dynamical quark flavors on an anisotropic lattice: Light-hadron spectroscopy and setting the strange-quark mass, Phys. Rev. D79 (2009) 034502, arXiv:0810.3588. 
[22] M. Lüscher and P. Weisz, On-shell improved lattice gauge theories, Comm. Math. Phys. 97 (1985), no. 1-2 59-77.

[23] M. Luscher and P. Weisz, Definition and General Properties of the Transfer Matrix in Continuum Limit Improved Lattice Gauge Theories, Nucl. Phys. B240 (1984) 349-361.

[24] C. Best, M. Göckeler, R. Horsley, E.-M. Ilgenfritz, H. Perlt, P. Rakow, A. Schäfer, G. Schierholz, A. Schiller, and S. Schramm, $\pi$ and $\rho$ structure functions from lattice qcd, Phys. Rev. D56 (Sep, 1997) 2743-2754.

[25] Hadron Spectrum Collaboration, M. Peardon, J. Bulava, J. Foley, C. Morningstar, J. Dudek, R. G. Edwards, B. Joo, H.-W. Lin, D. G. Richards, and K. J. Juge, A Novel quark-field creation operator construction for hadronic physics in lattice QCD, Phys. Rev. D80 (2009) 054506, arXiv:0905.2160].

[26] Hadron Spectrum Collaboration, J. J. Dudek, R. G. Edwards, P. Guo, and C. E. Thomas, Toward the excited isoscalar meson spectrum from lattice QCD, Phys. Rev. D88 (2013), no. 9 094505, arXiv:1309.2608|.

[27] D. C. Moore and G. T. Fleming, Angular momentum on the lattice: The Case of non-zero linear momentum, Phys. Rev. D73 (2006) 014504, hep-lat/0507018]. [Erratum: Phys. Rev.D74,079905(2006)].

[28] J. J. Dudek, R. G. Edwards, and C. E. Thomas, S and D-wave phase shifts in isospin-2 pi pi scattering from lattice QCD, Phys. Rev. D86 (2012) 034031, arXiv:1203.6041.

[29] J. J. Dudek, R. G. Edwards, M. J. Peardon, D. G. Richards, and C. E. Thomas, Toward the excited meson spectrum of dynamical QCD, Phys. Rev. D82 (2010) 034508, arXiv:1004.4930.

[30] Hadron Spectrum Collaboration, J. J. Dudek, R. G. Edwards, and C. E. Thomas, Energy dependence of the $\rho$ resonance in $\pi \pi$ elastic scattering from lattice QCD, Phys. Rev. D87 (2013), no. 3 034505, arXiv:1212.0830]. [Erratum: Phys. Rev.D90,no.9,099902(2014)]. 
[31] C. Michael, Adjoint Sources in Lattice Gauge Theory, Nucl. Phys. B259 (1985) 58-76.

[32] J. J. Dudek, R. G. Edwards, N. Mathur, and D. G. Richards, Charmonium excited state spectrum in lattice QCD, Phys. Rev. D77 (2008) 034501, arXiv:0707.4162.

[33] L. Maiani and M. Testa, Final state interactions from euclidean correlation functions, Physics Letters B245 (1990), no. 3585 - 590.

[34] M. Luscher, Volume Dependence of the Energy Spectrum in Massive Quantum Field Theories. 1. Stable Particle States, Commun. Math. Phys. 104 (1986) 177.

[35] M. Luscher, Volume Dependence of the Energy Spectrum in Massive Quantum Field Theories. 2. Scattering States, Commun. Math. Phys. 105 (1986) 153-188.

[36] M. Luscher, Two particle states on a torus and their relation to the scattering matrix, Nucl. Phys. B354 (1991) 531-578.

[37] K. Rummukainen and S. A. Gottlieb, Resonance scattering phase shifts on a non-rest frame lattice, Nucl. Phys. B450 (1995) 397-436, hep-lat/9503028.

[38] S. He, X. Feng, and C. Liu, Two particle states and the S-matrix elements in multi-channel scattering, JHEP 07 (2005) 011, hep-lat/0504019.

[39] N. H. Christ, C. Kim, and T. Yamazaki, Finite volume corrections to the two-particle decay of states with non-zero momentum, Phys. Rev. D72 (2005) 114506, hep-lat/0507009.

[40] C. H. Kim, C. T. Sachrajda, and S. R. Sharpe, Finite-volume effects for two-hadron states in moving frames, Nucl. Phys. B727 (2005) 218-243, hep-lat/0507006.

[41] P. Guo, J. Dudek, R. Edwards, and A. P. Szczepaniak, Coupled-channel scattering on a torus, Phys. Rev. D88 (2013), no. 1 014501, arXiv:1211.0929].

[42] M. T. Hansen and S. R. Sharpe, Multiple-channel generalization of Lellouch-Luscher formula, Phys. Rev. D86 (2012) 016007, arXiv:1204.0826]. 
[43] R. A. Briceño and Z. Davoudi, Moving multichannel systems in a finite volume with application to proton-proton fusion, Phys. Rev. D88 (2013), no. 9094507, arXiv:1204.1110.

[44] R. A. Briceño, Two-particle multichannel systems in a finite volume with arbitrary spin, Phys. Rev. D89 (2014), no. 7 074507, arXiv:1401.3312.

[45] M. Gockeler, R. Horsley, M. Lage, U. G. Meissner, P. E. L. Rakow, A. Rusetsky, G. Schierholz, and J. M. Zanotti, Scattering phases for meson and baryon resonances on general moving-frame lattices, Phys. Rev. D86 (2012) 094513, arXiv:1206.4141.

[46] FOCUS Collaboration, J. M. Link et al., Study of the $D^{0} \rightarrow \pi^{-} \pi^{+} \pi^{-} \pi^{+}$decay, Phys. Rev. D75 (2007) 052003, hep-ex/0701001].

[47] E852 Collaboration, M. Nozar et al., A Study of the reaction $\pi^{-} p \rightarrow \omega \pi^{-} p$ at 18 $\mathrm{GeV} / \mathrm{c}$ : The $D$ and $S$ decay amplitudes for $b_{1}(1235) \rightarrow \omega \pi$, Phys. Lett. B541 (2002) 35-44, hep-ex/0206026.

[48] R. A. Briceno, J. J. Dudek, and R. D. Young, Scattering processes and resonances from lattice QCD, Rev. Mod. Phys. 90 (2018), no. 2 025001, arXiv:1706.06223.

[49] C. B. Lang, L. Leskovec, D. Mohler, and S. Prelovsek, Axial resonances $a_{1}(1260)$, $b_{1}(1235)$ and their decays from the lattice, JHEP 04 (2014) 162, arXiv:1401.2088.

[50] R. A. Briceño, Z. Davoudi, T. Luu, and M. J. Savage, Two-nucleon systems in a finite volume. II. ${ }^{3} S_{1}-{ }^{3} D_{1}$ coupled channels and the deuteron, Phys. Rev. D88 (2013), no. 11 114507, arXiv:1309.3556.

[51] K. Orginos, A. Parreno, M. J. Savage, S. R. Beane, E. Chang, and W. Detmold, Two nucleon systems at $m_{\pi} \sim 450 \mathrm{MeV}$ from lattice QCD, Phys. Rev. D92 (2015), no. 11 114512, arXiv:1508.07583.

[52] R. A. Briceño and Z. Davoudi, Three-particle scattering amplitudes from a finite volume formalism, Phys. Rev. D87 (2013), no. 9 094507, arXiv:1212.3398.

[53] M. T. Hansen and S. R. Sharpe, Relativistic, model-independent, three-particle quantization condition, Phys. Rev. D90 (2014), no. 11 116003, arXiv:1408.5933. 
[54] M. T. Hansen and S. R. Sharpe, Expressing the three-particle finite-volume spectrum in terms of the three-to-three scattering amplitude, Phys. Rev. D92 (2015), no. 11 114509, arXiv:1504.04248].

[55] K. Polejaeva and A. Rusetsky, Three particles in a finite volume, Eur. Phys. J. A48 (2012) 67, arXiv:1203.1241.

[56] R. A. Briceño, M. T. Hansen, and S. R. Sharpe, Relating the finite-volume spectrum and the two-and-three-particle $S$ matrix for relativistic systems of identical scalar particles, Phys. Rev. D95 (2017), no. 7 074510, arXiv:1701.07465].

[57] H. W. Hammer, J. Y. Pang, and A. Rusetsky, Three particle quantization condition in a finite volume: 2. general formalism and the analysis of data, JHEP 10 (2017) 115, arXiv:1707.02176.

[58] M. Mai and M. Döring, Three-body Unitarity in the Finite Volume, Eur. Phys. J. A53 (2017), no. 12 240, arXiv:1709.08222.

[59] M. Döring, H. W. Hammer, M. Mai, J. Y. Pang, A. Rusetsky, and J. Wu, Three-body spectrum in a finite volume: the role of cubic symmetry, Phys. Rev. D97 (2018), no. 11 114508, arXiv:1802.03362.

[60] M. T. Hansen and S. R. Sharpe, Lattice QCD and Three-particle Decays of Resonances, arXiv:1901.00483.

[61] T. Barnes, N. Black, and E. S. Swanson, Meson meson scattering in the quark model: Spin dependence and exotic channels, Phys. Rev. C63 (2001) 025204, nucl-th/0007025.

[62] Hadron Spectrum Collaboration, J. J. Dudek, R. G. Edwards, and D. J. Wilson, An $a_{0}$ resonance in strongly coupled $\pi \eta, K \bar{K}$ scattering from lattice $Q C D$, Phys. Rev. D93 (2016), no. 9 094506, arXiv:1602.05122.

[63] G. Moir, M. Peardon, S. M. Ryan, C. E. Thomas, and D. J. Wilson, Coupled-Channel D $\pi, D \eta$ and $D_{s} \bar{K}$ Scattering from Lattice QCD, JHEP 10 (2016) 011, $\operatorname{arXiv:1607.07093.}$. 
[64] D. J. Wilson, J. J. Dudek, R. G. Edwards, and C. E. Thomas, Resonances in coupled $\pi K, \eta K$ scattering from lattice QCD, Phys. Rev. D91 (2015), no. 5 054008, arXiv:1411.2004.

[65] H. P. Stapp, T. J. Ypsilantis, and N. Metropolis, Phase shift analysis of 310-MeV proton proton scattering experiments, Phys. Rev. 105 (1957) 302-310.

[66] Hadron Spectrum Collaboration, J. J. Dudek, R. G. Edwards, C. E. Thomas, and D. J. Wilson, Resonances in coupled $\pi K-\eta K$ scattering from quantum chromodynamics, Phys. Rev. Lett. 113 (2014), no. 18 182001, arXiv:1406.4158.

[67] J. J. de Swart, The Octet model and its Clebsch-Gordan coefficients, Rev. Mod. Phys. 35 (1963) 916-939. [Erratum: Rev. Mod. Phys.37,326(1965)].

[68] Hadron Spectrum Collaboration, G. K. C. Cheung, C. E. Thomas, J. J. Dudek, and R. G. Edwards, Tetraquark operators in lattice $Q C D$ and exotic flavour states in the charm sector, JHEP 11 (2017) 033, arXiv:1709.01417.

[69] Hadron Spectrum Collaboration, R. G. Edwards, N. Mathur, D. G. Richards, and S. J. Wallace, Flavor structure of the excited baryon spectra from lattice QCD, Phys. Rev. D87 (2013), no. 5 054506, arXiv:1212.5236.

[70] C. J. Shultz, J. J. Dudek, and R. G. Edwards, Excited meson radiative transitions from lattice QCD using variationally optimized operators, Phys. Rev. D91 (2015), no. 11 114501, arXiv:1501.07457.

[71] C. W. Andersen, J. Bulava, B. Hörz, and C. Morningstar, Elastic I = 3/2 p-wave nucleon-pion scattering amplitude and the $\Delta$ (1232) resonance from $N_{f}=2+1$ lattice QCD, Phys. Rev. D97 (2018), no. 1 014506, arXiv:1710.01557.

[72] G. F. Chew and S. Mandelstam, Theory of low-energy pion pion interactions, Phys. Rev. 119 (1960) 467-477.

[73] CLEO Collaboration, D. M. Asner et al., Hadronic structure in the decay $\tau^{-} \rightarrow \nu_{\tau} \pi^{-} \pi^{0} \pi^{0}$ and the sign of the $\nu_{\tau}$ helicity, Phys. Rev. D61 (2000) 012002, hep-ex/9902022. 
[74] S. Prelovsek, C. B. Lang, L. Leskovec, and D. Mohler, Study of the $Z_{c}^{+}$channel using lattice QCD, Phys. Rev. D91 (2015), no. 1 014504, arXiv:1405.7623.

[75] J. L. Miramontes, Hermitian analyticity versus real analyticity in two-dimensional factorized S matrix theories, Phys. Lett. B455 (1999) 231-238, hep-th/9901145.

[76] Chung, Suh Urk, On SU(3) Representations of $q \bar{q}+q \bar{q}$ mesons http: //suchung. web. cern. ch/suchung/, .

[77] J. J. Dudek, R. G. Edwards, B. Joo, M. J. Peardon, D. G. Richards, and C. E. Thomas, Isoscalar meson spectroscopy from lattice QCD, Phys. Rev. D83 (2011) 111502, arXiv:1102.4299.

[78] UKQCD Collaboration, C. McNeile and C. Michael, Decay width of light quark hybrid meson from the lattice, Phys. Rev. D73 (2006) 074506, hep-lat/0603007.

[79] BESIII Collaboration, M. Ablikim et al., Search for a strangeonium-like structure $Z_{s}$ decaying into $\phi \pi$ and a measurement of the cross section $e^{+} e^{-} \rightarrow \phi \pi \pi$, Phys. Rev. D99 (2019), no. 1 011101, arXiv:1801.10384.

[80] Belle Collaboration, Z. Q. Liu et al., Study of $e^{+} e^{-} \rightarrow \pi^{+} \pi^{-} J \psi$ and Observation of a Charged Charmonium like State at Belle, Phys. Rev. Lett. 110 (2013) 252002, arXiv:1304.0121.

[81] BESIII Collaboration, M. Ablikim et al., Observation of a Charged Charmonium like Structure in $e^{+} e^{-} \rightarrow \pi^{+} \pi^{-} J \psi$ at $\sqrt{s}=4.26$ GeV, Phys. Rev. Lett. 110 (2013) 252001, arXiv: 1303.5949.

[82] M. Padmanath, C. B. Lang, and S. Prelovsek, X(3872) and Y(4140) using diquark-antidiquark operators with lattice QCD, Phys. Rev. D92 (2015), no. 3 034501, arXiv:1503.03257.

[83] D. J. Wilson, R. A. Briceno, J. J. Dudek, R. G. Edwards, and C. E. Thomas, The quark-mass dependence of elastic $\pi K$ scattering from $Q C D$, arXiv:1904.03188.

[84] A. Woss, C. E. Thomas, J. J. Dudek, R. G. Edwards, and D. J. Wilson, Dynamically-coupled partial-waves in $\rho \pi$ isospin-2 scattering from lattice $Q C D$, JHEP 07 (2018) 043, arXiv: 1802.05580]. 
[85] R. G. Edwards, J. J. Dudek, D. G. Richards, and S. J. Wallace, Excited state baryon spectroscopy from lattice QCD, Phys. Rev. D84 (2011) 074508, arXiv:1104.5152].

[86] C. Andersen, J. Bulava, B. Hörz, and C. Morningstar, The $I=1$ pion-pion scattering amplitude and timelike pion form factor from $N_{\mathrm{f}}=2+1$ lattice $Q C D$, Nucl. Phys. B939 (2019) 145-173, arXiv:1808.05007].

[87] L. Leskovec, C. Alexandrou, S. Meinel, J. W. Negele, S. Paul, M. Petschlies, A. Pochinsky, G. Rendon, and S. Syritsyn, A Lattice QCD study of the $\rho$ resonance, in 13th Conference on the Intersections of Particle and Nuclear Physics (CIPANP 2018) Palm Springs, California, USA, May 29-June 3, 2018, 2018. arXiv:1810.01927.

[88] C. Alexandrou, L. Leskovec, S. Meinel, J. Negele, S. Paul, M. Petschlies, A. Pochinsky, G. Rendon, and S. Syritsyn, P-wave $\pi \pi$ scattering and the $\rho$ resonance from lattice QCD, Phys. Rev. D96 (2017), no. 3 034525, arXiv: 1704.05439].

[89] RQCD Collaboration, G. S. Bali, S. Collins, A. Cox, G. Donald, M. Göckeler, C. B. Lang, and A. Schäfer, $\rho$ and $K^{*}$ resonances on the lattice at nearly physical quark masses and $N_{f}=2$, Phys. Rev. D93 (2016), no. 5 054509, arXiv:1512.08678].

[90] CS Collaboration, S. Aoki et al., $\rho$ Meson Decay in 2+1 Flavor Lattice QCD, Phys. Rev. D84 (2011) 094505, arXiv:1106.5365.

[91] X. Feng, K. Jansen, and D. B. Renner, Resonance Parameters of the rho-Meson from Lattice QCD, Phys. Rev. D83 (2011) 094505, arXiv:1011.5288.

[92] CP-PACS Collaboration, S. Aoki et al., Lattice QCD Calculation of the rho Meson Decay Width, Phys. Rev. D76 (2007) 094506, [arXiv:0708.3705.

[93] J. Bulava, B. Fahy, B. Hörz, K. J. Juge, C. Morningstar, and C. H. Wong, $I=1$ and $I=2 \pi-\pi$ scattering phase shifts from $N_{\mathrm{f}}=2+1$ lattice QCD, Nucl. Phys. B910 (2016) 842-867, arXiv: 1604.05593.

[94] D. Guo, A. Alexandru, R. Molina, and M. Döring, Rho resonance parameters from lattice QCD, Phys. Rev. D94 (2016), no. 3 034501, arXiv:1605.03993. 
[95] Z. Fu and L. Wang, Studying the $\rho$ resonance parameters with staggered fermions, Phys. Rev. D94 (2016), no. 3 034505, arXiv:1608.07478|.

[96] S. Prelovsek, L. Leskovec, C. B. Lang, and D. Mohler, $K \pi$ Scattering and the $K^{*}$ Decay width from Lattice QCD, Phys. Rev. D88 (2013), no. 5 054508, arXiv:1307.0736.

[97] R. Brett, J. Bulava, J. Fallica, A. Hanlon, B. Hörz, and C. Morningstar, Determination of $s$ - and p-wave $I=1 / 2 K \pi$ scattering amplitudes in $N_{\mathrm{f}}=2+1$ lattice QCD, Nucl. Phys. B932 (2018) 29-51, arXiv:1802.03100.

[98] G. Rendon, L. Leskovec, S. Meinel, J. Negele, S. Paul, M. Petschlies, A. Pochinsky, G. Silvi, and S. Syritsyn, $K \pi$ scattering and the $K^{*}(892)$ resonance in $2+1$ flavor QCD, in 36th International Symposium on Lattice Field Theory (Lattice 2018) East Lansing, MI, United States, July 22-28, 2018, 2018. arXiv:1811.10750.

[99] C. A. Meyer and E. S. Swanson, Hybrid Mesons, Prog. Part. Nucl. Phys. 82 (2015) 21-58, arXiv:1502.07276].

[100] COMPASS Collaboration, C. Adolph et al., Odd and even partial waves of $\eta \pi^{-}$ and $\eta^{\prime} \pi^{-}$in $\pi^{-} p \rightarrow \eta^{(\prime)} \pi^{-} p$ at 191 GeV/c, Phys. Lett. B740 (2015) 303-311, arXiv:1408.4286.

[101] J. M. Blatt and L. C. Biedenharn, The Angular Distribution of Scattering and Reaction Cross Sections, Rev. Mod. Phys. 24 (1952) 258-272. 